PCP-2011-0001

Revision 0

Keywords: Packaging

SGQ

Retention: Permanent

\title{
Packaging Certification Program Methodology for Determining Dose Rates for Small Gram Quantities in Shipping Packagings
}

\author{
S. J. Nathan, J. M. Risner*, S. Sitaraman** \\ Savannah River Nuclear Solutions, LLC \\ Aiken, SC 29808 \\ * Oak Ridge National Laboratory \\ Oak Ridge, TN 37831 \\ **Lawrence Livermore National Laboratory \\ Livermore, CA 94550
}

August 2011

Prepared for

U.S. Department of Energy

under contract number DE-AC09-08SR22470.

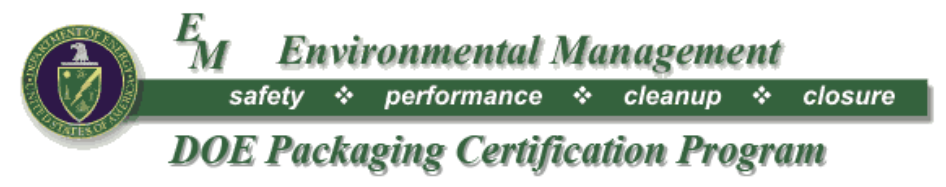


PCP-2011-0001

Revision 0

\title{
DISCLAIMER
}

This work was prepared under an agreement with and funded by the U.S. Government. Neither the U.S. Government or its employees, nor any of its contractors, subcontractors or their employees, makes any express or implied:

1. warranty or assumes any legal liability for the accuracy, completeness, or for the use or results of such use of any information, product, or process disclosed; or

2. representation that such use or results of such use would not infringe privately owned rights; or

3. endorsement or recommendation of any specifically identified commercial product, process, or service.

Any views and opinions of authors expressed in this work do not necessarily state or reflect those of the United States Government, or its contractors, or subcontractors.

\section{Printed in the United States of America}

\author{
Prepared for \\ U.S. Department of Energy
}


PCP-2011-0001

Revision 0

\section{REVIEWS AND APPROVALS}

AUTHORS:

S. J. Nathan, Savannah River Nuclear Solutions

Date

J. M. Risner, Oak Ridge National Laboratory

Date

S. Sitaraman, Lawrence Livermore National Laboratory

Date

TECHNICAL REVIEW:

A. H. Bridges, Savannah River Nuclear Solutions

Date

APPROVAL:

J. L. England, Packaging Technology \& Pressurized Systems

Date

Program Manager, DOE Packaging Certification Program

Dr. J. Shuler, U. S. Department of Energy, EM-45

Date

Manager, DOE Packaging Certification Program 
PCP-2011-0001

Revision 0

This page intentionally left blank 
PCP-2011-0001

Revision 0

\section{EXECUTIVE SUMMARY}

The Small Gram Quantity (SGQ) concept is based on the understanding that small amounts of hazardous materials, in this case radioactive materials, are significantly less hazardous than large amounts of the same materials. This study describes a methodology designed to estimate an SGQ for several neutron and gamma emitting isotopes that can be shipped in a package compliant with 10 CFR Part 71 external radiation level limits regulations. These regulations require packaging for the shipment of radioactive materials perform, under both normal and accident conditions, the essential functions of material containment, subcriticality, and maintain external radiation levels within regulatory limits. 10 CFR $71.33(b)(1)(2) \&(3)$ state radioactive and fissile materials must be identified and their maximum quantity, chemical and physical forms be included in an application. Furthermore, the U.S. Federal Regulations require application contain an evaluation demonstrating the package (i.e., the packaging and its contents) satisfies the external radiation standards for all packages (10 CFR 71.31(2), 71.35(a), \& 71.47). By placing the contents in a He leak-tight containment vessel, and limiting the mass to ensure subcriticality, the first two essential functions are readily met. Some isotopes emit sufficiently strong photon radiation that small amounts of material can yield a large external dose rate. Quantifying of the dose rate for a proposed content is a challenging issue for the SGQ approach. It is essential to quantify external radiation levels from several common gamma and neutron sources that can be safely placed in a specific packaging, to ensure compliance with federal regulations.

The Packaging Certification Program (PCP) Methodology for Determining Dose Rate for Small Gram Quantities in Shipping Packagings described in this report provides bounding mass limits for a set of proposed SGQ isotopes. Methodology calculations were performed to estimate external radiation levels for the 9977 shipping package using the MCNP radiation transport code to develop a set of response multipliers (Green's functions) for "dose per particle" for each neutron and photon spectral group. The source spectrum for each isotope generated using the ORIGEN-S and RASTA computer codes was folded with the response multipliers to generate the dose rate per gram of each isotope in the 9977 shipping package and its associated shielded containers.

The maximum amount of a single isotope that could be shipped within the regulatory limits contained in 10 CFR 71.47 for dose rate at the surface of the package is determined. If a package contains a mixture of isotopes, the acceptability for shipment can be determined by a sum of fractions approach. Furthermore, the results of this analysis can be easily extended to additional radioisotopes by simply evaluating the neutron and/or photon spectra of those isotopes and folding the spectral data with the Green's functions provided. 
PCP-2011-0001

Revision 0

\section{TABLE OF CONTENTS}

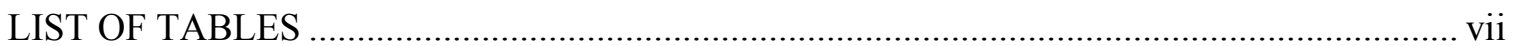

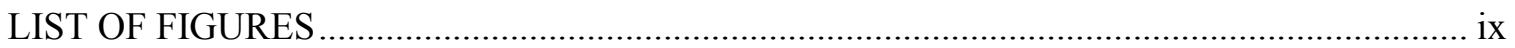

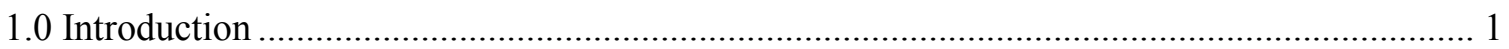

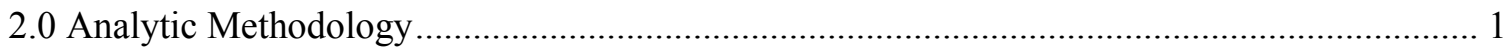

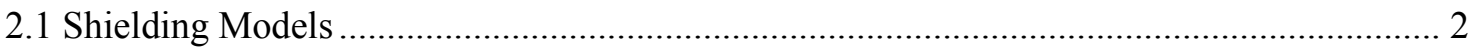

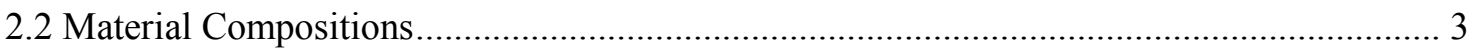

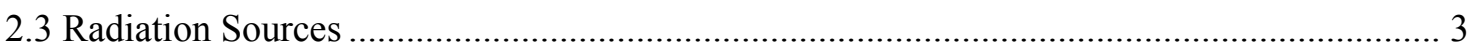

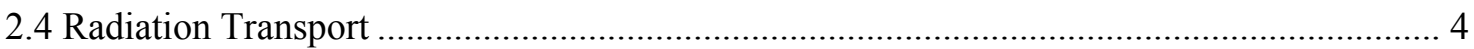

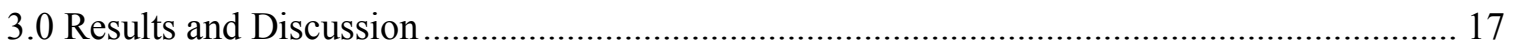

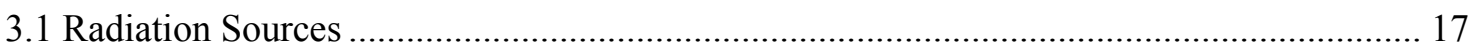

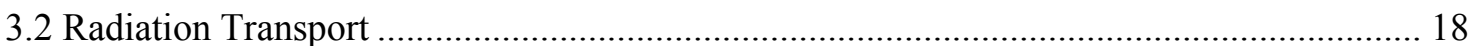

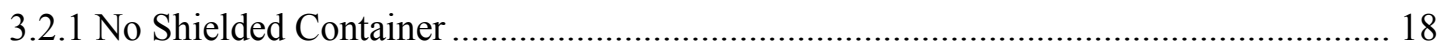

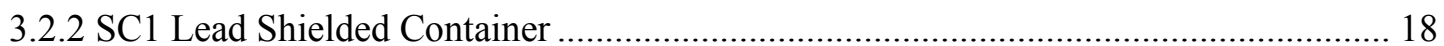

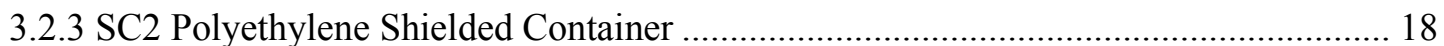

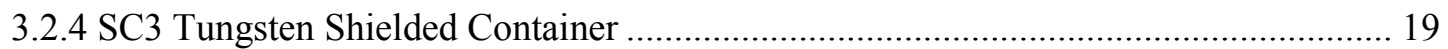

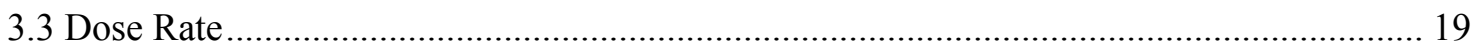

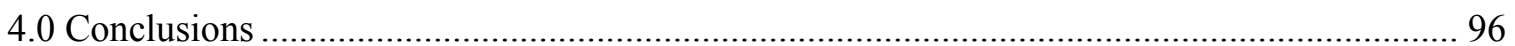

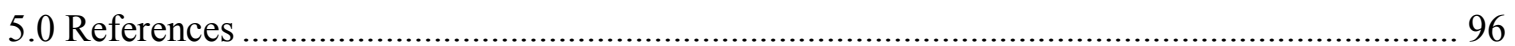

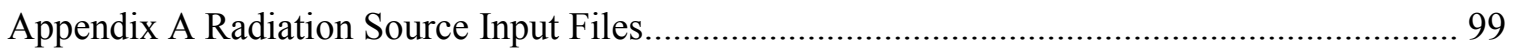

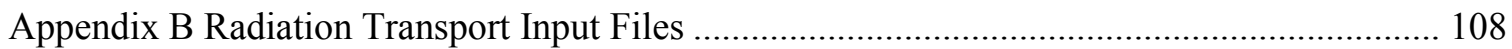

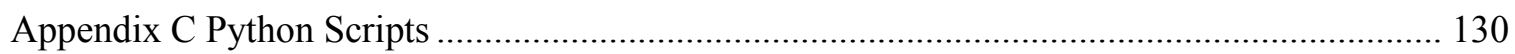


PCP-2011-0001

Revision 0

\section{LIST OF TABLES}

Table 2-1. Material Compositions 13

Table 2-2. Typical Sealed Source Isotopes 13

Table 2-3. Neutron Spectra 47 Group Structure...... 14

Table 2-4. Photon Spectra 77 Group Structure..... 15

Table 2-5. Flux-to-Dose-Rate Conversion Factors. 16

Table 3-1. Neutron Source from ORIGEN-S w/o Beryllium (Decay Time $=0$ days) …............. 25

Table 3-2. Neutron Source from ORIGEN-S w/o Beryllium (Decay Time $=30$ days) ............... 26

Table 3-3. Neutron Source from ORIGEN-S w/o Beryllium (Decay Time $=100$ days) ............. 27

Table 3-4. Neutron Source from ORIGEN-S w/o Beryllium (Decay Time $=30,000$ days) ........ 28

Table 3-5. Neutron Source from ORIGEN-S w/90\% Beryllium (Decay Time $=0$ days)............ 29

Table 3-6. Neutron Source from ORIGEN-S w/90\% Beryllium (Decay Time $=30$ days) .......... 30

Table 3-7. Neutron Source from ORIGEN-S w/90\% Beryllium (Decay Time $=100$ days) $\ldots . . . . .31$

Table 3-8. Neutron Source from ORIGEN-S w/90\% Beryllium (Decay Time = 30,000 days) .. 32

Table 3-9. Neutron Source from RASTA w/o Beryllium (Decay Time $=0$ days)...................... 33

Table 3-10. Neutron Source from RASTA w/90\% Beryllium (Decay Time $=0$ days) ............... 34

Table 3-11. Total Neutron Source (Decay Time $=0$ days) ....................................................... 35

Table 3-12. Neutron Source as a Function of Decay Time ....................................................... 35

Table 3-13. Photon Source from ORIGEN-S (Decay Time = 0 days) ….................................... 36

Table 3-14. Photon Source from ORIGEN-S (Decay Time $=0$ days) ........................................ 38

Table 3-15. Photon Source from ORIGEN-S (Decay Time $=0$ days) ......................................... 40

Table 3-16. Photon Source from ORIGEN-S (Decay Time = 0 days) ......................................... 42

Table 3-17. Photon Source from ORIGEN-S (Decay Time $=0$ days) ......................................... 44

Table 3-18. Photon Source from RASTA (Decay Time $=0$ days) ............................................... 46

Table 3-19. Photon Source from RASTA (Decay Time $=0$ days) ............................................... 48

Table 3-20. Photon Source from RASTA (Decay Time $=0$ days) .............................................. 50

Table 3-21. Photon Source from RASTA (Decay Time $=0$ days)............................................. 52

Table 3-22. Photon Source from RASTA (Decay Time $=0$ days) ............................................... 54

Table 3-23. Photon Source from ORIGEN-S (Decay Time = 30 days) …..................................56

Table 3-24. Photon Source from ORIGEN-S (Decay Time = 100 days) …............................... 58

Table 3-25. Photon Source from ORIGEN-S (Decay Time = 30,000 days) ............................... 60

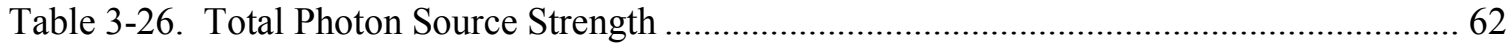

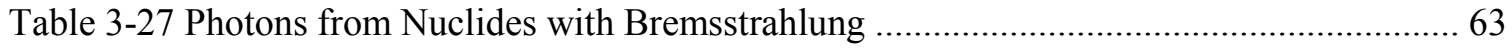


PCP-2011-0001

Revision 0

Table 3-28. Neutron Dose Rate per Unit Neutron Source in Each of the 47 Source Groups - No Shielded Container - No Self-shielding

Table 3-29. Secondary Photon Dose Rate per Unit Neutron Source in Each of the 47 Source Groups - No Shielded Container - No Self-shielding.

Table 3-30. Photon Dose Rate per Unit Source in Each of the 77 Source Groups - No Shielded Container - No Self-shielding

Table 3-31. Neutron Dose Rate per Unit Neutron Source in Each of the 47 Source Groups - No Shielded Container - $\mathrm{PuO}_{2}$ Self-shielding. 71

Table 3-32. Secondary Photon Dose Rate per Unit Neutron Source in Each of the 47 Source Groups - No Shielded Container - $\mathrm{PuO}_{2}$ Self-shielding .....

Table 3-33. Photon Dose Rate per Unit Source in Each of the 77 Source Groups - No Shielded Container $-\mathrm{PuO}_{2}$ Self-shielding .

Table 3-34. Photon Dose Rate per Unit Source in Each of the 77 Source Groups - SC1 Lead Shielded Container.

Table 3-35. Neutron Dose Rate per Unit Neutron Source in Each of the 47 Source Groups - SC2 Polyethylene Shielded Container 77

Table 3-36. Secondary Photon Dose Rate per Unit Neutron Source in Each of the 47 Source Groups - SC2 Polyethylene Shielded Container..... .78

Table 3-37. Photon Dose Rate per Unit Source in Each of the 77 Source Groups - SC2 Polyethylene Shielded Container .....

Table 3-38. Photon Dose Rate per Unit Source in Each of the 77 Source Groups - SC3 Tungsten Shielded Container. 81

Table 3-39. Actinide Total Dose Rate - No Shielded Container 83

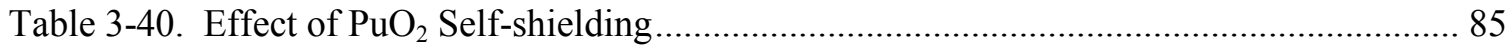

Table 3-41. Actinide Total Dose Rate - SC2 Polyethylene Shielded Container.......................... 86

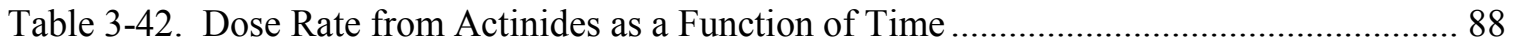

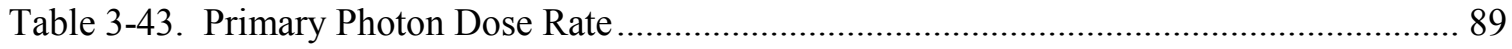

Table 3-44. Effect of Self-shielding by Iron on Primary Photon Dose Rate ................................ 90

Table 3-45. Maximum Dose Rate without Impurities ................................................................... 91

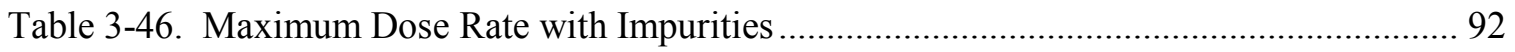

Table 3-47. Allowed Mass for Shipment - NCT without Impurities ........................................... 93

Table 3-48. Allowed Mass for Shipment - Exclusive Use without Impurities ............................ 94

Table 3-49. Allowed Actinide Mass for Shipment - NCT with Impurities ................................ 95

Table 3-50. Allowed Actinide Mass for Shipment - Exclusive Use with Impurities ................. 95 
PCP-2011-0001

Revision 0

\section{LIST OF FIGURES}

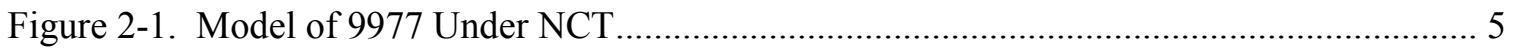

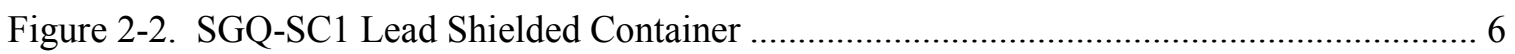

Figure 2-3. SGQ-SC2 HDPE Shielded Container ..................................................................... 7

Figure 2-4. SGQ-SC3 Tungsten Shielded Container................................................................ 8

Figure 2-5. 9977 NCT Neutron Source without Shielded Container ........................................... 9

Figure 2-6. 9977 NCT Photon Source in SC1 Lead Container ..................................................... 10

Figure 2-7. 9977 NCT Neutron Source in SC2 Polyethylene Container...................................... 11

Figure 2-8. 9977 NCT Photon Source in SC3 Tungsten Container ............................................. 12

Figure 3-1. Am-243 Fission Neutron Spectrum ........................................................................ 21

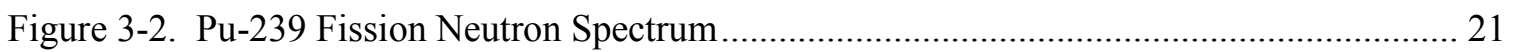

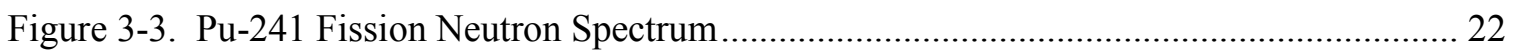

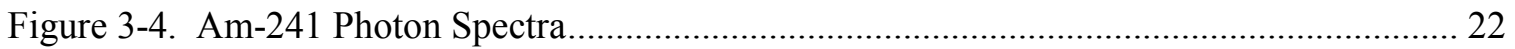

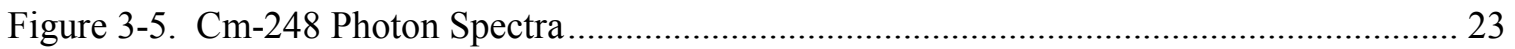

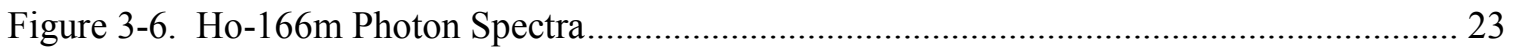

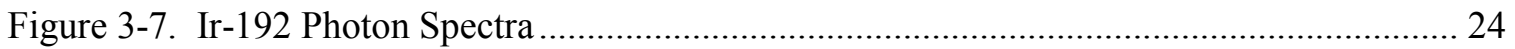

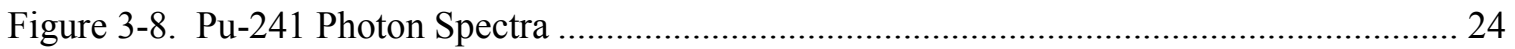


PCP-2011-0001

Revision 0

\section{LIST OF ABBREVIATIONS}

\begin{tabular}{|c|c|}
\hline $6 \mathrm{CV}$ & six-inch containment vessel \\
\hline ANL & Argonne National Laboratory \\
\hline ANS & American Nuclear Society \\
\hline BUGLE & 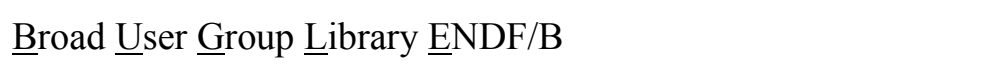 \\
\hline $\mathrm{CV}$ & Containment Vessel \\
\hline HDPE & High Density Polyethylene \\
\hline LLNL & Lawrence Livermore National Laboratory \\
\hline MCNP & Monte $\underline{\text { Carlo }} \underline{\mathrm{N}}-\underline{\text { Particle }}$ \\
\hline MCTAL & ASCII tally results file \\
\hline $\mathrm{NCT}$ & Normal Conditions of Transport \\
\hline ORIGEN & Oak Ridge Isotope GENeration Code \\
\hline ORIGEN-S & ORIGEN for SCALE \\
\hline ORNL & Oak Ridge National Laboratory \\
\hline PCP & Packaging Certification Program \\
\hline RAM & Radioactive Material \\
\hline RASTA & Radiation Source Term Analysis \\
\hline $\mathrm{SC}$ & Shielded Container \\
\hline SCALE & $\begin{array}{l}\text { A Modular Code System for Performing } \underline{\text { Standardized Computer }} \\
\text { Analyses for Licensing Evaluation }\end{array}$ \\
\hline SGQ & Small Gram Quantity \\
\hline SRNL & Savannah River National Laboratory \\
\hline SRNS & Savannah River Nuclear Solutions \\
\hline SS & Stainless Steel \\
\hline WSMS & Westinghouse Safety Management Solutions \\
\hline
\end{tabular}


PCP-2011-0001

Revision 0

\subsection{Introduction}

The Small Gram Quantity (SGQ) concept is based on the understanding that small amounts of hazardous materials, in this case radioactive materials (RAM), are significantly less hazardous than large amounts of the same materials. This study describes a methodology designed to estimate an SGQ for several neutron and gamma emitting isotopes that can be shipped in a package in compliant with 10 CFR Part 71 external radiation level limits regulations. These regulations require packaging for the shipment of radioactive materials perform, under both normal and accident conditions, the essential functions of material containment, subcriticality, and maintain external radiation levels within the regulatory limits. $10 \mathrm{CFR}$ $71.33(b)(1)(2) \&(3)$ state radioactive and fissile materials must be identified and their maximum quantity, chemical and physical forms be included in an application. Furthermore, the U.S. Federal Regulations require application contain an evaluation demonstrating the package (i.e., the packaging and its contents) satisfies the external radiation standards for all packages (10 CFR 71.31(2), 71.35(a), \& 71.47). By placing the contents in a He leak-tight containment vessel, and limiting the mass to ensure subcriticality, the first two essential functions are readily met. Some isotopes emit sufficiently strong photon radiation that small amounts of material can yield a large external dose rate. Quantifying of the dose rate for a proposed content is a challenging issue for the SGQ approach. It is essential to quantify external radiation levels from several common gamma and neutron sources that can be safely placed in a specific packaging, to ensure compliance with federal regulations. The Packaging Certification Program (PCP) Methodology for Determining Dose Rate for Small Gram Quantities in Shipping Packagings provides bounding shielding calculations that define mass limits compliant with 10 CFR 71.47 for a set of proposed SGQ isotopes. The approach is based on energy superposition with dose response calculated for a set of spectral groups for a baseline physical configuration. The methodology includes using the MCNP radiation transport code to evaluate a family of neutron and photon spectral groups using the 9977 shipping package and its associated shielded containers as the base case. This results in a set of multipliers for "dose per particle" for each spectral group. For a given isotope, the source spectrum is folded with the response for each group. The summed contribution determines the total dose from the RAM in the container.

\subsection{Analytic Methodology}

The MCNP( Monte $\underline{\text { Carlo }} \underline{\text { N-Particle })[L A-U R-03-1987] ~ c o d e ~ p a c k a g e ~ w a s ~ u s e d ~ f o r ~ t h r e e ~}$ dimensional Monte Carlo transport calculations to determine the radiation absorbed dose rates outside the package under Normal Conditions of Transport (NCT). The neutron transport calculations were performed as a series of calculations starting a unit source in each group using the BUGLE forty seven group structure [DLC-75]. Similarly, photon transport calculations were performed as a series of calculations staring a unit source in each group using a seventy seven group structure. Radiation source spectra and strengths were characterized using ORIGEN-S [ORNL/TM-2005/39, Section F7] and RASTA (Radiation Source Term Analysis) [SRNS-RP-2009-00275]. The neutron sources were defined using energy groups from the BUGLE 47 group structure.

The following paragraphs summarize features and use of the ORIGEN-S, RASTA, and MCNP codes:

ORIGEN-S computes time-dependent concentrations and source terms of a large number of isotopes, which are simultaneously generated and depleted through neutronic transmutation, fission, radioactive decay, input feed rates, and physical or chemical removal rates. The matrix exponential model of the ORIGEN code is unaltered in ORIGEN-S. The version of ORIGEN applied in the SCALE system [ORNL/TM-2005/39], ORIGEN-S, has several improvements over the original program. The code has been modified to include dynamic storage allocation, free-form input processing, and flexible dimensioning. 
PCP-2011-0001

Revision 0

RASTA (Radiation Source Term Analysis) is a code that computes neutron and photon source terms arising from ( $\alpha-n)$ events, spontaneous fission, bremsstrahlung, and decay. The code was written to consolidate existing capabilities into a single, easy to use code with flexible, extensive output edits, while also adding new capabilities. Specifically, the gamma decay calculation from the GAMSRC code, bremsstrahlung production calculations from the BREMRAD code [HW-82784], and the $(\alpha-n)$ and spontaneous fission neutron calculations from the SOURCES code [LA-13639] have been incorporated into RASTA. In addition, RASTA provides calculational routines to find the photon source arising from decay of the product isotope resulting from an $(\alpha-n)$ calculation, and to find the photon source arising from both prompt and delayed spontaneous fission events. The RASTA methodology for all processes is generalized and applicable to any isotopes for which data is available.

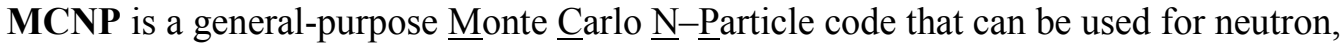
photon, electron, or coupled neutron/photon/electron transport, including the capability to calculate eigenvalues for critical systems. The code treats an arbitrary three-dimensional configuration of materials in geometric cells bounded by first- and second-degree surfaces and fourth-degree elliptical tori. Pointwise cross-section data are used. For neutrons, all reactions given in a particular cross-section evaluation (such as ENDF/B-VI) are accounted for. Thermal neutrons are described by both the free gas and $S(\alpha, \beta)$ models. For photons, the code accounts for incoherent and coherent scattering, the possibility of fluorescent emission after photoelectric absorption, absorption in pair production with local emission of annihilation radiation, and bremsstrahlung. A continuous-slowing-down model is used for electron transport that includes positrons, kshell x-rays, and bremsstrahlung, but does not include external or self-induced fields.

The ORIGEN-S, RASTA, and MCNP, calculations were performed on the SRNS Criticality Safety Advanced Computing Center stand-alone computers, using code and libraries under configuration control [SRNS-RP-2008-00150, SRNS-RP-2009-00276, and SRNS-RP-2008-00117] and verified on that system [SRNS-RP-2008-00151, SRNS-RP-2009-00277, SRNS-RP-2009-00285].

\subsection{Shielding Models}

The 9977 is a single containment drum type package with a bolted flange closure and a right circular cylinder Containment Vessel (CV) enclosed by LAST-A-FOAM ${ }^{\circledR}$ and Fiberfrax ${ }^{\circledR}$ insulation. Major materials of construction include stainless steel, polyurethane, and aluminum.

The drum consists of a SS outer shell with a SS liner, aluminum load distribution fixtures and miscellaneous other hardware, as shown in Figure 2-1. The drum is modeled as a right circular cylinder, simplifying some of the components. The simplifications are conservative since they place the source material closer to the surface of the drum being analyzed. These simplifications include:

- The bottom of the drum is modeled as flat rather than convex.

- The drum top rim and bottom wear ring are not modeled.

- The drum rolling hoops are not modeled.

The six-inch containment vessel $(6 \mathrm{CV})$ is modeled as a cylindrical main portion with a conical transition at the top, a short upper cylinder, a Cone-Seal Plug, and the Cone-Seal Nut. Some of the components of the $6 \mathrm{CV}$ that are simple to model (e.g., the conical transition at the top) are 
PCP-2011-0001

Revision 0

included exactly. Other more complex components are modeled as simpler shapes; these are discussed below.

- The Gland Nut is a complex set of cones and cylinders inside the Gland Nut Plug. For simplicity the nut and plug are modeled together as a single cylinder of 304L stainless steel with a small cylindrical cavity at the top to account for the nut being shorter than the plug. This does not impact the radiation transport calculations, since the limiting dose point is at the bottom of the package.

- The End Cap of the $6 \mathrm{CV}$ is modeled as a $2 / 1$ ellipse with the axis of rotation (the "z-axis") set to half the radius of the $6 \mathrm{CV}$ cylindrical portion and the minor axis set to the radius of the $6 \mathrm{CV}$ cylindrical portion. The bottom of the cylindrical portion of the $6 \mathrm{CV}$ is extended down to the point at which the ellipse intersects it.

The SGQ-SC1 Lead Shielded Container [R-R1-G-00037] (Figure 2-2) was modeled as a right circular cylinder (9.354" tall and 5.625" in diameter). The content cavity was modeled as $5.5 "$ tall by $1.625 "$ diameter, 2 " above the outside bottom of the container.

The SGQ-SC2 Polyethylene Shielded Container [R-R1-G-00038] (Figure 2-3) was modeled as a right circular cylinder (12.25" tall and 5.875" in diameter). The content cavity was modeled as $8.00 "$ tall by $1.625^{\prime \prime}$ diameter, $2.0{ }^{\prime \prime}$ above the outside bottom ${ }^{1}$ of the container.

The SGQ-SC3 Tungsten Shielded Container [R-R1-G-00039] (Figure 2-4) was modeled as a right circular cylinder (7.480" tall and 5.480" in diameter $\left.{ }^{2}\right)$. The content cavity was modeled as 3.330" tall by $1.330 "$ diameter, $2.075^{\prime \prime}$ above the outside bottom of the container.

Representing the shielded containers as right circular cylinders simplifies the geometric modeling without impacting the calculated dose rates since the detectors are located on the source axis or mid-plane.

\subsection{Material Compositions}

The 9977 Package consists of a stainless steel cylindrical CV, a 35 gallon steel drum, Fiberfrax ${ }^{\circledR}$, LAST-A-FOAM ${ }^{\circledR}$ insulation, aluminum load distribution fixtures, and a lid filled with TR-19 ${ }^{\circledR}$ Block/Min K ${ }^{\circledR} 2000$.

Ten material compositions (Table 2-1), other than the source region, are used to model the 9977. [WSMS CRT 02 0060, LA 12827 M, Midwest Tungsten Service]

\subsection{Radiation Sources}

A set of thirty six source isotopes was proposed by personnel at Lawrence Livermore National Laboratory [LLNL-TR-461255]. Thirty five of these isotopes (with the exception of the very short lived Pd-103), listed in Table 2-2, were analyzed to determine the neutron and photon source strengths. The 47 group structure used to calculate neutron spectra is shown in Table 2-3. The 77 group structure used to calculate photon spectra is shown in Table 2-4. The sources were computed as a function of decay time using ORIGEN-S and without decay using RASTA. Actinides were analyzed with varying amounts of beryllium to determine the effects of $(\alpha-n)$

1 These dimensions are based on an earlier design of the SGQ-SC2 Shielded Container. The impact of the differences on calculated dose is conservative since the thickness of polyethylene is less than the current design.

2 These dimensions are based on an earlier design of the SGQ-SC3 Shielded Container. The impact of the differences on calculated dose is minimal since the thickness of tungsten is the same as the current design. 
PCP-2011-0001

Revision 0

interactions on the neutron source. The added beryllium is modeled as a percent of the actinide mass (e.g., ten percent added beryllium in $\mathrm{Pu}-238$ is modeled as 1 gram of $\mathrm{Pu}-238$ and 0.1 grams of beryllium).

The input files used are described in Appendix A.

\subsection{Radiation Transport}

For NCT, the package is considered to be intact and dose rates are calculated on contact at the side, bottom, and top of the package. NCT models were developed for no shielded container (Figure 2-5), the SGQ-SC1 lead shielded container (Figure 2-6), the SGQ-SC2 polyethylene shielded container (Figure 2-7), and the SGQ-SC3 tungsten shielded container (Figure 2-8). The source region is modeled as a void for each of the shielded containers.

The neutron source was modeled as a cylinder centered at the bottom of the CV. The cylinder was $2.5 \mathrm{~cm}$ in diameter by $4 \mathrm{~cm}$ tall based on the average source dimensions as presented by Monsanto [ANS-1981]. The source cylinders were run initially as void and then as reduced density plutonium oxide without shielded containers to determine the effects of self-shielding.

The gamma source was modeled as a cylinder centered at the bottom of the CV. The cylinder dimensions were $1 \mathrm{~cm}$ in diameter by $2 \mathrm{~cm}$ tall based on the average dimensions obtained from sources listed in a catalog from AEA Technology [AEA Technology QSA]. The source cylinders were run initially as void and then as reduced density $(1 \mathrm{~g} / \mathrm{cc})$ iron $^{3}$ without shielded containers to determine the effects of self-shielding.

The source cylinders are placed at the bottom of the $\mathrm{CV}$ or at the base of the shielded containers with the cylinder centerline on the package centerline. The source region is modeled as a void for the shielded containers.

Separate MCNP cases were run with a unit source in each of the neutron groups (Table 2-3) and each of the photon groups (Table 2-4). The input files are described in Appendix B.

Neutron and photon flux-to-dose-rate conversion factors were obtained from the 1977 American Nuclear Society Standard [ANSI/ANS-6.1.1-1977] and are given in Table 2-5. The 1977 values were used rather than those from the 1991 standard because the neutron dose conversion factors more closely reflect those provided in federal regulations [49 CFR 173.403], and the photon dose conversion factors more closely correspond to the response measured by instrumentation.

Dose points were modeled as both segmented surface tallies and point detectors located:

- At the bottom surface on the centerline of the package

- At the top surface on the centerline of the package

- At the side surface of the package axially centered on the source

Output from the MCNP cases was written to MCTAL files to facilitate collecting the response per unit source. The MCTAL files were collected using a set of Python scripts (see Appendix C).

3 Iron was chosen for the self-shielding material since it should be representative of several of the isotopes being analyzed. 
PCP-2011-0001

Revision 0

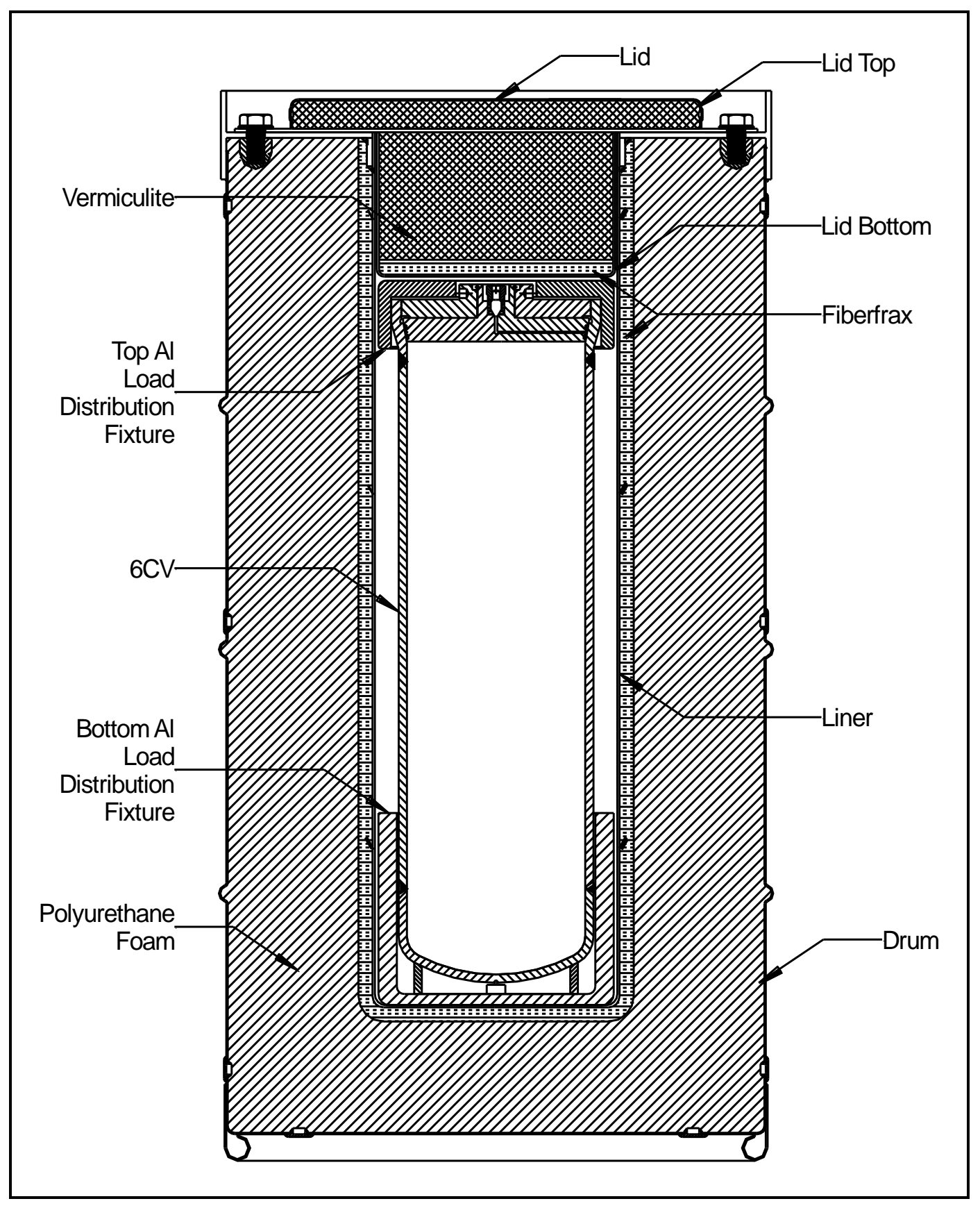

Figure 2-1. Model of 9977 Under NCT 
PCP-2011-0001

Revision 0

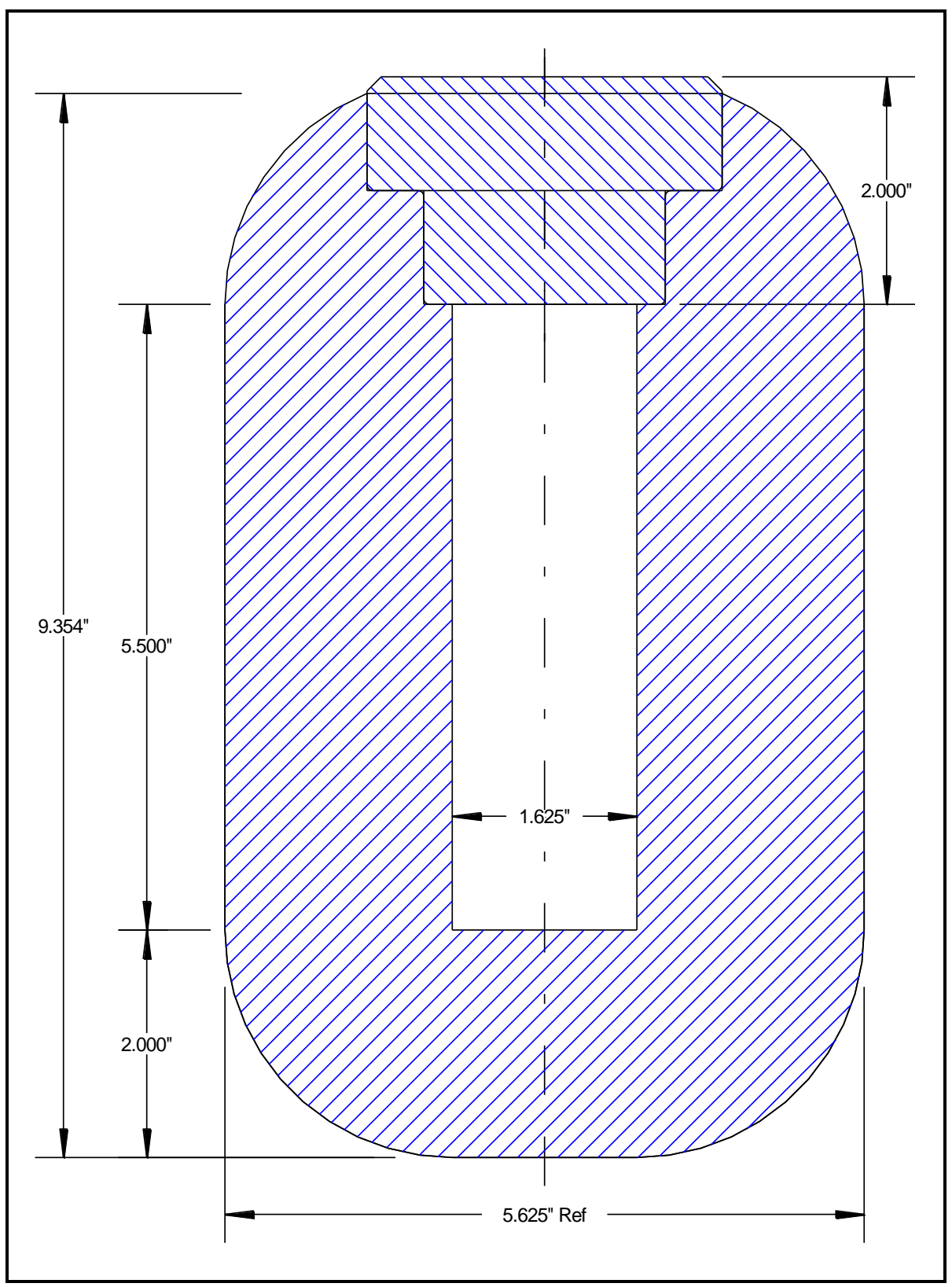

Figure 2-2. SGQ-SC1 Lead Shielded Container 
PCP-2011-0001

Revision 0

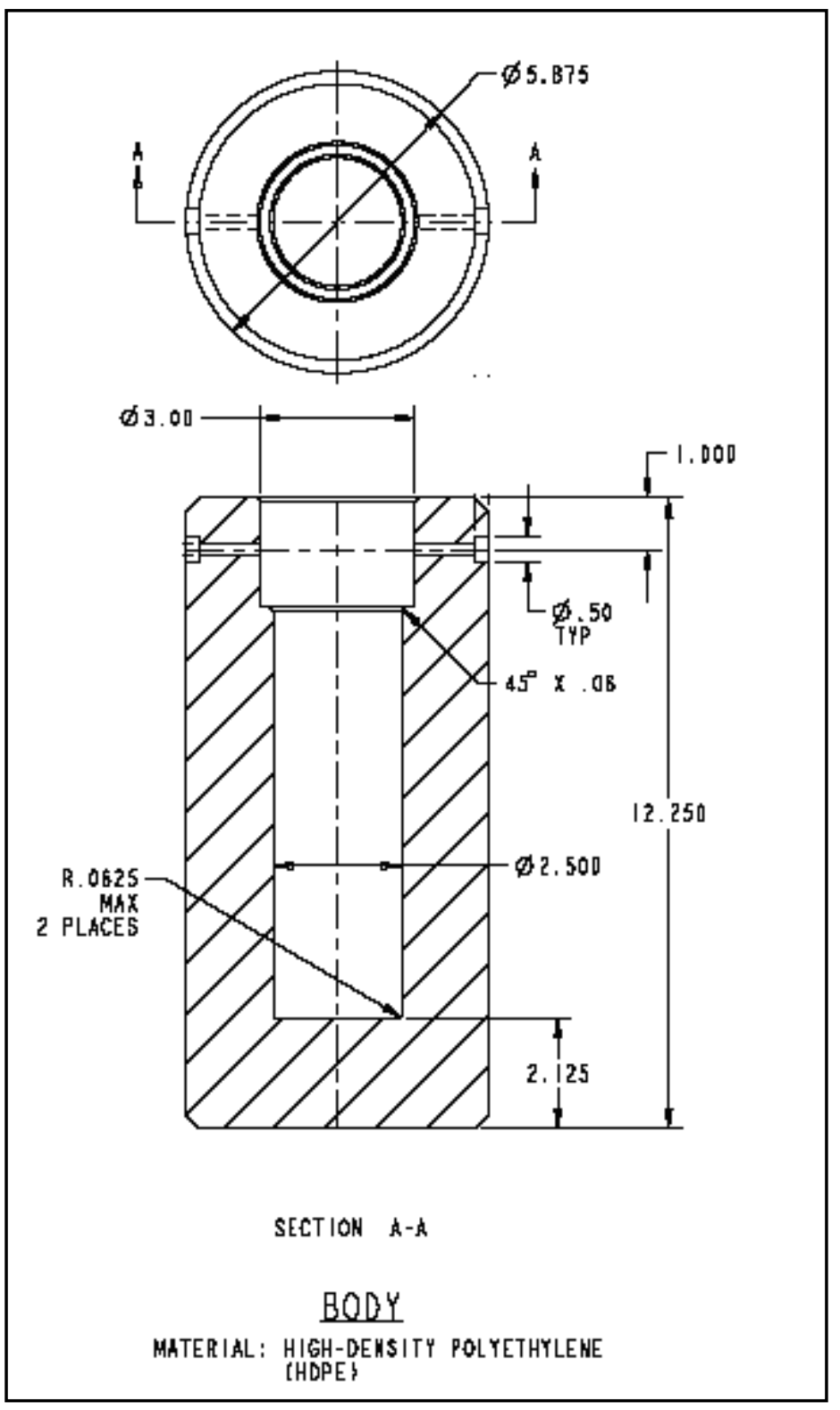

Figure 2-3. SGQ-SC2 HDPE Shielded Container 
PCP-2011-0001

Revision 0

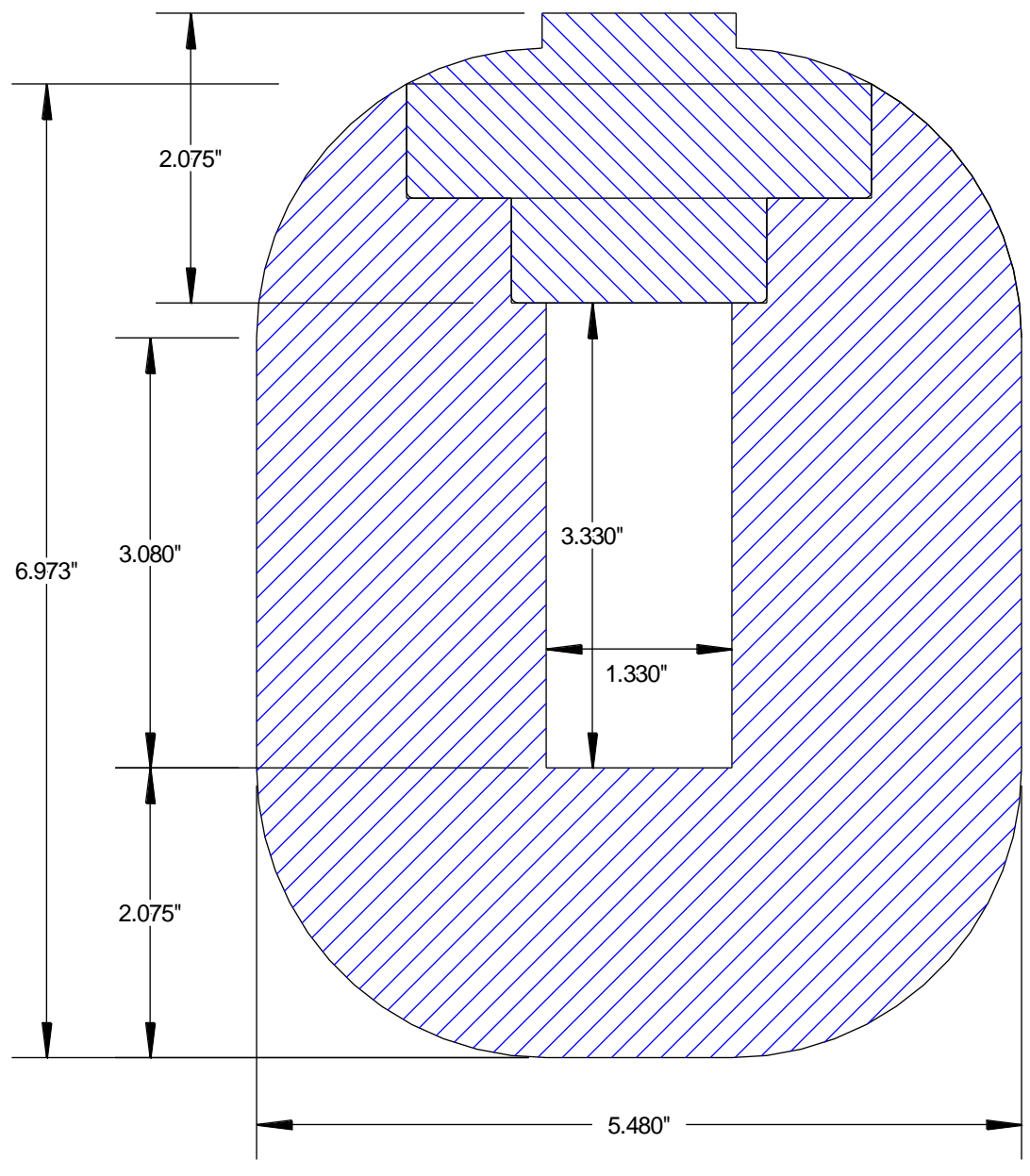

Figure 2-4. SGQ-SC3 Tungsten Shielded Container 
PCP-2011-0001

Revision 0

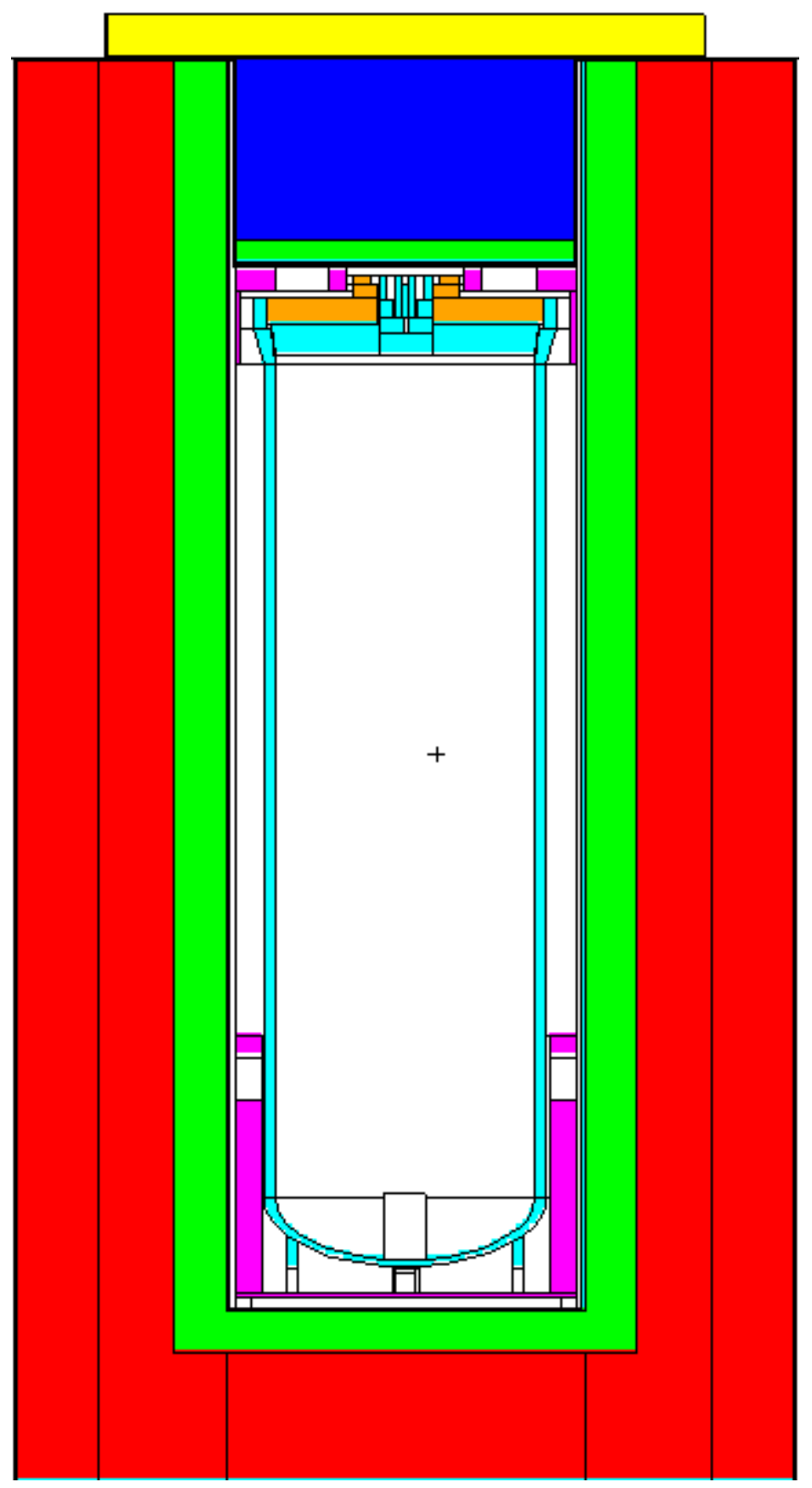

Figure 2-5. 9977 NCT Neutron Source without Shielded Container 
PCP-2011-0001

Revision 0

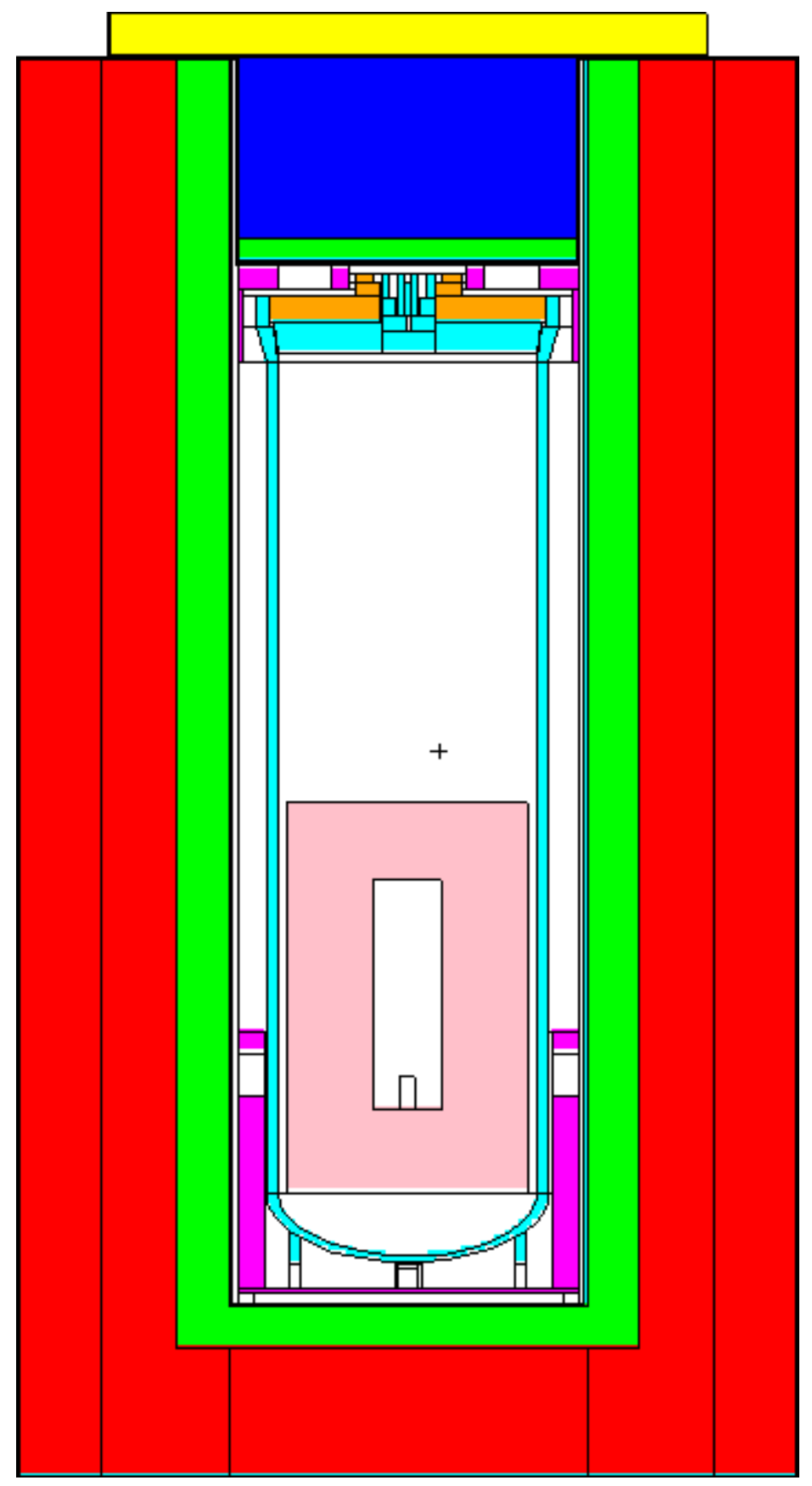

Figure 2-6. 9977 NCT Photon Source in SC1 Lead Container 
PCP-2011-0001

Revision 0

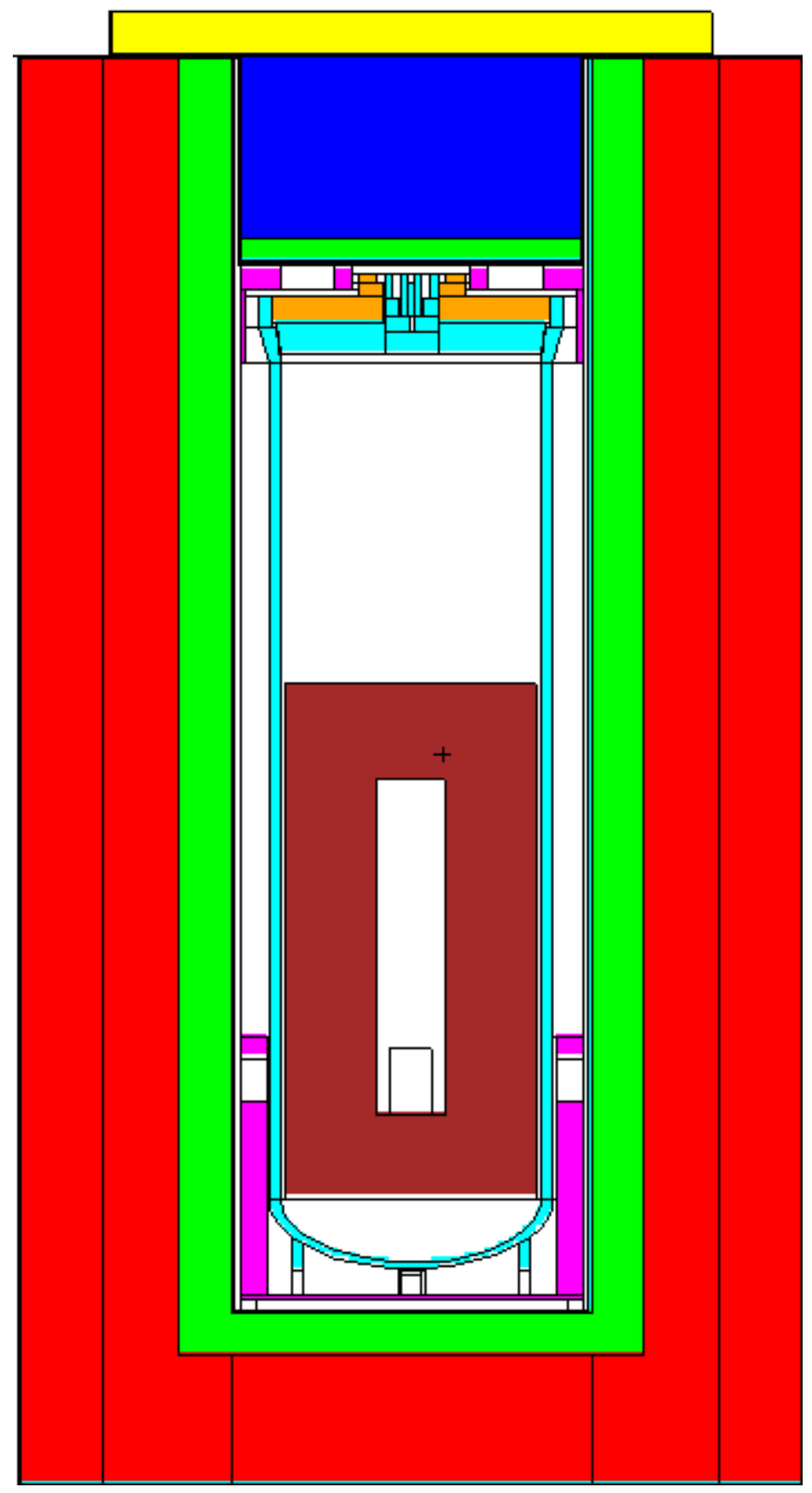

Figure 2-7. 9977 NCT Neutron Source in SC2 Polyethylene Container 
PCP-2011-0001

Revision 0

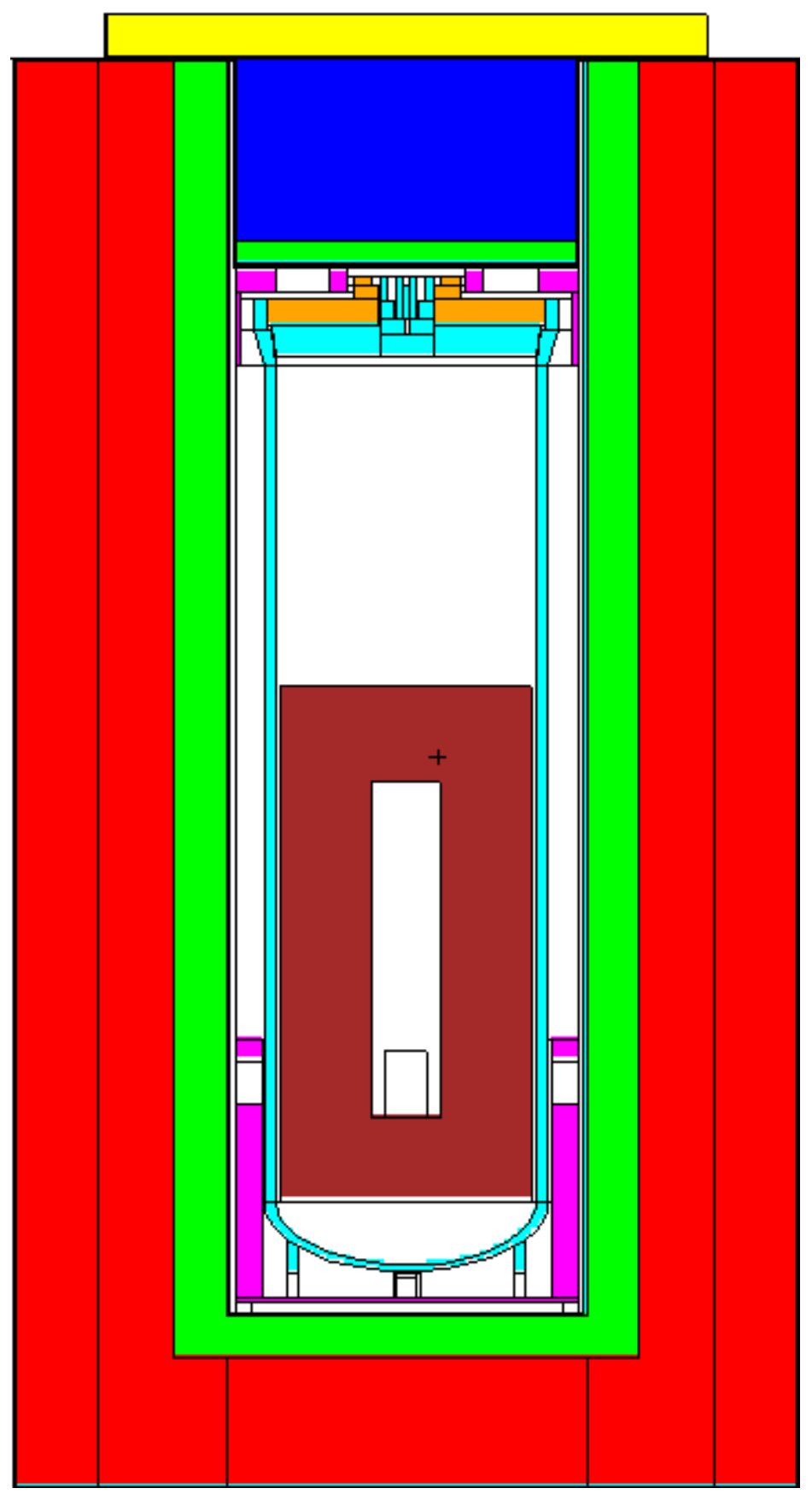

Figure 2-8. 9977 NCT Photon Source in SC3 Tungsten Container 
PCP-2011-0001

Revision 0

Table 2-1. Material Compositions

\begin{tabular}{|c|c|c|c|c|c|c|c|c|c|c|}
\hline & $\begin{array}{c}\text { Nitronic }^{\circledR} \\
60\end{array}$ & SS 304L & $\begin{array}{c}6061 \\
\text { Aluminum }\end{array}$ & Foam & Vermiculite & $\begin{array}{c}\text { Min-K }^{\circledR} \\
2000\end{array}$ & Fiberfrax $^{\circledR}$ & Lead & Tungsten & HDPE \\
\hline $\begin{array}{c}\text { density } \\
\text { (g/cc) }\end{array}$ & 7.584 & 7.9 & 2.7 & 0.256 & 0.3684 & 0.3204 & 0.2563 & 11.34 & 17 & 0.95 \\
\hline Element & \multicolumn{10}{|c|}{ Weight Percent } \\
\hline $\mathrm{H}$ & & & & 4.1 & & 0.84 & & & & 14.37 \\
\hline $\mathrm{C}$ & & 0.03 & & 54.4 & & 1.28 & & & & 85.63 \\
\hline $\mathrm{O}$ & & & & 29.4 & 45.53 & 48.01 & 50.03 & & & \\
\hline $\mathrm{N}$ & 0.14 & & & 12.1 & & & & & & \\
\hline $\mathrm{Si}$ & 4 & 1 & 0.6 & & 21.22 & 37.71 & 23.14 & & & \\
\hline $\mathrm{Ni}$ & 8.5 & 10 & & & & & & & 6 & \\
\hline $\mathrm{Cr}$ & 17 & 19 & 0.2 & & & & & & & \\
\hline $\mathrm{Mn}$ & 8 & 2 & & & & & & & & \\
\hline $\mathrm{Fe}$ & 62.36 & 67.895 & & & 5.4 & & & & & \\
\hline $\mathrm{P}$ & & 0.045 & & & & & & & & \\
\hline $\mathrm{S}$ & & & & & 0.18 & & & & & \\
\hline $\mathrm{Ca}$ & & & & & 12.17 & & & & & \\
\hline$\overline{\mathrm{Al}}$ & & & 97.9 & & 5.47 & 6.39 & 26.46 & & & \\
\hline $\mathrm{Cu}$ & & & 0.28 & & & & & & 4 & \\
\hline $\mathrm{Mg}$ & & & 1 & & 8.9 & & & & & \\
\hline $\mathrm{Ti}$ & & & & & 0.82 & 5.53 & & & & \\
\hline $\mathrm{Na}$ & & & & & 1.09 & & 0.37 & & & \\
\hline $\mathrm{K}$ & & & & & 1.22 & & & & & \\
\hline $\mathrm{Ga}$ & & & & & & 0.15 & & & & \\
\hline $\mathrm{Sb}$ & & & & & & 0.09 & & & & \\
\hline $\mathrm{W}$ & & & & & & & & & 90 & \\
\hline $\mathrm{Pb}$ & & & & & & & & 100 & & \\
\hline
\end{tabular}

Table 2-2. Typical Sealed Source Isotopes

\begin{tabular}{|c|c|c|c|c|c|c|c|}
\hline \multirow{2}{*}{ Nuclide } & \multirow{2}{*}{$\begin{array}{l}\text { Half Life } \\
\text { (days) }\end{array}$} & \multicolumn{2}{|c|}{ E-max $(\mathrm{MeV})$} & \multirow{2}{*}{ Nuclide } & \multirow{2}{*}{$\begin{array}{l}\text { Half Life } \\
\text { (days) }\end{array}$} & \multicolumn{2}{|c|}{ E-max $(\mathrm{MeV})$} \\
\hline & & Gamma & Alpha & & & Gamma & Alpha \\
\hline Ac-227 & $7.95 \mathrm{E}+03$ & 0.242 & 4.953 & Pm-147 & $9.58 \mathrm{E}+02$ & 0.121 & 0 \\
\hline Am-241 & $1.58 \mathrm{E}+05$ & 0.060 & 5.49 & Рo-210 & $1.38 \mathrm{E}+02$ & - & 5.304 \\
\hline $\mathrm{Am}-241 / \mathrm{Be}$ & $1.58 \mathrm{E}+05$ & 0.060 & 5.49 & $\mathrm{Pu}-238$ & $3.20 \mathrm{E}+04$ & 0.014 & 5.5 \\
\hline Am-243 & $2.69 \mathrm{E}+06$ & 0.075 & 5.28 & $\mathrm{Pu}-238 / \mathrm{Be}$ & $3.20 \mathrm{E}+04$ & 0.014 & 5.5 \\
\hline $\mathrm{Am}-243 / \mathrm{Be}$ & $2.69 \mathrm{E}+06$ & 0.075 & 5.28 & Pu-239 & $8.80 \mathrm{E}+06$ & 0.014 & 5.16 \\
\hline Cd-109 & $4.63 \mathrm{E}+02$ & 0.088 & 0 & $\mathrm{Pu}-239 / \mathrm{Be}$ & $8.80 \mathrm{E}+06$ & 0.014 & 5.16 \\
\hline Cf-252 & $9.66 \mathrm{E}+02$ & 0.160 & 6.12 & $\mathrm{Pu}-240$ & $2.40 \mathrm{E}+06$ & 0.014 & 5.168 \\
\hline $\mathrm{Cm}-244$ & $6.61 \mathrm{E}+03$ & 0.153 & 5.81 & $\mathrm{Pu}-240 / \mathrm{Be}$ & $2.40 \mathrm{E}+06$ & 0.014 & 5.168 \\
\hline $\mathrm{Cm}-244 / \mathrm{Be}$ & $6.61 \mathrm{E}+03$ & 0.153 & 5.81 & $\mathrm{Pu}-241$ & $5.26 \mathrm{E}+03$ & $0.060 *$ & 4.896 \\
\hline Cm-248 & $1.27 \mathrm{E}+08$ & - & 5.078 & $\mathrm{Pu}-241 / \mathrm{Be}$ & $5.26 \mathrm{E}+03$ & $0.060 *$ & 4.896 \\
\hline Co-60 & $1.93 \mathrm{E}+03$ & 1.333 & 0 & $\mathrm{Pu}-242$ & $1.37 \mathrm{E}+08$ & 0.014 & 4.9 \\
\hline Cs-137 & $1.10 \mathrm{E}+04$ & $0.662 *$ & 0 & $\mathrm{Pu}-242 / \mathrm{Be}$ & $1.37 \mathrm{E}+08$ & 0.014 & 4.9 \\
\hline Eu-152 & $4.93 \mathrm{E}+03$ & 1.410 & 0 & $\mathrm{Ra}-226$ & $5.84 \mathrm{E}+05$ & 0.186 & 4.78 \\
\hline Fe-59 & $4.45 \mathrm{E}+01$ & 1.292 & 0 & Ru-106 & $3.73 \mathrm{E}+02$ & $0.622 *$ & 0 \\
\hline Gd-153 & $2.42 \mathrm{E}+02$ & 0.103 & 0 & Sc-46 & $8.38 \mathrm{E}+01$ & 1.120 & 0 \\
\hline Hf-181 & $4.24 \mathrm{E}+01$ & 0.482 & 0 & Se-75 & $1.20 \mathrm{E}+02$ & 0.401 & 0 \\
\hline Ho-166m & $4.38 \mathrm{E}+05$ & 0.951 & 0 & Sm-145 & $3.40 \mathrm{E}+02$ & 0.061 & 0 \\
\hline Ir-192 & $7.38 \mathrm{E}+01$ & 0.612 & 0 & Sr-90 & $1.05 \mathrm{E}+04$ & $0.018^{*}$ & 0 \\
\hline Mn-54 & $3.12 \mathrm{E}+02$ & 0.835 & 0 & Tm-170 & $1.29 \mathrm{E}+02$ & 0.084 & 0 \\
\hline Np-237 & $7.82 \mathrm{E}+08$ & $0.415^{*}$ & 4.79 & Yb-169 & $3.20 \mathrm{E}+01$ & 0.198 & 0 \\
\hline $\mathrm{Np}-237 / \mathrm{Be}$ & $7.82 \mathrm{E}+08$ & $0.415^{*}$ & 4.79 & $\mathrm{Zn}-65$ & $2.44 \mathrm{E}+02$ & 1.116 & 0 \\
\hline $\mathrm{Pb}-210$ & $8.15 \mathrm{E}+03$ & 0.047 & 0 & Zr-95 & $6.40 \mathrm{E}+01$ & 0.757 & 0 \\
\hline
\end{tabular}

* Radiation from daughter isotope 
PCP-2011-0001

Revision 0

Table 2-3. Neutron Spectra 47 Group Structure

\begin{tabular}{|c|c|c|c|c|c|}
\hline Group & $\begin{array}{c}\text { E-upper } \\
(\mathrm{MeV})\end{array}$ & $\begin{array}{c}\text { E-lower } \\
(\mathrm{MeV})\end{array}$ & Group & $\begin{array}{c}\text { E-upper } \\
(\mathrm{MeV})\end{array}$ & $\begin{array}{c}\text { E-lower } \\
(\mathrm{MeV})\end{array}$ \\
\hline 47 & $1.00 \mathrm{E}-07$ & $1.00 \mathrm{E}-11$ & 23 & $4.98 \mathrm{E}-01$ & $3.69 \mathrm{E}-01$ \\
\hline 46 & 4.14E-07 & $1.00 \mathrm{E}-07$ & 22 & 6.08E-01 & $4.98 \mathrm{E}-01$ \\
\hline 45 & $8.76 \mathrm{E}-07$ & $4.14 \mathrm{E}-07$ & 21 & $7.43 \mathrm{E}-01$ & $6.08 \mathrm{E}-01$ \\
\hline 44 & $1.86 \mathrm{E}-06$ & $8.76 \mathrm{E}-07$ & 20 & $8.21 \mathrm{E}-01$ & $7.43 \mathrm{E}-01$ \\
\hline 43 & $5.04 \mathrm{E}-06$ & $1.86 \mathrm{E}-06$ & 19 & $1.00 \mathrm{E}+00$ & $8.21 \mathrm{E}-01$ \\
\hline 42 & $1.07 \mathrm{E}-05$ & $5.04 \mathrm{E}-06$ & 18 & $1.35 \mathrm{E}+00$ & $1.00 \mathrm{E}+00$ \\
\hline 41 & $3.73 \mathrm{E}-05$ & $1.07 \mathrm{E}-05$ & 17 & $1.65 \mathrm{E}+00$ & $1.35 \mathrm{E}+00$ \\
\hline 40 & $1.01 \mathrm{E}-04$ & $3.73 \mathrm{E}-05$ & 16 & $1.92 \mathrm{E}+00$ & $1.65 \mathrm{E}+00$ \\
\hline 39 & $2.14 \mathrm{E}-04$ & $1.01 \mathrm{E}-04$ & 15 & $2.23 \mathrm{E}+00$ & $1.92 \mathrm{E}+00$ \\
\hline 38 & $4.54 \mathrm{E}-04$ & $2.14 \mathrm{E}-04$ & 14 & $2.35 \mathrm{E}+00$ & $2.23 \mathrm{E}+00$ \\
\hline 37 & $1.58 \mathrm{E}-03$ & $4.54 \mathrm{E}-04$ & 13 & $2.37 \mathrm{E}+00$ & $2.35 \mathrm{E}+00$ \\
\hline 36 & $3.35 \mathrm{E}-03$ & $1.58 \mathrm{E}-03$ & 12 & $2.47 \mathrm{E}+00$ & $2.37 \mathrm{E}+00$ \\
\hline 35 & $7.10 \mathrm{E}-03$ & $3.35 \mathrm{E}-03$ & 11 & $2.73 \mathrm{E}+00$ & $2.47 \mathrm{E}+00$ \\
\hline 34 & $1.50 \mathrm{E}-02$ & $7.10 \mathrm{E}-03$ & 10 & $3.01 \mathrm{E}+00$ & $2.73 \mathrm{E}+00$ \\
\hline 33 & $2.19 \mathrm{E}-02$ & $1.50 \mathrm{E}-02$ & 9 & $3.68 \mathrm{E}+00$ & $3.01 \mathrm{E}+00$ \\
\hline 32 & $2.42 \mathrm{E}-02$ & $2.19 \mathrm{E}-02$ & 8 & $4.97 \mathrm{E}+00$ & $3.68 \mathrm{E}+00$ \\
\hline 31 & $2.61 \mathrm{E}-02$ & $2.42 \mathrm{E}-02$ & 7 & $6.07 \mathrm{E}+00$ & $4.97 \mathrm{E}+00$ \\
\hline 30 & $3.18 \mathrm{E}-02$ & $2.61 \mathrm{E}-02$ & 6 & $7.41 \mathrm{E}+00$ & $6.07 \mathrm{E}+00$ \\
\hline 29 & $4.09 \mathrm{E}-02$ & $3.18 \mathrm{E}-02$ & 5 & $8.61 \mathrm{E}+00$ & $7.41 \mathrm{E}+00$ \\
\hline 28 & $5.74 \mathrm{E}-02$ & $4.09 \mathrm{E}-02$ & 4 & $1.00 \mathrm{E}+01$ & $8.61 \mathrm{E}+00$ \\
\hline 27 & $1.11 \mathrm{E}-01$ & $5.74 \mathrm{E}-02$ & 3 & $1.22 \mathrm{E}+01$ & $1.00 \mathrm{E}+01$ \\
\hline 26 & $1.83 \mathrm{E}-01$ & $1.11 \mathrm{E}-01$ & 2 & $1.42 \mathrm{E}+01$ & $1.22 \mathrm{E}+01$ \\
\hline 25 & $2.97 \mathrm{E}-01$ & $1.83 \mathrm{E}-01$ & 1 & $1.73 \mathrm{E}+01$ & $1.42 \mathrm{E}+01$ \\
\hline 24 & $3.69 \mathrm{E}-01$ & $2.97 \mathrm{E}-01$ & & & \\
\hline
\end{tabular}

Note: Group structure is for RASTA. ORIGEN-S group structure differs for groups 1, 27, and 28. ORIGEN-ARP Group 1 Upper Bound is 1.96E+01, Group 27 Lower Bound/ Group 28 Upper Bound is 6.74 E-02.

Values are reported to three significant figures. Full precision is used in calculations. 
PCP-2011-0001

Revision 0

Table 2-4. Photon Spectra 77 Group Structure

\begin{tabular}{|r|r|r||r|r|r||r|r|r||}
\hline Group & $\begin{array}{c}\text { E-upper } \\
\text { (MeV) }\end{array}$ & $\begin{array}{c}\text { E-lower } \\
\text { (MeV) }\end{array}$ & Group & $\begin{array}{c}\text { E-upper } \\
\text { (MeV) }\end{array}$ & $\begin{array}{c}\text { E-lower } \\
\text { (MeV) }\end{array}$ & Group & $\begin{array}{c}\text { E-upper } \\
\text { (MeV) }\end{array}$ & $\begin{array}{c}\text { E-lower } \\
\text { (MeV) }\end{array}$ \\
\hline 77 & 0.020 & 0.010 & 51 & 0.280 & 0.270 & 25 & 0.900 & 0.875 \\
\hline 76 & 0.030 & 0.020 & 50 & 0.290 & 0.280 & 24 & 0.925 & 0.900 \\
\hline 75 & 0.040 & 0.030 & 49 & 0.300 & 0.290 & 23 & 0.950 & 0.925 \\
\hline 74 & 0.050 & 0.040 & 48 & 0.325 & 0.300 & 22 & 0.975 & 0.950 \\
\hline 73 & 0.060 & 0.050 & 47 & 0.350 & 0.325 & 21 & 1.000 & 0.975 \\
\hline 72 & 0.070 & 0.060 & 46 & 0.375 & 0.350 & 20 & 1.250 & 1.000 \\
\hline 71 & 0.080 & 0.070 & 45 & 0.400 & 0.375 & 19 & 1.500 & 1.250 \\
\hline 70 & 0.090 & 0.080 & 44 & 0.425 & 0.400 & 18 & 1.750 & 1.500 \\
\hline 69 & 0.100 & 0.090 & 43 & 0.450 & 0.425 & 17 & 2.000 & 1.750 \\
\hline 68 & 0.110 & 0.100 & 42 & 0.475 & 0.450 & 16 & 2.250 & 2.000 \\
\hline 67 & 0.120 & 0.110 & 41 & 0.500 & 0.475 & 15 & 2.500 & 2.250 \\
\hline 66 & 0.130 & 0.120 & 40 & 0.525 & 0.500 & 14 & 2.750 & 2.500 \\
\hline 65 & 0.140 & 0.130 & 39 & 0.550 & 0.525 & 13 & 3.000 & 2.750 \\
\hline 64 & 0.150 & 0.140 & 38 & 0.575 & 0.550 & 12 & 3.500 & 3.000 \\
\hline 63 & 0.160 & 0.150 & 37 & 0.600 & 0.575 & 11 & 4.000 & 3.500 \\
\hline 62 & 0.170 & 0.160 & 36 & 0.625 & 0.600 & 10 & 4.500 & 4.000 \\
\hline 61 & 0.180 & 0.170 & 35 & 0.650 & 0.625 & 9 & 5.000 & 4.500 \\
\hline 60 & 0.190 & 0.180 & 34 & 0.675 & 0.650 & 8 & 5.500 & 5.000 \\
\hline 59 & 0.200 & 0.190 & 33 & 0.700 & 0.675 & 7 & 6.000 & 5.500 \\
\hline 58 & 0.210 & 0.200 & 32 & 0.725 & 0.700 & 6 & 6.500 & 6.000 \\
\hline 57 & 0.220 & 0.210 & 31 & 0.750 & 0.725 & 5 & 7.000 & 6.500 \\
\hline 56 & 0.230 & 0.220 & 30 & 0.775 & 0.750 & 4 & 7.500 & 7.000 \\
\hline 55 & 0.240 & 0.230 & 29 & 0.800 & 0.775 & 3 & 8.000 & 7.500 \\
\hline 54 & 0.250 & 0.240 & 28 & 0.825 & 0.800 & 2 & 9.000 & 8.000 \\
\hline 53 & 0.260 & 0.250 & 27 & 0.850 & 0.825 & 1 & 10.000 & 9.000 \\
\hline 52 & 0.270 & 0.260 & 26 & 0.875 & 0.850 & & & \\
\hline
\end{tabular}


PCP-2011-0001

Revision 0

Table 2-5. Flux-to-Dose-Rate Conversion Factors

\begin{tabular}{|c|c|c|c|}
\hline \multicolumn{2}{|c|}{ Neutron } & \multicolumn{2}{|c|}{ Photon } \\
\hline $\begin{array}{l}\text { Energy } \\
(\mathrm{MeV})\end{array}$ & $\begin{array}{l}\text { Factor }(\text { rem } / \mathrm{hr} \\
\left.\text { per } \mathrm{n} / \mathrm{cm}^{2} / \mathrm{sec}\right)\end{array}$ & $\begin{array}{l}\text { Energy } \\
(\mathrm{MeV})\end{array}$ & $\begin{array}{l}\text { Factor }(\mathrm{rem} / \mathrm{hr} \\
\left.\text { per p } / \mathrm{cm}^{2} / \mathrm{sec}\right)\end{array}$ \\
\hline $2.50 \mathrm{E}-08$ & $3.67 \mathrm{E}-06$ & $1.00 \mathrm{E}-02$ & $3.96 \mathrm{E}-06$ \\
\hline $1.00 \mathrm{E}-07$ & $3.67 \mathrm{E}-06$ & $3.00 \mathrm{E}-02$ & $5.82 \mathrm{E}-07$ \\
\hline $1.00 \mathrm{E}-06$ & $4.46 \mathrm{E}-06$ & $5.00 \mathrm{E}-02$ & $2.90 \mathrm{E}-07$ \\
\hline $1.00 \mathrm{E}-05$ & 4.54E-06 & $7.00 \mathrm{E}-02$ & $2.58 \mathrm{E}-07$ \\
\hline $1.00 \mathrm{E}-04$ & $4.18 \mathrm{E}-06$ & $1.00 \mathrm{E}-01$ & $2.83 \mathrm{E}-07$ \\
\hline $1.00 \mathrm{E}-03$ & $3.76 \mathrm{E}-06$ & $1.50 \mathrm{E}-01$ & $3.79 \mathrm{E}-07$ \\
\hline $1.00 \mathrm{E}-02$ & $3.56 \mathrm{E}-06$ & $2.00 \mathrm{E}-01$ & $5.01 \mathrm{E}-07$ \\
\hline $1.00 \mathrm{E}-01$ & $2.17 \mathrm{E}-05$ & $2.50 \mathrm{E}-01$ & $6.31 \mathrm{E}-07$ \\
\hline $5.00 \mathrm{E}-01$ & $9.26 \mathrm{E}-05$ & $3.00 \mathrm{E}-01$ & $7.59 \mathrm{E}-07$ \\
\hline $1.00 \mathrm{E}+00$ & $1.32 \mathrm{E}-04$ & $3.50 \mathrm{E}-01$ & $8.78 \mathrm{E}-07$ \\
\hline $2.50 \mathrm{E}+00$ & $1.25 \mathrm{E}-04$ & $4.00 \mathrm{E}-01$ & $9.85 \mathrm{E}-07$ \\
\hline $5.00 \mathrm{E}+00$ & $1.56 \mathrm{E}-04$ & $4.50 \mathrm{E}-01$ & $1.08 \mathrm{E}-06$ \\
\hline $7.00 \mathrm{E}+00$ & $1.47 \mathrm{E}-04$ & $5.00 \mathrm{E}-01$ & $1.17 \mathrm{E}-06$ \\
\hline $1.00 \mathrm{E}+01$ & $1.47 \mathrm{E}-04$ & $5.50 \mathrm{E}-01$ & $1.27 \mathrm{E}-06$ \\
\hline $1.40 \mathrm{E}+01$ & $2.08 \mathrm{E}-04$ & $6.00 \mathrm{E}-01$ & $1.36 \mathrm{E}-06$ \\
\hline $2.00 \mathrm{E}+01$ & $2.27 \mathrm{E}-04$ & $6.50 \mathrm{E}-01$ & $1.44 \mathrm{E}-06$ \\
\hline & & $7.00 \mathrm{E}-01$ & $1.52 \mathrm{E}-06$ \\
\hline & & $8.00 \mathrm{E}-01$ & $1.68 \mathrm{E}-06$ \\
\hline & & $1.00 \mathrm{E}+00$ & $1.98 \mathrm{E}-06$ \\
\hline & & $1.40 \mathrm{E}+00$ & $2.51 \mathrm{E}-06$ \\
\hline & & $1.80 \mathrm{E}+00$ & $2.99 \mathrm{E}-06$ \\
\hline & & $2.20 \mathrm{E}+00$ & $3.42 \mathrm{E}-06$ \\
\hline & & $2.60 \mathrm{E}+00$ & $3.82 \mathrm{E}-06$ \\
\hline & & $2.80 \mathrm{E}+00$ & $4.01 \mathrm{E}-06$ \\
\hline & & $3.25 \mathrm{E}+00$ & $4.41 \mathrm{E}-06$ \\
\hline & & $3.75 \mathrm{E}+00$ & $4.83 \mathrm{E}-06$ \\
\hline & & $4.25 \mathrm{E}+00$ & $5.23 \mathrm{E}-06$ \\
\hline & & $4.75 \mathrm{E}+00$ & $5.60 \mathrm{E}-06$ \\
\hline & & $5.00 \mathrm{E}+00$ & $5.80 \mathrm{E}-06$ \\
\hline & & $5.25 \mathrm{E}+00$ & $6.01 \mathrm{E}-06$ \\
\hline & & $5.75 \mathrm{E}+00$ & $6.37 \mathrm{E}-06$ \\
\hline & & $6.25 \mathrm{E}+00$ & $6.74 \mathrm{E}-06$ \\
\hline & & $6.75 \mathrm{E}+00$ & $7.11 \mathrm{E}-06$ \\
\hline & & $7.50 \mathrm{E}+00$ & $7.66 \mathrm{E}-06$ \\
\hline & & $9.00 \mathrm{E}+00$ & $8.77 \mathrm{E}-06$ \\
\hline & & $1.10 \mathrm{E}+01$ & $1.03 \mathrm{E}-05$ \\
\hline & & $1.30 \mathrm{E}+01$ & $1.18 \mathrm{E}-05$ \\
\hline & & $1.50 \mathrm{E}+01$ & $1.33 \mathrm{E}-05$ \\
\hline
\end{tabular}


PCP-2011-0001

Revision 0

\subsection{Results and Discussion}

The neutron and photon sources were calculated in the group structure shown in Table 2-3 and Table 2-4 using both ORIGEN-S and RASTA. The response from a unit source in each neutron and photon group was calculated using MCNP5 with each unshielded and shielded container configuration. Effects of self-shielding on both neutron and photon response were evaluated by including either plutonium oxide or iron in the source region for the case with no shielded container.

\subsection{Radiation Sources}

The neutron source strengths are presented in Table 3-1 through Table 3-10. The total neutron source strengths as a function of added beryllium as calculated by ORIGEN-S and RASTA are compared in Table 3-11. It can be seen from Table 3-11 that the sources calculated by ORIGEN$\mathrm{S}$ are significantly greater than the sources calculated by RASTA except for Am-243, Pu-239, and $\mathrm{Pu}-241$ without any beryllium. Examination of the spectra for these isotopes (Figure 3-1, Figure 3-2, and Figure 3-3) indicates that the RASTA source and ORIGEN-S source have similar energy dependence with the RASTA source magnitude being higher in each. For these three isotopes, the RASTA source was used for conservatism.

The total neutron source strength as a function of decay time as calculated using ORIGEN-S is shown in Table 3-12. This table indicates that the total neutron source is steady or declines with increasing decay time for all isotopes except $\mathrm{Pu}-241$. The monotonic increase in total neutron source strength with decay time for Pu-241 is due to the formation of Am-241. The source strength for Pu-241 after 30,000 days decay was used for conservatism.

Photon source strengths are presented in Table 3-13 through Table 3-25. The total photon source strength as a function of decay time is presented in Table 3-26 which also compares the source strength calculated by ORIGEN-S and RASTA. Table 3-26 shows that RASTA calculates higher source strength than ORIGEN-S for Am-241, Cm-248, Ho-166m, Ir-192, and Pu-241.

Examination of the spectra for Am-241 (Figure 3-4) indicates that the RASTA source is higher than the ORIGEN-S source at low energies $(0.01$ to $1.0 \mathrm{MeV})$ but the ORIGEN-S source is higher than the RASTA source at high energies (1.0 to $10.0 \mathrm{MeV})$. Since the high energies will dominate the dose rate, the ORIGEN-S source was retained for conservatism.

Examination of the spectra for Cm-248 (Figure 3-5) shows the ORIGEN-S source significantly below the RASTA source for energies below $1 \mathrm{MeV}$. The RASTA source was retained for conservatism.

Examination of the spectra for Ho-166m (Figure 3-6) shows that the ORIGEN-S source is lower than the RASTA source over the entire range of energies. The RASTA source was retained for conservatism.

Examination of the spectra for Ir-192 (Figure 3-7) shows that ORIGEN-S is missing data over much of the range of energies. This is a known problem with this isotope in the ORIGEN data library. The RASTA source was used for conservatism.

Examination of the spectra for Pu-241 (Figure 3-8) shows that the ORIGEN-S source is significantly lower than the RASTA source for energies below $1 \mathrm{MeV}$, while the ORIGEN-S source is significantly higher than the RASTA source for most energies above $1 \mathrm{MeV}$. The ORIGEN-S source was retained for conservatism. 
PCP-2011-0001

Revision 0

Table 3-26 shows that the sources for Ac-227, Am-243, Np-237, Pb-201, Pu-241, Ra-226, Sr-90, and Zr-95 increase more than two percent with decay time. This is due to the build-in of daughter isotopes. Source strength for these isotopes will be at the time the total source reaches its peak:

$\begin{array}{lr}\text { Ac-227 } & 300 \text { days } \\ \text { Am-243 } & 100 \text { days } \\ \mathrm{Np}-237 & 3000 \text { days } \\ \mathrm{Pb}-210 & 100 \text { days } \\ \mathrm{Pu}-241 & 30,000 \text { days } \\ \mathrm{Ra}-226 & 30,000 \text { days } \\ \mathrm{Sr}-90 & 30 \text { days } \\ \text { Zr-95 } & 30 \text { days }\end{array}$

Five isotopes (Ac-227, Cs-137, Pb-210, Ru-106, and Sr-90) are beta emitters and have significant contributions to the photon source from bremsstrahlung. For these isotopes, the ORIGEN-S calculated isotopic concentration at the time of peak source strength was used in RASTA to calculate the photon source. The resulting photon sources which were used in the dose rate computation are presented in Table 3-27.

\subsection{Radiation Transport}

The dose rate outside the shipping package was calculated for a unit source in each neutron and photon group. Several configurations were evaluated for placing the sources in the package:

- Source without any shielded container

- $\quad$ Source in an SC1 Lead Shielded Container

- Actinide Source in an SC2 Polyethylene Shielded Container

- Source in an SC3 Tungsten Shielded Container

\subsubsection{No Shielded Container}

The dose rates without any shielded container were evaluated without any self-shielding. Table 3-28 and Table 3-29 present the neutron and secondary photon dose rates from a unit source in each neutron group. Table 3-30 lists photon dose rates from a unit source in each primary photon group.

The neutron and secondary photon dose rates from a unit source in each neutron group with self-shielding by twenty-five grams of $\mathrm{PuO}_{2}$ in the source volume are presented in Table 3-31 and Table 3-32. Table 3-33 contains the primary photon dose rate with self-shielding by $1 \mathrm{~g} / \mathrm{cc}$ of iron in the source volume for a unit source in each photon group.

\subsubsection{SC1 Lead Shielded Container}

The primary photon dose rates from a unit source in each photon group in the SC1 Lead Shielded Container are presented in Table 3-34.

\subsubsection{SC2 Polyethylene Shielded Container}

The neutron and secondary photon dose rates from a unit source in each neutron group in the SC2 Polyethylene Shielded Container are shown in Table 3-35 and Table 3-36. Table 3-37 lists dose rates from a unit source in each primary photon group. 
PCP-2011-0001

Revision 0

\subsubsection{SC3 Tungsten Shielded Container}

The primary photon dose rates from a unit source in each photon group in the SC1 Tungsten Shielded Container are presented in Table 3-38.

\subsection{Dose Rate}

The dose rate from one gram of each of the isotopes listed in Table 2-2 is computed by folding the source strength for the isotope with the dose rate from a unit source for the appropriate shielded container:

$$
D_{j}^{c}=\sum_{g=1}^{N G} S_{j}^{g} * R_{g}^{c}
$$

Where:

$$
\begin{aligned}
& D_{j}^{c} \text { is the dose rate for isotope " } \mathrm{j} \text { " in container "c" } \\
& S_{j}^{g} \text { is the source strength for isotope " } \mathrm{j} \text { " in group " } \mathrm{g} \text { " } \\
& R_{g}^{c} \text { is the dose rate from a unit source in group " } \mathrm{g} \text { " for container "c" }
\end{aligned}
$$$$
\text { NG is the number of groups, } 77 \text { for photons or } 47 \text { for neutrons }
$$

The results are presented in Table 3-39 (No Shielded Container), Table 3-41 (SC2 Polyethylene Shielded Container), Table 3-43 (Primary Photon Dose Rate all Containers), Table 3-45 (Maximum Dose Rate without Impurities), and Table 3-46 (Maximum Dose Rate with Impurities).

The effects of self-shielding by $\mathrm{PuO}_{2}$ on the actinide dose rates are shown in Table 3-40. A comparison of the results in this table indicates that self-shielding by twenty-five grams of $\mathrm{PuO}_{2}$ has a negligible effect on dose rate.

Table 3-44 compares the dose rate from primary photons with and without shielding by low density iron $(1 \mathrm{~g} / \mathrm{cc})$. Examination of the results in this table indicates that the effects on primary photon dose rate are not significant for most isotopes. The exceptions are: Am-241, Cd-109, $\mathrm{Pu}-241$, and Sm-145.

The dose rates from Am-241 and Pu-241 (which decays to Am-241) are dominated by the neutron dose rate when impurities are present. Therefore, the effects of self-shielding on dose rate are not significant for these two isotopes.

The other two isotopes emit only low energy photons: less than $0.09 \mathrm{MeV}$ from Cd-109 and less than $0.07 \mathrm{MeV}$ from Sm-145. Both of these isotopes have allowed mass limits in excess of $1 \mathrm{~kg}$ when shipped in the SC-1 Lead Shielded Container or the SC-3 Tungsten Shielded Container.

The allowable mass for shipment under NCT (dose rate at the surface of the package $<2 \mathrm{mSv} / \mathrm{hr}$ [200 mrem/hr]) is listed in Table 3-47 (isotopes without impurities) and Table 3-49 (actinide isotopes with impurities). Previous calculations have shown the dose rate at the surface package is the limiting case for non-exclusive use shipments. The dose rate at the package surface also bounds the dose rate under hypothetical accident conditions.

The allowable mass for shipment under Exclusive Use (dose rate at the surface of the package $<10 \mathrm{mSv} / \mathrm{hr}$ [1000 mrem/hr]) is listed in Table 3-48 (isotopes without impurities) and Table 3-50 (actinide isotopes with impurities). 
PCP-2011-0001

Revision 0

Table 3-47 through Table 3-50 present the maximum amount of a single isotope that could be shipped within the regulatory dose limits at the surface of the package. If a package contains a mixture of isotopes, the acceptability for shipment can be determined by a sum of fractions approach. The package would be acceptable if:

$$
\sum_{i} \frac{M_{i}}{\text { Limit }_{i}} \leq
$$

Where:

$M_{i}$ is the mass of isotope $\mathrm{i}$ in the package

Limit $_{i}$ is the allowed mass from Table 3-47 through Table 3-50. 
PCP-2011-0001

Revision 0

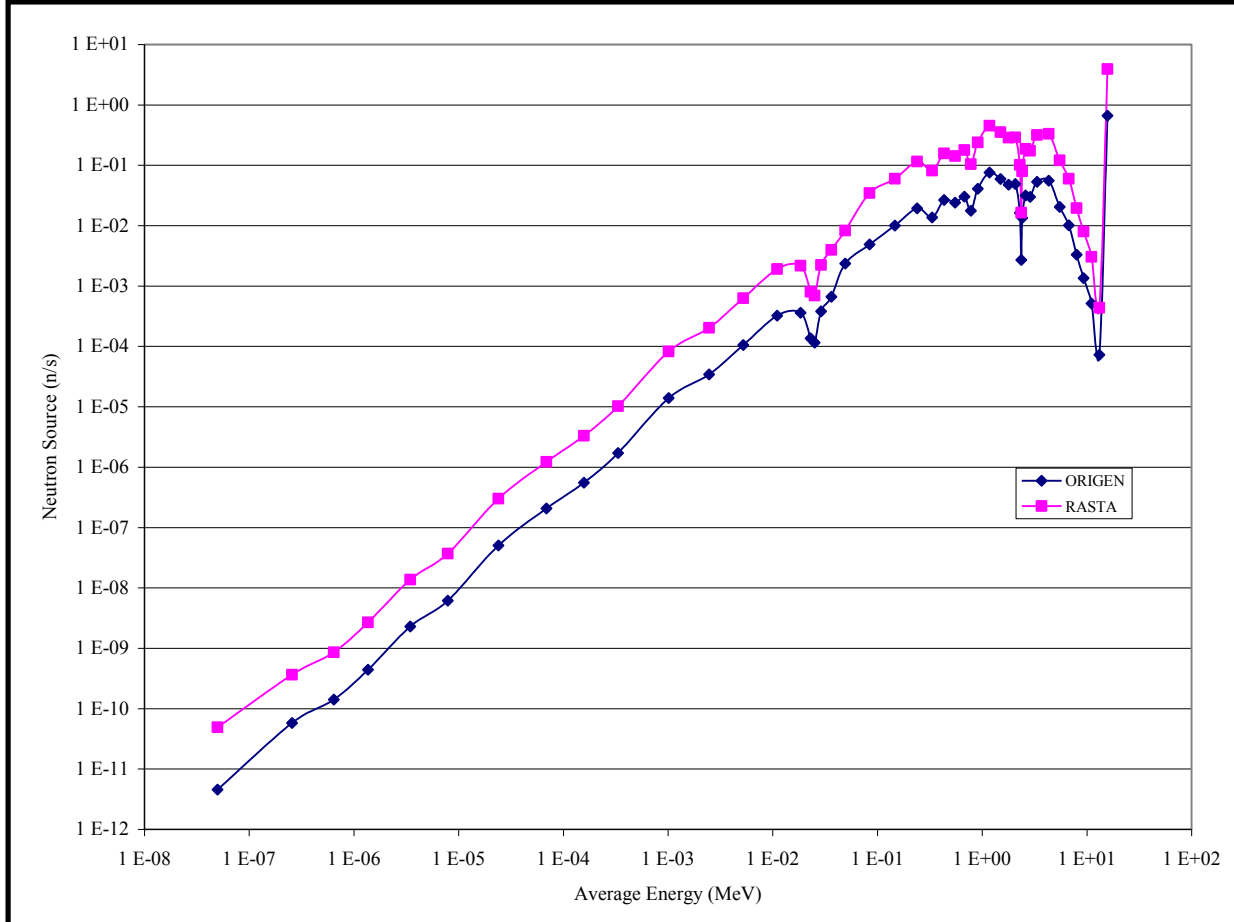

Figure 3-1. Am-243 Fission Neutron Spectrum

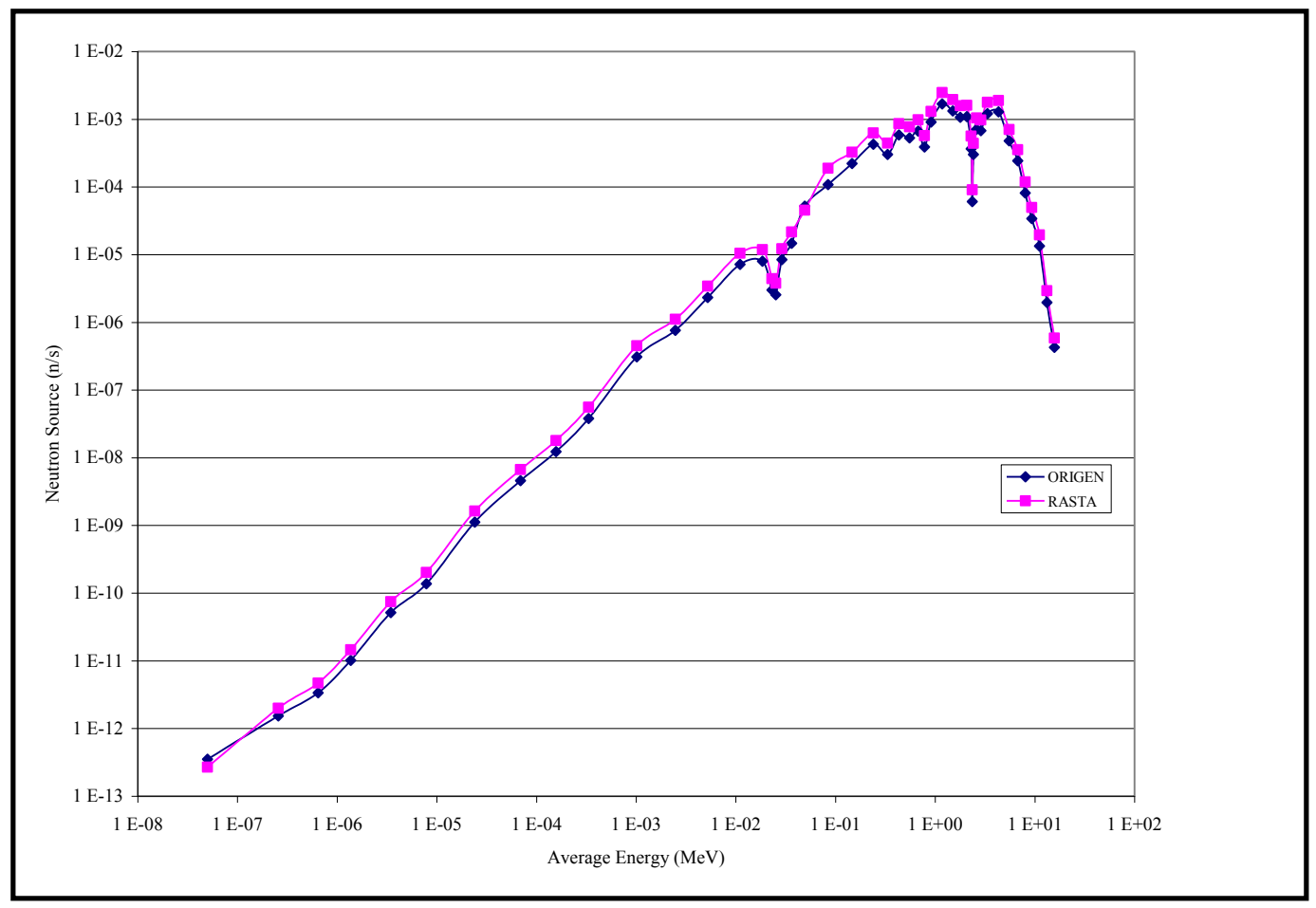

Figure 3-2. Pu-239 Fission Neutron Spectrum 
PCP-2011-0001

Revision 0

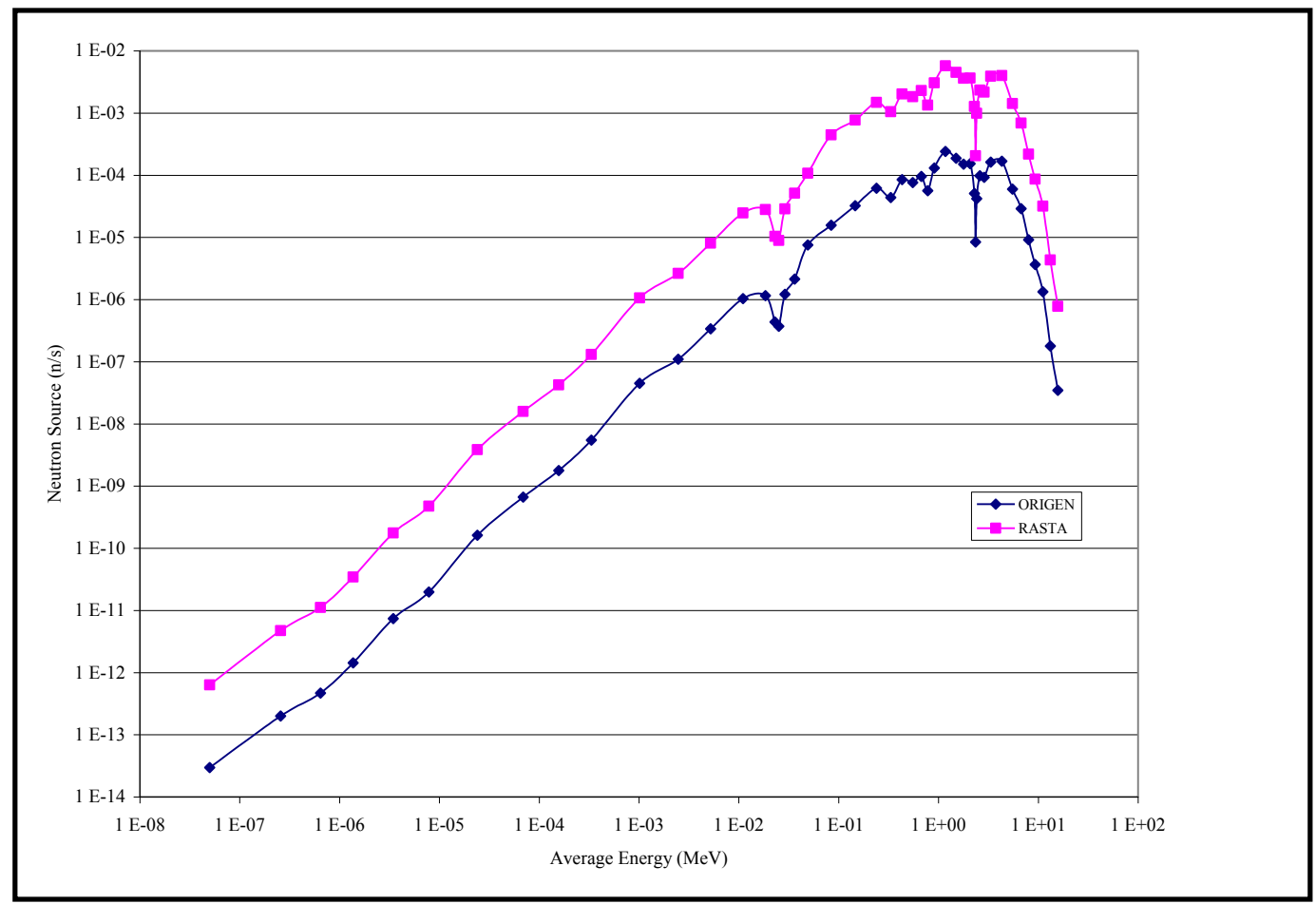

Figure 3-3. Pu-241 Fission Neutron Spectrum

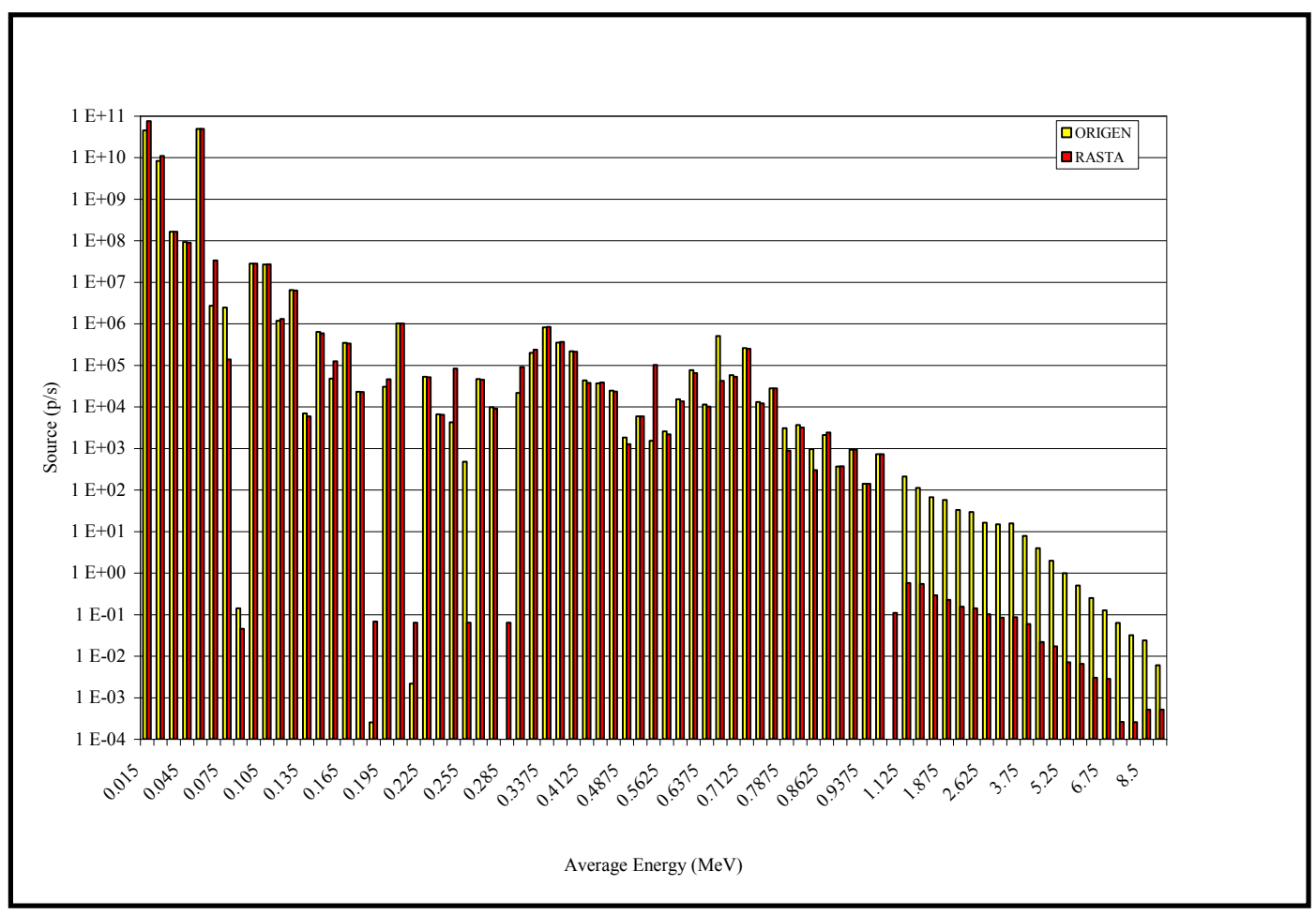

Figure 3-4. Am-241 Photon Spectra 
PCP-2011-0001

Revision 0

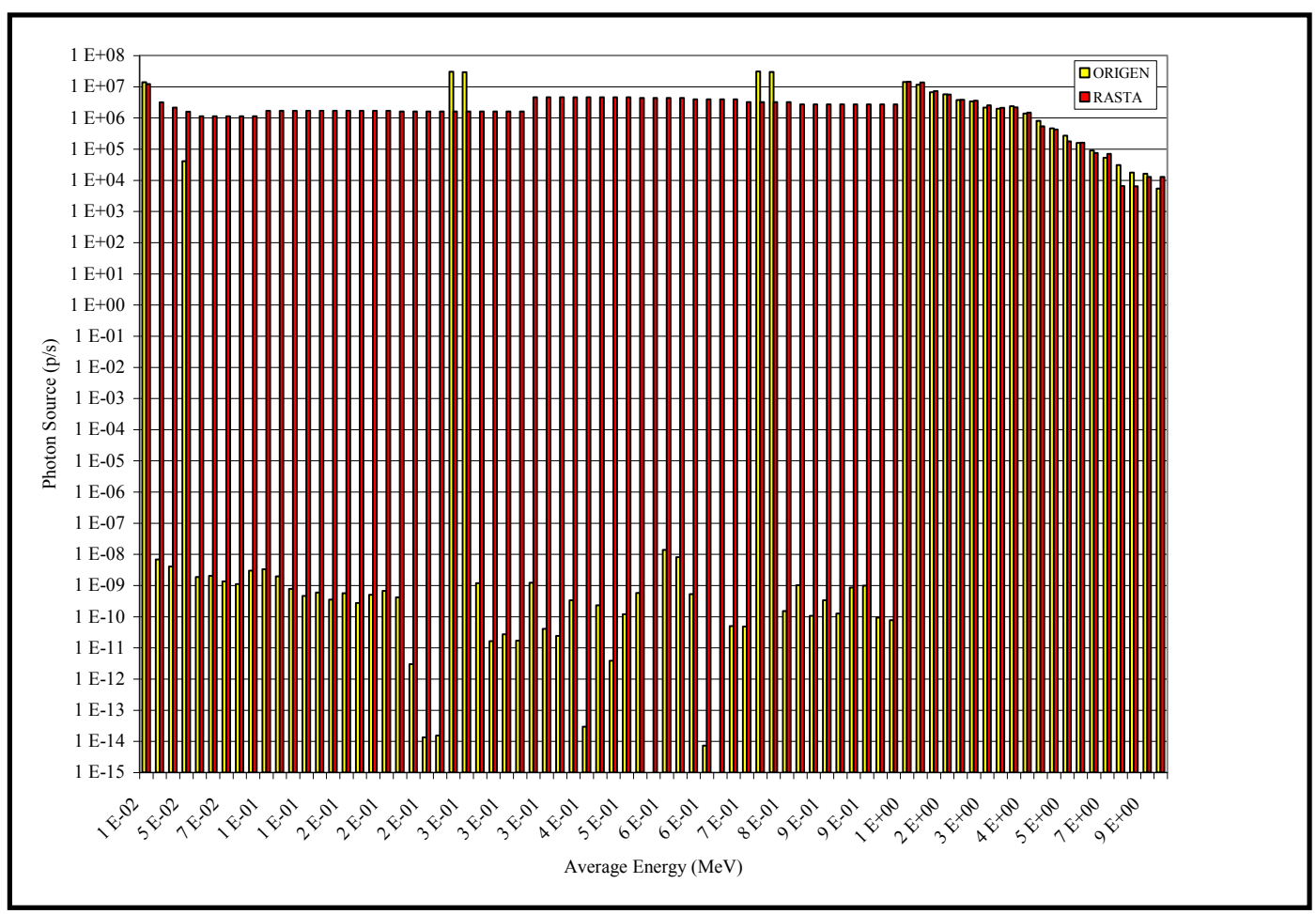

Figure 3-5. Cm-248 Photon Spectra

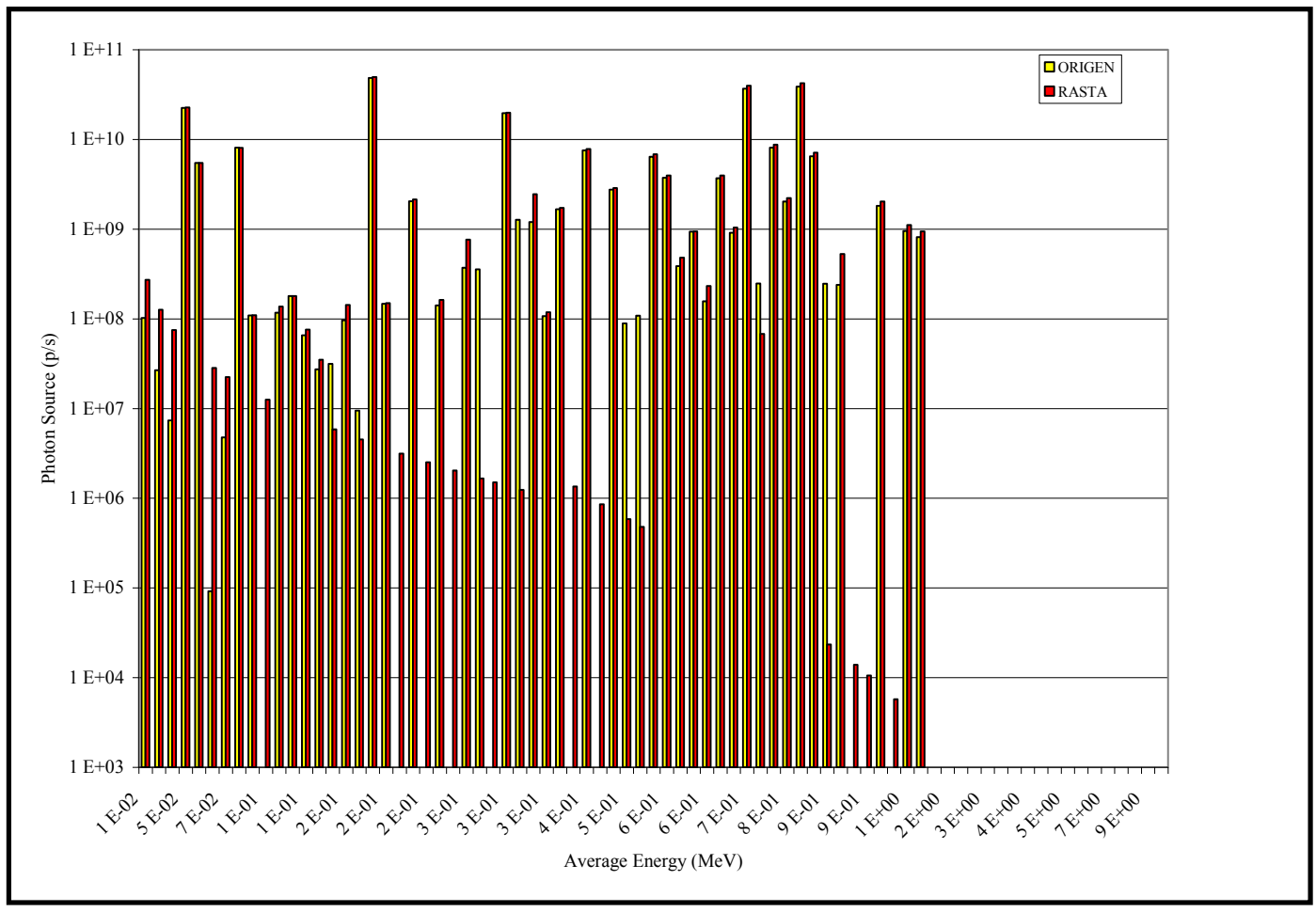

Figure 3-6. Ho-166m Photon Spectra 
PCP-2011-0001

Revision 0

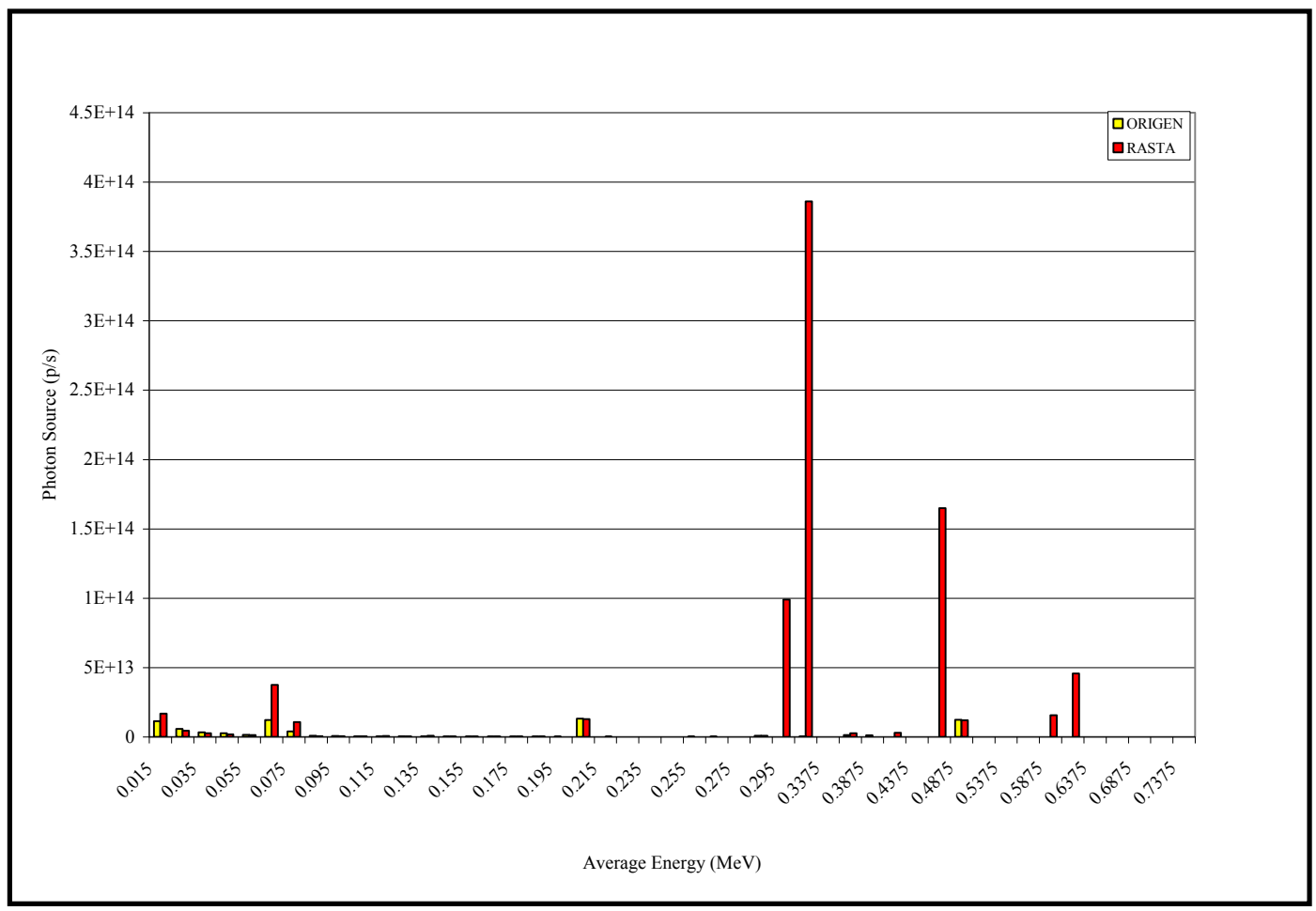

Figure 3-7. Ir-192 Photon Spectra

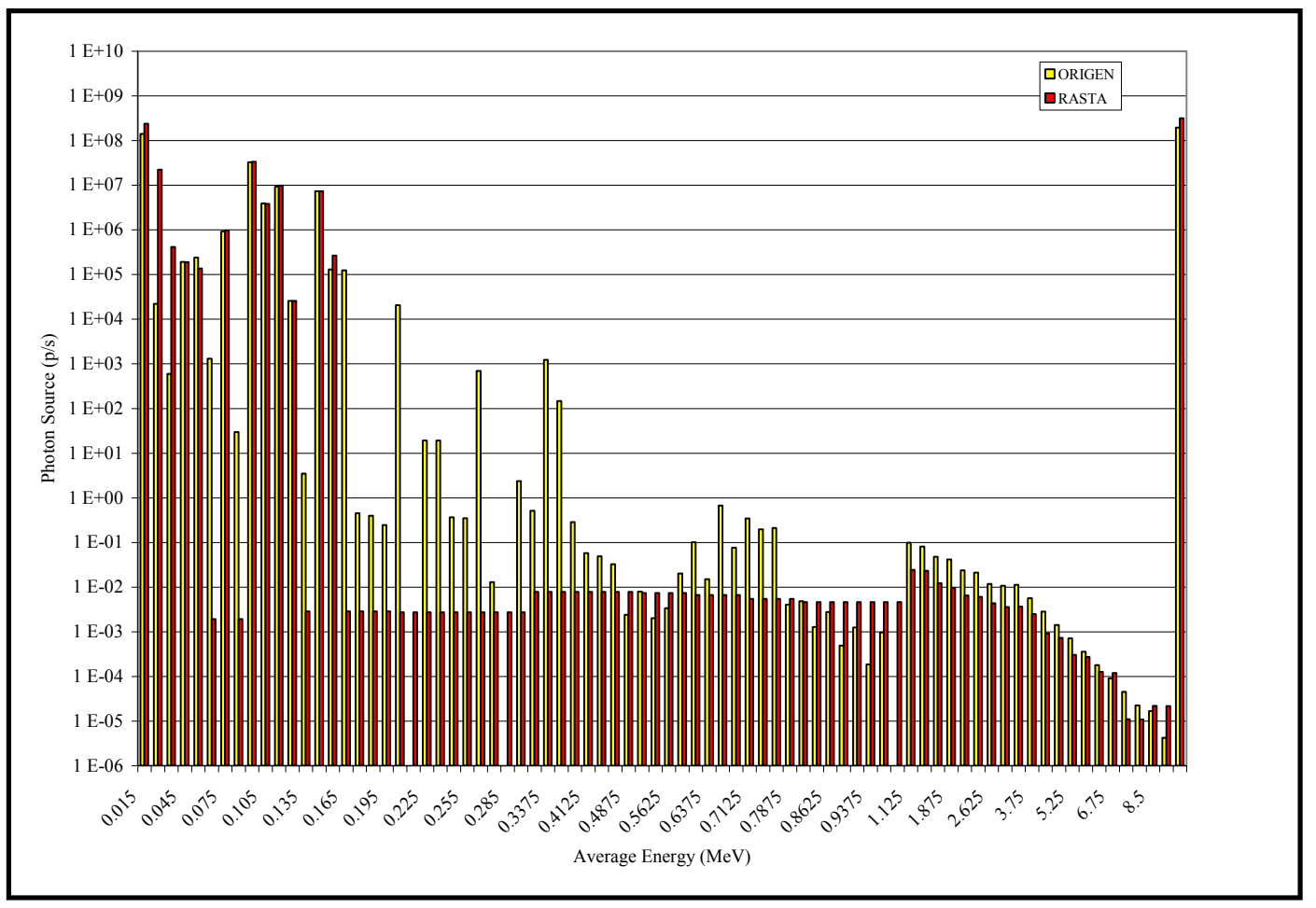

Figure 3-8. Pu-241 Photon Spectra 
PCP-2011-0001

Revision 0

Table 3-1. Neutron Source from ORIGEN-S w/o Beryllium (Decay Time $=0$ days)

\begin{tabular}{|c|c|c|c|c|c|c|c|c|c|c|c|c|}
\hline \multirow{2}{*}{$\begin{array}{l}\text { Energy } \\
\text { (MeV) } \\
\text { Upper }\end{array}$} & \multirow{2}{*}{\begin{tabular}{|l} 
Bound \\
Lower
\end{tabular}} & \multicolumn{11}{|c|}{ Neutron Source (n/s/g-actinide) } \\
\hline & & Am-241 & Am-243 & 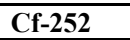 & Cm-244 & Cm-248 & Np-237 & Pu-238 & Pu-239 & Pu-240 & Pu-241 & Pu-242 \\
\hline $1.00 \mathrm{E}-07$ & $1.00 \mathrm{E}-11$ & $2.34 \mathrm{E}-11$ & $4.52 \mathrm{E}-12$ & $1.58 \mathrm{E}+01$ & $1.32 \mathrm{E}-04$ & $3.55 \mathrm{E}-04$ & $1.34 \mathrm{E}-15$ & $3.09 \mathrm{E}-08$ & $3.52 \mathrm{E}-13$ & $1.47 \mathrm{E}-08$ & $2.97 \mathrm{E}-14$ & $2.92 \mathrm{E}-08$ \\
\hline $4.14 \mathrm{E}-07$ & $1.00 \mathrm{E}-07$ & $1.17 \mathrm{E}-10$ & $5.75 \mathrm{E}-11$ & $1.76 \mathrm{E}+02$ & $9.83 \mathrm{E}-04$ & $3.79 \mathrm{E}-03$ & $1.07 \mathrm{E}-14$ & $2.41 \mathrm{E}-07$ & $1.54 \mathrm{E}-12$ & $1.03 \mathrm{E}-07$ & $2.00 \mathrm{E}-13$ & $1.76 \mathrm{E}-07$ \\
\hline $8.76 \mathrm{E}-07$ & $4.14 \mathrm{E}-07$ & $2.64 \mathrm{E}-10$ & $1.41 \mathrm{E}-10$ & $4.30 \mathrm{E}+02$ & $2.32 \mathrm{E}-03$ & $9.22 \mathrm{E}-03$ & $2.53 \mathrm{E}-14$ & $5.73 \mathrm{E}-07$ & $3.37 \mathrm{E}-12$ & $2.43 \mathrm{E}-07$ & $4.68 \mathrm{E}-13$ & $4.07 \mathrm{E}-07$ \\
\hline $1.86 \mathrm{E}-06$ & $8.76 \mathrm{E}-07$ & $7.99 \mathrm{E}-10$ & $4.41 \mathrm{E}-10$ & $1.34 \mathrm{E}+03$ & $7.15 \mathrm{E}-03$ & $2.87 \mathrm{E}-02$ & $7.82 \mathrm{E}-14$ & $1.77 \mathrm{E}-06$ & $1.01 \mathrm{E}-11$ & $7.46 \mathrm{E}-07$ & $1.44 \mathrm{E}-12$ & $1.24 \mathrm{E}-06$ \\
\hline $5.04 \mathrm{E}-06$ & $1.86 \mathrm{E}-06$ & $4.08 \mathrm{E}-09$ & $2.29 \mathrm{E}-09$ & $6.94 \mathrm{E}+03$ & $3.68 \mathrm{E}-02$ & $1.49 \mathrm{E}-01$ & $4.03 \mathrm{E}-13$ & $9.11 \mathrm{E}-06$ & $5.14 \mathrm{E}-11$ & $3.84 \mathrm{E}-06$ & $7.39 \mathrm{E}-12$ & $6.38 \mathrm{E}-06$ \\
\hline $1.07 \mathrm{E}-05$ & $5.04 \mathrm{E}-06$ & $1.09 \mathrm{E}-08$ & $6.15 \mathrm{E}-09$ & $1.86 \mathrm{E}+04$ & $9.86 \mathrm{E}-02$ & $3.99 \mathrm{E}-01$ & $1.08 \mathrm{E}-12$ & $2.44 \mathrm{E}-05$ & $1.37 \mathrm{E}-10$ & $1.03 \mathrm{E}-05$ & $1.98 \mathrm{E}-11$ & $1.71 \mathrm{E}-05$ \\
\hline $3.73 \mathrm{E}-05$ & & $8.89 \mathrm{E}-08$ & & $1.52 \mathrm{E}+05$ & & $3.26 \mathrm{E}+00$ & & $2.00 \mathrm{E}-04$ & & $8.40 \mathrm{E}-05$ & & \\
\hline $1.01 \mathrm{E}-04$ & $3.73 \mathrm{E}-05$ & $3.65 \mathrm{E}-07$ & $2.07 \mathrm{E}-07$ & $6.27 \mathrm{E}+05$ & $3.32 \mathrm{E}+00$ & $1.34 \mathrm{E}+01$ & & $8.21 \mathrm{E}-04$ & $4.59 \mathrm{E}-09$ & $3.45 \mathrm{E}-04$ & & \\
\hline $2.14 \mathrm{E}-04$ & $1.01 \mathrm{E}-04$ & $9.78 \mathrm{E}-07$ & $5.55 \mathrm{E}-07$ & $1.68 \mathrm{E}+06$ & $8.88 \mathrm{E}+00$ & $3.59 \mathrm{E}+01$ & $9.73 \mathrm{E}-11$ & $2.20 \mathrm{E}-03$ & $1.23 \mathrm{E}-08$ & $9.25 \mathrm{E}-04$ & $1.78 \mathrm{E}-09$ & $1.53 \mathrm{E}-03$ \\
\hline $4.54 \mathrm{E}-04$ & 14E-04 & $3.01 \mathrm{E}-06$ & $1.71 \mathrm{E}-06$ & $5.17 \mathrm{E}+06$ & $2.74 \mathrm{E}+01$ & $1.11 \mathrm{E}+02$ & $3.00 \mathrm{E}-10$ & $6.77 \mathrm{E}-03$ & $3.78 \mathrm{E}-08$ & $2.85 \mathrm{E}-03$ & $5.48 \mathrm{E}-09$ & $4.72 \mathrm{E}-03$ \\
\hline $1.58 \mathrm{E}-03$ & $4.54 \mathrm{E}-04$ & $2.46 \mathrm{E}-05$ & $1.40 \mathrm{E}-05$ & $4.22 \mathrm{E}+07$ & $2.24 \mathrm{E}+02$ & $9.04 \mathrm{E}+02$ & $2.45 \mathrm{E}-09$ & $5.53 \mathrm{E}-02$ & $3.09 \mathrm{E}-07$ & $2.33 \mathrm{E}-02$ & $4.48 \mathrm{E}-08$ & $3.86 \mathrm{E}-02$ \\
\hline $3.35 \mathrm{E}-03$ & $1.58 \mathrm{E}-03$ & 6.04E-05 & $3.43 \mathrm{E}-05$ & $1.04 \mathrm{E}+08$ & $5.49 \mathrm{E}+02$ & $2.22 \mathrm{E}+03$ & $6.01 \mathrm{E}-09$ & $1.36 \mathrm{E}-01$ & $7.59 \mathrm{E}-07$ & $5.72 \mathrm{E}-02$ & $1.10 \mathrm{E}-07$ & $9.48 \mathrm{E}-02$ \\
\hline $7.10 \mathrm{E}-03$ & $3.35 \mathrm{E}-03$ & $1.86 \mathrm{E}-04$ & $1.05 \mathrm{E}-04$ & $3.19 \mathrm{E}+08$ & $1.69 \mathrm{E}+03$ & $6.83 \mathrm{E}+03$ & $1.85 \mathrm{E}-08$ & $4.18 \mathrm{E}-01$ & $2.34 \mathrm{E}-06$ & $1.76 \mathrm{E}-01$ & $3.38 \mathrm{E}-07$ & $2.92 \mathrm{E}-01$ \\
\hline $1.50 \mathrm{E}-02$ & $7.10 \mathrm{E}-03$ & $5.71 \mathrm{E}-04$ & $3.24 \mathrm{E}-04$ & $9.80 \mathrm{E}+08$ & $5.18 \mathrm{E}+03$ & $2.10 \mathrm{E}+04$ & $5.68 \mathrm{E}-08$ & $1.28 \mathrm{E}+00$ & 7.17E-06 & $5.40 \mathrm{E}-01$ & $1.04 \mathrm{E}-06$ & $8.95 \mathrm{E}-01$ \\
\hline $2.19 \mathrm{E}-02$ & $1.50 \mathrm{E}-02$ & $6.36 \mathrm{E}-04$ & $3.61 \mathrm{E}-04$ & $1.09 \mathrm{E}+09$ & $5.78 \mathrm{E}+03$ & $2.34 \mathrm{E}+04$ & $6.33 \mathrm{E}-08$ & $1.43 \mathrm{E}+00$ & $7.99 \mathrm{E}-06$ & $6.02 \mathrm{E}-01$ & $1.16 \mathrm{E}-06$ & $9.97 \mathrm{E}-01$ \\
\hline $2.42 \mathrm{E}-02$ & $2.19 \mathrm{E}-02$ & $2.39 \mathrm{E}-04$ & $1.35 \mathrm{E}-04$ & $4.10 \mathrm{E}+08$ & $2.17 \mathrm{E}+03$ & $8.77 \mathrm{E}+03$ & $2.38 \mathrm{E}-08$ & $5.37 \mathrm{E}-01$ & $3.00 \mathrm{E}-06$ & $2.26 \mathrm{E}-01$ & $4.34 \mathrm{E}-07$ & $3.74 \mathrm{E}-01$ \\
\hline $2.61 \mathrm{E}-02$ & $2.42 \mathrm{E}-02$ & $2.04 \mathrm{E}-04$ & $1.16 \mathrm{E}-04$ & $3.50 \mathrm{E}+08$ & $1.85 \mathrm{E}+03$ & $7.49 \mathrm{E}+03$ & $2.03 \mathrm{E}-08$ & $4.58 \mathrm{E}-01$ & $2.56 \mathrm{E}-06$ & $1.93 \mathrm{E}-01$ & $3.71 \mathrm{E}-07$ & $3.20 \mathrm{E}-01$ \\
\hline $3.18 \mathrm{E}-02$ & $2.61 \mathrm{E}-02$ & $6.69 \mathrm{E}-04$ & $3.79 \mathrm{E}-04$ & $1.15 \mathrm{E}+09$ & $6.08 \mathrm{E}+03$ & $2.46 \mathrm{E}+04$ & $6.66 \mathrm{E}-08$ & $1.50 \mathrm{E}+00$ & $8.40 \mathrm{E}-06$ & $6.33 \mathrm{E}-01$ & & \\
\hline $4.09 \mathrm{E}-02$ & & & & $2.01 \mathrm{E}+09$ & & & & & & & & \\
\hline $6.74 \mathrm{E}-02$ & $4.09 \mathrm{E}-02$ & $4.14 \mathrm{E}-03$ & $2.35 \mathrm{E}-03$ & $7.11 \mathrm{E}+09$ & $3.76 \mathrm{E}+04$ & $1.52 \mathrm{E}+05$ & $4.12 \mathrm{E}-07$ & $9.31 \mathrm{E}+00$ & $5.20 \mathrm{E}-05$ & $3.92 \mathrm{E}+00$ & $7.53 \mathrm{E}-06$ & $6.49 \mathrm{E}+00$ \\
\hline $1.11 \mathrm{E}-01$ & $74 \mathrm{E}-02$ & $8.61 \mathrm{E}-03$ & E-03 & $1.48 \mathrm{E}+10$ & $7.82 \mathrm{E}+04$ & $3.17 \mathrm{E}+05$ & $8.57 \mathrm{E}-07$ & $\mathrm{E}+01$ & $1.08 \mathrm{E}-04$ & $\mathrm{E}+00$ & E-05 & $\mathrm{E}+01$ \\
\hline $1.83 \mathrm{E}-01$ & $\mathrm{E}-01$ & $1.77 \mathrm{E}-02$ & E-02 & $3.04 \mathrm{E}+10$ & +05 & $6.51 \mathrm{E}+05$ & $1.76 \mathrm{E}-06$ & $\mathrm{E}+01$ & $2.22 \mathrm{E}-04$ & $\mathrm{E}+01$ & E-05 & $8+01$ \\
\hline $2.97 \mathrm{E}-01$ & $\overline{E-01}$ & $3.41 \mathrm{E}-02$ & 1.9 & $5.87 \mathrm{E}+10$ & i+05 & $1.25 \mathrm{E}+06$ & E-06 & $\mathrm{E}+01$ & $8 \mathrm{E}-04$ & $\overline{\mathrm{E}+01}$ & $\mathrm{E}-05$ & $5.34 \mathrm{E}+01$ \\
\hline $3.69 \mathrm{E}-01$ & $2.97 \mathrm{E}-01$ & $2.41 \mathrm{E}-02$ & $1.36 \mathrm{E}-02$ & $4.15 \mathrm{E}+10$ & $2.19 \mathrm{E}+05$ & $8.84 \mathrm{E}+05$ & $2.39 \mathrm{E}-06$ & $5.41 \mathrm{E}+01$ & $3.02 \mathrm{E}-04$ & $2.28 \mathrm{E}+01$ & $4.36 \mathrm{E}-05$ & $3.76 \mathrm{E}+01$ \\
\hline $4.98 \mathrm{E}-01$ & $3.69 \mathrm{E}-01$ & $4.68 \mathrm{E}-02$ & $2.65 \mathrm{E}-02$ & $8.08 \mathrm{E}+10$ & $4.25 \mathrm{E}+05$ & $1.72 \mathrm{E}+06$ & 4.64E-06 & $1.05 \mathrm{E}+02$ & $5.86 \mathrm{E}-04$ & $4.42 \mathrm{E}+01$ & $8.47 \mathrm{E}-05$ & $7.30 \mathrm{E}+01$ \\
\hline $6.08 \mathrm{E}-01$ & $4.98 \mathrm{E}-01$ & $4.23 \mathrm{E}-02$ & $2.39 \mathrm{E}-02$ & $7.33 \mathrm{E}+10$ & $3.85 \mathrm{E}+05$ & $1.55 \mathrm{E}+06$ & $4.18 \mathrm{E}-06$ & $9.49 \mathrm{E}+01$ & $5.29 \mathrm{E}-04$ & $3.99 \mathrm{E}+01$ & $7.63 \mathrm{E}-05$ & $6.58 \mathrm{E}+01$ \\
\hline $7.43 \mathrm{E}-01$ & $6.08 \mathrm{E}-01$ & $5.33 \mathrm{E}-02$ & $3.00 \mathrm{E}-02$ & $9.26 \mathrm{E}+10$ & $4.84 \mathrm{E}+05$ & $1.94 \mathrm{E}+06$ & $5.25 \mathrm{E}-06$ & $1.19 \mathrm{E}+02$ & $6.66 \mathrm{E}-04$ & $5.00 \mathrm{E}+01$ & $9.59 \mathrm{E}-05$ & $8.25 \mathrm{E}+01$ \\
\hline $8.21 \mathrm{E}-01$ & $7.43 \mathrm{E}-01$ & $3.13 \mathrm{E}-02$ & $1.76 \mathrm{E}-02$ & $5.46 \mathrm{E}+10$ & $2.85 \mathrm{E}+05$ & $1.14 \mathrm{E}+06$ & $3.07 \mathrm{E}-06$ & $7.00 \mathrm{E}+01$ & $3.91 \mathrm{E}-04$ & $2.93 \mathrm{E}+01$ & $5.62 \mathrm{E}-05$ & $4.83 \mathrm{E}+01$ \\
\hline $1.00 \mathrm{E}+00$ & $8.21 \mathrm{E}-01$ & $7.29 \mathrm{E}-02$ & $4.09 \mathrm{E}-02$ & $1.28 \mathrm{E}+11$ & $6.62 \mathrm{E}+05$ & $2.63 \mathrm{E}+06$ & $7.12 \mathrm{E}-06$ & $1.62 \mathrm{E}+02$ & $9.07 \mathrm{E}-04$ & $6.78 \mathrm{E}+01$ & $1.30 \mathrm{E}-04$ & $1.12 \mathrm{E}+02$ \\
\hline $1.35 \mathrm{E}+00$ & $1.00 \mathrm{E}+00$ & $1.36 \mathrm{E}-01$ & $7.59 \mathrm{E}-02$ & $2.40 \mathrm{E}+11$ & $1.23 \mathrm{E}+06$ & $4.86 \mathrm{E}+06$ & $1.32 \mathrm{E}-05$ & $3.01 \mathrm{E}+02$ & $1.69 \mathrm{E}-03$ & $1.25 \mathrm{E}+02$ & $2.41 \mathrm{E}-04$ & $2.06 \mathrm{E}+02$ \\
\hline $1.65 \mathrm{E}+00$ & $1.35 \mathrm{E}+00$ & $1.08 \mathrm{E}-01$ & $5.93 \mathrm{E}-02$ & $1.92 \mathrm{E}+11$ & $9.72 \mathrm{E}+05$ & $3.77 \mathrm{E}+06$ & $1.02 \mathrm{E}-05$ & $\mathrm{E}+02$ & $1.32 \mathrm{E}-03$ & $9.66 \mathrm{E}+01$ & $1.87 \mathrm{E}-04$ & $1.60 \mathrm{E}+02$ \\
\hline $1.92 \mathrm{E}+00$ & $1.65 \mathrm{E}+00$ & $8.75 \mathrm{E}-02$ & $4.77 \mathrm{E}-02$ & $1.58 \mathrm{E}+11$ & & & & $\mathrm{E}+02$ & & $7.69 \mathrm{E}+01$ & & $1.28 \mathrm{E}+02$ \\
\hline $2.23 \mathrm{E}+00$ & $1.92 \mathrm{E}+00$ & $9.07 \mathrm{E}-02$ & $4.90 \mathrm{E}-02$ & $1.66 \mathrm{E}+11$ & $8.14 \mathrm{E}+05$ & 3.0 & $8.34 \mathrm{E}-06$ & $\mathrm{E}+02$ & $1.10 \mathrm{E}-03$ & $7.79 \mathrm{E}+01$ & $\overline{E-04}$ & $1.30 \mathrm{E}+02$ \\
\hline $2.35 \mathrm{E}+00$ & $2.23 \mathrm{E}+00$ & $3.04 \mathrm{E}-02$ & $1.63 \mathrm{E}-02$ & $5.63 \mathrm{E}+10$ & $2.72 \mathrm{E}+05$ & $\overline{E+06}$ & $2.76 \mathrm{E}-06$ & $\mathrm{E}+01$ & $3.66 \mathrm{E}-04$ & $2.57 \mathrm{E}+01$ & BE-05 & $4.28 \mathrm{E}+01$ \\
\hline $2.37 \mathrm{E}+00$ & $2.35 \mathrm{E}+00$ & $5.04 \mathrm{E}-03$ & $2.69 \mathrm{E}-03$ & $9.36 \mathrm{E}+09$ & $4.51 \mathrm{E}+04$ & $1.66 \mathrm{E}+05$ & $4.56 \mathrm{E}-07$ & $1.06 \mathrm{E}+01$ & $6.06 \mathrm{E}-05$ & $4.23 \mathrm{E}+00$ & $8.39 \mathrm{E}-06$ & $7.06 \mathrm{E}+00$ \\
\hline $2.47 \mathrm{E}+00$ & $2.37 \mathrm{E}+00$ & $2.53 \mathrm{E}-02$ & $1.35 \mathrm{E}-02$ & $4.70 \mathrm{E}+10$ & $2.26 \mathrm{E}+05$ & $8.28 \mathrm{E}+05$ & $2.27 \mathrm{E}-06$ & $5.29 \mathrm{E}+01$ & $3.03 \mathrm{E}-04$ & $2.11 \mathrm{E}+01$ & $4.19 \mathrm{E}-05$ & $3.52 \mathrm{E}+01$ \\
\hline $2.73 \mathrm{E}+00$ & $2.47 \mathrm{E}+00$ & $5.97 \mathrm{E}-02$ & $3.15 \mathrm{E}-02$ & $1.12 \mathrm{E}+11$ & $5.31 \mathrm{E}+05$ & $1.93 \mathrm{E}+06$ & $5.31 \mathrm{E}-06$ & $1.24 \mathrm{E}+02$ & $7.11 \mathrm{E}-04$ & $4.90 \mathrm{E}+01$ & $9.78 \mathrm{E}-05$ & $8.20 \mathrm{E}+01$ \\
\hline $3.01 \mathrm{E}+00$ & $2.73 \mathrm{E}+00$ & $5.75 \mathrm{E}-02$ & $3.00 \mathrm{E}-02$ & $1.10 \mathrm{E}+11$ & $5.09 \mathrm{E}+05$ & $1.81 \mathrm{E}+06$ & $5.02 \mathrm{E}-06$ & $1.18 \mathrm{E}+02$ & $6.79 \mathrm{E}-04$ & $4.59 \mathrm{E}+01$ & $9.26 \mathrm{E}-05$ & $7.72 \mathrm{E}+01$ \\
\hline $3.68 \mathrm{E}+00$ & $3.01 \mathrm{E}+00$ & $1.04 \mathrm{E}-01$ & $5.29 \mathrm{E}-02$ & $2.02 \mathrm{E}+11$ & $9.11 \mathrm{E}+05$ & $3.14 \mathrm{E}+06$ & $8.75 \mathrm{E}-06$ & $2.07 \mathrm{E}+02$ & $1.21 \mathrm{E}-03$ & $7.91 \mathrm{E}+01$ & $1.62 \mathrm{E}-04$ & $1.34 \mathrm{E}+02$ \\
\hline $4.97 \mathrm{E}+00$ & $3.68 \mathrm{E}+00$ & $1.14 \mathrm{E}-01$ & $5.56 \mathrm{E}-02$ & $2.35 \mathrm{E}+11$ & $9.85 \mathrm{E}+05$ & $3.16 \mathrm{E}+06$ & $8.97 \mathrm{E}-06$ & $2.15 \mathrm{E}+02$ & $1.29 \mathrm{E}-03$ & $7.88 \mathrm{E}+01$ & $1.67 \mathrm{E}-04$ & $1.36 \mathrm{E}+02$ \\
\hline $6.07 \mathrm{E}+00$ & $4.97 \mathrm{E}+00$ & $4.47 \mathrm{E}-02$ & $2.04 \mathrm{E}-02$ & $9.92 \mathrm{E}+10$ & $3.77 \mathrm{E}+05$ & $1.09 \mathrm{E}+06$ & $3.18 \mathrm{E}-06$ & $7.77 \mathrm{E}+01$ & $4.81 \mathrm{E}-04$ & $2.68 \mathrm{E}+01$ & $5.98 \mathrm{E}-05$ & $4.73 \mathrm{E}+01$ \\
\hline $7.41 \mathrm{E}+00$ & $6.07 \mathrm{E}+00$ & $2.37 \mathrm{E}-02$ & $1.01 \mathrm{E}-02$ & $5.69 \mathrm{E}+10$ & $1.94 \mathrm{E}+05$ & $5.09 \mathrm{E}+05$ & $1.52 \mathrm{E}-06$ & $3.79 \mathrm{E}+01$ & $2.44 \mathrm{E}-04$ & $1.23 \mathrm{E}+01$ & $2.89 \mathrm{E}-05$ & $2.22 \mathrm{E}+01$ \\
\hline $8.61 \mathrm{E}+00$ & $7.41 \mathrm{E}+00$ & $8.34 \mathrm{E}-03$ & $3.30 \mathrm{E}-03$ & $2.19 \mathrm{E}+10$ & & $1.54 \mathrm{E}+05$ & $4.76 \mathrm{E}-07$ & $1.21 \mathrm{E}+01$ & $8.13 \mathrm{E}-05$ & $3.66 \mathrm{E}+00$ & $9.15 \mathrm{E}-06$ & $6.82 \mathrm{E}+00$ \\
\hline $1.00 \mathrm{E}+01$ & $8.61 \mathrm{E}+00$ & $3.69 \mathrm{E}-03$ & $1.35 \mathrm{E}-03$ & $1.06 \mathrm{E}+10$ & $2.85 \mathrm{E}+04$ & $5.88 \mathrm{E}+04$ & $1.87 \mathrm{E}-07$ & $4.87 \mathrm{E}+00$ & $3.41 \mathrm{E}-05$ & $1.37 \mathrm{E}+00$ & $3.64 \mathrm{E}-06$ & $2.63 \mathrm{E}+00$ \\
\hline $1.22 \mathrm{E}+01$ & $1.00 \mathrm{E}+01$ & $1.55 \mathrm{E}-03$ & $5.12 \mathrm{E}-04$ & $5.00 \mathrm{E}+09$ & $1.15 \mathrm{E}+04$ & $2.03 \mathrm{E}+04$ & $6.73 \mathrm{E}-08$ & $1.80 \mathrm{E}+00$ & $1.34 \mathrm{E}-05$ & $4.61 \mathrm{E}-01$ & $1.33 \mathrm{E}-06$ & $9.22 \mathrm{E}-01$ \\
\hline $1.42 \mathrm{E}+01$ & $1.22 \mathrm{E}+01$ & $2.52 \mathrm{E}-04$ & $7.22 \mathrm{E}-05$ & $9.53 \mathrm{E}+08$ & $1.76 \mathrm{E}+03$ & $2.51 \mathrm{E}+03$ & $8.83 \mathrm{E}-09$ & $2.46 \mathrm{E}-01$ & $1.97 \mathrm{E}-06$ & $5.52 \mathrm{E}-02$ & $1.78 \mathrm{E}-07$ & $1.17 \mathrm{E}-01$ \\
\hline \multirow[t]{2}{*}{$1.96 \mathrm{E}+01$} & $1.42 \mathrm{E}+01$ & $6.06 \mathrm{E}-05$ & $1.48 \mathrm{E}-05$ & $2.76 \mathrm{E}+08$ & $3.98 \mathrm{E}+02$ & $4.47 \mathrm{E}+02$ & $1.68 \mathrm{E}-09$ & $4.86 \mathrm{E}-02$ & $4.25 \mathrm{E}-07$ & $9.46 \mathrm{E}-03$ & $3.46 \mathrm{E}-08$ & $2.13 \mathrm{E}-02$ \\
\hline & Total & $1.24 \mathrm{E}+00$ & $6.61 \mathrm{E}-01$ & $2.31 \mathrm{E}+12$ & $1.10 \mathrm{E}+07$ & $4.09 \mathrm{E}+07$ & $1.12 \mathrm{E}-04$ & $2.60 \mathrm{E}+03$ & 1.49E-02 & $1.04 \mathrm{E}+03$ & $2.07 \mathrm{E}-03$ & $1.74 \mathrm{E}+03$ \\
\hline
\end{tabular}


PCP-2011-0001

Revision 0

Table 3-2. Neutron Source from ORIGEN-S w/o Beryllium (Decay Time $=30$ days)

\begin{tabular}{|c|c|c|c|c|c|c|c|c|c|c|c|c|}
\hline \multicolumn{2}{|l|}{$\begin{array}{l}\text { Energy } \\
(\mathrm{MeV})\end{array}$} & \multicolumn{11}{|c|}{ Neutron Source (n/s/g-actinide) } \\
\hline Upper & Lower & Am-241 & Am-243 & Cf-252 & Cm-244 & Cm-248 & Np-237 & Pu-238 & Pu-239 & Pu-240 & Pu-241 & Pu-242 \\
\hline $1.00 \mathrm{E}-07$ & $1.00 \mathrm{E}-11$ & $2.34 \mathrm{E}-11$ & $4.52 \mathrm{E}-12$ & $1.54 \mathrm{E}+01$ & $1.32 \mathrm{E}-04$ & $3.55 \mathrm{E}-04$ & $1.34 \mathrm{E}-15$ & $3.09 \mathrm{E}-08$ & $3.52 \mathrm{E}-13$ & $1.47 \mathrm{E}-08$ & $1.22 \mathrm{E}-13$ & $2.92 \mathrm{E}-08$ \\
\hline $4.14 \mathrm{E}-07$ & $1.00 \mathrm{E}-07$ & $1.17 \mathrm{E}-10$ & $5.75 \mathrm{E}-11$ & $1.72 \mathrm{E}+02$ & $9.80 \mathrm{E}-04$ & $3.79 \mathrm{E}-03$ & $1.07 \mathrm{E}-14$ & $2.41 \mathrm{E}-07$ & $1.54 \mathrm{E}-12$ & $1.03 \mathrm{E}-07$ & $6.64 \mathrm{E}-13$ & $1.76 \mathrm{E}-07$ \\
\hline $8.76 \mathrm{E}-07$ & $4.14 \mathrm{E}-07$ & $2.64 \mathrm{E}-10$ & $1.41 \mathrm{E}-10$ & $4.21 \mathrm{E}+02$ & $2.31 \mathrm{E}-03$ & $9.22 \mathrm{E}-03$ & $2.53 \mathrm{E}-14$ & $5.72 \mathrm{E}-07$ & $3.37 \mathrm{E}-12$ & $2.43 \mathrm{E}-07$ & $1.51 \mathrm{E}-12$ & $4.07 \mathrm{E}-07$ \\
\hline $1.86 \mathrm{E}-06$ & $8.76 \mathrm{E}-07$ & $7.99 \mathrm{E}-10$ & $4.41 \mathrm{E}-10$ & $1.31 \mathrm{E}+03$ & $7.12 \mathrm{E}-03$ & $2.87 \mathrm{E}-02$ & $7.82 \mathrm{E}-14$ & $1.77 \mathrm{E}-06$ & $1.01 \mathrm{E}-11$ & $7.46 \mathrm{E}-07$ & $4.59 \mathrm{E}-12$ & $1.24 \mathrm{E}-06$ \\
\hline $5.04 \mathrm{E}-06$ & $1.86 \mathrm{E}-06$ & 4.08E-09 & $2.29 \mathrm{E}-09$ & $6.79 \mathrm{E}+03$ & $3.67 \mathrm{E}-02$ & $1.49 \mathrm{E}-01$ & $4.03 \mathrm{E}-13$ & $9.11 \mathrm{E}-06$ & $5.14 \mathrm{E}-11$ & $3.84 \mathrm{E}-06$ & $2.35 \mathrm{E}-11$ & $6.38 \mathrm{E}-06$ \\
\hline $1.07 \mathrm{E}-05$ & $5.04 \mathrm{E}-06$ & $1.09 \mathrm{E}-08$ & $6.15 \mathrm{E}-09$ & $1.82 \mathrm{E}+04$ & $9.83 \mathrm{E}-02$ & $3.99 \mathrm{E}-01$ & & $2.44 \mathrm{E}-05$ & $1.37 \mathrm{E}-10$ & & & \\
\hline $3.73 \mathrm{E}-05$ & $1.07 \mathrm{E}-05$ & $8.89 \mathrm{E}-08$ & & $1.49 \mathrm{E}+05$ & $8.04 \mathrm{E}-01$ & $3.26 \mathrm{E}+00$ & & $2.00 \mathrm{E}-04$ & & & & $1.39 \mathrm{E}-04$ \\
\hline $1.01 \mathrm{E}-04$ & $3.73 \mathrm{E}-05$ & $3.65 \mathrm{E}-07$ & $2.07 \mathrm{E}-07$ & $6.13 \mathrm{E}+05$ & $3.31 \mathrm{E}+00$ & $1.34 \mathrm{E}+01$ & $3.63 \mathrm{E}-11$ & $8.20 \mathrm{E}-04$ & $4.59 \mathrm{E}-09$ & $3.45 \mathrm{E}-04$ & $2.11 \mathrm{E}-09$ & $5.73 \mathrm{E}-04$ \\
\hline $2.14 \mathrm{E}-04$ & $1.01 \mathrm{E}-04$ & $9.78 \mathrm{E}-07$ & $5.55 \mathrm{E}-07$ & $1.64 \mathrm{E}+06$ & $8.85 \mathrm{E}+00$ & $3.59 \mathrm{E}+01$ & $9.73 \mathrm{E}-11$ & $2.20 \mathrm{E}-03$ & $1.23 \mathrm{E}-08$ & $9.25 \mathrm{E}-04$ & $5.64 \mathrm{E}-09$ & $1.53 \mathrm{E}-03$ \\
\hline $4.54 \mathrm{E}-04$ & $2.14 \mathrm{E}-04$ & $3.01 \mathrm{E}-06$ & $1.71 \mathrm{E}-06$ & $5.06 \mathrm{E}+06$ & $2.73 \mathrm{E}+01$ & $1.11 \mathrm{E}+02$ & $3.00 \mathrm{E}-10$ & $6.77 \mathrm{E}-03$ & $3.78 \mathrm{E}-08$ & $2.85 \mathrm{E}-03$ & $1.74 \mathrm{E}-08$ & $4.72 \mathrm{E}-03$ \\
\hline $1.58 \mathrm{E}-03$ & $4.54 \mathrm{E}-04$ & $2.46 \mathrm{E}-05$ & $1.40 \mathrm{E}-05$ & $4.13 \mathrm{E}+07$ & $2.23 \mathrm{E}+02$ & $9.04 \mathrm{E}+02$ & $2.45 \mathrm{E}-09$ & $5.53 \mathrm{E}-02$ & $3.09 \mathrm{E}-07$ & $2.33 \mathrm{E}-02$ & $1.42 \mathrm{E}-07$ & $3.86 \mathrm{E}-02$ \\
\hline $3.35 \mathrm{E}-03$ & $1.58 \mathrm{E}-03$ & $6.04 \mathrm{E}-05$ & $3.43 \mathrm{E}-05$ & $1.02 \mathrm{E}+08$ & $5.47 \mathrm{E}+02$ & $2.22 \mathrm{E}+03$ & $6.01 \mathrm{E}-09$ & $1.36 \mathrm{E}-01$ & $7.59 \mathrm{E}-07$ & $5.72 \mathrm{E}-02$ & $3.49 \mathrm{E}-07$ & $9.48 \mathrm{E}-02$ \\
\hline $7.10 \mathrm{E}-03$ & $3.35 \mathrm{E}-03$ & $1.86 \mathrm{E}-04$ & $1.05 \mathrm{E}-04$ & $3.12 \mathrm{E}+08$ & $1.68 \mathrm{E}+03$ & $6.83 \mathrm{E}+03$ & $1.85 \mathrm{E}-08$ & $4.18 \mathrm{E}-01$ & $2.34 \mathrm{E}-06$ & $1.76 \mathrm{E}-01$ & $1.07 \mathrm{E}-06$ & $2.92 \mathrm{E}-01$ \\
\hline $1.50 \mathrm{E}-02$ & $7.10 \mathrm{E}-03$ & $5.71 \mathrm{E}-04$ & $3.24 \mathrm{E}-04$ & $9.59 \mathrm{E}+08$ & $5.17 \mathrm{E}+03$ & $2.10 \mathrm{E}+04$ & $5.68 \mathrm{E}-08$ & $1.28 \mathrm{E}+00$ & 7.17E-06 & $5.40 \mathrm{E}-01$ & $3.29 \mathrm{E}-06$ & $8.95 \mathrm{E}-01$ \\
\hline $2.19 \mathrm{E}-02$ & $1.50 \mathrm{E}-02$ & $6.36 \mathrm{E}-04$ & $3.61 \mathrm{E}-04$ & $1.07 \mathrm{E}+09$ & $5.76 \mathrm{E}+03$ & $2.34 \mathrm{E}+04$ & $6.33 \mathrm{E}-08$ & $1.43 \mathrm{E}+00$ & 7.99E-06 & $6.02 \mathrm{E}-01$ & $3.67 \mathrm{E}-06$ & $9.97 \mathrm{E}-01$ \\
\hline $2.42 \mathrm{E}-02$ & $2.19 \mathrm{E}-02$ & $2.39 \mathrm{E}-04$ & $1.35 \mathrm{E}-04$ & $4.01 \mathrm{E}+08$ & $2.16 \mathrm{E}+03$ & $8.77 \mathrm{E}+03$ & $2.38 \mathrm{E}-08$ & $5.36 \mathrm{E}-01$ & $3.00 \mathrm{E}-06$ & $2.26 \mathrm{E}-01$ & $1.38 \mathrm{E}-06$ & $3.74 \mathrm{E}-01$ \\
\hline $2.61 \mathrm{E}-02$ & $2.42 \mathrm{E}-02$ & $2.04 \mathrm{E}-04$ & $1.16 \mathrm{E}-04$ & $3.42 \mathrm{E}+08$ & $1.85 \mathrm{E}+03$ & $7.49 \mathrm{E}+03$ & $2.03 \mathrm{E}-08$ & $4.58 \mathrm{E}-01$ & $2.56 \mathrm{E}-06$ & $1.93 \mathrm{E}-01$ & & \\
\hline $3.18 \mathrm{E}-02$ & $2.61 \mathrm{E}-02$ & $6.69 \mathrm{E}-04$ & $3.79 \mathrm{E}-04$ & $1.12 \mathrm{E}+09$ & $6.06 \mathrm{E}+03$ & $2.46 \mathrm{E}+04$ & & $1.50 \mathrm{E}+00$ & $8.40 \mathrm{E}-06$ & & & $\overline{\mathrm{E}+00}$ \\
\hline $4.09 \mathrm{E}-02$ & $\mathrm{E}-02$ & $1.17 \mathrm{E}-03$ & $6.64 \mathrm{E}-04$ & $1.97 \mathrm{E}+09$ & $1.06 \mathrm{E}+04$ & $4.30 \mathrm{E}+04$ & $1.16 \mathrm{E}-07$ & $2.63 \mathrm{E}+00$ & $1.47 \mathrm{E}-05$ & $1.11 \mathrm{E}+00$ & $6.75 \mathrm{E}-06$ & $1.84 \mathrm{E}+00$ \\
\hline $6.74 \mathrm{E}-02$ & $\mathrm{E}-02$ & $4.14 \mathrm{E}-03$ & $2.35 \mathrm{E}-03$ & $6.96 \mathrm{E}+09$ & $3.75 \mathrm{E}+04$ & $1.52 \mathrm{E}+05$ & E-07 & $9.31 \mathrm{E}+00$ & $5.20 \mathrm{E}-05$ & $\overline{E+00}$ & $\overline{E-05}$ & $\overline{\mathrm{E}+00}$ \\
\hline $1.11 \mathrm{E}-01$ & $74 \mathrm{E}-02$ & $8.61 \mathrm{E}-03$ & $4.88 \mathrm{E}-03$ & $1.45 \mathrm{E}+10$ & $7.80 \mathrm{E}+04$ & $3.17 \mathrm{E}+05$ & $8.57 \mathrm{E}-07$ & $1.94 \mathrm{E}+01$ & $1.08 \mathrm{E}-04$ & $\mathrm{E}+00$ & $4.97 \mathrm{E}-05$ & $\mathrm{E}+01$ \\
\hline $1.83 \mathrm{E}-01$ & $11 \mathrm{E}-01$ & $1.77 \mathrm{E}-02$ & $1.00 \mathrm{E}-02$ & $\overline{\mathrm{E}+10}$ & $1.60 \mathrm{E}+05$ & $6.51 \mathrm{E}+05$ & $\mathrm{E}-06$ & $\mathrm{E}+01$ & $2.22 \mathrm{E}-04$ & $\mathrm{E}+01$ & $2 \mathrm{E}-04$ & $E+01$ \\
\hline $2.97 \mathrm{E}-01$ & 1.83 & 3.411 & $1.93 \mathrm{E}-02$ & $5.74 \mathrm{E}+10$ & $3.09 \mathrm{E}+05$ & $\mathrm{E}+06$ & & $6 \mathrm{E}+01$ & $8 \mathrm{E}-04$ & & E-04 & $\mathrm{E}+01$ \\
\hline $3.69 \mathrm{E}-01$ & $2.97 \mathrm{E}-01$ & $2.41 \mathrm{E}-02$ & $1.36 \mathrm{E}-02$ & $4.06 \mathrm{E}+10$ & $2.18 \mathrm{E}+05$ & $8.84 \mathrm{E}+05$ & $2.39 \mathrm{E}-06$ & $5.41 \mathrm{E}+01$ & $3.02 \mathrm{E}-04$ & $\overline{\mathrm{E}+01}$ & $1.39 \mathrm{E}-04$ & $3.76 \mathrm{E}+01$ \\
\hline $4.98 \mathrm{E}-01$ & $3.69 \mathrm{E}-01$ & $4.68 \mathrm{E}-02$ & $2.65 \mathrm{E}-02$ & $7.91 \mathrm{E}+10$ & $4.24 \mathrm{E}+05$ & $1.72 \mathrm{E}+06$ & $4.64 \mathrm{E}-06$ & $1.05 \mathrm{E}+02$ & $5.86 \mathrm{E}-04$ & $4.42 \mathrm{E}+01$ & $2.70 \mathrm{E}-04$ & $7.30 \mathrm{E}+01$ \\
\hline $6.08 \mathrm{E}-01$ & $4.98 \mathrm{E}-01$ & $4.23 \mathrm{E}-02$ & $2.39 \mathrm{E}-02$ & $7.17 \mathrm{E}+10$ & $3.83 \mathrm{E}+05$ & $1.55 \mathrm{E}+06$ & $4.18 \mathrm{E}-06$ & $9.49 \mathrm{E}+01$ & $5.29 \mathrm{E}-04$ & $3.99 \mathrm{E}+01$ & $2.44 \mathrm{E}-04$ & $6.58 \mathrm{E}+01$ \\
\hline $7.43 \mathrm{E}-01$ & $6.08 \mathrm{E}-01$ & $5.33 \mathrm{E}-02$ & $3.00 \mathrm{E}-02$ & $9.06 \mathrm{E}+10$ & $4.83 \mathrm{E}+05$ & $1.94 \mathrm{E}+06$ & $5.25 \mathrm{E}-06$ & $1.19 \mathrm{E}+02$ & $6.66 \mathrm{E}-04$ & $5.00 \mathrm{E}+01$ & $3.07 \mathrm{E}-04$ & $8.25 \mathrm{E}+01$ \\
\hline $8.21 \mathrm{E}-01$ & $7.43 \mathrm{E}-01$ & $3.13 \mathrm{E}-02$ & $1.76 \mathrm{E}-02$ & $5.34 \mathrm{E}+10$ & $2.84 \mathrm{E}+05$ & $1.14 \mathrm{E}+06$ & $3.07 \mathrm{E}-06$ & $6.99 \mathrm{E}+01$ & $3.91 \mathrm{E}-04$ & $2.93 \mathrm{E}+01$ & $1.80 \mathrm{E}-04$ & $4.83 \mathrm{E}+01$ \\
\hline $1.00 \mathrm{E}+00$ & $8.21 \mathrm{E}-01$ & $7.29 \mathrm{E}-02$ & $4.09 \mathrm{E}-02$ & $1.25 \mathrm{E}+11$ & $6.60 \mathrm{E}+05$ & $2.63 \mathrm{E}+06$ & $7.12 \mathrm{E}-06$ & $1.62 \mathrm{E}+02$ & $9.07 \mathrm{E}-04$ & $6.78 \mathrm{E}+01$ & $4.18 \mathrm{E}-04$ & $1.12 \mathrm{E}+02$ \\
\hline $1.35 \mathrm{E}+00$ & $1.00 \mathrm{E}+00$ & $1.36 \mathrm{E}-01$ & $7.59 \mathrm{E}-02$ & $2.35 \mathrm{E}+11$ & $1.23 \mathrm{E}+06$ & $4.86 \mathrm{E}+06$ & $1.32 \mathrm{E}-05$ & $3.01 \mathrm{E}+02$ & $1.69 \mathrm{E}-03$ & $1.25 \mathrm{E}+02$ & $7.79 \mathrm{E}-04$ & $2.06 \mathrm{E}+02$ \\
\hline $1.65 \mathrm{E}+00$ & & $1.08 \mathrm{E}-01$ & $5.93 \mathrm{E}-02$ & $1.88 \mathrm{E}+11$ & $9.69 \mathrm{E}+05$ & $3.77 \mathrm{E}+06$ & & $2.35 \mathrm{E}+02$ & & & & $1.60 \mathrm{E}+02$ \\
\hline $1.92 \mathrm{E}+00$ & $1.65 \mathrm{E}+00$ & $8.75 \mathrm{E}-02$ & $4.77 \mathrm{E}-02$ & $1.55 \mathrm{E}+11$ & $7.85 \mathrm{E}+05$ & $3.01 \mathrm{E}+06$ & $8.18 \mathrm{E}-06$ & $1.89 \mathrm{E}+02$ & $1.07 \mathrm{E}-03$ & $7.69 \mathrm{E}+01$ & $4.96 \mathrm{E}-04$ & $\mathrm{E}+02$ \\
\hline $2.23 \mathrm{E}+00$ & $1.92 \mathrm{E}+00$ & $9.07 \mathrm{E}-02$ & $\overline{E-02}$ & $1.63 \mathrm{E}+11$ & $8.11 \mathrm{E}+05$ & $3.05 \mathrm{E}+06$ & $8.34 \mathrm{E}-06$ & $1.93 \mathrm{E}+02$ & $\mathrm{E}-03$ & $7.79 \mathrm{E}+01$ & $2 \mathrm{E}-04$ & $\mathrm{E}+02$ \\
\hline $2.35 \mathrm{E}+00$ & $2.23 \mathrm{E}+00$ & $3.04 \mathrm{E}-02$ & $\mathrm{E}-02$ & $5.51 \mathrm{E}+10$ & $2.71 \mathrm{E}+05$ & $\mathrm{E}+06$ & 2.7 & $\bar{E}+01$ & $6 \mathrm{E}-04$ & $7 \mathrm{E}+01$ & E-04 & $\mathrm{E}+01$ \\
\hline $2.37 \mathrm{E}+00$ & $2.35 \mathrm{E}+00$ & $5.04 \mathrm{E}-03$ & $2.69 \mathrm{E}-03$ & $9.16 \mathrm{E}+09$ & $4.49 \mathrm{E}+04$ & $1.66 \mathrm{E}+05$ & $4.56 \mathrm{E}-07$ & $1.06 \mathrm{E}+01$ & $6.06 \mathrm{E}-05$ & $4.23 \mathrm{E}+00$ & $2.83 \mathrm{E}-05$ & $7.06 \mathrm{E}+00$ \\
\hline $2.47 \mathrm{E}+00$ & $2.37 \mathrm{E}+00$ & $2.53 \mathrm{E}-02$ & $1.35 \mathrm{E}-02$ & $4.60 \mathrm{E}+10$ & $2.25 \mathrm{E}+05$ & $8.28 \mathrm{E}+05$ & $2.27 \mathrm{E}-06$ & $5.29 \mathrm{E}+01$ & $3.03 \mathrm{E}-04$ & $2.11 \mathrm{E}+01$ & $1.42 \mathrm{E}-04$ & $3.52 \mathrm{E}+01$ \\
\hline $2.73 \mathrm{E}+00$ & $2.47 \mathrm{E}+00$ & $5.97 \mathrm{E}-02$ & $3.15 \mathrm{E}-02$ & $1.10 \mathrm{E}+11$ & $5.30 \mathrm{E}+05$ & $1.93 \mathrm{E}+06$ & $5.31 \mathrm{E}-06$ & $1.24 \mathrm{E}+02$ & $7.11 \mathrm{E}-04$ & $4.90 \mathrm{E}+01$ & $3.34 \mathrm{E}-04$ & $8.20 \mathrm{E}+01$ \\
\hline $3.01 \mathrm{E}+00$ & $2.73 \mathrm{E}+00$ & $5.75 \mathrm{E}-02$ & $3.00 \mathrm{E}-02$ & $1.07 \mathrm{E}+11$ & $5.08 \mathrm{E}+05$ & $1.81 \mathrm{E}+06$ & $5.02 \mathrm{E}-06$ & $1.18 \mathrm{E}+02$ & $6.79 \mathrm{E}-04$ & $4.59 \mathrm{E}+01$ & $3.20 \mathrm{E}-04$ & $7.72 \mathrm{E}+01$ \\
\hline $3.68 \mathrm{E}+00$ & $3.01 \mathrm{E}+00$ & $1.04 \mathrm{E}-01$ & $5.29 \mathrm{E}-02$ & $1.98 \mathrm{E}+11$ & $9.09 \mathrm{E}+05$ & $3.14 \mathrm{E}+06$ & $8.75 \mathrm{E}-06$ & $2.06 \mathrm{E}+02$ & $1.21 \mathrm{E}-03$ & $7.91 \mathrm{E}+01$ & $5.72 \mathrm{E}-04$ & $1.34 \mathrm{E}+02$ \\
\hline $4.97 \mathrm{E}+00$ & $3.68 \mathrm{E}+00$ & $1.14 \mathrm{E}-01$ & $5.56 \mathrm{E}-02$ & $2.30 \mathrm{E}+11$ & $9.82 \mathrm{E}+05$ & $3.16 \mathrm{E}+06$ & $8.97 \mathrm{E}-06$ & $2.15 \mathrm{E}+02$ & $1.29 \mathrm{E}-03$ & $7.88 \mathrm{E}+01$ & $6.18 \mathrm{E}-04$ & $1.36 \mathrm{E}+02$ \\
\hline $6.07 \mathrm{E}+00$ & $4.97 \mathrm{E}+00$ & $4.47 \mathrm{E}-02$ & $2.04 \mathrm{E}-02$ & $9.71 \mathrm{E}+10$ & $3.75 \mathrm{E}+05$ & $1.09 \mathrm{E}+06$ & $3.18 \mathrm{E}-06$ & $7.76 \mathrm{E}+01$ & $4.81 \mathrm{E}-04$ & $2.68 \mathrm{E}+01$ & $2.36 \mathrm{E}-04$ & $4.73 \mathrm{E}+01$ \\
\hline $7.41 \mathrm{E}+00$ & $6.07 \mathrm{E}+00$ & $2.37 \mathrm{E}-02$ & $1.01 \mathrm{E}-02$ & $5.56 \mathrm{E}+10$ & $1.94 \mathrm{E}+05$ & $5.09 \mathrm{E}+05$ & $1.52 \mathrm{E}-06$ & $3.79 \mathrm{E}+01$ & $2.44 \mathrm{E}-04$ & $1.23 \mathrm{E}+01$ & $1.23 \mathrm{E}-04$ & $2.22 \mathrm{E}+01$ \\
\hline $8.61 \mathrm{E}+00$ & $7.41 \mathrm{E}+00$ & $8.34 \mathrm{E}-03$ & & $2.14 \mathrm{E}+10$ & $6.62 \mathrm{E}+04$ & $1.54 \mathrm{E}+05$ & $4.76 \mathrm{E}-07$ & $1.21 \mathrm{E}+01$ & $8.13 \mathrm{E}-05$ & $3.66 \mathrm{E}+00$ & $4.21 \mathrm{E}-05$ & $6.82 \mathrm{E}+00$ \\
\hline $1.00 \mathrm{E}+01$ & & & & & & & & & & & $1.82 \mathrm{E}-05$ & $2.63 \mathrm{E}+00$ \\
\hline $1.22 \mathrm{E}+01$ & $1.00 \mathrm{E}+01$ & & & & & & & $1.80 \mathrm{E}+00$ & $1.34 \mathrm{E}-05$ & & $7.46 \mathrm{E}-06$ & $9.22 \mathrm{E}-01$ \\
\hline $1.42 \mathrm{E}+01$ & $1.22 \mathrm{E}+01$ & $2.52 \mathrm{E}-04$ & $7.22 \mathrm{E}-05$ & $9.33 \mathrm{E}+08$ & $1.76 \mathrm{E}+03$ & $2.51 \mathrm{E}+03$ & $8.83 \mathrm{E}-09$ & $2.46 \mathrm{E}-01$ & $1.97 \mathrm{E}-06$ & $5.52 \mathrm{E}-02$ & $1.18 \mathrm{E}-06$ & $1.17 \mathrm{E}-01$ \\
\hline \multirow[t]{2}{*}{$1.96 \mathrm{E}+01$} & $1.42 \mathrm{E}+01$ & $6.06 \mathrm{E}-05$ & $1.48 \mathrm{E}-05$ & $2.70 \mathrm{E}+08$ & $3.97 \mathrm{E}+02$ & $4.47 \mathrm{E}+02$ & $1.68 \mathrm{E}-09$ & $4.86 \mathrm{E}-02$ & $4.25 \mathrm{E}-07$ & $9.46 \mathrm{E}-03$ & $2.74 \mathrm{E}-07$ & $2.13 \mathrm{E}-02$ \\
\hline & Total & $1.24 \mathrm{E}+00$ & $6.61 \mathrm{E}-01$ & $2.26 \mathrm{E}+12$ & $1.10 \mathrm{E}+07$ & $4.09 \mathrm{E}+07$ & $1.12 \mathrm{E}-04$ & $2.60 \mathrm{E}+03$ & $1.49 \mathrm{E}-02$ & $1.04 \mathrm{E}+03$ & $6.96 \mathrm{E}-03$ & $1.74 \mathrm{E}+03$ \\
\hline
\end{tabular}


PCP-2011-0001

Revision 0

Table 3-3. Neutron Source from ORIGEN-S w/o Beryllium (Decay Time $=100$ days)

\begin{tabular}{|c|c|c|c|c|c|c|c|c|c|c|c|c|}
\hline \multirow{2}{*}{$\begin{array}{l}\text { Energy } \\
\text { (MeV) } \\
\text { Upper }\end{array}$} & \multirow{2}{*}{$\begin{array}{l}\text { Bound } \\
\text { Lower }\end{array}$} & \multicolumn{11}{|c|}{ Neutron Source (n/s/g-actinide) } \\
\hline & & Am-241 & Am-243 & Cf-252 & $\mathrm{Cm}-244$ & Cm-248 & Np-237 & Pu-238 & Pu-239 & Pu-240 & Pu-241 & Pu-242 \\
\hline $1.00 \mathrm{E}-07$ & $1.00 \mathrm{E}-11$ & $2.34 \mathrm{E}-11$ & $4.52 \mathrm{E}-12$ & $1.47 \mathrm{E}+01$ & $1.31 \mathrm{E}-04$ & $3.55 \mathrm{E}-04$ & $1.34 \mathrm{E}-15$ & $3.09 \mathrm{E}-08$ & $3.52 \mathrm{E}-13$ & $1.47 \mathrm{E}-08$ & $3.36 \mathrm{E}-13$ & $2.92 \mathrm{E}-08$ \\
\hline $4.14 \mathrm{E}-07$ & $1.00 \mathrm{E}-07$ & $1.17 \mathrm{E}-10$ & $5.75 \mathrm{E}-11$ & $1.64 \mathrm{E}+02$ & $9.73 \mathrm{E}-04$ & $3.79 \mathrm{E}-03$ & $1.07 \mathrm{E}-14$ & $2.41 \mathrm{E}-07$ & $1.54 \mathrm{E}-12$ & $1.03 \mathrm{E}-07$ & $1.74 \mathrm{E}-12$ & $1.76 \mathrm{E}-07$ \\
\hline $8.76 \mathrm{E}-07$ & $4.14 \mathrm{E}-07$ & $2.64 \mathrm{E}-10$ & $1.41 \mathrm{E}-10$ & $4.00 \mathrm{E}+02$ & $2.30 \mathrm{E}-03$ & $9.22 \mathrm{E}-03$ & $2.53 \mathrm{E}-14$ & $5.71 \mathrm{E}-07$ & $3.37 \mathrm{E}-12$ & $2.43 \mathrm{E}-07$ & $3.93 \mathrm{E}-12$ & $4.07 \mathrm{E}-07$ \\
\hline $1.86 \mathrm{E}-06$ & $8.76 \mathrm{E}-07$ & $7.99 \mathrm{E}-10$ & $4.41 \mathrm{E}-10$ & $1.25 \mathrm{E}+03$ & $7.07 \mathrm{E}-03$ & $2.87 \mathrm{E}-02$ & $7.82 \mathrm{E}-14$ & $1.76 \mathrm{E}-06$ & $1.01 \mathrm{E}-11$ & $7.46 \mathrm{E}-07$ & $1.19 \mathrm{E}-11$ & $1.24 \mathrm{E}-06$ \\
\hline $5.04 \mathrm{E}-06$ & $1.86 \mathrm{E}-06$ & $4.08 \mathrm{E}-09$ & $2.29 \mathrm{E}-09$ & $6.46 \mathrm{E}+03$ & $3.65 \mathrm{E}-02$ & $1.49 \mathrm{E}-01$ & $4.03 \mathrm{E}-13$ & $9.09 \mathrm{E}-06$ & $5.14 \mathrm{E}-11$ & $3.84 \mathrm{E}-06$ & $6.09 \mathrm{E}-11$ & $6.38 \mathrm{E}-06$ \\
\hline $1.07 \mathrm{E}-05$ & $5.04 \mathrm{E}-06$ & $1.09 \mathrm{E}-08$ & $6.15 \mathrm{E}-09$ & $1.73 \mathrm{E}+04$ & $9.76 \mathrm{E}-02$ & $3.99 \mathrm{E}-01$ & $1.08 \mathrm{E}-12$ & $2.44 \mathrm{E}-05$ & $1.37 \mathrm{E}-10$ & $1.03 \mathrm{E}-05$ & $1.63 \mathrm{E}-10$ & $1.71 \mathrm{E}-05$ \\
\hline $3.73 \mathrm{E}-05$ & $1.07 \mathrm{E}-05$ & $8.89 \mathrm{E}-08$ & $5.03 \mathrm{E}-08$ & $1.42 \mathrm{E}+05$ & $7.98 \mathrm{E}-01$ & $3.26 \mathrm{E}+00$ & $8.84 \mathrm{E}-12$ & $1.99 \mathrm{E}-04$ & $1.12 \mathrm{E}-09$ & $8.40 \mathrm{E}-05$ & $1.33 \mathrm{E}-09$ & $1.39 \mathrm{E}-04$ \\
\hline $1.01 \mathrm{E}-04$ & $3.73 \mathrm{E}-05$ & $3.65 \mathrm{E}-07$ & $2.07 \mathrm{E}-07$ & $5.83 \mathrm{E}+05$ & $3.28 \mathrm{E}+00$ & & & $8.19 \mathrm{E}-04$ & & $3.45 \mathrm{E}-04$ & & \\
\hline $2.14 \mathrm{E}-04$ & $1.01 \mathrm{E}-04$ & $9.78 \mathrm{E}-07$ & $5.55 \mathrm{E}-07$ & $1.56 \mathrm{E}+06$ & $8.79 \mathrm{E}+00$ & & & $2.19 \mathrm{E}-03$ & & $9.25 \mathrm{E}-04$ & & \\
\hline $4.54 \mathrm{E}-04$ & $2.14 \mathrm{E}-04$ & $3.01 \mathrm{E}-06$ & $1.71 \mathrm{E}-06$ & $4.81 \mathrm{E}+06$ & $2.71 \mathrm{E}+01$ & $1.11 \mathrm{E}+02$ & $3.00 \mathrm{E}-10$ & $6.76 \mathrm{E}-03$ & $3.78 \mathrm{E}-08$ & $2.85 \mathrm{E}-03$ & $4.50 \mathrm{E}-08$ & $4.72 \mathrm{E}-03$ \\
\hline $1.58 \mathrm{E}-03$ & $4.54 \mathrm{E}-04$ & $2.46 \mathrm{E}-05$ & $1.40 \mathrm{E}-05$ & $3.93 \mathrm{E}+07$ & $2.21 \mathrm{E}+02$ & $9.04 \mathrm{E}+02$ & $2.45 \mathrm{E}-09$ & $5.52 \mathrm{E}-02$ & $3.09 \mathrm{E}-07$ & $2.33 \mathrm{E}-02$ & $3.68 \mathrm{E}-07$ & E-02 \\
\hline $3.35 \mathrm{E}-03$ & $58 \mathrm{E}-03$ & $6.04 \mathrm{E}-05$ & $3.43 \mathrm{E}-05$ & $9.65 \mathrm{E}+07$ & $5.43 \mathrm{E}+02$ & $2.22 \mathrm{E}+03$ & $6.01 \mathrm{E}-09$ & $1.36 \mathrm{E}-01$ & $7.59 \mathrm{E}-07$ & $5.71 \mathrm{E}-02$ & $9.02 \mathrm{E}-07$ & $\mathrm{E}-02$ \\
\hline $7.10 \mathrm{E}-03$ & $35 \mathrm{E}-03$ & $1.86 \mathrm{E}-04$ & $1.05 \mathrm{E}-04$ & $2.97 \mathrm{E}+08$ & $1.67 \mathrm{E}+03$ & $6.83 \mathrm{E}+03$ & $1.85 \mathrm{E}-08$ & $4.17 \mathrm{E}-01$ & $2.34 \mathrm{E}-06$ & $1.76 \mathrm{E}-01$ & $2.78 \mathrm{E}-06$ & $2 \mathrm{E}-01$ \\
\hline $1.50 \mathrm{E}-02$ & $7.10 \mathrm{E}-03$ & $5.71 \mathrm{E}-04$ & $3.24 \mathrm{E}-04$ & $9.12 \mathrm{E}+08$ & $5.13 \mathrm{E}+03$ & $2.10 \mathrm{E}+04$ & $5.68 \mathrm{E}-08$ & $1.28 \mathrm{E}+00$ & $7.17 \mathrm{E}-06$ & $5.40 \mathrm{E}-01$ & $8.52 \mathrm{E}-06$ & $8.95 \mathrm{E}-01$ \\
\hline $2.19 \mathrm{E}-02$ & $1.50 \mathrm{E}-02$ & $6.36 \mathrm{E}-04$ & $3.61 \mathrm{E}-04$ & $1.02 \mathrm{E}+09$ & $5.72 \mathrm{E}+03$ & $2.34 \mathrm{E}+04$ & $6.33 \mathrm{E}-08$ & $1.43 \mathrm{E}+00$ & 7.99E-06 & $6.02 \mathrm{E}-01$ & $9.50 \mathrm{E}-06$ & $9.97 \mathrm{E}-01$ \\
\hline $2.42 \mathrm{E}-02$ & $2.19 \mathrm{E}-02$ & $2.39 \mathrm{E}-04$ & $1.35 \mathrm{E}-04$ & $3.81 \mathrm{E}+08$ & $2.15 \mathrm{E}+03$ & $8.77 \mathrm{E}+03$ & $2.38 \mathrm{E}-08$ & $5.35 \mathrm{E}-01$ & $3.00 \mathrm{E}-06$ & $2.26 \mathrm{E}-01$ & $3.56 \mathrm{E}-06$ & $3.74 \mathrm{E}-01$ \\
\hline $2.61 \mathrm{E}-02$ & $2.42 \mathrm{E}-02$ & $2.04 \mathrm{E}-04$ & $1.16 \mathrm{E}-04$ & $3.26 \mathrm{E}+08$ & $1.83 \mathrm{E}+03$ & $7.49 \mathrm{E}+03$ & $2.03 \mathrm{E}-08$ & $4.57 \mathrm{E}-01$ & $2.56 \mathrm{E}-06$ & $1.93 \mathrm{E}-01$ & $3.04 \mathrm{E}-06$ & $3.20 \mathrm{E}-01$ \\
\hline $3.18 \mathrm{E}-02$ & $2.61 \mathrm{E}-02$ & $6.69 \mathrm{E}-04$ & $3.79 \mathrm{E}-04$ & $1.07 \mathrm{E}+09$ & $6.01 \mathrm{E}+03$ & $2.46 \mathrm{E}+04$ & $6.66 \mathrm{E}-08$ & $1.50 \mathrm{E}+00$ & $8.40 \mathrm{E}-06$ & $6.33 \mathrm{E}-01$ & $9.99 \mathrm{E}-06$ & $1.05 \mathrm{E}+00$ \\
\hline $4.09 \mathrm{E}-02$ & $3.18 \mathrm{E}-02$ & $1.17 \mathrm{E}-03$ & $6.64 \mathrm{E}-04$ & $1.87 \mathrm{E}+09$ & $1.05 \mathrm{E}+04$ & $4.30 \mathrm{E}+04$ & $1.16 \mathrm{E}-07$ & $2.63 \mathrm{E}+00$ & $1.47 \mathrm{E}-05$ & $1.11 \mathrm{E}+00$ & $1.75 \mathrm{E}-05$ & \\
\hline $6.74 \mathrm{E}-02$ & & $4.14 \mathrm{E}-03$ & & $6.62 \mathrm{E}+09$ & $3.72 \mathrm{E}+04$ & & & $9.29 \mathrm{E}+00$ & & $3.92 \mathrm{E}+00$ & & \\
\hline $1.11 \mathrm{E}-01$ & 74E-02 & $8.61 \mathrm{E}-03$ & $4.88 \mathrm{E}-03$ & $1.38 \mathrm{E}+10$ & $7.74 \mathrm{E}+04$ & $3.17 \mathrm{E}+05$ & $8.57 \mathrm{E}-07$ & $1.93 \mathrm{E}+01$ & $1.08 \mathrm{E}-04$ & $8.15 \mathrm{E}+00$ & $1.29 \mathrm{E}-04$ & $1.35 \mathrm{E}+01$ \\
\hline $1.83 \mathrm{E}-01$ & $11 \mathrm{E}-01$ & $1.77 \mathrm{E}-02$ & $1.00 \mathrm{E}-02$ & $2.83 \mathrm{E}+10$ & $1.59 \mathrm{E}+05$ & $6.51 \mathrm{E}+05$ & $1.76 \mathrm{E}-06$ & $3.97 \mathrm{E}+01$ & $2.22 \mathrm{E}-04$ & $1.68 \mathrm{E}+01$ & E-04 & $2.77 \mathrm{E}+01$ \\
\hline $2.97 \mathrm{E}-01$ & -01 & $3.41 \mathrm{E}-02$ & -02 & +10 & +05 & $\mathrm{E}+06$ & E-06 & $7.65 \mathrm{E}+01$ & E-04 & +01 & E-04 & +01 \\
\hline $3.69 \mathrm{E}-01$ & $97 \mathrm{E}-01$ & $2.41 \mathrm{E}-02$ & $1.36 \mathrm{E}-02$ & $3.86 \mathrm{E}+10$ & $\mathrm{E}+05$ & $8.84 \mathrm{E}+05$ & $2.39 \mathrm{E}-06$ & $5.40 \mathrm{E}+01$ & $3.02 \mathrm{E}-04$ & $2.28 \mathrm{E}+01$ & $3.59 \mathrm{E}-04$ & $3.76 \mathrm{E}+01$ \\
\hline $4.98 \mathrm{E}-01$ & $3.69 \mathrm{E}-01$ & $4.68 \mathrm{E}-02$ & $2.65 \mathrm{E}-02$ & $7.52 \mathrm{E}+10$ & $4.21 \mathrm{E}+05$ & $1.72 \mathrm{E}+06$ & $4.64 \mathrm{E}-06$ & $1.05 \mathrm{E}+02$ & $5.86 \mathrm{E}-04$ & $4.42 \mathrm{E}+01$ & $6.98 \mathrm{E}-04$ & $7.30 \mathrm{E}+01$ \\
\hline $6.08 \mathrm{E}-01$ & $4.98 \mathrm{E}-01$ & 4.23E-02 & $2.39 \mathrm{E}-02$ & $6.82 \mathrm{E}+10$ & $3.81 \mathrm{E}+05$ & $1.55 \mathrm{E}+06$ & $4.18 \mathrm{E}-06$ & $9.47 \mathrm{E}+01$ & 5.29E-04 & $3.99 \mathrm{E}+01$ & $6.31 \mathrm{E}-04$ & $6.58 \mathrm{E}+01$ \\
\hline $7.43 \mathrm{E}-01$ & $6.08 \mathrm{E}-01$ & $5.33 \mathrm{E}-02$ & $3.00 \mathrm{E}-02$ & $8.62 \mathrm{E}+10$ & $4.79 \mathrm{E}+05$ & $1.94 \mathrm{E}+06$ & $5.25 \mathrm{E}-06$ & $1.19 \mathrm{E}+02$ & $6.66 \mathrm{E}-04$ & $5.00 \mathrm{E}+01$ & $7.95 \mathrm{E}-04$ & $8.25 \mathrm{E}+01$ \\
\hline $8.21 \mathrm{E}-01$ & $7.43 \mathrm{E}-01$ & $3.13 \mathrm{E}-02$ & $1.76 \mathrm{E}-02$ & $5.08 \mathrm{E}+10$ & $2.82 \mathrm{E}+05$ & $1.14 \mathrm{E}+06$ & $3.07 \mathrm{E}-06$ & $6.98 \mathrm{E}+01$ & $3.91 \mathrm{E}-04$ & $2.93 \mathrm{E}+01$ & $4.67 \mathrm{E}-04$ & $4.83 \mathrm{E}+01$ \\
\hline $1.00 \mathrm{E}+00$ & $8.21 \mathrm{E}-01$ & $7.29 \mathrm{E}-02$ & $4.09 \mathrm{E}-02$ & $1.19 \mathrm{E}+11$ & $6.55 \mathrm{E}+05$ & $2.63 \mathrm{E}+06$ & $7.12 \mathrm{E}-06$ & $1.62 \mathrm{E}+02$ & $9.07 \mathrm{E}-04$ & $6.78 \mathrm{E}+01$ & $1.09 \mathrm{E}-03$ & $1.12 \mathrm{E}+02$ \\
\hline $1.35 \mathrm{E}+00$ & $1.00 \mathrm{E}+00$ & $1.36 \mathrm{E}-01$ & $7.59 \mathrm{E}-02$ & $2.24 \mathrm{E}+11$ & $1.22 \mathrm{E}+06$ & $4.86 \mathrm{E}+06$ & $1.32 \mathrm{E}-05$ & $3.00 \mathrm{E}+02$ & $1.69 \mathrm{E}-03$ & $1.25 \mathrm{E}+02$ & $2.03 \mathrm{E}-03$ & $2.06 \mathrm{E}+02$ \\
\hline $1.65 \mathrm{E}+00$ & $1.35 \mathrm{E}+00$ & $1.08 \mathrm{E}-01$ & $5.93 \mathrm{E}-02$ & $1.79 \mathrm{E}+11$ & $9.62 \mathrm{E}+05$ & $3.77 \mathrm{E}+06$ & $1.02 \mathrm{E}-05$ & $2.35 \mathrm{E}+02$ & $1.32 \mathrm{E}-03$ & $9.66 \mathrm{E}+01$ & $1.60 \mathrm{E}-03$ & $1.60 \mathrm{E}+02$ \\
\hline $1.92 \mathrm{E}+00$ & $1.65 \mathrm{E}+00$ & $8.74 \mathrm{E}-02$ & $4.77 \mathrm{E}-02$ & $1.47 \mathrm{E}+11$ & $7.79 \mathrm{E}+05$ & $3.01 \mathrm{E}+06$ & $8.18 \mathrm{E}-06$ & $1.88 \mathrm{E}+02$ & $1.07 \mathrm{E}-03$ & $7.69 \mathrm{E}+01$ & $1.30 \mathrm{E}-03$ & $1.28 \mathrm{E}+02$ \\
\hline $2.23 \mathrm{E}+00$ & $1.92 \mathrm{E}+00$ & $9.07 \mathrm{E}-02$ & $4.90 \mathrm{E}-02$ & $1.55 \mathrm{E}+11$ & & & E-06 & $1.93 \mathrm{E}+02$ & & $7.79 \mathrm{E}+01$ & & $1.30 \mathrm{E}+02$ \\
\hline $2.35 \mathrm{E}+00$ & $2.23 \mathrm{E}+00$ & -02 & & 5.24 & 2.69 & & E-06 & $6.40 \mathrm{E}+01$ & & & & \\
\hline $2.37 \mathrm{E}+00$ & $2.35 \mathrm{E}+00$ & $5.04 \mathrm{E}-03$ & $2.69 \mathrm{E}-03$ & $8.71 \mathrm{E}+09$ & $4.46 \mathrm{E}+04$ & $1.66 \mathrm{E}+05$ & E-07 & $\mathrm{E}+01$ & E-05 & $4.23 \mathrm{E}+00$ & & $7.06 \mathrm{E}+00$ \\
\hline $2.47 \mathrm{E}+00$ & $2.37 \mathrm{E}+00$ & $2.53 \mathrm{E}-02$ & $1.35 \mathrm{E}-02$ & $4.38 \mathrm{E}+10$ & $2.23 \mathrm{E}+05$ & $8.28 \mathrm{E}+05$ & $2.27 \mathrm{E}-06$ & $5.28 \mathrm{E}+01$ & $3.03 \mathrm{E}-04$ & $2.11 \mathrm{E}+01$ & $3.73 \mathrm{E}-04$ & $3.52 \mathrm{E}+01$ \\
\hline $2.73 \mathrm{E}+00$ & $2.47 \mathrm{E}+00$ & $5.97 \mathrm{E}-02$ & $3.15 \mathrm{E}-02$ & $1.04 \mathrm{E}+11$ & $5.26 \mathrm{E}+05$ & $1.93 \mathrm{E}+06$ & $5.31 \mathrm{E}-06$ & $1.24 \mathrm{E}+02$ & $7.11 \mathrm{E}-04$ & $4.90 \mathrm{E}+01$ & $8.81 \mathrm{E}-04$ & $8.20 \mathrm{E}+01$ \\
\hline $3.01 \mathrm{E}+00$ & $2.73 \mathrm{E}+00$ & $5.74 \mathrm{E}-02$ & $3.00 \mathrm{E}-02$ & $1.02 \mathrm{E}+11$ & $5.04 \mathrm{E}+05$ & $1.81 \mathrm{E}+06$ & $5.02 \mathrm{E}-06$ & $1.17 \mathrm{E}+02$ & $6.79 \mathrm{E}-04$ & $4.59 \mathrm{E}+01$ & $8.46 \mathrm{E}-04$ & $7.72 \mathrm{E}+01$ \\
\hline $3.68 \mathrm{E}+00$ & $3.01 \mathrm{E}+00$ & $1.04 \mathrm{E}-01$ & $5.29 \mathrm{E}-02$ & $1.88 \mathrm{E}+11$ & $9.02 \mathrm{E}+05$ & $3.14 \mathrm{E}+06$ & $8.75 \mathrm{E}-06$ & $2.06 \mathrm{E}+02$ & $1.21 \mathrm{E}-03$ & $7.91 \mathrm{E}+01$ & $1.52 \mathrm{E}-03$ & $1.34 \mathrm{E}+02$ \\
\hline $4.97 \mathrm{E}+00$ & $3.68 \mathrm{E}+00$ & $1.14 \mathrm{E}-01$ & $5.56 \mathrm{E}-02$ & $2.19 \mathrm{E}+11$ & $9.75 \mathrm{E}+05$ & $3.16 \mathrm{E}+06$ & $8.97 \mathrm{E}-06$ & $2.14 \mathrm{E}+02$ & $1.29 \mathrm{E}-03$ & $7.88 \mathrm{E}+01$ & $1.66 \mathrm{E}-03$ & $1.36 \mathrm{E}+02$ \\
\hline $6.07 \mathrm{E}+00$ & $4.97 \mathrm{E}+00$ & $4.46 \mathrm{E}-02$ & $2.04 \mathrm{E}-02$ & $9.23 \mathrm{E}+10$ & $3.73 \mathrm{E}+05$ & $1.09 \mathrm{E}+06$ & $3.18 \mathrm{E}-06$ & $7.75 \mathrm{E}+01$ & $4.81 \mathrm{E}-04$ & $2.68 \mathrm{E}+01$ & $6.46 \mathrm{E}-04$ & $4.73 \mathrm{E}+01$ \\
\hline $7.41 \mathrm{E}+00$ & $6.07 \mathrm{E}+00$ & $2.37 \mathrm{E}-02$ & $1.01 \mathrm{E}-02$ & $5.29 \mathrm{E}+10$ & $1.92 \mathrm{E}+05$ & $5.09 \mathrm{E}+05$ & $1.52 \mathrm{E}-06$ & $3.78 \mathrm{E}+01$ & $2.44 \mathrm{E}-04$ & $1.23 \mathrm{E}+01$ & $3.40 \mathrm{E}-04$ & $2.22 \mathrm{E}+01$ \\
\hline $8.61 \mathrm{E}+00$ & $7.41 \mathrm{E}+00$ & $8.33 \mathrm{E}-03$ & $3.30 \mathrm{E}-03$ & $2.03 \mathrm{E}+10$ & $6.57 \mathrm{E}+04$ & $1.54 \mathrm{E}+05$ & $4.76 \mathrm{E}-07$ & $1.21 \mathrm{E}+01$ & $8.13 \mathrm{E}-05$ & $3.66 \mathrm{E}+00$ & $1.19 \mathrm{E}-04$ & $6.82 \mathrm{E}+00$ \\
\hline $1.00 \mathrm{E}+01$ & $8.61 \mathrm{E}+00$ & & $1.35 \mathrm{E}-03$ & $9.85 \mathrm{E}+09$ & $2.82 \mathrm{E}+04$ & & $1.87 \mathrm{E}-07$ & $4.86 \mathrm{E}+00$ & $3.41 \mathrm{E}-05$ & $1.37 \mathrm{E}+00$ & $5.21 \mathrm{E}-05$ & $2.63 \mathrm{E}+00$ \\
\hline $1.22 \mathrm{E}+01$ & $1.00 \mathrm{E}+01$ & $1.55 \mathrm{E}-03$ & $5.12 \mathrm{E}-04$ & $4.65 \mathrm{E}+09$ & $1.14 \mathrm{E}+04$ & $2.03 \mathrm{E}+04$ & $6.73 \mathrm{E}-08$ & $1.80 \mathrm{E}+00$ & $1.34 \mathrm{E}-05$ & $4.61 \mathrm{E}-01$ & $2.17 \mathrm{E}-05$ & $9.22 \mathrm{E}-01$ \\
\hline $1.42 \mathrm{E}+01$ & $1.22 \mathrm{E}+01$ & $2.52 \mathrm{E}-04$ & $7.22 \mathrm{E}-05$ & $8.87 \mathrm{E}+08$ & $1.75 \mathrm{E}+03$ & $2.51 \mathrm{E}+03$ & 8.83E-09 & $2.45 \mathrm{E}-01$ & $1.97 \mathrm{E}-06$ & $5.52 \mathrm{E}-02$ & $3.49 \mathrm{E}-06$ & $1.17 \mathrm{E}-01$ \\
\hline \multirow[t]{2}{*}{$1.96 \mathrm{E}+01$} & $1.42 \mathrm{E}+01$ & $6.06 \mathrm{E}-05$ & $1.48 \mathrm{E}-05$ & $2.56 \mathrm{E}+08$ & $3.94 \mathrm{E}+02$ & $4.47 \mathrm{E}+02$ & $1.68 \mathrm{E}-09$ & $4.85 \mathrm{E}-02$ & $4.25 \mathrm{E}-07$ & $9.46 \mathrm{E}-03$ & $8.30 \mathrm{E}-07$ & $2.13 \mathrm{E}-02$ \\
\hline & Total & $1.24 \mathrm{E}+00$ & $6.61 \mathrm{E}-01$ & $2.15 \mathrm{E}+12$ & $1.09 \mathrm{E}+07$ & $4.09 \mathrm{E}+07$ & $1.12 \mathrm{E}-04$ & $2.60 \mathrm{E}+03$ & $1.49 \mathrm{E}-02$ & $1.04 \mathrm{E}+03$ & $1.83 \mathrm{E}-02$ & $1.74 \mathrm{E}+03$ \\
\hline
\end{tabular}


PCP-2011-0001

Revision 0

Table 3-4. Neutron Source from ORIGEN-S w/o Beryllium (Decay Time $=\mathbf{3 0 , 0 0 0}$ days)

\begin{tabular}{|c|c|c|c|c|c|c|c|c|c|c|c|c|}
\hline \multicolumn{2}{|l|}{$\begin{array}{l}\text { Energy } \\
(\mathrm{MeV})\end{array}$} & \multicolumn{11}{|c|}{ Neutron Source (n/s/g-actinide) } \\
\hline Upper & Lower & Am-241 & Am-243 & Cf-252 & Cm-244 & Cm-248 & Np-237 & Pu-238 & Pu-239 & Pu-240 & Pu-241 & Pu-242 \\
\hline $1.00 \mathrm{E}-07$ & $1.00 \mathrm{E}-11$ & $2.05 \mathrm{E}-11$ & $4.49 \mathrm{E}-12$ & $3.38 \mathrm{E}-04$ & $5.71 \mathrm{E}-06$ & $3.55 \mathrm{E}-04$ & $1.34 \mathrm{E}-15$ & $1.62 \mathrm{E}-08$ & $3.51 \mathrm{E}-13$ & $1.46 \mathrm{E}-08$ & $2.07 \mathrm{E}-11$ & $2.92 \mathrm{E}-08$ \\
\hline $4.14 \mathrm{E}-07$ & $1.00 \mathrm{E}-07$ & $1.03 \mathrm{E}-10$ & $5.70 \mathrm{E}-11$ & $3.61 \mathrm{E}-03$ & $4.24 \mathrm{E}-05$ & $3.79 \mathrm{E}-03$ & $1.07 \mathrm{E}-14$ & $1.26 \mathrm{E}-07$ & $1.53 \mathrm{E}-12$ & $1.03 \mathrm{E}-07$ & $1.04 \mathrm{E}-10$ & $1.76 \mathrm{E}-07$ \\
\hline $8.76 \mathrm{E}-07$ & $4.14 \mathrm{E}-07$ & $2.31 \mathrm{E}-10$ & $1.40 \mathrm{E}-10$ & $8.79 \mathrm{E}-03$ & $1.00 \mathrm{E}-04$ & $9.22 \mathrm{E}-03$ & $2.53 \mathrm{E}-14$ & $2.99 \mathrm{E}-07$ & $3.36 \mathrm{E}-12$ & $2.40 \mathrm{E}-07$ & $2.34 \mathrm{E}-10$ & $4.07 \mathrm{E}-07$ \\
\hline $1.86 \mathrm{E}-06$ & $8.76 \mathrm{E}-07$ & $7.01 \mathrm{E}-10$ & $4.38 \mathrm{E}-10$ & $2.73 \mathrm{E}-02$ & $3.08 \mathrm{E}-04$ & $2.87 \mathrm{E}-02$ & $7.82 \mathrm{E}-14$ & $9.23 \mathrm{E}-07$ & $1.01 \mathrm{E}-11$ & $7.39 \mathrm{E}-07$ & $7.09 \mathrm{E}-10$ & $1.24 \mathrm{E}-06$ \\
\hline $5.04 \mathrm{E}-06$ & $1.86 \mathrm{E}-06$ & $3.58 \mathrm{E}-09$ & $2.27 \mathrm{E}-09$ & $1.42 \mathrm{E}-01$ & $1.59 \mathrm{E}-03$ & $1.49 \mathrm{E}-01$ & $4.03 \mathrm{E}-13$ & $4.76 \mathrm{E}-06$ & $5.13 \mathrm{E}-11$ & $3.81 \mathrm{E}-06$ & $3.62 \mathrm{E}-09$ & \\
\hline $1.07 \mathrm{E}-05$ & $5.04 \mathrm{E}-06$ & $9.55 \mathrm{E}-09$ & $6.10 \mathrm{E}-09$ & $3.80 \mathrm{E}-01$ & $4.26 \mathrm{E}-03$ & 3.99E-01 & $1.08 \mathrm{E}-12$ & $1.28 \mathrm{E}-05$ & $1.37 \mathrm{E}-10$ & $1.02 \mathrm{E}-05$ & $9.67 \mathrm{E}-09$ & $1.71 \mathrm{E}-05$ \\
\hline $3.73 \mathrm{E}-05$ & $1.07 \mathrm{E}-05$ & $7.79 \mathrm{E}-08$ & $5.00 \mathrm{E}-08$ & $3.11 \mathrm{E}+00$ & $3.48 \mathrm{E}-02$ & $3.26 \mathrm{E}+00$ & $8.84 \mathrm{E}-12$ & $1.04 \mathrm{E}-04$ & $1.11 \mathrm{E}-09$ & $8.33 \mathrm{E}-05$ & $7.89 \mathrm{E}-08$ & $1.39 \mathrm{E}-04$ \\
\hline $1.01 \mathrm{E}-04$ & $3.73 \mathrm{E}-05$ & $3.20 \mathrm{E}-07$ & $2.06 \mathrm{E}-07$ & $1.28 \mathrm{E}+01$ & $1.43 \mathrm{E}-01$ & $1.34 \mathrm{E}+01$ & $3.63 \mathrm{E}-11$ & $4.29 \mathrm{E}-04$ & $4.58 \mathrm{E}-09$ & $3.42 \mathrm{E}-04$ & $3.24 \mathrm{E}-07$ & $5.73 \mathrm{E}-04$ \\
\hline $2.14 \mathrm{E}-04$ & $01 \mathrm{E}-04$ & $8.57 \mathrm{E}-07$ & $5.50 \mathrm{E}-07$ & $3.43 \mathrm{E}+01$ & $3.83 \mathrm{E}-01$ & $3.59 \mathrm{E}+01$ & $9.73 \mathrm{E}-11$ & $1.15 \mathrm{E}-03$ & $1.23 \mathrm{E}-08$ & $9.17 \mathrm{E}-04$ & $8.68 \mathrm{E}-07$ & $1.53 \mathrm{E}-03$ \\
\hline $4.54 \mathrm{E}-04$ & $2.14 \mathrm{E}-04$ & $2.64 \mathrm{E}-06$ & $1.70 \mathrm{E}-06$ & $1.06 \mathrm{E}+02$ & $1.18 \mathrm{E}+00$ & $1.11 \mathrm{E}+02$ & $3.00 \mathrm{E}-10$ & $3.54 \mathrm{E}-03$ & $3.78 \mathrm{E}-08$ & $2.82 \mathrm{E}-03$ & $2.67 \mathrm{E}-06$ & $4.72 \mathrm{E}-03$ \\
\hline $1.58 \mathrm{E}-03$ & $4.54 \mathrm{E}-04$ & $2.16 \mathrm{E}-05$ & $1.39 \mathrm{E}-05$ & $8.62 \mathrm{E}+02$ & $9.64 \mathrm{E}+00$ & $9.04 \mathrm{E}+02$ & $2.45 \mathrm{E}-09$ & $2.89 \mathrm{E}-02$ & $3.08 \mathrm{E}-07$ & $2.31 \mathrm{E}-02$ & $2.18 \mathrm{E}-05$ & $3.86 \mathrm{E}-02$ \\
\hline $3.35 \mathrm{E}-03$ & $1.58 \mathrm{E}-03$ & $5.30 \mathrm{E}-05$ & $3.40 \mathrm{E}-05$ & $2.12 \mathrm{E}+03$ & $2.37 \mathrm{E}+01$ & $2.22 \mathrm{E}+03$ & $6.01 \mathrm{E}-09$ & $7.10 \mathrm{E}-02$ & $7.57 \mathrm{E}-07$ & $5.67 \mathrm{E}-02$ & $5.36 \mathrm{E}-05$ & $9.48 \mathrm{E}-02$ \\
\hline $7.10 \mathrm{E}-03$ & $3.35 \mathrm{E}-03$ & $1.63 \mathrm{E}-04$ & $1.05 \mathrm{E}-04$ & $6.51 \mathrm{E}+03$ & $7.28 \mathrm{E}+01$ & $6.83 \mathrm{E}+03$ & $1.85 \mathrm{E}-08$ & $2.18 \mathrm{E}-01$ & $2.33 \mathrm{E}-06$ & $1.74 \mathrm{E}-01$ & $1.65 \mathrm{E}-04$ & $2.92 \mathrm{E}-01$ \\
\hline $1.50 \mathrm{E}-02$ & $7.10 \mathrm{E}-03$ & $5.01 \mathrm{E}-04$ & $3.21 \mathrm{E}-04$ & $2.00 \mathrm{E}+04$ & $2.24 \mathrm{E}+02$ & $2.10 \mathrm{E}+04$ & $5.68 \mathrm{E}-08$ & $6.71 \mathrm{E}-01$ & $7.15 \mathrm{E}-06$ & $5.35 \mathrm{E}-01$ & $5.07 \mathrm{E}-04$ & $8.95 \mathrm{E}-01$ \\
\hline $2.19 \mathrm{E}-02$ & $1.50 \mathrm{E}-02$ & $5.58 \mathrm{E}-04$ & $3.58 \mathrm{E}-04$ & $2.23 \mathrm{E}+04$ & $2.49 \mathrm{E}+02$ & $2.34 \mathrm{E}+04$ & $6.33 \mathrm{E}-08$ & $7.47 \mathrm{E}-01$ & $7.97 \mathrm{E}-06$ & $5.96 \mathrm{E}-01$ & $5.64 \mathrm{E}-04$ & \\
\hline $2.42 \mathrm{E}-02$ & $2.19 \mathrm{E}-02$ & $2.09 \mathrm{E}-04$ & $1.34 \mathrm{E}-04$ & $8.36 \mathrm{E}+03$ & $9.35 \mathrm{E}+01$ & $8.77 \mathrm{E}+03$ & $2.38 \mathrm{E}-08$ & $2.80 \mathrm{E}-01$ & $2.99 \mathrm{E}-06$ & & & \\
\hline $2.61 \mathrm{E}-02$ & & & & & & & & $2.39 \mathrm{E}-01$ & & & & \\
\hline $3.18 \mathrm{E}-02$ & $2.61 \mathrm{E}-02$ & $5.86 \mathrm{E}-04$ & $3.77 \mathrm{E}-04$ & $2.34 \mathrm{E}+04$ & $2.62 \mathrm{E}+02$ & $2.46 \mathrm{E}+04$ & $6.66 \mathrm{E}-08$ & $7.86 \mathrm{E}-01$ & $8.38 \mathrm{E}-06$ & $6.27 \mathrm{E}-01$ & $5.94 \mathrm{E}-04$ & $1.05 \mathrm{E}+00$ \\
\hline $4.09 \mathrm{E}-02$ & $18 \mathrm{E}-02$ & $1.03 \mathrm{E}-03$ & $6.59 \mathrm{E}-04$ & $4.10 \mathrm{E}+04$ & $4.58 \mathrm{E}+02$ & $4.30 \mathrm{E}+04$ & $1.16 \mathrm{E}-07$ & $1.37 \mathrm{E}+00$ & $7 \mathrm{E}-05$ & $1.10 \mathrm{E}+00$ & $1.04 \mathrm{E}-03$ & $\overline{\mathrm{E}+00}$ \\
\hline $6.74 \mathrm{E}-02$ & $\mathrm{E}-02$ & $3.63 \mathrm{E}-03$ & E-03 & $1.45 \mathrm{E}+05$ & $\mathrm{E}+03$ & $1.52 \mathrm{E}+05$ & $4.12 \mathrm{E}-07$ & $\overline{\mathrm{E}+00}$ & E-05 & $\mathrm{E}+00$ & E-03 & $8+00$ \\
\hline $1.11 \mathrm{E}-01$ & $74 \mathrm{E}-02$ & $7.55 \mathrm{E}-03$ & $4.85 \mathrm{E}-03$ & $3.02 \mathrm{E}+05$ & $3.38 \mathrm{E}+03$ & $3.17 \mathrm{E}+05$ & $8.57 \mathrm{E}-07$ & $1.01 \mathrm{E}+01$ & $1.08 \mathrm{E}-04$ & $8.08 \mathrm{E}+00$ & 7.64E-03 & $1.35 \mathrm{E}+01$ \\
\hline $1.83 \mathrm{E}-01$ & $1.11 \mathrm{E}-01$ & $1.55 \mathrm{E}-02$ & $9.96 \mathrm{E}-03$ & $6.21 \mathrm{E}+05$ & $6.94 \mathrm{E}+03$ & $6.51 \mathrm{E}+05$ & $1.76 \mathrm{E}-06$ & $2.08 \mathrm{E}+01$ & $2.22 \mathrm{E}-04$ & $1.66 \mathrm{E}+01$ & $1.57 \mathrm{E}-02$ & $2.77 \mathrm{E}+01$ \\
\hline $2.97 \mathrm{E}-01$ & $1.83 \mathrm{E}-01$ & $2.99 \mathrm{E}-02$ & $1.92 \mathrm{E}-02$ & $1.20 \mathrm{E}+06$ & $1.34 \mathrm{E}+04$ & $1.25 \mathrm{E}+06$ & $3.39 \mathrm{E}-06$ & $4.01 \mathrm{E}+01$ & $4.27 \mathrm{E}-04$ & $\mathrm{E}+01$ & $3.02 \mathrm{E}-02$ & $5.34 \mathrm{E}+01$ \\
\hline 3.69E-01 & $2.97 \mathrm{E}-01$ & $2.11 \mathrm{E}-02$ & $1.35 \mathrm{E}-02$ & $8.43 \mathrm{E}+05$ & $9.44 \mathrm{E}+03$ & $8.84 \mathrm{E}+05$ & $2.39 \mathrm{E}-06$ & $2.83 \mathrm{E}+01$ & $3.01 \mathrm{E}-04$ & $2.26 \mathrm{E}+01$ & $2.14 \mathrm{E}-02$ & $3.76 \mathrm{E}+01$ \\
\hline $4.98 \mathrm{E}-01$ & $3.69 \mathrm{E}-01$ & $4.10 \mathrm{E}-02$ & $2.63 \mathrm{E}-02$ & $1.64 \mathrm{E}+06$ & $1.84 \mathrm{E}+04$ & $1.72 \mathrm{E}+06$ & $4.64 \mathrm{E}-06$ & $5.49 \mathrm{E}+01$ & $5.85 \mathrm{E}-04$ & $4.38 \mathrm{E}+01$ & $4.15 \mathrm{E}-02$ & $7.30 \mathrm{E}+01$ \\
\hline $6.08 \mathrm{E}-01$ & $4.98 \mathrm{E}-01$ & $3.71 \mathrm{E}-02$ & $2.37 \mathrm{E}-02$ & $1.48 \mathrm{E}+06$ & $1.66 \mathrm{E}+04$ & $1.55 \mathrm{E}+06$ & $4.18 \mathrm{E}-06$ & $4.96 \mathrm{E}+01$ & $5.28 \mathrm{E}-04$ & $3.95 \mathrm{E}+01$ & $3.75 \mathrm{E}-02$ & $6.58 \mathrm{E}+01$ \\
\hline $7.43 \mathrm{E}-01$ & $6.08 \mathrm{E}-01$ & $4.67 \mathrm{E}-02$ & $2.98 \mathrm{E}-02$ & $1.85 \mathrm{E}+06$ & $2.09 \mathrm{E}+04$ & $1.94 \mathrm{E}+06$ & $5.25 \mathrm{E}-06$ & $6.24 \mathrm{E}+01$ & $6.64 \mathrm{E}-04$ & $4.96 \mathrm{E}+01$ & $4.73 \mathrm{E}-02$ & $8.25 \mathrm{E}+01$ \\
\hline $8.21 \mathrm{E}-01$ & $7.43 \mathrm{E}-01$ & $2.75 \mathrm{E}-02$ & $1.75 \mathrm{E}-02$ & $1.09 \mathrm{E}+06$ & $1.23 \mathrm{E}+04$ & $1.14 \mathrm{E}+06$ & $3.07 \mathrm{E}-06$ & $3.66 \mathrm{E}+01$ & $3.90 \mathrm{E}-04$ & $2.90 \mathrm{E}+01$ & $2.78 \mathrm{E}-02$ & $4.83 \mathrm{E}+01$ \\
\hline $1.00 \mathrm{E}+00$ & $8.21 \mathrm{E}-01$ & $6.39 \mathrm{E}-02$ & $4.06 \mathrm{E}-02$ & $2.51 \mathrm{E}+06$ & $2.86 \mathrm{E}+04$ & $2.63 \mathrm{E}+06$ & $7.12 \mathrm{E}-06$ & $8.48 \mathrm{E}+01$ & $9.05 \mathrm{E}-04$ & $6.72 \mathrm{E}+01$ & $6.47 \mathrm{E}-02$ & $1.12 \mathrm{E}+02$ \\
\hline $1.35 \mathrm{E}+00$ & $1.00 \mathrm{E}+00$ & $1.19 \mathrm{E}-01$ & $7.53 \mathrm{E}-02$ & $4.63 \mathrm{E}+06$ & $5.33 \mathrm{E}+04$ & $4.86 \mathrm{E}+06$ & $1.32 \mathrm{E}-05$ & $1.57 \mathrm{E}+02$ & $1.68 \mathrm{E}-03$ & $1.24 \mathrm{E}+02$ & E-01 & $2.06 \mathrm{E}+02$ \\
\hline $1.65 \mathrm{E}+00$ & $1.35 \mathrm{E}+00$ & $9.44 \mathrm{E}-02$ & $5.89 \mathrm{E}-02$ & $3.59 \mathrm{E}+06$ & $4.20 \mathrm{E}+04$ & $3.77 \mathrm{E}+06$ & $2 \mathrm{E}-05$ & $1.23 \mathrm{E}+02$ & $1.32 \mathrm{E}-03$ & $9.58 \mathrm{E}+01$ & $\mathrm{E}-02$ & $1.60 \mathrm{E}+02$ \\
\hline $1.92 \mathrm{E}+00$ & 1.65 & 7.67 & 4.74 & $2.87 \mathrm{E}+06$ & $E+04$ & $E+06$ & $\mathrm{E}-06$ & $\mathrm{E}+01$ & E-03 & $\mathrm{E}+01$ & $7.76 \mathrm{E}-02$ & $8 \mathrm{E}+02$ \\
\hline $2.23 \mathrm{E}+00$ & $1.92 \mathrm{E}+00$ & $7.95 \mathrm{E}-02$ & 4.86 & 2.91 & $3.51 \mathrm{E}+04$ & $\mathrm{E}+06$ & -06 & $\overline{E+02}$ & E-03 & $8+01$ & $\mathrm{E}-02$ & $\overline{\mathrm{E}+02}$ \\
\hline $2.35 \mathrm{E}+00$ & $2.23 \mathrm{E}+00$ & $2.67 \mathrm{E}-02$ & $1.62 \mathrm{E}-02$ & $9.61 \mathrm{E}+05$ & $\mathrm{E}+04$ & $\overline{E+06}$ & $2.76 \mathrm{E}-06$ & $3.35 \mathrm{E}+01$ & E-04 & $\mathrm{E}+01$ & $2.70 \mathrm{E}-02$ & $4.28 \mathrm{E}+01$ \\
\hline $2.37 \mathrm{E}+00$ & $2.35 \mathrm{E}+00$ & $4.42 \mathrm{E}-03$ & $2.67 \mathrm{E}-03$ & $1.58 \mathrm{E}+05$ & $1.94 \mathrm{E}+03$ & $1.66 \mathrm{E}+05$ & $4.56 \mathrm{E}-07$ & $5.54 \mathrm{E}+00$ & $6.04 \mathrm{E}-05$ & $4.19 \mathrm{E}+00$ & $4.47 \mathrm{E}-03$ & $7.06 \mathrm{E}+00$ \\
\hline $2.47 \mathrm{E}+00$ & $2.37 \mathrm{E}+00$ & $2.22 \mathrm{E}-02$ & $1.34 \mathrm{E}-02$ & $7.90 \mathrm{E}+05$ & $9.73 \mathrm{E}+03$ & $8.28 \mathrm{E}+05$ & $2.27 \mathrm{E}-06$ & $2.77 \mathrm{E}+01$ & $3.02 \mathrm{E}-04$ & $2.09 \mathrm{E}+01$ & $2.24 \mathrm{E}-02$ & $3.52 \mathrm{E}+01$ \\
\hline $2.73 \mathrm{E}+00$ & $2.47 \mathrm{E}+00$ & $5.23 \mathrm{E}-02$ & $3.13 \mathrm{E}-02$ & $1.84 \mathrm{E}+06$ & $2.29 \mathrm{E}+04$ & $1.93 \mathrm{E}+06$ & $5.31 \mathrm{E}-06$ & $6.48 \mathrm{E}+01$ & $7.10 \mathrm{E}-04$ & $4.85 \mathrm{E}+01$ & $5.30 \mathrm{E}-02$ & $8.20 \mathrm{E}+01$ \\
\hline $3.01 \mathrm{E}+00$ & $2.73 \mathrm{E}+00$ & $5.04 \mathrm{E}-02$ & $2.98 \mathrm{E}-02$ & $1.73 \mathrm{E}+06$ & $2.20 \mathrm{E}+04$ & $1.81 \mathrm{E}+06$ & $5.02 \mathrm{E}-06$ & $6.14 \mathrm{E}+01$ & $6.78 \mathrm{E}-04$ & $4.55 \mathrm{E}+01$ & $5.10 \mathrm{E}-02$ & $7.72 \mathrm{E}+01$ \\
\hline $3.68 \mathrm{E}+00$ & $3.01 \mathrm{E}+00$ & $9.09 \mathrm{E}-02$ & $5.25 \mathrm{E}-02$ & $2.99 \mathrm{E}+06$ & $3.93 \mathrm{E}+04$ & $3.14 \mathrm{E}+06$ & $8.75 \mathrm{E}-06$ & $1.08 \mathrm{E}+02$ & $1.20 \mathrm{E}-03$ & $7.84 \mathrm{E}+01$ & $9.19 \mathrm{E}-02$ & $1.34 \mathrm{E}+02$ \\
\hline $4.97 \mathrm{E}+00$ & $3.68 \mathrm{E}+00$ & 9.99E-02 & $5.52 \mathrm{E}-02$ & $3.02 \mathrm{E}+06$ & $4.25 \mathrm{E}+04$ & $3.16 \mathrm{E}+06$ & $8.97 \mathrm{E}-06$ & $1.12 \mathrm{E}+02$ & $1.28 \mathrm{E}-03$ & $7.81 \mathrm{E}+01$ & $1.01 \mathrm{E}-01$ & $1.36 \mathrm{E}+02$ \\
\hline $6.07 \mathrm{E}+00$ & $4.97 \mathrm{E}+00$ & $3.92 \mathrm{E}-02$ & $2.03 \mathrm{E}-02$ & $1.04 \mathrm{E}+06$ & & & $3.18 \mathrm{E}-06$ & $4.06 \mathrm{E}+01$ & & $2.66 \mathrm{E}+01$ & $3.96 \mathrm{E}-02$ & $4.73 \mathrm{E}+01$ \\
\hline & & & & & & & & $1.98 \mathrm{E}+01$ & & & & $2.22 \mathrm{E}+01$ \\
\hline $8.61 \mathrm{E}+00$ & $7.41 \mathrm{E}+00$ & $7.31 \mathrm{E}-03$ & $3.27 \mathrm{E}-03$ & $1.47 \mathrm{E}+05$ & $2.86 \mathrm{E}+03$ & $1.54 \mathrm{E}+05$ & $4.76 \mathrm{E}-07$ & $6.33 \mathrm{E}+00$ & $8.11 \mathrm{E}-05$ & $3.63 \mathrm{E}+00$ & $7.40 \mathrm{E}-03$ & $6.82 \mathrm{E}+00$ \\
\hline $1.00 \mathrm{E}+01$ & $8.61 \mathrm{E}+00$ & $3.24 \mathrm{E}-03$ & $1.34 \mathrm{E}-03$ & $5.61 \mathrm{E}+04$ & $1.23 \mathrm{E}+03$ & $5.88 \mathrm{E}+04$ & $1.87 \mathrm{E}-07$ & $2.55 \mathrm{E}+00$ & $3.40 \mathrm{E}-05$ & $1.36 \mathrm{E}+00$ & $3.28 \mathrm{E}-03$ & $2.63 \mathrm{E}+00$ \\
\hline $1.22 \mathrm{E}+01$ & $1.00 \mathrm{E}+01$ & $1.36 \mathrm{E}-03$ & $5.08 \mathrm{E}-04$ & $1.94 \mathrm{E}+04$ & $4.95 \mathrm{E}+02$ & $2.03 E+04$ & $6.73 \mathrm{E}-08$ & $9.42 \mathrm{E}-01$ & $1.33 \mathrm{E}-05$ & $4.57 \mathrm{E}-01$ & $1.38 \mathrm{E}-03$ & $9.21 \mathrm{E}-01$ \\
\hline $1.42 \mathrm{E}+01$ & $1.22 \mathrm{E}+01$ & $2.21 \mathrm{E}-04$ & $7.17 \mathrm{E}-05$ & $2.40 \mathrm{E}+03$ & $7.60 \mathrm{E}+01$ & $2.51 \mathrm{E}+03$ & 8.83E-09 & $1.29 \mathrm{E}-01$ & $1.97 \mathrm{E}-06$ & $5.47 \mathrm{E}-02$ & $2.24 \mathrm{E}-04$ & $1.17 \mathrm{E}-01$ \\
\hline \multirow[t]{2}{*}{$1.96 \mathrm{E}+01$} & $1.42 \mathrm{E}+01$ & $5.31 \mathrm{E}-05$ & $1.47 \mathrm{E}-05$ & $4.26 \mathrm{E}+02$ & $1.71 \mathrm{E}+01$ & $4.47 \mathrm{E}+02$ & $1.68 \mathrm{E}-09$ & $2.54 \mathrm{E}-02$ & $4.24 \mathrm{E}-07$ & $9.38 \mathrm{E}-03$ & $5.38 \mathrm{E}-05$ & $2.13 \mathrm{E}-02$ \\
\hline & Total & $1.09 \mathrm{E}+00$ & $6.56 \mathrm{E}-01$ & $3.90 \mathrm{E}+07$ & $4.77 \mathrm{E}+05$ & $4.09 \mathrm{E}+07$ & $1.12 \mathrm{E}-04$ & $1.36 \mathrm{E}+03$ & $1.48 \mathrm{E}-02$ & $1.03 E+03$ & $1.10 \mathrm{E}+00$ & $1.74 \mathrm{E}+03$ \\
\hline
\end{tabular}


PCP-2011-0001

Revision 0

Table 3-5. Neutron Source from ORIGEN-S w/90\% Beryllium (Decay Time $=0$ days)

\begin{tabular}{|c|c|c|c|c|c|c|c|c|c|c|}
\hline \multicolumn{2}{|c|}{ Energy Bound (MeV) } & \multicolumn{9}{|c|}{ Neutron Source (n/s/g-actinide) } \\
\hline Upper & Upper & Am-241 & Am-243 & Cm-244 & Np-237 & Pu-238 & Pu-239 & Pu-240 & Pu-241 & Pu-242 \\
\hline $1.00 \mathrm{E}-07$ & $1.00 \mathrm{E}-11$ & $2.34 \mathrm{E}-11$ & $4.52 \mathrm{E}-12$ & $1.32 \mathrm{E}-04$ & $1.34 \mathrm{E}-15$ & $3.09 \mathrm{E}-08$ & $3.52 \mathrm{E}-13$ & $1.47 \mathrm{E}-08$ & $2.97 \mathrm{E}-14$ & $2.92 \mathrm{E}-08$ \\
\hline $4.14 \mathrm{E}-07$ & $1.00 \mathrm{E}-07$ & $1.17 \mathrm{E}-10$ & $5.75 \mathrm{E}-11$ & $9.83 \mathrm{E}-04$ & $1.07 \mathrm{E}-14$ & $2.41 \mathrm{E}-07$ & $1.54 \mathrm{E}-12$ & $1.03 \mathrm{E}-07$ & $2.00 \mathrm{E}-13$ & $1.76 \mathrm{E}-07$ \\
\hline $8.76 \mathrm{E}-07$ & $4.14 \mathrm{E}-07$ & $2.64 \mathrm{E}-10$ & $1.41 \mathrm{E}-10$ & $2.32 \mathrm{E}-03$ & $2.53 \mathrm{E}-14$ & $5.73 \mathrm{E}-07$ & $3.37 \mathrm{E}-12$ & $2.42 \mathrm{E}-07$ & $4.68 \mathrm{E}-13$ & $4.07 \mathrm{E}-07$ \\
\hline $1.86 \mathrm{E}-06$ & $8.76 \mathrm{E}-07$ & $7.99 \mathrm{E}-10$ & $4.41 \mathrm{E}-10$ & $7.15 \mathrm{E}-03$ & $7.82 \mathrm{E}-14$ & $1.77 \mathrm{E}-06$ & $1.01 \mathrm{E}-11$ & $7.46 \mathrm{E}-07$ & $1.44 \mathrm{E}-12$ & $1.24 \mathrm{E}-06$ \\
\hline $5.04 \mathrm{E}-06$ & $1.86 \mathrm{E}-06$ & $4.08 \mathrm{E}-09$ & 2.29E-09 & $3.68 \mathrm{E}-02$ & $4.03 \mathrm{E}-13$ & $9.11 \mathrm{E}-06$ & $5.14 \mathrm{E}-11$ & $3.84 \mathrm{E}-06$ & $7.38 \mathrm{E}-12$ & $6.38 \mathrm{E}-06$ \\
\hline $1.07 \mathrm{E}-05$ & $5.04 \mathrm{E}-06$ & $1.09 \mathrm{E}-08$ & $6.15 \mathrm{E}-09$ & $9.86 \mathrm{E}-02$ & $1.08 \mathrm{E}-12$ & $2.44 \mathrm{E}-05$ & $1.37 \mathrm{E}-10$ & $1.03 \mathrm{E}-05$ & $1.98 \mathrm{E}-11$ & $1.71 \mathrm{E}-05$ \\
\hline $3.73 \mathrm{E}-05$ & $1.07 \mathrm{E}-05$ & $8.89 \mathrm{E}-08$ & 5.03E-08 & $8.06 \mathrm{E}-01$ & $8.84 \mathrm{E}-12$ & $2.00 \mathrm{E}-04$ & $1.12 \mathrm{E}-09$ & $8.40 \mathrm{E}-05$ & $1.61 \mathrm{E}-10$ & $1.39 \mathrm{E}-04$ \\
\hline $1.01 \mathrm{E}-04$ & $3.73 \mathrm{E}-05$ & $3.65 \mathrm{E}-07$ & $2.07 \mathrm{E}-07$ & $3.32 \mathrm{E}+00$ & $3.63 \mathrm{E}-11$ & $8.21 \mathrm{E}-04$ & $4.59 \mathrm{E}-09$ & $3.45 \mathrm{E}-04$ & $6.64 \mathrm{E}-10$ & $5.73 \mathrm{E}-04$ \\
\hline $2.14 \mathrm{E}-04$ & $1.01 \mathrm{E}-04$ & $9.78 \mathrm{E}-07$ & $5.54 \mathrm{E}-07$ & $8.88 \mathrm{E}+00$ & $9.73 \mathrm{E}-11$ & $2.20 \mathrm{E}-03$ & $1.23 \mathrm{E}-08$ & $9.25 \mathrm{E}-04$ & $1.78 \mathrm{E}-09$ & $1.53 \mathrm{E}-03$ \\
\hline $4.54 \mathrm{E}-04$ & $2.14 \mathrm{E}-04$ & $3.01 \mathrm{E}-06$ & $1.71 \mathrm{E}-06$ & $2.74 \mathrm{E}+01$ & $3.00 \mathrm{E}-10$ & $6.77 \mathrm{E}-03$ & $3.78 \mathrm{E}-08$ & $2.85 \mathrm{E}-03$ & $5.48 \mathrm{E}-09$ & $4.72 \mathrm{E}-03$ \\
\hline $1.58 \mathrm{E}-03$ & $4.54 \mathrm{E}-04$ & $2.70 \mathrm{E}-03$ & $1.40 \mathrm{E}-05$ & $2.24 \mathrm{E}+02$ & $3.82 \mathrm{E}-08$ & $5.53 \mathrm{E}-02$ & $2.19 \mathrm{E}-04$ & $2.33 \mathrm{E}-02$ & $2.17 \mathrm{E}-05$ & $3.86 \mathrm{E}-02$ \\
\hline $3.35 \mathrm{E}-03$ & $1.58 \mathrm{E}-03$ & $2.05 \mathrm{E}-01$ & $1.18 \mathrm{E}-02$ & $5.53 \mathrm{E}+02$ & $3.47 \mathrm{E}-05$ & $1.17 \mathrm{E}+00$ & $3.12 \mathrm{E}-03$ & $6.98 \mathrm{E}-02$ & $1.57 \mathrm{E}-04$ & $9.50 \mathrm{E}-02$ \\
\hline $7.10 \mathrm{E}-03$ & $3.35 \mathrm{E}-03$ & $1.40 \mathrm{E}+00$ & $7.85 \mathrm{E}-02$ & $1.72 \mathrm{E}+03$ & $2.87 \mathrm{E}-04$ & $6.94 \mathrm{E}+00$ & $2.68 \mathrm{E}-02$ & $2.64 \mathrm{E}-01$ & $1.03 \mathrm{E}-03$ & $2.93 \mathrm{E}-01$ \\
\hline $1.50 \mathrm{E}-02$ & $7.10 \mathrm{E}-03$ & $7.20 \mathrm{E}+00$ & $4.18 \mathrm{E}-01$ & $5.35 \mathrm{E}+03$ & $1.39 \mathrm{E}-03$ & $3.70 \mathrm{E}+01$ & $1.30 \mathrm{E}-01$ & $1.01 \mathrm{E}+00$ & & $9.04 \mathrm{E}-01$ \\
\hline 2.19E-02 & $1.50 \mathrm{E}-02$ & $1.00 \mathrm{E}+01$ & $5.77 \mathrm{E}-01$ & $6.01 \mathrm{E}+03$ & & & $1.81 \mathrm{E}-01$ & $1.25 \mathrm{E}+00$ & & $1.01 \mathrm{E}+00$ \\
\hline $2.42 \mathrm{E}-02$ & $2.19 \mathrm{E}-02$ & $4.00 \mathrm{E}+00$ & $2.38 \mathrm{E}-01$ & $2.26 \mathrm{E}+03$ & $7.89 \mathrm{E}-04$ & $2.01 \mathrm{E}+01$ & $7.32 \mathrm{E}-02$ & $5.04 \mathrm{E}-01$ & $3.05 \mathrm{E}-03$ & $3.79 \mathrm{E}-01$ \\
\hline $2.61 \mathrm{E}-02$ & $2.42 \mathrm{E}-02$ & $3.68 \mathrm{E}+00$ & $2.08 \mathrm{E}-01$ & $1.94 \mathrm{E}+03$ & $7.09 \mathrm{E}-04$ & $1.90 \mathrm{E}+01$ & $6.47 \mathrm{E}-02$ & $4.21 \mathrm{E}-01$ & $2.63 \mathrm{E}-03$ & $3.24 \mathrm{E}-01$ \\
\hline $3.18 \mathrm{E}-02$ & $2.61 \mathrm{E}-02$ & $1.24 \mathrm{E}+01$ & $7.15 \mathrm{E}-01$ & $6.37 \mathrm{E}+03$ & $2.40 \mathrm{E}-03$ & $6.31 \mathrm{E}+01$ & $2.25 \mathrm{E}-01$ & $1.45 \mathrm{E}+00$ & $9.09 \mathrm{E}-03$ & $1.06 \mathrm{E}+00$ \\
\hline 4.09E-02 & $3.18 \mathrm{E}-02$ & $2.36 \mathrm{E}+01$ & $1.38 \mathrm{E}+00$ & $1.12 \mathrm{E}+04$ & $4.64 \mathrm{E}-03$ & $1.20 \mathrm{E}+02$ & $4.26 \mathrm{E}-01$ & $2.67 \mathrm{E}+00$ & $1.76 \mathrm{E}-02$ & $1.86 \mathrm{E}+00$ \\
\hline $6.74 \mathrm{E}-02$ & $4.09 \mathrm{E}-02$ & $9.72 \mathrm{E}+01$ & $5.63 \mathrm{E}+00$ & $3.99 \mathrm{E}+04$ & $1.90 \mathrm{E}-02$ & $4.93 \mathrm{E}+02$ & $1.76 \mathrm{E}+00$ & $1.03 \mathrm{E}+01$ & $7.20 \mathrm{E}-02$ & $6.61 \mathrm{E}+00$ \\
\hline $1.11 \mathrm{E}-01$ & $6.74 \mathrm{E}-02$ & $2.33 \mathrm{E}+02$ & $1.36 \mathrm{E}+01$ & $8.38 \mathrm{E}+04$ & $4.58 \mathrm{E}-02$ & $1.18 \mathrm{E}+03$ & $4.22 \mathrm{E}+00$ & $2.36 \mathrm{E}+01$ & $1.73 \mathrm{E}-01$ & $1.38 \mathrm{E}+01$ \\
\hline $1.83 \mathrm{E}-01$ & $1.11 \mathrm{E}-01$ & $5.11 \mathrm{E}+02$ & $2.97 \mathrm{E}+01$ & $1.73 \mathrm{E}+05$ & $1.00 \mathrm{E}-01$ & $2.59 \mathrm{E}+03$ & $9.25 \mathrm{E}+00$ & $5.06 \mathrm{E}+01$ & $3.79 \mathrm{E}-01$ & $2.83 \mathrm{E}+01$ \\
\hline $2.97 \mathrm{E}-01$ & $1.83 \mathrm{E}-01$ & $2.05 \mathrm{E}+03$ & $1.19 \mathrm{E}+02$ & $3.58 \mathrm{E}+05$ & $4.01 \mathrm{E}-01$ & $1.03 \mathrm{E}+04$ & $3.71 \mathrm{E}+01$ & $1.68 \mathrm{E}+02$ & $1.52 \mathrm{E}+00$ & $5.57 \mathrm{E}+01$ \\
\hline 3.69E-01 & $2.97 \mathrm{E}-01$ & $6.37 \mathrm{E}+03$ & $3.72 \mathrm{E}+02$ & $3.70 \mathrm{E}+05$ & $1.25 \mathrm{E}+00$ & $3.18 \mathrm{E}+04$ & $1.15 \mathrm{E}+02$ & $4.46 \mathrm{E}+02$ & $4.73 \mathrm{E}+00$ & $4.50 \mathrm{E}+01$ \\
\hline $4.98 \mathrm{E}-01$ & $3.69 \mathrm{E}-01$ & $2.71 \mathrm{E}+04$ & $1.58 \mathrm{E}+03$ & $1.07 \mathrm{E}+06$ & $5.32 \mathrm{E}+00$ & $1.35 \mathrm{E}+05$ & $4.90 \mathrm{E}+02$ & $1.84 \mathrm{E}+03$ & $2.01 \mathrm{E}+01$ & $1.04 \mathrm{E}+02$ \\
\hline $6.08 \mathrm{E}-01$ & $4.98 \mathrm{E}-01$ & $3.53 \mathrm{E}+04$ & $2.05 \mathrm{E}+03$ & $1.22 \mathrm{E}+06$ & $6.89 \mathrm{E}+00$ & $1.76 \mathrm{E}+05$ & $6.36 \mathrm{E}+02$ & $2.38 \mathrm{E}+03$ & $2.61 \mathrm{E}+01$ & $1.07 \mathrm{E}+02$ \\
\hline $7.43 \mathrm{E}-01$ & $6.08 \mathrm{E}-01$ & $5.45 \mathrm{E}+04$ & $3.17 \mathrm{E}+03$ & $1.77 \mathrm{E}+06$ & $9.43 \mathrm{E}+00$ & $2.72 \mathrm{E}+05$ & $9.83 \mathrm{E}+02$ & $3.66 \mathrm{E}+03$ & $3.85 \mathrm{E}+01$ & $1.43 \mathrm{E}+02$ \\
\hline $8.21 \mathrm{E}-01$ & $7.43 \mathrm{E}-01$ & $3.62 \mathrm{E}+04$ & $2.10 \mathrm{E}+03$ & $1.14 \mathrm{E}+06$ & $5.45 \mathrm{E}+00$ & $1.81 \mathrm{E}+05$ & $6.36 \mathrm{E}+02$ & $2.37 \mathrm{E}+03$ & $2.27 \mathrm{E}+01$ & $8.36 \mathrm{E}+01$ \\
\hline $1.00 \mathrm{E}+00$ & $8.21 \mathrm{E}-01$ & $9.30 \mathrm{E}+04$ & $5.03 \mathrm{E}+03$ & $2.93 \mathrm{E}+06$ & $1.27 \mathrm{E}+01$ & $4.65 \mathrm{E}+05$ & $1.48 \mathrm{E}+03$ & $5.51 \mathrm{E}+03$ & $5.26 \mathrm{E}+01$ & $1.94 \mathrm{E}+02$ \\
\hline $1.35 \mathrm{E}+00$ & $1.00 \mathrm{E}+00$ & $1.78 \mathrm{E}+05$ & $9.47 \mathrm{E}+03$ & $5.91 \mathrm{E}+06$ & $2.36 \mathrm{E}+01$ & $8.89 \mathrm{E}+05$ & $2.78 \mathrm{E}+03$ & $1.04 \mathrm{E}+04$ & $9.85 \mathrm{E}+01$ & $3.60 \mathrm{E}+02$ \\
\hline $1.65 \mathrm{E}+00$ & $1.35 \mathrm{E}+00$ & $1.20 \mathrm{E}+05$ & $6.25 \mathrm{E}+03$ & $4.22 \mathrm{E}+06$ & $1.40 \mathrm{E}+01$ & $6.02 \mathrm{E}+05$ & $1.81 \mathrm{E}+03$ & $6.75 \mathrm{E}+03$ & $6.07 \mathrm{E}+01$ & $2.55 \mathrm{E}+02$ \\
\hline $1.92 \mathrm{E}+00$ & $1.65 \mathrm{E}+00$ & $1.10 \mathrm{E}+05$ & $5.72 \mathrm{E}+03$ & $3.75 \mathrm{E}+06$ & $1.30 \mathrm{E}+01$ & $5.50 \mathrm{E}+05$ & $1.66 \mathrm{E}+03$ & $6.18 \mathrm{E}+03$ & $5.61 \mathrm{E}+01$ & $2.15 \mathrm{E}+02$ \\
\hline $2.23 \mathrm{E}+00$ & $1.92 \mathrm{E}+00$ & $1.71 \mathrm{E}+05$ & $9.20 \mathrm{E}+03$ & $5.29 \mathrm{E}+06$ & $2.42 \mathrm{E}+01$ & $8.58 \mathrm{E}+05$ & $2.72 \mathrm{E}+03$ & $1.01 \mathrm{E}+04$ & $9.76 \mathrm{E}+01$ & $2.82 \mathrm{E}+02$ \\
\hline $2.35 \mathrm{E}+00$ & $2.23 \mathrm{E}+00$ & $6.99 \mathrm{E}+04$ & $3.79 \mathrm{E}+03$ & $2.08 \mathrm{E}+06$ & $1.13 \mathrm{E}+01$ & $3.50 \mathrm{E}+05$ & $1.13 \mathrm{E}+03$ & $4.16 \mathrm{E}+03$ & $4.26 \mathrm{E}+01$ & $1.09 \mathrm{E}+02$ \\
\hline $2.37 \mathrm{E}+00$ & $2.35 \mathrm{E}+00$ & $1.22 \mathrm{E}+04$ & $6.61 \mathrm{E}+02$ & $3.60 \mathrm{E}+05$ & $2.04 \mathrm{E}+00$ & $6.10 \mathrm{E}+04$ & $1.97 \mathrm{E}+02$ & $7.26 \mathrm{E}+02$ & $7.71 \mathrm{E}+00$ & $1.91 \mathrm{E}+01$ \\
\hline $2.47 \mathrm{E}+00$ & $2.37 \mathrm{E}+00$ & $6.44 \mathrm{E}+04$ & $3.50 \mathrm{E}+03$ & $1.89 \mathrm{E}+06$ & $1.11 \mathrm{E}+01$ & $3.22 \mathrm{E}+05$ & $1.04 \mathrm{E}+03$ & $3.84 \mathrm{E}+03$ & $4.19 \mathrm{E}+01$ & $1.01 \mathrm{E}+02$ \\
\hline $2.73 \mathrm{E}+00$ & $2.47 \mathrm{E}+00$ & $1.90 \mathrm{E}+05$ & $1.06 \mathrm{E}+04$ & $5.38 \mathrm{E}+06$ & $3.53 \mathrm{E}+01$ & $9.49 \mathrm{E}+05$ & $3.26 \mathrm{E}+03$ & $1.20 \mathrm{E}+04$ & $1.33 \mathrm{E}+02$ & $2.90 \mathrm{E}+02$ \\
\hline $3.01 \mathrm{E}+00$ & $2.73 \mathrm{E}+00$ & $3.13 \mathrm{E}+05$ & $1.82 \mathrm{E}+04$ & $8.16 \mathrm{E}+06$ & $6.05 \mathrm{E}+01$ & $1.56 \mathrm{E}+06$ & $5.65 \mathrm{E}+03$ & $2.08 \mathrm{E}+04$ & $2.31 \mathrm{E}+02$ & $4.39 \mathrm{E}+02$ \\
\hline $3.68 \mathrm{E}+00$ & $3.01 \mathrm{E}+00$ & $1.01 \mathrm{E}+06$ & $5.39 \mathrm{E}+04$ & $2.73 \mathrm{E}+07$ & $1.30 \mathrm{E}+02$ & $5.06 \mathrm{E}+06$ & $1.57 \mathrm{E}+04$ & $5.78 \mathrm{E}+04$ & $5.37 \mathrm{E}+02$ & $9.72 \mathrm{E}+02$ \\
\hline $4.97 \mathrm{E}+00$ & $3.68 \mathrm{E}+00$ & $1.71 \mathrm{E}+06$ & $8.58 \mathrm{E}+04$ & $4.97 \mathrm{E}+07$ & $1.82 \mathrm{E}+02$ & $8.55 \mathrm{E}+06$ & $2.42 \mathrm{E}+04$ & $8.93 \mathrm{E}+04$ & $7.76 \mathrm{E}+02$ & $1.34 \mathrm{E}+03$ \\
\hline $6.07 \mathrm{E}+00$ & $4.97 \mathrm{E}+00$ & $9.50 \mathrm{E}+05$ & $4.32 \mathrm{E}+04$ & $3.01 \mathrm{E}+07$ & $6.13 \mathrm{E}+01$ & $4.78 \mathrm{E}+06$ & $1.12 \mathrm{E}+04$ & $4.16 \mathrm{E}+04$ & $2.89 \mathrm{E}+02$ & $4.96 \mathrm{E}+02$ \\
\hline $7.41 \mathrm{E}+00$ & $6.07 \mathrm{E}+00$ & $9.51 \mathrm{E}+05$ & $4.74 \mathrm{E}+04$ & $3.03 \mathrm{E}+07$ & $1.27 \mathrm{E}+02$ & $4.77 \mathrm{E}+06$ & $1.38 \mathrm{E}+04$ & $5.06 \mathrm{E}+04$ & $5.05 \mathrm{E}+02$ & $8.11 \mathrm{E}+02$ \\
\hline $8.61 \mathrm{E}+00$ & $7.41 \mathrm{E}+00$ & $7.92 \mathrm{E}+05$ & $4.00 \mathrm{E}+04$ & $2.35 \mathrm{E}+07$ & $1.01 \mathrm{E}+02$ & $3.97 \mathrm{E}+06$ & $1.15 \mathrm{E}+04$ & $4.24 \mathrm{E}+04$ & $4.08 \mathrm{E}+02$ & $6.44 \mathrm{E}+02$ \\
\hline $1.00 \mathrm{E}+01$ & $8.61 \mathrm{E}+00$ & $5.99 \mathrm{E}+05$ & $2.82 \mathrm{E}+04$ & $1.96 \mathrm{E}+07$ & $6.21 \mathrm{E}+01$ & $3.01 \mathrm{E}+06$ & $7.83 \mathrm{E}+03$ & $2.89 \mathrm{E}+04$ & $2.60 \mathrm{E}+02$ & $4.07 \mathrm{E}+02$ \\
\hline $1.22 \mathrm{E}+01$ & $1.00 \mathrm{E}+01$ & $6.74 \mathrm{E}+04$ & $2.81 \mathrm{E}+03$ & $2.75 \mathrm{E}+06$ & $2.10 \mathrm{E}+00$ & $3.40 \mathrm{E}+05$ & $6.65 \mathrm{E}+02$ & $2.48 \mathrm{E}+03$ & $1.30 \mathrm{E}+01$ & $2.09 \mathrm{E}+01$ \\
\hline $1.42 \mathrm{E}+01$ & $1.22 \mathrm{E}+01$ & $2.52 \mathrm{E}-04$ & $7.22 \mathrm{E}-05$ & $1.76 \mathrm{E}+03$ & $8.83 \mathrm{E}-09$ & $2.46 \mathrm{E}-01$ & $1.97 \mathrm{E}-06$ & $5.52 \mathrm{E}-02$ & $1.78 \mathrm{E}-07$ & $1.17 \mathrm{E}-01$ \\
\hline \multirow[t]{2}{*}{$1.96 \mathrm{E}+01$} & $1.42 \mathrm{E}+01$ & $6.06 \mathrm{E}-05$ & $1.48 \mathrm{E}-05$ & $3.98 \mathrm{E}+02$ & $1.68 \mathrm{E}-09$ & $4.86 \mathrm{E}-02$ & $4.25 \mathrm{E}-07$ & $9.46 \mathrm{E}-03$ & $3.45 \mathrm{E}-08$ & $2.13 \mathrm{E}-02$ \\
\hline & Total & $7.56 \mathrm{E}+06$ & $3.83 \mathrm{E}+05$ & $2.29 \mathrm{E}+08$ & $9.03 \mathrm{E}+02$ & $3.79 \mathrm{E}+07$ & $1.09 \mathrm{E}+05$ & $4.04 \mathrm{E}+05$ & $3.72 \mathrm{E}+03$ & $7.55 \mathrm{E}+03$ \\
\hline
\end{tabular}

Note: $90 \%$ Beryllium is modeled as 1 gram of actinide and 0.9 grams of beryllium. 
PCP-2011-0001

Revision 0

Table 3-6. Neutron Source from ORIGEN-S w/90\% Beryllium (Decay Time $=30$ days)

\begin{tabular}{|c|c|c|c|c|c|c|c|c|c|c|}
\hline \multicolumn{2}{|c|}{ Energy Bound (MeV) } & \multicolumn{9}{|c|}{ Neutron Source (n/s/g-actinide) } \\
\hline Upper & Upper & Am-241 & Am-243 & Cm-244 & Np-237 & Pu-238 & Pu-239 & Pu-240 & Pu-241 & Pu-242 \\
\hline $1.00 \mathrm{E}-07$ & $1.00 \mathrm{E}-11$ & $2.34 \mathrm{E}-11$ & $4.52 \mathrm{E}-12$ & $1.32 \mathrm{E}-04$ & $1.34 \mathrm{E}-15$ & $3.09 \mathrm{E}-08$ & $3.52 \mathrm{E}-13$ & $1.47 \mathrm{E}-08$ & $1.22 \mathrm{E}-13$ & $2.92 \mathrm{E}-08$ \\
\hline $4.14 \mathrm{E}-07$ & $1.00 \mathrm{E}-07$ & $1.17 \mathrm{E}-10$ & $5.75 \mathrm{E}-11$ & $9.80 \mathrm{E}-04$ & $1.07 \mathrm{E}-14$ & $2.41 \mathrm{E}-07$ & $1.54 \mathrm{E}-12$ & $1.03 \mathrm{E}-07$ & $6.64 \mathrm{E}-13$ & $1.76 \mathrm{E}-07$ \\
\hline $8.76 \mathrm{E}-07$ & $4.14 \mathrm{E}-07$ & $2.64 \mathrm{E}-10$ & $1.41 \mathrm{E}-10$ & $2.31 \mathrm{E}-03$ & $2.53 \mathrm{E}-14$ & $5.72 \mathrm{E}-07$ & $3.37 \mathrm{E}-12$ & $2.42 \mathrm{E}-07$ & $1.51 \mathrm{E}-12$ & $4.07 \mathrm{E}-07$ \\
\hline $1.86 \mathrm{E}-06$ & $8.76 \mathrm{E}-07$ & $7.99 \mathrm{E}-10$ & $4.41 \mathrm{E}-10$ & $7.12 \mathrm{E}-03$ & $7.82 \mathrm{E}-14$ & $1.77 \mathrm{E}-06$ & $1.01 \mathrm{E}-11$ & $7.46 \mathrm{E}-07$ & $4.59 \mathrm{E}-12$ & $1.24 \mathrm{E}-06$ \\
\hline $5.04 \mathrm{E}-06$ & $1.86 \mathrm{E}-06$ & $4.08 \mathrm{E}-09$ & 2.29E-09 & $3.67 \mathrm{E}-02$ & $4.03 \mathrm{E}-13$ & $9.11 \mathrm{E}-06$ & $5.14 \mathrm{E}-11$ & $3.84 \mathrm{E}-06$ & $2.35 \mathrm{E}-11$ & $6.38 \mathrm{E}-06$ \\
\hline $1.07 \mathrm{E}-05$ & $5.04 \mathrm{E}-06$ & $1.09 \mathrm{E}-08$ & $6.15 \mathrm{E}-09$ & $9.83 \mathrm{E}-02$ & $1.08 \mathrm{E}-12$ & $2.44 \mathrm{E}-05$ & $1.37 \mathrm{E}-10$ & $1.03 \mathrm{E}-05$ & $6.28 \mathrm{E}-11$ & $1.71 \mathrm{E}-05$ \\
\hline $3.73 \mathrm{E}-05$ & $1.07 \mathrm{E}-05$ & $8.89 \mathrm{E}-08$ & 5.03E-08 & $8.04 \mathrm{E}-01$ & $8.84 \mathrm{E}-12$ & $2.00 \mathrm{E}-04$ & $1.12 \mathrm{E}-09$ & $8.40 \mathrm{E}-05$ & $5.13 \mathrm{E}-10$ & $1.39 \mathrm{E}-04$ \\
\hline $1.01 \mathrm{E}-04$ & $3.73 \mathrm{E}-05$ & $3.65 \mathrm{E}-07$ & $2.07 \mathrm{E}-07$ & $3.31 \mathrm{E}+00$ & $3.63 \mathrm{E}-11$ & $8.20 \mathrm{E}-04$ & $4.59 \mathrm{E}-09$ & $3.45 \mathrm{E}-04$ & $2.11 \mathrm{E}-09$ & $5.73 \mathrm{E}-04$ \\
\hline $2.14 \mathrm{E}-04$ & $1.01 \mathrm{E}-04$ & $9.78 \mathrm{E}-07$ & $5.54 \mathrm{E}-07$ & $8.85 \mathrm{E}+00$ & $9.73 \mathrm{E}-11$ & $2.20 \mathrm{E}-03$ & $1.23 \mathrm{E}-08$ & $9.25 \mathrm{E}-04$ & $5.64 \mathrm{E}-09$ & $1.53 \mathrm{E}-03$ \\
\hline $4.54 \mathrm{E}-04$ & $2.14 \mathrm{E}-04$ & $3.01 \mathrm{E}-06$ & $1.71 \mathrm{E}-06$ & $2.73 \mathrm{E}+01$ & $3.00 \mathrm{E}-10$ & $6.77 \mathrm{E}-03$ & $3.78 \mathrm{E}-08$ & $2.85 \mathrm{E}-03$ & $1.74 \mathrm{E}-08$ & $4.72 \mathrm{E}-03$ \\
\hline $1.58 \mathrm{E}-03$ & $4.54 \mathrm{E}-04$ & $2.70 \mathrm{E}-03$ & $1.40 \mathrm{E}-05$ & $2.24 \mathrm{E}+02$ & $3.82 \mathrm{E}-08$ & $5.53 \mathrm{E}-02$ & $2.19 \mathrm{E}-04$ & $2.33 \mathrm{E}-02$ & $3.23 \mathrm{E}-05$ & $3.86 \mathrm{E}-02$ \\
\hline $3.35 \mathrm{E}-03$ & $1.58 \mathrm{E}-03$ & $2.05 \mathrm{E}-01$ & $1.18 \mathrm{E}-02$ & $5.51 \mathrm{E}+02$ & $3.47 \mathrm{E}-05$ & $1.17 \mathrm{E}+00$ & $3.12 \mathrm{E}-03$ & $6.98 \mathrm{E}-02$ & $9.68 \mathrm{E}-04$ & $9.50 \mathrm{E}-02$ \\
\hline $7.10 \mathrm{E}-03$ & $3.35 \mathrm{E}-03$ & $1.40 \mathrm{E}+00$ & $7.85 \mathrm{E}-02$ & $1.72 \mathrm{E}+03$ & $2.87 \mathrm{E}-04$ & $6.94 \mathrm{E}+00$ & $2.68 \mathrm{E}-02$ & $2.64 \mathrm{E}-01$ & $6.58 \mathrm{E}-03$ & $2.93 \mathrm{E}-01$ \\
\hline $1.50 \mathrm{E}-02$ & $7.10 \mathrm{E}-03$ & $7.20 \mathrm{E}+00$ & $4.18 \mathrm{E}-01$ & $5.34 \mathrm{E}+03$ & $1.39 \mathrm{E}-03$ & $3.70 \mathrm{E}+01$ & $1.30 \mathrm{E}-01$ & $1.01 \mathrm{E}+00$ & & $9.04 \mathrm{E}-01$ \\
\hline 2.19E-02 & $1.50 \mathrm{E}-02$ & $1.00 \mathrm{E}+01$ & $5.77 \mathrm{E}-01$ & $5.99 \mathrm{E}+03$ & & & $1.81 \mathrm{E}-01$ & $1.25 \mathrm{E}+00$ & & $1.01 \mathrm{E}+00$ \\
\hline $2.42 \mathrm{E}-02$ & $2.19 \mathrm{E}-02$ & $4.00 \mathrm{E}+00$ & $2.38 \mathrm{E}-01$ & $2.26 \mathrm{E}+03$ & $7.89 \mathrm{E}-04$ & $2.01 \mathrm{E}+01$ & $7.32 \mathrm{E}-02$ & $5.04 \mathrm{E}-01$ & $1.89 \mathrm{E}-02$ & $3.79 \mathrm{E}-01$ \\
\hline $2.61 \mathrm{E}-02$ & $2.42 \mathrm{E}-02$ & $3.68 \mathrm{E}+00$ & $2.08 \mathrm{E}-01$ & $1.93 \mathrm{E}+03$ & $7.09 \mathrm{E}-04$ & $1.90 \mathrm{E}+01$ & $6.47 \mathrm{E}-02$ & $4.21 \mathrm{E}-01$ & $1.72 \mathrm{E}-02$ & $3.24 \mathrm{E}-01$ \\
\hline $3.18 \mathrm{E}-02$ & $2.61 \mathrm{E}-02$ & $1.24 \mathrm{E}+01$ & $7.15 \mathrm{E}-01$ & $6.35 \mathrm{E}+03$ & $2.40 \mathrm{E}-03$ & $6.31 \mathrm{E}+01$ & $2.25 \mathrm{E}-01$ & $1.45 \mathrm{E}+00$ & $5.81 \mathrm{E}-02$ & $1.06 \mathrm{E}+00$ \\
\hline 4.09E-02 & $3.18 \mathrm{E}-02$ & $2.36 \mathrm{E}+01$ & $1.38 \mathrm{E}+00$ & $1.11 \mathrm{E}+04$ & $4.64 \mathrm{E}-03$ & $1.20 \mathrm{E}+02$ & $4.26 \mathrm{E}-01$ & $2.67 \mathrm{E}+00$ & $1.11 \mathrm{E}-01$ & $1.86 \mathrm{E}+00$ \\
\hline $6.74 \mathrm{E}-02$ & $4.09 \mathrm{E}-02$ & $9.71 \mathrm{E}+01$ & $5.63 \mathrm{E}+00$ & $3.98 \mathrm{E}+04$ & $1.90 \mathrm{E}-02$ & $4.93 \mathrm{E}+02$ & $1.76 \mathrm{E}+00$ & $1.03 \mathrm{E}+01$ & $4.57 \mathrm{E}-01$ & $6.61 \mathrm{E}+00$ \\
\hline $1.11 \mathrm{E}-01$ & $6.74 \mathrm{E}-02$ & $2.33 \mathrm{E}+02$ & $1.36 \mathrm{E}+01$ & $8.35 \mathrm{E}+04$ & $4.58 \mathrm{E}-02$ & $1.18 \mathrm{E}+03$ & $4.22 \mathrm{E}+00$ & $2.36 \mathrm{E}+01$ & $1.10 \mathrm{E}+00$ & $1.38 \mathrm{E}+01$ \\
\hline $1.83 \mathrm{E}-01$ & $1.11 \mathrm{E}-01$ & $5.11 \mathrm{E}+02$ & $2.97 \mathrm{E}+01$ & $1.72 \mathrm{E}+05$ & $1.00 \mathrm{E}-01$ & $2.59 \mathrm{E}+03$ & $9.25 \mathrm{E}+00$ & $5.06 \mathrm{E}+01$ & $2.41 \mathrm{E}+00$ & $2.83 \mathrm{E}+01$ \\
\hline $2.97 \mathrm{E}-01$ & $1.83 \mathrm{E}-01$ & $2.05 \mathrm{E}+03$ & $1.19 \mathrm{E}+02$ & $3.57 \mathrm{E}+05$ & $4.01 \mathrm{E}-01$ & $1.03 \mathrm{E}+04$ & $3.71 \mathrm{E}+01$ & $1.68 \mathrm{E}+02$ & $9.66 \mathrm{E}+00$ & $5.57 \mathrm{E}+01$ \\
\hline 3.69E-01 & $2.97 \mathrm{E}-01$ & $6.37 \mathrm{E}+03$ & $3.72 \mathrm{E}+02$ & $3.69 \mathrm{E}+05$ & $1.25 \mathrm{E}+00$ & $3.18 \mathrm{E}+04$ & $1.15 \mathrm{E}+02$ & $4.46 \mathrm{E}+02$ & $3.00 \mathrm{E}+01$ & $4.50 \mathrm{E}+01$ \\
\hline $4.98 \mathrm{E}-01$ & $3.69 \mathrm{E}-01$ & $2.71 \mathrm{E}+04$ & $1.58 \mathrm{E}+03$ & $1.06 \mathrm{E}+06$ & $5.32 \mathrm{E}+00$ & $1.35 \mathrm{E}+05$ & $4.90 \mathrm{E}+02$ & $1.84 \mathrm{E}+03$ & $1.28 \mathrm{E}+02$ & $1.04 \mathrm{E}+02$ \\
\hline $6.08 \mathrm{E}-01$ & $4.98 \mathrm{E}-01$ & $3.52 \mathrm{E}+04$ & $2.05 \mathrm{E}+03$ & $1.22 \mathrm{E}+06$ & $6.89 \mathrm{E}+00$ & $1.76 \mathrm{E}+05$ & $6.36 \mathrm{E}+02$ & $2.38 \mathrm{E}+03$ & $1.66 \mathrm{E}+02$ & $1.07 \mathrm{E}+02$ \\
\hline $7.43 \mathrm{E}-01$ & $6.08 \mathrm{E}-01$ & $5.44 \mathrm{E}+04$ & $3.17 \mathrm{E}+03$ & $1.77 \mathrm{E}+06$ & $9.43 \mathrm{E}+00$ & $2.71 \mathrm{E}+05$ & $9.83 \mathrm{E}+02$ & $3.66 \mathrm{E}+03$ & $2.54 \mathrm{E}+02$ & $1.43 \mathrm{E}+02$ \\
\hline $8.21 \mathrm{E}-01$ & $7.43 \mathrm{E}-01$ & $3.62 \mathrm{E}+04$ & $2.10 \mathrm{E}+03$ & $1.14 \mathrm{E}+06$ & $5.45 \mathrm{E}+00$ & $1.81 \mathrm{E}+05$ & $6.36 \mathrm{E}+02$ & $2.37 \mathrm{E}+03$ & $1.66 \mathrm{E}+02$ & $8.36 \mathrm{E}+01$ \\
\hline $1.00 \mathrm{E}+00$ & $8.21 \mathrm{E}-01$ & $9.30 \mathrm{E}+04$ & $5.03 \mathrm{E}+03$ & $2.92 \mathrm{E}+06$ & $1.27 \mathrm{E}+01$ & $4.64 \mathrm{E}+05$ & $1.48 \mathrm{E}+03$ & $5.51 \mathrm{E}+03$ & $4.21 \mathrm{E}+02$ & $1.94 \mathrm{E}+02$ \\
\hline $1.35 \mathrm{E}+00$ & $1.00 \mathrm{E}+00$ & $1.78 \mathrm{E}+05$ & $9.47 \mathrm{E}+03$ & $5.90 \mathrm{E}+06$ & $2.36 \mathrm{E}+01$ & $8.88 \mathrm{E}+05$ & $2.78 \mathrm{E}+03$ & $1.04 \mathrm{E}+04$ & $8.04 \mathrm{E}+02$ & $3.60 \mathrm{E}+02$ \\
\hline $1.65 \mathrm{E}+00$ & $1.35 \mathrm{E}+00$ & $1.20 \mathrm{E}+05$ & $6.25 \mathrm{E}+03$ & $4.21 \mathrm{E}+06$ & $1.40 \mathrm{E}+01$ & $6.02 \mathrm{E}+05$ & $1.81 \mathrm{E}+03$ & $6.75 \mathrm{E}+03$ & $5.38 \mathrm{E}+02$ & $2.55 \mathrm{E}+02$ \\
\hline $1.92 \mathrm{E}+00$ & $1.65 \mathrm{E}+00$ & $1.10 \mathrm{E}+05$ & $5.72 \mathrm{E}+03$ & $3.74 \mathrm{E}+06$ & $1.30 \mathrm{E}+01$ & $5.50 \mathrm{E}+05$ & $1.66 \mathrm{E}+03$ & $6.18 \mathrm{E}+03$ & $4.91 \mathrm{E}+02$ & $2.15 \mathrm{E}+02$ \\
\hline $2.23 \mathrm{E}+00$ & $1.92 \mathrm{E}+00$ & $1.71 \mathrm{E}+05$ & $9.20 \mathrm{E}+03$ & $5.27 \mathrm{E}+06$ & $2.42 \mathrm{E}+01$ & $8.57 \mathrm{E}+05$ & $2.72 \mathrm{E}+03$ & $1.01 \mathrm{E}+04$ & $7.77 \mathrm{E}+02$ & $2.82 \mathrm{E}+02$ \\
\hline $2.35 \mathrm{E}+00$ & $2.23 \mathrm{E}+00$ & $6.99 \mathrm{E}+04$ & $3.79 \mathrm{E}+03$ & $2.08 \mathrm{E}+06$ & $1.13 \mathrm{E}+01$ & $3.50 \mathrm{E}+05$ & $1.13 \mathrm{E}+03$ & $4.16 \mathrm{E}+03$ & $3.20 \mathrm{E}+02$ & $1.09 \mathrm{E}+02$ \\
\hline $2.37 \mathrm{E}+00$ & $2.35 \mathrm{E}+00$ & $1.22 \mathrm{E}+04$ & $6.61 \mathrm{E}+02$ & $3.59 \mathrm{E}+05$ & $2.04 \mathrm{E}+00$ & $6.10 \mathrm{E}+04$ & $1.97 \mathrm{E}+02$ & $7.26 \mathrm{E}+02$ & $5.61 \mathrm{E}+01$ & $1.91 \mathrm{E}+01$ \\
\hline $2.47 \mathrm{E}+00$ & $2.37 \mathrm{E}+00$ & $6.43 \mathrm{E}+04$ & $3.50 \mathrm{E}+03$ & $1.88 \mathrm{E}+06$ & $1.11 \mathrm{E}+01$ & $3.22 \mathrm{E}+05$ & $1.04 \mathrm{E}+03$ & $3.84 \mathrm{E}+03$ & $2.97 \mathrm{E}+02$ & $1.01 \mathrm{E}+02$ \\
\hline $2.73 \mathrm{E}+00$ & $2.47 \mathrm{E}+00$ & $1.90 \mathrm{E}+05$ & $1.06 \mathrm{E}+04$ & $5.36 \mathrm{E}+06$ & $3.53 \mathrm{E}+01$ & $9.49 \mathrm{E}+05$ & $3.26 \mathrm{E}+03$ & $1.20 \mathrm{E}+04$ & $8.86 \mathrm{E}+02$ & $2.90 \mathrm{E}+02$ \\
\hline $3.01 \mathrm{E}+00$ & $2.73 \mathrm{E}+00$ & $3.13 \mathrm{E}+05$ & $1.82 \mathrm{E}+04$ & $8.13 \mathrm{E}+06$ & $6.05 \mathrm{E}+01$ & $1.56 \mathrm{E}+06$ & $5.65 \mathrm{E}+03$ & $2.08 \mathrm{E}+04$ & $1.47 \mathrm{E}+03$ & $4.39 \mathrm{E}+02$ \\
\hline $3.68 \mathrm{E}+00$ & $3.01 \mathrm{E}+00$ & $1.01 \mathrm{E}+06$ & $5.39 \mathrm{E}+04$ & $2.72 \mathrm{E}+07$ & $1.30 \mathrm{E}+02$ & $5.06 \mathrm{E}+06$ & $1.57 \mathrm{E}+04$ & $5.78 \mathrm{E}+04$ & $4.55 \mathrm{E}+03$ & $9.72 \mathrm{E}+02$ \\
\hline $4.97 \mathrm{E}+00$ & $3.68 \mathrm{E}+00$ & $1.71 \mathrm{E}+06$ & $8.58 \mathrm{E}+04$ & $4.95 \mathrm{E}+07$ & $1.82 \mathrm{E}+02$ & $8.55 \mathrm{E}+06$ & $2.42 \mathrm{E}+04$ & $8.93 \mathrm{E}+04$ & $7.54 \mathrm{E}+03$ & $1.34 \mathrm{E}+03$ \\
\hline $6.07 \mathrm{E}+00$ & $4.97 \mathrm{E}+00$ & $9.50 \mathrm{E}+05$ & $4.32 \mathrm{E}+04$ & $3.01 \mathrm{E}+07$ & $6.13 \mathrm{E}+01$ & $4.77 \mathrm{E}+06$ & $1.12 \mathrm{E}+04$ & $4.16 \mathrm{E}+04$ & $4.06 \mathrm{E}+03$ & $4.96 \mathrm{E}+02$ \\
\hline $7.41 \mathrm{E}+00$ & $6.07 \mathrm{E}+00$ & $9.51 \mathrm{E}+05$ & $4.74 \mathrm{E}+04$ & $3.02 \mathrm{E}+07$ & $1.27 \mathrm{E}+02$ & $4.77 \mathrm{E}+06$ & $1.38 \mathrm{E}+04$ & $5.06 \mathrm{E}+04$ & $4.28 \mathrm{E}+03$ & $8.11 \mathrm{E}+02$ \\
\hline $8.61 \mathrm{E}+00$ & $7.41 \mathrm{E}+00$ & $7.91 \mathrm{E}+05$ & $4.00 \mathrm{E}+04$ & $2.34 \mathrm{E}+07$ & $1.01 \mathrm{E}+02$ & $3.97 \mathrm{E}+06$ & $1.15 \mathrm{E}+04$ & $4.24 \mathrm{E}+04$ & $3.55 \mathrm{E}+03$ & $6.44 \mathrm{E}+02$ \\
\hline $1.00 \mathrm{E}+01$ & $8.61 \mathrm{E}+00$ & $5.99 \mathrm{E}+05$ & $2.82 \mathrm{E}+04$ & $1.96 \mathrm{E}+07$ & $6.21 \mathrm{E}+01$ & $3.01 \mathrm{E}+06$ & $7.83 \mathrm{E}+03$ & $2.89 \mathrm{E}+04$ & $2.64 \mathrm{E}+03$ & $4.07 \mathrm{E}+02$ \\
\hline $1.22 \mathrm{E}+01$ & $1.00 \mathrm{E}+01$ & $6.74 \mathrm{E}+04$ & $2.81 \mathrm{E}+03$ & $2.74 \mathrm{E}+06$ & $2.10 \mathrm{E}+00$ & $3.40 \mathrm{E}+05$ & $6.65 \mathrm{E}+02$ & $2.48 \mathrm{E}+03$ & $2.81 \mathrm{E}+02$ & $2.09 \mathrm{E}+01$ \\
\hline $1.42 \mathrm{E}+01$ & $1.22 \mathrm{E}+01$ & $2.52 \mathrm{E}-04$ & $7.22 \mathrm{E}-05$ & $1.76 \mathrm{E}+03$ & $8.83 \mathrm{E}-09$ & $2.46 \mathrm{E}-01$ & $1.97 \mathrm{E}-06$ & $5.52 \mathrm{E}-02$ & $1.18 \mathrm{E}-06$ & $1.17 \mathrm{E}-01$ \\
\hline \multirow[t]{2}{*}{$1.96 \mathrm{E}+01$} & $1.42 \mathrm{E}+01$ & $6.06 \mathrm{E}-05$ & $1.48 \mathrm{E}-05$ & $3.97 \mathrm{E}+02$ & $1.68 \mathrm{E}-09$ & $4.86 \mathrm{E}-02$ & $4.25 \mathrm{E}-07$ & $9.46 \mathrm{E}-03$ & $2.74 \mathrm{E}-07$ & $2.13 \mathrm{E}-02$ \\
\hline & Total & $7.56 \mathrm{E}+06$ & $3.83 \mathrm{E}+05$ & $2.29 \mathrm{E}+08$ & $9.03 \mathrm{E}+02$ & $3.79 \mathrm{E}+07$ & $1.09 \mathrm{E}+05$ & $4.04 \mathrm{E}+05$ & $3.37 \mathrm{E}+04$ & $7.55 \mathrm{E}+03$ \\
\hline
\end{tabular}

Note: $90 \%$ Beryllium is modeled as 1 gram of actinide and 0.9 grams of beryllium. 
PCP-2011-0001

Revision 0

Table 3-7. Neutron Source from ORIGEN-S w/90\% Beryllium (Decay Time $=100$ days)

\begin{tabular}{|c|c|c|c|c|c|c|c|c|c|c|}
\hline \multicolumn{2}{|c|}{ Energy Bound (MeV) } & \multicolumn{9}{|c|}{ Neutron Source (n/s/g-actinide) } \\
\hline Upper & Upper & Am-241 & Am-243 & Cm-244 & Np-237 & Pu-238 & Pu-239 & Pu-240 & Pu-241 & Pu-242 \\
\hline $1.00 \mathrm{E}-07$ & $1.00 \mathrm{E}-11$ & $2.34 \mathrm{E}-11$ & $4.52 \mathrm{E}-12$ & $1.31 \mathrm{E}-04$ & $1.34 \mathrm{E}-15$ & $3.09 \mathrm{E}-08$ & $3.52 \mathrm{E}-13$ & $1.47 \mathrm{E}-08$ & $3.36 \mathrm{E}-13$ & $2.92 \mathrm{E}-08$ \\
\hline $4.14 \mathrm{E}-07$ & $1.00 \mathrm{E}-07$ & $1.17 \mathrm{E}-10$ & $5.75 \mathrm{E}-11$ & $9.73 \mathrm{E}-04$ & $1.07 \mathrm{E}-14$ & $2.41 \mathrm{E}-07$ & $1.54 \mathrm{E}-12$ & $1.03 \mathrm{E}-07$ & $1.74 \mathrm{E}-12$ & $1.76 \mathrm{E}-07$ \\
\hline $8.76 \mathrm{E}-07$ & $4.14 \mathrm{E}-07$ & $2.64 \mathrm{E}-10$ & $1.41 \mathrm{E}-10$ & $2.30 \mathrm{E}-03$ & $2.53 \mathrm{E}-14$ & $5.71 \mathrm{E}-07$ & $3.37 \mathrm{E}-12$ & $2.42 \mathrm{E}-07$ & $3.93 \mathrm{E}-12$ & $4.07 \mathrm{E}-07$ \\
\hline $1.86 \mathrm{E}-06$ & $8.76 \mathrm{E}-07$ & $7.99 \mathrm{E}-10$ & $4.41 \mathrm{E}-10$ & $7.07 \mathrm{E}-03$ & $7.82 \mathrm{E}-14$ & $1.76 \mathrm{E}-06$ & $1.01 \mathrm{E}-11$ & $7.46 \mathrm{E}-07$ & $1.19 \mathrm{E}-11$ & $1.24 \mathrm{E}-06$ \\
\hline $5.04 \mathrm{E}-06$ & $1.86 \mathrm{E}-06$ & $4.08 \mathrm{E}-09$ & $2.29 \mathrm{E}-09$ & $3.65 \mathrm{E}-02$ & $4.03 \mathrm{E}-13$ & $9.09 \mathrm{E}-06$ & $5.14 \mathrm{E}-11$ & $3.84 \mathrm{E}-06$ & $6.09 \mathrm{E}-11$ & $6.38 \mathrm{E}-06$ \\
\hline $1.07 \mathrm{E}-05$ & $5.04 \mathrm{E}-06$ & $1.09 \mathrm{E}-08$ & $6.15 \mathrm{E}-09$ & $9.76 \mathrm{E}-02$ & $1.08 \mathrm{E}-12$ & $2.44 \mathrm{E}-05$ & $1.37 \mathrm{E}-10$ & $1.03 \mathrm{E}-05$ & $1.63 \mathrm{E}-10$ & $1.71 \mathrm{E}-05$ \\
\hline $3.73 \mathrm{E}-05$ & $1.07 \mathrm{E}-05$ & $8.89 \mathrm{E}-08$ & $5.03 \mathrm{E}-08$ & $7.98 \mathrm{E}-01$ & $8.84 \mathrm{E}-12$ & $1.99 \mathrm{E}-04$ & $1.12 \mathrm{E}-09$ & $8.40 \mathrm{E}-05$ & $1.33 \mathrm{E}-09$ & $1.39 \mathrm{E}-04$ \\
\hline $1.01 \mathrm{E}-04$ & $3.73 \mathrm{E}-05$ & $3.65 \mathrm{E}-07$ & $2.07 \mathrm{E}-07$ & $3.28 \mathrm{E}+00$ & $3.63 \mathrm{E}-11$ & $8.19 \mathrm{E}-04$ & $4.59 \mathrm{E}-09$ & $3.45 \mathrm{E}-04$ & $5.45 \mathrm{E}-09$ & $5.73 \mathrm{E}-04$ \\
\hline $2.14 \mathrm{E}-04$ & $1.01 \mathrm{E}-04$ & $9.78 \mathrm{E}-07$ & $5.54 \mathrm{E}-07$ & $8.79 \mathrm{E}+00$ & $9.73 \mathrm{E}-11$ & $2.19 \mathrm{E}-03$ & $1.23 \mathrm{E}-08$ & $9.25 \mathrm{E}-04$ & $1.46 \mathrm{E}-08$ & $1.53 \mathrm{E}-03$ \\
\hline $4.54 \mathrm{E}-04$ & $2.14 \mathrm{E}-04$ & $3.01 \mathrm{E}-06$ & $1.71 \mathrm{E}-06$ & $2.71 \mathrm{E}+01$ & $3.00 \mathrm{E}-10$ & $6.76 \mathrm{E}-03$ & $3.78 \mathrm{E}-08$ & $2.85 \mathrm{E}-03$ & $4.50 \mathrm{E}-08$ & $4.72 \mathrm{E}-03$ \\
\hline $1.58 \mathrm{E}-03$ & $4.54 \mathrm{E}-04$ & $2.70 \mathrm{E}-03$ & $1.40 \mathrm{E}-05$ & $2.22 \mathrm{E}+02$ & $3.82 \mathrm{E}-08$ & $5.52 \mathrm{E}-02$ & $2.19 \mathrm{E}-04$ & $2.33 \mathrm{E}-02$ & $5.69 \mathrm{E}-05$ & $3.86 \mathrm{E}-02$ \\
\hline $3.35 \mathrm{E}-03$ & $1.58 \mathrm{E}-03$ & $2.04 \mathrm{E}-01$ & $1.18 \mathrm{E}-02$ & $5.47 \mathrm{E}+02$ & $3.47 \mathrm{E}-05$ & $1.17 \mathrm{E}+00$ & $3.12 \mathrm{E}-03$ & $6.98 \mathrm{E}-02$ & $2.85 \mathrm{E}-03$ & $9.50 \mathrm{E}-02$ \\
\hline $7.10 \mathrm{E}-03$ & $3.35 \mathrm{E}-03$ & $1.40 \mathrm{E}+00$ & $7.85 \mathrm{E}-02$ & $1.71 \mathrm{E}+03$ & $2.87 \mathrm{E}-04$ & $6.93 \mathrm{E}+00$ & $2.68 \mathrm{E}-02$ & $2.64 \mathrm{E}-01$ & $1.94 \mathrm{E}-02$ & $2.93 \mathrm{E}-01$ \\
\hline $1.50 \mathrm{E}-02$ & $7.10 \mathrm{E}-03$ & $7.20 \mathrm{E}+00$ & $4.18 \mathrm{E}-01$ & $5.30 \mathrm{E}+03$ & $1.39 \mathrm{E}-03$ & $3.69 \mathrm{E}+01$ & $1.30 \mathrm{E}-01$ & $1.01 \mathrm{E}+00$ & $1.00 \mathrm{E}-01$ & $9.04 \mathrm{E}-01$ \\
\hline $2.19 \mathrm{E}-02$ & $1.50 \mathrm{E}-02$ & $1.00 \mathrm{E}+01$ & $5.77 \mathrm{E}-01$ & $5.95 \mathrm{E}+03$ & $1.97 \mathrm{E}-03$ & $5.14 \mathrm{E}+01$ & $1.81 \mathrm{E}-01$ & $1.25 \mathrm{E}+00$ & $1.39 \mathrm{E}-01$ & $1.01 \mathrm{E}+00$ \\
\hline $2.42 \mathrm{E}-02$ & $2.19 \mathrm{E}-02$ & $3.99 \mathrm{E}+00$ & $2.38 \mathrm{E}-01$ & $2.24 \mathrm{E}+03$ & $7.89 \mathrm{E}-04$ & $2.01 \mathrm{E}+01$ & $7.32 \mathrm{E}-02$ & $5.04 \mathrm{E}-01$ & $5.56 \mathrm{E}-02$ & $3.79 \mathrm{E}-01$ \\
\hline $2.61 \mathrm{E}-02$ & $2.42 \mathrm{E}-02$ & $3.68 \mathrm{E}+00$ & $2.08 \mathrm{E}-01$ & $1.92 \mathrm{E}+03$ & $7.09 \mathrm{E}-04$ & $1.89 \mathrm{E}+01$ & $6.47 \mathrm{E}-02$ & $4.21 \mathrm{E}-01$ & $5.10 \mathrm{E}-02$ & $3.24 \mathrm{E}-01$ \\
\hline $3.18 \mathrm{E}-02$ & $2.61 \mathrm{E}-02$ & $1.24 \mathrm{E}+01$ & $7.15 \mathrm{E}-01$ & $6.30 \mathrm{E}+03$ & $2.40 \mathrm{E}-03$ & $6.30 \mathrm{E}+01$ & $2.25 \mathrm{E}-01$ & $1.45 \mathrm{E}+00$ & $1.72 \mathrm{E}-01$ & $1.06 \mathrm{E}+00$ \\
\hline 4.09E-02 & $3.18 \mathrm{E}-02$ & $2.36 \mathrm{E}+01$ & $1.38 \mathrm{E}+00$ & $1.11 \mathrm{E}+04$ & $4.64 \mathrm{E}-03$ & $1.20 \mathrm{E}+02$ & $4.26 \mathrm{E}-01$ & $2.67 \mathrm{E}+00$ & $3.28 \mathrm{E}-01$ & $1.86 \mathrm{E}+00$ \\
\hline $6.74 \mathrm{E}-02$ & $4.09 \mathrm{E}-02$ & $9.71 \mathrm{E}+01$ & $5.63 \mathrm{E}+00$ & $3.95 \mathrm{E}+04$ & $1.90 \mathrm{E}-02$ & $4.92 \mathrm{E}+02$ & $1.76 \mathrm{E}+00$ & $1.03 \mathrm{E}+01$ & $1.35 \mathrm{E}+00$ & $6.61 \mathrm{E}+00$ \\
\hline $1.11 \mathrm{E}-01$ & $6.74 \mathrm{E}-02$ & $2.33 \mathrm{E}+02$ & $1.36 \mathrm{E}+01$ & $8.29 \mathrm{E}+04$ & $4.58 \mathrm{E}-02$ & $1.18 \mathrm{E}+03$ & $4.22 \mathrm{E}+00$ & $2.36 \mathrm{E}+01$ & $3.24 \mathrm{E}+00$ & $1.38 \mathrm{E}+01$ \\
\hline $1.83 \mathrm{E}-01$ & $1.11 \mathrm{E}-01$ & $5.11 \mathrm{E}+02$ & $2.97 \mathrm{E}+01$ & $1.71 \mathrm{E}+05$ & $1.00 \mathrm{E}-01$ & $2.58 \mathrm{E}+03$ & $9.25 \mathrm{E}+00$ & $5.06 \mathrm{E}+01$ & $7.10 \mathrm{E}+00$ & $2.83 \mathrm{E}+01$ \\
\hline $2.97 \mathrm{E}-01$ & $1.83 \mathrm{E}-01$ & $2.05 \mathrm{E}+03$ & $1.19 \mathrm{E}+02$ & $3.55 \mathrm{E}+05$ & $4.01 \mathrm{E}-01$ & $1.03 \mathrm{E}+04$ & $3.71 \mathrm{E}+01$ & $1.68 \mathrm{E}+02$ & $2.85 \mathrm{E}+01$ & $5.57 \mathrm{E}+01$ \\
\hline $3.69 \mathrm{E}-01$ & $2.97 \mathrm{E}-01$ & $6.37 \mathrm{E}+03$ & $3.72 \mathrm{E}+02$ & $3.66 \mathrm{E}+05$ & $1.25 \mathrm{E}+00$ & $3.17 \mathrm{E}+04$ & $1.15 \mathrm{E}+02$ & $4.46 \mathrm{E}+02$ & $8.85 \mathrm{E}+01$ & $4.50 \mathrm{E}+01$ \\
\hline $4.98 \mathrm{E}-01$ & $3.69 \mathrm{E}-01$ & $2.71 \mathrm{E}+04$ & $1.58 \mathrm{E}+03$ & $1.06 \mathrm{E}+06$ & $5.32 \mathrm{E}+00$ & $1.35 \mathrm{E}+05$ & $4.90 \mathrm{E}+02$ & $1.84 \mathrm{E}+03$ & $3.77 \mathrm{E}+02$ & $1.04 \mathrm{E}+02$ \\
\hline $6.08 \mathrm{E}-01$ & $4.98 \mathrm{E}-01$ & $3.52 \mathrm{E}+04$ & $2.05 \mathrm{E}+03$ & $1.21 \mathrm{E}+06$ & $6.89 \mathrm{E}+00$ & $1.75 \mathrm{E}+05$ & $6.36 \mathrm{E}+02$ & $2.38 \mathrm{E}+03$ & $4.90 \mathrm{E}+02$ & $1.07 \mathrm{E}+02$ \\
\hline $7.43 \mathrm{E}-01$ & $6.08 \mathrm{E}-01$ & $5.44 \mathrm{E}+04$ & $3.17 \mathrm{E}+03$ & $1.75 \mathrm{E}+06$ & $9.43 \mathrm{E}+00$ & $2.71 \mathrm{E}+05$ & $9.83 \mathrm{E}+02$ & $3.66 \mathrm{E}+03$ & $7.55 \mathrm{E}+02$ & $1.43 \mathrm{E}+02$ \\
\hline $8.21 \mathrm{E}-01$ & $7.43 \mathrm{E}-01$ & $3.62 \mathrm{E}+04$ & $2.10 \mathrm{E}+03$ & $1.13 \mathrm{E}+06$ & $5.45 \mathrm{E}+00$ & $1.80 \mathrm{E}+05$ & $6.36 \mathrm{E}+02$ & $2.37 \mathrm{E}+03$ & $4.99 \mathrm{E}+02$ & $8.36 \mathrm{E}+01$ \\
\hline $1.00 \mathrm{E}+00$ & $8.21 \mathrm{E}-01$ & $9.29 \mathrm{E}+04$ & $5.03 \mathrm{E}+03$ & $2.90 \mathrm{E}+06$ & $1.27 \mathrm{E}+01$ & $4.63 \mathrm{E}+05$ & $1.48 \mathrm{E}+03$ & $5.51 \mathrm{E}+03$ & $1.28 \mathrm{E}+03$ & $1.94 \mathrm{E}+02$ \\
\hline $1.35 \mathrm{E}+00$ & $1.00 \mathrm{E}+00$ & $1.78 \mathrm{E}+05$ & $9.47 \mathrm{E}+03$ & $5.85 \mathrm{E}+06$ & $2.36 \mathrm{E}+01$ & $8.87 \mathrm{E}+05$ & $2.78 \mathrm{E}+03$ & $1.04 \mathrm{E}+04$ & $2.44 \mathrm{E}+03$ & $3.60 \mathrm{E}+02$ \\
\hline $1.65 \mathrm{E}+00$ & $1.35 \mathrm{E}+00$ & $1.20 \mathrm{E}+05$ & $6.25 \mathrm{E}+03$ & $4.18 \mathrm{E}+06$ & $1.40 \mathrm{E}+01$ & $6.01 \mathrm{E}+05$ & $1.81 \mathrm{E}+03$ & $6.75 \mathrm{E}+03$ & $1.64 \mathrm{E}+03$ & $2.55 \mathrm{E}+02$ \\
\hline $1.92 \mathrm{E}+00$ & $1.65 \mathrm{E}+00$ & $1.10 \mathrm{E}+05$ & $5.72 \mathrm{E}+03$ & $3.71 \mathrm{E}+06$ & $1.30 \mathrm{E}+01$ & $5.49 \mathrm{E}+05$ & $1.66 \mathrm{E}+03$ & $6.18 \mathrm{E}+03$ & $1.50 \mathrm{E}+03$ & $2.15 \mathrm{E}+02$ \\
\hline $2.23 \mathrm{E}+00$ & $1.92 \mathrm{E}+00$ & $1.71 \mathrm{E}+05$ & $9.20 \mathrm{E}+03$ & $5.24 \mathrm{E}+06$ & $2.42 \mathrm{E}+01$ & $8.56 \mathrm{E}+05$ & $2.72 \mathrm{E}+03$ & $1.01 \mathrm{E}+04$ & $2.35 \mathrm{E}+03$ & $2.82 \mathrm{E}+02$ \\
\hline $2.35 \mathrm{E}+00$ & $2.23 \mathrm{E}+00$ & $6.99 \mathrm{E}+04$ & $3.79 \mathrm{E}+03$ & $2.06 \mathrm{E}+06$ & $1.13 \mathrm{E}+01$ & $3.49 \mathrm{E}+05$ & $1.13 \mathrm{E}+03$ & $4.16 \mathrm{E}+03$ & $9.63 \mathrm{E}+02$ & $1.09 \mathrm{E}+02$ \\
\hline $2.37 \mathrm{E}+00$ & $2.35 \mathrm{E}+00$ & $1.22 \mathrm{E}+04$ & $6.61 \mathrm{E}+02$ & $3.56 \mathrm{E}+05$ & $2.04 \mathrm{E}+00$ & $6.09 \mathrm{E}+04$ & $1.97 \mathrm{E}+02$ & $7.26 \mathrm{E}+02$ & $1.68 \mathrm{E}+02$ & $1.91 \mathrm{E}+01$ \\
\hline $2.47 \mathrm{E}+00$ & $2.37 \mathrm{E}+00$ & $6.43 \mathrm{E}+04$ & $3.50 \mathrm{E}+03$ & $1.87 \mathrm{E}+06$ & $1.11 \mathrm{E}+01$ & $3.21 \mathrm{E}+05$ & $1.04 \mathrm{E}+03$ & $3.84 \mathrm{E}+03$ & $8.88 \mathrm{E}+02$ & $1.01 \mathrm{E}+02$ \\
\hline $2.73 \mathrm{E}+00$ & $2.47 \mathrm{E}+00$ & $1.90 \mathrm{E}+05$ & $1.06 \mathrm{E}+04$ & $5.32 \mathrm{E}+06$ & $3.53 \mathrm{E}+01$ & $9.47 \mathrm{E}+05$ & $3.26 \mathrm{E}+03$ & $1.20 \mathrm{E}+04$ & $2.63 \mathrm{E}+03$ & $2.90 \mathrm{E}+02$ \\
\hline $3.01 \mathrm{E}+00$ & $2.73 \mathrm{E}+00$ & $3.13 \mathrm{E}+05$ & $1.82 \mathrm{E}+04$ & $8.07 \mathrm{E}+06$ & $6.05 \mathrm{E}+01$ & $1.56 \mathrm{E}+06$ & $5.65 \mathrm{E}+03$ & $2.08 \mathrm{E}+04$ & $4.35 \mathrm{E}+03$ & $4.39 \mathrm{E}+02$ \\
\hline $3.68 \mathrm{E}+00$ & $3.01 \mathrm{E}+00$ & $1.01 \mathrm{E}+06$ & $5.39 \mathrm{E}+04$ & $2.70 \mathrm{E}+07$ & $1.30 \mathrm{E}+02$ & $5.05 \mathrm{E}+06$ & $1.57 \mathrm{E}+04$ & $5.78 \mathrm{E}+04$ & $1.39 \mathrm{E}+04$ & $9.72 \mathrm{E}+02$ \\
\hline $4.97 \mathrm{E}+00$ & $3.68 \mathrm{E}+00$ & $1.71 \mathrm{E}+06$ & $8.58 \mathrm{E}+04$ & $4.92 \mathrm{E}+07$ & $1.82 \mathrm{E}+02$ & $8.53 \mathrm{E}+06$ & $2.42 \mathrm{E}+04$ & $8.93 \mathrm{E}+04$ & $2.32 \mathrm{E}+04$ & $1.34 \mathrm{E}+03$ \\
\hline $6.07 \mathrm{E}+00$ & & & & & & $4.77 \mathrm{E}+06$ & $1.12 \mathrm{E}+04$ & $4.16 \mathrm{E}+04$ & $1.28 \mathrm{E}+04$ & $4.96 \mathrm{E}+02$ \\
\hline $7.41 \mathrm{E}+00$ & $6.07 \mathrm{E}+00$ & $9.51 \mathrm{E}+05$ & $4.74 \mathrm{E}+04$ & $2.99 \mathrm{E}+07$ & $1.27 \mathrm{E}+02$ & $4.76 \mathrm{E}+06$ & $1.38 \mathrm{E}+04$ & $5.06 \mathrm{E}+04$ & $1.30 \mathrm{E}+04$ & $8.11 \mathrm{E}+02$ \\
\hline $8.61 \mathrm{E}+00$ & $7.41 \mathrm{E}+00$ & $7.91 \mathrm{E}+05$ & $4.00 \mathrm{E}+04$ & $2.32 \mathrm{E}+07$ & $1.01 \mathrm{E}+02$ & $3.96 \mathrm{E}+06$ & $1.15 \mathrm{E}+04$ & $4.24 \mathrm{E}+04$ & $1.08 \mathrm{E}+04$ & $6.44 \mathrm{E}+02$ \\
\hline $1.00 \mathrm{E}+01$ & $8.61 \mathrm{E}+00$ & $5.99 \mathrm{E}+05$ & $2.82 \mathrm{E}+04$ & $1.94 \mathrm{E}+07$ & $6.21 \mathrm{E}+01$ & $3.01 \mathrm{E}+06$ & $7.83 \mathrm{E}+03$ & $2.89 \mathrm{E}+04$ & $8.14 \mathrm{E}+03$ & $4.07 \mathrm{E}+02$ \\
\hline $1.22 \mathrm{E}+01$ & $1.00 \mathrm{E}+01$ & $6.74 \mathrm{E}+04$ & $2.81 \mathrm{E}+03$ & $2.72 \mathrm{E}+06$ & $2.10 \mathrm{E}+00$ & $3.39 \mathrm{E}+05$ & $6.65 \mathrm{E}+02$ & $2.48 \mathrm{E}+03$ & $9.01 \mathrm{E}+02$ & $2.09 \mathrm{E}+01$ \\
\hline $1.42 \mathrm{E}+01$ & $1.22 \mathrm{E}+01$ & $2.52 \mathrm{E}-04$ & $7.22 \mathrm{E}-05$ & $1.75 \mathrm{E}+03$ & $8.83 \mathrm{E}-09$ & $2.45 \mathrm{E}-01$ & $1.97 \mathrm{E}-06$ & $5.52 \mathrm{E}-02$ & $3.49 \mathrm{E}-06$ & $1.17 \mathrm{E}-01$ \\
\hline \multirow[t]{2}{*}{$1.96 \mathrm{E}+01$} & $1.42 \mathrm{E}+01$ & $6.06 \mathrm{E}-05$ & $1.48 \mathrm{E}-05$ & $3.94 \mathrm{E}+02$ & $1.68 \mathrm{E}-09$ & $4.85 \mathrm{E}-02$ & $4.25 \mathrm{E}-07$ & $9.46 \mathrm{E}-03$ & $8.30 \mathrm{E}-07$ & $2.13 \mathrm{E}-02$ \\
\hline & Total & $7.56 \mathrm{E}+06$ & $3.83 \mathrm{E}+05$ & $2.27 \mathrm{E}+08$ & $9.03 \mathrm{E}+02$ & $3.78 \mathrm{E}+07$ & $1.09 \mathrm{E}+05$ & $4.04 \mathrm{E}+05$ & $1.03 \mathrm{E}+05$ & $7.55 \mathrm{E}+03$ \\
\hline
\end{tabular}

Note: $90 \%$ Beryllium is modeled as 1 gram of actinide and 0.9 grams of beryllium. 
PCP-2011-0001

Revision 0

Table 3-8. Neutron Source from ORIGEN-S w/90\% Beryllium (Decay Time $=\mathbf{3 0 , 0 0 0}$ days)

\begin{tabular}{|c|c|c|c|c|c|c|c|c|c|c|}
\hline \multicolumn{2}{|c|}{ Energy Bound (MeV) } & \multicolumn{9}{|c|}{ Neutron Source (n/s/g-actinide) } \\
\hline Upper & Upper & Am-241 & Am-243 & Cm-244 & Np-237 & Pu-238 & Pu-239 & Pu-240 & Pu-241 & Pu-242 \\
\hline $1.00 \mathrm{E}-07$ & $1.00 \mathrm{E}-11$ & $2.05 \mathrm{E}-11$ & $4.49 \mathrm{E}-12$ & $5.71 \mathrm{E}-06$ & $1.34 \mathrm{E}-15$ & $1.62 \mathrm{E}-08$ & $3.51 \mathrm{E}-13$ & $1.46 \mathrm{E}-08$ & $2.07 \mathrm{E}-11$ & $2.92 \mathrm{E}-08$ \\
\hline $4.14 \mathrm{E}-07$ & $1.00 \mathrm{E}-07$ & $1.03 \mathrm{E}-10$ & $5.70 \mathrm{E}-11$ & $4.24 \mathrm{E}-05$ & $1.07 \mathrm{E}-14$ & $1.26 \mathrm{E}-07$ & $1.53 \mathrm{E}-12$ & $1.03 \mathrm{E}-07$ & $1.04 \mathrm{E}-10$ & $1.76 \mathrm{E}-07$ \\
\hline $8.76 \mathrm{E}-07$ & $4.14 \mathrm{E}-07$ & $2.31 \mathrm{E}-10$ & $1.40 \mathrm{E}-10$ & $1.00 \mathrm{E}-04$ & $2.53 \mathrm{E}-14$ & 2.99E-07 & $3.36 \mathrm{E}-12$ & $2.40 \mathrm{E}-07$ & $2.34 \mathrm{E}-10$ & $4.07 \mathrm{E}-07$ \\
\hline $1.86 \mathrm{E}-06$ & $8.76 \mathrm{E}-07$ & $7.00 \mathrm{E}-10$ & $4.38 \mathrm{E}-10$ & $3.08 \mathrm{E}-04$ & $7.82 \mathrm{E}-14$ & $9.23 \mathrm{E}-07$ & $1.01 \mathrm{E}-11$ & $7.39 \mathrm{E}-07$ & $7.09 \mathrm{E}-10$ & $1.24 \mathrm{E}-06$ \\
\hline $5.04 \mathrm{E}-06$ & $1.86 \mathrm{E}-06$ & $3.58 \mathrm{E}-09$ & $2.27 \mathrm{E}-09$ & $1.59 \mathrm{E}-03$ & $4.03 \mathrm{E}-13$ & $4.76 \mathrm{E}-06$ & $5.13 \mathrm{E}-11$ & $3.81 \mathrm{E}-06$ & $3.62 \mathrm{E}-09$ & $6.38 \mathrm{E}-06$ \\
\hline $1.07 \mathrm{E}-05$ & $5.04 \mathrm{E}-06$ & $9.55 \mathrm{E}-09$ & $6.10 \mathrm{E}-09$ & $4.26 \mathrm{E}-03$ & $1.08 \mathrm{E}-12$ & $1.28 \mathrm{E}-05$ & $1.37 \mathrm{E}-10$ & $1.02 \mathrm{E}-05$ & $9.67 \mathrm{E}-09$ & $1.71 \mathrm{E}-05$ \\
\hline $3.73 \mathrm{E}-05$ & $1.07 \mathrm{E}-05$ & $7.79 \mathrm{E}-08$ & $5.00 \mathrm{E}-08$ & $3.48 \mathrm{E}-02$ & $8.84 \mathrm{E}-12$ & $1.04 \mathrm{E}-04$ & $1.11 \mathrm{E}-09$ & $8.33 \mathrm{E}-05$ & $7.89 \mathrm{E}-08$ & $1.39 \mathrm{E}-04$ \\
\hline $1.01 \mathrm{E}-04$ & $3.73 \mathrm{E}-05$ & $3.20 \mathrm{E}-07$ & $2.06 \mathrm{E}-07$ & $1.43 \mathrm{E}-01$ & $3.63 \mathrm{E}-11$ & $4.29 \mathrm{E}-04$ & $4.58 \mathrm{E}-09$ & $3.42 \mathrm{E}-04$ & $3.24 \mathrm{E}-07$ & $5.73 \mathrm{E}-04$ \\
\hline $2.14 \mathrm{E}-04$ & $1.01 \mathrm{E}-04$ & $8.57 \mathrm{E}-07$ & $5.50 \mathrm{E}-07$ & $3.83 \mathrm{E}-01$ & $9.73 \mathrm{E}-11$ & $1.15 \mathrm{E}-03$ & $1.23 \mathrm{E}-08$ & $9.17 \mathrm{E}-04$ & $8.68 \mathrm{E}-07$ & $1.53 \mathrm{E}-03$ \\
\hline $4.54 \mathrm{E}-04$ & $2.14 \mathrm{E}-04$ & $2.64 \mathrm{E}-06$ & $1.70 \mathrm{E}-06$ & $1.18 \mathrm{E}+00$ & $3.00 \mathrm{E}-10$ & $3.54 \mathrm{E}-03$ & $3.77 \mathrm{E}-08$ & $2.82 \mathrm{E}-03$ & $2.67 \mathrm{E}-06$ & $4.72 \mathrm{E}-03$ \\
\hline $1.58 \mathrm{E}-03$ & $4.54 \mathrm{E}-04$ & $2.36 \mathrm{E}-03$ & $1.39 \mathrm{E}-05$ & $9.68 \mathrm{E}+00$ & $3.82 \mathrm{E}-08$ & $2.89 \mathrm{E}-02$ & $2.18 \mathrm{E}-04$ & $2.31 \mathrm{E}-02$ & $2.40 \mathrm{E}-03$ & $3.86 \mathrm{E}-02$ \\
\hline $3.35 \mathrm{E}-03$ & $1.58 \mathrm{E}-03$ & $1.79 \mathrm{E}-01$ & $1.17 \mathrm{E}-02$ & $2.39 \mathrm{E}+01$ & $3.47 \mathrm{E}-05$ & $6.14 \mathrm{E}-01$ & $3.11 \mathrm{E}-03$ & $6.92 \mathrm{E}-02$ & $1.82 \mathrm{E}-01$ & $9.50 \mathrm{E}-02$ \\
\hline $7.10 \mathrm{E}-03$ & $3.35 \mathrm{E}-03$ & $1.23 \mathrm{E}+00$ & $7.79 \mathrm{E}-02$ & $7.44 \mathrm{E}+01$ & $2.87 \mathrm{E}-04$ & $3.63 \mathrm{E}+00$ & $2.67 \mathrm{E}-02$ & $2.62 \mathrm{E}-01$ & $1.24 \mathrm{E}+00$ & $2.93 \mathrm{E}-01$ \\
\hline $1.50 \mathrm{E}-02$ & $7.10 \mathrm{E}-03$ & $6.31 \mathrm{E}+00$ & $4.15 \mathrm{E}-01$ & $2.32 \mathrm{E}+02$ & $1.39 \mathrm{E}-03$ & $1.93 \mathrm{E}+01$ & $1.30 \mathrm{E}-01$ & $1.01 \mathrm{E}+00$ & $6.40 \mathrm{E}+00$ & $9.04 \mathrm{E}-01$ \\
\hline $2.19 \mathrm{E}-02$ & $1.50 \mathrm{E}-02$ & $8.79 \mathrm{E}+00$ & $5.73 \mathrm{E}-01$ & $2.60 \mathrm{E}+02$ & $1.97 \mathrm{E}-03$ & $2.69 \mathrm{E}+01$ & $1.80 \mathrm{E}-01$ & $1.24 \mathrm{E}+00$ & $8.92 \mathrm{E}+00$ & $1.01 \mathrm{E}+00$ \\
\hline $2.42 \mathrm{E}-02$ & $2.19 \mathrm{E}-02$ & $3.50 \mathrm{E}+00$ & $2.36 \mathrm{E}-01$ & $9.78 \mathrm{E}+01$ & $7.89 \mathrm{E}-04$ & $1.05 \mathrm{E}+01$ & $7.31 \mathrm{E}-02$ & $4.99 \mathrm{E}-01$ & $3.55 \mathrm{E}+00$ & $3.79 \mathrm{E}-01$ \\
\hline $2.61 \mathrm{E}-02$ & $2.42 \mathrm{E}-02$ & $3.22 \mathrm{E}+00$ & $2.06 \mathrm{E}-01$ & $8.39 \mathrm{E}+01$ & $7.09 \mathrm{E}-04$ & $9.91 \mathrm{E}+00$ & $6.46 \mathrm{E}-02$ & $4.17 \mathrm{E}-01$ & $3.27 \mathrm{E}+00$ & $3.24 \mathrm{E}-01$ \\
\hline $3.18 \mathrm{E}-02$ & $2.61 \mathrm{E}-02$ & $1.08 \mathrm{E}+01$ & $7.10 \mathrm{E}-01$ & $2.76 \mathrm{E}+02$ & $2.40 \mathrm{E}-03$ & $3.30 \mathrm{E}+01$ & $2.25 \mathrm{E}-01$ & $1.44 \mathrm{E}+00$ & $1.10 \mathrm{E}+01$ & $1.06 \mathrm{E}+00$ \\
\hline 4.09E-02 & $3.18 \mathrm{E}-02$ & $2.07 \mathrm{E}+01$ & $1.37 \mathrm{E}+00$ & $4.84 \mathrm{E}+02$ & $4.64 \mathrm{E}-03$ & $6.26 \mathrm{E}+01$ & $4.25 \mathrm{E}-01$ & $2.64 \mathrm{E}+00$ & $2.10 \mathrm{E}+01$ & $1.86 \mathrm{E}+00$ \\
\hline $6.74 \mathrm{E}-02$ & $4.09 \mathrm{E}-02$ & $8.52 \mathrm{E}+01$ & $5.59 \mathrm{E}+00$ & $1.73 \mathrm{E}+03$ & $1.90 \mathrm{E}-02$ & $2.58 \mathrm{E}+02$ & $1.75 \mathrm{E}+00$ & $1.03 \mathrm{E}+01$ & $8.64 \mathrm{E}+01$ & $6.61 \mathrm{E}+00$ \\
\hline $1.11 \mathrm{E}-01$ & $6.74 \mathrm{E}-02$ & $2.04 \mathrm{E}+02$ & $1.35 \mathrm{E}+01$ & $3.63 \mathrm{E}+03$ & $4.58 \mathrm{E}-02$ & $6.17 \mathrm{E}+02$ & $4.21 \mathrm{E}+00$ & $2.34 \mathrm{E}+01$ & $2.07 \mathrm{E}+02$ & $1.38 \mathrm{E}+01$ \\
\hline $1.83 \mathrm{E}-01$ & $1.11 \mathrm{E}-01$ & $4.48 \mathrm{E}+02$ & $2.95 \mathrm{E}+01$ & $7.49 \mathrm{E}+03$ & $1.00 \mathrm{E}-01$ & $1.35 \mathrm{E}+03$ & $9.22 \mathrm{E}+00$ & $5.02 \mathrm{E}+01$ & $4.54 \mathrm{E}+02$ & $2.83 \mathrm{E}+01$ \\
\hline $2.97 \mathrm{E}-01$ & $1.83 \mathrm{E}-01$ & $1.80 \mathrm{E}+03$ & $1.19 \mathrm{E}+02$ & $1.56 \mathrm{E}+04$ & $4.01 \mathrm{E}-01$ & $5.38 \mathrm{E}+03$ & $3.70 \mathrm{E}+01$ & $1.67 \mathrm{E}+02$ & $1.83 \mathrm{E}+03$ & $5.57 \mathrm{E}+01$ \\
\hline $3.69 \mathrm{E}-01$ & $2.97 \mathrm{E}-01$ & $5.59 \mathrm{E}+03$ & $3.69 \mathrm{E}+02$ & $1.63 \mathrm{E}+04$ & $1.25 \mathrm{E}+00$ & $1.66 \mathrm{E}+04$ & $1.15 \mathrm{E}+02$ & $4.42 \mathrm{E}+02$ & $5.66 \mathrm{E}+03$ & $4.50 \mathrm{E}+01$ \\
\hline $4.98 \mathrm{E}-01$ & $3.69 \mathrm{E}-01$ & $2.38 \mathrm{E}+04$ & $1.57 \mathrm{E}+03$ & $4.77 \mathrm{E}+04$ & $5.32 \mathrm{E}+00$ & $7.06 \mathrm{E}+04$ & $4.88 \mathrm{E}+02$ & $1.83 \mathrm{E}+03$ & $2.41 \mathrm{E}+04$ & $1.04 \mathrm{E}+02$ \\
\hline $6.08 \mathrm{E}-01$ & $4.98 \mathrm{E}-01$ & $3.09 \mathrm{E}+04$ & $2.04 \mathrm{E}+03$ & $5.47 \mathrm{E}+04$ & $6.89 \mathrm{E}+00$ & $9.18 \mathrm{E}+04$ & $6.35 \mathrm{E}+02$ & $2.36 \mathrm{E}+03$ & $3.13 \mathrm{E}+04$ & $1.07 \mathrm{E}+02$ \\
\hline $7.43 \mathrm{E}-01$ & $6.08 \mathrm{E}-01$ & $4.77 \mathrm{E}+04$ & $3.15 \mathrm{E}+03$ & $7.98 \mathrm{E}+04$ & $9.43 \mathrm{E}+00$ & $1.42 \mathrm{E}+05$ & $9.81 \mathrm{E}+02$ & $3.63 \mathrm{E}+03$ & $4.84 \mathrm{E}+04$ & $1.43 \mathrm{E}+02$ \\
\hline $8.21 \mathrm{E}-01$ & $7.43 \mathrm{E}-01$ & $3.17 \mathrm{E}+04$ & $2.09 \mathrm{E}+03$ & $5.14 \mathrm{E}+04$ & $5.45 \mathrm{E}+00$ & $9.44 \mathrm{E}+04$ & $6.34 \mathrm{E}+02$ & $2.35 \mathrm{E}+03$ & $3.22 \mathrm{E}+04$ & $8.36 \mathrm{E}+01$ \\
\hline $1.00 \mathrm{E}+00$ & $8.21 \mathrm{E}-01$ & $8.15 \mathrm{E}+04$ & $4.99 \mathrm{E}+03$ & $1.31 \mathrm{E}+05$ & $1.27 \mathrm{E}+01$ & $2.43 \mathrm{E}+05$ & $1.48 \mathrm{E}+03$ & $5.46 \mathrm{E}+03$ & $8.27 \mathrm{E}+04$ & $1.94 \mathrm{E}+02$ \\
\hline $1.35 \mathrm{E}+00$ & $1.00 \mathrm{E}+00$ & $1.56 \mathrm{E}+05$ & $9.40 \mathrm{E}+03$ & $2.64 \mathrm{E}+05$ & $2.36 \mathrm{E}+01$ & $4.64 \mathrm{E}+05$ & $2.77 \mathrm{E}+03$ & $1.03 \mathrm{E}+04$ & $1.58 \mathrm{E}+05$ & $3.60 \mathrm{E}+02$ \\
\hline $1.65 \mathrm{E}+00$ & $1.35 \mathrm{E}+00$ & $1.05 \mathrm{E}+05$ & $6.20 \mathrm{E}+03$ & $1.88 \mathrm{E}+05$ & $1.40 \mathrm{E}+01$ & $3.15 \mathrm{E}+05$ & $1.80 \mathrm{E}+03$ & $6.69 \mathrm{E}+03$ & $1.07 \mathrm{E}+05$ & $2.55 \mathrm{E}+02$ \\
\hline $1.92 \mathrm{E}+00$ & $1.65 \mathrm{E}+00$ & $9.62 \mathrm{E}+04$ & $5.68 \mathrm{E}+03$ & $1.67 \mathrm{E}+05$ & $1.30 \mathrm{E}+01$ & $2.88 \mathrm{E}+05$ & $1.65 \mathrm{E}+03$ & $6.12 \mathrm{E}+03$ & $9.76 \mathrm{E}+04$ & $2.15 \mathrm{E}+02$ \\
\hline $2.23 \mathrm{E}+00$ & $1.92 \mathrm{E}+00$ & $1.50 \mathrm{E}+05$ & $9.13 \mathrm{E}+03$ & $2.37 \mathrm{E}+05$ & $2.42 \mathrm{E}+01$ & $4.48 \mathrm{E}+05$ & $2.71 \mathrm{E}+03$ & $9.98 \mathrm{E}+03$ & $1.52 \mathrm{E}+05$ & $2.82 \mathrm{E}+02$ \\
\hline $2.35 \mathrm{E}+00$ & $2.23 \mathrm{E}+00$ & $6.13 \mathrm{E}+04$ & $3.76 \mathrm{E}+03$ & $9.36 \mathrm{E}+04$ & $1.13 \mathrm{E}+01$ & $1.83 \mathrm{E}+05$ & $1.12 \mathrm{E}+03$ & $4.12 \mathrm{E}+03$ & $6.22 \mathrm{E}+04$ & $1.09 \mathrm{E}+02$ \\
\hline $2.37 \mathrm{E}+00$ & $2.35 \mathrm{E}+00$ & $1.07 \mathrm{E}+04$ & $6.56 \mathrm{E}+02$ & $1.62 \mathrm{E}+04$ & $2.04 \mathrm{E}+00$ & $3.19 \mathrm{E}+04$ & $1.96 \mathrm{E}+02$ & $7.20 \mathrm{E}+02$ & $1.08 \mathrm{E}+04$ & $1.91 \mathrm{E}+01$ \\
\hline $2.47 \mathrm{E}+00$ & $2.37 \mathrm{E}+00$ & $5.64 \mathrm{E}+04$ & $3.47 \mathrm{E}+03$ & $8.48 \mathrm{E}+04$ & $1.11 \mathrm{E}+01$ & $1.68 \mathrm{E}+05$ & $1.04 \mathrm{E}+03$ & $3.81 \mathrm{E}+03$ & $5.72 \mathrm{E}+04$ & $1.01 \mathrm{E}+02$ \\
\hline $2.73 \mathrm{E}+00$ & $2.47 \mathrm{E}+00$ & $1.66 \mathrm{E}+05$ & $1.05 \mathrm{E}+04$ & $2.43 \mathrm{E}+05$ & $3.53 \mathrm{E}+01$ & $4.96 \mathrm{E}+05$ & $3.25 \mathrm{E}+03$ & $1.19 \mathrm{E}+04$ & $1.69 \mathrm{E}+05$ & $2.90 \mathrm{E}+02$ \\
\hline $3.01 \mathrm{E}+00$ & $2.73 \mathrm{E}+00$ & $2.74 \mathrm{E}+05$ & $1.81 \mathrm{E}+04$ & $3.71 \mathrm{E}+05$ & $6.05 \mathrm{E}+01$ & $8.16 \mathrm{E}+05$ & $5.64 \mathrm{E}+03$ & $2.06 \mathrm{E}+04$ & $2.78 \mathrm{E}+05$ & $4.39 \mathrm{E}+02$ \\
\hline $3.68 \mathrm{E}+00$ & $3.01 \mathrm{E}+00$ & $8.87 \mathrm{E}+05$ & $5.34 \mathrm{E}+04$ & $1.23 \mathrm{E}+06$ & $1.30 \mathrm{E}+02$ & $2.65 \mathrm{E}+06$ & $1.56 \mathrm{E}+04$ & $5.73 \mathrm{E}+04$ & $9.00 \mathrm{E}+05$ & $9.71 \mathrm{E}+02$ \\
\hline $4.97 \mathrm{E}+00$ & $3.68 \mathrm{E}+00$ & $1.50 \mathrm{E}+06$ & $8.52 \mathrm{E}+04$ & $2.22 \mathrm{E}+06$ & $1.82 \mathrm{E}+02$ & $4.47 \mathrm{E}+06$ & $2.41 \mathrm{E}+04$ & $8.85 \mathrm{E}+04$ & $1.52 \mathrm{E}+06$ & $1.34 \mathrm{E}+03$ \\
\hline $6.07 \mathrm{E}+00$ & & & $4.29 \mathrm{E}+04$ & & & $2.50 \mathrm{E}+06$ & $1.12 \mathrm{E}+04$ & $4.12 \mathrm{E}+04$ & $8.45 \mathrm{E}+05$ & $4.96 \mathrm{E}+02$ \\
\hline $7.41 \mathrm{E}+00$ & $6.07 \mathrm{E}+00$ & $8.34 \mathrm{E}+05$ & $4.70 \mathrm{E}+04$ & $1.35 \mathrm{E}+06$ & $1.27 \mathrm{E}+02$ & $2.49 \mathrm{E}+06$ & $1.37 \mathrm{E}+04$ & $5.02 \mathrm{E}+04$ & $8.46 \mathrm{E}+05$ & $8.10 \mathrm{E}+02$ \\
\hline $8.61 \mathrm{E}+00$ & $7.41 \mathrm{E}+00$ & $6.94 \mathrm{E}+05$ & $3.97 \mathrm{E}+04$ & $1.05 \mathrm{E}+06$ & $1.01 \mathrm{E}+02$ & $2.07 \mathrm{E}+06$ & $1.15 \mathrm{E}+04$ & $4.20 \mathrm{E}+04$ & $7.04 \mathrm{E}+05$ & $6.43 \mathrm{E}+02$ \\
\hline $1.00 \mathrm{E}+01$ & $8.61 \mathrm{E}+00$ & $5.25 \mathrm{E}+05$ & $2.80 \mathrm{E}+04$ & $8.71 \mathrm{E}+05$ & $6.21 \mathrm{E}+01$ & $1.57 \mathrm{E}+06$ & $7.81 \mathrm{E}+03$ & $2.86 \mathrm{E}+04$ & $5.33 \mathrm{E}+05$ & $4.07 \mathrm{E}+02$ \\
\hline $1.22 \mathrm{E}+01$ & $1.00 \mathrm{E}+01$ & $5.91 \mathrm{E}+04$ & $2.79 \mathrm{E}+03$ & $1.21 \mathrm{E}+05$ & $2.10 \mathrm{E}+00$ & $1.78 \mathrm{E}+05$ & $6.64 \mathrm{E}+02$ & $2.46 \mathrm{E}+03$ & $6.00 \mathrm{E}+04$ & $2.09 \mathrm{E}+01$ \\
\hline $1.42 \mathrm{E}+01$ & $1.22 \mathrm{E}+01$ & $2.21 \mathrm{E}-04$ & $7.17 \mathrm{E}-05$ & $7.60 \mathrm{E}+01$ & $8.84 \mathrm{E}-09$ & $1.29 \mathrm{E}-01$ & $1.96 \mathrm{E}-06$ & $5.47 \mathrm{E}-02$ & $2.24 \mathrm{E}-04$ & $1.17 \mathrm{E}-01$ \\
\hline \multirow[t]{2}{*}{$1.96 \mathrm{E}+01$} & $1.42 \mathrm{E}+01$ & $5.31 \mathrm{E}-05$ & $1.47 \mathrm{E}-05$ & $1.71 \mathrm{E}+01$ & $1.68 \mathrm{E}-09$ & $2.54 \mathrm{E}-02$ & $4.24 \mathrm{E}-07$ & $9.38 \mathrm{E}-03$ & $5.38 \mathrm{E}-05$ & $2.13 \mathrm{E}-02$ \\
\hline & Total & $6.63 \mathrm{E}+06$ & $3.80 \mathrm{E}+05$ & $1.03 \mathrm{E}+07$ & $9.03 \mathrm{E}+02$ & $1.98 \mathrm{E}+07$ & $1.09 \mathrm{E}+05$ & $4.01 \mathrm{E}+05$ & $6.72 \mathrm{E}+06$ & $7.55 \mathrm{E}+03$ \\
\hline
\end{tabular}

Note: $90 \%$ Beryllium is modeled as 1 gram of actinide and 0.9 grams of beryllium. 
PCP-2011-0001

Revision 0

Table 3-9. Neutron Source from RASTA w/o Beryllium (Decay Time $=0$ days)

\begin{tabular}{|c|c|c|c|c|c|c|c|c|c|c|c|c|}
\hline \multicolumn{2}{|c|}{ Energy Bound (MeV) } & \multicolumn{11}{|c|}{ Neutron Source (n/s/g-actinide) } \\
\hline Upper & Lower & Am-241 & Am-243 & Cf-252 & $\mathrm{Cm}-244$ & Cm-248 & Np-237 & Pu-238 & Pu-239 & Pu-240 & Pu-241 & Pu-242 \\
\hline $1.00 \mathrm{E}-07$ & $1.00 \mathrm{E}-11$ & $1.39 \mathrm{E}-11$ & $4.91 \mathrm{E}-11$ & \#N/A & $1.30 \mathrm{E}-04$ & $5.28 \mathrm{E}-04$ & $1.48 \mathrm{E}-15$ & $3.26 \mathrm{E}-08$ & $5.84 \mathrm{E}-07$ & $1.36 \mathrm{E}-08$ & $6.34 \mathrm{E}-13$ & $2.25 \mathrm{E}-08$ \\
\hline $4.14 \mathrm{E}-07$ & $1.00 \mathrm{E}-07$ & $1.03 \mathrm{E}-10$ & $3.64 \mathrm{E}-10$ & \#N/A & $9.65 \mathrm{E}-04$ & $3.92 \mathrm{E}-03$ & $1.10 \mathrm{E}-14$ & $2.42 \mathrm{E}-07$ & $2.94 \mathrm{E}-06$ & $1.01 \mathrm{E}-07$ & $4.70 \mathrm{E}-12$ & $1.67 \mathrm{E}-07$ \\
\hline $8.76 \mathrm{E}-07$ & $4.14 \mathrm{E}-07$ & $2.43 \mathrm{E}-10$ & $8.59 \mathrm{E}-10$ & \#N/A & $2.27 \mathrm{E}-03$ & $9.24 \mathrm{E}-03$ & $2.59 \mathrm{E}-14$ & $5.70 \mathrm{E}-07$ & $1.95 \mathrm{E}-05$ & $2.37 \mathrm{E}-07$ & $1.11 \mathrm{E}-11$ & $3.94 \mathrm{E}-07$ \\
\hline $1.86 \mathrm{E}-06$ & $8.76 \mathrm{E}-07$ & $7.55 \mathrm{E}-10$ & $2.66 \mathrm{E}-09$ & $\# \mathrm{~N} / \mathrm{A}$ & $7.06 \mathrm{E}-03$ & $2.86 \mathrm{E}-02$ & $8.02 \mathrm{E}-14$ & $1.77 \mathrm{E}-06$ & $4.98 \mathrm{E}-05$ & $7.36 \mathrm{E}-07$ & $3.44 \mathrm{E}-11$ & $1.22 \mathrm{E}-06$ \\
\hline $5.04 \mathrm{E}-06$ & $1.86 \mathrm{E}-06$ & $3.86 \mathrm{E}-09$ & $1.36 \mathrm{E}-08$ & \#N/A & $3.61 \mathrm{E}-02$ & $1.46 \mathrm{E}-01$ & $4.10 \mathrm{E}-13$ & $9.04 \mathrm{E}-06$ & $1.19 \mathrm{E}-04$ & $3.76 \mathrm{E}-06$ & $1.76 \mathrm{E}-10$ & $6.25 \mathrm{E}-06$ \\
\hline $1.07 \mathrm{E}-05$ & $5.04 \mathrm{E}-06$ & $1.04 \mathrm{E}-08$ & $3.68 \mathrm{E}-08$ & \#N/A & $9.73 \mathrm{E}-02$ & $3.95 \mathrm{E}-01$ & $1.11 \mathrm{E}-12$ & $2.44 \mathrm{E}-05$ & $3.55 \mathrm{E}-04$ & $1.02 \mathrm{E}-05$ & $4.75 \mathrm{E}-10$ & $1.69 \mathrm{E}-05$ \\
\hline $3.73 \mathrm{E}-05$ & $1.07 \mathrm{E}-05$ & $8.48 \mathrm{E}-08$ & $2.99 \mathrm{E}-07$ & $\# \mathrm{~N} / \mathrm{A}$ & $7.92 \mathrm{E}-01$ & $3.22 \mathrm{E}+00$ & $9.01 \mathrm{E}-12$ & $1.99 \mathrm{E}-04$ & $7.04 \mathrm{E}-04$ & $8.27 \mathrm{E}-05$ & $3.86 \mathrm{E}-09$ & $1.37 \mathrm{E}-04$ \\
\hline $1.01 \mathrm{E}-04$ & $3.73 \mathrm{E}-05$ & $3.46 \mathrm{E}-07$ & $1.22 \mathrm{E}-06$ & $\# \mathrm{~N} / \mathrm{A}$ & $3.24 \mathrm{E}+00$ & $1.31 \mathrm{E}+01$ & $3.68 \mathrm{E}-11$ & $8.11 \mathrm{E}-04$ & $1.88 \mathrm{E}-03$ & $3.38 \mathrm{E}-04$ & $1.58 \mathrm{E}-08$ & $5.61 \mathrm{E}-04$ \\
\hline $2.14 \mathrm{E}-04$ & $1.01 \mathrm{E}-04$ & $9.30 \mathrm{E}-07$ & $3.28 \mathrm{E}-06$ & \#N/A & $8.69 \mathrm{E}+00$ & $3.53 \mathrm{E}+01$ & $9.89 \mathrm{E}-11$ & $2.18 \mathrm{E}-03$ & $1.78 \mathrm{E}-03$ & $9.07 \mathrm{E}-04$ & $4.24 \mathrm{E}-08$ & $1.51 \mathrm{E}-03$ \\
\hline $4.54 \mathrm{E}-04$ & $2.14 \mathrm{E}-04$ & $2.88 \mathrm{E}-06$ & $1.02 \mathrm{E}-05$ & $\# \mathrm{~N} / \mathrm{A}$ & $2.69 \mathrm{E}+01$ & $1.09 \mathrm{E}+02$ & $3.06 \mathrm{E}-10$ & $6.74 \mathrm{E}-03$ & $9.72 \mathrm{E}-04$ & $2.81 \mathrm{E}-03$ & $1.31 \mathrm{E}-07$ & $4.66 \mathrm{E}-03$ \\
\hline $1.58 \mathrm{E}-03$ & $4.54 \mathrm{E}-04$ & $2.34 \mathrm{E}-05$ & $8.24 \mathrm{E}-05$ & $\# \mathrm{~N} / \mathrm{A}$ & $2.18 \mathrm{E}+02$ & $8.86 \mathrm{E}+02$ & $2.48 \mathrm{E}-09$ & $5.47 \mathrm{E}-02$ & $1.04 \mathrm{E}-03$ & $2.28 \mathrm{E}-02$ & $1.06 \mathrm{E}-06$ & $3.78 \mathrm{E}-02$ \\
\hline $3.35 \mathrm{E}-03$ & $1.58 \mathrm{E}-03$ & $5.76 \mathrm{E}-05$ & $2.03 \mathrm{E}-04$ & \#N/A & $5.38 \mathrm{E}+02$ & $2.18 \mathrm{E}+03$ & $6.12 \mathrm{E}-09$ & $1.35 \mathrm{E}-01$ & $4.40 \mathrm{E}-04$ & $5.62 \mathrm{E}-02$ & $2.62 \mathrm{E}-06$ & $9.33 \mathrm{E}-02$ \\
\hline $7.10 \mathrm{E}-03$ & $3.35 \mathrm{E}-03$ & $1.77 \mathrm{E}-04$ & $6.26 \mathrm{E}-04$ & \#N/A & $1.66 \mathrm{E}+03$ & $6.73 \mathrm{E}+03$ & $1.88 \mathrm{E}-08$ & $4.15 \mathrm{E}-01$ & $9.06 \mathrm{E}-05$ & $1.73 \mathrm{E}-01$ & $8.08 \mathrm{E}-06$ & $2.87 \mathrm{E}-01$ \\
\hline $1.50 \mathrm{E}-02$ & $7.10 \mathrm{E}-03$ & $5.42 \mathrm{E}-04$ & $1.91 \mathrm{E}-03$ & \#N/A & $5.06 \mathrm{E}+03$ & $2.06 \mathrm{E}+04$ & $5.76 \mathrm{E}-08$ & $1.27 \mathrm{E}+00$ & $5.63 \mathrm{E}-04$ & $5.28 \mathrm{E}-01$ & $2.47 \mathrm{E}-05$ & $8.78 \mathrm{E}-01$ \\
\hline $2.19 \mathrm{E}-02$ & $1.50 \mathrm{E}-02$ & $6.12 \mathrm{E}-04$ & $2.16 \mathrm{E}-03$ & $\# \mathrm{~N} / \mathrm{A}$ & $5.72 \mathrm{E}+03$ & $2.32 \mathrm{E}+04$ & $6.50 \mathrm{E}-08$ & $1.43 \mathrm{E}+00$ & $1.61 \mathrm{E}-03$ & $5.97 \mathrm{E}-01$ & $2.79 \mathrm{E}-05$ & $9.91 \mathrm{E}-01$ \\
\hline $2.42 \mathrm{E}-02$ & $2.19 \mathrm{E}-02$ & $2.28 \mathrm{E}-04$ & $8.04 \mathrm{E}-04$ & $\# \mathrm{~N} / \mathrm{A}$ & $2.13 \mathrm{E}+03$ & $8.64 \mathrm{E}+03$ & $2.42 \mathrm{E}-08$ & $5.33 \mathrm{E}-01$ & $1.58 \mathrm{E}-03$ & $2.22 \mathrm{E}-01$ & $1.04 \mathrm{E}-05$ & $3.69 \mathrm{E}-01$ \\
\hline $2.61 \mathrm{E}-02$ & $2.42 \mathrm{E}-02$ & $1.96 \mathrm{E}-04$ & $6.93 \mathrm{E}-04$ & $\# \mathrm{~N} / \mathrm{A}$ & $1.83 \mathrm{E}+03$ & $7.45 \mathrm{E}+03$ & $2.09 \mathrm{E}-08$ & $4.60 \mathrm{E}-01$ & $1.94 \mathrm{E}-03$ & $1.91 \mathrm{E}-01$ & $8.94 \mathrm{E}-06$ & \\
\hline $3.18 \mathrm{E}-02$ & $2.61 \mathrm{E}-02$ & $6.30 \mathrm{E}-04$ & $2.22 \mathrm{E}-03$ & \#N/A & $5.89 \mathrm{E}+03$ & $2.39 \mathrm{E}+04$ & & $1.48 \mathrm{E}+00$ & $2.47 \mathrm{E}-03$ & $6.15 \mathrm{E}-01$ & $2.87 \mathrm{E}-05$ & $1.02 \mathrm{E}+00$ \\
\hline $4.09 \mathrm{E}-02$ & & & & \#N/A & $1.05 \mathrm{E}+04$ & & & & & & & \\
\hline $5.74 \mathrm{E}-02$ & $4.09 \mathrm{E}-02$ & $2.35 \mathrm{E}-03$ & $8.30 \mathrm{E}-03$ & $\# \mathrm{~N} / \mathrm{A}$ & $2.20 \mathrm{E}+04$ & $8.93 \mathrm{E}+04$ & $2.50 \mathrm{E}-07$ & $5.51 \mathrm{E}+00$ & $5.72 \mathrm{E}-04$ & $2.30 \mathrm{E}+00$ & $1.07 \mathrm{E}-04$ & $3.81 \mathrm{E}+00$ \\
\hline $1.11 \mathrm{E}-01$ & $5.74 \mathrm{E}-02$ & $9.79 \mathrm{E}-03$ & $3.45 \mathrm{E}-02$ & $\# \mathrm{~N} / \mathrm{A}$ & $9.15 \mathrm{E}+04$ & $3.72 \mathrm{E}+05$ & $1.04 \mathrm{E}-06$ & $2.29 \mathrm{E}+01$ & $9.79 \mathrm{E}-04$ & $9.56 \mathrm{E}+00$ & $4.46 \mathrm{E}-04$ & $1.59 \mathrm{E}+01$ \\
\hline $1.83 \mathrm{E}-01$ & -01 & $1.69 \mathrm{E}-02$ & $5.95 \mathrm{E}-02$ & $\# \mathrm{~N} / \mathrm{A}$ & $1.58 \mathrm{E}+05$ & $8+05$ & $\overline{E-06}$ & $8+01$ & $7.74 \mathrm{E}-04$ & $1.65 \mathrm{E}+01$ & E-04 & $2.73 \mathrm{E}+01$ \\
\hline $2.97 \mathrm{E}-01$ & $1.83 \mathrm{E}-01$ & $3.25 \mathrm{E}-02$ & $1.15 \mathrm{E}-01$ & $\# \mathrm{~N} / \mathrm{A}$ & $3.04 \mathrm{E}+05$ & $1.23 \mathrm{E}+06$ & $3.45 \mathrm{E}-06$ & $\overline{\mathrm{E}+01}$ & $8.59 \mathrm{E}-04$ & $3.17 \mathrm{E}+01$ & $1.48 \mathrm{E}-03$ & $5.25 \mathrm{E}+01$ \\
\hline $3.69 \mathrm{E}-01$ & $2.97 \mathrm{E}-01$ & $2.31 \mathrm{E}-02$ & $8.13 \mathrm{E}-02$ & $\# \mathrm{~N} / \mathrm{A}$ & $2.16 \mathrm{E}+05$ & $8.75 E+05$ & $2.44 \mathrm{E}-06$ & $5.40 \mathrm{E}+01$ & $4.45 \mathrm{E}-04$ & $2.25 \mathrm{E}+01$ & $1.05 \mathrm{E}-03$ & $3.72 \mathrm{E}+01$ \\
\hline $4.98 \mathrm{E}-01$ & $3.69 \mathrm{E}-01$ & $4.46 \mathrm{E}-02$ & $1.57 \mathrm{E}-01$ & \#N/A & $4.18 \mathrm{E}+05$ & $1.69 \mathrm{E}+06$ & $4.72 \mathrm{E}-06$ & $1.04 \mathrm{E}+02$ & $6.27 \mathrm{E}-04$ & $4.35 \mathrm{E}+01$ & $2.02 \mathrm{E}-03$ & $7.19 \mathrm{E}+01$ \\
\hline $6.08 \mathrm{E}-01$ & $4.98 \mathrm{E}-01$ & $4.03 \mathrm{E}-02$ & $1.42 \mathrm{E}-01$ & \#N/A & $3.77 \mathrm{E}+05$ & $1.52 \mathrm{E}+06$ & $4.25 \mathrm{E}-06$ & $9.41 \mathrm{E}+01$ & $3.25 \mathrm{E}-04$ & $3.91 \mathrm{E}+01$ & $1.82 \mathrm{E}-03$ & $6.47 \mathrm{E}+01$ \\
\hline $7.43 \mathrm{E}-01$ & $6.08 \mathrm{E}-01$ & $5.10 \mathrm{E}-02$ & $1.79 \mathrm{E}-01$ & \#N/A & $4.77 \mathrm{E}+05$ & $1.92 \mathrm{E}+06$ & $5.36 \mathrm{E}-06$ & $1.19 \mathrm{E}+02$ & $1.89 \mathrm{E}-04$ & $4.93 \mathrm{E}+01$ & $2.30 \mathrm{E}-03$ & $8.15 \mathrm{E}+01$ \\
\hline $8.21 \mathrm{E}-01$ & $7.43 \mathrm{E}-01$ & $2.98 \mathrm{E}-02$ & $1.04 \mathrm{E}-01$ & $\# \mathrm{~N} / \mathrm{A}$ & $2.79 \mathrm{E}+05$ & $1.12 \mathrm{E}+06$ & $3.13 \mathrm{E}-06$ & $6.94 \mathrm{E}+01$ & $4.54 \mathrm{E}-05$ & $2.88 \mathrm{E}+01$ & $1.34 \mathrm{E}-03$ & $4.75 \mathrm{E}+01$ \\
\hline $1.00 \mathrm{E}+00$ & $8.21 \mathrm{E}-01$ & $6.85 \mathrm{E}-02$ & $2.39 \mathrm{E}-01$ & $\# \mathrm{~N} / \mathrm{A}$ & $6.40 \mathrm{E}+05$ & $2.56 \mathrm{E}+06$ & $7.14 \mathrm{E}-06$ & $1.59 \mathrm{E}+02$ & $2.17 \mathrm{E}-05$ & $6.56 \mathrm{E}+01$ & $3.06 \mathrm{E}-03$ & $1.08 \mathrm{E}+02$ \\
\hline $1.35 \mathrm{E}+00$ & $1.00 \mathrm{E}+00$ & $1.30 \mathrm{E}-01$ & $4.50 \mathrm{E}-01$ & $\# \mathrm{~N} / \mathrm{A}$ & $1.21 \mathrm{E}+06$ & $4.78 \mathrm{E}+06$ & $1.34 \mathrm{E}-05$ & $2.99 \mathrm{E}+02$ & $1.22 \mathrm{E}-05$ & $1.23 \mathrm{E}+02$ & $5.74 \mathrm{E}-03$ & $2.03 \mathrm{E}+02$ \\
\hline $1.65 \mathrm{E}+00$ & $1.35 \mathrm{E}+00$ & $1.03 \mathrm{E}-01$ & $3.53 \mathrm{E}-01$ & \#N/A & $9.56 \mathrm{E}+05$ & $3.72 \mathrm{E}+06$ & $1.04 \mathrm{E}-05$ & $2.34 \mathrm{E}+02$ & $3.79 \mathrm{E}-06$ & $9.52 \mathrm{E}+01$ & $4.49 \mathrm{E}-03$ & $1.58 \mathrm{E}+02$ \\
\hline $1.92 \mathrm{E}+00$ & $1.65 \mathrm{E}+00$ & $8.43 \mathrm{E}-02$ & $2.86 \mathrm{E}-01$ & \#N/A & $7.81 \mathrm{E}+05$ & $2.99 \mathrm{E}+06$ & $8.42 \mathrm{E}-06$ & $1.90 \mathrm{E}+02$ & $4.40 \mathrm{E}-06$ & $7.64 \mathrm{E}+01$ & $3.63 \mathrm{E}-03$ & $1.27 \mathrm{E}+02$ \\
\hline $2.23 \mathrm{E}+00$ & $1.92 \mathrm{E}+00$ & 8.631 & $2.90 \mathrm{E}-01$ & $\# \mathrm{~N} / \mathrm{A}$ & $7.97 \mathrm{E}+05$ & $3.00 \mathrm{E}+06$ & $8.48 \mathrm{E}-06$ & $1.92 \mathrm{E}+02$ & $1.18 \mathrm{E}-05$ & $7.65 \mathrm{E}+01$ & $3.66 \mathrm{E}-03$ & $1.27 \mathrm{E}+02$ \\
\hline $2.35 \mathrm{E}+00$ & $2.23 \mathrm{E}+00$ & $3.04 \mathrm{E}-02$ & $1.01 \mathrm{E}-01$ & $\# \mathrm{~N} / \mathrm{A}$ & $2.80 \mathrm{E}+05$ & $1.04 \mathrm{E}+06$ & $2.95 \mathrm{E}-06$ & $\mathrm{E}+01$ & $1.05 \mathrm{E}-05$ & $2.65 \mathrm{E}+01$ & $1.27 \mathrm{E}-03$ & $4.42 \mathrm{E}+01$ \\
\hline $2.37 \mathrm{E}+00$ & $2.35 \mathrm{E}+00$ & $4.91 \mathrm{E}-03$ & $1.63 \mathrm{E}-02$ & $\# \mathrm{~N} / \mathrm{A}$ & $4.52 \mathrm{E}+04$ & $1.67 \mathrm{E}+05$ & $4.74 \mathrm{E}-07$ & $1.08 \mathrm{E}+01$ & $3.42 \mathrm{E}-06$ & $4.24 \mathrm{E}+00$ & $2.05 \mathrm{E}-04$ & $7.10 \mathrm{E}+00$ \\
\hline $2.47 \mathrm{E}+00$ & $2.37 \mathrm{E}+00$ & $2.39 \mathrm{E}-02$ & $7.91 \mathrm{E}-02$ & $\# \mathrm{~N} / \mathrm{A}$ & $2.19 \mathrm{E}+05$ & $8.08 \mathrm{E}+05$ & $2.29 \mathrm{E}-06$ & $5.21 \mathrm{E}+01$ & $1.11 \mathrm{E}-06$ & $2.05 \mathrm{E}+01$ & $9.91 \mathrm{E}-04$ & $3.43 \mathrm{E}+01$ \\
\hline $2.73 \mathrm{E}+00$ & $2.47 \mathrm{E}+00$ & $5.69 \mathrm{E}-02$ & $1.87 \mathrm{E}-01$ & \#N/A & $5.22 \mathrm{E}+05$ & $1.90 \mathrm{E}+06$ & $5.41 \mathrm{E}-06$ & $1.23 \mathrm{E}+02$ & $4.51 \mathrm{E}-07$ & $4.82 \mathrm{E}+01$ & $2.34 \mathrm{E}-03$ & $8.08 \mathrm{E}+01$ \\
\hline $3.01 \mathrm{E}+00$ & $2.73 \mathrm{E}+00$ & $5.35 \mathrm{E}-02$ & $1.74 \mathrm{E}-01$ & $\# \mathrm{~N} / \mathrm{A}$ & $4.88 \mathrm{E}+05$ & $1.74 \mathrm{E}+06$ & $4.99 \mathrm{E}-06$ & $1.14 \mathrm{E}+02$ & $5.55 \mathrm{E}-08$ & $4.41 \mathrm{E}+01$ & $2.16 \mathrm{E}-03$ & $7.43 \mathrm{E}+01$ \\
\hline $3.68 \mathrm{E}+00$ & $3.01 \mathrm{E}+00$ & $9.93 \mathrm{E}-02$ & $3.16 \mathrm{E}-01$ & $\# \mathrm{~N} / \mathrm{A}$ & $8.99 \mathrm{E}+05$ & $3.11 \mathrm{E}+06$ & $8.96 \mathrm{E}-06$ & $2.06 \mathrm{E}+02$ & $1.80 \mathrm{E}-08$ & $7.82 \mathrm{E}+01$ & $3.89 \mathrm{E}-03$ & $1.33 \mathrm{E}+02$ \\
\hline $4.97 \mathrm{E}+00$ & $3.68 \mathrm{E}+00$ & $1.09 \mathrm{E}-01$ & $3.30 \mathrm{E}-01$ & \#N/A & $9.67 \mathrm{E}+05$ & $3.12 \mathrm{E}+06$ & $9.14 \mathrm{E}-06$ & $2.14 \mathrm{E}+02$ & $6.68 \mathrm{E}-09$ & $7.76 \mathrm{E}+01$ & $4.00 \mathrm{E}-03$ & $1.34 \mathrm{E}+02$ \\
\hline $6.07 \mathrm{E}+00$ & $4.97 \mathrm{E}+00$ & $4.25 \mathrm{E}-02$ & $1.21 \mathrm{E}-01$ & \#N/A & $3.69 \mathrm{E}+05$ & $1.07 \mathrm{E}+06$ & $3.23 \mathrm{E}-06$ & $7.70 \mathrm{E}+01$ & $1.64 \mathrm{E}-09$ & $2.63 \mathrm{E}+01$ & $1.43 \mathrm{E}-03$ & $4.64 \mathrm{E}+01$ \\
\hline $7.41 \mathrm{E}+00$ & $6.07 \mathrm{E}+00$ & $2.25 \mathrm{E}-02$ & $5.97 \mathrm{E}-02$ & \#N/A & $1.90 \mathrm{E}+05$ & $4.98 \mathrm{E}+05$ & $1.54 \mathrm{E}-06$ & $3.75 \mathrm{E}+01$ & $2.01 \mathrm{E}-10$ & $1.20 \mathrm{E}+01$ & $6.87 \mathrm{E}-04$ & $2.18 \mathrm{E}+01$ \\
\hline $8.61 \mathrm{E}+00$ & $7.41 \mathrm{E}+00$ & $7.95 \mathrm{E}-03$ & $1.95 \mathrm{E}-02$ & \#N/A & $6.51 \mathrm{E}+04$ & $1.52 \mathrm{E}+05$ & $4.84 \mathrm{E}-07$ & $1.20 \mathrm{E}+01$ & $7.45 \mathrm{E}-11$ & $3.59 \mathrm{E}+00$ & $2.18 \mathrm{E}-04$ & $6.71 \mathrm{E}+00$ \\
\hline $1.00 \mathrm{E}+01$ & & & & & $2.79 \mathrm{E}+04$ & & & $4.83 \mathrm{E}+00$ & $1.46 \mathrm{E}-11$ & $1.34 \mathrm{E}+00$ & $8.67 \mathrm{E}-05$ & $2.58 \mathrm{E}+00$ \\
\hline $1.22 \mathrm{E}+01$ & $1.00 \mathrm{E}+01$ & $1.48 \mathrm{E}-03$ & $3.03 \mathrm{E}-03$ & $\# \mathrm{~N} / \mathrm{A}$ & $1.13 \mathrm{E}+04$ & $1.99 \mathrm{E}+04$ & $6.84 \mathrm{E}-08$ & $1.79 \mathrm{E}+00$ & $4.70 \mathrm{E}-12$ & $4.52 \mathrm{E}-01$ & $3.17 \mathrm{E}-05$ & $9.06 \mathrm{E}-01$ \\
\hline $1.42 \mathrm{E}+01$ & $1.22 \mathrm{E}+01$ & $2.44 \mathrm{E}-04$ & $4.36 \mathrm{E}-04$ & $\# \mathrm{~N} / \mathrm{A}$ & $1.76 \mathrm{E}+03$ & $2.52 \mathrm{E}+03$ & $9.15 \mathrm{E}-09$ & $2.48 \mathrm{E}-01$ & $1.99 \mathrm{E}-12$ & $5.52 \mathrm{E}-02$ & $4.33 \mathrm{E}-06$ & $1.17 \mathrm{E}-01$ \\
\hline \multirow[t]{2}{*}{$1.73 \mathrm{E}+01$} & $1.42 \mathrm{E}+01$ & $5.37 \mathrm{E}-05$ & $8.27 \mathrm{E}-05$ & $\# \mathrm{~N} / \mathrm{A}$ & $3.65 \mathrm{E}+02$ & $4.17 \mathrm{E}+02$ & $1.61 \mathrm{E}-09$ & $4.55 \mathrm{E}-02$ & $2.69 \mathrm{E}-13$ & $8.83 \mathrm{E}-03$ & $7.79 \mathrm{E}-07$ & $1.98 \mathrm{E}-02$ \\
\hline & Total & $1.18 \mathrm{E}+00$ & $3.93 \mathrm{E}+00$ & $\# \mathrm{~N} / \mathrm{A}$ & $1.08 \mathrm{E}+07$ & $4.03 \mathrm{E}+07$ & $1.14 \mathrm{E}-04$ & $2.59 \mathrm{E}+03$ & $2.18 \mathrm{E}-02$ & $1.03 \mathrm{E}+03$ & $4.94 \mathrm{E}-02$ & $1.72 \mathrm{E}+03$ \\
\hline
\end{tabular}


PCP-2011-0001

Revision 0

Table 3-10. Neutron Source from RASTA w/90\% Beryllium (Decay Time $=0$ days)

\begin{tabular}{|c|c|c|c|c|c|c|c|c|c|c|}
\hline \multicolumn{2}{|c|}{ Energy Bound (MeV) } & \multicolumn{9}{|c|}{ Neutron Source (n/s/g-actinide) } \\
\hline Upper & Lower & Am-241 & Am-243 & Cm-244 & Np-237 & Pu-238 & Pu-239 & Pu-240 & Pu-241 & Pu-242 \\
\hline $1.00 \mathrm{E}-07$ & $1.00 \mathrm{E}-11$ & $1.39 \mathrm{E}-11$ & $4.91 \mathrm{E}-11$ & $1.30 \mathrm{E}-04$ & $1.48 \mathrm{E}-15$ & $3.26 \mathrm{E}-08$ & 5.84E-07 & $1.36 \mathrm{E}-08$ & $6.34 \mathrm{E}-13$ & $2.25 \mathrm{E}-08$ \\
\hline $4.14 \mathrm{E}-07$ & $1.00 \mathrm{E}-07$ & $1.03 \mathrm{E}-10$ & $3.64 \mathrm{E}-10$ & $9.65 \mathrm{E}-04$ & $1.10 \mathrm{E}-14$ & $2.42 \mathrm{E}-07$ & $2.94 \mathrm{E}-06$ & $1.01 \mathrm{E}-07$ & $4.70 \mathrm{E}-12$ & $1.67 \mathrm{E}-07$ \\
\hline $8.76 \mathrm{E}-07$ & $4.14 \mathrm{E}-07$ & $2.43 \mathrm{E}-10$ & $8.59 \mathrm{E}-10$ & $2.27 \mathrm{E}-03$ & $2.59 \mathrm{E}-14$ & $5.70 \mathrm{E}-07$ & $6.63 \mathrm{E}+02$ & $2.37 \mathrm{E}-07$ & $1.11 \mathrm{E}-11$ & $3.94 \mathrm{E}-07$ \\
\hline $1.86 \mathrm{E}-06$ & $8.76 \mathrm{E}-07$ & $7.55 \mathrm{E}-10$ & $2.66 \mathrm{E}-09$ & $7.06 \mathrm{E}-03$ & $8.02 \mathrm{E}-14$ & $1.77 \mathrm{E}-06$ & $7.80 \mathrm{E}+03$ & $7.36 \mathrm{E}-07$ & $3.44 \mathrm{E}-11$ & $1.22 \mathrm{E}-06$ \\
\hline $5.04 \mathrm{E}-06$ & $1.86 \mathrm{E}-06$ & $3.86 \mathrm{E}-09$ & $1.36 \mathrm{E}-08$ & $3.61 \mathrm{E}-02$ & $4.10 \mathrm{E}-13$ & $9.04 \mathrm{E}-06$ & $1.15 \mathrm{E}+04$ & $3.76 \mathrm{E}-06$ & $1.76 \mathrm{E}-10$ & $6.25 \mathrm{E}-06$ \\
\hline $1.07 \mathrm{E}-05$ & $5.04 \mathrm{E}-06$ & $1.04 \mathrm{E}-08$ & $3.68 \mathrm{E}-08$ & $9.73 \mathrm{E}-02$ & $1.11 \mathrm{E}-12$ & $2.44 \mathrm{E}-05$ & $1.37 \mathrm{E}+04$ & $1.02 \mathrm{E}-05$ & $4.75 \mathrm{E}-10$ & $1.69 \mathrm{E}-05$ \\
\hline $3.73 \mathrm{E}-05$ & $1.07 \mathrm{E}-05$ & $8.48 \mathrm{E}-08$ & $2.99 \mathrm{E}-07$ & 7.92E-01 & $9.01 \mathrm{E}-12$ & $1.99 \mathrm{E}-04$ & $1.12 \mathrm{E}+04$ & $8.27 \mathrm{E}-05$ & $3.86 \mathrm{E}-09$ & $1.37 \mathrm{E}-04$ \\
\hline $1.01 \mathrm{E}-04$ & $3.73 \mathrm{E}-05$ & $3.46 \mathrm{E}-07$ & $1.22 \mathrm{E}-06$ & $3.24 \mathrm{E}+00$ & $3.68 \mathrm{E}-11$ & $8.11 \mathrm{E}-04$ & $2.42 \mathrm{E}+04$ & $3.38 \mathrm{E}-04$ & $1.58 \mathrm{E}-08$ & $5.61 \mathrm{E}-04$ \\
\hline $2.14 \mathrm{E}-04$ & $1.01 \mathrm{E}-04$ & $9.30 \mathrm{E}-07$ & $3.28 \mathrm{E}-06$ & $8.69 \mathrm{E}+00$ & $9.89 \mathrm{E}-11$ & $2.18 \mathrm{E}-03$ & $1.57 \mathrm{E}+04$ & $9.07 \mathrm{E}-04$ & $4.24 \mathrm{E}-08$ & $1.51 \mathrm{E}-03$ \\
\hline $4.54 \mathrm{E}-04$ & $2.14 \mathrm{E}-04$ & $2.88 \mathrm{E}-06$ & $1.02 \mathrm{E}-05$ & $2.69 \mathrm{E}+01$ & $3.06 \mathrm{E}-10$ & $6.74 \mathrm{E}-03$ & $5.54 \mathrm{E}+03$ & $2.81 \mathrm{E}-03$ & $1.31 \mathrm{E}-07$ & $4.66 \mathrm{E}-03$ \\
\hline $1.58 \mathrm{E}-03$ & $4.54 \mathrm{E}-04$ & $1.58 \mathrm{E}-02$ & $9.97 \mathrm{E}-04$ & $2.19 \mathrm{E}+02$ & $3.24 \mathrm{E}-06$ & $1.33 \mathrm{E}-01$ & $3.29 \mathrm{E}+03$ & $2.38 \mathrm{E}-02$ & $1.24 \mathrm{E}-05$ & $3.79 \mathrm{E}-02$ \\
\hline $3.35 \mathrm{E}-03$ & $1.58 \mathrm{E}-03$ & $1.94 \mathrm{E}-01$ & $1.15 \mathrm{E}-02$ & $5.43 \mathrm{E}+02$ & $3.98 \mathrm{E}-05$ & $1.10 \mathrm{E}+00$ & $1.04 \mathrm{E}+03$ & $6.90 \mathrm{E}-02$ & $1.43 \mathrm{E}-04$ & $9.35 \mathrm{E}-02$ \\
\hline $7.10 \mathrm{E}-03$ & $3.35 \mathrm{E}-03$ & $1.41 \mathrm{E}+00$ & $8.26 \mathrm{E}-02$ & $1.69 \mathrm{E}+03$ & $2.89 \mathrm{E}-04$ & $7.43 \mathrm{E}+00$ & $2.01 \mathrm{E}+02$ & $2.66 \mathrm{E}-01$ & $1.02 \mathrm{E}-03$ & $2.89 \mathrm{E}-01$ \\
\hline $1.50 \mathrm{E}-02$ & $7.10 \mathrm{E}-03$ & $7.13 \mathrm{E}+00$ & $4.17 \mathrm{E}-01$ & $5.23 \mathrm{E}+03$ & $1.46 \mathrm{E}-03$ & $3.68 \mathrm{E}+01$ & $1.18 \mathrm{E}+03$ & $1.00 \mathrm{E}+00$ & $5.17 \mathrm{E}-03$ & $8.86 \mathrm{E}-01$ \\
\hline $2.19 \mathrm{E}-02$ & $1.50 \mathrm{E}-02$ & $1.01 \mathrm{E}+01$ & $5.87 \mathrm{E}-01$ & $5.95 \mathrm{E}+03$ & $2.06 \mathrm{E}-03$ & $5.15 \mathrm{E}+01$ & $2.71 \mathrm{E}+03$ & $1.26 \mathrm{E}+00$ & $7.28 \mathrm{E}-03$ & $1.00 \mathrm{E}+00$ \\
\hline $2.42 \mathrm{E}-02$ & $2.19 \mathrm{E}-02$ & $4.06 \mathrm{E}+00$ & $2.37 \mathrm{E}-01$ & $2.22 \mathrm{E}+03$ & $8.33 \mathrm{E}-04$ & $2.08 \mathrm{E}+01$ & $1.67 \mathrm{E}+03$ & $4.92 \mathrm{E}-01$ & $2.94 \mathrm{E}-03$ & $3.74 \mathrm{E}-01$ \\
\hline $2.61 \mathrm{E}-02$ & $2.42 \mathrm{E}-02$ & $3.61 \mathrm{E}+00$ & $2.11 \mathrm{E}-01$ & $1.92 \mathrm{E}+03$ & $7.41 \mathrm{E}-04$ & $1.85 \mathrm{E}+01$ & $1.82 \mathrm{E}+03$ & $4.31 \mathrm{E}-01$ & $2.62 \mathrm{E}-03$ & $3.22 \mathrm{E}-01$ \\
\hline $3.18 \mathrm{E}-02$ & $2.61 \mathrm{E}-02$ & $1.22 \mathrm{E}+01$ & $7.14 \mathrm{E}-01$ & $6.18 \mathrm{E}+03$ & $2.51 \mathrm{E}-03$ & $6.24 \mathrm{E}+01$ & $2.78 \mathrm{E}+03$ & $1.43 \mathrm{E}+00$ & $8.86 \mathrm{E}-03$ & $1.04 \mathrm{E}+00$ \\
\hline 4.09E-02 & $3.18 \mathrm{E}-02$ & $2.38 \mathrm{E}+01$ & $1.39 \mathrm{E}+00$ & $1.11 \mathrm{E}+04$ & $4.87 \mathrm{E}-03$ & $1.21 \mathrm{E}+02$ & $1.46 \mathrm{E}+03$ & $2.67 \mathrm{E}+00$ & $1.72 \mathrm{E}-02$ & $1.85 \mathrm{E}+00$ \\
\hline $5.74 \mathrm{E}-02$ & $4.09 \mathrm{E}-02$ & $5.58 \mathrm{E}+01$ & $3.26 \mathrm{E}+00$ & $2.33 \mathrm{E}+04$ & $1.15 \mathrm{E}-02$ & $2.84 \mathrm{E}+02$ & $6.35 \mathrm{E}+02$ & $6.00 \mathrm{E}+00$ & $4.04 \mathrm{E}-02$ & $3.87 \mathrm{E}+00$ \\
\hline $1.11 \mathrm{E}-01$ & $5.74 \mathrm{E}-02$ & $2.74 \mathrm{E}+02$ & $1.60 \mathrm{E}+01$ & $9.80 \mathrm{E}+04$ & $5.62 \mathrm{E}-02$ & $1.39 \mathrm{E}+03$ & $9.87 \mathrm{E}+02$ & $2.77 \mathrm{E}+01$ & $1.98 \mathrm{E}-01$ & $1.62 \mathrm{E}+01$ \\
\hline $1.83 \mathrm{E}-01$ & $1.11 \mathrm{E}-01$ & $5.10 \mathrm{E}+02$ & $2.98 \mathrm{E}+01$ & $1.70 \mathrm{E}+05$ & $1.05 \mathrm{E}-01$ & $2.58 \mathrm{E}+03$ & $6.36 \mathrm{E}+02$ & $5.03 \mathrm{E}+01$ & $3.69 \mathrm{E}-01$ & $2.79 \mathrm{E}+01$ \\
\hline $2.97 \mathrm{E}-01$ & $1.83 \mathrm{E}-01$ & $2.04 \mathrm{E}+03$ & $1.19 \mathrm{E}+02$ & $3.52 \mathrm{E}+05$ & $4.18 \mathrm{E}-01$ & $1.02 \mathrm{E}+04$ & $4.90 \mathrm{E}+02$ & $1.67 \mathrm{E}+02$ & $1.47 \mathrm{E}+00$ & $5.49 \mathrm{E}+01$ \\
\hline $3.69 \mathrm{E}-01$ & $2.97 \mathrm{E}-01$ & $6.40 \mathrm{E}+03$ & $3.73 \mathrm{E}+02$ & $3.67 \mathrm{E}+05$ & $1.31 \mathrm{E}+00$ & $3.19 \mathrm{E}+04$ & $1.16 \mathrm{E}+02$ & $4.47 \mathrm{E}+02$ & $4.62 \mathrm{E}+00$ & $4.46 \mathrm{E}+01$ \\
\hline $4.98 \mathrm{E}-01$ & $3.69 \mathrm{E}-01$ & $2.71 \mathrm{E}+04$ & $1.58 \mathrm{E}+03$ & $1.06 \mathrm{E}+06$ & $5.56 \mathrm{E}+00$ & $1.35 \mathrm{E}+05$ & $3.69 \mathrm{E}+01$ & $1.84 \mathrm{E}+03$ & $1.96 \mathrm{E}+01$ & $1.03 \mathrm{E}+02$ \\
\hline $6.08 \mathrm{E}-01$ & $4.98 \mathrm{E}-01$ & $3.52 \mathrm{E}+04$ & $2.05 \mathrm{E}+03$ & $1.21 \mathrm{E}+06$ & $7.19 \mathrm{E}+00$ & $1.75 \mathrm{E}+05$ & $9.23 \mathrm{E}+00$ & $2.37 \mathrm{E}+03$ & $2.54 \mathrm{E}+01$ & $1.05 \mathrm{E}+02$ \\
\hline $7.43 \mathrm{E}-01$ & $6.08 \mathrm{E}-01$ & $5.46 \mathrm{E}+04$ & $3.18 \mathrm{E}+03$ & $1.77 \mathrm{E}+06$ & $9.90 \mathrm{E}+00$ & $2.72 \mathrm{E}+05$ & $4.95 \mathrm{E}+00$ & $3.67 \mathrm{E}+03$ & $3.76 \mathrm{E}+01$ & $1.41 \mathrm{E}+02$ \\
\hline $8.21 \mathrm{E}-01$ & $7.43 \mathrm{E}-01$ & $3.62 \mathrm{E}+04$ & $2.10 \mathrm{E}+03$ & $1.13 \mathrm{E}+06$ & $5.70 \mathrm{E}+00$ & $1.80 \mathrm{E}+05$ & $1.01 \mathrm{E}+00$ & $2.37 \mathrm{E}+03$ & $2.21 \mathrm{E}+01$ & $8.25 \mathrm{E}+01$ \\
\hline $1.00 \mathrm{E}+00$ & $8.21 \mathrm{E}-01$ & $9.15 \mathrm{E}+04$ & $4.95 \mathrm{E}+03$ & $2.87 \mathrm{E}+06$ & $1.30 \mathrm{E}+01$ & $4.57 \mathrm{E}+05$ & $4.30 \mathrm{E}-01$ & $5.43 \mathrm{E}+03$ & $5.05 \mathrm{E}+01$ & $1.89 \mathrm{E}+02$ \\
\hline $1.35 \mathrm{E}+00$ & $1.00 \mathrm{E}+00$ & $1.77 \mathrm{E}+05$ & $9.45 \mathrm{E}+03$ & $5.87 \mathrm{E}+06$ & $2.47 \mathrm{E}+01$ & $8.87 \mathrm{E}+05$ & $2.21 \mathrm{E}-01$ & $1.04 \mathrm{E}+04$ & $9.59 \mathrm{E}+01$ & $3.55 \mathrm{E}+02$ \\
\hline $1.65 \mathrm{E}+00$ & $1.35 \mathrm{E}+00$ & $1.21 \mathrm{E}+05$ & $6.29 \mathrm{E}+03$ & $4.22 \mathrm{E}+06$ & $1.48 \mathrm{E}+01$ & $6.05 E+05$ & $6.53 \mathrm{E}-02$ & $6.79 \mathrm{E}+03$ & $5.97 \mathrm{E}+01$ & $2.52 \mathrm{E}+02$ \\
\hline $1.92 \mathrm{E}+00$ & $1.65 \mathrm{E}+00$ & $1.11 \mathrm{E}+05$ & $5.76 \mathrm{E}+03$ & $3.76 \mathrm{E}+06$ & $1.37 \mathrm{E}+01$ & $5.53 \mathrm{E}+05$ & $7.34 \mathrm{E}-02$ & $6.22 \mathrm{E}+03$ & $5.50 \mathrm{E}+01$ & $2.14 \mathrm{E}+02$ \\
\hline $2.23 \mathrm{E}+00$ & $1.92 \mathrm{E}+00$ & $1.71 \mathrm{E}+05$ & $9.17 \mathrm{E}+03$ & $5.26 \mathrm{E}+06$ & $2.52 \mathrm{E}+01$ & $8.54 \mathrm{E}+05$ & $1.82 \mathrm{E}-01$ & $1.01 \mathrm{E}+04$ & $9.49 \mathrm{E}+01$ & $2.78 \mathrm{E}+02$ \\
\hline $2.35 \mathrm{E}+00$ & $2.23 \mathrm{E}+00$ & $7.34 \mathrm{E}+04$ & $3.97 \mathrm{E}+03$ & $2.18 \mathrm{E}+06$ & $1.24 \mathrm{E}+01$ & $3.67 \mathrm{E}+05$ & $1.29 \mathrm{E}-01$ & $4.37 \mathrm{E}+03$ & $4.37 \mathrm{E}+01$ & $1.14 \mathrm{E}+02$ \\
\hline $2.37 \mathrm{E}+00$ & $2.35 \mathrm{E}+00$ & $1.25 \mathrm{E}+04$ & $6.78 \mathrm{E}+02$ & $3.68 \mathrm{E}+05$ & $2.19 \mathrm{E}+00$ & $6.25 \mathrm{E}+04$ & $2.55 \mathrm{E}-02$ & $7.46 \mathrm{E}+02$ & $7.73 \mathrm{E}+00$ & $1.94 \mathrm{E}+01$ \\
\hline $2.47 \mathrm{E}+00$ & $2.37 \mathrm{E}+00$ & $6.41 \mathrm{E}+04$ & $3.48 \mathrm{E}+03$ & $1.87 \mathrm{E}+06$ & $1.16 \mathrm{E}+01$ & $3.20 \mathrm{E}+05$ & $3.51 \mathrm{E}-03$ & $3.83 \mathrm{E}+03$ & $4.07 \mathrm{E}+01$ & $9.90 \mathrm{E}+01$ \\
\hline $2.73 \mathrm{E}+00$ & $2.47 \mathrm{E}+00$ & $1.91 \mathrm{E}+05$ & $1.07 \mathrm{E}+04$ & $5.40 \mathrm{E}+06$ & $3.73 \mathrm{E}+01$ & $9.56 \mathrm{E}+05$ & $2.86 \mathrm{E}-04$ & $1.21 \mathrm{E}+04$ & $1.31 \mathrm{E}+02$ & $2.89 \mathrm{E}+02$ \\
\hline $3.01 \mathrm{E}+00$ & $2.73 \mathrm{E}+00$ & $3.06 \mathrm{E}+05$ & $1.78 \mathrm{E}+04$ & $7.97 \mathrm{E}+06$ & $6.20 \mathrm{E}+01$ & $1.53 \mathrm{E}+06$ & $5.55 \mathrm{E}-08$ & $2.04 \mathrm{E}+04$ & $2.21 \mathrm{E}+02$ & $4.25 \mathrm{E}+02$ \\
\hline $3.68 \mathrm{E}+00$ & $3.01 \mathrm{E}+00$ & $1.02 \mathrm{E}+06$ & $5.41 \mathrm{E}+04$ & $2.74 \mathrm{E}+07$ & $1.37 \mathrm{E}+02$ & $5.08 \mathrm{E}+06$ & $1.80 \mathrm{E}-08$ & $5.81 \mathrm{E}+04$ & $5.27 \mathrm{E}+02$ & $9.68 \mathrm{E}+02$ \\
\hline $4.97 \mathrm{E}+00$ & $3.68 \mathrm{E}+00$ & $1.71 \mathrm{E}+06$ & $8.60 \mathrm{E}+04$ & $4.97 \mathrm{E}+07$ & $1.91 \mathrm{E}+02$ & $8.56 \mathrm{E}+06$ & $6.68 \mathrm{E}-09$ & $8.95 \mathrm{E}+04$ & $7.58 \mathrm{E}+02$ & $1.33 \mathrm{E}+03$ \\
\hline $6.07 \mathrm{E}+00$ & & & & & & $4.77 \mathrm{E}+06$ & $1.64 \mathrm{E}-09$ & $4.15 \mathrm{E}+04$ & $2.81 \mathrm{E}+02$ & $4.90 \mathrm{E}+02$ \\
\hline $7.41 \mathrm{E}+00$ & $6.07 \mathrm{E}+00$ & $9.49 \mathrm{E}+05$ & $4.73 \mathrm{E}+04$ & $3.01 \mathrm{E}+07$ & $1.33 \mathrm{E}+02$ & $4.76 \mathrm{E}+06$ & $2.01 \mathrm{E}-10$ & $5.06 \mathrm{E}+04$ & $4.92 \mathrm{E}+02$ & $8.03 \mathrm{E}+02$ \\
\hline $8.61 \mathrm{E}+00$ & $7.41 \mathrm{E}+00$ & $7.92 \mathrm{E}+05$ & $4.00 \mathrm{E}+04$ & $2.34 \mathrm{E}+07$ & $1.06 \mathrm{E}+02$ & $3.97 \mathrm{E}+06$ & $7.45 \mathrm{E}-11$ & $4.24 \mathrm{E}+04$ & $3.98 \mathrm{E}+02$ & $6.38 \mathrm{E}+02$ \\
\hline $1.00 \mathrm{E}+01$ & $8.61 \mathrm{E}+00$ & $5.98 \mathrm{E}+05$ & $2.81 \mathrm{E}+04$ & $1.95 \mathrm{E}+07$ & $6.48 \mathrm{E}+01$ & $3.00 \mathrm{E}+06$ & $1.46 \mathrm{E}-11$ & $2.88 \mathrm{E}+04$ & $2.52 \mathrm{E}+02$ & $4.03 \mathrm{E}+02$ \\
\hline $1.22 \mathrm{E}+01$ & $1.00 \mathrm{E}+01$ & $6.75 \mathrm{E}+04$ & $2.81 \mathrm{E}+03$ & $2.75 \mathrm{E}+06$ & $2.22 \mathrm{E}+00$ & $3.40 \mathrm{E}+05$ & $4.70 \mathrm{E}-12$ & $2.48 \mathrm{E}+03$ & $1.26 \mathrm{E}+01$ & $2.08 \mathrm{E}+01$ \\
\hline $1.42 \mathrm{E}+01$ & $1.22 \mathrm{E}+01$ & $2.44 \mathrm{E}-04$ & $4.36 \mathrm{E}-04$ & $1.76 \mathrm{E}+03$ & $9.15 \mathrm{E}-09$ & $2.48 \mathrm{E}-01$ & $1.99 \mathrm{E}-12$ & $5.52 \mathrm{E}-02$ & $4.33 \mathrm{E}-06$ & $1.17 \mathrm{E}-01$ \\
\hline $1.73 \mathrm{E}+01$ & $1.42 \mathrm{E}+01$ & $5.37 \mathrm{E}-05$ & $8.27 \mathrm{E}-05$ & $3.65 \mathrm{E}+02$ & $1.61 \mathrm{E}-09$ & $4.55 \mathrm{E}-02$ & $2.69 \mathrm{E}-13$ & $8.83 \mathrm{E}-03$ & 7.79E-07 & $1.98 \mathrm{E}-02$ \\
\hline & Total & $7.56 \mathrm{E}+06$ & $3.83 \mathrm{E}+05$ & $2.29 \mathrm{E}+08$ & $9.45 \mathrm{E}+02$ & $3.79 \mathrm{E}+07$ & $1.09 \mathrm{E}+05$ & $4.05 \mathrm{E}+05$ & $3.63 \mathrm{E}+03$ & $7.48 \mathrm{E}+03$ \\
\hline
\end{tabular}

Note: $90 \%$ Beryllium is modeled as 1 gram of actinide and 0.9 grams of beryllium. 
PCP-2011-0001

Revision 0

Table 3-11. Total Neutron Source (Decay Time $=0$ days)

\begin{tabular}{|c|c|c|c|c|c|c|c|}
\hline Isotope & Code & $0 \% \mathrm{Be}$ & $10 \% \mathrm{Be}$ & $30 \% \mathrm{Be}$ & $50 \% \mathrm{Be}$ & $70 \% \mathrm{Be}$ & $90 \% \mathrm{Be}$ \\
\hline \multirow{2}{*}{ Am-241 } & ORIGEN & $1.24 \mathrm{E}+00$ & $2.55 \mathrm{E}+06$ & $5.07 \mathrm{E}+06$ & $6.32 \mathrm{E}+06$ & $7.06 \mathrm{E}+06$ & $7.56 \mathrm{E}+06$ \\
\hline & RASTA & $1.18 \mathrm{E}+00$ & $2.55 \mathrm{E}+06$ & $5.07 \mathrm{E}+06$ & $6.32 \mathrm{E}+06$ & $7.07 \mathrm{E}+06$ & $7.56 \mathrm{E}+06$ \\
\hline \multirow{2}{*}{ Am-243 } & ORIGEN & $6.61 \mathrm{E}-01$ & $1.30 \mathrm{E}+05$ & $2.58 \mathrm{E}+05$ & $3.21 \mathrm{E}+05$ & $3.58 \mathrm{E}+05$ & $3.83 \mathrm{E}+05$ \\
\hline & RASTA & $3.93 \mathrm{E}+00$ & $1.30 \mathrm{E}+05$ & $2.58 \mathrm{E}+05$ & $3.21 \mathrm{E}+05$ & $3.58 \mathrm{E}+05$ & $3.83 \mathrm{E}+05$ \\
\hline \multirow{2}{*}{$\mathrm{Cm}-244$} & ORIGEN & $1.10 \mathrm{E}+07$ & $8.49 \mathrm{E}+07$ & $1.58 \mathrm{E}+08$ & $1.94 \mathrm{E}+08$ & $2.15 \mathrm{E}+08$ & $2.29 \mathrm{E}+08$ \\
\hline & RASTA & $1.08 \mathrm{E}+07$ & $8.44 \mathrm{E}+07$ & $1.57 \mathrm{E}+08$ & $1.93 \mathrm{E}+08$ & $2.15 \mathrm{E}+08$ & $2.29 \mathrm{E}+08$ \\
\hline \multirow{2}{*}{ Np-237 } & ORIGEN & $1.12 \mathrm{E}-04$ & $3.06 \mathrm{E}+02$ & $6.07 \mathrm{E}+02$ & $7.55 \mathrm{E}+02$ & $8.44 \mathrm{E}+02$ & $9.03 \mathrm{E}+02$ \\
\hline & RASTA & 1.14E-04 & $3.20 \mathrm{E}+02$ & $6.35 \mathrm{E}+02$ & $7.91 \mathrm{E}+02$ & $8.84 \mathrm{E}+02$ & $9.45 \mathrm{E}+02$ \\
\hline \multirow{2}{*}{$\mathrm{Pu}-238$} & ORIGEN & $2.60 \mathrm{E}+03$ & $1.28 \mathrm{E}+07$ & $2.54 \mathrm{E}+07$ & $3.17 \mathrm{E}+07$ & $3.54 \mathrm{E}+07$ & $3.79 E+07$ \\
\hline & RASTA & $2.59 \mathrm{E}+03$ & $1.27 \mathrm{E}+07$ & $2.54 \mathrm{E}+07$ & $3.16 \mathrm{E}+07$ & $3.54 \mathrm{E}+07$ & $3.79 \mathrm{E}+07$ \\
\hline \multirow{2}{*}{$\mathrm{Pu}-239$} & ORIGEN & 1.49E-02 & $3.70 \mathrm{E}+04$ & $7.35 \mathrm{E}+04$ & $9.15 \mathrm{E}+04$ & $1.02 \mathrm{E}+05$ & $1.09 \mathrm{E}+05$ \\
\hline & RASTA & $2.18 \mathrm{E}-02$ & $3.70 \mathrm{E}+04$ & $7.35 \mathrm{E}+04$ & $9.15 \mathrm{E}+04$ & $1.02 \mathrm{E}+05$ & $1.09 \mathrm{E}+05$ \\
\hline \multirow{2}{*}{$\mathrm{Pu}-240$} & ORIGEN & $1.04 \mathrm{E}+03$ & $1.38 \mathrm{E}+05$ & $2.72 \mathrm{E}+05$ & $3.38 \mathrm{E}+05$ & $3.78 \mathrm{E}+05$ & $4.04 \mathrm{E}+05$ \\
\hline & RASTA & $1.03 \mathrm{E}+03$ & $1.38 \mathrm{E}+05$ & $2.72 \mathrm{E}+05$ & $3.39 \mathrm{E}+05$ & $3.78 \mathrm{E}+05$ & $4.05 \mathrm{E}+05$ \\
\hline \multirow{2}{*}{$\mathrm{Pu}-241$} & ORIGEN & $2.07 \mathrm{E}-03$ & $1.27 \mathrm{E}+03$ & $2.51 \mathrm{E}+03$ & $3.12 \mathrm{E}+03$ & $3.48 \mathrm{E}+03$ & $3.72 \mathrm{E}+03$ \\
\hline & RASTA & $4.94 \mathrm{E}-02$ & $1.24 \mathrm{E}+03$ & $2.45 \mathrm{E}+03$ & $3.04 \mathrm{E}+03$ & $3.40 \mathrm{E}+03$ & $3.63 \mathrm{E}+03$ \\
\hline \multirow{2}{*}{$\mathrm{Pu}-242$} & ORIGEN & $1.74 \mathrm{E}+03$ & $3.73 \mathrm{E}+03$ & $8.38 \mathrm{E}+04$ & $6.61 \mathrm{E}+03$ & $7.17 \mathrm{E}+03$ & $7.55 \mathrm{E}+03$ \\
\hline & RASTA & $1.72 \mathrm{E}+03$ & $3.68 \mathrm{E}+03$ & $5.60 \mathrm{E}+03$ & $6.55 \mathrm{E}+03$ & $7.11 \mathrm{E}+03$ & $7.48 \mathrm{E}+03$ \\
\hline
\end{tabular}

Note: $90 \%$ Beryllium is modeled as 1 gram of actinide and 0.9 grams of beryllium.

Table 3-12. Neutron Source as a Function of Decay Time

\begin{tabular}{||c|c|c|c|c|c|c|c|c||}
\hline & \multicolumn{4}{|c|}{$\begin{array}{c}\text { No Beryllium } \\
\text { Decay Time (in days) }\end{array}$} & \multicolumn{3}{c|}{$\begin{array}{c}\text { 90\% Beryllium Added } \\
\text { Decay Time (in days) }\end{array}$} \\
\hline Isotope & $\mathbf{0}$ & $\mathbf{3 0}$ & $\mathbf{1 0 0}$ & $\mathbf{3 0 0 0 0}$ & $\mathbf{0}$ & $\mathbf{3 0}$ & $\mathbf{1 0 0}$ & $\mathbf{3 0 0 0 0}$ \\
\hline Am-241 & $1.24 \mathrm{E}+00$ & $1.24 \mathrm{E}+00$ & $1.24 \mathrm{E}+00$ & $1.09 \mathrm{E}+00$ & $7.56 \mathrm{E}+06$ & $7.56 \mathrm{E}+06$ & $7.56 \mathrm{E}+06$ & $6.63 \mathrm{E}+06$ \\
\hline $\mathrm{Am}-243$ & $6.61 \mathrm{E}-01$ & $6.61 \mathrm{E}-01$ & $6.61 \mathrm{E}-01$ & $6.56 \mathrm{E}-01$ & $3.83 \mathrm{E}+05$ & $3.83 \mathrm{E}+05$ & $3.83 \mathrm{E}+05$ & $3.80 \mathrm{E}+05$ \\
\hline $\mathrm{Cf}-252$ & $2.31 \mathrm{E}+12$ & $2.26 \mathrm{E}+12$ & $2.15 \mathrm{E}+12$ & $3.90 \mathrm{E}+07$ & & & & \\
\hline $\mathrm{Cm}-244$ & $1.10 \mathrm{E}+07$ & $1.10 \mathrm{E}+07$ & $1.09 \mathrm{E}+07$ & $4.77 \mathrm{E}+05$ & $2.29 \mathrm{E}+08$ & $2.29 \mathrm{E}+08$ & $2.27 \mathrm{E}+08$ & $1.03 \mathrm{E}+07$ \\
\hline $\mathrm{Cm}-248$ & $4.09 \mathrm{E}+07$ & $4.09 \mathrm{E}+07$ & $4.09 \mathrm{E}+07$ & $4.09 \mathrm{E}+07$ & & & & \\
\hline $\mathrm{Np}-237$ & $1.12 \mathrm{E}-04$ & $1.12 \mathrm{E}-04$ & $1.12 \mathrm{E}-04$ & $1.12 \mathrm{E}-04$ & $9.03 \mathrm{E}+02$ & $9.03 \mathrm{E}+02$ & $9.03 \mathrm{E}+02$ & $9.03 \mathrm{E}+02$ \\
\hline $\mathrm{Pu}-238$ & $2.60 \mathrm{E}+03$ & $2.60 \mathrm{E}+03$ & $2.60 \mathrm{E}+03$ & $1.36 \mathrm{E}+03$ & $3.79 \mathrm{E}+07$ & $3.79 \mathrm{E}+07$ & $3.78 \mathrm{E}+07$ & $1.98 \mathrm{E}+07$ \\
\hline $\mathrm{Pu}-239$ & $1.49 \mathrm{E}-02$ & $1.49 \mathrm{E}-02$ & $1.49 \mathrm{E}-02$ & $1.48 \mathrm{E}-02$ & $1.09 \mathrm{E}+05$ & $1.09 \mathrm{E}+05$ & $1.09 \mathrm{E}+05$ & $1.09 \mathrm{E}+05$ \\
\hline $\mathrm{Pu}-240$ & $1.04 \mathrm{E}+03$ & $1.04 \mathrm{E}+03$ & $1.04 \mathrm{E}+03$ & $1.03 \mathrm{E}+03$ & $4.04 \mathrm{E}+05$ & $4.04 \mathrm{E}+05$ & $4.04 \mathrm{E}+05$ & $4.01 \mathrm{E}+05$ \\
\hline $\mathrm{Pu}-241$ & $2.07 \mathrm{E}-03$ & $6.96 \mathrm{E}-03$ & $1.83 \mathrm{E}-02$ & $1.10 \mathrm{E}+00$ & $3.72 \mathrm{E}+03$ & $3.37 \mathrm{E}+04$ & $1.03 \mathrm{E}+05$ & $6.72 \mathrm{E}+06$ \\
\hline $\mathrm{Pu}-242$ & $1.74 \mathrm{E}+03$ & $1.74 \mathrm{E}+03$ & $1.74 \mathrm{E}+03$ & $1.74 \mathrm{E}+03$ & $7.55 \mathrm{E}+03$ & $7.55 \mathrm{E}+03$ & $7.55 \mathrm{E}+03$ & $7.55 \mathrm{E}+03$ \\
\hline
\end{tabular}

Note: $90 \%$ Beryllium is modeled as 1 gram of actinide and 0.9 grams of beryllium. 
PCP-2011-0001

Revision 0

\begin{tabular}{|c|c|c|c|c|c|c|c|c|}
\hline \multicolumn{2}{|c|}{ Energy (MeV) } & \multicolumn{7}{|c|}{ Photon Source $(\mathrm{p} / \mathrm{s} / \mathrm{g})$} \\
\hline Upper & Lower & Ac-227 & Am-241 & Am-243 & Cd-109 & Cf-252 & Cm-244 & Cm-248 \\
\hline 0.020 & 0.010 & $2.04 \mathrm{E}+10$ & $4.54 \mathrm{E}+10$ & $1.95 \mathrm{E}+09$ & $0.00 \mathrm{E}+00$ & $1.84 \mathrm{E}+12$ & $3.65 \mathrm{E}+11$ & $1.40 \mathrm{E}+07$ \\
\hline 0.030 & 0.020 & $7.48 \mathrm{E}+08$ & $8.33 \mathrm{E}+09$ & $2.51 \mathrm{E}+05$ & $8.57 \mathrm{E}+13$ & $1.33 \mathrm{E}-14$ & $4.19 \mathrm{E}+01$ & $6.85 \mathrm{E}-09$ \\
\hline 0.040 & 0.030 & $3.10 \mathrm{E}+08$ & $1.65 \mathrm{E}+08$ & $5.06 \mathrm{E}+06$ & $0.00 \mathrm{E}+00$ & $7.80 \mathrm{E}-15$ & $0.00 \mathrm{E}+00$ & $4.03 \mathrm{E}-09$ \\
\hline 0.050 & 0.040 & $2.97 \mathrm{E}+09$ & $9.36 \mathrm{E}+07$ & $4.29 \mathrm{E}+08$ & $0.00 \mathrm{E}+00$ & $2.84 \mathrm{E}+09$ & $7.07 \mathrm{E}+08$ & $4.12 \mathrm{E}+04$ \\
\hline 0.060 & 0.050 & $2.33 \mathrm{E}+09$ & $4.93 \mathrm{E}+10$ & $1.07 \mathrm{E}+06$ & $0.00 \mathrm{E}+00$ & $3.56 \mathrm{E}-15$ & $0.00 \mathrm{E}+00$ & $1.86 \mathrm{E}-09$ \\
\hline 0.070 & 0.060 & $5.31 \mathrm{E}+08$ & $2.71 \mathrm{E}+06$ & $3.35 \mathrm{E}+05$ & $0.00 \mathrm{E}+00$ & $4.01 \mathrm{E}-15$ & $4.14 \mathrm{E}-27$ & $2.04 \mathrm{E}-09$ \\
\hline 0.080 & 0.070 & $9.83 \mathrm{E}+08$ & $2.47 \mathrm{E}+06$ & $5.02 \mathrm{E}+09$ & $0.00 \mathrm{E}+00$ & $2.56 \mathrm{E}-15$ & $0.00 \mathrm{E}+00$ & $1.35 \mathrm{E}-09$ \\
\hline 0.090 & 0.080 & $1.35 \mathrm{E}+09$ & $1.42 \mathrm{E}-01$ & $2.55 \mathrm{E}+07$ & $3.58 \mathrm{E}+12$ & $2.09 \mathrm{E}-15$ & $2.41 \mathrm{E}-14$ & $1.10 \mathrm{E}-09$ \\
\hline 0.100 & 0.090 & $6.55 \mathrm{E}+08$ & $2.81 \mathrm{E}+07$ & $4.71 \mathrm{E}+06$ & $0.00 \mathrm{E}+00$ & $1.32 \mathrm{E}+09$ & $3.62 \mathrm{E}+07$ & $3.00 \mathrm{E}-09$ \\
\hline 0.110 & 0.100 & $6.31 \mathrm{E}+08$ & $2.68 \mathrm{E}+07$ & $1.20 \mathrm{E}+07$ & $0.00 \mathrm{E}+00$ & $1.25 \mathrm{E}+09$ & $2.84 \mathrm{E}+06$ & $3.30 \mathrm{E}-09$ \\
\hline 0.120 & 0.110 & $5.63 \mathrm{E}+07$ & $1.19 \mathrm{E}+06$ & $4.56 \mathrm{E}+07$ & $0.00 \mathrm{E}+00$ & $4.16 \mathrm{E}-15$ & $1.20 \mathrm{E}+06$ & $1.95 \mathrm{E}-09$ \\
\hline 0.130 & 0.120 & $1.88 \mathrm{E}+08$ & $6.47 \mathrm{E}+06$ & $3.28 \mathrm{E}+05$ & $0.00 \mathrm{E}+00$ & $1.50 \mathrm{E}+07$ & $1.69 \mathrm{E}+05$ & $7.74 \mathrm{E}-10$ \\
\hline 0.140 & 0.130 & $1.81 \mathrm{E}+08$ & $6.95 \mathrm{E}+03$ & $7.28 \mathrm{E}+03$ & $0.00 \mathrm{E}+00$ & $8.61 \mathrm{E}-16$ & $0.00 \mathrm{E}+00$ & $4.64 \mathrm{E}-10$ \\
\hline 0.150 & 0.140 & $2.59 \mathrm{E}+08$ & $6.39 \mathrm{E}+05$ & $8.70 \mathrm{E}+06$ & $0.00 \mathrm{E}+00$ & $1.17 \mathrm{E}-15$ & $4.14 \mathrm{E}-28$ & $5.94 \mathrm{E}-10$ \\
\hline 0.160 & 0.150 & $2.57 \mathrm{E}+07$ & $4.80 \mathrm{E}+04$ & $4.50 \mathrm{E}+03$ & $0.00 \mathrm{E}+00$ & $1.99 \mathrm{E}+08$ & $2.92 \mathrm{E}+07$ & $3.51 \mathrm{E}-10$ \\
\hline 0.170 & 0.160 & $5.27 \mathrm{E}+08$ & $3.47 \mathrm{E}+05$ & $1.14 \mathrm{E}+05$ & $0.00 \mathrm{E}+00$ & $1.87 \mathrm{E}+08$ & $3.38 \mathrm{E}-02$ & $5.66 \mathrm{E}-10$ \\
\hline 0.180 & 0.170 & $1.47 \mathrm{E}+08$ & $2.31 \mathrm{E}+04$ & $2.82 \mathrm{E}+03$ & $0.00 \mathrm{E}+00$ & $5.04 \mathrm{E}-16$ & $0.00 \mathrm{E}+00$ & $2.74 \mathrm{E}-10$ \\
\hline 0.190 & 0.180 & $6.00 \mathrm{E}+07$ & $2.54 \mathrm{E}-04$ & $1.99 \mathrm{E}+04$ & $0.00 \mathrm{E}+00$ & $1.04 \mathrm{E}-15$ & $0.00 \mathrm{E}+00$ & $5.06 \mathrm{E}-10$ \\
\hline 0.200 & 0.190 & $2.99 \mathrm{E}+07$ & $3.03 \mathrm{E}+04$ & $7.37 \mathrm{E}+04$ & $0.00 \mathrm{E}+00$ & $1.33 \mathrm{E}-15$ & $0.00 \mathrm{E}+00$ & $6.71 \mathrm{E}-10$ \\
\hline 0.210 & 0.200 & $1.64 \mathrm{E}+08$ & $1.02 \mathrm{E}+06$ & $3.83 \mathrm{E}+05$ & $0.00 \mathrm{E}+00$ & $7.62 \mathrm{E}-16$ & $0.00 \mathrm{E}+00$ & $4.16 \mathrm{E}-10$ \\
\hline 0.220 & 0.210 & $1.27 \mathrm{E}+07$ & $2.18 \mathrm{E}-03$ & $3.62 \mathrm{E}+05$ & $0.00 \mathrm{E}+00$ & $6.78 \mathrm{E}-18$ & $2.48 \mathrm{E}-03$ & $2.97 \mathrm{E}-12$ \\
\hline 0.230 & 0.220 & $1.49 \mathrm{E}+05$ & $5.30 \mathrm{E}+04$ & $2.54 \mathrm{E}+06$ & $0.00 \mathrm{E}+00$ & $1.91 \mathrm{E}-20$ & $0.00 \mathrm{E}+00$ & $1.33 \mathrm{E}-14$ \\
\hline 0.240 & 0.230 & $5.76 \mathrm{E}+08$ & $6.62 \mathrm{E}+03$ & $1.25 \mathrm{E}-09$ & $0.00 \mathrm{E}+00$ & $2.21 \mathrm{E}-20$ & $0.00 \mathrm{E}+00$ & $1.54 \mathrm{E}-14$ \\
\hline 0.250 & 0.240 & $1.11 \mathrm{E}+07$ & $4.26 \mathrm{E}+03$ & $2.96 \mathrm{E}+01$ & $0.00 \mathrm{E}+00$ & $1.44 \mathrm{E}+12$ & $9.48 \mathrm{E}+06$ & $3.04 \mathrm{E}+07$ \\
\hline 0.260 & 0.250 & $1.15 \mathrm{E}+08$ & $4.80 \mathrm{E}+02$ & $2.60 \mathrm{E}+04$ & $0.00 \mathrm{E}+00$ & $1.38 \mathrm{E}+12$ & $9.40 \mathrm{E}+06$ & $2.92 \mathrm{E}+07$ \\
\hline 0.270 & 0.260 & $2.84 \mathrm{E}+07$ & $4.66 \mathrm{E}+04$ & $2.07 \mathrm{E}+03$ & $0.00 \mathrm{E}+00$ & $2.16 \mathrm{E}-15$ & $1.60 \mathrm{E}+06$ & 1.19E-09 \\
\hline 0.280 & 0.270 & $5.96 \mathrm{E}+06$ & $9.76 \mathrm{E}+03$ & $3.17 \mathrm{E}+06$ & $0.00 \mathrm{E}+00$ & $3.76 \mathrm{E}-17$ & $0.00 \mathrm{E}+00$ & $1.65 \mathrm{E}-11$ \\
\hline 0.290 & 0.280 & $5.85 \mathrm{E}+07$ & $6.21 \mathrm{E}-20$ & $1.72 \mathrm{E}+05$ & $0.00 \mathrm{E}+00$ & $5.72 \mathrm{E}-17$ & $1.43 \mathrm{E}+04$ & $2.74 \mathrm{E}-11$ \\
\hline 0.300 & 0.290 & $1.88 \mathrm{E}+07$ & $2.18 \mathrm{E}+04$ & $4.29 \mathrm{E}-09$ & $0.00 \mathrm{E}+00$ & $3.89 \mathrm{E}-17$ & $0.00 \mathrm{E}+00$ & $1.71 \mathrm{E}-11$ \\
\hline 0.325 & 0.300 & $1.52 \mathrm{E}+08$ & $2.00 \mathrm{E}+05$ & $3.52 \mathrm{E}+05$ & $0.00 \mathrm{E}+00$ & $2.25 \mathrm{E}-15$ & $4.82 \mathrm{E}+05$ & \begin{tabular}{|l}
$1.23 \mathrm{E}-09$ \\
\end{tabular} \\
\hline 0.350 & 0.325 & $6.70 \mathrm{E}+07$ & $8.23 \mathrm{E}+05$ & $4.45 \mathrm{E}+05$ & $0.00 \mathrm{E}+00$ & 7.44E-17 & $5.14 \mathrm{E}+04$ & $4.09 \mathrm{E}-11$ \\
\hline 0.375 & 0.350 & $2.28 \mathrm{E}+07$ & $3.53 \mathrm{E}+05$ & $8.53 \mathrm{E}-08$ & $0.00 \mathrm{E}+00$ & $4.41 \mathrm{E}-17$ & $0.00 \mathrm{E}+00$ & $2.43 \mathrm{E}-11$ \\
\hline 0.400 & 0.375 & $1.60 \mathrm{E}+07$ & $2.17 \mathrm{E}+05$ & $6.64 \mathrm{E}+02$ & $0.00 \mathrm{E}+00$ & $6.06 \mathrm{E}-16$ & $0.00 \mathrm{E}+00$ & $3.34 \mathrm{E}-10$ \\
\hline 0.425 & 0.400 & $4.52 \mathrm{E}+05$ & $4.34 \mathrm{E}+04$ & $1.37 \mathrm{E}-07$ & $0.00 \mathrm{E}+00$ & $4.21 \mathrm{E}-20$ & $0.00 \mathrm{E}+00$ & $2.94 \mathrm{E}-14$ \\
\hline 0.450 & 0.425 & $8.83 \mathrm{E}+06$ & $3.70 \mathrm{E}+04$ & $3.65 \mathrm{E}+03$ & $0.00 \mathrm{E}+00$ & $4.21 \mathrm{E}-16$ & $0.00 \mathrm{E}+00$ & $2.32 \mathrm{E}-10$ \\
\hline 0.475 & 0.450 & $2.62 \mathrm{E}+03$ & $2.46 \mathrm{E}+04$ & $5.81 \mathrm{E}+02$ & $0.00 \mathrm{E}+00$ & $6.99 \mathrm{E}-18$ & $0.00 \mathrm{E}+00$ & $3.86 \mathrm{E}-12$ \\
\hline 0.500 & 0.475 & $3.32 \mathrm{E}+06$ & $1.82 \mathrm{E}+03$ & $2.94 \mathrm{E}+03$ & $0.00 \mathrm{E}+00$ & $2.18 \mathrm{E}-16$ & $0.00 \mathrm{E}+00$ & $1.20 \mathrm{E}-10$ \\
\hline 0.525 & 0.500 & $3.16 \mathrm{E}+06$ & $5.93 \mathrm{E}+03$ & $2.26 \mathrm{E}+01$ & $0.00 \mathrm{E}+00$ & $1.05 \mathrm{E}-15$ & $2.37 \mathrm{E}+05$ & $5.76 \mathrm{E}-10$ \\
\hline 0.550 & 0.525 & $1.60 \mathrm{E}+04$ & $1.53 \mathrm{E}+03$ & $4.89 \mathrm{E}-11$ & $0.00 \mathrm{E}+00$ & $1.09 \mathrm{E}-31$ & $1.27 \mathrm{E}-05$ & $7.63 \mathrm{E}-26$ \\
\hline 0.575 & 0.550 & $1.10 \mathrm{E}+04$ & $2.56 \mathrm{E}+03$ & $2.08 \mathrm{E}-11$ & $0.00 \mathrm{E}+00$ & $2.53 \mathrm{E}-14$ & $2.33 E+06$ & $1.39 \mathrm{E}-08$ \\
\hline 0.600 & 0.575 & $1.87 \mathrm{E}+06$ & $1.52 \mathrm{E}+04$ & $4.61 \mathrm{E}+00$ & $0.00 \mathrm{E}+00$ & $1.48 \mathrm{E}-14$ & $1.49 \mathrm{E}+06$ & $8.13 \mathrm{E}-09$ \\
\hline 0.625 & 0.600 & $1.80 \mathrm{E}+06$ & $7.62 \mathrm{E}+04$ & $4.42 \mathrm{E}+00$ & $0.00 \mathrm{E}+00$ & $9.65 \mathrm{E}-16$ & $2.19 \mathrm{E}+05$ & $5.31 \mathrm{E}-10$ \\
\hline 0.650 & 0.625 & $2.25 \mathrm{E}+03$ & $1.14 \mathrm{E}+04$ & $2.19 \mathrm{E}+04$ & $0.00 \mathrm{E}+00$ & $1.05 \mathrm{E}-20$ & $1.13 \mathrm{E}-03$ & $7.34 \mathrm{E}-15$ \\
\hline 0.675 & 0.650 & $6.01 \mathrm{E}+02$ & $5.05 \mathrm{E}+05$ & $8.86 \mathrm{E}+04$ & $0.00 \mathrm{E}+00$ & $0.00 \mathrm{E}+00$ & $0.00 \mathrm{E}+00$ & $0.00 \mathrm{E}+00$ \\
\hline 0.700 & 0.675 & $6.42 \mathrm{E}+05$ & $5.76 \mathrm{E}+04$ & $5.64 \mathrm{E}-02$ & $0.00 \mathrm{E}+00$ & $9.14 \mathrm{E}-17$ & $3.03 \mathrm{E}-04$ & $5.03 \mathrm{E}-11$ \\
\hline 0.725 & 0.700 & $7.66 \mathrm{E}+06$ & $2.60 \mathrm{E}+05$ & $5.45 \mathrm{E}-02$ & $0.00 \mathrm{E}+00$ & 8.82E-17 & $0.00 \mathrm{E}+00$ & $4.85 \mathrm{E}-11$ \\
\hline 0.750 & 0.725 & $3.28 \mathrm{E}+06$ & $1.31 \mathrm{E}+04$ & $1.51 \mathrm{E}+01$ & $0.00 \mathrm{E}+00$ & $1.45 \mathrm{E}+12$ & $9.51 \mathrm{E}+06$ & $3.05 \mathrm{E}+07$ \\
\hline
\end{tabular}


PCP-2011-0001

Revision 0

\begin{tabular}{|c|c|c|c|c|c|c|c|c|}
\hline \multicolumn{9}{|c|}{ Table 3-13. Photon Source from ORIGEN-S (Decay Time = 0 days) } \\
\hline \multicolumn{2}{|c|}{ Energy (MeV) } & \multicolumn{7}{|c|}{ Photon Source (p/s/g) } \\
\hline Upper & Lower & Ac-227 & Am-241 & Am-243 & Cd-109 & Cf-252 & Cm-244 & Cm-248 \\
\hline 0.775 & 0.750 & $3.81 \mathrm{E}+07$ & $2.81 \mathrm{E}+04$ & $1.46 \mathrm{E}+01$ & $0.00 \mathrm{E}+00$ & $1.40 \mathrm{E}+12$ & $9.58 \mathrm{E}+06$ & $2.95 \mathrm{E}+07$ \\
\hline 0.800 & 0.775 & $3.46 \mathrm{E}+07$ & $3.07 \mathrm{E}+03$ & $2.72 \mathrm{E}-11$ & $0.00 \mathrm{E}+00$ & $2.76 \mathrm{E}-16$ & $0.00 \mathrm{E}+00$ & $1.52 \mathrm{E}-10$ \\
\hline 0.825 & 0.800 & $1.22 \mathrm{E}+07$ & $3.66 \mathrm{E}+03$ & $2.82 \mathrm{E}-11$ & $0.00 \mathrm{E}+00$ & $1.86 \mathrm{E}-15$ & $1.92 \mathrm{E}+06$ & $1.02 \mathrm{E}-09$ \\
\hline 0.850 & 0.825 & $1.45 \mathrm{E}+07$ & $9.65 \mathrm{E}+02$ & $3.26 \mathrm{E}-11$ & $0.00 \mathrm{E}+00$ & $1.95 \mathrm{E}-16$ & $0.00 \mathrm{E}+00$ & $1.07 \mathrm{E}-10$ \\
\hline 0.875 & 0.850 & $9.27 \mathrm{E}+05$ & $2.09 \mathrm{E}+03$ & $0.00 \mathrm{E}+00$ & $0.00 \mathrm{E}+00$ & $6.10 \mathrm{E}-16$ & $1.61 \mathrm{E}+05$ & $3.36 \mathrm{E}-10$ \\
\hline 0.900 & 0.875 & $8.85 \mathrm{E}+06$ & $3.69 \mathrm{E}+02$ & $1.17 \mathrm{E}-11$ & $0.00 \mathrm{E}+00$ & $2.30 \mathrm{E}-16$ & $6.96 \mathrm{E}+04$ & $1.27 \mathrm{E}-10$ \\
\hline 0.925 & 0.900 & $1.90 \mathrm{E}+06$ & $9.37 \mathrm{E}+02$ & $2.55 \mathrm{E}-12$ & $0.00 \mathrm{E}+00$ & $1.56 \mathrm{E}-15$ & $1.77 \mathrm{E}+04$ & $8.57 \mathrm{E}-10$ \\
\hline 0.950 & 0.925 & $8.72 \mathrm{E}+02$ & $1.41 \mathrm{E}+02$ & $5.39 \mathrm{E}-12$ & $0.00 \mathrm{E}+00$ & $1.79 \mathrm{E}-15$ & $0.00 \mathrm{E}+00$ & $9.84 \mathrm{E}-10$ \\
\hline 0.975 & 0.950 & $1.09 \mathrm{E}+03$ & $7.31 \mathrm{E}+02$ & $7.76 \mathrm{E}-12$ & $0.00 \mathrm{E}+00$ & $1.70 \mathrm{E}-16$ & $0.00 \mathrm{E}+00$ & $9.37 \mathrm{E}-11$ \\
\hline 1.000 & 0.975 & $5.12 \mathrm{E}+03$ & $2.39 \mathrm{E}-22$ & $8.21 \mathrm{E}-12$ & $0.00 \mathrm{E}+00$ & $1.41 \mathrm{E}-16$ & $0.00 \mathrm{E}+00$ & $7.74 \mathrm{E}-11$ \\
\hline 1.250 & 1.000 & $5.52 \mathrm{E}+03$ & $2.10 \mathrm{E}+02$ & $8.24 \mathrm{E}+00$ & $0.00 \mathrm{E}+00$ & $6.74 \mathrm{E}+11$ & $4.43 \mathrm{E}+06$ & $1.42 \mathrm{E}+07$ \\
\hline 1.500 & 1.250 & $6.80 \mathrm{E}+01$ & $1.12 \mathrm{E}+02$ & $6.74 \mathrm{E}+00$ & $0.00 \mathrm{E}+00$ & $5.51 \mathrm{E}+11$ & $3.63 \mathrm{E}+06$ & $1.16 \mathrm{E}+07$ \\
\hline 1.750 & 1.500 & $1.98 \mathrm{E}+01$ & $6.66 \mathrm{E}+01$ & $4.00 \mathrm{E}+00$ & $0.00 \mathrm{E}+00$ & $3.13 \mathrm{E}+11$ & $2.06 \mathrm{E}+06$ & $6.61 \mathrm{E}+06$ \\
\hline 2.000 & 1.750 & $1.72 \mathrm{E}+01$ & $5.77 \mathrm{E}+01$ & $3.47 \mathrm{E}+00$ & $0.00 \mathrm{E}+00$ & $2.71 \mathrm{E}+11$ & $1.78 \mathrm{E}+06$ & $5.73 \mathrm{E}+06$ \\
\hline 2.250 & 2.000 & $9.78 \mathrm{E}+00$ & $3.29 \mathrm{E}+01$ & $1.98 \mathrm{E}+00$ & $0.00 \mathrm{E}+00$ & $1.78 \mathrm{E}+11$ & $1.17 \mathrm{E}+06$ & $3.77 \mathrm{E}+06$ \\
\hline 2.500 & 2.250 & $8.75 \mathrm{E}+00$ & $2.94 \mathrm{E}+01$ & $1.77 \mathrm{E}+00$ & $0.00 \mathrm{E}+00$ & $1.60 \mathrm{E}+11$ & $1.05 \mathrm{E}+06$ & $3.37 \mathrm{E}+06$ \\
\hline 2.750 & 2.500 & $4.85 \mathrm{E}+00$ & $1.63 \mathrm{E}+01$ & $9.90 \mathrm{E}-01$ & $0.00 \mathrm{E}+00$ & $1.02 \mathrm{E}+11$ & $6.73 \mathrm{E}+05$ & $2.16 \mathrm{E}+06$ \\
\hline 3.000 & 2.750 & $4.43 \mathrm{E}+00$ & $1.49 \mathrm{E}+01$ & $9.04 \mathrm{E}-01$ & $0.00 \mathrm{E}+00$ & $9.34 \mathrm{E}+10$ & $6.15 \mathrm{E}+05$ & $1.97 \mathrm{E}+06$ \\
\hline 3.500 & 3.000 & $4.65 \mathrm{E}+00$ & $1.56 \mathrm{E}+01$ & $9.55 \mathrm{E}-01$ & $0.00 \mathrm{E}+00$ & $1.13 \mathrm{E}+11$ & $7.45 \mathrm{E}+05$ & $2.39 \mathrm{E}+06$ \\
\hline 4.000 & 3.500 & $2.33 \mathrm{E}+00$ & $7.85 \mathrm{E}+00$ & $4.83 \mathrm{E}-01$ & $0.00 \mathrm{E}+00$ & $6.57 \mathrm{E}+10$ & $4.32 \mathrm{E}+05$ & $1.39 \mathrm{E}+06$ \\
\hline 4.500 & 4.000 & $1.17 \mathrm{E}+00$ & $3.94 \mathrm{E}+00$ & $2.45 \mathrm{E}-01$ & $0.00 \mathrm{E}+00$ & $3.81 \mathrm{E}+10$ & $2.51 \mathrm{E}+05$ & $8.04 \mathrm{E}+05$ \\
\hline 5.000 & 4.500 & $5.86 \mathrm{E}-01$ & $1.98 \mathrm{E}+00$ & $1.24 \mathrm{E}-01$ & $0.00 \mathrm{E}+00$ & $2.21 \mathrm{E}+10$ & $1.45 \mathrm{E}+05$ & $4.66 \mathrm{E}+05$ \\
\hline 5.500 & 5.000 & $2.94 \mathrm{E}-01$ & $9.94 \mathrm{E}-01$ & $6.30 \mathrm{E}-02$ & $0.00 \mathrm{E}+00$ & $1.28 \mathrm{E}+10$ & $8.42 \mathrm{E}+04$ & $2.70 \mathrm{E}+05$ \\
\hline 6.000 & 5.500 & $1.48 \mathrm{E}-01$ & 4.99E-01 & $3.20 \mathrm{E}-02$ & $0.00 \mathrm{E}+00$ & $7.43 \mathrm{E}+09$ & $4.88 \mathrm{E}+04$ & $1.57 \mathrm{E}+05$ \\
\hline 6.500 & 6.000 & $7.40 \mathrm{E}-02$ & $2.51 \mathrm{E}-01$ & $1.63 \mathrm{E}-02$ & $0.00 \mathrm{E}+00$ & $4.31 \mathrm{E}+09$ & $2.83 \mathrm{E}+04$ & $9.09 \mathrm{E}+04$ \\
\hline 7.000 & 6.500 & $3.71 \mathrm{E}-02$ & $1.26 \mathrm{E}-01$ & $8.34 \mathrm{E}-03$ & $0.00 \mathrm{E}+00$ & $2.50 \mathrm{E}+09$ & $1.64 \mathrm{E}+04$ & $5.27 \mathrm{E}+04$ \\
\hline 7.500 & 7.000 & $1.86 \mathrm{E}-02$ & $6.34 \mathrm{E}-02$ & $4.27 \mathrm{E}-03$ & $0.00 \mathrm{E}+00$ & $1.45 \mathrm{E}+09$ & $9.52 \mathrm{E}+03$ & $3.06 \mathrm{E}+04$ \\
\hline 8.000 & 7.500 & $9.34 \mathrm{E}-03$ & $3.19 \mathrm{E}-02$ & $2.19 \mathrm{E}-03$ & $0.00 \mathrm{E}+00$ & $8.39 \mathrm{E}+08$ & $5.52 \mathrm{E}+03$ & $1.77 \mathrm{E}+04$ \\
\hline 9.000 & 8.000 & $6.96 \mathrm{E}-03$ & $2.39 \mathrm{E}-02$ & $1.70 \mathrm{E}-03$ & $0.00 \mathrm{E}+00$ & $7.63 \mathrm{E}+08$ & $5.02 \mathrm{E}+03$ & $1.61 \mathrm{E}+04$ \\
\hline \multirow[t]{2}{*}{10.000} & 9.000 & $1.75 \mathrm{E}-03$ & $6.07 \mathrm{E}-03$ & $4.56 \mathrm{E}-04$ & $0.00 \mathrm{E}+00$ & $2.57 \mathrm{E}+08$ & $1.69 \mathrm{E}+03$ & $5.42 \mathrm{E}+03$ \\
\hline & Total & $3.38 \mathrm{E}+10$ & $1.03 \mathrm{E}+11$ & $7.51 \mathrm{E}+09$ & $8.93 \mathrm{E}+13$ & $1.01 \mathrm{E}+13$ & $3.66 \mathrm{E}+11$ & $1.89 \mathrm{E}+08$ \\
\hline
\end{tabular}


PCP-2011-0001

Revision 0

\begin{tabular}{|c|c|c|c|c|c|c|c|c|}
\hline \multicolumn{2}{|c|}{ Energy (MeV) } & \multicolumn{7}{|c|}{ Photon Source (p/s/g) } \\
\hline Upper & Lower & Co-60 & Cs-137 & Eu-152 & Fe-59 & Gd-153 & Hf-181 & Ho-166m \\
\hline 0.020 & 0.010 & $7.62 \mathrm{E}+11$ & $1.12 \mathrm{E}+11$ & $3.27 \mathrm{E}+10$ & $4.19 \mathrm{E}+13$ & $2.23 \mathrm{E}+10$ & $1.47 \mathrm{E}+13$ & $1.02 \mathrm{E}+08$ \\
\hline 0.030 & 0.020 & $3.66 \mathrm{E}+11$ & $5.71 \mathrm{E}+10$ & $1.74 \mathrm{E}+10$ & $2.06 \mathrm{E}+13$ & $0.00 \mathrm{E}+00$ & $7.26 \mathrm{E}+12$ & $2.67 \mathrm{E}+07$ \\
\hline 0.040 & 0.030 & $1.97 \mathrm{E}+11$ & $2.37 \mathrm{E}+11$ & $2.98 \mathrm{E}+12$ & $1.14 \mathrm{E}+13$ & $0.00 \mathrm{E}+00$ & $4.01 \mathrm{E}+12$ & $7.36 \mathrm{E}+06$ \\
\hline 0.050 & 0.040 & $1.45 \mathrm{E}+11$ & $2.56 \mathrm{E}+10$ & $2.14 \mathrm{E}+12$ & $8.65 \mathrm{E}+12$ & $1.52 \mathrm{E}+14$ & $3.04 \mathrm{E}+12$ & $2.25 \mathrm{E}+10$ \\
\hline 0.060 & 0.050 & $7.49 \mathrm{E}+10$ & $1.40 \mathrm{E}+10$ & $5.97 \mathrm{E}+09$ & $4.59 \mathrm{E}+12$ & $3.89 \mathrm{E}+10$ & $1.64 \mathrm{E}+14$ & $5.48 \mathrm{E}+09$ \\
\hline 0.070 & 0.060 & $6.42 \mathrm{E}+10$ & $1.26 \mathrm{E}+10$ & $4.43 \mathrm{E}+09$ & $4.04 \mathrm{E}+12$ & $3.57 \mathrm{E}+12$ & $4.34 \mathrm{E}+13$ & $9.17 \mathrm{E}+04$ \\
\hline 0.080 & 0.070 & $4.46 \mathrm{E}+10$ & $9.42 \mathrm{E}+09$ & $3.45 \mathrm{E}+09$ & $2.91 \mathrm{E}+12$ & $1.03 \mathrm{E}+11$ & $1.01 \mathrm{E}+12$ & $4.75 \mathrm{E}+06$ \\
\hline 0.090 & 0.080 & $3.16 \mathrm{E}+10$ & $7.22 \mathrm{E}+09$ & $2.76 \mathrm{E}+09$ & $2.15 \mathrm{E}+12$ & $3.67 \mathrm{E}+11$ & $7.45 \mathrm{E}+11$ & $8.09 \mathrm{E}+09$ \\
\hline 0.100 & 0.090 & $2.78 \mathrm{E}+10$ & $6.96 \mathrm{E}+09$ & $2.78 \mathrm{E}+09$ & $1.99 \mathrm{E}+12$ & $4.05 \mathrm{E}+13$ & $6.85 \mathrm{E}+11$ & $1.09 \mathrm{E}+08$ \\
\hline 0.110 & 0.100 & $1.37 \mathrm{E}+10$ & $3.58 \mathrm{E}+09$ & $1.46 \mathrm{E}+09$ & $1.00 \mathrm{E}+12$ & $2.75 \mathrm{E}+13$ & $3.45 \mathrm{E}+11$ & $0.00 \mathrm{E}+00$ \\
\hline 0.120 & 0.110 & $1.25 \mathrm{E}+10$ & $3.74 \mathrm{E}+09$ & $1.63 \mathrm{E}+09$ & $9.89 \mathrm{E}+11$ & $0.00 \mathrm{E}+00$ & $3.36 \mathrm{E}+11$ & $1.17 \mathrm{E}+08$ \\
\hline 0.130 & 0.120 & $1.15 \mathrm{E}+10$ & $3.44 \mathrm{E}+09$ & $1.81 \mathrm{E}+12$ & $9.09 \mathrm{E}+11$ & $0.00 \mathrm{E}+00$ & $3.09 \mathrm{E}+11$ & $1.79 \mathrm{E}+08$ \\
\hline 0.140 & 0.130 & $6.63 \mathrm{E}+09$ & $2.46 \mathrm{E}+09$ & $1.18 \mathrm{E}+09$ & $5.99 \mathrm{E}+11$ & $0.00 \mathrm{E}+00$ & $3.12 \mathrm{E}+14$ & $6.56 \mathrm{E}+07$ \\
\hline 0.150 & 0.140 & $6.18 \mathrm{E}+09$ & $2.29 \mathrm{E}+09$ & $3.74 \mathrm{E}+09$ & $1.90 \mathrm{E}+13$ & $0.00 \mathrm{E}+00$ & $1.85 \mathrm{E}+11$ & $2.73 \mathrm{E}+07$ \\
\hline 0.160 & 0.150 & $3.51 \mathrm{E}+09$ & $1.67 \mathrm{E}+09$ & $8.78 \mathrm{E}+08$ & $3.72 \mathrm{E}+11$ & $0.00 \mathrm{E}+00$ & $1.19 \mathrm{E}+11$ & $3.15 \mathrm{E}+07$ \\
\hline 0.170 & 0.160 & $3.30 \mathrm{E}+09$ & $1.57 \mathrm{E}+09$ & $8.25 \mathrm{E}+08$ & $3.49 \mathrm{E}+11$ & $0.00 \mathrm{E}+00$ & $1.12 \mathrm{E}+11$ & $9.62 \mathrm{E}+07$ \\
\hline 0.180 & 0.170 & $1.82 \mathrm{E}+09$ & $1.15 \mathrm{E}+09$ & $6.99 \mathrm{E}+08$ & $2.34 \mathrm{E}+11$ & $3.90 \mathrm{E}+10$ & $7.14 \mathrm{E}+10$ & $9.44 \mathrm{E}+06$ \\
\hline 0.190 & 0.180 & $1.72 \mathrm{E}+09$ & $1.09 \mathrm{E}+09$ & $6.36 \mathrm{E}+08$ & $4.66 \mathrm{E}+11$ & $0.00 \mathrm{E}+00$ & $6.75 \mathrm{E}+10$ & $4.83 \mathrm{E}+10$ \\
\hline 0.200 & 0.190 & $1.83 \mathrm{E}+09$ & $1.62 \mathrm{E}+09$ & $1.49 \mathrm{E}+09$ & $5.62 \mathrm{E}+13$ & $0.00 \mathrm{E}+00$ & $8.51 \mathrm{E}+10$ & $1.47 \mathrm{E}+08$ \\
\hline 0.210 & 0.200 & $1.74 \mathrm{E}+09$ & $1.54 \mathrm{E}+09$ & $1.82 \mathrm{E}+09$ & $2.84 \mathrm{E}+11$ & $0.00 \mathrm{E}+00$ & $8.09 \mathrm{E}+10$ & $0.00 \mathrm{E}+00$ \\
\hline 0.220 & 0.210 & $0.00 \mathrm{E}+00$ & $0.00 \mathrm{E}+00$ & $1.27 \mathrm{E}+09$ & $0.00 \mathrm{E}+00$ & $0.00 \mathrm{E}+00$ & $0.00 \mathrm{E}+00$ & $2.04 \mathrm{E}+09$ \\
\hline 0.230 & 0.220 & $0.00 \mathrm{E}+00$ & $0.00 \mathrm{E}+00$ & $0.00 \mathrm{E}+00$ & $0.00 \mathrm{E}+00$ & $0.00 \mathrm{E}+00$ & $0.00 \mathrm{E}+00$ & $0.00 \mathrm{E}+00$ \\
\hline 0.240 & 0.230 & $0.00 \mathrm{E}+00$ & $0.00 \mathrm{E}+00$ & $8.96 \mathrm{E}+08$ & $0.00 \mathrm{E}+00$ & $0.00 \mathrm{E}+00$ & $0.00 \mathrm{E}+00$ & $1.41 \mathrm{E}+08$ \\
\hline 0.250 & 0.240 & $0.00 \mathrm{E}+00$ & $0.00 \mathrm{E}+00$ & $4.90 \mathrm{E}+11$ & $0.00 \mathrm{E}+00$ & $0.00 \mathrm{E}+00$ & $0.00 \mathrm{E}+00$ & $0.00 \mathrm{E}+00$ \\
\hline 0.260 & 0.250 & $6.29 \mathrm{E}+08$ & $1.23 \mathrm{E}+09$ & $5.03 \mathrm{E}+09$ & $1.85 \mathrm{E}+11$ & $0.00 \mathrm{E}+00$ & $4.25 \mathrm{E}+10$ & $3.70 \mathrm{E}+08$ \\
\hline 0.270 & 0.260 & $6.05 \mathrm{E}+08$ & $1.19 \mathrm{E}+09$ & $1.23 \mathrm{E}+09$ & $1.78 \mathrm{E}+11$ & $0.00 \mathrm{E}+00$ & $4.09 \mathrm{E}+10$ & $3.56 \mathrm{E}+08$ \\
\hline 0.280 & 0.270 & $0.00 \mathrm{E}+00$ & $0.00 \mathrm{E}+00$ & $7.15 \mathrm{E}+09$ & $0.00 \mathrm{E}+00$ & $0.00 \mathrm{E}+00$ & $0.00 \mathrm{E}+00$ & $0.00 \mathrm{E}+00$ \\
\hline 0.290 & 0.280 & $0.00 \mathrm{E}+00$ & $0.00 \mathrm{E}+00$ & $7.24 \mathrm{E}+08$ & $0.00 \mathrm{E}+00$ & $0.00 \mathrm{E}+00$ & $0.00 \mathrm{E}+00$ & $1.96 \mathrm{E}+10$ \\
\hline 0.300 & 0.290 & $0.00 \mathrm{E}+00$ & $0.00 \mathrm{E}+00$ & $2.88 \mathrm{E}+10$ & $0.00 \mathrm{E}+00$ & $0.00 \mathrm{E}+00$ & $0.00 \mathrm{E}+00$ & $1.27 \mathrm{E}+09$ \\
\hline 0.325 & 0.300 & $2.58 \mathrm{E}+08$ & $9.67 \mathrm{E}+08$ & $7.24 \mathrm{E}+09$ & $9.82 \mathrm{E}+10$ & $0.00 \mathrm{E}+00$ & $1.18 \mathrm{E}+10$ & $1.20 \mathrm{E}+09$ \\
\hline 0.350 & 0.325 & $3.27 \mathrm{E}+09$ & $0.00 \mathrm{E}+00$ & $1.78 \mathrm{E}+12$ & $4.93 \mathrm{E}+12$ & $0.00 \mathrm{E}+00$ & $9.76 \mathrm{E}+13$ & $1.07 \mathrm{E}+08$ \\
\hline 0.375 & 0.350 & $0.00 \mathrm{E}+00$ & $0.00 \mathrm{E}+00$ & $5.78 \mathrm{E}+10$ & $0.00 \mathrm{E}+00$ & $0.00 \mathrm{E}+00$ & $0.00 \mathrm{E}+00$ & $1.66 \mathrm{E}+09$ \\
\hline 0.400 & 0.375 & $1.55 \mathrm{E}+08$ & $3.97 \mathrm{E}+08$ & $1.27 \mathrm{E}+09$ & $3.51 \mathrm{E}+11$ & $0.00 \mathrm{E}+00$ & $4.97 \mathrm{E}+08$ & $0.00 \mathrm{E}+00$ \\
\hline 0.425 & 0.400 & $0.00 \mathrm{E}+00$ & $0.00 \mathrm{E}+00$ & $1.53 \mathrm{E}+11$ & $0.00 \mathrm{E}+00$ & $0.00 \mathrm{E}+00$ & $0.00 \mathrm{E}+00$ & $7.55 \mathrm{E}+09$ \\
\hline 0.450 & 0.425 & $1.04 \mathrm{E}+08$ & $2.01 \mathrm{E}+08$ & $2.08 \mathrm{E}+11$ & $9.96 \mathrm{E}+09$ & $0.00 \mathrm{E}+00$ & $0.00 \mathrm{E}+00$ & $0.00 \mathrm{E}+00$ \\
\hline 0.475 & 0.450 & $0.00 \mathrm{E}+00$ & $0.00 \mathrm{E}+00$ & $0.00 \mathrm{E}+00$ & $0.00 \mathrm{E}+00$ & $0.00 \mathrm{E}+00$ & $0.00 \mathrm{E}+00$ & $2.75 \mathrm{E}+09$ \\
\hline 0.500 & 0.475 & $4.73 \mathrm{E}+07$ & $7.68 \mathrm{E}+07$ & $3.18 \mathrm{E}+10$ & $4.31 \mathrm{E}+09$ & $0.00 \mathrm{E}+00$ & $5.06 \mathrm{E}+14$ & $8.89 \mathrm{E}+07$ \\
\hline 0.525 & 0.500 & $4.50 \mathrm{E}+07$ & $7.30 \mathrm{E}+07$ & $1.79 \mathrm{E}+10$ & $4.10 \mathrm{E}+09$ & $0.00 \mathrm{E}+00$ & $0.00 \mathrm{E}+00$ & $1.08 \mathrm{E}+08$ \\
\hline 0.550 & 0.525 & $0.00 \mathrm{E}+00$ & $0.00 \mathrm{E}+00$ & $4.42 \mathrm{E}+09$ & $0.00 \mathrm{E}+00$ & $0.00 \mathrm{E}+00$ & $0.00 \mathrm{E}+00$ & $6.40 \mathrm{E}+09$ \\
\hline 0.575 & 0.550 & $0.00 \mathrm{E}+00$ & $0.00 \mathrm{E}+00$ & $4.26 \mathrm{E}+10$ & $0.00 \mathrm{E}+00$ & $0.00 \mathrm{E}+00$ & $0.00 \mathrm{E}+00$ & $3.74 \mathrm{E}+09$ \\
\hline 0.600 & 0.575 & & $4.75 \mathrm{E}+07$ & $3.01 \mathrm{E}+10$ & $3.19 \mathrm{E}+09$ & $0.00 \mathrm{E}+00$ & $0.00 \mathrm{E}+00$ & $3.87 \mathrm{E}+08$ \\
\hline 0.625 & 0.600 & $3.39 \mathrm{E}+07$ & $4.56 \mathrm{E}+07$ & $7.00 \mathrm{E}+08$ & $3.06 \mathrm{E}+09$ & $0.00 \mathrm{E}+00$ & $1.64 \mathrm{E}+12$ & $9.34 \mathrm{E}+08$ \\
\hline 0.650 & 0.625 & $0.00 \mathrm{E}+00$ & $0.00 \mathrm{E}+00$ & $3.85 \mathrm{E}+08$ & $0.00 \mathrm{E}+00$ & $0.00 \mathrm{E}+00$ & $0.00 \mathrm{E}+00$ & $1.57 \mathrm{E}+08$ \\
\hline 0.675 & 0.650 & $0.00 \mathrm{E}+00$ & $2.68 \mathrm{E}+12$ & $1.82 \mathrm{E}+10$ & $0.00 \mathrm{E}+00$ & $0.00 \mathrm{E}+00$ & $0.00 \mathrm{E}+00$ & $3.70 \mathrm{E}+09$ \\
\hline 0.700 & 0.675 & $1.89 \mathrm{E}+07$ & $1.90 \mathrm{E}+07$ & $9.33 \mathrm{E}+10$ & $1.69 \mathrm{E}+09$ & $0.00 \mathrm{E}+00$ & $0.00 \mathrm{E}+00$ & $9.11 \mathrm{E}+08$ \\
\hline 0.725 & 0.700 & $1.82 \mathrm{E}+07$ & $1.83 \mathrm{E}+07$ & $2.81 \mathrm{E}+10$ & $1.63 \mathrm{E}+09$ & $0.00 \mathrm{E}+00$ & $0.00 \mathrm{E}+00$ & $3.68 \mathrm{E}+10$ \\
\hline 0.750 & 0.725 & $0.00 \mathrm{E}+00$ & $0.00 \mathrm{E}+00$ & $1.11 \mathrm{E}+09$ & $0.00 \mathrm{E}+00$ & $0.00 \mathrm{E}+00$ & $0.00 \mathrm{E}+00$ & $2.47 \mathrm{E}+08$ \\
\hline
\end{tabular}


PCP-2011-0001

Revision 0

\begin{tabular}{|c|c|c|c|c|c|c|c|c|}
\hline \multicolumn{9}{|c|}{ Table 3-14. Photon Source from ORIGEN-S (Decay Time = 0 days) } \\
\hline \multicolumn{2}{|c|}{ Energy $(\mathrm{MeV})$} & \multicolumn{7}{|c|}{ Photon Source (p/s/g) } \\
\hline Upper & Lower & Co-60 & Cs-137 & Eu-152 & Fe-59 & Gd-153 & Hf-181 & Ho-166m \\
\hline 0.775 & 0.750 & $0.00 \mathrm{E}+00$ & $0.00 \mathrm{E}+00$ & $1.77 \mathrm{E}+10$ & $0.00 \mathrm{E}+00$ & $0.00 \mathrm{E}+00$ & $0.00 \mathrm{E}+00$ & $8.08 \mathrm{E}+09$ \\
\hline 0.800 & 0.775 & $1.00 \mathrm{E}+07$ & $6.86 \mathrm{E}+06$ & $8.39 \mathrm{E}+11$ & $8.86 \mathrm{E}+08$ & $0.00 \mathrm{E}+00$ & $0.00 \mathrm{E}+00$ & $2.03 \mathrm{E}+09$ \\
\hline 0.825 & 0.800 & $9.71 \mathrm{E}+06$ & $6.65 \mathrm{E}+06$ & $2.15 \mathrm{E}+10$ & $8.59 \mathrm{E}+08$ & $0.00 \mathrm{E}+00$ & $0.00 \mathrm{E}+00$ & $3.86 \mathrm{E}+10$ \\
\hline 0.850 & 0.825 & $3.14 \mathrm{E}+09$ & $0.00 \mathrm{E}+00$ & $1.18 \mathrm{E}+10$ & $0.00 \mathrm{E}+00$ & $0.00 \mathrm{E}+00$ & $0.00 \mathrm{E}+00$ & $6.49 \mathrm{E}+09$ \\
\hline 0.875 & 0.850 & $0.00 \mathrm{E}+00$ & $0.00 \mathrm{E}+00$ & $2.77 \mathrm{E}+11$ & $0.00 \mathrm{E}+00$ & $0.00 \mathrm{E}+00$ & $0.00 \mathrm{E}+00$ & $2.46 \mathrm{E}+08$ \\
\hline 0.900 & 0.875 & $5.16 \mathrm{E}+06$ & $2.06 \mathrm{E}+06$ & $1.35 \mathrm{E}+07$ & $4.53 \mathrm{E}+08$ & $0.00 \mathrm{E}+00$ & $0.00 \mathrm{E}+00$ & $2.39 \mathrm{E}+08$ \\
\hline 0.925 & 0.900 & $5.02 \mathrm{E}+06$ & $2.00 \mathrm{E}+06$ & $3.56 \mathrm{E}+10$ & $4.40 \mathrm{E}+08$ & $0.00 \mathrm{E}+00$ & $0.00 \mathrm{E}+00$ & $0.00 \mathrm{E}+00$ \\
\hline 0.950 & 0.925 & $0.00 \mathrm{E}+00$ & $0.00 \mathrm{E}+00$ & $2.20 \mathrm{E}+10$ & $0.00 \mathrm{E}+00$ & $0.00 \mathrm{E}+00$ & $0.00 \mathrm{E}+00$ & $0.00 \mathrm{E}+00$ \\
\hline 0.975 & 0.950 & $0.00 \mathrm{E}+00$ & $0.00 \mathrm{E}+00$ & $9.59 \mathrm{E}+11$ & $0.00 \mathrm{E}+00$ & $0.00 \mathrm{E}+00$ & $0.00 \mathrm{E}+00$ & $1.82 \mathrm{E}+09$ \\
\hline 1.000 & 0.975 & $2.50 \mathrm{E}+06$ & $4.30 \mathrm{E}+05$ & $2.36 \mathrm{E}+10$ & $2.20 \mathrm{E}+08$ & $0.00 \mathrm{E}+00$ & $0.00 \mathrm{E}+00$ & $0.00 \mathrm{E}+00$ \\
\hline 1.250 & 1.000 & $4.36 \mathrm{E}+13$ & $4.54 \mathrm{E}+05$ & $1.76 \mathrm{E}+12$ & $1.02 \mathrm{E}+15$ & $0.00 \mathrm{E}+00$ & $0.00 \mathrm{E}+00$ & $9.52 \mathrm{E}+08$ \\
\hline 1.500 & 1.250 & $4.05 \mathrm{E}+13$ & $0.00 \mathrm{E}+00$ & $1.55 \mathrm{E}+12$ & $7.48 \mathrm{E}+14$ & $0.00 \mathrm{E}+00$ & $0.00 \mathrm{E}+00$ & $8.11 \mathrm{E}+08$ \\
\hline 1.750 & 1.500 & $5.61 \mathrm{E}+01$ & $0.00 \mathrm{E}+00$ & $1.75 \mathrm{E}+10$ & $2.07 \mathrm{E}+05$ & $0.00 \mathrm{E}+00$ & $0.00 \mathrm{E}+00$ & $0.00 \mathrm{E}+00$ \\
\hline 2.000 & 1.750 & $0.00 \mathrm{E}+00$ & $0.00 \mathrm{E}+00$ & $5.40 \mathrm{E}+08$ & $0.00 \mathrm{E}+00$ & $0.00 \mathrm{E}+00$ & $0.00 \mathrm{E}+00$ & $0.00 \mathrm{E}+00$ \\
\hline 2.250 & 2.000 & $4.72 \mathrm{E}+08$ & $0.00 \mathrm{E}+00$ & $8.26 \mathrm{E}-17$ & $0.00 \mathrm{E}+00$ & $0.00 \mathrm{E}+00$ & $0.00 \mathrm{E}+00$ & $0.00 \mathrm{E}+00$ \\
\hline 2.500 & 2.250 & $4.41 \mathrm{E}+05$ & $0.00 \mathrm{E}+00$ & 7.39E-17 & $0.00 \mathrm{E}+00$ & $0.00 \mathrm{E}+00$ & $0.00 \mathrm{E}+00$ & $0.00 \mathrm{E}+00$ \\
\hline 2.750 & 2.500 & $3.99 \mathrm{E}+05$ & $0.00 \mathrm{E}+00$ & $4.10 \mathrm{E}-17$ & $0.00 \mathrm{E}+00$ & $0.00 \mathrm{E}+00$ & $0.00 \mathrm{E}+00$ & $0.00 \mathrm{E}+00$ \\
\hline 3.000 & 2.750 & $0.00 \mathrm{E}+00$ & $0.00 \mathrm{E}+00$ & $3.74 \mathrm{E}-17$ & $0.00 \mathrm{E}+00$ & $0.00 \mathrm{E}+00$ & $0.00 \mathrm{E}+00$ & $0.00 \mathrm{E}+00$ \\
\hline 3.500 & 3.000 & $0.00 \mathrm{E}+00$ & $0.00 \mathrm{E}+00$ & $3.92 \mathrm{E}-17$ & $0.00 \mathrm{E}+00$ & $0.00 \mathrm{E}+00$ & $0.00 \mathrm{E}+00$ & $0.00 \mathrm{E}+00$ \\
\hline 4.000 & 3.500 & $0.00 \mathrm{E}+00$ & $0.00 \mathrm{E}+00$ & $1.97 \mathrm{E}-17$ & $0.00 \mathrm{E}+00$ & $0.00 \mathrm{E}+00$ & $0.00 \mathrm{E}+00$ & $0.00 \mathrm{E}+00$ \\
\hline 4.500 & 4.000 & $0.00 \mathrm{E}+00$ & $0.00 \mathrm{E}+00$ & $9.87 \mathrm{E}-18$ & $0.00 \mathrm{E}+00$ & $0.00 \mathrm{E}+00$ & $0.00 \mathrm{E}+00$ & $0.00 \mathrm{E}+00$ \\
\hline 5.000 & 4.500 & $0.00 \mathrm{E}+00$ & $0.00 \mathrm{E}+00$ & $4.95 \mathrm{E}-18$ & $0.00 \mathrm{E}+00$ & $0.00 \mathrm{E}+00$ & $0.00 \mathrm{E}+00$ & $0 \mathrm{E}+00$ \\
\hline 5.500 & 5.000 & $0.00 \mathrm{E}+00$ & $0.00 \mathrm{E}+00$ & $2.48 \mathrm{E}-18$ & $0.00 \mathrm{E}+00$ & $0.00 \mathrm{E}+00$ & $0.00 \mathrm{E}+00$ & $0.00 \mathrm{E}+00$ \\
\hline 6.000 & 5.500 & $0.00 \mathrm{E}+00$ & $0.00 \mathrm{E}+00$ & $1.25 \mathrm{E}-18$ & $0.00 \mathrm{E}+00$ & $0.00 \mathrm{E}+00$ & $0.00 \mathrm{E}+00$ & $0.00 \mathrm{E}+00$ \\
\hline 6.500 & 6.000 & $0.00 \mathrm{E}+00$ & $0.00 \mathrm{E}+00$ & $6.25 \mathrm{E}-19$ & $0.00 \mathrm{E}+00$ & $0.00 \mathrm{E}+00$ & $0.00 \mathrm{E}+00$ & $0.00 \mathrm{E}+00$ \\
\hline 7.000 & 6.500 & $0.00 \mathrm{E}+00$ & $0.00 \mathrm{E}+00$ & $3.13 \mathrm{E}-19$ & $0.00 \mathrm{E}+00$ & $0.00 \mathrm{E}+00$ & $0.00 \mathrm{E}+00$ & $0.00 \mathrm{E}+00$ \\
\hline 7.500 & 7.000 & $0.00 \mathrm{E}+00$ & $0.00 \mathrm{E}+00$ & $1.57 \mathrm{E}-19$ & $0.00 \mathrm{E}+00$ & $0.00 \mathrm{E}+00$ & $0.00 \mathrm{E}+00$ & $0.00 \mathrm{E}+00$ \\
\hline 8.000 & 7.500 & $0.00 \mathrm{E}+00$ & $0.00 \mathrm{E}+00$ & $7.89 \mathrm{E}-20$ & $0.00 \mathrm{E}+00$ & $0.00 \mathrm{E}+00$ & $0.00 \mathrm{E}+00$ & $0.00 \mathrm{E}+00$ \\
\hline 9.000 & 8.000 & $0.00 \mathrm{E}+00$ & $0.00 \mathrm{E}+00$ & $5.88 \mathrm{E}-20$ & $0.00 \mathrm{E}+00$ & $0.00 \mathrm{E}+00$ & $0.00 \mathrm{E}+00$ & $0.00 \mathrm{E}+00$ \\
\hline \multirow[t]{2}{*}{10.000} & 9.000 & $0.00 \mathrm{E}+00$ & $0.00 \mathrm{E}+00$ & $1.48 \mathrm{E}-20$ & $0.00 \mathrm{E}+00$ & $0.00 \mathrm{E}+00$ & $0.00 \mathrm{E}+00$ & $0.00 \mathrm{E}+00$ \\
\hline & Total & $8.59 \mathrm{E}+13$ & $3.19 \mathrm{E}+12$ & $1.56 \mathrm{E}+13$ & $1.95 \mathrm{E}+15$ & $2.24 \mathrm{E}+14$ & $1.16 \mathrm{E}+15$ & $2.35 \mathrm{E}+11$ \\
\hline
\end{tabular}


PCP-2011-0001

Revision 0

\begin{tabular}{|c|c|c|c|c|c|c|c|c|}
\hline \multicolumn{9}{|c|}{ Table 3-15. Photon Source from ORIGEN-S (Decay Time $=0$ days) } \\
\hline \multicolumn{2}{|c|}{ Energy (MeV) } & \multicolumn{7}{|c|}{ Photon Source $(\mathrm{p} / \mathrm{s} / \mathrm{g})$} \\
\hline Upper & Lower & Ir-192 & Mn-54 & Np-237 & Pb-210 & Pm-147 & Po-210 & Pu-238 \\
\hline 0.020 & 0.010 & $1.14 \mathrm{E}+13$ & $0.00 \mathrm{E}+00$ & $1.66 \mathrm{E}+07$ & $5.97 \mathrm{E}+11$ & $3.72 \mathrm{E}+11$ & $5.05 \mathrm{E}+06$ & $7.31 \mathrm{E}+10$ \\
\hline 0.030 & 0.020 & $5.83 \mathrm{E}+12$ & $0.00 \mathrm{E}+00$ & $4.69 \mathrm{E}+06$ & $3.38 \mathrm{E}+08$ & $1.67 \mathrm{E}+11$ & $0.00 \mathrm{E}+00$ & $3.53 \mathrm{E}+09$ \\
\hline 0.040 & 0.030 & $3.33 \mathrm{E}+12$ & $0.00 \mathrm{E}+00$ & $1.98 \mathrm{E}+01$ & $1.39 \mathrm{E}+08$ & $8.45 \mathrm{E}+10$ & $0.00 \mathrm{E}+00$ & $4.80 \mathrm{E}-24$ \\
\hline 0.050 & 0.040 & $2.63 \mathrm{E}+12$ & $0.00 \mathrm{E}+00$ & $2.86 \mathrm{E}+04$ & $1.18 \mathrm{E}+11$ & $5.71 \mathrm{E}+10$ & $0.00 \mathrm{E}+00$ & $2.42 \mathrm{E}+08$ \\
\hline 0.060 & 0.050 & $1.44 \mathrm{E}+12$ & $0.00 \mathrm{E}+00$ & $1.03 E+05$ & $4.88 \mathrm{E}+07$ & $2.73 \mathrm{E}+10$ & $0.00 \mathrm{E}+00$ & \\
\hline 0.070 & 0.060 & $1.21 \mathrm{E}+13$ & $0.00 \mathrm{E}+00$ & $4.59 \mathrm{E}+03$ & $4.52 \mathrm{E}+07$ & $2.18 \mathrm{E}+10$ & $0.00 \mathrm{E}+00$ & \\
\hline 0.080 & 0.070 & $3.89 \mathrm{E}+12$ & $0.00 \mathrm{E}+00$ & $5.89 \mathrm{E}+03$ & $3.53 \mathrm{E}+07$ & $1.37 \mathrm{E}+10$ & $1.07 \mathrm{E}+07$ & $5.12 \mathrm{E}-23$ \\
\hline 0.090 & 0.080 & $7.53 \mathrm{E}+11$ & $0.00 \mathrm{E}+00$ & $3.30 \mathrm{E}+06$ & $2.83 \mathrm{E}+07$ & $8.72 \mathrm{E}+09$ & $3.02 \mathrm{E}+06$ & $6.68 \mathrm{E}-04$ \\
\hline 0.100 & 0.090 & $7.29 \mathrm{E}+11$ & $0.00 \mathrm{E}+00$ & $1.43 \mathrm{E}+06$ & $2.87 \mathrm{E}+07$ & $6.70 \mathrm{E}+09$ & $0.00 \mathrm{E}+00$ & $2.55 \mathrm{E}+07$ \\
\hline 0.110 & 0.100 & $3.75 \mathrm{E}+11$ & $0.00 \mathrm{E}+00$ & $3.09 \mathrm{E}+05$ & $1.51 \mathrm{E}+07$ & $3.12 \mathrm{E}+09$ & $0.00 \mathrm{E}+00$ & $2.21 \mathrm{E}+07$ \\
\hline 0.120 & 0.110 & $3.95 \mathrm{E}+11$ & $0.00 \mathrm{E}+00$ & $1.36 \mathrm{E}+05$ & $1.69 \mathrm{E}+07$ & $2.26 \mathrm{E}+09$ & $0.00 \mathrm{E}+00$ & $2.81 \mathrm{E}+05$ \\
\hline 0.130 & 0.120 & $3.63 \mathrm{E}+11$ & $0.00 \mathrm{E}+00$ & $1.03 \mathrm{E}+00$ & $1.56 \mathrm{E}+07$ & $3.03 \mathrm{E}+09$ & $0.00 \mathrm{E}+00$ & \begin{tabular}{|l|}
$1.62 \mathrm{E}-02$ \\
\end{tabular} \\
\hline 0.140 & 0.130 & $2.62 \mathrm{E}+11$ & $0.00 \mathrm{E}+00$ & $4.09 \mathrm{E}+04$ & $1.23 \mathrm{E}+07$ & $8.24 \mathrm{E}+08$ & $0.00 \mathrm{E}+00$ & $6.52 \mathrm{E}-25$ \\
\hline 0.150 & 0.140 & $2.44 \mathrm{E}+11$ & $0.00 \mathrm{E}+00$ & $1.12 \mathrm{E}+05$ & $1.14 \mathrm{E}+07$ & $7.67 \mathrm{E}+08$ & $0.00 \mathrm{E}+00$ & $6.13 \mathrm{E}+01$ \\
\hline 0.160 & 0.150 & $1.79 \mathrm{E}+11$ & $0.00 \mathrm{E}+00$ & $8.50 \mathrm{E}+04$ & $9.20 \mathrm{E}+06$ & $2.59 \mathrm{E}+08$ & $0.00 \mathrm{E}+00$ & $5.85 \mathrm{E}+06$ \\
\hline 0.170 & 0.160 & $1.68 \mathrm{E}+11$ & $0.00 \mathrm{E}+00$ & $2.72 \mathrm{E}+04$ & $8.64 \mathrm{E}+06$ & $2.43 \mathrm{E}+08$ & $0.00 \mathrm{E}+00$ & $3.29 \mathrm{E}-25$ \\
\hline 0.180 & 0.170 & $1.24 \mathrm{E}+11$ & $0.00 \mathrm{E}+00$ & $9.81 \mathrm{E}+03$ & $7.05 \mathrm{E}+06$ & $6.20 \mathrm{E}+07$ & $0.00 \mathrm{E}+00$ & $3.16 \mathrm{E}+01$ \\
\hline 0.190 & 0.180 & $1.18 \mathrm{E}+11$ & $0.00 \mathrm{E}+00$ & $5.89 \mathrm{E}+03$ & $6.67 \mathrm{E}+06$ & $5.87 \mathrm{E}+07$ & $0.00 \mathrm{E}+00$ & $5.43 \mathrm{E}-13$ \\
\hline 0.200 & 0.190 & $1.75 \mathrm{E}+11$ & $0.00 \mathrm{E}+00$ & $7.31 \mathrm{E}+04$ & $1.10 \mathrm{E}+07$ & $1.50 \mathrm{E}+07$ & $0.00 \mathrm{E}+00$ & 5.34E-25 \\
\hline 0.210 & 0.200 & $1.32 \mathrm{E}+13$ & $0.00 \mathrm{E}+00$ & $1.73 \mathrm{E}+04$ & $1.05 \mathrm{E}+07$ & $1.41 \mathrm{E}+07$ & $0.00 \mathrm{E}+00$ & $2.56 \mathrm{E}+04$ \\
\hline 0.220 & 0.210 & $0.00 \mathrm{E}+00$ & $0.00 \mathrm{E}+00$ & $5.06 \mathrm{E}+04$ & $0.00 \mathrm{E}+00$ & $0.00 \mathrm{E}+00$ & $0.00 \mathrm{E}+00$ & $0.00 \mathrm{E}+00$ \\
\hline 0.230 & 0.220 & $0.00 \mathrm{E}+00$ & $0.00 \mathrm{E}+00$ & $2.38 \mathrm{E}+03$ & $0.00 \mathrm{E}+00$ & $0.00 \mathrm{E}+00$ & $0.00 \mathrm{E}+00$ & $0.00 \mathrm{E}+00$ \\
\hline 0.240 & 0.230 & $0.00 \mathrm{E}+00$ & $0.00 \mathrm{E}+00$ & $1.74 \mathrm{E}+04$ & $0.00 \mathrm{E}+00$ & $0.00 \mathrm{E}+00$ & $0.00 \mathrm{E}+00$ & $6.36 \mathrm{E}+01$ \\
\hline 0.250 & 0.240 & $0.00 \mathrm{E}+00$ & $0.00 \mathrm{E}+00$ & $1.33 \mathrm{E}+03$ & $6.15 \mathrm{E}-04$ & $2.41 \mathrm{E}-11$ & $6.52 \mathrm{E}+05$ & $5.31 \mathrm{E}+03$ \\
\hline 0.260 & 0.250 & $1.34 \mathrm{E}+11$ & $0.00 \mathrm{E}+00$ & $1.68 \mathrm{E}+03$ & $1.05 \mathrm{E}+07$ & $2.32 \mathrm{E}-11$ & $6.27 \mathrm{E}+05$ & $5.81 \mathrm{E}+03$ \\
\hline 0.270 & 0.260 & $1.29 \mathrm{E}+11$ & $0.00 \mathrm{E}+00$ & $1.76 \mathrm{E}+03$ & $1.01 \mathrm{E}+07$ & $0.00 \mathrm{E}+00$ & $0.00 \mathrm{E}+00$ & $1.18 \mathrm{E}-21$ \\
\hline 0.280 & 0.270 & $0.00 \mathrm{E}+00$ & $0.00 \mathrm{E}+00$ & $5.52 \mathrm{E}+02$ & $0.00 \mathrm{E}+00$ & $0.00 \mathrm{E}+00$ & $0.00 \mathrm{E}+00$ & $9.48 \mathrm{E}-25$ \\
\hline 0.290 & 0.280 & $9.01 \mathrm{E}+11$ & $0.00 \mathrm{E}+00$ & $4.31 \mathrm{E}-12$ & $0.00 \mathrm{E}+00$ & $0.00 \mathrm{E}+00$ & $0.00 \mathrm{E}+00$ & $2.25 \mathrm{E}-26$ \\
\hline 0.300 & 0.290 & $0.00 \mathrm{E}+00$ & $0.00 \mathrm{E}+00$ & $2.28 \mathrm{E}+02$ & $0.00 \mathrm{E}+00$ & $0.00 \mathrm{E}+00$ & $0.00 \mathrm{E}+00$ & $4.50 \mathrm{E}+02$ \\
\hline 0.325 & 0.300 & $9.89 \mathrm{E}+10$ & $0.00 \mathrm{E}+00$ & $2.80 \mathrm{E}+03$ & $1.14 \mathrm{E}+07$ & $0.00 \mathrm{E}+00$ & $0.00 \mathrm{E}+00$ & $6.80 \mathrm{E}-25$ \\
\hline 0.350 & 0.325 & $0.00 \mathrm{E}+00$ & $0.00 \mathrm{E}+00$ & $3.04 \mathrm{E}+02$ & $0.00 \mathrm{E}+00$ & $0.00 \mathrm{E}+00$ & $0.00 \mathrm{E}+00$ & $7.70 \mathrm{E}-26$ \\
\hline 0.375 & 0.350 & $1.28 \mathrm{E}+12$ & $0.00 \mathrm{E}+00$ & $2.36 \mathrm{E}+01$ & $0.00 \mathrm{E}+00$ & $0.00 \mathrm{E}+00$ & $0.00 \mathrm{E}+00$ & $1.01 \mathrm{E}-22$ \\
\hline 0.400 & 0.375 & $1.23 \mathrm{E}+12$ & $0.00 \mathrm{E}+00$ & $1.19 \mathrm{E}+02$ & $6.19 \mathrm{E}+06$ & $0.00 \mathrm{E}+00$ & $0.00 \mathrm{E}+00$ & $3.52 \mathrm{E}-25$ \\
\hline 0.425 & 0.400 & $0.00 \mathrm{E}+00$ & $0.00 \mathrm{E}+00$ & $1.17 \mathrm{E}+02$ & $0.00 \mathrm{E}+00$ & $0.00 \mathrm{E}+00$ & $0.00 \mathrm{E}+00$ & $7.35 \mathrm{E}-23$ \\
\hline 0.450 & 0.425 & $1.11 \mathrm{E}+10$ & $0.00 \mathrm{E}+00$ & $9.50 \mathrm{E}-03$ & $3.69 \mathrm{E}+06$ & $0.00 \mathrm{E}+00$ & $0.00 \mathrm{E}+00$ & $2.39 \mathrm{E}-23$ \\
\hline 0.475 & 0.450 & $0.00 \mathrm{E}+00$ & $0.00 \mathrm{E}+00$ & $1.13 \mathrm{E}-13$ & $0.00 \mathrm{E}+00$ & $0.00 \mathrm{E}+00$ & $0.00 \mathrm{E}+00$ & $1.20 \mathrm{E}-05$ \\
\hline 0.500 & 0.475 & $1.23 \mathrm{E}+13$ & $0.00 \mathrm{E}+00$ & $8.27 \mathrm{E}-04$ & $1.47 \mathrm{E}+06$ & $0.00 \mathrm{E}+00$ & $0.00 \mathrm{E}+00$ & $2.24 \mathrm{E}-24$ \\
\hline 0.525 & 0.500 & $1.99 \mathrm{E}+09$ & $0.00 \mathrm{E}+00$ & $7.86 \mathrm{E}-04$ & $1.40 \mathrm{E}+06$ & $0.00 \mathrm{E}+00$ & $0.00 \mathrm{E}+00$ & 7.74E-06 \\
\hline 0.550 & 0.525 & $0.00 \mathrm{E}+00$ & $0.00 \mathrm{E}+00$ & $3.06 \mathrm{E}-14$ & $0.00 \mathrm{E}+00$ & $0.00 \mathrm{E}+00$ & $0.00 \mathrm{E}+00$ & $6.49 \mathrm{E}-25$ \\
\hline 0.575 & 0.550 & $0.00 \mathrm{E}+00$ & $0.00 \mathrm{E}+00$ & $1.54 \mathrm{E}-14$ & $0.00 \mathrm{E}+00$ & $0.00 \mathrm{E}+00$ & $0.00 \mathrm{E}+00$ & $2.41 \mathrm{E}-16$ \\
\hline 0.600 & 0.575 & $2.88 \mathrm{E}+08$ & $0.00 \mathrm{E}+00$ & $4.92 \mathrm{E}-07$ & $8.97 \mathrm{E}+05$ & $0.00 \mathrm{E}+00$ & $0.00 \mathrm{E}+00$ & $5.82 \mathrm{E}-06$ \\
\hline 0.625 & 0.600 & $2.76 \mathrm{E}+08$ & $0.00 \mathrm{E}+00$ & $4.72 \mathrm{E}-07$ & $8.60 \mathrm{E}+05$ & $0.00 \mathrm{E}+00$ & $0.00 \mathrm{E}+00$ & $2.05 \mathrm{E}-07$ \\
\hline 0.650 & 0.625 & $0.00 \mathrm{E}+00$ & $0.00 \mathrm{E}+00$ & $0.00 \mathrm{E}+00$ & $0.00 \mathrm{E}+00$ & $0.00 \mathrm{E}+00$ & $0.00 \mathrm{E}+00$ & $1.97 \mathrm{E}-07$ \\
\hline 0.675 & 0.650 & $0.00 \mathrm{E}+00$ & $0.00 \mathrm{E}+00$ & $1.19 \mathrm{E}-14$ & $0.00 \mathrm{E}+00$ & $0.00 \mathrm{E}+00$ & $0.00 \mathrm{E}+00$ & $3.24 \mathrm{E}-25$ \\
\hline 0.700 & 0.675 & $7.48 \mathrm{E}+04$ & $0.00 \mathrm{E}+00$ & $0.00 \mathrm{E}+00$ & $3.49 \mathrm{E}+05$ & $0.00 \mathrm{E}+00$ & $0.00 \mathrm{E}+00$ & $4.82 \mathrm{E}-07$ \\
\hline 0.725 & 0.700 & $1.78 \mathrm{E}+10$ & $0.00 \mathrm{E}+00$ & $1.11 \mathrm{E}-14$ & $3.37 \mathrm{E}+05$ & $0.00 \mathrm{E}+00$ & $0.00 \mathrm{E}+00$ & $3.27 \mathrm{E}+03$ \\
\hline 0.750 & 0.725 & $0.00 \mathrm{E}+00$ & $0.00 \mathrm{E}+00$ & $5.12 \mathrm{E}-02$ & $3.07 \mathrm{E}-04$ & $1.21 \mathrm{E}-11$ & $3.26 \mathrm{E}+05$ & $5.26 \mathrm{E}+04$ \\
\hline
\end{tabular}


PCP-2011-0001

Revision 0

\begin{tabular}{|c|c|c|c|c|c|c|c|c|}
\hline \multicolumn{9}{|c|}{ Table 3-15. Photon Source from ORIGEN-S (Decay Time = 0 days) } \\
\hline \multicolumn{2}{|c|}{ Energy (MeV) } & \multicolumn{7}{|c|}{ Photon Source $(\mathbf{p} / \mathbf{s} / \mathbf{g})$} \\
\hline Upper & Lower & Ir-192 & Mn-54 & Np-237 & Pb-210 & Pm-147 & Po-210 & Pu-238 \\
\hline 0.775 & 0.750 & $0.00 \mathrm{E}+00$ & $0.00 \mathrm{E}+00$ & $4.95 \mathrm{E}-02$ & $2.97 \mathrm{E}-04$ & $1.17 \mathrm{E}-11$ & $3.15 \mathrm{E}+05$ & $2.14 \mathrm{E}+05$ \\
\hline 0.800 & 0.775 & $0.00 \mathrm{E}+00$ & $0.00 \mathrm{E}+00$ & $0.00 \mathrm{E}+00$ & $1.22 \mathrm{E}+05$ & $0.00 \mathrm{E}+00$ & $0.00 \mathrm{E}+00$ & $3.06 \mathrm{E}+04$ \\
\hline 0.825 & 0.800 & $0.00 \mathrm{E}+00$ & $0.00 \mathrm{E}+00$ & $0.00 \mathrm{E}+00$ & $1.18 \mathrm{E}+05$ & $0.00 \mathrm{E}+00$ & $1.74 \mathrm{E}+09$ & $8.07 \mathrm{E}+03$ \\
\hline 0.850 & 0.825 & $0.00 \mathrm{E}+00$ & $2.86 \mathrm{E}+14$ & $0.00 \mathrm{E}+00$ & $0.00 \mathrm{E}+00$ & $0.00 \mathrm{E}+00$ & $0.00 \mathrm{E}+00$ & $1.68 \mathrm{E}-24$ \\
\hline 0.875 & 0.850 & $0.00 \mathrm{E}+00$ & $0.00 \mathrm{E}+00$ & $8.43 \mathrm{E}-15$ & $0.00 \mathrm{E}+00$ & $0.00 \mathrm{E}+00$ & $0.00 \mathrm{E}+00$ & $1.19 \mathrm{E}+04$ \\
\hline 0.900 & 0.875 & $0.00 \mathrm{E}+00$ & $0.00 \mathrm{E}+00$ & $0.00 \mathrm{E}+00$ & $3.49 \mathrm{E}+04$ & $0.00 \mathrm{E}+00$ & $0.00 \mathrm{E}+00$ & $8.38 \mathrm{E}+03$ \\
\hline 0.925 & 0.900 & $0.00 \mathrm{E}+00$ & $0.00 \mathrm{E}+00$ & $0.00 \mathrm{E}+00$ & $3.39 \mathrm{E}+04$ & $0.00 \mathrm{E}+00$ & $0.00 \mathrm{E}+00$ & $6.28 \mathrm{E}+02$ \\
\hline 0.950 & 0.925 & $0.00 \mathrm{E}+00$ & $0.00 \mathrm{E}+00$ & $0.00 \mathrm{E}+00$ & $0.00 \mathrm{E}+00$ & $0.00 \mathrm{E}+00$ & $0.00 \mathrm{E}+00$ & $1.03 \mathrm{E}+04$ \\
\hline 0.975 & 0.950 & $0.00 \mathrm{E}+00$ & $0.00 \mathrm{E}+00$ & $0.00 \mathrm{E}+00$ & $0.00 \mathrm{E}+00$ & $0.00 \mathrm{E}+00$ & $0.00 \mathrm{E}+00$ & $7.71 \mathrm{E}-26$ \\
\hline 1.000 & 0.975 & & $0.00 \mathrm{E}+00$ & $1.66 \mathrm{E}-14$ & $6.76 \mathrm{E}+03$ & $0.00 \mathrm{E}+00$ & $0.00 \mathrm{E}+00$ & $5.00 \mathrm{E}+03$ \\
\hline 1.250 & 1.000 & $0.00 \mathrm{E}+00$ & $0.00 \mathrm{E}+00$ & $2.80 \mathrm{E}-02$ & $6.92 \mathrm{E}+03$ & $6.60 \mathrm{E}-12$ & $1.79 \mathrm{E}+05$ & $8.27 \mathrm{E}+03$ \\
\hline 1.500 & 1.250 & $0.00 \mathrm{E}+00$ & $0.00 \mathrm{E}+00$ & $2.29 \mathrm{E}-02$ & $1.38 \mathrm{E}-04$ & $5.40 \mathrm{E}-12$ & $1.46 \mathrm{E}+05$ & $1.64 \mathrm{E}+03$ \\
\hline 1.750 & 1.500 & $0.00 \mathrm{E}+00$ & $0.00 \mathrm{E}+00$ & $1.36 \mathrm{E}-02$ & $8.19 \mathrm{E}-05$ & $3.21 \mathrm{E}-12$ & $8.69 \mathrm{E}+04$ & $9.45 \mathrm{E}+02$ \\
\hline 2.000 & 1.750 & $0.00 \mathrm{E}+00$ & $0.00 \mathrm{E}+00$ & $1.18 \mathrm{E}-02$ & 7.09E-05 & $2.78 \mathrm{E}-12$ & $7.53 \mathrm{E}+04$ & $8.19 \mathrm{E}+02$ \\
\hline 2.250 & 2.000 & $0.00 \mathrm{E}+00$ & $0.00 \mathrm{E}+00$ & $6.72 \mathrm{E}-03$ & 4.04E-05 & $1.58 \mathrm{E}-12$ & $4.28 \mathrm{E}+04$ & $5.13 \mathrm{E}+02$ \\
\hline 2.500 & 2.250 & $0.00 \mathrm{E}+00$ & $0.00 \mathrm{E}+00$ & $6.02 \mathrm{E}-03$ & $3.61 \mathrm{E}-05$ & $1.42 \mathrm{E}-12$ & $3.83 \mathrm{E}+04$ & $4.59 \mathrm{E}+02$ \\
\hline 2.750 & 2.500 & $0.00 \mathrm{E}+00$ & $0.00 \mathrm{E}+00$ & $3.34 \mathrm{E}-03$ & $2.00 \mathrm{E}-05$ & $7.86 \mathrm{E}-13$ & $2.13 \mathrm{E}+04$ & $2.82 \mathrm{E}+02$ \\
\hline 3.000 & 2.750 & $0.00 \mathrm{E}+00$ & $0.00 \mathrm{E}+00$ & $3.05 \mathrm{E}-03$ & $1.83 \mathrm{E}-05$ & $7.17 \mathrm{E}-13$ & $1.94 \mathrm{E}+04$ & $2.57 \mathrm{E}+02$ \\
\hline 3.500 & 3.000 & $0.00 \mathrm{E}+00$ & $0.00 \mathrm{E}+00$ & $3.20 \mathrm{E}-03$ & $1.92 \mathrm{E}-05$ & $7.52 \mathrm{E}-13$ & $2.04 \mathrm{E}+04$ & $3.00 \mathrm{E}+02$ \\
\hline 4.000 & 3.500 & $0.00 \mathrm{E}+00$ & $0.00 \mathrm{E}+00$ & $1.60 \mathrm{E}-03$ & $9.62 \mathrm{E}-06$ & $3.77 \mathrm{E}-13$ & $1.02 \mathrm{E}+04$ & $1.68 \mathrm{E}+02$ \\
\hline 4.500 & 4.000 & $0.00 \mathrm{E}+00$ & $0.00 \mathrm{E}+00$ & $8.04 \mathrm{E}-04$ & $4.83 \mathrm{E}-06$ & $1.89 \mathrm{E}-13$ & $5.12 \mathrm{E}+03$ & $9.43 \mathrm{E}+01$ \\
\hline 5.000 & 4.500 & $0.00 \mathrm{E}+00$ & $0.00 \mathrm{E}+00$ & $4.03 \mathrm{E}-04$ & $2.42 \mathrm{E}-06$ & $9.49 \mathrm{E}-14$ & $2.57 \mathrm{E}+03$ & $5.31 \mathrm{E}+01$ \\
\hline 5.500 & 5.000 & $0.00 \mathrm{E}+00$ & $0.00 \mathrm{E}+00$ & $2.02 \mathrm{E}-04$ & $1.21 \mathrm{E}-06$ & $4.76 \mathrm{E}-14$ & $1.29 \mathrm{E}+03$ & $3.00 \mathrm{E}+01$ \\
\hline 6.000 & 5.500 & $0.00 \mathrm{E}+00$ & $0.00 \mathrm{E}+00$ & $1.01 \mathrm{E}-04$ & 6.09E-07 & $2.39 \mathrm{E}-14$ & $6.46 \mathrm{E}+02$ & $1.70 \mathrm{E}+01$ \\
\hline 6.500 & 6.000 & $0.00 \mathrm{E}+00$ & $0.00 \mathrm{E}+00$ & 5.09E-05 & $3.05 \mathrm{E}-07$ & $1.20 \mathrm{E}-14$ & $3.24 \mathrm{E}+02$ & $9.68 \mathrm{E}+00$ \\
\hline 7.000 & 6.500 & $0.00 \mathrm{E}+00$ & $0.00 \mathrm{E}+00$ & $2.55 \mathrm{E}-05$ & $1.53 \mathrm{E}-07$ & $6.01 \mathrm{E}-15$ & $1.63 \mathrm{E}+02$ & $5.52 \mathrm{E}+00$ \\
\hline 7.500 & 7.000 & $0.00 \mathrm{E}+00$ & $0.00 \mathrm{E}+00$ & $1.28 \mathrm{E}-05$ & $7.68 \mathrm{E}-08$ & $3.01 \mathrm{E}-15$ & $8.16 \mathrm{E}+01$ & $3.15 \mathrm{E}+00$ \\
\hline 8.000 & 7.500 & $0.00 \mathrm{E}+00$ & $0.00 \mathrm{E}+00$ & $6.42 \mathrm{E}-06$ & $3.85 \mathrm{E}-08$ & $1.51 \mathrm{E}-15$ & $4.09 \mathrm{E}+01$ & $1.80 \mathrm{E}+00$ \\
\hline 9.000 & 8.000 & $0.00 \mathrm{E}+00$ & $0.00 \mathrm{E}+00$ & $4.79 \mathrm{E}-06$ & $2.87 \mathrm{E}-08$ & $1.13 \mathrm{E}-15$ & $3.05 \mathrm{E}+01$ & $1.61 \mathrm{E}+00$ \\
\hline \multirow[t]{2}{*}{10.000} & 9.000 & $0.00 \mathrm{E}+00$ & $0.00 \mathrm{E}+00$ & $1.21 \mathrm{E}-06$ & 7.24E-09 & $2.84 \mathrm{E}-16$ & $7.68 \mathrm{E}+00$ & 5.33E-01 \\
\hline & Total & $7.38 \mathrm{E}+13$ & $2.86 \mathrm{E}+14$ & $2.71 \mathrm{E}+07$ & $7.16 \mathrm{E}+11$ & $7.69 \mathrm{E}+11$ & $1.76 \mathrm{E}+09$ & $7.69 \mathrm{E}+10$ \\
\hline
\end{tabular}


PCP-2011-0001

Revision 0

\begin{tabular}{|c|c|c|c|c|c|c|c|c|}
\hline \multicolumn{9}{|c|}{ Table 3-16. Photon Source from ORIGEN-S (Decay Time $=0$ days) } \\
\hline \multicolumn{2}{|c|}{ Energy $(\mathrm{MeV})$} & \multicolumn{7}{|c|}{ Photon Source $(\mathrm{p} / \mathrm{s} / \mathrm{g})$} \\
\hline Upper & Lower & Pu-239 & Pu-240 & Pu-241 & Pu-242 & Ra-226 & Ru-106 & Sc-46 \\
\hline 0.020 & 0.010 & $1.05 \mathrm{E}+08$ & $8.38 \mathrm{E}+08$ & $1.41 \mathrm{E}+08$ & $1.24 \mathrm{E}+07$ & $3.01 \mathrm{E}+08$ & $3.77 \mathrm{E}+13$ & $2.69 \mathrm{E}+13$ \\
\hline 0.030 & 0.020 & $4.91 \mathrm{E}+06$ & $4.07 \mathrm{E}+07$ & $2.19 \mathrm{E}+04$ & $6.37 \mathrm{E}+05$ & $2.11 \mathrm{E}+05$ & $2.13 \mathrm{E}+13$ & $1.32 \mathrm{E}+13$ \\
\hline 0.040 & 0.030 & $2.68 \mathrm{E}+05$ & $8.07 \mathrm{E}-28$ & $5.94 \mathrm{E}+02$ & $5.49 \mathrm{E}-12$ & $1.24 \mathrm{E}+05$ & $1.31 \mathrm{E}+13$ & $7.22 \mathrm{E}+12$ \\
\hline 0.050 & 0.040 & $2.70 \mathrm{E}+04$ & $3.77 \mathrm{E}+06$ & $1.90 \mathrm{E}+05$ & $5.45 \mathrm{E}+04$ & $1.01 \mathrm{E}+05$ & $1.13 \mathrm{E}+13$ & $5.41 \mathrm{E}+12$ \\
\hline 0.060 & 0.050 & $6.15 \mathrm{E}+05$ & $5.19 \mathrm{E}-28$ & $2.38 \mathrm{E}+05$ & $2.67 \mathrm{E}-12$ & $1.24 \mathrm{E}+05$ & $6.60 \mathrm{E}+12$ & \\
\hline 0.070 & 0.060 & $1.49 \mathrm{E}+04$ & $1.20 \mathrm{E}-20$ & $1.30 \mathrm{E}+03$ & $6.44 \mathrm{E}-12$ & $5.24 \mathrm{E}+04$ & $6.35 \mathrm{E}+12$ & \\
\hline 0.080 & 0.070 & $1.40 \mathrm{E}+04$ & $2.86 \mathrm{E}-28$ & $9.18 \mathrm{E}+05$ & $2.03 \mathrm{E}-12$ & $1.15 \mathrm{E}+06$ & $5.15 \mathrm{E}+12$ & \\
\hline 0.090 & 0.080 & $7.26 \mathrm{E}+02$ & $4.68 \mathrm{E}-08$ & $2.96 \mathrm{E}+01$ & $4.04 \mathrm{E}-12$ & $1.82 \mathrm{E}+08$ & $4.28 \mathrm{E}+12$ & $1.27 \mathrm{E}+12$ \\
\hline 0.100 & 0.090 & $1.57 \mathrm{E}+05$ & $1.42 \mathrm{E}+04$ & $3.28 \mathrm{E}+07$ & $7.26 \mathrm{E}+01$ & $5.35 \mathrm{E}+07$ & $4.51 \mathrm{E}+12$ & $1.14 \mathrm{E}+12$ \\
\hline 0.110 & 0.100 & $5.18 \mathrm{E}+03$ & $5.95 \mathrm{E}+05$ & $3.88 \mathrm{E}+06$ & $3.68 \mathrm{E}+03$ & $1.68 \mathrm{E}+04$ & $2.40 \mathrm{E}+12$ & $5.72 \mathrm{E}+11$ \\
\hline 0.120 & 0.110 & $6.03 \mathrm{E}+04$ & $3.61 \mathrm{E}+03$ & $9.24 \mathrm{E}+06$ & $1.99 \mathrm{E}+01$ & $1.85 \mathrm{E}+04$ & $2.84 \mathrm{E}+12$ & $5.40 \mathrm{E}+11$ \\
\hline 0.130 & 0.120 & $1.53 \mathrm{E}+05$ & $7.92 \mathrm{E}-28$ & $2.56 \mathrm{E}+04$ & $9.75 \mathrm{E}-13$ & $1.71 \mathrm{E}+04$ & $2.61 \mathrm{E}+12$ & $4.97 \mathrm{E}+11$ \\
\hline 0.140 & 0.130 & $7.76 \mathrm{E}-09$ & $1.11 \mathrm{E}-28$ & $3.49 \mathrm{E}+00$ & $8.05 \mathrm{E}-13$ & $1.70 \mathrm{E}+04$ & $2.20 \mathrm{E}+12$ & $3.05 \mathrm{E}+11$ \\
\hline 0.150 & 0.140 & $1.05 \mathrm{E}+04$ & $1.21 \mathrm{E}-21$ & $7.29 \mathrm{E}+06$ & $7.50 \mathrm{E}-13$ & $1.47 \mathrm{E}+04$ & $2.05 \mathrm{E}+12$ & $2.84 \mathrm{E}+11$ \\
\hline 0.160 & 0.150 & $9.70 \mathrm{E}+01$ & $2.69 \mathrm{E}-28$ & $1.30 \mathrm{E}+05$ & $4.50 \mathrm{E}+02$ & $9.70 \mathrm{E}+03$ & $1.77 \mathrm{E}+12$ & $1.73 \mathrm{E}+11$ \\
\hline 0.170 & 0.160 & $2.90 \mathrm{E}+03$ & $3.28 \mathrm{E}+04$ & $1.24 \mathrm{E}+05$ & $5.93 \mathrm{E}-13$ & $9.12 \mathrm{E}+03$ & $1.66 \mathrm{E}+12$ & $1.62 \mathrm{E}+11$ \\
\hline 0.180 & 0.170 & $4.10 \mathrm{E}+03$ & $6.79 \mathrm{E}-29$ & $4.47 \mathrm{E}-01$ & $5.08 \mathrm{E}-13$ & $7.32 \mathrm{E}+03$ & $1.45 \mathrm{E}+12$ & $9.66 \mathrm{E}+10$ \\
\hline 0.190 & 0.180 & $2.25 \mathrm{E}+03$ & $7.42 \mathrm{E}-29$ & $3.94 \mathrm{E}-01$ & $4.92 \mathrm{E}-13$ & $1.29 \mathrm{E}+09$ & $1.37 \mathrm{E}+12$ & $9.14 \mathrm{E}+10$ \\
\hline 0.200 & 0.190 & $2.75 \mathrm{E}+03$ & $2.13 \mathrm{E}-28$ & $2.46 \mathrm{E}-01$ & $8.33 \mathrm{E}-13$ & $1.44 \mathrm{E}+04$ & $2.43 \mathrm{E}+12$ & $1.05 \mathrm{E}+11$ \\
\hline 0.210 & 0.200 & $1.30 \mathrm{E}+04$ & $1.17 \mathrm{E}-27$ & $2.06 \mathrm{E}+04$ & $7.93 \mathrm{E}-13$ & $1.07 \mathrm{E}+04$ & $2.31 \mathrm{E}+12$ & $1.00 \mathrm{E}+11$ \\
\hline 0.220 & 0.210 & $2.79 \mathrm{E}+01$ & $2.41 \mathrm{E}+03$ & $2.25 \mathrm{E}-09$ & $4.03 \mathrm{E}-16$ & $0.00 \mathrm{E}+00$ & $0.00 \mathrm{E}+00$ & $0.00 \mathrm{E}+00$ \\
\hline 0.230 & 0.220 & $3.47 \mathrm{E}+02$ & $1.47 \mathrm{E}-29$ & $1.90 \mathrm{E}+01$ & $9.78 \mathrm{E}-17$ & $0.00 \mathrm{E}+00$ & $0.00 \mathrm{E}+00$ & $0.00 \mathrm{E}+00$ \\
\hline 0.240 & 0.230 & $3.35 \mathrm{E}+02$ & $6.64 \mathrm{E}-30$ & $1.92 \mathrm{E}+01$ & $2.87 \mathrm{E}-17$ & $0.00 \mathrm{E}+00$ & $0.00 \mathrm{E}+00$ & $0.00 \mathrm{E}+00$ \\
\hline 0.250 & 0.240 & $1.04 \mathrm{E}+03$ & $1.16 \mathrm{E}+03$ & $3.65 \mathrm{E}-01$ & $1.89 \mathrm{E}+03$ & $4.63 \mathrm{E}+05$ & $0.00 \mathrm{E}+00$ & $0.00 \mathrm{E}+00$ \\
\hline 0.260 & 0.250 & $1.85 \mathrm{E}+03$ & $1.11 \mathrm{E}+03$ & $3.46 \mathrm{E}-01$ & $1.81 \mathrm{E}+03$ & $4.57 \mathrm{E}+04$ & $2.65 \mathrm{E}+12$ & $4.09 \mathrm{E}+10$ \\
\hline 0.270 & 0.260 & $6.43 \mathrm{E}+02$ & $5.60 \mathrm{E}-28$ & $6.92 \mathrm{E}+02$ & $8.40 \mathrm{E}-13$ & $1.78 \mathrm{E}+06$ & $2.55 \mathrm{E}+12$ & $3.93 \mathrm{E}+10$ \\
\hline 0.280 & 0.270 & $2.00 \mathrm{E}-07$ & $4.98 \mathrm{E}-28$ & $1.29 \mathrm{E}-02$ & $2.08 \mathrm{E}-16$ & $2.18 \mathrm{E}+04$ & $0.00 \mathrm{E}+00$ & $0.00 \mathrm{E}+00$ \\
\hline 0.290 & 0.280 & $9.12 \mathrm{E}+01$ & $1.67 \mathrm{E}-29$ & $3.20 \mathrm{E}-26$ & $2.13 \mathrm{E}-18$ & $9.12 \mathrm{E}+02$ & $0.00 \mathrm{E}+00$ & $0.00 \mathrm{E}+00$ \\
\hline 0.300 & 0.290 & $1.15 \mathrm{E}+03$ & $0.00 \mathrm{E}+00$ & $2.36 \mathrm{E}+00$ & $4.40 \mathrm{E}-16$ & $1.21 \mathrm{E}+06$ & $0.00 \mathrm{E}+00$ & $0.00 \mathrm{E}+00$ \\
\hline 0.325 & 0.300 & $3.81 \mathrm{E}+03$ & $1.68 \mathrm{E}-28$ & $5.14 \mathrm{E}-01$ & $1.11 \mathrm{E}-12$ & $1.85 \mathrm{E}+04$ & $3.57 \mathrm{E}+12$ & $5.06 \mathrm{E}+09$ \\
\hline 0.350 & 0.325 & $2.83 \mathrm{E}+04$ & $3.97 \mathrm{E}-27$ & $1.22 \mathrm{E}+03$ & $7.15 \mathrm{E}-16$ & $2.64 \mathrm{E}+03$ & $6.54 \mathrm{E}+09$ & $0.00 \mathrm{E}+00$ \\
\hline 0.375 & 0.350 & $2.29 \mathrm{E}+04$ & $6.45 \mathrm{E}-30$ & $1.46 \mathrm{E}+02$ & $9.64 \mathrm{E}-16$ & $2.26 \mathrm{E}+06$ & $0.00 \mathrm{E}+00$ & $0.00 \mathrm{E}+00$ \\
\hline 0.400 & 0.375 & $4.30 \mathrm{E}+04$ & $8.35 \mathrm{E}-29$ & $2.87 \mathrm{E}-01$ & $7.15 \mathrm{E}-13$ & $1.29 \mathrm{E}+04$ & $2.43 \mathrm{E}+12$ & $1.70 \mathrm{E}+08$ \\
\hline 0.425 & 0.400 & $3.69 \mathrm{E}+04$ & $5.21 \mathrm{E}-28$ & $5.74 \mathrm{E}-02$ & $4.20 \mathrm{E}-18$ & $1.12 \mathrm{E}+05$ & $0.00 \mathrm{E}+00$ & $0.00 \mathrm{E}+00$ \\
\hline 0.450 & 0.425 & $8.67 \mathrm{E}+02$ & $8.59 \mathrm{E}-29$ & 4.89E-02 & $5.11 \mathrm{E}-13$ & $4.07 \mathrm{E}+04$ & $1.97 \mathrm{E}+12$ & $1.12 \mathrm{E}+08$ \\
\hline 0.475 & 0.450 & $4.34 \mathrm{E}+03$ & $1.21 \mathrm{E}-27$ & $3.25 \mathrm{E}-02$ & $6.19 \mathrm{E}-15$ & $4.90 \mathrm{E}+04$ & $0.00 \mathrm{E}+00$ & $0.00 \mathrm{E}+00$ \\
\hline 0.500 & 0.475 & $1.32 \mathrm{E}+02$ & $9.36 \mathrm{E}-29$ & $2.41 \mathrm{E}-03$ & $2.50 \mathrm{E}-13$ & $5.09 \mathrm{E}+04$ & $9.54 \mathrm{E}+11$ & $4.99 \mathrm{E}+07$ \\
\hline 0.525 & 0.500 & $2.50 \mathrm{E}-10$ & $2.48 \mathrm{E}-28$ & $7.85 \mathrm{E}-03$ & $2.39 \mathrm{E}-13$ & $5.39 \mathrm{E}+04$ & $2.59 \mathrm{E}+13$ & $4.75 \mathrm{E}+07$ \\
\hline 0.550 & 0.525 & $1.31 \mathrm{E}+01$ & $1.24 \mathrm{E}+01$ & $2.02 \mathrm{E}-03$ & $2.38 \mathrm{E}-15$ & $1.52 \mathrm{E}+04$ & $0.00 \mathrm{E}+00$ & $0.00 \mathrm{E}+00$ \\
\hline 0.575 & 0.550 & $5.58 \mathrm{E}+00$ & $3.29 \mathrm{E}-28$ & $3.38 \mathrm{E}-03$ & $1.16 \mathrm{E}-15$ & $6.76 \mathrm{E}+02$ & $0.00 \mathrm{E}+00$ & $0.00 \mathrm{E}+00$ \\
\hline 0.600 & 0.575 & $6.17 \mathrm{E}+01$ & $4.26 \mathrm{E}-29$ & $2.01 \mathrm{E}-02$ & $2.05 \mathrm{E}-13$ & $1.16 \mathrm{E}+05$ & $8.63 \mathrm{E}+11$ & $3.61 \mathrm{E}+07$ \\
\hline 0.625 & 0.600 & $1.41 \mathrm{E}+02$ & $6.54 \mathrm{E}-29$ & $1.01 \mathrm{E}-01$ & $1.97 \mathrm{E}-13$ & $4.63 \mathrm{E}+05$ & $1.41 \mathrm{E}+13$ & $3.46 \mathrm{E}+07$ \\
\hline 0.650 & 0.625 & $6.87 \mathrm{E}+02$ & $1.10 \mathrm{E}+03$ & $1.50 \mathrm{E}-02$ & $2.14 \mathrm{E}-15$ & $1.40 \mathrm{E}+03$ & $0.00 \mathrm{E}+00$ & $0.00 \mathrm{E}+00$ \\
\hline 0.675 & 0.650 & $4.84 \mathrm{E}+02$ & $7.62 \mathrm{E}-29$ & $6.68 \mathrm{E}-01$ & $1.56 \mathrm{E}-15$ & $1.31 \mathrm{E}+04$ & $0.00 \mathrm{E}+00$ & $0.00 \mathrm{E}+00$ \\
\hline 0.700 & 0.675 & $4.66 \mathrm{E}+01$ & $2.94 \mathrm{E}+02$ & $7.62 \mathrm{E}-02$ & $1.33 \mathrm{E}-13$ & $1.85 \mathrm{E}+03$ & $6.05 \mathrm{E}+11$ & $1.83 \mathrm{E}+07$ \\
\hline 0.725 & 0.700 & $1.71 \mathrm{E}+02$ & 9.97E-29 & $3.44 \mathrm{E}-01$ & $1.30 \mathrm{E}-13$ & $8.86 \mathrm{E}+03$ & $5.85 \mathrm{E}+11$ & $1.77 \mathrm{E}+07$ \\
\hline 0.750 & 0.725 & $1.08 \mathrm{E}+01$ & $1.14 \mathrm{E}+03$ & $1.97 \mathrm{E}-01$ & $1.89 \mathrm{E}+03$ & $9.02 \mathrm{E}+02$ & $0.00 \mathrm{E}+00$ & $0.00 \mathrm{E}+00$ \\
\hline
\end{tabular}


PCP-2011-0001

Revision 0

\begin{tabular}{|c|c|c|c|c|c|c|c|c|}
\hline \multicolumn{9}{|c|}{ Table 3-16. Photon Source from ORIGEN-S (Decay Time = 0 days) } \\
\hline \multicolumn{2}{|c|}{ Energy (MeV) } & \multicolumn{7}{|c|}{ Photon Source $(\mathbf{p} / \mathbf{s} / \mathbf{g})$} \\
\hline Upper & Lower & Pu-239 & Pu-240 & Pu-241 & Pu-242 & Ra-226 & Ru-106 & Sc-46 \\
\hline 0.775 & 0.750 & $3.66 \mathrm{E}+02$ & $1.11 \mathrm{E}+03$ & $2.11 \mathrm{E}-01$ & $1.83 \mathrm{E}+03$ & $4.62 \mathrm{E}+04$ & $1.31 \mathrm{E}+09$ & $0.00 \mathrm{E}+00$ \\
\hline 0.800 & 0.775 & $7.31 \mathrm{E}+00$ & $1.30 \mathrm{E}-27$ & $4.05 \mathrm{E}-03$ & $1.15 \mathrm{E}-13$ & $7.18 \mathrm{E}+04$ & $4.14 \mathrm{E}+11$ & $9.18 \mathrm{E}+06$ \\
\hline 0.825 & 0.800 & $7.58 \mathrm{E}+00$ & $2.05 \mathrm{E}-29$ & $4.84 \mathrm{E}-03$ & $8.30 \mathrm{E}-14$ & $1.22 \mathrm{E}+04$ & $4.01 \mathrm{E}+11$ & $8.90 \mathrm{E}+06$ \\
\hline 0.850 & 0.825 & $8.75 \mathrm{E}+00$ & $8.43 \mathrm{E}-28$ & $1.28 \mathrm{E}-03$ & $3.42 \mathrm{E}-15$ & $3.80 \mathrm{E}+04$ & $0.00 \mathrm{E}+00$ & $0.00 \mathrm{E}+00$ \\
\hline 0.875 & 0.850 & $3.32 \mathrm{E}-33$ & $4.94 \mathrm{E}+01$ & $2.77 \mathrm{E}-03$ & $4.56 \mathrm{E}-15$ & $0.00 \mathrm{E}+00$ & $5.45 \mathrm{E}+11$ & $0.00 \mathrm{E}+00$ \\
\hline 0.900 & 0.875 & $3.14 \mathrm{E}+00$ & $2.11 \mathrm{E}-29$ & $4.88 \mathrm{E}-04$ & $6.06 \mathrm{E}-14$ & $3.45 \mathrm{E}+02$ & $2.99 \mathrm{E}+11$ & $1.26 \mathrm{E}+15$ \\
\hline 0.925 & 0.900 & $6.86 \mathrm{E}-01$ & $7.24 \mathrm{E}-27$ & $1.24 \mathrm{E}-03$ & $5.71 \mathrm{E}-14$ & $1.37 \mathrm{E}+03$ & $2.91 \mathrm{E}+11$ & $4.29 \mathrm{E}+06$ \\
\hline 0.950 & 0.925 & $1.45 \mathrm{E}+00$ & $6.11 \mathrm{E}-29$ & $1.86 \mathrm{E}-04$ & $1.05 \mathrm{E}-14$ & $2.58 \mathrm{E}+04$ & $7.04 \mathrm{E}+08$ & $0.00 \mathrm{E}+00$ \\
\hline 0.975 & 0.950 & $2.08 \mathrm{E}+00$ & $5.73 \mathrm{E}-27$ & $9.67 \mathrm{E}-04$ & $5.41 \mathrm{E}-16$ & $3.12 \mathrm{E}+03$ & $0.00 \mathrm{E}+00$ & $0.00 \mathrm{E}+00$ \\
\hline 1.000 & 0.975 & $2.20 \mathrm{E}+00$ & $7.01 \mathrm{E}-29$ & $1.23 \mathrm{E}-28$ & $3.03 \mathrm{E}-13$ & $5.14 \mathrm{E}+02$ & $2.20 \mathrm{E}+11$ & $1.96 \mathrm{E}+06$ \\
\hline 1.250 & 1.000 & $3.92 \mathrm{E}+00$ & $5.35 \mathrm{E}+02$ & $9.86 \mathrm{E}-02$ & $8.82 \mathrm{E}+02$ & $2.05 \mathrm{E}+05$ & $3.22 \mathrm{E}+12$ & $1.25 \mathrm{E}+15$ \\
\hline 1.500 & 1.250 & $2.03 \mathrm{E}+00$ & $4.38 \mathrm{E}+02$ & $8.06 \mathrm{E}-02$ & $7.22 \mathrm{E}+02$ & $8.56 \mathrm{E}+04$ & $4.09 \mathrm{E}+11$ & $1.19 \mathrm{E}+05$ \\
\hline 1.750 & 1.500 & $1.21 \mathrm{E}+00$ & $2.48 \mathrm{E}+02$ & 4.79E-02 & $4.10 \mathrm{E}+02$ & $7.18 \mathrm{E}+04$ & $4.04 \mathrm{E}+11$ & $4.77 \mathrm{E}+00$ \\
\hline 2.000 & 1.750 & $1.04 \mathrm{E}+00$ & $2.15 \mathrm{E}+02$ & $4.15 \mathrm{E}-02$ & $3.55 \mathrm{E}+02$ & $1.47 \mathrm{E}+05$ & $2.41 \mathrm{E}+11$ & $0.00 \mathrm{E}+00$ \\
\hline 2.250 & 2.000 & $5.95 \mathrm{E}-01$ & $1.41 \mathrm{E}+02$ & $2.36 \mathrm{E}-02$ & $2.34 \mathrm{E}+02$ & $5.50 \mathrm{E}+04$ & $7.92 \mathrm{E}+10$ & $1.54 \mathrm{E}+08$ \\
\hline 2.500 & 2.250 & 5.32E-01 & $1.26 \mathrm{E}+02$ & $2.11 \mathrm{E}-02$ & $2.09 \mathrm{E}+02$ & $1.64 \mathrm{E}+04$ & $1.23 \mathrm{E}+11$ & $0.00 \mathrm{E}+00$ \\
\hline 2.750 & 2.500 & $2.95 \mathrm{E}-01$ & $8.09 \mathrm{E}+01$ & $1.17 \mathrm{E}-02$ & $1.34 \mathrm{E}+02$ & $3.53 \mathrm{E}+02$ & $1.45 \mathrm{E}+10$ & $0.00 \mathrm{E}+00$ \\
\hline 3.000 & 2.750 & $2.70 \mathrm{E}-01$ & $7.39 \mathrm{E}+01$ & $1.07 \mathrm{E}-02$ & $1.22 \mathrm{E}+02$ & $7.34 \mathrm{E}+02$ & $1.23 \mathrm{E}+10$ & $0.00 \mathrm{E}+00$ \\
\hline 3.500 & 3.000 & $2.83 \mathrm{E}-01$ & $8.94 \mathrm{E}+01$ & $1.12 \mathrm{E}-02$ & $1.48 \mathrm{E}+02$ & $2.81 \mathrm{E}+02$ & $2.71 \mathrm{E}+09$ & $0.00 \mathrm{E}+00$ \\
\hline 4.000 & 3.500 & $1.42 \mathrm{E}-01$ & $5.18 \mathrm{E}+01$ & $5.63 \mathrm{E}-03$ & $8.60 \mathrm{E}+01$ & $2.25 \mathrm{E}+00$ & $1.63 \mathrm{E}+06$ & $0.00 \mathrm{E}+00$ \\
\hline 4.500 & 4.000 & $7.14 \mathrm{E}-02$ & $3.00 \mathrm{E}+01$ & $2.82 \mathrm{E}-03$ & $4.99 \mathrm{E}+01$ & $1.13 \mathrm{E}+00$ & $0.00 \mathrm{E}+00$ & $0.00 \mathrm{E}+00$ \\
\hline 5.000 & 4.500 & 3.59E-02 & $1.74 \mathrm{E}+01$ & $1.42 \mathrm{E}-03$ & $2.89 \mathrm{E}+01$ & $5.67 \mathrm{E}-01$ & $0.00 \mathrm{E}+00$ & $0.00 \mathrm{E}+00$ \\
\hline 5.500 & 5.000 & $1.80 \mathrm{E}-02$ & $1.01 \mathrm{E}+01$ & $7.10 \mathrm{E}-04$ & $1.68 \mathrm{E}+01$ & $2.85 \mathrm{E}-01$ & $0.00 \mathrm{E}+00$ & $0.00 \mathrm{E}+00$ \\
\hline 6.000 & 5.500 & $9.05 \mathrm{E}-03$ & $5.83 \mathrm{E}+00$ & $3.56 \mathrm{E}-04$ & $9.72 \mathrm{E}+00$ & $1.43 \mathrm{E}-01$ & $0.00 \mathrm{E}+00$ & $0.00 \mathrm{E}+00$ \\
\hline 6.500 & 6.000 & $4.55 \mathrm{E}-03$ & $3.38 \mathrm{E}+00$ & $1.79 \mathrm{E}-04$ & $5.64 \mathrm{E}+00$ & $7.16 \mathrm{E}-02$ & $0.00 \mathrm{E}+00$ & $0.00 \mathrm{E}+00$ \\
\hline 7.000 & 6.500 & $2.29 \mathrm{E}-03$ & $1.96 \mathrm{E}+00$ & $8.96 \mathrm{E}-05$ & $3.27 \mathrm{E}+00$ & $3.59 \mathrm{E}-02$ & $0.00 \mathrm{E}+00$ & $0.00 \mathrm{E}+00$ \\
\hline 7.500 & 7.000 & $1.15 \mathrm{E}-03$ & $1.13 \mathrm{E}+00$ & $4.50 \mathrm{E}-05$ & $1.89 \mathrm{E}+00$ & $1.80 \mathrm{E}-02$ & $0.00 \mathrm{E}+00$ & $0.00 \mathrm{E}+00$ \\
\hline 8.000 & 7.500 & 5.79E-04 & $6.57 \mathrm{E}-01$ & $2.25 \mathrm{E}-05$ & $1.10 \mathrm{E}+00$ & $9.03 \mathrm{E}-03$ & $0.00 \mathrm{E}+00$ & $0.00 \mathrm{E}+00$ \\
\hline 9.000 & 8.000 & $4.34 \mathrm{E}-04$ & $5.97 \mathrm{E}-01$ & $1.68 \mathrm{E}-05$ & 9.99E-01 & $6.74 \mathrm{E}-03$ & $0.00 \mathrm{E}+00$ & $0.00 \mathrm{E}+00$ \\
\hline \multirow[t]{2}{*}{10.000} & 9.000 & $1.10 \mathrm{E}-04$ & $2.01 \mathrm{E}-01$ & $4.23 \mathrm{E}-06$ & $3.36 \mathrm{E}-01$ & $1.70 \mathrm{E}-03$ & $0.00 \mathrm{E}+00$ & $0.00 \mathrm{E}+00$ \\
\hline & Total & $1.11 \mathrm{E}+08$ & $8.83 \mathrm{E}+08$ & $1.96 \mathrm{E}+08$ & $1.31 \mathrm{E}+07$ & $1.84 \mathrm{E}+09$ & $1.96 \mathrm{E}+14$ & $2.58 \mathrm{E}+15$ \\
\hline
\end{tabular}


PCP-2011-0001

Revision 0

\begin{tabular}{|c|c|c|c|c|c|c|c|c|}
\hline \multicolumn{9}{|c|}{ Table 3-17. Photon Source from ORIGEN-S (Decay Time $=0$ days) } \\
\hline \multicolumn{2}{|c|}{ Energy (MeV) } & \multicolumn{7}{|c|}{ Photon Source $(\mathrm{p} / \mathrm{s} / \mathrm{g})$} \\
\hline Upper & Lower & Se-75 & Sm-145 & Sr-90 & Tm-170 & Yb-169 & Zn-65 & Zr-95 \\
\hline 0.020 & 0.010 & $2.15 \mathrm{E}+14$ & $0.00 \mathrm{E}+00$ & $2.13 \mathrm{E}+11$ & $1.47 \mathrm{E}+13$ & $0.00 \mathrm{E}+00$ & $5.34 \mathrm{E}+11$ & $1.88 \mathrm{E}+13$ \\
\hline 0.030 & 0.020 & $1.72 \mathrm{E}+11$ & $0.00 \mathrm{E}+00$ & $1.10 \mathrm{E}+11$ & $7.80 \mathrm{E}+12$ & $1.41 \mathrm{E}+12$ & $2.91 \mathrm{E}+11$ & $8.81 \mathrm{E}+12$ \\
\hline 0.040 & 0.030 & $0.00 \mathrm{E}+00$ & $1.22 \mathrm{E}+14$ & $6.30 \mathrm{E}+10$ & $4.60 \mathrm{E}+12$ & $0.00 \mathrm{E}+00$ & $1.76 \mathrm{E}+11$ & $4.85 \mathrm{E}+12$ \\
\hline 0.050 & 0.040 & $0.00 \mathrm{E}+00$ & $2.68 \mathrm{E}+13$ & $4.99 \mathrm{E}+10$ & $3.95 \mathrm{E}+12$ & $2.62 \mathrm{E}+14$ & $1.47 \mathrm{E}+11$ & $3.66 \mathrm{E}+12$ \\
\hline 0.060 & 0.050 & $0.00 \mathrm{E}+00$ & $0.00 \mathrm{E}+00$ & $2.74 \mathrm{E}+10$ & $2.16 \mathrm{E}+12$ & $1.35 \mathrm{E}+15$ & $8.44 \mathrm{E}+10$ & \\
\hline 0.070 & 0.060 & $6.23 \mathrm{E}+12$ & $1.17 \mathrm{E}+13$ & $2.49 \mathrm{E}+10$ & $1.96 \mathrm{E}+12$ & $3.83 \mathrm{E}+14$ & $7.98 \mathrm{E}+10$ & \\
\hline 0.080 & 0.070 & $0.00 \mathrm{E}+00$ & $2.38 \mathrm{E}+06$ & $1.88 \mathrm{E}+10$ & $1.52 \mathrm{E}+12$ & $0.00 \mathrm{E}+00$ & $6.32 \mathrm{E}+10$ & $1.21 \mathrm{E}+12$ \\
\hline 0.090 & 0.080 & $3.63 \mathrm{E}+10$ & $0.00 \mathrm{E}+00$ & $1.45 \mathrm{E}+10$ & $1.21 \mathrm{E}+12$ & $1.28 \mathrm{E}+10$ & $5.14 \mathrm{E}+10$ & $8.85 \mathrm{E}+11$ \\
\hline 0.100 & 0.090 & $1.91 \mathrm{E}+13$ & $0.00 \mathrm{E}+00$ & $1.41 \mathrm{E}+10$ & $1.21 \mathrm{E}+12$ & $2.29 \mathrm{E}+13$ & $5.29 \mathrm{E}+10$ & $8.09 \mathrm{E}+11$ \\
\hline 0.110 & 0.100 & $0.00 \mathrm{E}+00$ & $0.00 \mathrm{E}+00$ & $7.25 \mathrm{E}+09$ & $6.32 \mathrm{E}+11$ & $8.16 \mathrm{E}+13$ & $2.78 \mathrm{E}+10$ & $4.06 \mathrm{E}+11$ \\
\hline 0.120 & 0.110 & $0.00 \mathrm{E}+00$ & $0.00 \mathrm{E}+00$ & $7.65 \mathrm{E}+09$ & $7.00 \mathrm{E}+11$ & $9.20 \mathrm{E}+13$ & $3.19 \mathrm{E}+10$ & $3.92 \mathrm{E}+11$ \\
\hline 0.130 & 0.120 & $9.02 \mathrm{E}+13$ & $0.00 \mathrm{E}+00$ & $7.04 \mathrm{E}+09$ & $6.44 \mathrm{E}+11$ & $1.33 \mathrm{E}+12$ & $2.93 \mathrm{E}+10$ & $3.61 \mathrm{E}+11$ \\
\hline 0.140 & 0.130 & $3.20 \mathrm{E}+14$ & $0.00 \mathrm{E}+00$ & $5.10 \mathrm{E}+09$ & $4.98 \mathrm{E}+11$ & $9.88 \mathrm{E}+13$ & $2.37 \mathrm{E}+10$ & $2.29 \mathrm{E}+11$ \\
\hline 0.150 & 0.140 & $0.00 \mathrm{E}+00$ & $0.00 \mathrm{E}+00$ & $4.74 \mathrm{E}+09$ & $4.64 \mathrm{E}+11$ & $0.00 \mathrm{E}+00$ & $2.21 \mathrm{E}+10$ & $2.14 \mathrm{E}+11$ \\
\hline 0.160 & 0.150 & $0.00 \mathrm{E}+00$ & $0.00 \mathrm{E}+00$ & $3.48 \mathrm{E}+09$ & $3.66 \mathrm{E}+11$ & $9.01 \mathrm{E}+10$ & $1.82 \mathrm{E}+10$ & $1.36 \mathrm{E}+11$ \\
\hline 0.170 & 0.160 & $0.00 \mathrm{E}+00$ & $0.00 \mathrm{E}+00$ & $3.27 \mathrm{E}+09$ & $3.43 \mathrm{E}+11$ & $0.00 \mathrm{E}+00$ & $1.71 \mathrm{E}+10$ & $1.28 \mathrm{E}+11$ \\
\hline 0.180 & 0.170 & $0.00 \mathrm{E}+00$ & $0.00 \mathrm{E}+00$ & $2.42 \mathrm{E}+09$ & $2.75 \mathrm{E}+11$ & $2.00 \mathrm{E}+14$ & $1.43 \mathrm{E}+10$ & $8.07 \mathrm{E}+10$ \\
\hline 0.190 & 0.180 & $0.00 \mathrm{E}+00$ & $0.00 \mathrm{E}+00$ & $2.29 \mathrm{E}+09$ & $2.60 \mathrm{E}+11$ & $0.00 \mathrm{E}+00$ & $1.35 \mathrm{E}+10$ & $7.63 \mathrm{E}+10$ \\
\hline 0.200 & 0.190 & $8.06 \mathrm{E}+12$ & $0.00 \mathrm{E}+00$ & $3.40 \mathrm{E}+09$ & $4.20 \mathrm{E}+11$ & $3.25 \mathrm{E}+14$ & $2.28 \mathrm{E}+10$ & $9.52 \mathrm{E}+10$ \\
\hline 0.210 & 0.200 & $0.00 \mathrm{E}+00$ & $0.00 \mathrm{E}+00$ & $3.24 \mathrm{E}+09$ & $3.99 \mathrm{E}+11$ & $1.14 \mathrm{E}+11$ & $2.17 \mathrm{E}+10$ & $9.10 \mathrm{E}+10$ \\
\hline 0.220 & 0.210 & $0.00 \mathrm{E}+00$ & $0.00 \mathrm{E}+00$ & $0.00 \mathrm{E}+00$ & $0.00 \mathrm{E}+00$ & $2.58 \mathrm{E}+10$ & $0.00 \mathrm{E}+00$ & $0.00 \mathrm{E}+00$ \\
\hline 0.230 & 0.220 & $0.00 \mathrm{E}+00$ & $0.00 \mathrm{E}+00$ & $0.00 \mathrm{E}+00$ & $0.00 \mathrm{E}+00$ & $0.00 \mathrm{E}+00$ & $0.00 \mathrm{E}+00$ & $0.00 \mathrm{E}+00$ \\
\hline 0.240 & 0.230 & $0.00 \mathrm{E}+00$ & $0.00 \mathrm{E}+00$ & $0.00 \mathrm{E}+00$ & $0.00 \mathrm{E}+00$ & $0.00 \mathrm{E}+00$ & $0.00 \mathrm{E}+00$ & $4.09 \mathrm{E}+09$ \\
\hline 0.250 & 0.240 & $5.17 \mathrm{E}+08$ & $1.15 \mathrm{E}-09$ & $0.00 \mathrm{E}+00$ & $0.00 \mathrm{E}+00$ & $9.97 \mathrm{E}+11$ & $0.00 \mathrm{E}+00$ & $0.00 \mathrm{E}+00$ \\
\hline 0.260 & 0.250 & $0.00 \mathrm{E}+00$ & $1.11 \mathrm{E}-09$ & $2.56 \mathrm{E}+09$ & $3.80 \mathrm{E}+11$ & $0.00 \mathrm{E}+00$ & $2.28 \mathrm{E}+10$ & $4.80 \mathrm{E}+10$ \\
\hline 0.270 & 0.260 & $3.17 \mathrm{E}+14$ & $0.00 \mathrm{E}+00$ & $2.46 \mathrm{E}+09$ & $3.66 \mathrm{E}+11$ & $1.51 \mathrm{E}+13$ & $2.19 \mathrm{E}+10$ & $4.62 \mathrm{E}+10$ \\
\hline 0.280 & 0.270 & $1.38 \mathrm{E}+14$ & $0.00 \mathrm{E}+00$ & $0.00 \mathrm{E}+00$ & $0.00 \mathrm{E}+00$ & $0.00 \mathrm{E}+00$ & $0.00 \mathrm{E}+00$ & $0.00 \mathrm{E}+00$ \\
\hline 0.290 & 0.280 & $0.00 \mathrm{E}+00$ & $0.00 \mathrm{E}+00$ & $0.00 \mathrm{E}+00$ & $0.00 \mathrm{E}+00$ & $0.00 \mathrm{E}+00$ & $0.00 \mathrm{E}+00$ & $0.00 \mathrm{E}+00$ \\
\hline 0.300 & 0.290 & $0.00 \mathrm{E}+00$ & $0.00 \mathrm{E}+00$ & $0.00 \mathrm{E}+00$ & $0.00 \mathrm{E}+00$ & $3.79 \mathrm{E}+10$ & $0.00 \mathrm{E}+00$ & $0.00 \mathrm{E}+00$ \\
\hline 0.325 & 0.300 & $7.02 \mathrm{E}+12$ & $0.00 \mathrm{E}+00$ & $1.82 \mathrm{E}+09$ & $3.83 \mathrm{E}+11$ & $9.06 \mathrm{E}+13$ & $2.66 \mathrm{E}+10$ & $1.82 \mathrm{E}+10$ \\
\hline 0.350 & 0.325 & $0.00 \mathrm{E}+00$ & $0.00 \mathrm{E}+00$ & $0.00 \mathrm{E}+00$ & $0.00 \mathrm{E}+00$ & $9.68 \mathrm{E}+10$ & $9.35 \mathrm{E}+09$ & $0.00 \mathrm{E}+00$ \\
\hline 0.375 & 0.350 & $1.38 \mathrm{E}+10$ & $0.00 \mathrm{E}+00$ & $0.00 \mathrm{E}+00$ & $0.00 \mathrm{E}+00$ & $6.57 \mathrm{E}+10$ & $0.00 \mathrm{E}+00$ & $0.00 \mathrm{E}+00$ \\
\hline 0.400 & 0.375 & $3.21 \mathrm{E}+13$ & $0.00 \mathrm{E}+00$ & $5.80 \mathrm{E}+08$ & $1.90 \mathrm{E}+11$ & $1.06 \mathrm{E}+10$ & $1.56 \mathrm{E}+10$ & $5.28 \mathrm{E}+09$ \\
\hline 0.425 & 0.400 & $3.03 \mathrm{E}+13$ & $0.00 \mathrm{E}+00$ & $0.00 \mathrm{E}+00$ & $0.00 \mathrm{E}+00$ & $0.00 \mathrm{E}+00$ & $0.00 \mathrm{E}+00$ & $0.00 \mathrm{E}+00$ \\
\hline 0.450 & 0.425 & $0.00 \mathrm{E}+00$ & $4.91 \mathrm{E}+07$ & $1.93 \mathrm{E}+08$ & $1.02 \mathrm{E}+11$ & $0.00 \mathrm{E}+00$ & $1.01 \mathrm{E}+10$ & $2.74 \mathrm{E}+09$ \\
\hline 0.475 & 0.450 & $1.93 \mathrm{E}+09$ & $0.00 \mathrm{E}+00$ & $0.00 \mathrm{E}+00$ & $0.00 \mathrm{E}+00$ & $3.21 \mathrm{E}+07$ & $0.00 \mathrm{E}+00$ & $0.00 \mathrm{E}+00$ \\
\hline 0.500 & 0.475 & $0.00 \mathrm{E}+00$ & $3.40 \mathrm{E}+09$ & $5.46 \mathrm{E}+07$ & $3.57 \mathrm{E}+10$ & $1.35 \mathrm{E}+10$ & $4.39 \mathrm{E}+09$ & $9.44 \mathrm{E}+08$ \\
\hline 0.525 & 0.500 & $0.00 \mathrm{E}+00$ & $0.00 \mathrm{E}+00$ & $5.19 \mathrm{E}+07$ & $3.39 \mathrm{E}+10$ & $3.72 \mathrm{E}+10$ & $8.61 \mathrm{E}+12$ & $8.98 \mathrm{E}+08$ \\
\hline 0.550 & 0.525 & $7.05 \mathrm{E}+08$ & $0.00 \mathrm{E}+00$ & $0.00 \mathrm{E}+00$ & $0.00 \mathrm{E}+00$ & $1.33 \mathrm{E}+07$ & $0.00 \mathrm{E}+00$ & $0.00 \mathrm{E}+00$ \\
\hline 0.575 & 0.550 & $1.62 \mathrm{E}+11$ & $0.00 \mathrm{E}+00$ & $0.00 \mathrm{E}+00$ & $0.00 \mathrm{E}+00$ & $0.00 \mathrm{E}+00$ & $0.00 \mathrm{E}+00$ & $1.71 \mathrm{E}+07$ \\
\hline 0.600 & 0.575 & $0.00 \mathrm{E}+00$ & $0.00 \mathrm{E}+00$ & $4.11 \mathrm{E}+07$ & $1.74 \mathrm{E}+10$ & $0.00 \mathrm{E}+00$ & $3.05 \mathrm{E}+09$ & $4.46 \mathrm{E}+08$ \\
\hline 0.625 & 0.600 & $1.99 \mathrm{E}+10$ & $0.00 \mathrm{E}+00$ & $3.94 \mathrm{E}+07$ & $1.67 \mathrm{E}+10$ & $2.23 \mathrm{E}+10$ & $2.92 \mathrm{E}+09$ & $4.19 \mathrm{E}+08$ \\
\hline 0.650 & 0.625 & $0.00 \mathrm{E}+00$ & $0.00 \mathrm{E}+00$ & $0.00 \mathrm{E}+00$ & $0.00 \mathrm{E}+00$ & $2.15 \mathrm{E}+10$ & $0.00 \mathrm{E}+00$ & $0.00 \mathrm{E}+00$ \\
\hline 0.675 & 0.650 & $0.00 \mathrm{E}+00$ & $0.00 \mathrm{E}+00$ & $0.00 \mathrm{E}+00$ & $0.00 \mathrm{E}+00$ & $0.00 \mathrm{E}+00$ & $0.00 \mathrm{E}+00$ & $0.00 \mathrm{E}+00$ \\
\hline 0.700 & 0.675 & $0.00 \mathrm{E}+00$ & $0.00 \mathrm{E}+00$ & $2.59 \mathrm{E}+07$ & $4.56 \mathrm{E}+09$ & $7.80 \mathrm{E}+07$ & $1.45 \mathrm{E}+09$ & $9.69 \mathrm{E}+07$ \\
\hline 0.725 & 0.700 & $0.00 \mathrm{E}+00$ & $0.00 \mathrm{E}+00$ & $2.50 \mathrm{E}+07$ & $4.40 \mathrm{E}+09$ & $0.00 \mathrm{E}+00$ & $1.40 \mathrm{E}+09$ & $3.56 \mathrm{E}+14$ \\
\hline 0.750 & 0.725 & $0.00 \mathrm{E}+00$ & $5.77 \mathrm{E}-10$ & $0.00 \mathrm{E}+00$ & $0.00 \mathrm{E}+00$ & $1.63 \mathrm{E}+07$ & $0.00 \mathrm{E}+00$ & $0.00 \mathrm{E}+00$ \\
\hline
\end{tabular}


PCP-2011-0001

Revision 0

\begin{tabular}{|c|c|c|c|c|c|c|c|c|}
\hline \multicolumn{9}{|c|}{ Table 3-17. Photon Source from ORIGEN-S (Decay Time = 0 days) } \\
\hline \multicolumn{2}{|c|}{ Energy (MeV) } & \multicolumn{7}{|c|}{ Photon Source (p/s/g) } \\
\hline Upper & Lower & Se-75 & Sm-145 & Sr-90 & Tm-170 & Yb-169 & Zn-65 & Zr-95 \\
\hline 0.775 & 0.750 & $0.00 \mathrm{E}+00$ & $5.58 \mathrm{E}-10$ & $0.00 \mathrm{E}+00$ & $0.00 \mathrm{E}+00$ & $7.33 \mathrm{E}+06$ & $9.24 \mathrm{E}+09$ & $4.30 \mathrm{E}+14$ \\
\hline 0.800 & 0.775 & $0.00 \mathrm{E}+00$ & $0.00 \mathrm{E}+00$ & $1.68 \mathrm{E}+07$ & $8.23 \mathrm{E}+08$ & $2.66 \mathrm{E}+07$ & $6.65 \mathrm{E}+08$ & $1.33 \mathrm{E}+07$ \\
\hline 0.825 & 0.800 & $9.00 \mathrm{E}+08$ & $0.00 \mathrm{E}+00$ & $1.63 \mathrm{E}+07$ & $7.98 \mathrm{E}+08$ & $0.00 \mathrm{E}+00$ & $6.45 \mathrm{E}+08$ & $1.05 \mathrm{E}+07$ \\
\hline 0.850 & 0.825 & $0.00 \mathrm{E}+00$ & $0.00 \mathrm{E}+00$ & $0.00 \mathrm{E}+00$ & $0.00 \mathrm{E}+00$ & $0.00 \mathrm{E}+00$ & $0.00 \mathrm{E}+00$ & $2.03 \mathrm{E}+03$ \\
\hline 0.875 & 0.850 & $0.00 \mathrm{E}+00$ & $0.00 \mathrm{E}+00$ & $0.00 \mathrm{E}+00$ & $0.00 \mathrm{E}+00$ & $0.00 \mathrm{E}+00$ & $0.00 \mathrm{E}+00$ & $0.00 \mathrm{E}+00$ \\
\hline 0.900 & 0.875 & $0.00 \mathrm{E}+00$ & $0.00 \mathrm{E}+00$ & $1.11 \mathrm{E}+07$ & $6.24 \mathrm{E}+07$ & $0.00 \mathrm{E}+00$ & $2.82 \mathrm{E}+08$ & $2.61 \mathrm{E}+04$ \\
\hline 0.925 & 0.900 & $0.00 \mathrm{E}+00$ & $0.00 \mathrm{E}+00$ & $1.08 \mathrm{E}+07$ & $6.07 \mathrm{E}+07$ & $0.00 \mathrm{E}+00$ & $2.74 \mathrm{E}+08$ & $2.54 \mathrm{E}+04$ \\
\hline 0.950 & 0.925 & $0.00 \mathrm{E}+00$ & $0.00 \mathrm{E}+00$ & $0.00 \mathrm{E}+00$ & $0.00 \mathrm{E}+00$ & $0.00 \mathrm{E}+00$ & $0.00 \mathrm{E}+00$ & $0.00 \mathrm{E}+00$ \\
\hline 0.975 & 0.950 & $0.00 \mathrm{E}+00$ & $0.00 \mathrm{E}+00$ & $0.00 \mathrm{E}+00$ & $0.00 \mathrm{E}+00$ & $0.00 \mathrm{E}+00$ & $0.00 \mathrm{E}+00$ & $0.00 \mathrm{E}+00$ \\
\hline 1.000 & 0.975 & $0.00 \mathrm{E}+00$ & $0.00 \mathrm{E}+00$ & $7.32 \mathrm{E}+06$ & $7.99 \mathrm{E}+03$ & $0.00 \mathrm{E}+00$ & $1.05 \mathrm{E}+08$ & $0.00 \mathrm{E}+00$ \\
\hline 1.250 & 1.000 & $0.00 \mathrm{E}+00$ & $3.16 \mathrm{E}-10$ & $2.25 \mathrm{E}+07$ & $7.02 \mathrm{E}+03$ & $0.00 \mathrm{E}+00$ & $1.53 \mathrm{E}+14$ & $0.00 \mathrm{E}+00$ \\
\hline 1.500 & 1.250 & $0.00 \mathrm{E}+00$ & $2.59 \mathrm{E}-10$ & $7.32 \mathrm{E}+06$ & $0.00 \mathrm{E}+00$ & $0.00 \mathrm{E}+00$ & $7.58 \mathrm{E}+05$ & $0.00 \mathrm{E}+00$ \\
\hline 1.750 & 1.500 & $0.00 \mathrm{E}+00$ & $1.54 \mathrm{E}-10$ & $2.16 \mathrm{E}+06$ & $0.00 \mathrm{E}+00$ & $0.00 \mathrm{E}+00$ & $0.00 \mathrm{E}+00$ & $0.00 \mathrm{E}+00$ \\
\hline 2.000 & 1.750 & $0.00 \mathrm{E}+00$ & $1.33 \mathrm{E}-10$ & $4.13 \mathrm{E}+05$ & $0.00 \mathrm{E}+00$ & $0.00 \mathrm{E}+00$ & $0.00 \mathrm{E}+00$ & $0.00 \mathrm{E}+00$ \\
\hline 2.250 & 2.000 & $0.00 \mathrm{E}+00$ & $7.58 \mathrm{E}-11$ & $4.83 \mathrm{E}+04$ & $0.00 \mathrm{E}+00$ & $0.00 \mathrm{E}+00$ & $0.00 \mathrm{E}+00$ & $0.00 \mathrm{E}+00$ \\
\hline 2.500 & 2.250 & $0.00 \mathrm{E}+00$ & $6.78 \mathrm{E}-11$ & $1.91 \mathrm{E}+02$ & $0.00 \mathrm{E}+00$ & $0.00 \mathrm{E}+00$ & $0.00 \mathrm{E}+00$ & $0.00 \mathrm{E}+00$ \\
\hline 2.750 & 2.500 & $0.00 \mathrm{E}+00$ & $3.76 \mathrm{E}-11$ & $0.00 \mathrm{E}+00$ & $0.00 \mathrm{E}+00$ & $0.00 \mathrm{E}+00$ & $0.00 \mathrm{E}+00$ & $0.00 \mathrm{E}+00$ \\
\hline 3.000 & 2.750 & $0.00 \mathrm{E}+00$ & $3.44 \mathrm{E}-11$ & $0.00 \mathrm{E}+00$ & $0.00 \mathrm{E}+00$ & $0.00 \mathrm{E}+00$ & $0.00 \mathrm{E}+00$ & $0.00 \mathrm{E}+00$ \\
\hline 3.500 & 3.000 & $0.00 \mathrm{E}+00$ & $3.60 \mathrm{E}-11$ & $0.00 \mathrm{E}+00$ & $0.00 \mathrm{E}+00$ & $0.00 \mathrm{E}+00$ & $0.00 \mathrm{E}+00$ & $0.00 \mathrm{E}+00$ \\
\hline 4.000 & 3.500 & $0.00 \mathrm{E}+00$ & $1.81 \mathrm{E}-11$ & $0.00 \mathrm{E}+00$ & $0.00 \mathrm{E}+00$ & $0.00 \mathrm{E}+00$ & $0.00 \mathrm{E}+00$ & $0.00 \mathrm{E}+00$ \\
\hline 4.500 & 4.000 & $0.00 \mathrm{E}+00$ & $9.07 \mathrm{E}-12$ & $0.00 \mathrm{E}+00$ & $0.00 \mathrm{E}+00$ & $0.00 \mathrm{E}+00$ & $0.00 \mathrm{E}+00$ & $0.00 \mathrm{E}+00$ \\
\hline 5.000 & 4.500 & $0.00 \mathrm{E}+00$ & $4.55 \mathrm{E}-12$ & $0.00 \mathrm{E}+00$ & $0.00 \mathrm{E}+00$ & $0.00 \mathrm{E}+00$ & $0.00 \mathrm{E}+00$ & $0.00 \mathrm{E}+00$ \\
\hline 5.500 & 5.000 & $0.00 \mathrm{E}+00$ & $2.28 \mathrm{E}-12$ & $0.00 \mathrm{E}+00$ & $0.00 \mathrm{E}+00$ & $0.00 \mathrm{E}+00$ & $0.00 \mathrm{E}+00$ & $0.00 \mathrm{E}+00$ \\
\hline 6.000 & 5.500 & $0.00 \mathrm{E}+00$ & $1.14 \mathrm{E}-12$ & $0.00 \mathrm{E}+00$ & $0.00 \mathrm{E}+00$ & $0.00 \mathrm{E}+00$ & $0.00 \mathrm{E}+00$ & $0.00 \mathrm{E}+00$ \\
\hline 6.500 & 6.000 & $0.00 \mathrm{E}+00$ & $5.74 \mathrm{E}-13$ & $0.00 \mathrm{E}+00$ & $0.00 \mathrm{E}+00$ & $0.00 \mathrm{E}+00$ & $0.00 \mathrm{E}+00$ & $0.00 \mathrm{E}+00$ \\
\hline 7.000 & 6.500 & $0.00 \mathrm{E}+00$ & $2.88 \mathrm{E}-13$ & $0.00 \mathrm{E}+00$ & $0.00 \mathrm{E}+00$ & $0.00 \mathrm{E}+00$ & $0.00 \mathrm{E}+00$ & $0.00 \mathrm{E}+00$ \\
\hline 7.500 & 7.000 & $0.00 \mathrm{E}+00$ & $1.44 \mathrm{E}-13$ & $0.00 \mathrm{E}+00$ & $0.00 \mathrm{E}+00$ & $0.00 \mathrm{E}+00$ & $0.00 \mathrm{E}+00$ & $0.00 \mathrm{E}+00$ \\
\hline 8.000 & 7.500 & $0.00 \mathrm{E}+00$ & $7.24 \mathrm{E}-14$ & $0.00 \mathrm{E}+00$ & $0.00 \mathrm{E}+00$ & $0.00 \mathrm{E}+00$ & $0.00 \mathrm{E}+00$ & $0.00 \mathrm{E}+00$ \\
\hline 9.000 & 8.000 & $0.00 \mathrm{E}+00$ & $5.40 \mathrm{E}-14$ & $0.00 \mathrm{E}+00$ & $0.00 \mathrm{E}+00$ & $0.00 \mathrm{E}+00$ & $0.00 \mathrm{E}+00$ & $0.00 \mathrm{E}+00$ \\
\hline \multirow[t]{2}{*}{10.000} & 9.000 & $0.00 \mathrm{E}+00$ & $1.36 \mathrm{E}-14$ & $0.00 \mathrm{E}+00$ & $0.00 \mathrm{E}+00$ & $0.00 \mathrm{E}+00$ & $0.00 \mathrm{E}+00$ & $0.00 \mathrm{E}+00$ \\
\hline & Total & $1.18 \mathrm{E}+15$ & $1.61 \mathrm{E}+14$ & $5.93 \mathrm{E}+11$ & $4.56 \mathrm{E}+13$ & $2.93 \mathrm{E}+15$ & $1.63 \mathrm{E}+14$ & $8.31 \mathrm{E}+14$ \\
\hline
\end{tabular}


PCP-2011-0001

Revision 0

\begin{tabular}{|c|c|c|c|c|c|c|c|c|}
\hline \multicolumn{2}{|c|}{ Energy (MeV) } & \multicolumn{7}{|c|}{ Photon Source (p/s/g) } \\
\hline Upper & Lower & Ac-227 & Am-241 & Am-243 & Cd-109 & Cf-252 & Cm-244 & Cm-248 \\
\hline 0.020 & 0.010 & $1.96 \mathrm{E}+10$ & $7.58 \mathrm{E}+10$ & $1.32 \mathrm{E}+09$ & $0.00 \mathrm{E}+00$ & $1.33 \mathrm{E}+12$ & $2.89 \mathrm{E}+11$ & $1.22 \mathrm{E}+07$ \\
\hline 0.030 & 0.020 & $1.48 \mathrm{E}+08$ & $1.10 \mathrm{E}+10$ & $1.39 \mathrm{E}+08$ & $8.79 \mathrm{E}+13$ & $1.38 \mathrm{E}+11$ & $2.81 \mathrm{E}+10$ & $3.14 \mathrm{E}+06$ \\
\hline 0.040 & 0.030 & $2.04 \mathrm{E}+06$ & $1.63 \mathrm{E}+08$ & $4.76 \mathrm{E}+06$ & $0.00 \mathrm{E}+00$ & $0.00 \mathrm{E}+00$ & $6.73 \mathrm{E}+05$ & $2.16 \mathrm{E}+06$ \\
\hline 0.050 & 0.040 & $3.82 \mathrm{E}+07$ & $8.97 \mathrm{E}+07$ & $4.00 \mathrm{E}+08$ & $0.00 \mathrm{E}+00$ & $2.84 \mathrm{E}+09$ & $7.41 \mathrm{E}+08$ & $1.57 \mathrm{E}+06$ \\
\hline 0.060 & 0.050 & $0.00 \mathrm{E}+00$ & $4.91 \mathrm{E}+10$ & $9.53 \mathrm{E}+05$ & $0.00 \mathrm{E}+00$ & $0.00 \mathrm{E}+00$ & $3.51 \mathrm{E}+05$ & $1.13 \mathrm{E}+06$ \\
\hline 0.070 & 0.060 & $1.58 \mathrm{E}+08$ & $3.36 \mathrm{E}+07$ & $1.41 \mathrm{E}-01$ & $0.00 \mathrm{E}+00$ & $0.00 \mathrm{E}+00$ & $3.51 \mathrm{E}+05$ & $1.13 \mathrm{E}+06$ \\
\hline 0.080 & 0.070 & $4.15 \mathrm{E}+08$ & $1.38 \mathrm{E}+05$ & $4.85 \mathrm{E}+09$ & $0.00 \mathrm{E}+00$ & $0.00 \mathrm{E}+00$ & $3.51 \mathrm{E}+05$ & $1.13 \mathrm{E}+06$ \\
\hline 0.090 & 0.080 & $2.47 \mathrm{E}+08$ & $4.54 \mathrm{E}-02$ & $2.54 \mathrm{E}+07$ & $3.58 \mathrm{E}+12$ & $0.00 \mathrm{E}+00$ & $3.51 \mathrm{E}+05$ & $1.13 \mathrm{E}+06$ \\
\hline 0.100 & 0.090 & $9.46 \mathrm{E}+08$ & $2.80 \mathrm{E}+07$ & $1.19 \mathrm{E}+06$ & $0.00 \mathrm{E}+00$ & $0.00 \mathrm{E}+00$ & $3.51 \mathrm{E}+05$ & $1.13 \mathrm{E}+06$ \\
\hline 0.110 & 0.100 & $5.32 \mathrm{E}+07$ & $2.69 \mathrm{E}+07$ & $7.22 \mathrm{E}+05$ & $0.00 \mathrm{E}+00$ & $2.44 \mathrm{E}+09$ & $5.27 \mathrm{E}+05$ & $1.69 \mathrm{E}+06$ \\
\hline 0.120 & 0.110 & $0.00 \mathrm{E}+00$ & $1.31 \mathrm{E}+06$ & $4.22 \mathrm{E}+07$ & $0.00 \mathrm{E}+00$ & $0.00 \mathrm{E}+00$ & $5.27 \mathrm{E}+05$ & $1.69 \mathrm{E}+06$ \\
\hline 0.130 & 0.120 & $1.44 \mathrm{E}+08$ & $6.33 \mathrm{E}+06$ & $2.12 \mathrm{E}-01$ & $0.00 \mathrm{E}+00$ & $1.54 \mathrm{E}+07$ & $5.27 \mathrm{E}+05$ & $1.69 \mathrm{E}+06$ \\
\hline 0.140 & 0.130 & $7.34 \mathrm{E}+07$ & $5.89 \mathrm{E}+03$ & $2.12 \mathrm{E}-01$ & $0.00 \mathrm{E}+00$ & $0.00 \mathrm{E}+00$ & $5.27 \mathrm{E}+05$ & $1.69 \mathrm{E}+06$ \\
\hline 0.150 & 0.140 & $2.33 \mathrm{E}+08$ & $5.91 \mathrm{E}+05$ & $9.08 \mathrm{E}+06$ & $0.00 \mathrm{E}+00$ & $0.00 \mathrm{E}+00$ & $5.27 \mathrm{E}+05$ & $1.69 \mathrm{E}+06$ \\
\hline 0.160 & 0.150 & $0.00 \mathrm{E}+00$ & $1.26 \mathrm{E}+05$ & $2.12 \mathrm{E}-01$ & $0.00 \mathrm{E}+00$ & $3.97 \mathrm{E}+08$ & $5.27 \mathrm{E}+05$ & $1.69 \mathrm{E}+06$ \\
\hline 0.170 & 0.160 & $5.06 \mathrm{E}+08$ & $3.35 \mathrm{E}+05$ & $9.98 \mathrm{E}+04$ & $0.00 \mathrm{E}+00$ & $0.00 \mathrm{E}+00$ & $5.27 \mathrm{E}+05$ & $1.69 \mathrm{E}+06$ \\
\hline 0.180 & 0.170 & $1.09 \mathrm{E}+08$ & $2.28 \mathrm{E}+04$ & $2.12 \mathrm{E}-01$ & $0.00 \mathrm{E}+00$ & $0.00 \mathrm{E}+00$ & $5.27 \mathrm{E}+05$ & $1.69 \mathrm{E}+06$ \\
\hline 0.190 & 0.180 & $0.00 \mathrm{E}+00$ & $6.80 \mathrm{E}-02$ & $2.12 \mathrm{E}-01$ & $0.00 \mathrm{E}+00$ & $0.00 \mathrm{E}+00$ & $5.27 \mathrm{E}+05$ & $1.69 \mathrm{E}+06$ \\
\hline 0.200 & 0.190 & $0.00 \mathrm{E}+00$ & $4.62 \mathrm{E}+04$ & $6.82 \mathrm{E}+04$ & $0.00 \mathrm{E}+00$ & $0.00 \mathrm{E}+00$ & $5.27 \mathrm{E}+05$ & $1.69 \mathrm{E}+06$ \\
\hline 0.210 & 0.200 & $0.00 \mathrm{E}+00$ & $1.02 \mathrm{E}+06$ & & & $0.00 \mathrm{E}+00$ & $\mathrm{E}+05$ & $\mathrm{E}+06$ \\
\hline 0.220 & 0.210 & $0.00 \mathrm{E}+00$ & $6.44 \mathrm{E}-02$ & & & $\mathrm{E}+00$ & $E+05$ & $\mathrm{E}+06$ \\
\hline 0.230 & 0.220 & $3.68 \mathrm{E}+06$ & $5.17 \mathrm{E}+04$ & & & $\mathrm{E}+00$ & $E+05$ & \\
\hline 0.240 & 0.230 & $0.00 \mathrm{E}+00$ & $6.45 \mathrm{E}+03$ & 2.01 & $0.00 \mathrm{E}+00$ & $0.00 \mathrm{E}+00$ & $4.98 \mathrm{E}+05$ & $1.60 \mathrm{E}+06$ \\
\hline 0.250 & 0.240 & $0.00 \mathrm{E}+00$ & $8.38 \mathrm{E}+04$ & 2.01 & $0.00 \mathrm{E}+00$ & $0.00 \mathrm{E}+00$ & $4.98 \mathrm{E}+05$ & $\mathrm{E}+06$ \\
\hline 0.260 & 0.250 & $0.00 \mathrm{E}+00$ & $6.44 \mathrm{E}-02$ & $2.01 \mathrm{E}-01$ & $0.00 \mathrm{E}+00$ & $0.00 \mathrm{E}+00$ & $4.98 \mathrm{E}+05$ & $\mathrm{E}+06$ \\
\hline 0.270 & 0.260 & $0.00 \mathrm{E}+00$ & $4.49 \mathrm{E}+04$ & $2.01 \mathrm{E}-01$ & $0.00 \mathrm{E}+00$ & $0.00 \mathrm{E}+00$ & $4.98 \mathrm{E}+05$ & $1.60 \mathrm{E}+06$ \\
\hline 0.280 & 0.270 & $0.00 \mathrm{E}+00$ & $9.01 \mathrm{E}+03$ & $2.01 \mathrm{E}-01$ & $0.00 \mathrm{E}+00$ & $0.00 \mathrm{E}+00$ & $4.98 \mathrm{E}+05$ & $1.60 \mathrm{E}+06$ \\
\hline 0.290 & 0.280 & $0.00 \mathrm{E}+00$ & $6.44 \mathrm{E}-02$ & $2.01 \mathrm{E}-01$ & $0.00 \mathrm{E}+00$ & $0.00 \mathrm{E}+00$ & $4.98 \mathrm{E}+05$ & $1.60 \mathrm{E}+06$ \\
\hline 0.300 & 0.290 & $0.00 \mathrm{E}+00$ & $9.11 \mathrm{E}+04$ & $2.01 \mathrm{E}-01$ & $0.00 \mathrm{E}+00$ & $0.00 \mathrm{E}+00$ & $4.98 \mathrm{E}+05$ & $1.60 \mathrm{E}+06$ \\
\hline 0.325 & 0.300 & $3.77 \mathrm{E}+06$ & $2.37 \mathrm{E}+05$ & $5.73 \mathrm{E}-01$ & $0.00 \mathrm{E}+00$ & $0.00 \mathrm{E}+00$ & $1.42 \mathrm{E}+06$ & $4.58 \mathrm{E}+06$ \\
\hline 0.350 & 0.325 & $0.00 \mathrm{E}+00$ & $8.43 \mathrm{E}+05$ & $5.73 \mathrm{E}-01$ & $0.00 \mathrm{E}+00$ & $0.00 \mathrm{E}+00$ & $1.42 \mathrm{E}+06$ & $4.58 \mathrm{E}+06$ \\
\hline 0.375 & 0.350 & $1.72 \mathrm{E}+07$ & $3.65 \mathrm{E}+05$ & $5.73 \mathrm{E}-01$ & $0.00 \mathrm{E}+00$ & $0.00 \mathrm{E}+00$ & $1.42 \mathrm{E}+06$ & $4.58 \mathrm{E}+06$ \\
\hline 0.400 & 0.375 & $0.00 \mathrm{E}+00$ & $2.13 \mathrm{E}+05$ & $5.73 \mathrm{E}-01$ & $0.00 \mathrm{E}+00$ & $0.00 \mathrm{E}+00$ & $1.42 \mathrm{E}+06$ & $4.58 \mathrm{E}+06$ \\
\hline 0.425 & 0.400 & $0.00 \mathrm{E}+00$ & $3.77 \mathrm{E}+04$ & $5.75 \mathrm{E}-01$ & $0.00 \mathrm{E}+00$ & $0.00 \mathrm{E}+00$ & $1.43 \mathrm{E}+06$ & $4.60 \mathrm{E}+06$ \\
\hline 0.450 & 0.425 & $0.00 \mathrm{E}+00$ & $3.89 \mathrm{E}+04$ & $5.75 \mathrm{E}-01$ & $0.00 \mathrm{E}+00$ & $0.00 \mathrm{E}+00$ & $1.43 \mathrm{E}+06$ & $4.60 \mathrm{E}+06$ \\
\hline 0.475 & 0.450 & $0.00 \mathrm{E}+00$ & $2.35 \mathrm{E}+04$ & $5.75 \mathrm{E}-01$ & $0.00 \mathrm{E}+00$ & $0.00 \mathrm{E}+00$ & $1.43 \mathrm{E}+06$ & $4.60 \mathrm{E}+06$ \\
\hline 0.500 & 0.475 & $0.00 \mathrm{E}+00$ & $1.27 \mathrm{E}+03$ & $5.75 \mathrm{E}-01$ & $0.00 \mathrm{E}+00$ & $0.00 \mathrm{E}+00$ & $1.43 \mathrm{E}+06$ & $4.60 \mathrm{E}+06$ \\
\hline 0.525 & 0.500 & $0.00 \mathrm{E}+00$ & $5.89 \mathrm{E}+03$ & $5.45 \mathrm{E}-01$ & $0.00 \mathrm{E}+00$ & $0.00 \mathrm{E}+00$ & $1.35 \mathrm{E}+06$ & $4.35 \mathrm{E}+06$ \\
\hline 0.550 & 0.525 & & & & & $0.00 \mathrm{E}+00$ & $1.35 \mathrm{E}+06$ & $4.35 \mathrm{E}+06$ \\
\hline 0.575 & 0.550 & $0.00 \mathrm{E}+00$ & $2.18 \mathrm{E}+03$ & & $0.00 \mathrm{E}+00$ & $0.00 \mathrm{E}+00$ & $1.35 \mathrm{E}+06$ & $4.35 \mathrm{E}+06$ \\
\hline 0.600 & 0.575 & $0.00 \mathrm{E}+00$ & $1.36 \mathrm{E}+04$ & $5.45 \mathrm{E}-01$ & $0.00 \mathrm{E}+00$ & $0.00 \mathrm{E}+00$ & $1.35 \mathrm{E}+06$ & $4.35 \mathrm{E}+06$ \\
\hline 0.625 & 0.600 & $0.00 \mathrm{E}+00$ & $6.50 \mathrm{E}+04$ & $2.32 \mathrm{E}+04$ & $0.00 \mathrm{E}+00$ & $0.00 \mathrm{E}+00$ & $1.21 \mathrm{E}+06$ & $3.90 \mathrm{E}+06$ \\
\hline 0.650 & 0.625 & $0.00 \mathrm{E}+00$ & $1.01 \mathrm{E}+04$ & $4.88 \mathrm{E}-01$ & $0.00 \mathrm{E}+00$ & $0.00 \mathrm{E}+00$ & $1.21 \mathrm{E}+06$ & $3.90 \mathrm{E}+06$ \\
\hline 0.675 & 0.650 & $0.00 \mathrm{E}+00$ & $4.21 \mathrm{E}+04$ & $7.22 \mathrm{E}+04$ & $0.00 \mathrm{E}+00$ & $0.00 \mathrm{E}+00$ & $1.21 \mathrm{E}+06$ & $3.90 \mathrm{E}+06$ \\
\hline 0.700 & 0.675 & $0.00 \mathrm{E}+00$ & $5.30 \mathrm{E}+04$ & $4.88 \mathrm{E}-01$ & $0.00 \mathrm{E}+00$ & $0.00 \mathrm{E}+00$ & $1.21 \mathrm{E}+06$ & $3.90 \mathrm{E}+06$ \\
\hline 0.725 & 0.700 & $0.00 \mathrm{E}+00$ & $2.51 \mathrm{E}+05$ & $4.02 \mathrm{E}-01$ & $0.00 \mathrm{E}+00$ & $0.00 \mathrm{E}+00$ & $9.99 \mathrm{E}+05$ & $3.21 \mathrm{E}+06$ \\
\hline 0.750 & 0.725 & $0.00 \mathrm{E}+00$ & $1.22 \mathrm{E}+04$ & $4.02 \mathrm{E}-01$ & $0.00 \mathrm{E}+00$ & $0.00 \mathrm{E}+00$ & $9.99 \mathrm{E}+05$ & $3.21 \mathrm{E}+06$ \\
\hline
\end{tabular}


PCP-2011-0001

Revision 0

\begin{tabular}{|c|c|c|c|c|c|c|c|c|}
\hline \multicolumn{9}{|c|}{ Table 3-18. Photon Source from RASTA (Decay Time $=0$ days) } \\
\hline \multicolumn{2}{|c|}{ Energy (MeV) } & \multicolumn{7}{|c|}{ Photon Source $(\mathbf{p} / \mathbf{s} / \mathrm{g})$} \\
\hline Upper & Lower & Ac-227 & Am-241 & Am-243 & Cd-109 & Cf-252 & Cm-244 & Cm-248 \\
\hline 0.775 & 0.750 & $0.00 \mathrm{E}+00$ & $2.79 \mathrm{E}+04$ & 4.02E-01 & $0.00 \mathrm{E}+00$ & $0.00 \mathrm{E}+00$ & $9.99 \mathrm{E}+05$ & $3.21 \mathrm{E}+06$ \\
\hline 0.800 & 0.775 & $0.00 \mathrm{E}+00$ & $8.86 \mathrm{E}+02$ & $4.02 \mathrm{E}-01$ & $0.00 \mathrm{E}+00$ & $0.00 \mathrm{E}+00$ & $9.99 \mathrm{E}+05$ & $3.21 \mathrm{E}+06$ \\
\hline 0.825 & 0.800 & $0.00 \mathrm{E}+00$ & $3.18 \mathrm{E}+03$ & $3.43 \mathrm{E}-01$ & $0.00 \mathrm{E}+00$ & $0.00 \mathrm{E}+00$ & $8.51 \mathrm{E}+05$ & \\
\hline 0.850 & 0.825 & $0.00 \mathrm{E}+00$ & $3.01 \mathrm{E}+02$ & $3.43 \mathrm{E}-01$ & $0.00 \mathrm{E}+00$ & $0.00 \mathrm{E}+00$ & $8.51 \mathrm{E}+05$ & \\
\hline 0.875 & 0.850 & $0.00 \mathrm{E}+00$ & $2.40 \mathrm{E}+03$ & $3.43 \mathrm{E}-01$ & $0.00 \mathrm{E}+00$ & $0.00 \mathrm{E}+00$ & $8.51 \mathrm{E}+05$ & \\
\hline 0.900 & 0.875 & $0.00 \mathrm{E}+00$ & $3.72 \mathrm{E}+02$ & $3.43 \mathrm{E}-01$ & $0.00 \mathrm{E}+00$ & $0.00 \mathrm{E}+00$ & $8.51 \mathrm{E}+05$ & $2.74 \mathrm{E}+06$ \\
\hline 0.925 & 0.900 & $0.00 \mathrm{E}+00$ & $9.37 \mathrm{E}+02$ & $3.43 \mathrm{E}-01$ & $0.00 \mathrm{E}+00$ & $0.00 \mathrm{E}+00$ & $8.51 \mathrm{E}+05$ & $2.74 \mathrm{E}+06$ \\
\hline 0.950 & 0.925 & $0.00 \mathrm{E}+00$ & $1.41 \mathrm{E}+02$ & $3.43 \mathrm{E}-01$ & $0.00 \mathrm{E}+00$ & $0.00 \mathrm{E}+00$ & $8.51 \mathrm{E}+05$ & $2.74 \mathrm{E}+06$ \\
\hline 0.975 & 0.950 & $0.00 \mathrm{E}+00$ & $7.31 \mathrm{E}+02$ & $3.43 \mathrm{E}-01$ & $0.00 \mathrm{E}+00$ & $0.00 \mathrm{E}+00$ & $8.51 \mathrm{E}+05$ & \\
\hline 1.000 & 0.975 & $.00 \mathrm{E}+00$ & & $3.43 \mathrm{E}-01$ & & $0.00 \mathrm{E}+00$ & $8.51 \mathrm{E}+05$ & \\
\hline 1.250 & 1.000 & $0.00 \mathrm{E}+00$ & $5.76 \mathrm{E}-01$ & $1.80 \mathrm{E}+00$ & $0.00 \mathrm{E}+00$ & $0.00 \mathrm{E}+00$ & $4.46 \mathrm{E}+06$ & $1.44 \mathrm{E}+07$ \\
\hline 1.500 & 1.250 & $0.00 \mathrm{E}+00$ & $5.47 \mathrm{E}-01$ & $1.71 \mathrm{E}+00$ & $0.00 \mathrm{E}+00$ & $0.00 \mathrm{E}+00$ & $4.24 \mathrm{E}+06$ & $1.36 \mathrm{E}+07$ \\
\hline 1.750 & 1.500 & $0.00 \mathrm{E}+00$ & $2.89 \mathrm{E}-01$ & $9.01 \mathrm{E}-01$ & $0.00 \mathrm{E}+00$ & $0.00 \mathrm{E}+00$ & $2.24 \mathrm{E}+06$ & $7.20 \mathrm{E}+06$ \\
\hline 2.000 & 1.750 & $0.00 \mathrm{E}+00$ & $2.26 \mathrm{E}-01$ & $7.05 \mathrm{E}-01$ & $0.00 \mathrm{E}+00$ & $0.00 \mathrm{E}+00$ & $1.75 \mathrm{E}+06$ & $5.63 \mathrm{E}+06$ \\
\hline 2.250 & 2.000 & $0.00 \mathrm{E}+00$ & $1.54 \mathrm{E}-01$ & $4.80 \mathrm{E}-01$ & $0.00 \mathrm{E}+00$ & $0.00 \mathrm{E}+00$ & $1.19 \mathrm{E}+06$ & \\
\hline 2.500 & 2.250 & $0.00 \mathrm{E}+00$ & $1.43 \mathrm{E}-01$ & $4.44 \mathrm{E}-01$ & $0.00 \mathrm{E}+00$ & $0.00 \mathrm{E}+00$ & $1.10 \mathrm{E}+06$ & $3.55 \mathrm{E}+06$ \\
\hline 2.750 & 2.500 & $0.00 \mathrm{E}+00$ & $1.03 \mathrm{E}-01$ & $3.20 \mathrm{E}-01$ & $0.00 \mathrm{E}+00$ & $0.00 \mathrm{E}+00$ & $7.94 \mathrm{E}+05$ & $2.56 \mathrm{E}+06$ \\
\hline 3.000 & 2.750 & $0.00 \mathrm{E}+00$ & $8.49 \mathrm{E}-02$ & $2.65 \mathrm{E}-01$ & $0.00 \mathrm{E}+00$ & $0.00 \mathrm{E}+00$ & $6.58 \mathrm{E}+05$ & $2.12 \mathrm{E}+06$ \\
\hline 3.500 & 3.000 & $0.00 \mathrm{E}+00$ & $8.70 \mathrm{E}-02$ & $2.71 \mathrm{E}-01$ & $0.00 \mathrm{E}+00$ & $0.00 \mathrm{E}+00$ & $6.73 \mathrm{E}+05$ & $2.17 \mathrm{E}+06$ \\
\hline 4.000 & 3.500 & $0.00 \mathrm{E}+00$ & $5.90 \mathrm{E}-02$ & $1.84 \mathrm{E}-01$ & $0.00 \mathrm{E}+00$ & $0.00 \mathrm{E}+00$ & $4.57 \mathrm{E}+05$ & $1.47 \mathrm{E}+06$ \\
\hline 4.500 & 4.000 & $0.00 \mathrm{E}+00$ & $2.18 \mathrm{E}-02$ & $6.79 \mathrm{E}-02$ & $0.00 \mathrm{E}+00$ & $0.00 \mathrm{E}+00$ & $1.69 \mathrm{E}+05$ & $5.43 \mathrm{E}+05$ \\
\hline 5.000 & 4.500 & $0.00 \mathrm{E}+00$ & $1.72 \mathrm{E}-02$ & $5.35 \mathrm{E}-02$ & $0.00 \mathrm{E}+00$ & $0.00 \mathrm{E}+00$ & $1.33 \mathrm{E}+05$ & $4.27 \mathrm{E}+05$ \\
\hline 5.500 & 5.000 & $0.00 \mathrm{E}+00$ & $7.16 \mathrm{E}-03$ & $2.23 \mathrm{E}-02$ & $0.00 \mathrm{E}+00$ & $0.00 \mathrm{E}+00$ & $5.55 \mathrm{E}+04$ & $3 \mathrm{E}+05$ \\
\hline 6.000 & 5.500 & $0.00 \mathrm{E}+00$ & $6.48 \mathrm{E}-03$ & $2.02 \mathrm{E}-02$ & $0.00 \mathrm{E}+00$ & $0.00 \mathrm{E}+00$ & $5.02 \mathrm{E}+04$ & $1.61 \mathrm{E}+05$ \\
\hline 6.500 & 6.000 & $0.00 \mathrm{E}+00$ & $3.01 \mathrm{E}-03$ & $9.37 \mathrm{E}-03$ & $0.00 \mathrm{E}+00$ & $0.00 \mathrm{E}+00$ & $2.33 \mathrm{E}+04$ & $7.49 \mathrm{E}+04$ \\
\hline 7.000 & 6.500 & $0.00 \mathrm{E}+00$ & $2.84 \mathrm{E}-03$ & $8.84 \mathrm{E}-03$ & $0.00 \mathrm{E}+00$ & $0.00 \mathrm{E}+00$ & $2.20 \mathrm{E}+04$ & $7.06 \mathrm{E}+04$ \\
\hline 7.500 & 7.000 & $0.00 \mathrm{E}+00$ & $2.62 \mathrm{E}-04$ & $8.15 \mathrm{E}-04$ & $0.00 \mathrm{E}+00$ & $0.00 \mathrm{E}+00$ & $2.03 \mathrm{E}+03$ & $6.52 \mathrm{E}+03$ \\
\hline 8.000 & 7.500 & $0.00 \mathrm{E}+00$ & $2.59 \mathrm{E}-04$ & $8.06 \mathrm{E}-04$ & $0.00 \mathrm{E}+00$ & $0.00 \mathrm{E}+00$ & $2.00 \mathrm{E}+03$ & $6.44 \mathrm{E}+03$ \\
\hline 9.000 & 8.000 & $0.00 \mathrm{E}+00$ & $5.16 \mathrm{E}-04$ & $1.61 \mathrm{E}-03$ & $0.00 \mathrm{E}+00$ & $0.00 \mathrm{E}+00$ & $3.99 \mathrm{E}+03$ & $1.28 \mathrm{E}+04$ \\
\hline \multirow[t]{2}{*}{10.000} & 9.000 & $0.00 \mathrm{E}+00$ & $5.15 \mathrm{E}-04$ & $1.61 \mathrm{E}-03$ & $0.00 \mathrm{E}+00$ & $0.00 \mathrm{E}+00$ & $3.99 \mathrm{E}+03$ & $1.28 \mathrm{E}+04$ \\
\hline & Total & $2.27 \mathrm{E}+10$ & $1.36 \mathrm{E}+11$ & $6.80 \mathrm{E}+09$ & $9.15 \mathrm{E}+13$ & $1.47 \mathrm{E}+12$ & $3.18 \mathrm{E}+11$ & $2.20 \mathrm{E}+08$ \\
\hline
\end{tabular}


PCP-2011-0001

Revision 0

\begin{tabular}{|c|c|c|c|c|c|c|c|c|}
\hline \multicolumn{9}{|c|}{ Table 3-19. Photon Source from RASTA (Decay Time $=0$ days) } \\
\hline \multicolumn{2}{|c|}{ Energy (MeV) } & \multicolumn{7}{|c|}{ Photon Source (p/s/g) } \\
\hline Upper & Lower & Co-60 & Cs-137 & \begin{tabular}{|l|} 
Eu-152 \\
\end{tabular} & Fe-59 & Gd-153 & Hf-181 & Ho-166m \\
\hline 0.020 & 0.010 & $1.75 \mathrm{E}+11$ & $6.73 \mathrm{E}+10$ & $7.38 \mathrm{E}+10$ & $9.61 \mathrm{E}+12$ & $2.00 \mathrm{E}+10$ & $1.51 \mathrm{E}+13$ & $2.72 \mathrm{E}+08$ \\
\hline 0.030 & 0.020 & $8.05 \mathrm{E}+10$ & $3.31 \mathrm{E}+10$ & $3.74 \mathrm{E}+10$ & $4.53 \mathrm{E}+12$ & $0.00 \mathrm{E}+00$ & $4.71 \mathrm{E}+12$ & $1.26 \mathrm{E}+08$ \\
\hline 0.040 & 0.030 & $4.56 \mathrm{E}+10$ & $2.02 \mathrm{E}+10$ & $1.59 \mathrm{E}+12$ & $2.64 \mathrm{E}+12$ & $0.00 \mathrm{E}+00$ & $2.76 \mathrm{E}+12$ & $7.48 \mathrm{E}+07$ \\
\hline 0.050 & 0.040 & $2.85 \mathrm{E}+10$ & $1.36 \mathrm{E}+10$ & $3.31 \mathrm{E}+12$ & $1.70 \mathrm{E}+12$ & $1.50 \mathrm{E}+14$ & $1.79 \mathrm{E}+12$ & $2.28 \mathrm{E}+10$ \\
\hline 0.060 & 0.050 & $1.89 \mathrm{E}+10$ & $9.74 \mathrm{E}+09$ & $1.20 \mathrm{E}+10$ & $1.17 \mathrm{E}+12$ & $0.00 \mathrm{E}+00$ & $1.72 \mathrm{E}+14$ & $5.47 \mathrm{E}+09$ \\
\hline 0.070 & 0.060 & $1.30 \mathrm{E}+10$ & $7.27 \mathrm{E}+09$ & $9.20 \mathrm{E}+09$ & $8.34 \mathrm{E}+11$ & $3.39 \mathrm{E}+12$ & $4.47 \mathrm{E}+13$ & $2.83 \mathrm{E}+07$ \\
\hline 0.080 & 0.070 & $9.17 \mathrm{E}+09$ & $5.59 \mathrm{E}+09$ & $7.29 \mathrm{E}+09$ & $6.12 \mathrm{E}+11$ & $1.01 \mathrm{E}+11$ & $6.45 \mathrm{E}+11$ & $2.24 \mathrm{E}+07$ \\
\hline 0.090 & 0.080 & $6.57 \mathrm{E}+09$ & $4.39 \mathrm{E}+09$ & $5.91 \mathrm{E}+09$ & $4.59 \mathrm{E}+11$ & $3.58 \mathrm{E}+11$ & $4.83 \mathrm{E}+11$ & $8.04 \mathrm{E}+09$ \\
\hline 0.100 & 0.090 & $4.75 \mathrm{E}+09$ & $3.50 \mathrm{E}+09$ & $4.87 \mathrm{E}+09$ & $3.49 \mathrm{E}+11$ & $3.96 \mathrm{E}+13$ & $3.67 \mathrm{E}+11$ & $1.09 \mathrm{E}+08$ \\
\hline 0.110 & 0.100 & $3.47 \mathrm{E}+09$ & $2.83 \mathrm{E}+09$ & $4.08 \mathrm{E}+09$ & $2.69 \mathrm{E}+11$ & $2.71 \mathrm{E}+13$ & $2.81 \mathrm{E}+11$ & $1.25 \mathrm{E}+07$ \\
\hline 0.120 & 0.110 & $2.54 \mathrm{E}+09$ & $2.31 \mathrm{E}+09$ & $3.45 \mathrm{E}+09$ & $2.09 \mathrm{E}+11$ & $0.00 \mathrm{E}+00$ & $2.17 \mathrm{E}+11$ & $1.37 \mathrm{E}+08$ \\
\hline 0.130 & 0.120 & $1.86 \mathrm{E}+09$ & $1.90 \mathrm{E}+09$ & $1.81 \mathrm{E}+12$ & $1.64 \mathrm{E}+11$ & $0.00 \mathrm{E}+00$ & $1.69 \mathrm{E}+11$ & $1.80 \mathrm{E}+08$ \\
\hline 0.140 & 0.130 & $1.36 \mathrm{E}+09$ & $1.57 \mathrm{E}+09$ & $2.54 \mathrm{E}+09$ & $1.29 \mathrm{E}+11$ & $0.00 \mathrm{E}+00$ & $3.17 \mathrm{E}+14$ & $7.56 \mathrm{E}+07$ \\
\hline 0.150 & 0.140 & $9.92 \mathrm{E}+08$ & $1.31 \mathrm{E}+09$ & $4.84 \mathrm{E}+09$ & $1.82 \mathrm{E}+13$ & $0.00 \mathrm{E}+00$ & $1.03 \mathrm{E}+11$ & $3.50 \mathrm{E}+07$ \\
\hline 0.160 & 0.150 & $7.21 \mathrm{E}+08$ & $1.10 \mathrm{E}+09$ & $1.92 \mathrm{E}+09$ & $8.14 \mathrm{E}+10$ & $0.00 \mathrm{E}+00$ & $8.05 \mathrm{E}+10$ & $5.85 \mathrm{E}+06$ \\
\hline 0.170 & 0.160 & $5.20 \mathrm{E}+08$ & $9.24 \mathrm{E}+08$ & $1.69 \mathrm{E}+09$ & $6.50 \mathrm{E}+10$ & $0.00 \mathrm{E}+00$ & $6.30 \mathrm{E}+10$ & $1.43 \mathrm{E}+08$ \\
\hline 0.180 & 0.170 & $3.73 \mathrm{E}+08$ & $7.79 \mathrm{E}+08$ & $1.51 \mathrm{E}+09$ & $5.21 \mathrm{E}+10$ & $5.79 \mathrm{E}+10$ & $4.92 \mathrm{E}+10$ & $4.51 \mathrm{E}+06$ \\
\hline 0.190 & 0.180 & $2.64 \mathrm{E}+08$ & $6.59 \mathrm{E}+08$ & $1.32 \mathrm{E}+09$ & $4.18 \mathrm{E}+10$ & $0.00 \mathrm{E}+00$ & $3.83 \mathrm{E}+10$ & $4.94 \mathrm{E}+10$ \\
\hline 0.200 & 0.190 & $1.85 \mathrm{E}+08$ & $5.58 \mathrm{E}+08$ & $1.61 \mathrm{E}+09$ & $5.45 \mathrm{E}+13$ & $0.00 \mathrm{E}+00$ & $2.97 \mathrm{E}+10$ & $1.49 \mathrm{E}+08$ \\
\hline 0.210 & 0.200 & $1.29 \mathrm{E}+08$ & $4.74 \mathrm{E}+08$ & $1.87 \mathrm{E}+09$ & $2.72 \mathrm{E}+10$ & $0.00 \mathrm{E}+00$ & $2.30 \mathrm{E}+10$ & $3.15 \mathrm{E}+06$ \\
\hline 0.220 & 0.210 & $8.82 \mathrm{E}+07$ & $4.03 \mathrm{E}+08$ & $2.20 \mathrm{E}+09$ & $2.19 \mathrm{E}+10$ & $0.00 \mathrm{E}+00$ & $1.77 \mathrm{E}+10$ & $2.14 \mathrm{E}+09$ \\
\hline 0.230 & 0.220 & $5.99 \mathrm{E}+07$ & $3.43 \mathrm{E}+08$ & $8.39 \mathrm{E}+08$ & $1.77 \mathrm{E}+10$ & $0.00 \mathrm{E}+00$ & $1.35 \mathrm{E}+10$ & $2.52 \mathrm{E}+06$ \\
\hline 0.240 & 0.230 & $4.05 \mathrm{E}+07$ & $2.93 \mathrm{E}+08$ & $1.37 \mathrm{E}+09$ & $1.43 \mathrm{E}+10$ & $0.00 \mathrm{E}+00$ & $1.02 \mathrm{E}+10$ & $1.62 \mathrm{E}+08$ \\
\hline 0.250 & 0.240 & $2.76 \mathrm{E}+07$ & $2.50 \mathrm{E}+08$ & $4.90 \mathrm{E}+11$ & $1.16 \mathrm{E}+10$ & $0.00 \mathrm{E}+00$ & $7.66 \mathrm{E}+09$ & $2.04 \mathrm{E}+06$ \\
\hline 0.260 & 0.250 & $1.91 \mathrm{E}+07$ & $2.13 \mathrm{E}+08$ & $4.65 \mathrm{E}+09$ & $9.36 \mathrm{E}+09$ & $0.00 \mathrm{E}+00$ & $5.69 \mathrm{E}+09$ & $7.61 \mathrm{E}+08$ \\
\hline 0.270 & 0.260 & $1.38 \mathrm{E}+07$ & $1.82 \mathrm{E}+08$ & $1.10 \mathrm{E}+09$ & $7.56 \mathrm{E}+09$ & $0.00 \mathrm{E}+00$ & $4.17 \mathrm{E}+09$ & $1.66 \mathrm{E}+06$ \\
\hline 0.280 & 0.270 & $1.06 \mathrm{E}+07$ & $1.56 \mathrm{E}+08$ & $7.31 \mathrm{E}+09$ & $6.10 \mathrm{E}+09$ & $0.00 \mathrm{E}+00$ & $3.01 \mathrm{E}+09$ & $1.50 \mathrm{E}+06$ \\
\hline 0.290 & 0.280 & $8.73 \mathrm{E}+06$ & $1.34 \mathrm{E}+08$ & $1.19 \mathrm{E}+09$ & $4.91 \mathrm{E}+09$ & $0.00 \mathrm{E}+00$ & $2.13 \mathrm{E}+09$ & $1.99 \mathrm{E}+10$ \\
\hline 0.300 & 0.290 & $7.61 \mathrm{E}+06$ & $1.15 \mathrm{E}+08$ & $2.86 \mathrm{E}+10$ & $3.95 \mathrm{E}+09$ & $0.00 \mathrm{E}+00$ & $1.48 \mathrm{E}+09$ & $1.24 \mathrm{E}+06$ \\
\hline 0.325 & 0.300 & $1.63 \mathrm{E}+07$ & $2.21 \mathrm{E}+08$ & $9.59 \mathrm{E}+09$ & $6.84 \mathrm{E}+09$ & $0.00 \mathrm{E}+00$ & $1.89 \mathrm{E}+09$ & $2.45 \mathrm{E}+09$ \\
\hline 0.350 & 0.325 & $3.28 \mathrm{E}+09$ & $1.52 \mathrm{E}+08$ & $1.78 \mathrm{E}+12$ & $4.93 \mathrm{E}+12$ & $0.00 \mathrm{E}+00$ & $9.04 \mathrm{E}+13$ & $1.18 \mathrm{E}+08$ \\
\hline 0.375 & 0.350 & $1.10 \mathrm{E}+07$ & $1.05 \mathrm{E}+08$ & $5.87 \mathrm{E}+10$ & $2.45 \mathrm{E}+09$ & $0.00 \mathrm{E}+00$ & $1.29 \mathrm{E}+08$ & $1.72 \mathrm{E}+09$ \\
\hline 0.400 & 0.375 & $9.27 \mathrm{E}+06$ & $7.58 \mathrm{E}+07$ & $1.12 \mathrm{E}+09$ & $3.64 \mathrm{E}+11$ & $0.00 \mathrm{E}+00$ & $2.75 \mathrm{E}+07$ & $1.35 \mathrm{E}+06$ \\
\hline 0.425 & 0.400 & $7.71 \mathrm{E}+06$ & $5.51 \mathrm{E}+07$ & $1.53 \mathrm{E}+11$ & $1.24 \mathrm{E}+09$ & $0.00 \mathrm{E}+00$ & $1.01 \mathrm{E}+07$ & $7.83 \mathrm{E}+09$ \\
\hline 0.450 & 0.425 & $7.92 \mathrm{E}+06$ & $4.04 \mathrm{E}+07$ & $2.08 \mathrm{E}+11$ & $1.26 \mathrm{E}+09$ & $0.00 \mathrm{E}+00$ & $2.22 \mathrm{E}+06$ & $8.57 \mathrm{E}+05$ \\
\hline 0.475 & 0.450 & $5.51 \mathrm{E}+06$ & $3.32 \mathrm{E}+07$ & $2.65 \mathrm{E}+08$ & $8.57 \mathrm{E}+08$ & $0.00 \mathrm{E}+00$ & $1.77 \mathrm{E}+06$ & $2.86 \mathrm{E}+09$ \\
\hline 0.500 & 0.475 & $4.63 \mathrm{E}+06$ & $2.63 \mathrm{E}+07$ & $3.28 \mathrm{E}+10$ & $7.26 \mathrm{E}+08$ & $0.00 \mathrm{E}+00$ & $5.38 \mathrm{E}+14$ & $5.83 \mathrm{E}+05$ \\
\hline 0.525 & 0.500 & $3.95 \mathrm{E}+06$ & $2.14 \mathrm{E}+07$ & $1.79 \mathrm{E}+10$ & $6.24 \mathrm{E}+08$ & $0.00 \mathrm{E}+00$ & $2.13 \mathrm{E}+05$ & $4.80 \mathrm{E}+05$ \\
\hline 0.550 & 0.525 & $3.34 \mathrm{E}+06$ & $1.71 \mathrm{E}+07$ & $4.06 \mathrm{E}+09$ & $5.31 \mathrm{E}+08$ & $0.00 \mathrm{E}+00$ & $1.31 \mathrm{E}+05$ & $6.81 \mathrm{E}+09$ \\
\hline 0.575 & 0.550 & $2.86 \mathrm{E}+06$ & $1.39 \mathrm{E}+07$ & $4.27 \mathrm{E}+10$ & $4.58 \mathrm{E}+08$ & $0.00 \mathrm{E}+00$ & $1.01 \mathrm{E}+05$ & $3.95 \mathrm{E}+09$ \\
\hline 0.600 & 0.575 & $2.43 \mathrm{E}+06$ & $1.12 \mathrm{E}+07$ & $1.08 \mathrm{E}+08$ & $3.93 \mathrm{E}+08$ & $0.00 \mathrm{E}+00$ & $7.66 \mathrm{E}+04$ & $4.80 \mathrm{E}+08$ \\
\hline 0.625 & 0.600 & $2.06 \mathrm{E}+06$ & $8.84 \mathrm{E}+06$ & $6.79 \mathrm{E}+08$ & $3.35 \mathrm{E}+08$ & $0.00 \mathrm{E}+00$ & $1.77 \mathrm{E}+12$ & $9.40 \mathrm{E}+08$ \\
\hline 0.650 & 0.625 & $1.76 \mathrm{E}+06$ & $7.10 \mathrm{E}+06$ & $4.62 \mathrm{E}+08$ & $2.89 \mathrm{E}+08$ & $0.00 \mathrm{E}+00$ & $4.29 \mathrm{E}+04$ & $2.32 \mathrm{E}+08$ \\
\hline 0.675 & 0.650 & $1.49 \mathrm{E}+06$ & $5.57 \mathrm{E}+06$ & $1.64 \mathrm{E}+10$ & $2.47 \mathrm{E}+08$ & $0.00 \mathrm{E}+00$ & $3.11 \mathrm{E}+04$ & $3.96 \mathrm{E}+09$ \\
\hline 0.700 & 0.675 & $1.27 \mathrm{E}+06$ & $4.38 \mathrm{E}+06$ & $3.19 \mathrm{E}+10$ & $2.12 \mathrm{E}+08$ & $0.00 \mathrm{E}+00$ & $2.25 \mathrm{E}+04$ & $1.04 \mathrm{E}+09$ \\
\hline 0.725 & 0.700 & $1.08 \mathrm{E}+06$ & $3.45 \mathrm{E}+06$ & $2.81 \mathrm{E}+10$ & $1.83 \mathrm{E}+08$ & $0.00 \mathrm{E}+00$ & $1.62 \mathrm{E}+04$ & $3.97 \mathrm{E}+10$ \\
\hline 0.750 & 0.725 & $9.11 \mathrm{E}+05$ & $2.64 \mathrm{E}+06$ & $1.14 \mathrm{E}+09$ & $1.56 \mathrm{E}+08$ & $0.00 \mathrm{E}+00$ & $1.11 \mathrm{E}+04$ & $6.77 \mathrm{E}+07$ \\
\hline
\end{tabular}


PCP-2011-0001

Revision 0

\begin{tabular}{|c|c|c|c|c|c|c|c|c|}
\hline \multicolumn{9}{|c|}{ Table 3-19. Photon Source from RASTA (Decay Time $=0$ days) } \\
\hline \multicolumn{2}{|c|}{ Energy (MeV) } & \multicolumn{7}{|c|}{ Photon Source $(\mathbf{p} / \mathbf{s} / \mathrm{g})$} \\
\hline Upper & Lower & Co-60 & Cs-137 & Eu-152 & Fe-59 & Gd-153 & Hf-181 & Ho-166m \\
\hline 0.775 & 0.750 & $7.75 \mathrm{E}+05$ & $2.04 \mathrm{E}+06$ & $1.78 \mathrm{E}+10$ & $1.34 \mathrm{E}+08$ & $0.00 \mathrm{E}+00$ & $7.64 \mathrm{E}+03$ & $8.75 \mathrm{E}+09$ \\
\hline 0.800 & 0.775 & $6.50 \mathrm{E}+05$ & $1.53 \mathrm{E}+06$ & $8.39 \mathrm{E}+11$ & $1.14 \mathrm{E}+08$ & $0.00 \mathrm{E}+00$ & $4.93 \mathrm{E}+03$ & $2.21 \mathrm{E}+09$ \\
\hline 0.825 & 0.800 & $5.51 \mathrm{E}+05$ & $1.16 \mathrm{E}+06$ & $2.18 \mathrm{E}+10$ & $9.75 \mathrm{E}+07$ & $0.00 \mathrm{E}+00$ & $3.17 \mathrm{E}+03$ & $4.24 \mathrm{E}+10$ \\
\hline 0.850 & 0.825 & $3.14 \mathrm{E}+09$ & $8.49 \mathrm{E}+05$ & $1.18 \mathrm{E}+10$ & $8.29 \mathrm{E}+07$ & $0.00 \mathrm{E}+00$ & $1.90 \mathrm{E}+03$ & \\
\hline 0.875 & 0.850 & $3.84 \mathrm{E}+05$ & $6.04 \mathrm{E}+05$ & $2.77 \mathrm{E}+11$ & $6.99 \mathrm{E}+07$ & $0.00 \mathrm{E}+00$ & $1.05 \mathrm{E}+03$ & \\
\hline 0.900 & 0.875 & $3.22 \mathrm{E}+05$ & $4.32 \mathrm{E}+05$ & $1.24 \mathrm{E}+07$ & $5.95 \mathrm{E}+07$ & $0.00 \mathrm{E}+00$ & $5.56 \mathrm{E}+02$ & $5.28 \mathrm{E}+08$ \\
\hline 0.925 & 0.900 & $2.65 \mathrm{E}+05$ & $2.92 \mathrm{E}+05$ & $3.56 \mathrm{E}+10$ & $4.98 \mathrm{E}+07$ & $0.00 \mathrm{E}+00$ & $2.47 \mathrm{E}+02$ & $1.39 \mathrm{E}+04$ \\
\hline 0.950 & 0.925 & $2.18 \mathrm{E}+05$ & $1.94 \mathrm{E}+05$ & $2.20 \mathrm{E}+10$ & $4.18 \mathrm{E}+07$ & $0.00 \mathrm{E}+00$ & $9.46 \mathrm{E}+01$ & \\
\hline 0.975 & 0.950 & $1.80 \mathrm{E}+05$ & & $9.59 \mathrm{E}+11$ & $3.52 \mathrm{E}+07$ & $0.00 \mathrm{E}+00$ & $2.84 \mathrm{E}+01$ & \\
\hline 1.000 & 0.975 & $1.45 \mathrm{E}+05$ & $7.50 \mathrm{E}+04$ & $2.03 \mathrm{E}+09$ & $2.90 \mathrm{E}+07$ & $0.00 \mathrm{E}+00$ & $4.21 \mathrm{E}+00$ & \\
\hline 1.250 & 1.000 & $4.36 \mathrm{E}+13$ & $2.77 \mathrm{E}+04$ & $1.80 \mathrm{E}+12$ & $1.01 \mathrm{E}+15$ & $0.00 \mathrm{E}+00$ & $9.29 \mathrm{E}-02$ & $E+09$ \\
\hline 1.500 & 1.250 & $4.05 \mathrm{E}+13$ & $0.00 \mathrm{E}+00$ & $1.54 \mathrm{E}+12$ & $7.55 \mathrm{E}+14$ & $0.00 \mathrm{E}+00$ & $0.00 \mathrm{E}+00$ & $9.39 \mathrm{E}+08$ \\
\hline 1.750 & 1.500 & $0.00 \mathrm{E}+00$ & $0.00 \mathrm{E}+00$ & $1.75 \mathrm{E}+10$ & $1.57 \mathrm{E}+03$ & $0.00 \mathrm{E}+00$ & $0.00 \mathrm{E}+00$ & $0 \mathrm{E}+00$ \\
\hline 2.000 & 1.750 & $0.00 \mathrm{E}+00$ & $0.00 \mathrm{E}+00$ & $5.40 \mathrm{E}+08$ & $0.00 \mathrm{E}+00$ & $0.00 \mathrm{E}+00$ & $0.00 \mathrm{E}+00$ & \\
\hline 2.250 & 2.000 & $4.68 \mathrm{E}+08$ & $0.00 \mathrm{E}+00$ & $0.00 \mathrm{E}+00$ & $0.00 \mathrm{E}+00$ & $0.00 \mathrm{E}+00$ & $0.00 \mathrm{E}+00$ & \\
\hline 2.500 & 2.250 & $0.00 \mathrm{E}+00$ & $0.00 \mathrm{E}+00$ & $0.00 \mathrm{E}+00$ & $0.00 \mathrm{E}+00$ & $0.00 \mathrm{E}+00$ & $0.00 \mathrm{E}+00$ & \\
\hline 2.750 & 2.500 & $1.44 \mathrm{E}+06$ & $0.00 \mathrm{E}+00$ & $0.00 \mathrm{E}+00$ & $0.00 \mathrm{E}+00$ & $0.00 \mathrm{E}+00$ & $0.00 \mathrm{E}+00$ & $\mathrm{E}+00$ \\
\hline 3.000 & 2.750 & $0.00 \mathrm{E}+00$ & $0.00 \mathrm{E}+00$ & $0.00 \mathrm{E}+00$ & $0.00 \mathrm{E}+00$ & $0.00 \mathrm{E}+00$ & $0.00 \mathrm{E}+00$ & $i+00$ \\
\hline 3.500 & 3.000 & $0.00 \mathrm{E}+00$ & $0.00 \mathrm{E}+00$ & $0.00 \mathrm{E}+00$ & $0.00 \mathrm{E}+00$ & $0.00 \mathrm{E}+00$ & $0.00 \mathrm{E}+00$ & $0 \mathrm{E}+00$ \\
\hline 4.000 & 3.500 & $0.00 \mathrm{E}+00$ & $0.00 \mathrm{E}+00$ & $0.00 \mathrm{E}+00$ & $0.00 \mathrm{E}+00$ & $0.00 \mathrm{E}+00$ & $0.00 \mathrm{E}+00$ & $0 \mathrm{E}+00$ \\
\hline 4.500 & 4.000 & $0.00 \mathrm{E}+00$ & $0.00 \mathrm{E}+00$ & $0.00 \mathrm{E}+00$ & $0.00 \mathrm{E}+00$ & $0.00 \mathrm{E}+00$ & $0.00 \mathrm{E}+00$ & $\overline{\mathrm{E}+00}$ \\
\hline 5.000 & 4.500 & $0.00 \mathrm{E}+00$ & $0.00 \mathrm{E}+00$ & $0.00 \mathrm{E}+00$ & $0.00 \mathrm{E}+00$ & $0.00 \mathrm{E}+00$ & $0.00 \mathrm{E}+00$ & \\
\hline 5.500 & 5.000 & $0.00 \mathrm{E}+00$ & $0.00 \mathrm{E}+00$ & $0.00 \mathrm{E}+00$ & $0.00 \mathrm{E}+00$ & $0.00 \mathrm{E}+00$ & $0.00 \mathrm{E}+00$ & $0 \mathrm{E}+00$ \\
\hline 6.000 & 5.500 & $0.00 \mathrm{E}+00$ & $0.00 \mathrm{E}+00$ & $0.00 \mathrm{E}+00$ & $0.00 \mathrm{E}+00$ & $0.00 \mathrm{E}+00$ & $0.00 \mathrm{E}+00$ & $0.00 \mathrm{E}+00$ \\
\hline 6.500 & 6.000 & $0.00 \mathrm{E}+00$ & $0.00 \mathrm{E}+00$ & $0.00 \mathrm{E}+00$ & $0.00 \mathrm{E}+00$ & $0.00 \mathrm{E}+00$ & $0.00 \mathrm{E}+00$ & $0.00 \mathrm{E}+00$ \\
\hline 7.000 & 6.500 & $0.00 \mathrm{E}+00$ & $0.00 \mathrm{E}+00$ & $0.00 \mathrm{E}+00$ & $0.00 \mathrm{E}+00$ & $0.00 \mathrm{E}+00$ & $0.00 \mathrm{E}+00$ & $0.00 \mathrm{E}+00$ \\
\hline 7.500 & 7.000 & $0.00 \mathrm{E}+00$ & $0.00 \mathrm{E}+00$ & $0.00 \mathrm{E}+00$ & $0.00 \mathrm{E}+00$ & $0.00 \mathrm{E}+00$ & $0.00 \mathrm{E}+00$ & $0.00 \mathrm{E}+00$ \\
\hline 8.000 & 7.500 & $0.00 \mathrm{E}+00$ & $0.00 \mathrm{E}+00$ & $0.00 \mathrm{E}+00$ & $0.00 \mathrm{E}+00$ & $0.00 \mathrm{E}+00$ & $0.00 \mathrm{E}+00$ & $0.00 \mathrm{E}+00$ \\
\hline 9.000 & 8.000 & $0.00 \mathrm{E}+00$ & $0.00 \mathrm{E}+00$ & $0.00 \mathrm{E}+00$ & $0.00 \mathrm{E}+00$ & $0.00 \mathrm{E}+00$ & $0.00 \mathrm{E}+00$ & $0.00 \mathrm{E}+00$ \\
\hline \multirow[t]{2}{*}{10.000} & 9.000 & $0.00 \mathrm{E}+00$ & $0.00 \mathrm{E}+00$ & $0.00 \mathrm{E}+00$ & $0.00 \mathrm{E}+00$ & $0.00 \mathrm{E}+00$ & $0.00 \mathrm{E}+00$ & $0.00 \mathrm{E}+00$ \\
\hline & Total & $8.45 \mathrm{E}+13$ & $1.82 \mathrm{E}+11$ & $1.54 \mathrm{E}+13$ & $1.86 \mathrm{E}+15$ & $2.21 \mathrm{E}+14$ & $1.19 \mathrm{E}+15$ & $2.47 \mathrm{E}+11$ \\
\hline
\end{tabular}


PCP-2011-0001

Revision 0

\begin{tabular}{|c|c|c|c|c|c|c|c|c|}
\hline \multicolumn{2}{|c|}{ Energy (MeV) } & \multicolumn{7}{|c|}{ Photon Source $(\mathrm{p} / \mathrm{s} / \mathrm{g})$} \\
\hline Upper & Lower & Ir-192 & Mn-54 & Np-237 & Pb-210 & Pm-147 & Po-210 & Pu-238 \\
\hline 0.020 & 0.010 & $1.67 \mathrm{E}+13$ & $0.00 \mathrm{E}+00$ & $1.52 \mathrm{E}+07$ & $5.45 \mathrm{E}+11$ & $1.88 \mathrm{E}+11$ & $4.25 \mathrm{E}+06$ & $6.46 \mathrm{E}+10$ \\
\hline 0.030 & 0.020 & $4.44 \mathrm{E}+12$ & $0.00 \mathrm{E}+00$ & $4.59 \mathrm{E}+06$ & $7.50 \mathrm{E}+07$ & $8.13 \mathrm{E}+10$ & $0.00 \mathrm{E}+00$ & $6.59 \mathrm{E}+09$ \\
\hline 0.040 & 0.030 & $2.68 \mathrm{E}+12$ & $0.00 \mathrm{E}+00$ & $9.31 \mathrm{E}-06$ & $1.46 \mathrm{E}+07$ & $4.34 \mathrm{E}+10$ & $0.00 \mathrm{E}+00$ & $2.06 \mathrm{E}+02$ \\
\hline 0.050 & 0.040 & $1.80 \mathrm{E}+12$ & $0.00 \mathrm{E}+00$ & $3.77 \mathrm{E}+04$ & $1.18 \mathrm{E}+11$ & $2.56 \mathrm{E}+10$ & $0.00 \mathrm{E}+00$ & \\
\hline 0.060 & 0.050 & $1.29 \mathrm{E}+12$ & $0.00 \mathrm{E}+00$ & $1.13 \mathrm{E}+05$ & $5.71 \mathrm{E}+04$ & $1.54 \mathrm{E}+10$ & $0.00 \mathrm{E}+00$ & \\
\hline 0.070 & 0.060 & $3.74 \mathrm{E}+13$ & $0.00 \mathrm{E}+00$ & $7.36 \mathrm{E}+03$ & $2.10 \mathrm{E}+01$ & $9.70 \mathrm{E}+09$ & $0.00 \mathrm{E}+00$ & \\
\hline 0.080 & 0.070 & $1.08 \mathrm{E}+13$ & $0.00 \mathrm{E}+00$ & $4.03 \mathrm{E}+03$ & $0.00 \mathrm{E}+00$ & $6.20 \mathrm{E}+09$ & $1.06 \mathrm{E}+07$ & $1.02 \mathrm{E}+02$ \\
\hline 0.090 & 0.080 & $5.72 \mathrm{E}+11$ & $0.00 \mathrm{E}+00$ & $3.39 \mathrm{E}+06$ & $0.00 \mathrm{E}+00$ & $3.98 \mathrm{E}+09$ & $3.09 \mathrm{E}+06$ & $1.02 \mathrm{E}+02$ \\
\hline 0.100 & 0.090 & $4.55 \mathrm{E}+11$ & $0.00 \mathrm{E}+00$ & $1.41 \mathrm{E}+06$ & $0.00 \mathrm{E}+00$ & $2.55 \mathrm{E}+09$ & $0.00 \mathrm{E}+00$ & $5.11 \mathrm{E}+07$ \\
\hline 0.110 & 0.100 & $3.66 \mathrm{E}+11$ & $0.00 \mathrm{E}+00$ & $3.14 \mathrm{E}+05$ & $0.00 \mathrm{E}+00$ & $1.62 \mathrm{E}+09$ & $0.00 \mathrm{E}+00$ & $1.52 \mathrm{E}+02$ \\
\hline 0.120 & 0.110 & $6.74 \mathrm{E}+11$ & $0.00 \mathrm{E}+00$ & $1.34 \mathrm{E}+05$ & $0.00 \mathrm{E}+00$ & $1.01 \mathrm{E}+09$ & $0.00 \mathrm{E}+00$ & $3.97 \mathrm{E}+05$ \\
\hline 0.130 & 0.120 & $2.45 \mathrm{E}+11$ & $0.00 \mathrm{E}+00$ & $7.29 \mathrm{E}-06$ & $0.00 \mathrm{E}+00$ & $1.56 \mathrm{E}+09$ & $0.00 \mathrm{E}+00$ & $1.52 \mathrm{E}+02$ \\
\hline 0.140 & 0.130 & $8.24 \mathrm{E}+11$ & $0.00 \mathrm{E}+00$ & $3.94 \mathrm{E}+04$ & $0.00 \mathrm{E}+00$ & $3.62 \mathrm{E}+08$ & $0.00 \mathrm{E}+00$ & $1.52 \mathrm{E}+02$ \\
\hline 0.150 & 0.140 & $1.68 \mathrm{E}+11$ & $0.00 \mathrm{E}+00$ & $1.12 \mathrm{E}+05$ & $0.00 \mathrm{E}+00$ & $2.05 \mathrm{E}+08$ & $0.00 \mathrm{E}+00$ & $1.89 \mathrm{E}+02$ \\
\hline 0.160 & 0.150 & $1.40 \mathrm{E}+11$ & $0.00 \mathrm{E}+00$ & $9.08 \mathrm{E}+04$ & $0.00 \mathrm{E}+00$ & $1.10 \mathrm{E}+08$ & $0.00 \mathrm{E}+00$ & $6.25 \mathrm{E}+06$ \\
\hline 0.170 & 0.160 & $1.18 \mathrm{E}+11$ & $0.00 \mathrm{E}+00$ & $3.02 \mathrm{E}+04$ & $0.00 \mathrm{E}+00$ & $5.43 \mathrm{E}+07$ & $0.00 \mathrm{E}+00$ & $1.52 \mathrm{E}+02$ \\
\hline 0.180 & 0.170 & $9.89 \mathrm{E}+10$ & $0.00 \mathrm{E}+00$ & $1.22 \mathrm{E}+04$ & $0.00 \mathrm{E}+00$ & $2.42 \mathrm{E}+07$ & $0.00 \mathrm{E}+00$ & $1.74 \mathrm{E}+02$ \\
\hline 0.190 & 0.180 & $8.34 \mathrm{E}+10$ & $0.00 \mathrm{E}+00$ & $7.96 \mathrm{E}+03$ & $0.00 \mathrm{E}+00$ & $9.18 \mathrm{E}+06$ & $0.00 \mathrm{E}+00$ & $1.52 \mathrm{E}+02$ \\
\hline 0.200 & 0.190 & $7.05 \mathrm{E}+10$ & $0.00 \mathrm{E}+00$ & $8.32 \mathrm{E}+04$ & $0.00 \mathrm{E}+00$ & $2.82 \mathrm{E}+06$ & $0.00 \mathrm{E}+00$ & $1.52 \mathrm{E}+02$ \\
\hline 0.210 & 0.200 & $1.29 \mathrm{E}+13$ & $0.00 \mathrm{E}+00$ & $1.76 \mathrm{E}+04$ & $0.00 \mathrm{E}+00$ & $4.98 \mathrm{E}+05$ & $0.00 \mathrm{E}+00$ & $2.57 \mathrm{E}+04$ \\
\hline 0.220 & 0.210 & $1.92 \mathrm{E}+11$ & $0.00 \mathrm{E}+00$ & $4.79 \mathrm{E}+04$ & $0.00 \mathrm{E}+00$ & $2.85 \mathrm{E}+04$ & $0.00 \mathrm{E}+00$ & $1.44 \mathrm{E}+02$ \\
\hline 0.230 & 0.220 & $4.29 \mathrm{E}+10$ & $0.00 \mathrm{E}+00$ & $4.55 \mathrm{E}+02$ & $0.00 \mathrm{E}+00$ & $4.94 \mathrm{E}+01$ & $0.00 \mathrm{E}+00$ & $1.44 \mathrm{E}+02$ \\
\hline 0.240 & 0.230 & $3.64 \mathrm{E}+10$ & $0.00 \mathrm{E}+00$ & $2.15 \mathrm{E}+04$ & $0.00 \mathrm{E}+00$ & $0.00 \mathrm{E}+00$ & $0.00 \mathrm{E}+00$ & $\mathrm{E}+02$ \\
\hline 0.250 & 0.240 & $3.09 \mathrm{E}+10$ & $0.00 \mathrm{E}+00$ & $1.34 \mathrm{E}+03$ & $0.00 \mathrm{E}+00$ & $0.00 \mathrm{E}+00$ & $0.00 \mathrm{E}+00$ & $\mathrm{E}+02$ \\
\hline 0.260 & 0.250 & $2.63 \mathrm{E}+10$ & $0.00 \mathrm{E}+00$ & $1.69 \mathrm{E}+03$ & $0.00 \mathrm{E}+00$ & $0.00 \mathrm{E}+00$ & $0.00 \mathrm{E}+00$ & $8.74 \mathrm{E}+02$ \\
\hline 0.270 & 0.260 & $2.23 \mathrm{E}+10$ & $0.00 \mathrm{E}+00$ & $1.71 \mathrm{E}+03$ & $0.00 \mathrm{E}+00$ & $0.00 \mathrm{E}+00$ & $0.00 \mathrm{E}+00$ & $1.44 \mathrm{E}+02$ \\
\hline 0.280 & 0.270 & $1.89 \mathrm{E}+10$ & $0.00 \mathrm{E}+00$ & $4.67 \mathrm{E}+02$ & $0.00 \mathrm{E}+00$ & $0.00 \mathrm{E}+00$ & $0.00 \mathrm{E}+00$ & $1.44 \mathrm{E}+02$ \\
\hline 0.290 & 0.280 & $9.00 \mathrm{E}+11$ & $0.00 \mathrm{E}+00$ & $6.90 \mathrm{E}-06$ & $0.00 \mathrm{E}+00$ & $0.00 \mathrm{E}+00$ & $0.00 \mathrm{E}+00$ & $1.44 \mathrm{E}+02$ \\
\hline 0.300 & 0.290 & $9.90 \mathrm{E}+13$ & $0.00 \mathrm{E}+00$ & $6.90 \mathrm{E}-06$ & $0.00 \mathrm{E}+00$ & $0.00 \mathrm{E}+00$ & $0.00 \mathrm{E}+00$ & $5.77 \mathrm{E}+02$ \\
\hline 0.325 & 0.300 & $3.86 \mathrm{E}+14$ & $0.00 \mathrm{E}+00$ & $1.97 \mathrm{E}-05$ & $0.00 \mathrm{E}+00$ & $0.00 \mathrm{E}+00$ & $0.00 \mathrm{E}+00$ & $4.12 \mathrm{E}+02$ \\
\hline 0.350 & 0.325 & $6.90 \mathrm{E}+10$ & $0.00 \mathrm{E}+00$ & $1.97 \mathrm{E}-05$ & $0.00 \mathrm{E}+00$ & $0.00 \mathrm{E}+00$ & $0.00 \mathrm{E}+00$ & $4.12 \mathrm{E}+02$ \\
\hline 0.375 & 0.350 & $2.58 \mathrm{E}+12$ & $0.00 \mathrm{E}+00$ & $1.97 \mathrm{E}-05$ & $0.00 \mathrm{E}+00$ & $0.00 \mathrm{E}+00$ & $0.00 \mathrm{E}+00$ & $4.12 \mathrm{E}+02$ \\
\hline 0.400 & 0.375 & $6.77 \mathrm{E}+09$ & $0.00 \mathrm{E}+00$ & $1.97 \mathrm{E}-05$ & $0.00 \mathrm{E}+00$ & $0.00 \mathrm{E}+00$ & $0.00 \mathrm{E}+00$ & $4.12 \mathrm{E}+02$ \\
\hline 0.425 & 0.400 & $2.98 \mathrm{E}+12$ & $0.00 \mathrm{E}+00$ & $1.98 \mathrm{E}-05$ & $0.00 \mathrm{E}+00$ & $0.00 \mathrm{E}+00$ & $0.00 \mathrm{E}+00$ & $4.13 \mathrm{E}+02$ \\
\hline 0.450 & 0.425 & $1.45 \mathrm{E}+09$ & $0.00 \mathrm{E}+00$ & $1.98 \mathrm{E}-05$ & $0.00 \mathrm{E}+00$ & $0.00 \mathrm{E}+00$ & $0.00 \mathrm{E}+00$ & $4.13 \mathrm{E}+02$ \\
\hline 0.475 & 0.450 & $1.65 \mathrm{E}+14$ & $0.00 \mathrm{E}+00$ & $1.98 \mathrm{E}-05$ & $0.00 \mathrm{E}+00$ & $0.00 \mathrm{E}+00$ & $0.00 \mathrm{E}+00$ & $4.13 \mathrm{E}+02$ \\
\hline 0.500 & 0.475 & $1.21 \mathrm{E}+13$ & $0.00 \mathrm{E}+00$ & $1.98 \mathrm{E}-05$ & $0.00 \mathrm{E}+00$ & $0.00 \mathrm{E}+00$ & $0.00 \mathrm{E}+00$ & $4.13 \mathrm{E}+02$ \\
\hline 0.525 & 0.500 & $4.36 \mathrm{E}+08$ & $0.00 \mathrm{E}+00$ & $1.87 \mathrm{E}-05$ & $0.00 \mathrm{E}+00$ & $0.00 \mathrm{E}+00$ & $0.00 \mathrm{E}+00$ & $3.91 \mathrm{E}+02$ \\
\hline 0.550 & 0.525 & $2.07 \mathrm{E}+08$ & $0.00 \mathrm{E}+00$ & $1.87 \mathrm{E}-05$ & $0.00 \mathrm{E}+00$ & $0.00 \mathrm{E}+00$ & $0.00 \mathrm{E}+00$ & $3.91 \mathrm{E}+02$ \\
\hline 0.575 & 0.550 & $9.24 \mathrm{E}+07$ & $0.00 \mathrm{E}+00$ & $1.87 \mathrm{E}-05$ & $0.00 \mathrm{E}+00$ & $0.00 \mathrm{E}+00$ & $0.00 \mathrm{E}+00$ & $3.91 \mathrm{E}+02$ \\
\hline 0.600 & 0.575 & & & & $0.00 \mathrm{E}+00$ & $0.00 \mathrm{E}+00$ & $0.00 \mathrm{E}+00$ & $3.91 \mathrm{E}+02$ \\
\hline 0.625 & 0.600 & $4.57 \mathrm{E}+13$ & $0.00 \mathrm{E}+00$ & $1.68 \mathrm{E}-05$ & $0.00 \mathrm{E}+00$ & $0.00 \mathrm{E}+00$ & $0.00 \mathrm{E}+00$ & $3.51 \mathrm{E}+02$ \\
\hline 0.650 & 0.625 & $1.19 \mathrm{E}+06$ & $0.00 \mathrm{E}+00$ & $1.68 \mathrm{E}-05$ & $0.00 \mathrm{E}+00$ & $0.00 \mathrm{E}+00$ & $0.00 \mathrm{E}+00$ & $3.51 \mathrm{E}+02$ \\
\hline 0.675 & 0.650 & $1.53 \mathrm{E}+04$ & $0.00 \mathrm{E}+00$ & $1.68 \mathrm{E}-05$ & $0.00 \mathrm{E}+00$ & $0.00 \mathrm{E}+00$ & $0.00 \mathrm{E}+00$ & $3.51 \mathrm{E}+02$ \\
\hline 0.700 & 0.675 & $0.00 \mathrm{E}+00$ & $0.00 \mathrm{E}+00$ & $1.68 \mathrm{E}-05$ & $0.00 \mathrm{E}+00$ & $0.00 \mathrm{E}+00$ & $0.00 \mathrm{E}+00$ & $3.51 \mathrm{E}+02$ \\
\hline 0.725 & 0.700 & $1.95 \mathrm{E}+10$ & $0.00 \mathrm{E}+00$ & $1.38 \mathrm{E}-05$ & $0.00 \mathrm{E}+00$ & $0.00 \mathrm{E}+00$ & $0.00 \mathrm{E}+00$ & $3.56 \mathrm{E}+03$ \\
\hline 0.750 & 0.725 & $0.00 \mathrm{E}+00$ & $0.00 \mathrm{E}+00$ & $1.38 \mathrm{E}-05$ & $0.00 \mathrm{E}+00$ & $0.00 \mathrm{E}+00$ & $0.00 \mathrm{E}+00$ & $4.85 \mathrm{E}+04$ \\
\hline
\end{tabular}


PCP-2011-0001

Revision 0

\begin{tabular}{|c|c|c|c|c|c|c|c|c|}
\hline \multicolumn{9}{|c|}{ Table 3-20. Photon Source from RASTA (Decay Time $=0$ days) } \\
\hline \multicolumn{2}{|c|}{ Energy (MeV) } & \multicolumn{7}{|c|}{ Photon Source $(\mathbf{p} / \mathbf{s} / \mathbf{g})$} \\
\hline Upper & Lower & Ir-192 & Mn-54 & Np-237 & Pb-210 & Pm-147 & Po-210 & Pu-238 \\
\hline 0.775 & 0.750 & $0.00 \mathrm{E}+00$ & $0.00 \mathrm{E}+00$ & $1.38 \mathrm{E}-05$ & $0.00 \mathrm{E}+00$ & $0.00 \mathrm{E}+00$ & $0.00 \mathrm{E}+00$ & $2.13 \mathrm{E}+05$ \\
\hline 0.800 & 0.775 & $0.00 \mathrm{E}+00$ & $0.00 \mathrm{E}+00$ & $1.38 \mathrm{E}-05$ & $0.00 \mathrm{E}+00$ & $0.00 \mathrm{E}+00$ & $0.00 \mathrm{E}+00$ & $3.05 \mathrm{E}+04$ \\
\hline 0.825 & 0.800 & $0.00 \mathrm{E}+00$ & $0.00 \mathrm{E}+00$ & $1.18 \mathrm{E}-05$ & $0.00 \mathrm{E}+00$ & $0.00 \mathrm{E}+00$ & $1.74 \mathrm{E}+09$ & $2.85 \mathrm{E}+04$ \\
\hline 0.850 & 0.825 & $0.00 \mathrm{E}+00$ & $2.86 \mathrm{E}+14$ & $1.18 \mathrm{E}-05$ & $0.00 \mathrm{E}+00$ & $0.00 \mathrm{E}+00$ & $0.00 \mathrm{E}+00$ & $2.46 \mathrm{E}+02$ \\
\hline 0.875 & 0.850 & $0.00 \mathrm{E}+00$ & $0.00 \mathrm{E}+00$ & $1.18 \mathrm{E}-05$ & $0.00 \mathrm{E}+00$ & $0.00 \mathrm{E}+00$ & $0.00 \mathrm{E}+00$ & \\
\hline 0.900 & 0.875 & $1.02 \mathrm{E}+12$ & $0.00 \mathrm{E}+00$ & $1.18 \mathrm{E}-05$ & $0.00 \mathrm{E}+00$ & $0.00 \mathrm{E}+00$ & $0.00 \mathrm{E}+00$ & $8.52 \mathrm{E}+03$ \\
\hline 0.925 & 0.900 & $0.00 \mathrm{E}+00$ & $0.00 \mathrm{E}+00$ & $1.18 \mathrm{E}-05$ & $0.00 \mathrm{E}+00$ & $0.00 \mathrm{E}+00$ & $0.00 \mathrm{E}+00$ & $8.65 \mathrm{E}+02$ \\
\hline 0.950 & 0.925 & $0.00 \mathrm{E}+00$ & $0.00 \mathrm{E}+00$ & $1.18 \mathrm{E}-05$ & $0.00 \mathrm{E}+00$ & $0.00 \mathrm{E}+00$ & $0.00 \mathrm{E}+00$ & $1.05 \mathrm{E}+04$ \\
\hline 0.975 & 0.950 & $0.00 \mathrm{E}+00$ & $0.00 \mathrm{E}+00$ & $1.18 \mathrm{E}-05$ & $0.00 \mathrm{E}+00$ & $0.00 \mathrm{E}+00$ & $0 \mathrm{E}+00$ & $2.46 \mathrm{E}+02$ \\
\hline 1.000 & 0.975 & $0.00 \mathrm{E}+00$ & & & & $0.00 \mathrm{E}+00$ & & \\
\hline 1.250 & 1.000 & $1.84 \mathrm{E}+11$ & $0.00 \mathrm{E}+00$ & $6.18 \mathrm{E}-05$ & $0.00 \mathrm{E}+00$ & $0.00 \mathrm{E}+00$ & $0 \mathrm{E}+00$ & $1.13 \mathrm{E}+04$ \\
\hline 1.500 & 1.250 & $4.24 \mathrm{E}+09$ & $0.00 \mathrm{E}+00$ & $5.87 \mathrm{E}-05$ & $0.00 \mathrm{E}+00$ & $0.00 \mathrm{E}+00$ & $0.00 \mathrm{E}+00$ & $1.22 \mathrm{E}+03$ \\
\hline 1.750 & 1.500 & $0.00 \mathrm{E}+00$ & $0.00 \mathrm{E}+00$ & $3.10 \mathrm{E}-05$ & $0.00 \mathrm{E}+00$ & $0.00 \mathrm{E}+00$ & $0.00 \mathrm{E}+00$ & $6.47 \mathrm{E}+02$ \\
\hline 2.000 & 1.750 & $0.00 \mathrm{E}+00$ & $0.00 \mathrm{E}+00$ & $2.42 \mathrm{E}-05$ & $0.00 \mathrm{E}+00$ & $0.00 \mathrm{E}+00$ & $0 \mathrm{E}+00$ & $5.06 \mathrm{E}+02$ \\
\hline 2.250 & 2.000 & $0.00 \mathrm{E}+00$ & $0.00 \mathrm{E}+00$ & $1.65 \mathrm{E}-05$ & $0.00 \mathrm{E}+00$ & $0.00 \mathrm{E}+00$ & $0 \mathrm{E}+00$ & $3.45 \mathrm{E}+02$ \\
\hline 2.500 & 2.250 & $0.00 \mathrm{E}+00$ & $0.00 \mathrm{E}+00$ & $1.53 \mathrm{E}-05$ & $0.00 \mathrm{E}+00$ & $0.00 \mathrm{E}+00$ & $0 \mathrm{E}+00$ & $9 \mathrm{E}+02$ \\
\hline 2.750 & 2.500 & $0.00 \mathrm{E}+00$ & $0.00 \mathrm{E}+00$ & $1.10 \mathrm{E}-05$ & $0.00 \mathrm{E}+00$ & $0.00 \mathrm{E}+00$ & $0 \mathrm{E}+00$ & $2.30 \mathrm{E}+02$ \\
\hline 3.000 & 2.750 & $0.00 \mathrm{E}+00$ & $0.00 \mathrm{E}+00$ & $9.10 \mathrm{E}-06$ & $0.00 \mathrm{E}+00$ & $0.00 \mathrm{E}+00$ & $0 \mathrm{E}+00$ & $1.90 \mathrm{E}+02$ \\
\hline 3.500 & 3.000 & $0.00 \mathrm{E}+00$ & $0.00 \mathrm{E}+00$ & $9.32 \mathrm{E}-06$ & $0.00 \mathrm{E}+00$ & $0.00 \mathrm{E}+00$ & $0 \mathrm{E}+00$ & $1.95 \mathrm{E}+02$ \\
\hline 4.000 & 3.500 & $0.00 \mathrm{E}+00$ & $0.00 \mathrm{E}+00$ & $6.33 \mathrm{E}-06$ & $0.00 \mathrm{E}+00$ & $0.00 \mathrm{E}+00$ & $0 \mathrm{E}+00$ & $1.32 \mathrm{E}+02$ \\
\hline 4.500 & 4.000 & $0.00 \mathrm{E}+00$ & $0.00 \mathrm{E}+00$ & $2.34 \mathrm{E}-06$ & $0.00 \mathrm{E}+00$ & $0.00 \mathrm{E}+00$ & $00 \mathrm{E}+00$ & $4.88 \mathrm{E}+01$ \\
\hline 5.000 & 4.500 & $0.00 \mathrm{E}+00$ & $0.00 \mathrm{E}+00$ & $1.84 \mathrm{E}-06$ & $0.00 \mathrm{E}+00$ & $0.00 \mathrm{E}+00$ & $0 \mathrm{E}+00$ & $3.84 \mathrm{E}+01$ \\
\hline 5.500 & 5.000 & $0.00 \mathrm{E}+00$ & $0.00 \mathrm{E}+00$ & $7.68 \mathrm{E}-07$ & $0.00 \mathrm{E}+00$ & $0.00 \mathrm{E}+00$ & $0.00 \mathrm{E}+00$ & $1.60 \mathrm{E}+01$ \\
\hline 6.000 & 5.500 & $0.00 \mathrm{E}+00$ & $0.00 \mathrm{E}+00$ & $6.94 \mathrm{E}-07$ & $0.00 \mathrm{E}+00$ & $0.00 \mathrm{E}+00$ & $0.00 \mathrm{E}+00$ & $1.45 \mathrm{E}+01$ \\
\hline 6.500 & 6.000 & $0.00 \mathrm{E}+00$ & $0.00 \mathrm{E}+00$ & $3.22 \mathrm{E}-07$ & $0.00 \mathrm{E}+00$ & $0.00 \mathrm{E}+00$ & $0.00 \mathrm{E}+00$ & $6.73 \mathrm{E}+00$ \\
\hline 7.000 & 6.500 & $0.00 \mathrm{E}+00$ & $0.00 \mathrm{E}+00$ & $3.04 \mathrm{E}-07$ & $0.00 \mathrm{E}+00$ & $0.00 \mathrm{E}+00$ & $0.00 \mathrm{E}+00$ & $6.35 \mathrm{E}+00$ \\
\hline 7.500 & 7.000 & $0.00 \mathrm{E}+00$ & $0.00 \mathrm{E}+00$ & $2.80 \mathrm{E}-08$ & $0.00 \mathrm{E}+00$ & $0.00 \mathrm{E}+00$ & $0.00 \mathrm{E}+00$ & $5.86 \mathrm{E}-01$ \\
\hline 8.000 & 7.500 & $0.00 \mathrm{E}+00$ & $0.00 \mathrm{E}+00$ & $2.77 \mathrm{E}-08$ & $0.00 \mathrm{E}+00$ & $0.00 \mathrm{E}+00$ & $0.00 \mathrm{E}+00$ & $5.79 \mathrm{E}-01$ \\
\hline 9.000 & 8.000 & $0.00 \mathrm{E}+00$ & $0.00 \mathrm{E}+00$ & $5.53 \mathrm{E}-08$ & $0.00 \mathrm{E}+00$ & $0.00 \mathrm{E}+00$ & $0.00 \mathrm{E}+00$ & $1.15 \mathrm{E}+00$ \\
\hline \multirow[t]{2}{*}{10.000} & 9.000 & $0.00 \mathrm{E}+00$ & $0.00 \mathrm{E}+00$ & $5.52 \mathrm{E}-08$ & $0.00 \mathrm{E}+00$ & $0.00 \mathrm{E}+00$ & $0.00 \mathrm{E}+00$ & $1.15 \mathrm{E}+00$ \\
\hline & Total & $8.23 \mathrm{E}+14$ & $2.86 \mathrm{E}+14$ & $2.57 \mathrm{E}+07$ & $6.64 \mathrm{E}+11$ & $3.81 \mathrm{E}+11$ & $1.76 \mathrm{E}+09$ & $7.15 \mathrm{E}+10$ \\
\hline
\end{tabular}


PCP-2011-0001

Revision 0

\begin{tabular}{|c|c|c|c|c|c|c|c|c|}
\hline \multicolumn{2}{|c|}{ Energy (MeV) } & \multicolumn{7}{|c|}{ Photon Source (p/s/g) } \\
\hline Upper & Lower & Pu-239 & Pu-240 & \begin{tabular}{|l|}
$\mathbf{P u}-241$ \\
\end{tabular} & Pu-242 & Ra-226 & Ru-106 & Sc-46 \\
\hline 0.020 & 0.010 & $8.75 \mathrm{E}+07$ & $8.16 \mathrm{E}+08$ & $2.38 \mathrm{E}+08$ & $1.18 \mathrm{E}+07$ & $2.56 \mathrm{E}+08$ & $1.26 \mathrm{E}+10$ & $4.81 \mathrm{E}+12$ \\
\hline 0.030 & 0.020 & $9.05 \mathrm{E}+06$ & $8.35 \mathrm{E}+07$ & $2.21 \mathrm{E}+07$ & $1.21 \mathrm{E}+06$ & $0.00 \mathrm{E}+00$ & $8.20 \mathrm{E}+08$ & $2.25 \mathrm{E}+12$ \\
\hline 0.040 & 0.030 & $1.51 \mathrm{E}+05$ & $7.93 \mathrm{E}+01$ & $4.13 \mathrm{E}+05$ & $1.33 \mathrm{E}+02$ & $0.00 \mathrm{E}+00$ & $5.87 \mathrm{E}+06$ & $1.29 \mathrm{E}+12$ \\
\hline 0.050 & 0.040 & $1.49 \mathrm{E}+04$ & $3.80 \mathrm{E}+06$ & $1.88 \mathrm{E}+05$ & $5.26 \mathrm{E}+04$ & $0.00 \mathrm{E}+00$ & $0.00 \mathrm{E}+00$ & $8.22 \mathrm{E}+11$ \\
\hline 0.060 & 0.050 & $4.74 \mathrm{E}+05$ & $4.14 \mathrm{E}+01$ & $1.37 \mathrm{E}+05$ & $6.96 \mathrm{E}+01$ & $0.00 \mathrm{E}+00$ & $0.00 \mathrm{E}+00$ & \\
\hline 0.070 & 0.060 & $1.87 \mathrm{E}+04$ & $4.14 \mathrm{E}+01$ & $1.91 \mathrm{E}-03$ & $6.96 \mathrm{E}+01$ & $0.00 \mathrm{E}+00$ & $0.00 \mathrm{E}+00$ & \\
\hline 0.080 & 0.070 & $1.51 \mathrm{E}+04$ & $4.14 \mathrm{E}+01$ & $9.71 \mathrm{E}+05$ & $6.96 \mathrm{E}+01$ & $0.00 \mathrm{E}+00$ & $0.00 \mathrm{E}+00$ & $2.79 \mathrm{E}+11$ \\
\hline 0.090 & 0.080 & $6.30 \mathrm{E}+02$ & $4.14 \mathrm{E}+01$ & $1.91 \mathrm{E}-03$ & $6.96 \mathrm{E}+01$ & $1.70 \mathrm{E}+08$ & $0.00 \mathrm{E}+00$ & $2.04 \mathrm{E}+11$ \\
\hline 0.100 & 0.090 & $1.80 \mathrm{E}+05$ & $6.03 \mathrm{E}+03$ & $3.33 \mathrm{E}+07$ & $1.78 \mathrm{E}+02$ & $5.12 \mathrm{E}+07$ & $0.00 \mathrm{E}+00$ & $1.52 \mathrm{E}+11$ \\
\hline 0.110 & 0.100 & $4.03 \mathrm{E}+03$ & $5.84 \mathrm{E}+05$ & $3.80 \mathrm{E}+06$ & $1.14 \mathrm{E}+04$ & $0.00 \mathrm{E}+00$ & $0.00 \mathrm{E}+00$ & $\mathrm{E}+11$ \\
\hline 0.120 & 0.110 & $7.35 \mathrm{E}+04$ & $1.78 \mathrm{E}+03$ & $9.82 \mathrm{E}+06$ & $1.35 \mathrm{E}+02$ & $0.00 \mathrm{E}+00$ & $0.00 \mathrm{E}+00$ & $8.52 \mathrm{E}+10$ \\
\hline 0.130 & 0.120 & $1.50 \mathrm{E}+05$ & $6.21 \mathrm{E}+01$ & $2.54 \mathrm{E}+04$ & $1.04 \mathrm{E}+02$ & $0.00 \mathrm{E}+00$ & $0.00 \mathrm{E}+00$ & $6.43 \mathrm{E}+10$ \\
\hline 0.140 & 0.130 & $1.27 \mathrm{E}-03$ & $6.21 \mathrm{E}+01$ & $2.87 \mathrm{E}-03$ & $1.04 \mathrm{E}+02$ & $0.00 \mathrm{E}+00$ & $0.00 \mathrm{E}+00$ & $4.86 \mathrm{E}+10$ \\
\hline 0.150 & 0.140 & $9.97 \mathrm{E}+03$ & $6.21 \mathrm{E}+01$ & $7.30 \mathrm{E}+06$ & $1.04 \mathrm{E}+02$ & $0.00 \mathrm{E}+00$ & $0.00 \mathrm{E}+00$ & $3.67 \mathrm{E}+10$ \\
\hline 0.160 & 0.150 & $1.88 \mathrm{E}+02$ & $6.21 \mathrm{E}+01$ & $2.65 \mathrm{E}+05$ & $7.83 \mathrm{E}+02$ & $0.00 \mathrm{E}+00$ & $0.00 \mathrm{E}+00$ & $2.77 \mathrm{E}+10$ \\
\hline 0.170 & 0.160 & $3.12 \mathrm{E}+03$ & $3.43 \mathrm{E}+04$ & $2.87 \mathrm{E}-03$ & $1.04 \mathrm{E}+02$ & $0.00 \mathrm{E}+00$ & $0.00 \mathrm{E}+00$ & $2.08 \mathrm{E}+10$ \\
\hline 0.180 & 0.170 & $4.04 \mathrm{E}+03$ & $6.21 \mathrm{E}+01$ & $2.87 \mathrm{E}-03$ & $1.04 \mathrm{E}+02$ & $0.00 \mathrm{E}+00$ & $0.00 \mathrm{E}+00$ & $1.56 \mathrm{E}+10$ \\
\hline 0.190 & 0.180 & $2.08 \mathrm{E}+03$ & $6.21 \mathrm{E}+01$ & $2.87 \mathrm{E}-03$ & $1.04 \mathrm{E}+02$ & $1.21 \mathrm{E}+09$ & $0.00 \mathrm{E}+00$ & $1.16 \mathrm{E}+10$ \\
\hline 0.200 & 0.190 & $2.58 \mathrm{E}+03$ & $6.21 \mathrm{E}+01$ & $2.87 \mathrm{E}-03$ & $1.04 \mathrm{E}+02$ & $0.00 \mathrm{E}+00$ & $0.00 \mathrm{E}+00$ & $8.52 \mathrm{E}+09$ \\
\hline 0.210 & 0.200 & $1.28 \mathrm{E}+04$ & $5.87 \mathrm{E}+01$ & $2.71 \mathrm{E}-03$ & $9.88 \mathrm{E}+01$ & $0.00 \mathrm{E}+00$ & $0.00 \mathrm{E}+00$ & $6.22 \mathrm{E}+09$ \\
\hline 0.220 & 0.210 & $1.16 \mathrm{E}-03$ & $2.46 \mathrm{E}+03$ & $2.71 \mathrm{E}-03$ & $9.88 \mathrm{E}+01$ & $0.00 \mathrm{E}+00$ & $0.00 \mathrm{E}+00$ & $4.48 \mathrm{E}+09$ \\
\hline 0.230 & 0.220 & $3.75 \mathrm{E}+02$ & $5.87 \mathrm{E}+01$ & $2.71 \mathrm{E}-03$ & $9.88 \mathrm{E}+01$ & $0.00 \mathrm{E}+00$ & $0.00 \mathrm{E}+00$ & $3+09$ \\
\hline 0.240 & 0.230 & $3.48 \mathrm{E}+02$ & $5.87 \mathrm{E}+01$ & $2.71 \mathrm{E}-03$ & $9.88 \mathrm{E}+01$ & $0.00 \mathrm{E}+00$ & $0.00 \mathrm{E}+00$ & $2.22 \mathrm{E}+09$ \\
\hline 0.250 & 0.240 & $1.01 \mathrm{E}+03$ & $5.87 \mathrm{E}+01$ & & $9.88 \mathrm{E}+01$ & $0.00 \mathrm{E}+00$ & $0.00 \mathrm{E}+00$ & \\
\hline 0.260 & 0.250 & $1.85 \mathrm{E}+03$ & $5.87 \mathrm{E}+01$ & $2.71 \mathrm{E}-03$ & $9.88 \mathrm{E}+01$ & $0.00 \mathrm{E}+00$ & $0.00 \mathrm{E}+00$ & $\overline{\mathrm{E}+09}$ \\
\hline 0.270 & 0.260 & $6.52 \mathrm{E}+02$ & $5.87 \mathrm{E}+01$ & $2.71 \mathrm{E}-03$ & $9.88 \mathrm{E}+01$ & $1.96 \mathrm{E}+06$ & $0.00 \mathrm{E}+00$ & $6.49 \mathrm{E}+08$ \\
\hline 0.280 & 0.270 & $1.16 \mathrm{E}-03$ & $5.87 \mathrm{E}+01$ & $2.71 \mathrm{E}-03$ & $9.88 \mathrm{E}+01$ & $0.00 \mathrm{E}+00$ & $0.00 \mathrm{E}+00$ & $4.03 \mathrm{E}+08$ \\
\hline 0.290 & 0.280 & $8.20 \mathrm{E}+01$ & $5.87 \mathrm{E}+01$ & $2.71 \mathrm{E}-03$ & $9.88 \mathrm{E}+01$ & $0.00 \mathrm{E}+00$ & $0.00 \mathrm{E}+00$ & $2.39 \mathrm{E}+08$ \\
\hline 0.300 & 0.290 & $1.16 \mathrm{E}+03$ & $5.87 \mathrm{E}+01$ & $2.71 \mathrm{E}-03$ & $9.88 \mathrm{E}+01$ & $0.00 \mathrm{E}+00$ & $0.00 \mathrm{E}+00$ & $1.34 \mathrm{E}+08$ \\
\hline 0.325 & 0.300 & $5.23 \mathrm{E}+03$ & $1.68 \mathrm{E}+02$ & $7.76 \mathrm{E}-03$ & $2.82 \mathrm{E}+02$ & $0.00 \mathrm{E}+00$ & $0.00 \mathrm{E}+00$ & $1.10 \mathrm{E}+08$ \\
\hline 0.350 & 0.325 & $2.87 \mathrm{E}+04$ & $1.68 \mathrm{E}+02$ & $7.76 \mathrm{E}-03$ & $2.82 \mathrm{E}+02$ & $0.00 \mathrm{E}+00$ & $0.00 \mathrm{E}+00$ & $2.32 \mathrm{E}+07$ \\
\hline 0.375 & 0.350 & $4.40 \mathrm{E}+03$ & $1.68 \mathrm{E}+02$ & $7.76 \mathrm{E}-03$ & $2.82 \mathrm{E}+02$ & $0.00 \mathrm{E}+00$ & $0.00 \mathrm{E}+00$ & $1.60 \mathrm{E}+07$ \\
\hline 0.400 & 0.375 & $6.70 \mathrm{E}+04$ & $1.68 \mathrm{E}+02$ & $7.76 \mathrm{E}-03$ & $2.82 \mathrm{E}+02$ & $0.00 \mathrm{E}+00$ & $0.00 \mathrm{E}+00$ & $1.35 \mathrm{E}+07$ \\
\hline 0.425 & 0.400 & $3.77 \mathrm{E}+04$ & $1.68 \mathrm{E}+02$ & $7.78 \mathrm{E}-03$ & $2.83 \mathrm{E}+02$ & $1.43 \mathrm{E}+05$ & $0.00 \mathrm{E}+00$ & $1.13 \mathrm{E}+07$ \\
\hline 0.450 & 0.425 & $1.05 \mathrm{E}+03$ & $1.68 \mathrm{E}+02$ & $7.78 \mathrm{E}-03$ & $2.83 \mathrm{E}+02$ & $1.01 \mathrm{E}+05$ & $0.00 \mathrm{E}+00$ & $1.23 \mathrm{E}+07$ \\
\hline 0.475 & 0.450 & $4.39 \mathrm{E}+03$ & $1.68 \mathrm{E}+02$ & $7.78 \mathrm{E}-03$ & $2.83 \mathrm{E}+02$ & $0.00 \mathrm{E}+00$ & $0.00 \mathrm{E}+00$ & $8.08 \mathrm{E}+06$ \\
\hline 0.500 & 0.475 & $1.34 \mathrm{E}+02$ & $1.68 \mathrm{E}+02$ & $7.78 \mathrm{E}-03$ & $2.83 \mathrm{E}+02$ & $0.00 \mathrm{E}+00$ & $0.00 \mathrm{E}+00$ & $6.80 \mathrm{E}+06$ \\
\hline 0.525 & 0.500 & $3.18 \mathrm{E}-03$ & $1.60 \mathrm{E}+02$ & $7.37 \mathrm{E}-03$ & $2.68 \mathrm{E}+02$ & $0.00 \mathrm{E}+00$ & $0.00 \mathrm{E}+00$ & $5.82 \mathrm{E}+06$ \\
\hline 0.550 & 0.525 & $6.91 \mathrm{E}+00$ & $1.72 \mathrm{E}+02$ & $7.37 \mathrm{E}-03$ & $2.68 \mathrm{E}+02$ & $0.00 \mathrm{E}+00$ & $0.00 \mathrm{E}+00$ & $4.92 \mathrm{E}+06$ \\
\hline 0.575 & 0.550 & & $1.60 \mathrm{E}+02$ & $7.37 \mathrm{E}-03$ & $2.68 \mathrm{E}+02$ & $0.00 \mathrm{E}+00$ & $0.00 \mathrm{E}+00$ & $4.21 \mathrm{E}+06$ \\
\hline 0.600 & 0.575 & & $1.60 \mathrm{E}+02$ & & $2.68 \mathrm{E}+02$ & $0.00 \mathrm{E}+00$ & $0.00 \mathrm{E}+00$ & $3.59 \mathrm{E}+06$ \\
\hline 0.625 & 0.600 & $1.45 \mathrm{E}+02$ & $1.43 \mathrm{E}+02$ & $6.61 \mathrm{E}-03$ & $2.41 \mathrm{E}+02$ & $2.19 \mathrm{E}+05$ & $0.00 \mathrm{E}+00$ & $3.04 \mathrm{E}+06$ \\
\hline 0.650 & 0.625 & $8.30 \mathrm{E}+02$ & $1.24 \mathrm{E}+03$ & $6.61 \mathrm{E}-03$ & $2.41 \mathrm{E}+02$ & $0.00 \mathrm{E}+00$ & $0.00 \mathrm{E}+00$ & $2.61 \mathrm{E}+06$ \\
\hline 0.675 & 0.650 & $4.57 \mathrm{E}+02$ & $1.43 \mathrm{E}+02$ & $6.61 \mathrm{E}-03$ & $2.41 \mathrm{E}+02$ & $0.00 \mathrm{E}+00$ & $0.00 \mathrm{E}+00$ & $2.20 \mathrm{E}+06$ \\
\hline 0.700 & 0.675 & $3.31 \mathrm{E}+01$ & $4.37 \mathrm{E}+02$ & $6.61 \mathrm{E}-03$ & $2.41 \mathrm{E}+02$ & $0.00 \mathrm{E}+00$ & $0.00 \mathrm{E}+00$ & $1.88 \mathrm{E}+06$ \\
\hline 0.725 & 0.700 & $1.64 \mathrm{E}+02$ & $1.18 \mathrm{E}+02$ & $5.44 \mathrm{E}-03$ & $1.98 \mathrm{E}+02$ & $0.00 \mathrm{E}+00$ & $0.00 \mathrm{E}+00$ & $1.60 \mathrm{E}+06$ \\
\hline 0.750 & 0.725 & $2.47 \mathrm{E}+01$ & $1.18 \mathrm{E}+02$ & $5.44 \mathrm{E}-03$ & $1.98 \mathrm{E}+02$ & $0.00 \mathrm{E}+00$ & $0.00 \mathrm{E}+00$ & $1.35 \mathrm{E}+06$ \\
\hline
\end{tabular}


PCP-2011-0001

Revision 0

\begin{tabular}{|c|c|c|c|c|c|c|c|c|}
\hline \multicolumn{9}{|c|}{ Table 3-21. Photon Source from RASTA (Decay Time $=0$ days) } \\
\hline \multicolumn{2}{|c|}{ Energy (MeV) } & \multicolumn{7}{|c|}{ Photon Source $(\mathrm{p} / \mathrm{s} / \mathrm{g})$} \\
\hline Upper & Lower & Pu-239 & Pu-240 & Pu-241 & Pu-242 & Ra-226 & Ru-106 & Sc-46 \\
\hline 0.775 & 0.750 & $3.31 \mathrm{E}+02$ & $1.18 \mathrm{E}+02$ & $5.44 \mathrm{E}-03$ & $1.98 \mathrm{E}+02$ & $0.00 \mathrm{E}+00$ & $0.00 \mathrm{E}+00$ & $1.15 \mathrm{E}+06$ \\
\hline 0.800 & 0.775 & $6.27 \mathrm{E}+00$ & $1.18 \mathrm{E}+02$ & $5.44 \mathrm{E}-03$ & $1.98 \mathrm{E}+02$ & $0.00 \mathrm{E}+00$ & $0.00 \mathrm{E}+00$ & $9.67 \mathrm{E}+05$ \\
\hline 0.825 & 0.800 & $6.96 \mathrm{E}+00$ & $1.00 \mathrm{E}+02$ & $4.64 \mathrm{E}-03$ & $1.69 \mathrm{E}+02$ & $0.00 \mathrm{E}+00$ & $0.00 \mathrm{E}+00$ & \\
\hline 0.850 & 0.825 & $8.33 \mathrm{E}+00$ & $1.00 \mathrm{E}+02$ & $4.64 \mathrm{E}-03$ & $1.69 \mathrm{E}+02$ & $0.00 \mathrm{E}+00$ & $0.00 \mathrm{E}+00$ & \\
\hline 0.875 & 0.850 & $1.95 \mathrm{E}-03$ & $1.50 \mathrm{E}+02$ & $4.64 \mathrm{E}-03$ & $1.69 \mathrm{E}+02$ & $0.00 \mathrm{E}+00$ & $0.00 \mathrm{E}+00$ & $5.72 \mathrm{E}+05$ \\
\hline 0.900 & 0.875 & $2.91 \mathrm{E}+00$ & $1.00 \mathrm{E}+02$ & $4.64 \mathrm{E}-03$ & $1.69 \mathrm{E}+02$ & $0.00 \mathrm{E}+00$ & $0.00 \mathrm{E}+00$ & $1.26 \mathrm{E}+15$ \\
\hline 0.925 & 0.900 & $1.95 \mathrm{E}-03$ & $1.00 \mathrm{E}+02$ & 4.64E-03 & $1.69 \mathrm{E}+02$ & $0.00 \mathrm{E}+00$ & $0.00 \mathrm{E}+00$ & $3.94 \mathrm{E}+05$ \\
\hline 0.950 & 0.925 & $9.69 \mathrm{E}-01$ & $1.00 \mathrm{E}+02$ & $4.64 \mathrm{E}-03$ & $1.69 \mathrm{E}+02$ & $0.00 \mathrm{E}+00$ & $0.00 \mathrm{E}+00$ & $3.25 \mathrm{E}+05$ \\
\hline 0.975 & 0.950 & $1.28 \mathrm{E}+00$ & $1.00 \mathrm{E}+02$ & $4.64 \mathrm{E}-03$ & $1.69 \mathrm{E}+02$ & $0.00 \mathrm{E}+00$ & $0 \mathrm{E}+00$ & \\
\hline 1.000 & 0.975 & $1.33 \mathrm{E}+00$ & & $4.64 \mathrm{E}-03$ & $1.69 \mathrm{E}+02$ & $0.00 \mathrm{E}+00$ & $0 \mathrm{E}+00$ & \\
\hline 1.250 & 1.000 & $1.23 \mathrm{E}+00$ & $5.26 \mathrm{E}+02$ & $2.43 \mathrm{E}-02$ & $8.84 \mathrm{E}+02$ & $0.00 \mathrm{E}+00$ & $0 \mathrm{E}+00$ & $1.25 \mathrm{E}+15$ \\
\hline 1.500 & 1.250 & $9.36 \mathrm{E}-03$ & $4.99 \mathrm{E}+02$ & $2.31 \mathrm{E}-02$ & $8.40 \mathrm{E}+02$ & $0.00 \mathrm{E}+00$ & $0.00 \mathrm{E}+00$ & $7.90 \mathrm{E}+03$ \\
\hline 1.750 & 1.500 & $5.11 \mathrm{E}-03$ & $2.64 \mathrm{E}+02$ & $1.22 \mathrm{E}-02$ & $4.44 \mathrm{E}+02$ & $0.00 \mathrm{E}+00$ & $0.00 \mathrm{E}+00$ & $0.00 \mathrm{E}+00$ \\
\hline 2.000 & 1.750 & $3.92 \mathrm{E}-03$ & $2.06 \mathrm{E}+02$ & $9.53 \mathrm{E}-03$ & $3.47 \mathrm{E}+02$ & $0.00 \mathrm{E}+00$ & $0 \mathrm{E}+00$ & $0.00 \mathrm{E}+00$ \\
\hline 2.250 & 2.000 & $2.66 \mathrm{E}-03$ & $1.41 \mathrm{E}+02$ & $6.50 \mathrm{E}-03$ & $2.36 \mathrm{E}+02$ & $0.00 \mathrm{E}+00$ & $0 \mathrm{E}+00$ & $1.42 \mathrm{E}+08$ \\
\hline 2.500 & 2.250 & $2.47 \mathrm{E}-03$ & $1.30 \mathrm{E}+02$ & $6.01 \mathrm{E}-03$ & $2.19 \mathrm{E}+02$ & $0.00 \mathrm{E}+00$ & $0 \mathrm{E}+00$ & $0.00 \mathrm{E}+00$ \\
\hline 2.750 & 2.500 & $1.76 \mathrm{E}-03$ & $9.36 \mathrm{E}+01$ & $4.33 \mathrm{E}-03$ & $1.57 \mathrm{E}+02$ & $0.00 \mathrm{E}+00$ & $0 \mathrm{E}+00$ & $0.00 \mathrm{E}+00$ \\
\hline 3.000 & 2.750 & $1.51 \mathrm{E}-03$ & $7.75 \mathrm{E}+01$ & $3.58 \mathrm{E}-03$ & $1.30 \mathrm{E}+02$ & $0.00 \mathrm{E}+00$ & $0 \mathrm{E}+00$ & $0.00 \mathrm{E}+00$ \\
\hline 3.500 & 3.000 & $1.57 \mathrm{E}-03$ & $7.94 \mathrm{E}+01$ & $3.67 \mathrm{E}-03$ & $1.33 \mathrm{E}+02$ & $0.00 \mathrm{E}+00$ & $0 \mathrm{E}+00$ & $0.00 \mathrm{E}+00$ \\
\hline 4.000 & 3.500 & $1.22 \mathrm{E}-03$ & $5.39 \mathrm{E}+01$ & $2.49 \mathrm{E}-03$ & $9.06 \mathrm{E}+01$ & $0.00 \mathrm{E}+00$ & $0 \mathrm{E}+00$ & $0.00 \mathrm{E}+00$ \\
\hline 4.500 & 4.000 & $5.08 \mathrm{E}-04$ & $1.99 \mathrm{E}+01$ & $9.19 \mathrm{E}-04$ & $3.34 \mathrm{E}+01$ & $0.00 \mathrm{E}+00$ & $0 \mathrm{E}+00$ & $0.00 \mathrm{E}+00$ \\
\hline 5.000 & 4.500 & $4.35 \mathrm{E}-04$ & $1.57 \mathrm{E}+01$ & $7.24 \mathrm{E}-04$ & $2.63 \mathrm{E}+01$ & $0.00 \mathrm{E}+00$ & $0 \mathrm{E}+00$ & $0.00 \mathrm{E}+00$ \\
\hline 5.500 & 5.000 & $1.66 \mathrm{E}-04$ & $6.53 \mathrm{E}+00$ & $3.02 \mathrm{E}-04$ & $1.10 \mathrm{E}+01$ & $0.00 \mathrm{E}+00$ & $0.00 \mathrm{E}+00$ & $0.00 \mathrm{E}+00$ \\
\hline 6.000 & 5.500 & $1.54 \mathrm{E}-04$ & $5.91 \mathrm{E}+00$ & $2.73 \mathrm{E}-04$ & $9.94 \mathrm{E}+00$ & $0.00 \mathrm{E}+00$ & $0.00 \mathrm{E}+00$ & $0.00 \mathrm{E}+00$ \\
\hline 6.500 & 6.000 & $6.03 \mathrm{E}-05$ & $2.74 \mathrm{E}+00$ & $1.27 \mathrm{E}-04$ & $4.61 \mathrm{E}+00$ & $0.00 \mathrm{E}+00$ & $0.00 \mathrm{E}+00$ & $0.00 \mathrm{E}+00$ \\
\hline 7.000 & 6.500 & $5.81 \mathrm{E}-05$ & $2.59 \mathrm{E}+00$ & $1.20 \mathrm{E}-04$ & $4.35 \mathrm{E}+00$ & $0.00 \mathrm{E}+00$ & $0.00 \mathrm{E}+00$ & $0.00 \mathrm{E}+00$ \\
\hline 7.500 & 7.000 & $2.80 \mathrm{E}-06$ & $2.39 \mathrm{E}-01$ & $1.10 \mathrm{E}-05$ & $4.01 \mathrm{E}-01$ & $0.00 \mathrm{E}+00$ & $0.00 \mathrm{E}+00$ & $0.00 \mathrm{E}+00$ \\
\hline 8.000 & 7.500 & $2.78 \mathrm{E}-06$ & $2.36 \mathrm{E}-01$ & $1.09 \mathrm{E}-05$ & $3.97 \mathrm{E}-01$ & $0.00 \mathrm{E}+00$ & $0.00 \mathrm{E}+00$ & $0.00 \mathrm{E}+00$ \\
\hline 9.000 & 8.000 & $5.56 \mathrm{E}-06$ & $4.70 \mathrm{E}-01$ & $2.17 \mathrm{E}-05$ & $7.91 \mathrm{E}-01$ & $0.00 \mathrm{E}+00$ & $0.00 \mathrm{E}+00$ & $0.00 \mathrm{E}+00$ \\
\hline \multirow[t]{2}{*}{10.000} & 9.000 & $5.55 \mathrm{E}-06$ & $4.70 \mathrm{E}-01$ & $2.17 \mathrm{E}-05$ & $7.91 \mathrm{E}-01$ & $0.00 \mathrm{E}+00$ & $0.00 \mathrm{E}+00$ & $0.00 \mathrm{E}+00$ \\
\hline & Total & $9.79 \mathrm{E}+07$ & $9.04 \mathrm{E}+08$ & $3.16 \mathrm{E}+08$ & $1.31 \mathrm{E}+07$ & $1.69 \mathrm{E}+09$ & $1.34 \mathrm{E}+10$ & $2.51 \mathrm{E}+15$ \\
\hline
\end{tabular}


PCP-2011-0001

Revision 0

\begin{tabular}{|c|c|c|c|c|c|c|c|c|}
\hline \multicolumn{9}{|c|}{ Table 3-22. Photon Source from RASTA (Decay Time $=0$ days) } \\
\hline \multicolumn{2}{|c|}{ Energy $(\mathrm{MeV})$} & \multicolumn{7}{|c|}{ Photon Source (p/s/g) } \\
\hline Upper & Lower & Se-75 & Sm-145 & \begin{tabular}{|l|} 
Sr-90 \\
\end{tabular} & Tm-170 & Yb-169 & Zn-65 & Zr-95 \\
\hline 0.020 & 0.010 & $2.13 \mathrm{E}+14$ & $0.00 \mathrm{E}+00$ & $7.62 \mathrm{E}+10$ & $1.03 \mathrm{E}+13$ & $0.00 \mathrm{E}+00$ & $3.24 \mathrm{E}+10$ & $7.26 \mathrm{E}+12$ \\
\hline 0.030 & 0.020 & $1.34 \mathrm{E}+11$ & $0.00 \mathrm{E}+00$ & $3.75 \mathrm{E}+10$ & $5.21 \mathrm{E}+12$ & $1.76 \mathrm{E}+12$ & $1.54 \mathrm{E}+10$ & $3.03 \mathrm{E}+12$ \\
\hline 0.040 & 0.030 & $0.00 \mathrm{E}+00$ & $1.22 \mathrm{E}+14$ & $2.28 \mathrm{E}+10$ & $3.25 \mathrm{E}+12$ & $0.00 \mathrm{E}+00$ & $8.99 \mathrm{E}+09$ & $1.77 \mathrm{E}+12$ \\
\hline 0.050 & 0.040 & $0.00 \mathrm{E}+00$ & $2.71 \mathrm{E}+13$ & $1.53 \mathrm{E}+10$ & $2.46 \mathrm{E}+12$ & $5.21 \mathrm{E}+14$ & $5.80 \mathrm{E}+09$ & $1.14 \mathrm{E}+12$ \\
\hline 0.060 & 0.050 & $0.00 \mathrm{E}+00$ & $0.00 \mathrm{E}+00$ & $1.10 \mathrm{E}+10$ & $1.08 \mathrm{E}+13$ & $1.12 \mathrm{E}+15$ & $3.96 \mathrm{E}+09$ & $7.82 \mathrm{E}+11$ \\
\hline 0.070 & 0.060 & $6.04 \mathrm{E}+12$ & $1.18 \mathrm{E}+13$ & $8.18 \mathrm{E}+09$ & $1.67 \mathrm{E}+12$ & $3.61 \mathrm{E}+14$ & $2.80 \mathrm{E}+09$ & $5.57 \mathrm{E}+11$ \\
\hline 0.080 & 0.070 & $0.00 \mathrm{E}+00$ & $0.00 \mathrm{E}+00$ & $6.27 \mathrm{E}+09$ & $1.01 \mathrm{E}+12$ & $0.00 \mathrm{E}+00$ & $2.02 \mathrm{E}+09$ & $4.08 \mathrm{E}+11$ \\
\hline 0.090 & 0.080 & $4.25 \mathrm{E}+10$ & $0.00 \mathrm{E}+00$ & $4.91 \mathrm{E}+09$ & $7.94 \mathrm{E}+12$ & $0.00 \mathrm{E}+00$ & $1.49 \mathrm{E}+09$ & $3.04 \mathrm{E}+11$ \\
\hline 0.100 & 0.090 & $1.92 \mathrm{E}+13$ & $0.00 \mathrm{E}+00$ & $3.91 \mathrm{E}+09$ & $6.60 \mathrm{E}+11$ & $2.24 \mathrm{E}+13$ & $1.10 \mathrm{E}+09$ & $2.30 \mathrm{E}+11$ \\
\hline 0.110 & 0.100 & $0.00 \mathrm{E}+00$ & $0.00 \mathrm{E}+00$ & $3.15 \mathrm{E}+09$ & $5.49 \mathrm{E}+11$ & $1.62 \mathrm{E}+14$ & $8.25 \mathrm{E}+08$ & $1.76 \mathrm{E}+11$ \\
\hline 0.120 & 0.110 & $0.00 \mathrm{E}+00$ & $0.00 \mathrm{E}+00$ & $2.56 \mathrm{E}+09$ & $4.61 \mathrm{E}+11$ & $1.78 \mathrm{E}+13$ & $6.18 \mathrm{E}+08$ & $1.35 \mathrm{E}+11$ \\
\hline 0.130 & 0.120 & $9.22 \mathrm{E}+13$ & $6.57 \mathrm{E}+08$ & $2.10 \mathrm{E}+09$ & $3.92 \mathrm{E}+11$ & $0.00 \mathrm{E}+00$ & $4.63 \mathrm{E}+08$ & $1.05 \mathrm{E}+11$ \\
\hline 0.140 & 0.130 & $3.28 \mathrm{E}+14$ & $0.00 \mathrm{E}+00$ & $1.73 \mathrm{E}+09$ & $3.35 \mathrm{E}+11$ & $9.91 \mathrm{E}+13$ & $3.47 \mathrm{E}+08$ & $8.16 \mathrm{E}+10$ \\
\hline 0.150 & 0.140 & $0.00 \mathrm{E}+00$ & $0.00 \mathrm{E}+00$ & $1.44 \mathrm{E}+09$ & $2.88 \mathrm{E}+11$ & $0.00 \mathrm{E}+00$ & $2.58 \mathrm{E}+08$ & $6.37 \mathrm{E}+10$ \\
\hline 0.160 & 0.150 & $0.00 \mathrm{E}+00$ & $0.00 \mathrm{E}+00$ & $1.20 \mathrm{E}+09$ & $2.50 \mathrm{E}+11$ & $7.45 \mathrm{E}+10$ & $1.92 \mathrm{E}+08$ & $4.98 \mathrm{E}+10$ \\
\hline 0.170 & 0.160 & $0.00 \mathrm{E}+00$ & $0.00 \mathrm{E}+00$ & $1.00 \mathrm{E}+09$ & $2.17 \mathrm{E}+11$ & $0.00 \mathrm{E}+00$ & $1.41 \mathrm{E}+08$ & $3.90 \mathrm{E}+10$ \\
\hline 0.180 & 0.170 & $0.00 \mathrm{E}+00$ & $0.00 \mathrm{E}+00$ & $8.39 \mathrm{E}+08$ & $1.90 \mathrm{E}+11$ & $2.02 \mathrm{E}+14$ & $1.03 \mathrm{E}+08$ & $3.05 \mathrm{E}+10$ \\
\hline 0.190 & 0.180 & $0.00 \mathrm{E}+00$ & $0.00 \mathrm{E}+00$ & $7.05 \mathrm{E}+08$ & $1.66 \mathrm{E}+11$ & $0.00 \mathrm{E}+00$ & $7.40 \mathrm{E}+07$ & $2.39 \mathrm{E}+10$ \\
\hline 0.200 & 0.190 & $8.23 \mathrm{E}+12$ & $0.00 \mathrm{E}+00$ & $5.92 \mathrm{E}+08$ & $1.46 \mathrm{E}+11$ & $3.25 \mathrm{E}+14$ & $5.25 \mathrm{E}+07$ & $1.87 \mathrm{E}+10$ \\
\hline 0.210 & 0.200 & $1.02 \mathrm{E}+10$ & $0.00 \mathrm{E}+00$ & $4.98 \mathrm{E}+08$ & $1.29 \mathrm{E}+11$ & $0.00 \mathrm{E}+00$ & $3.66 \mathrm{E}+07$ & $1.47 \mathrm{E}+10$ \\
\hline 0.220 & 0.210 & $0.00 \mathrm{E}+00$ & $0.00 \mathrm{E}+00$ & $4.19 \mathrm{E}+08$ & $1.14 \mathrm{E}+11$ & $0.00 \mathrm{E}+00$ & $2.49 \mathrm{E}+07$ & $1.15 \mathrm{E}+10$ \\
\hline 0.230 & 0.220 & $0.00 \mathrm{E}+00$ & $0.00 \mathrm{E}+00$ & $3.52 \mathrm{E}+08$ & $1.01 \mathrm{E}+11$ & $0.00 \mathrm{E}+00$ & $1.65 \mathrm{E}+07$ & $8.98 \mathrm{E}+09$ \\
\hline 0.240 & 0.230 & $0.00 \mathrm{E}+00$ & $0.00 \mathrm{E}+00$ & $2.96 \mathrm{E}+08$ & $9.01 \mathrm{E}+10$ & $0.00 \mathrm{E}+00$ & $1.06 \mathrm{E}+07$ & $7.03 \mathrm{E}+09$ \\
\hline 0.250 & 0.240 & $0.00 \mathrm{E}+00$ & $0.00 \mathrm{E}+00$ & $2.49 \mathrm{E}+08$ & $8.02 \mathrm{E}+10$ & $1.07 \mathrm{E}+12$ & $6.55 \mathrm{E}+06$ & $5.52 \mathrm{E}+09$ \\
\hline 0.260 & 0.250 & $0.00 \mathrm{E}+00$ & $0.00 \mathrm{E}+00$ & $2.08 \mathrm{E}+08$ & $7.15 \mathrm{E}+10$ & $0.00 \mathrm{E}+00$ & $3.84 \mathrm{E}+06$ & $4.35 \mathrm{E}+09$ \\
\hline 0.270 & 0.260 & $3.19 \mathrm{E}+14$ & $0.00 \mathrm{E}+00$ & $1.74 \mathrm{E}+08$ & $6.38 \mathrm{E}+10$ & $1.48 \mathrm{E}+13$ & $2.10 \mathrm{E}+06$ & $3.44 \mathrm{E}+09$ \\
\hline 0.280 & 0.270 & $1.38 \mathrm{E}+14$ & $0.00 \mathrm{E}+00$ & $1.45 \mathrm{E}+08$ & $5.70 \mathrm{E}+10$ & $0.00 \mathrm{E}+00$ & $1.05 \mathrm{E}+06$ & $2.75 \mathrm{E}+09$ \\
\hline 0.290 & 0.280 & $0.00 \mathrm{E}+00$ & $0.00 \mathrm{E}+00$ & $1.21 \mathrm{E}+08$ & $5.10 \mathrm{E}+10$ & $0.00 \mathrm{E}+00$ & $4.59 \mathrm{E}+05$ & $2.22 \mathrm{E}+09$ \\
\hline 0.300 & 0.290 & $1.90 \mathrm{E}+09$ & $0.00 \mathrm{E}+00$ & $1.00 \mathrm{E}+08$ & $4.57 \mathrm{E}+10$ & $0.00 \mathrm{E}+00$ & $1.65 \mathrm{E}+05$ & $1.81 \mathrm{E}+09$ \\
\hline 0.325 & 0.300 & $6.86 \mathrm{E}+12$ & $0.00 \mathrm{E}+00$ & $1.81 \mathrm{E}+08$ & $9.49 \mathrm{E}+10$ & $8.68 \mathrm{E}+13$ & $2.77 \mathrm{E}+04$ & $3.31 \mathrm{E}+09$ \\
\hline 0.350 & 0.325 & $2.24 \mathrm{E}+09$ & $0.00 \mathrm{E}+00$ & $1.08 \mathrm{E}+08$ & $7.25 \mathrm{E}+10$ & $1.04 \mathrm{E}+11$ & $9.32 \mathrm{E}+09$ & $2.27 \mathrm{E}+09$ \\
\hline 0.375 & 0.350 & $5.60 \mathrm{E}+09$ & $0.00 \mathrm{E}+00$ & $6.07 \mathrm{E}+07$ & $5.48 \mathrm{E}+10$ & $9.51 \mathrm{E}+09$ & $0.00 \mathrm{E}+00$ & $1.66 \mathrm{E}+09$ \\
\hline 0.400 & 0.375 & $0.00 \mathrm{E}+00$ & $0.00 \mathrm{E}+00$ & $3.36 \mathrm{E}+07$ & $4.24 \mathrm{E}+10$ & $6.33 \mathrm{E}+09$ & $0.00 \mathrm{E}+00$ & $1.29 \mathrm{E}+09$ \\
\hline 0.425 & 0.400 & $5.93 \mathrm{E}+13$ & $0.00 \mathrm{E}+00$ & $1.61 \mathrm{E}+07$ & $3.21 \mathrm{E}+10$ & $0.00 \mathrm{E}+00$ & $0.00 \mathrm{E}+00$ & $9.81 \mathrm{E}+08$ \\
\hline 0.450 & 0.425 & $0.00 \mathrm{E}+00$ & $0.00 \mathrm{E}+00$ & $3.51 \mathrm{E}+06$ & $2.06 \mathrm{E}+10$ & $0.00 \mathrm{E}+00$ & $0.00 \mathrm{E}+00$ & $7.75 \mathrm{E}+08$ \\
\hline 0.475 & 0.450 & $1.72 \mathrm{E}+09$ & $0.00 \mathrm{E}+00$ & $2.62 \mathrm{E}+06$ & $1.88 \mathrm{E}+10$ & $4.44 \mathrm{E}+09$ & $0.00 \mathrm{E}+00$ & $5.82 \mathrm{E}+08$ \\
\hline 0.500 & 0.475 & $1.26 \mathrm{E}+09$ & $2.71 \mathrm{E}+09$ & $6.33 \mathrm{E}+05$ & $1.41 \mathrm{E}+10$ & $1.33 \mathrm{E}+10$ & $0.00 \mathrm{E}+00$ & $4.38 \mathrm{E}+08$ \\
\hline 0.525 & 0.500 & $0.00 \mathrm{E}+00$ & $0.00 \mathrm{E}+00$ & $8.47 \mathrm{E}+04$ & $1.07 \mathrm{E}+10$ & $3.77 \mathrm{E}+10$ & $8.87 \mathrm{E}+12$ & $3.34 \mathrm{E}+08$ \\
\hline 0.550 & 0.525 & $6.12 \mathrm{E}+07$ & $0.00 \mathrm{E}+00$ & $8.33 \mathrm{E}+02$ & $7.87 \mathrm{E}+09$ & $1.07 \mathrm{E}+09$ & $0.00 \mathrm{E}+00$ & $2.47 \mathrm{E}+08$ \\
\hline 0.575 & 0.550 & $2.06 \mathrm{E}+11$ & $0.00 \mathrm{E}+00$ & $0.00 \mathrm{E}+00$ & $5.88 \mathrm{E}+09$ & $1.29 \mathrm{E}+10$ & $0.00 \mathrm{E}+00$ & $1.85 \mathrm{E}+08$ \\
\hline 0.600 & 0.575 & $0.00 \mathrm{E}+00$ & $0.00 \mathrm{E}+00$ & $0.00 \mathrm{E}+00$ & $4.29 \mathrm{E}+09$ & $1.68 \mathrm{E}+10$ & $0.00 \mathrm{E}+00$ & $1.35 \mathrm{E}+08$ \\
\hline 0.625 & 0.600 & $2.48 \mathrm{E}+10$ & $0.00 \mathrm{E}+00$ & $0.00 \mathrm{E}+00$ & $3.04 \mathrm{E}+09$ & $5.57 \mathrm{E}+10$ & $0.00 \mathrm{E}+00$ & $9.59 \mathrm{E}+07$ \\
\hline 0.650 & 0.625 & $0.00 \mathrm{E}+00$ & $0.00 \mathrm{E}+00$ & $0.00 \mathrm{E}+00$ & $2.18 \mathrm{E}+09$ & $7.11 \mathrm{E}+08$ & $0.00 \mathrm{E}+00$ & $6.87 \mathrm{E}+07$ \\
\hline 0.675 & 0.650 & $0.00 \mathrm{E}+00$ & $0.00 \mathrm{E}+00$ & $0.00 \mathrm{E}+00$ & $1.49 \mathrm{E}+09$ & $1.83 \mathrm{E}+09$ & $0.00 \mathrm{E}+00$ & $4.68 \mathrm{E}+07$ \\
\hline 0.700 & 0.675 & $0.00 \mathrm{E}+00$ & $0.00 \mathrm{E}+00$ & $0.00 \mathrm{E}+00$ & $1.01 \mathrm{E}+09$ & $0.00 \mathrm{E}+00$ & $0.00 \mathrm{E}+00$ & $3.16 \mathrm{E}+07$ \\
\hline 0.725 & 0.700 & $0.00 \mathrm{E}+00$ & $0.00 \mathrm{E}+00$ & $0.00 \mathrm{E}+00$ & $6.73 \mathrm{E}+08$ & $3.51 \mathrm{E}+08$ & $0.00 \mathrm{E}+00$ & $3.60 \mathrm{E}+14$ \\
\hline 0.750 & 0.725 & $0.00 \mathrm{E}+00$ & $0.00 \mathrm{E}+00$ & $0.00 \mathrm{E}+00$ & $4.19 \mathrm{E}+08$ & $0.00 \mathrm{E}+00$ & $0.00 \mathrm{E}+00$ & $1.33 \mathrm{E}+07$ \\
\hline
\end{tabular}


PCP-2011-0001

Revision 0

\begin{tabular}{|c|c|c|c|c|c|c|c|c|}
\hline \multicolumn{9}{|c|}{ Table 3-22. Photon Source from RASTA (Decay Time $=0$ days) } \\
\hline \multicolumn{2}{|c|}{ Energy (MeV) } & \multicolumn{7}{|c|}{ Photon Source $(\mathrm{p} / \mathrm{s} / \mathrm{g})$} \\
\hline Upper & Lower & Se-75 & Sm-145 & Sr-90 & Tm-170 & Yb-169 & Zn-65 & Zr-95 \\
\hline 0.775 & 0.750 & $0.00 \mathrm{E}+00$ & $0.00 \mathrm{E}+00$ & $0.00 \mathrm{E}+00$ & $2.60 \mathrm{E}+08$ & $1.98 \mathrm{E}+09$ & $9.25 \mathrm{E}+09$ & $4.34 \mathrm{E}+14$ \\
\hline 0.800 & 0.775 & $0.00 \mathrm{E}+00$ & $0.00 \mathrm{E}+00$ & $0.00 \mathrm{E}+00$ & $1.46 \mathrm{E}+08$ & $0.00 \mathrm{E}+00$ & $0.00 \mathrm{E}+00$ & $5.10 \mathrm{E}+06$ \\
\hline 0.825 & 0.800 & $7.11 \mathrm{E}+08$ & $0.00 \mathrm{E}+00$ & $0.00 \mathrm{E}+00$ & $8.04 \mathrm{E}+07$ & $0.00 \mathrm{E}+00$ & $0.00 \mathrm{E}+00$ & $3.20 \mathrm{E}+06$ \\
\hline 0.850 & 0.825 & $0.00 \mathrm{E}+00$ & $0.00 \mathrm{E}+00$ & $0.00 \mathrm{E}+00$ & $3.94 \mathrm{E}+07$ & $0.00 \mathrm{E}+00$ & $0.00 \mathrm{E}+00$ & $2.01 \mathrm{E}+06$ \\
\hline 0.875 & 0.850 & $0.00 \mathrm{E}+00$ & $0.00 \mathrm{E}+00$ & $0.00 \mathrm{E}+00$ & $1.63 \mathrm{E}+07$ & $0.00 \mathrm{E}+00$ & $0.00 \mathrm{E}+00$ & \\
\hline 0.900 & 0.875 & $0.00 \mathrm{E}+00$ & $0.00 \mathrm{E}+00$ & $0.00 \mathrm{E}+00$ & $5.89 \mathrm{E}+06$ & $0.00 \mathrm{E}+00$ & $0.00 \mathrm{E}+00$ & $8.66 \mathrm{E}+05$ \\
\hline 0.925 & 0.900 & $0.00 \mathrm{E}+00$ & $0.00 \mathrm{E}+00$ & $0.00 \mathrm{E}+00$ & $1.33 \mathrm{E}+06$ & $0.00 \mathrm{E}+00$ & $0.00 \mathrm{E}+00$ & $5.45 \mathrm{E}+05$ \\
\hline 0.950 & 0.925 & $0.00 \mathrm{E}+00$ & $0.00 \mathrm{E}+00$ & $0.00 \mathrm{E}+00$ & $1.34 \mathrm{E}+05$ & $0.00 \mathrm{E}+00$ & $0.00 \mathrm{E}+00$ & $3.32 \mathrm{E}+05$ \\
\hline 0.975 & 0.950 & $0.00 \mathrm{E}+00$ & $0.00 \mathrm{E}+00$ & $0.00 \mathrm{E}+00$ & $1.27 \mathrm{E}+03$ & $0.00 \mathrm{E}+00$ & $0.00 \mathrm{E}+00$ & \\
\hline 1.000 & 0.975 & $0.00 \mathrm{E}+00$ & & $0.00 \mathrm{E}+00$ & $0.00 \mathrm{E}+00$ & $0.00 \mathrm{E}+00$ & & \\
\hline 1.250 & 1.000 & $0.00 \mathrm{E}+00$ & $0.00 \mathrm{E}+00$ & $0.00 \mathrm{E}+00$ & $0.00 \mathrm{E}+00$ & $0.00 \mathrm{E}+00$ & $1.53 \mathrm{E}+14$ & $2.57 \mathrm{E}+04$ \\
\hline 1.500 & 1.250 & $0.00 \mathrm{E}+00$ & $0.00 \mathrm{E}+00$ & $0.00 \mathrm{E}+00$ & $0.00 \mathrm{E}+00$ & $0.00 \mathrm{E}+00$ & $0.00 \mathrm{E}+00$ & $0.00 \mathrm{E}+00$ \\
\hline 1.750 & 1.500 & $0.00 \mathrm{E}+00$ & $0.00 \mathrm{E}+00$ & $0.00 \mathrm{E}+00$ & $0.00 \mathrm{E}+00$ & $0.00 \mathrm{E}+00$ & $0.00 \mathrm{E}+00$ & $0.00 \mathrm{E}+00$ \\
\hline 2.000 & 1.750 & $0.00 \mathrm{E}+00$ & $0.00 \mathrm{E}+00$ & $0.00 \mathrm{E}+00$ & $0.00 \mathrm{E}+00$ & $0.00 \mathrm{E}+00$ & $0.00 \mathrm{E}+00$ & $0.00 \mathrm{E}+00$ \\
\hline 2.250 & 2.000 & $0.00 \mathrm{E}+00$ & $0.00 \mathrm{E}+00$ & $0.00 \mathrm{E}+00$ & $0.00 \mathrm{E}+00$ & $0.00 \mathrm{E}+00$ & $0.00 \mathrm{E}+00$ & $0.00 \mathrm{E}+00$ \\
\hline 2.500 & 2.250 & $0.00 \mathrm{E}+00$ & $0.00 \mathrm{E}+00$ & $0.00 \mathrm{E}+00$ & $0.00 \mathrm{E}+00$ & $0.00 \mathrm{E}+00$ & $0.00 \mathrm{E}+00$ & $0 \mathrm{E}+00$ \\
\hline 2.750 & 2.500 & $0.00 \mathrm{E}+00$ & $0.00 \mathrm{E}+00$ & $0.00 \mathrm{E}+00$ & $0.00 \mathrm{E}+00$ & $0.00 \mathrm{E}+00$ & $0.00 \mathrm{E}+00$ & $0.00 \mathrm{E}+00$ \\
\hline 3.000 & 2.750 & $0.00 \mathrm{E}+00$ & $0.00 \mathrm{E}+00$ & $0.00 \mathrm{E}+00$ & $0.00 \mathrm{E}+00$ & $0.00 \mathrm{E}+00$ & $0 \mathrm{E}+00$ & $0 \mathrm{E}+00$ \\
\hline 3.500 & 3.000 & $0.00 \mathrm{E}+00$ & $0.00 \mathrm{E}+00$ & $0.00 \mathrm{E}+00$ & $0.00 \mathrm{E}+00$ & $0.00 \mathrm{E}+00$ & $0.00 \mathrm{E}+00$ & $0.00 \mathrm{E}+00$ \\
\hline 4.000 & 3.500 & $0.00 \mathrm{E}+00$ & $0.00 \mathrm{E}+00$ & $0.00 \mathrm{E}+00$ & $0.00 \mathrm{E}+00$ & $0.00 \mathrm{E}+00$ & $0.00 \mathrm{E}+00$ & $0.00 \mathrm{E}+00$ \\
\hline 4.500 & 4.000 & $0.00 \mathrm{E}+00$ & $0.00 \mathrm{E}+00$ & $0.00 \mathrm{E}+00$ & $0.00 \mathrm{E}+00$ & $0.00 \mathrm{E}+00$ & $0.00 \mathrm{E}+00$ & $00 \mathrm{E}+00$ \\
\hline 5.000 & 4.500 & $0.00 \mathrm{E}+00$ & $0.00 \mathrm{E}+00$ & $0.00 \mathrm{E}+00$ & $0.00 \mathrm{E}+00$ & $0.00 \mathrm{E}+00$ & $0 \mathrm{E}+00$ & $0 \mathrm{E}+00$ \\
\hline 5.500 & 5.000 & $0.00 \mathrm{E}+00$ & $0.00 \mathrm{E}+00$ & $0.00 \mathrm{E}+00$ & $0.00 \mathrm{E}+00$ & $0.00 \mathrm{E}+00$ & $0.00 \mathrm{E}+00$ & $0.00 \mathrm{E}+00$ \\
\hline 6.000 & 5.500 & $0.00 \mathrm{E}+00$ & $0.00 \mathrm{E}+00$ & $0.00 \mathrm{E}+00$ & $0.00 \mathrm{E}+00$ & $0.00 \mathrm{E}+00$ & $0.00 \mathrm{E}+00$ & $0.00 \mathrm{E}+00$ \\
\hline 6.500 & 6.000 & $0.00 \mathrm{E}+00$ & $0.00 \mathrm{E}+00$ & $0.00 \mathrm{E}+00$ & $0.00 \mathrm{E}+00$ & $0.00 \mathrm{E}+00$ & $0.00 \mathrm{E}+00$ & $0.00 \mathrm{E}+00$ \\
\hline 7.000 & 6.500 & $0.00 \mathrm{E}+00$ & $0.00 \mathrm{E}+00$ & $0.00 \mathrm{E}+00$ & $0.00 \mathrm{E}+00$ & $0.00 \mathrm{E}+00$ & $0.00 \mathrm{E}+00$ & $0.00 \mathrm{E}+00$ \\
\hline 7.500 & 7.000 & $0.00 \mathrm{E}+00$ & $0.00 \mathrm{E}+00$ & $0.00 \mathrm{E}+00$ & $0.00 \mathrm{E}+00$ & $0.00 \mathrm{E}+00$ & $0.00 \mathrm{E}+00$ & $0.00 \mathrm{E}+00$ \\
\hline 8.000 & 7.500 & $0.00 \mathrm{E}+00$ & $0.00 \mathrm{E}+00$ & $0.00 \mathrm{E}+00$ & $0.00 \mathrm{E}+00$ & $0.00 \mathrm{E}+00$ & $0.00 \mathrm{E}+00$ & $0.00 \mathrm{E}+00$ \\
\hline 9.000 & 8.000 & $0.00 \mathrm{E}+00$ & $0.00 \mathrm{E}+00$ & $0.00 \mathrm{E}+00$ & $0.00 \mathrm{E}+00$ & $0.00 \mathrm{E}+00$ & $0.00 \mathrm{E}+00$ & $0.00 \mathrm{E}+00$ \\
\hline \multirow[t]{2}{*}{10.000} & 9.000 & $0.00 \mathrm{E}+00$ & $0.00 \mathrm{E}+00$ & $0.00 \mathrm{E}+00$ & $0.00 \mathrm{E}+00$ & $0.00 \mathrm{E}+00$ & $0.00 \mathrm{E}+00$ & $0.00 \mathrm{E}+00$ \\
\hline & Total & $1.19 \mathrm{E}+15$ & $1.61 \mathrm{E}+14$ & $2.04 \mathrm{E}+11$ & $4.75 \mathrm{E}+13$ & $2.93 \mathrm{E}+15$ & $1.62 \mathrm{E}+14$ & $8.10 \mathrm{E}+14$ \\
\hline
\end{tabular}


PCP-2011-0001

Revision 0

Table 3-23. Photon Source from ORIGEN-S (Decay Time $=30$ days)

\begin{tabular}{|c|c|c|c|c|c|c|c|c|c|}
\hline \multicolumn{2}{|c|}{ Energy (MeV) } & \multicolumn{8}{|c|}{ Photon Source (p/s/g) } \\
\hline Upper & & Ac-227 & Am-243 & Np-237 & Pb-210 & Pu-241 & Ra-226 & Sr-90 & Zr-95 \\
\hline 0.020 & & $.29 \mathrm{E}+12$ & $42 \mathrm{E}+09$ & $2.66 \mathrm{E}+07$ & & & & $1.28 \mathrm{E}+12$ & \\
\hline & 0.020 & $6 \mathrm{E}+11$ & $55 \mathrm{E}+07$ & $4.79 \mathrm{E}+06$ & & & $3.64 \mathrm{E}+09$ & $.02 \mathrm{E}+11$ & $7.20 \mathrm{E}+12$ \\
\hline & & & & $4.15 \mathrm{E}+04$ & & & & & \\
\hline & & +11 & $79 \mathrm{E}+08$ & $6.47 \mathrm{E}+04$ & & & $82 \mathrm{E}+09$ & & \\
\hline 60 & 0 & $\mathrm{E}+11$ & $85 \mathrm{E}+07$ & $1.19 \mathrm{E}+05$ & $3.43 \mathrm{E}+10$ & & $1.43 \mathrm{E}+09$ & $.06 \mathrm{E}+11$ & $1.50 \mathrm{E}+12$ \\
\hline 070 & & $\mathrm{E}+10$ & $14 \mathrm{E}+08$ & $1.70 \mathrm{E}+04$ & 3.21 & & 9.791 & & \\
\hline 80 & & $\mathrm{E}+10$ & $\mathrm{E}+09$ & $2.00 \mathrm{E}+05$ & & & $7.50 \mathrm{E}+09$ & $\mathrm{E}+11$ & \\
\hline 090 & 0 & $74 \mathrm{E}+11$ & $49 \mathrm{E}+07$ & $3.59 \mathrm{E}+06$ & $2.01 \mathrm{E}+10$ & & $2.70 \mathrm{E}+09$ & $1.28 \mathrm{E}+11$ & $6.60 \mathrm{E}+11$ \\
\hline & & $7 \mathrm{E}+11$ & $20 \mathrm{E}+09$ & +06 & & & $7.18 \mathrm{E}+08$ & +11 & $\mathrm{E}+11$ \\
\hline 110 & & $92 \mathrm{E}+10$ & $83 \mathrm{E}+09$ & $4.32 \mathrm{E}+05$ & 1.07 & 07 & $3.38 \mathrm{E}+08$ & $6.98 \mathrm{E}+10$ & $2.98 \mathrm{E}+11$ \\
\hline 120 & & $93 \mathrm{E}+10$ & $.09 \mathrm{E}+08$ & $1.09 \mathrm{E}+06$ & $1.20 \mathrm{E}+10$ & & $3.86 \mathrm{E}+08$ & $8.08 \mathrm{E}+10$ & $2.86 \mathrm{E}+11$ \\
\hline & & $1 \mathrm{E}+10$ & $12 \mathrm{E}+08$ & $2.16 \mathrm{E}+03$ & & & $3.55 \mathrm{E}+08$ & & \\
\hline 140 & & $16 \mathrm{E}+10$ & $\mathrm{E}+06$ & $4.22 \mathrm{E}+04$ & 8.73 & & $3.09 \mathrm{E}+08$ & $6.10 \mathrm{E}+10$ & $1.66 \mathrm{E}+11$ \\
\hline 150 & 0.140 & $78 \mathrm{E}+10$ & $.10 \mathrm{E}+07$ & $1.13 \mathrm{E}+05$ & $8.13 \mathrm{I}$ & 7.26 & $2.81 \mathrm{E}+08$ & $5.68 \mathrm{E}+10$ & $1.55 \mathrm{E}+11$ \\
\hline & & $7 \mathrm{E}+10$ & +06 & +04 & & & & & \\
\hline 170 & & $71 \mathrm{E}+09$ & +06 & +04 & & & $2.07 \mathrm{E}+08$ & $4.47 \mathrm{E}+10$ & $9.22 \mathrm{E}+10$ \\
\hline 180 & & $89 \mathrm{E}+09$ & $9.59 \mathrm{E}+05$ & $1.03 \mathrm{E}+04$ & 5.01 & 4.78 & $1.73 \mathrm{E}+08$ & +10 & $5.83 \mathrm{E}+10$ \\
\hline & & $99 \mathrm{E}+09$ & $6.78 \mathrm{E}+06$ & +03 & & & $8+09$ & & \\
\hline .200 & & $14 \mathrm{E}+09$ & +06 & $\mathrm{E}+04$ & 7.83 & & $2.97 \mathrm{E}+08$ & $6.22 \mathrm{E}+10$ & $6.88 \mathrm{E}+10$ \\
\hline & & $04 \mathrm{E}+10$ & +08 & 1.8 & & & $2.65 \mathrm{E}+08$ & 5.91 & 3.00 \\
\hline 20 & & $29 \mathrm{E}+10$ & -08 & +04 & & & +00 & & \\
\hline 0.230 & & $51 \mathrm{E}+08$ & & +03 & & & & & \\
\hline & & $8 \mathrm{E}+11$ & & +04 & & & $0.00 \mathrm{E}+00$ & 0.00 & 1.63 \\
\hline 0.250 & & $4.20 \mathrm{E}+09$ & +01 & 9.7 & & & $8+09$ & 0.00 & $0.00 \mathrm{E}+00$ \\
\hline 0.260 & & $1.52 \mathrm{E}+11$ & & & & & 4.85 & & $3.47 \mathrm{E}+10$ \\
\hline & & & & & & & 2.73 & & \\
\hline 280 & & $E+11$ & +09 & +04 & 0.0 & & 1.82 & 0.0 & $0.00 \mathrm{E}+00$ \\
\hline 0.290 & & $\mathrm{E}+10$ & -07 & 3.0 & & & +07 & 0.00 & \\
\hline & & +10 & & +05 & & & +09 & & 0.00 \\
\hline & & +11 & +08 & +06 & & & 3.95 & 8.2 & $1.31 \mathrm{E}+10$ \\
\hline & & +11 & & & & & 9.4 & & \\
\hline & & $\mathrm{E}+11$ & 01 & +04 & 0.0 & & 1.3 & 0.00 & 0.00 \\
\hline 0.400 & & $.53 \mathrm{E}+09$ & +05 & +05 & 4.4 & 8.5 & $5.11 \mathrm{E}+08$ & $5.29 \mathrm{E}+10$ & $3.82 \mathrm{E}+09$ \\
\hline & & $14 \mathrm{E}+11$ & & +05 & & & $6.00 \mathrm{E}+07$ & $0.00 \mathrm{E}+00$ & \\
\hline & 0.42 & $3.80 \mathrm{E}+10$ & +06 & $\mathrm{E}+01$ & 2.6 & & $1.97 \mathrm{E}+08$ & $3.81 \mathrm{E}+10$ & $1.98 \mathrm{E}+09$ \\
\hline 0.475 & & $4.73 \mathrm{E}+06$ & $1.98 \mathrm{E}+05$ & -07 & +00 & 9.7 & $2.50 \mathrm{E}+08$ & $0.00 \mathrm{E}+00$ & $0.00 \mathrm{E}+00$ \\
\hline & & & & $1.73 \mathrm{E}+00$ & & & $3.76 \mathrm{E}+08$ & & \\
\hline & & $2.22 \mathrm{E}+09$ & $7.69 \mathrm{E}+03$ & $1.65 \mathrm{E}+00$ & $9.97 \mathrm{E}+08$ & $2.35 \mathrm{E}+01$ & $1.17 \mathrm{E}+08$ & $1.79 \mathrm{E}+10$ & $6.49 \mathrm{E}+08$ \\
\hline 0.550 & & $8.61 \mathrm{E}+08$ & $8.84 \mathrm{E}-05$ & $2.16 \mathrm{E}$ & $0.00 \mathrm{E}+00$ & $6.05 \mathrm{E}+00$ & $1.50 \mathrm{E}+08$ & $0.00 \mathrm{E}+00$ & $0.00 \mathrm{E}+00$ \\
\hline & & & $3.76 \mathrm{E}-05$ & & $0.00 \mathrm{E}+00$ & & $3.03 \mathrm{E}+07$ & $0.00 \mathrm{E}+00$ & $3.28 \mathrm{E}+10$ \\
\hline 0.600 & 0.575 & $2.14 \mathrm{E}+09$ & $1.57 \mathrm{E}+03$ & $1.03 \mathrm{E}-03$ & $6.37 \mathrm{E}+08$ & $6.00 \mathrm{E}+01$ & $1.93 \mathrm{E}+08$ & $1.58 \mathrm{E}+10$ & $3.90 \mathrm{E}+09$ \\
\hline
\end{tabular}


PCP-2011-0001

Revision 0

Table 3-23. Photon Source from ORIGEN-S (Decay Time = 30 days)

\begin{tabular}{|c|c|c|c|c|c|c|c|c|c|}
\hline \multicolumn{2}{|c|}{ Energy (MeV) } & \multicolumn{8}{|c|}{ Photon Source (p/s/g) } \\
\hline Upper & Lower & Ac-227 & Am-243 & Np-237 & $\mathbf{P b}-210$ & Pu-241 & Ra-226 & Sr-90 & Zr-95 \\
\hline & & $2.16 \mathrm{E}+09$ & & & & & & & \\
\hline 650 & & $6 \mathrm{E}+06$ & $19 \mathrm{E}+04$ & $0.00 \mathrm{E}+00$ & $0 \mathrm{E}+00$ & & $6.28 \mathrm{E}+07$ & $.00 \mathrm{E}+00$ & $.00 \mathrm{E}+00$ \\
\hline 575 & 650 & $71 \mathrm{E}+07$ & $86 \mathrm{E}+04$ & $8.46 \mathrm{E}-08$ & $0.00 \mathrm{E}+00$ & & $5.87 \mathrm{E}+08$ & $0.00 \mathrm{E}+00$ & $0.00 \mathrm{E}+00$ \\
\hline 00 & & $34 \mathrm{E}+08$ & $92 \mathrm{E}+01$ & $1.26 \mathrm{E}-10$ & +08 & & $8.23 \mathrm{E}+07$ & $.99 E+09$ & $7.00 \mathrm{E}+07$ \\
\hline 725 & 0.700 & $86 \mathrm{E}+09$ & $85 \mathrm{E}+01$ & $7.86 \mathrm{E}-08$ & $9 \mathrm{E}+08$ & +03 & $3.96 \mathrm{E}+08$ & $9.64 \mathrm{E}+09$ & $2.58 \mathrm{E}+14$ \\
\hline 750 & 0.72 & $19 \mathrm{E}+07$ & $.51 \mathrm{E}+01$ & $5.12 \mathrm{E}-02$ & $5.97 \mathrm{E}+02$ & & $3.71 \mathrm{E}+07$ & $0.00 \mathrm{E}+00$ & $0.00 \mathrm{E}+00$ \\
\hline & & $05 \mathrm{E}+09$ & $1.46 \mathrm{E}+01$ & $4.95 \mathrm{E}-02$ & & & $1.87 \mathrm{E}+09$ & $0.00 \mathrm{E}+00$ & $6.10 \mathrm{E}+14$ \\
\hline 800 & 0.775 & $30 \mathrm{E}+08$ & $4.93 \mathrm{E}-05$ & $5.94 \mathrm{E}-11$ & +07 & 1.2 & $5.44 \mathrm{E}+08$ & $6.48 \mathrm{E}+09$ & $1.04 \mathrm{E}+09$ \\
\hline 825 & 0.800 & $36 \mathrm{E}+08$ & $5.11 \mathrm{E}-05$ & $1.94 \mathrm{E}-09$ & $8.73 \mathrm{E}+07$ & $1.45 \mathrm{E}+01$ & $5.46 \mathrm{E}+08$ & $6.28 \mathrm{E}+09$ & $3.37 \mathrm{E}+07$ \\
\hline & & $20 \mathrm{E}+10$ & & $0.00 \mathrm{E}+00$ & & & $2.62 \mathrm{E}+08$ & & \\
\hline 875 & 0.8 & $6 \mathrm{E}+07$ & $3.06 \mathrm{E}-22$ & 5.97E-08 & $0.00 \mathrm{E}+00$ & 8.2 & $0.00 \mathrm{E}+00$ & $0.00 \mathrm{E}+00$ & $0.00 \mathrm{E}+00$ \\
\hline 900 & 0.875 & $36 \mathrm{E}+09$ & 05 & $2.69 \mathrm{E}$ & $2.48 \mathrm{E}+07$ & $1.46 \mathrm{~F}$ & $1.54 \mathrm{E}+07$ & $4.26 \mathrm{E}+09$ & $1.89 \mathrm{E}$ \\
\hline & & +08 & & & & & $6.12 \mathrm{E}+07$ & $4.14 \mathrm{E}+09$ & \\
\hline 0.950 & 0.925 & $8 \mathrm{E}+06$ & $9.76 \mathrm{E}-06$ & $0.00 \mathrm{E}+00$ & 0.0 & 5.58 & $1.15 \mathrm{E}+09$ & $0.00 \mathrm{E}+00$ & $0.00 \mathrm{E}+00$ \\
\hline 0.975 & 0.950 & $40 \mathrm{E}+08$ & $1.40 \mathrm{E}-05$ & $0.00 \mathrm{E}+00$ & $0.00 \mathrm{E}+00$ & $2.89 \mathrm{H}$ & $1.40 \mathrm{E}+08$ & $0.00 \mathrm{E}+00$ & $0.00 \mathrm{E}+00$ \\
\hline 1.000 & 0.97 & $40 \mathrm{E}+07$ & $1.49 \mathrm{E}-05$ & $1.18 \mathrm{E}-07$ & & & $2.30 \mathrm{E}+07$ & $2.82 \mathrm{E}+09$ & $0.00 \mathrm{E}+00$ \\
\hline 1.250 & 1.000 & $28 \mathrm{E}+09$ & $8.24 \mathrm{E}+00$ & 2.80 & +06 & & $9.18 \mathrm{E}+09$ & $8.67 \mathrm{E}+09$ & $0.00 \mathrm{E}+00$ \\
\hline 1.500 & & $.25 \mathrm{E}+07$ & $6.74 \mathrm{E}+00$ & $2.29 \mathrm{E}$ & +02 & & $3.83 \mathrm{E}+09$ & $2.82 \mathrm{E}+09$ & $0.00 \mathrm{E}$ \\
\hline 1.750 & & $.26 \mathrm{E}+03$ & $4.00 \mathrm{E}+00$ & 1.3 & & & $3.21 \mathrm{E}+09$ & $8.32 \mathrm{E}+08$ & +00 \\
\hline 2.000 & & $3 \mathrm{E}+03$ & $3.47 \mathrm{E}+00$ & & & & $9 \mathrm{E}+09$ & & $0.00 \mathrm{E}$ \\
\hline 2.250 & & & $E+00$ & & & & $2.46 \mathrm{E}+09$ & & \\
\hline 2.500 & 2.250 & $4 \mathrm{E}+03$ & $1.77 \mathrm{E}+00$ & $8-03$ & & & $7.35 \mathrm{E}+08$ & $7.35 \mathrm{I}$ & $0.00 \mathrm{E}+00$ \\
\hline 2.750 & & & & & & & & & \\
\hline 3.000 & 2.75 & $9 \mathrm{E}+02$ & & 03 & & & $3.27 \mathrm{E}+07$ & $0.00 \mathrm{E}$ & $0.00 \mathrm{E}+00$ \\
\hline 3.500 & 3.000 & $4 \mathrm{E}+02$ & E-01 & -03 & & & $1.24 \mathrm{E}+07$ & $0.00 \mathrm{E}$ & $0.00 \mathrm{E}+00$ \\
\hline 4.000 & 3.500 & $\mathrm{E}+02$ & -01 & -03 & & & $8.96 \mathrm{E}+00$ & 0.00 & $0.00 \mathrm{E}+00$ \\
\hline 4.500 & 4.000 & $2 \mathrm{E}+02$ & & -04 & & & $4.49 \mathrm{E}+00$ & $0.00 \mathrm{~F}$ & $0.00 \mathrm{E}+00$ \\
\hline 5.000 & 4.500 & $9.64 \mathrm{E}+01$ & -01 & -04 & +00 & 9.2 & $2.25 \mathrm{E}+00$ & $0.00 \mathrm{E}+00$ & $0.00 \mathrm{E}+00$ \\
\hline 5.500 & & $4.84 \mathrm{E}+01$ & $\mathrm{E}-02$ & $2.02 \mathrm{E}-04$ & +00 & 4.6 & $1.13 \mathrm{E}+00$ & $0.00 \mathrm{E}+00$ & $0.00 \mathrm{E}+00$ \\
\hline 6.000 & 5.500 & $2.43 \mathrm{E}+01$ & $3.20 \mathrm{E}-02$ & $1.01 \mathrm{E}-04$ & +00 & 2.33 & $5.67 \mathrm{E}-01$ & $0.00 \mathrm{E}+00$ & $0.00 \mathrm{E}+00$ \\
\hline 6.500 & 6.000 & $2 \mathrm{E}+01$ & $1.63 \mathrm{E}-02$ & -05 & -01 & 03 & $2.84 \mathrm{E}-01$ & $0.00 \mathrm{E}+00$ & $0.00 \mathrm{E}+00$ \\
\hline & 6.500 & $6.10 \mathrm{E}+00$ & $8.34 \mathrm{E}-03$ & $2.55 \mathrm{E}-05$ & & & \begin{tabular}{|l|}
$1.43 \mathrm{E}-01$ \\
\end{tabular} & $0.00 \mathrm{E}+00$ & $0.00 \mathrm{E}+00$ \\
\hline 7.500 & 7.000 & $3.06 \mathrm{E}+00$ & $4.27 \mathrm{E}-03$ & $1.28 \mathrm{E}-05$ & 1.49E-01 & $2.96 \mathrm{E}-04$ & $7.16 \mathrm{E}-02$ & $0.00 \mathrm{E}+00$ & $0.00 \mathrm{E}+00$ \\
\hline 8.000 & 7.500 & $1.54 \mathrm{E}+00$ & $2.19 \mathrm{E}-03$ & $6.42 \mathrm{E}-06$ & 7.48 & 1.49 & $3.59 \mathrm{E}-02$ & $0.00 \mathrm{E}+00$ & $0.00 \mathrm{E}+00$ \\
\hline 9.000 & 8.000 & $1.15 \mathrm{E}+00$ & $1.70 \mathrm{E}-03$ & 4.79E-06 & $5.58 \mathrm{I}$ & $1.11 \mathrm{~F}$ & $2.68 \mathrm{E}-02$ & $0.00 \mathrm{E}+00$ & $0.00 \mathrm{E}+00$ \\
\hline 10.000 & 9.000 & $2.88 \mathrm{E}-01$ & $4.56 \mathrm{E}-04$ & $1.21 \mathrm{E}-06$ & $1.41 \mathrm{E}-02$ & $2.82 \mathrm{E}-05$ & 6.74E-03 & $0.00 \mathrm{E}+00$ & $0.00 \mathrm{E}+00$ \\
\hline & Total & $4.26 \mathrm{E}+12$ & $2.18 \mathrm{E}+10$ & $4.97 \mathrm{E}+07$ & $1.46 \mathrm{E}+12$ & $7.64 \mathrm{E}+08$ & $1.12 \mathrm{E}+11$ & $4.64 \mathrm{E}+12$ & $9.10 \mathrm{E}+14$ \\
\hline
\end{tabular}


PCP-2011-0001

Revision 0

Table 3-24. Photon Source from ORIGEN-S (Decay Time = 100 days)

\begin{tabular}{|c|c|c|c|c|c|c|c|c|c|}
\hline \multicolumn{2}{|c|}{ Energy (MeV) } & \multicolumn{8}{|c|}{ Photon Source (p/s/g) } \\
\hline Upper & Lower & Ac-227 & Am-243 & Np-237 & Pb-210 & Pu-241 & Ra-226 & Sr-90 & Zr-95 \\
\hline 0.020 & 0.010 & $2.27 \mathrm{E}+12$ & $7.42 \mathrm{E}+09$ & $3.38 \mathrm{E}+07$ & $8.25 \mathrm{E}+11$ & $7.86 \mathrm{E}+08$ & $1.17 \mathrm{E}+10$ & $1.27 \mathrm{E}+12$ & $1.06 \mathrm{E}+13$ \\
\hline 0.030 & 0.020 & $.81 \mathrm{E}+11$ & $8.55 \mathrm{E}+07$ & $4.87 \mathrm{E}+06$ & $25 \mathrm{E}+11$ & $1.17 \mathrm{E}+08$ & $3.66 \mathrm{E}+09$ & $6.99 \mathrm{E}+11$ & $3.96 \mathrm{E}+12$ \\
\hline 0.040 & 0.030 & $1.69 \mathrm{E}+11$ & $5.22 \mathrm{E}+07$ & $7.14 \mathrm{E}+04$ & $7.42 \mathrm{E}+10$ & $2.53 \mathrm{E}+06$ & $2.20 \mathrm{E}+09$ & $4.23 \mathrm{E}+11$ & $2.10 \mathrm{E}+12$ \\
\hline 0.050 & 0.040 & $78 \mathrm{E}+11$ & $4.79 \mathrm{E}+08$ & $9.06 \mathrm{E}+04$ & $.78 \mathrm{E}+11$ & $1.61 \mathrm{E}+06$ & $1.84 \mathrm{E}+09$ & $3.57 \mathrm{E}+11$ & $1.51 \mathrm{E}+12$ \\
\hline 0.060 & 0.050 & $1.87 \mathrm{E}+11$ & $2.85 \mathrm{E}+07$ & $1.30 \mathrm{E}+05$ & $3.47 \mathrm{E}+10$ & $6.83 \mathrm{E}+08$ & $1.44 \mathrm{E}+09$ & $2.06 \mathrm{E}+11$ & $7.71 \mathrm{E}+11$ \\
\hline 0.070 & 0.060 & $8.68 \mathrm{E}+10$ & $1.14 \mathrm{E}+08$ & $2.59 \mathrm{E}+04$ & $3.24 \mathrm{E}+10$ & $1.29 \mathrm{E}+06$ & $9.86 \mathrm{E}+08$ & $1.95 \mathrm{E}+11$ & $6.57 \mathrm{E}+11$ \\
\hline 0.080 & 0.070 & $1.89 \mathrm{E}+11$ & $5.03 \mathrm{E}+09$ & $3.39 \mathrm{E}+05$ & $2.54 \mathrm{E}+10$ & $9.83 \mathrm{E}+05$ & $7.54 \mathrm{E}+09$ & $1.55 \mathrm{E}+11$ & $4.54 \mathrm{E}+11$ \\
\hline 0.090 & 0.080 & $1.21 \mathrm{E}+12$ & $3.49 \mathrm{E}+07$ & $3.79 \mathrm{E}+06$ & $2.03 \mathrm{E}+10$ & $2.85 \mathrm{E}+04$ & $2.71 \mathrm{E}+09$ & $1.27 \mathrm{E}+11$ & $3.23 \mathrm{E}+11$ \\
\hline 0.100 & 0.090 & $3.91 \mathrm{E}+11$ & $1.20 \mathrm{E}+09$ & $7.20 \mathrm{E}+06$ & $2.06 \mathrm{E}+10$ & $4.76 \mathrm{E}+07$ & $7.23 \mathrm{E}+08$ & $1.32 \mathrm{E}+11$ & $2.88 \mathrm{E}+11$ \\
\hline .110 & 0.100 & $3 \mathrm{E}+10$ & $3.83 \mathrm{E}+09$ & $5.20 \mathrm{E}+05$ & $\mathrm{E}+10$ & $2.67 \mathrm{E}+07$ & $3.40 \mathrm{E}+08$ & $6.95 \mathrm{E}+10$ & $1.43 \mathrm{E}+11$ \\
\hline 0.120 & 0.110 & $5.32 \mathrm{E}+10$ & $8.09 \mathrm{E}+08$ & $1.77 \mathrm{E}+06$ & $1.21 \mathrm{E}+10$ & $2.01 \mathrm{E}+07$ & $3.89 \mathrm{E}+08$ & $8.04 \mathrm{E}+10$ & $1.35 \mathrm{E}+11$ \\
\hline 0.130 & 0.120 & $5.62 \mathrm{E}+10$ & $1.12 \mathrm{E}+08$ & $3.72 \mathrm{E}+03$ & $1.12 \mathrm{E}+10$ & $1.18 \mathrm{E}+05$ & $3.58 \mathrm{E}+08$ & $7.40 \mathrm{E}+10$ & $1.24 \mathrm{E}+11$ \\
\hline 0.140 & 0.130 & $2.54 \mathrm{E}+10$ & $2.48 \mathrm{E}+06$ & $4.32 \mathrm{E}+04$ & $8.82 \mathrm{E}+09$ & $3.45 \mathrm{E}+03$ & $3.11 \mathrm{E}+08$ & $6.07 \mathrm{E}+10$ & $7.79 \mathrm{E}+10$ \\
\hline 0.150 & 0.140 & $1.05 \mathrm{E}+11$ & $1.10 \mathrm{E}+07$ & $1.14 \mathrm{E}+05$ & $8.21 \mathrm{E}+09$ & $7.20 \mathrm{E}+06$ & $2.83 \mathrm{E}+08$ & $5.66 \mathrm{E}+10$ & $7.26 \mathrm{E}+10$ \\
\hline 0.160 & 0.150 & $73 \mathrm{E}+11$ & $1.53 \mathrm{E}+06$ & $8.64 \mathrm{E}+04$ & $6.60 \mathrm{E}+09$ & $1.30 \mathrm{E}+05$ & $2.22 \mathrm{E}+08$ & $4.74 \mathrm{E}+10$ & $4.60 \mathrm{E}+10$ \\
\hline 0.170 & 0.160 & $1.64 \mathrm{E}+10$ & $2.81 \mathrm{E}+06$ & $2.85 \mathrm{E}+04$ & $6.20 \mathrm{E}+09$ & $1.81 \mathrm{E}+06$ & $2.08 \mathrm{E}+08$ & $4.45 \mathrm{E}+10$ & $4.32 \mathrm{E}+10$ \\
\hline 0.180 & 0.170 & $1.93 \mathrm{E}+10$ & $9.59 \mathrm{E}+05$ & $1.07 \mathrm{E}+04$ & $5.07 \mathrm{E}+09$ & $7.05 \mathrm{E}+02$ & $1.75 \mathrm{E}+08$ & $3.79 \mathrm{E}+10$ & $2.73 \mathrm{E}+10$ \\
\hline 0.190 & 0.180 & $1.29 \mathrm{E}+10$ & $6.78 \mathrm{E}+06$ & $6.74 \mathrm{E}+03$ & $4.79 \mathrm{E}+09$ & $3.80 \mathrm{E}+02$ & $1.45 \mathrm{E}+09$ & $3.59 \mathrm{E}+10$ & $2.58 \mathrm{E}+10$ \\
\hline 0.200 & 0.190 & $2.04 \mathrm{E}+10$ & $1.28 \mathrm{E}+06$ & $7.43 \mathrm{E}+04$ & $7.91 \mathrm{E}+09$ & $5.97 \mathrm{E}+02$ & $2.98 \mathrm{E}+08$ & $6.19 \mathrm{E}+10$ & $3.23 \mathrm{E}+10$ \\
\hline 0.210 & 0.200 & $3.61 \mathrm{E}+10$ & $1.30 \mathrm{E}+08$ & $1.84 \mathrm{E}+04$ & $7.52 \mathrm{E}+09$ & $1.98 \mathrm{E}+07$ & $2.67 \mathrm{E}+08$ & $5.89 \mathrm{E}+10$ & $2.00 \mathrm{E}+11$ \\
\hline 0.220 & 0.210 & $3.31 \mathrm{E}+10$ & $1.23 \mathrm{E}+08$ & $5.06 \mathrm{E}+04$ & $0.00 \mathrm{E}+00$ & $1.58 \mathrm{E}-01$ & $0.00 \mathrm{E}+00$ & $0.00 \mathrm{E}+00$ & $0.00 \mathrm{E}+00$ \\
\hline 0.230 & 0.220 & $1.93 \mathrm{E}+09$ & $8.65 \mathrm{E}+08$ & $2.38 \mathrm{E}+03$ & $0.00 \mathrm{E}+00$ & $1.90 \mathrm{E}+04$ & $0.00 \mathrm{E}+00$ & $0.00 \mathrm{E}+00$ & $0.00 \mathrm{E}+00$ \\
\hline 0.240 & 0.230 & $3.01 \mathrm{E}+11$ & $8.18 \mathrm{E}-03$ & $1.74 \mathrm{E}+04$ & $0.00 \mathrm{E}+00$ & $1.86 \mathrm{E}+04$ & $0.00 \mathrm{E}+00$ & $0.00 \mathrm{E}+00$ & $7.65 \mathrm{E}+11$ \\
\hline 0.250 & 0.240 & $6.49 \mathrm{E}+09$ & $2.97 \mathrm{E}+01$ & $1.57 \mathrm{E}+04$ & $4.05 \mathrm{E}+03$ & $5.64 \mathrm{E}+01$ & $2.69 \mathrm{E}+09$ & $0.00 \mathrm{E}+00$ & $0.00 \mathrm{E}+00$ \\
\hline 0.260 & 0.250 & $2.28 \mathrm{E}+11$ & $8.84 \mathrm{E}+06$ & $3.46 \mathrm{E}+03$ & $7.52 \mathrm{E}+09$ & $7.46 \mathrm{E}+00$ & $4.88 \mathrm{E}+08$ & $6.45 \mathrm{E}+10$ & $1.63 \mathrm{E}+10$ \\
\hline 0.270 & 0.260 & $3.68 \mathrm{E}+11$ & $7.05 \mathrm{E}+05$ & $2.56 \mathrm{E}+03$ & $7.23 \mathrm{E}+09$ & $6.67 \mathrm{E}+05$ & $2.75 \mathrm{E}+08$ & $6.21 \mathrm{E}+10$ & $1.56 \mathrm{E}+10$ \\
\hline 0.280 & 0.270 & $2.59 \mathrm{E}+11$ & $1.08 \mathrm{E}+09$ & $7.66 \mathrm{E}+04$ & $0.00 \mathrm{E}+00$ & $1.28 \mathrm{E}+02$ & $1.83 \mathrm{E}+08$ & $0.00 \mathrm{E}+00$ & $0.00 \mathrm{E}+00$ \\
\hline 0.290 & 0.280 & $5.26 \mathrm{E}+10$ & $5.84 \mathrm{E}+07$ & $2.15 \mathrm{E}-04$ & $0.00 \mathrm{E}+00$ & $1.54 \mathrm{E}-10$ & $4.10 \mathrm{E}+07$ & $0.00 \mathrm{E}+00$ & $0.00 \mathrm{E}+00$ \\
\hline 0.300 & 0.290 & $4.28 \mathrm{E}+10$ & $2.82 \mathrm{E}-02$ & $8.21 \mathrm{E}+05$ & $0.00 \mathrm{E}+00$ & $2.53 \mathrm{E}+03$ & $7.01 \mathrm{E}+09$ & $0.00 \mathrm{E}+00$ & $0.00 \mathrm{E}+00$ \\
\hline 0.325 & 0.300 & $2.04 \mathrm{E}+11$ & $1.20 \mathrm{E}+08$ & $1.01 \mathrm{E}+07$ & $8.18 \mathrm{E}+09$ & $2.88 \mathrm{E}+03$ & $3.97 \mathrm{E}+08$ & $8.18 \mathrm{E}+10$ & $6.15 \mathrm{E}+09$ \\
\hline 0.350 & 0.325 & $1.88 \mathrm{E}+11$ & $1.51 \mathrm{E}+08$ & $1.09 \mathrm{E}+06$ & $0.00 \mathrm{E}+00$ & $1.18 \mathrm{E}+06$ & $9.52 \mathrm{E}+07$ & $0.00 \mathrm{E}+00$ & $0.00 \mathrm{E}+00$ \\
\hline 0.375 & 0.350 & $3.26 \mathrm{E}+11$ & $5.61 \mathrm{E}-01$ & $8.48 \mathrm{E}+04$ & $0.00 \mathrm{E}+00$ & $1.45 \mathrm{E}+05$ & $1.32 \mathrm{E}+10$ & $0.00 \mathrm{E}+00$ & $0.00 \mathrm{E}+00$ \\
\hline 0.400 & 0.375 & $1.58 \mathrm{E}+10$ & $2.26 \mathrm{E}+05$ & $4.29 \mathrm{E}+05$ & $4.45 \mathrm{E}+09$ & $2.85 \mathrm{E}+03$ & $5.14 \mathrm{E}+08$ & $5.27 \mathrm{E}+10$ & $1.79 \mathrm{E}+09$ \\
\hline 0.425 & 0.400 & $2.57 \mathrm{E}+11$ & 9.03E-01 & $4.22 \mathrm{E}+05$ & $0.00 \mathrm{E}+00$ & $5.71 \mathrm{E}+02$ & $6.02 \mathrm{E}+07$ & $0.00 \mathrm{E}+00$ & $0.00 \mathrm{E}+00$ \\
\hline 0.450 & 0.425 & $8.59 \mathrm{E}+10$ & $1.24 \mathrm{E}+06$ & $3.42 \mathrm{E}+01$ & $2.65 \mathrm{E}+09$ & $4.86 \mathrm{E}+02$ & $1.98 \mathrm{E}+08$ & $3.80 \mathrm{E}+10$ & $9.27 \mathrm{E}+08$ \\
\hline 0.475 & 0.450 & $6.84 \mathrm{E}+06$ & $1.98 \mathrm{E}+05$ & $7.26 \mathrm{E}-06$ & $0.00 \mathrm{E}+00$ & $3.23 \mathrm{E}+02$ & $2.51 \mathrm{E}+08$ & $0.00 \mathrm{E}+00$ & $0.00 \mathrm{E}+00$ \\
\hline 0.500 & 0.475 & $5.36 \mathrm{E}+09$ & $1.00 \mathrm{E}+06$ & $2.97 \mathrm{E}+00$ & $1.06 \mathrm{E}+09$ & $2.39 \mathrm{E}+01$ & $3.78 \mathrm{E}+08$ & $1.87 \mathrm{E}+10$ & $3.20 \mathrm{E}+08$ \\
\hline 0.525 & 0.500 & $5.01 \mathrm{E}+09$ & $7.69 \mathrm{E}+03$ & $2.83 \mathrm{E}+00$ & $1.01 \mathrm{E}+09$ & $7.79 \mathrm{E}+01$ & $1.17 \mathrm{E}+08$ & $1.78 \mathrm{E}+10$ & $3.04 \mathrm{E}+08$ \\
\hline 0.550 & 0.525 & $1.93 \mathrm{E}+09$ & $3.21 \mathrm{E}-04$ & $1.53 \mathrm{E}-06$ & $0.00 \mathrm{E}+00$ & $2.01 \mathrm{E}+01$ & $1.51 \mathrm{E}+08$ & $0.00 \mathrm{E}+00$ & $0.00 \mathrm{E}+00$ \\
\hline 0.575 & 0.550 & $1.16 \mathrm{E}+08$ & $1.37 \mathrm{E}-04$ & $7.66 \mathrm{E}-07$ & $0.00 \mathrm{E}+00$ & $3.36 \mathrm{E}+01$ & $3.04 \mathrm{E}+07$ & $0.00 \mathrm{E}+00$ & $3.85 \mathrm{E}+10$ \\
\hline 0.600 & 0.575 & $4.82 \mathrm{E}+09$ & $1.57 \mathrm{E}+03$ & $1.77 \mathrm{E}-03$ & $6.44 \mathrm{E}+08$ & $1.99 \mathrm{E}+02$ & $1.94 \mathrm{E}+08$ & $1.57 \mathrm{E}+10$ & $1.83 \mathrm{E}+09$ \\
\hline
\end{tabular}


PCP-2011-0001

Revision 0

Table 3-24. Photon Source from ORIGEN-S (Decay Time = 100 days)

\begin{tabular}{|c|c|c|c|c|c|c|c|c|c|}
\hline \multicolumn{2}{|c|}{ Energy (MeV) } & \multicolumn{8}{|c|}{ Photon Source $(\mathrm{p} / \mathrm{s} / \mathrm{g})$} \\
\hline Upper & Lower & Ac-227 & Am-243 & Np-237 & Pb-210 & Pu-241 & Ra-226 & Sr-90 & Zr-95 \\
\hline 0.625 & & $4.87 \mathrm{E}+09$ & $1.50 \mathrm{E}+03$ & $1.70 \mathrm{E}-03$ & $6.18 \mathrm{E}+08$ & $1.00 \mathrm{E}+03$ & $1.69 \mathrm{E}+10$ & $1.51 \mathrm{E}+10$ & $1.42 \mathrm{E}+08$ \\
\hline 0.650 & 0.625 & $88 \mathrm{E}+06$ & $2.19 \mathrm{E}+04$ & $0.00 \mathrm{E}+00$ & $0 \mathrm{E}+00$ & $49 \mathrm{E}+02$ & $6.31 \mathrm{E}+07$ & $0.00 \mathrm{E}+00$ & $0.00 \mathrm{E}+00$ \\
\hline 0.675 & 0.650 & $8.30 \mathrm{E}+07$ & $8.86 \mathrm{E}+04$ & $6.58 \mathrm{E}-07$ & $0.00 \mathrm{E}+00$ & $6.63 \mathrm{E}+03$ & $5.90 \mathrm{E}+08$ & $0.00 \mathrm{E}+00$ & $0.00 \mathrm{E}+00$ \\
\hline 0.700 & 0.675 & $1.43 \mathrm{E}+09$ & $1.92 \mathrm{E}+01$ & $1.48 \mathrm{E}-08$ & $2.51 \mathrm{E}+08$ & $7.57 \mathrm{E}+02$ & $8.27 \mathrm{E}+07$ & $9.95 \mathrm{E}+09$ & $3.28 \mathrm{E}+07$ \\
\hline 0.725 & 0.700 & $1.32 \mathrm{E}+10$ & $1.85 \mathrm{E}+01$ & $5.68 \mathrm{E}-07$ & $2.42 \mathrm{E}+08$ & $3.42 \mathrm{E}+03$ & $3.98 \mathrm{E}+08$ & $9.60 \mathrm{E}+09$ & $1.21 \mathrm{E}+14$ \\
\hline 0.750 & 0.725 & $2.76 \mathrm{E}+07$ & $1.51 \mathrm{E}+01$ & $5.12 \mathrm{E}-02$ & $2.03 \mathrm{E}+03$ & $1.72 \mathrm{E}+02$ & $3.73 \mathrm{E}+07$ & $0.00 \mathrm{E}+00$ & $0.00 \mathrm{E}+00$ \\
\hline 0.775 & 0.750 & $1.80 \mathrm{E}+10$ & $1.46 \mathrm{E}+01$ & $4.95 \mathrm{E}-02$ & $1.96 \mathrm{E}+03$ & $3.69 \mathrm{E}+02$ & $1.88 \mathrm{E}+09$ & $0.00 \mathrm{E}+00$ & $4.98 \mathrm{E}+14$ \\
\hline 0.800 & 0.775 & $7.09 \mathrm{E}+08$ & 1.79E-04 & $6.96 \mathrm{E}-09$ & $8.76 \mathrm{E}+07$ & $4.03 \mathrm{E}+01$ & $5.46 \mathrm{E}+08$ & $6.45 \mathrm{E}+09$ & $4.91 \mathrm{E}+08$ \\
\hline 0.825 & 0.800 & $7.38 \mathrm{E}+08$ & $1.85 \mathrm{E}-04$ & $2.27 \mathrm{E}-07$ & $9.58 \mathrm{E}+07$ & $4.81 \mathrm{E}+01$ & $5.48 \mathrm{E}+08$ & $6.25 \mathrm{E}+09$ & $1.58 \mathrm{E}+07$ \\
\hline 0.850 & 0.825 & $9.48 \mathrm{E}+10$ & $2.14 \mathrm{E}-04$ & $0.00 \mathrm{E}+00$ & $0.00 \mathrm{E}+00$ & $1.27 \mathrm{E}+01$ & $2.63 \mathrm{E}+08$ & $0.00 \mathrm{E}+00$ & $3.80 \mathrm{E}+05$ \\
\hline 0.875 & 0.850 & $1.75 \mathrm{E}+08$ & $8.87 \mathrm{E}-20$ & $4.21 \mathrm{E}-07$ & $0.00 \mathrm{E}+00$ & $2.75 \mathrm{E}+01$ & $0.00 \mathrm{E}+00$ & $0.00 \mathrm{E}+00$ & $0.00 \mathrm{E}+00$ \\
\hline 0.900 & 0.875 & $6.42 \mathrm{E}+09$ & $7.68 \mathrm{E}-05$ & $3.15 \mathrm{E}-09$ & $2.51 \mathrm{E}+07$ & $4.85 \mathrm{E}+00$ & $1.55 \mathrm{E}+07$ & $4.24 \mathrm{E}+09$ & $8.85 \mathrm{E}+03$ \\
\hline 0.925 & 0.900 & $2.98 \mathrm{E}+08$ & $1.68 \mathrm{E}-05$ & 3.07E-09 & $2.44 \mathrm{E}+07$ & $1.23 \mathrm{E}+01$ & $6.15 \mathrm{E}+07$ & $4.13 \mathrm{E}+09$ & $8.61 \mathrm{E}+03$ \\
\hline 0.950 & 0.925 & $2.28 \mathrm{E}+06$ & $3.54 \mathrm{E}-05$ & $0.00 \mathrm{E}+00$ & $0.00 \mathrm{E}+00$ & $1.85 \mathrm{E}+00$ & $1.16 \mathrm{E}+09$ & $0.00 \mathrm{E}+00$ & $0.00 \mathrm{E}+00$ \\
\hline 0.975 & 0.950 & $5.40 \mathrm{E}+08$ & $5.10 \mathrm{E}-05$ & $0.00 \mathrm{E}+00$ & $0.00 \mathrm{E}+00$ & $9.60 \mathrm{E}+00$ & $1.40 \mathrm{E}+08$ & $0.00 \mathrm{E}+00$ & $0.00 \mathrm{E}+00$ \\
\hline 1.000 & 0.975 & $9.87 \mathrm{E}+07$ & $5.39 \mathrm{E}-05$ & $8.31 \mathrm{E}-07$ & $4.85 \mathrm{E}+06$ & $5.95 \mathrm{E}-13$ & $2.31 \mathrm{E}+07$ & $2.81 \mathrm{E}+09$ & $0.00 \mathrm{E}+00$ \\
\hline 1.250 & 1.000 & $5.15 \mathrm{E}+09$ & $8.24 \mathrm{E}+00$ & $2.81 \mathrm{E}-02$ & $4.97 \mathrm{E}+06$ & $2.86 \mathrm{E}+00$ & $9.22 \mathrm{E}+09$ & $8.63 \mathrm{E}+09$ & $0.00 \mathrm{E}+00$ \\
\hline 1.500 & 1.250 & $2.09 \mathrm{E}+08$ & $6.74 \mathrm{E}+00$ & $2.29 \mathrm{E}-02$ & $9.08 \mathrm{E}+02$ & $1.55 \mathrm{E}+00$ & $3.85 \mathrm{E}+09$ & $2.81 \mathrm{E}+09$ & $0.00 \mathrm{E}+00$ \\
\hline 1.750 & 1.500 & $6.60 \mathrm{E}+03$ & $4.00 \mathrm{E}+00$ & $1.36 \mathrm{E}-02$ & $5.40 \mathrm{E}+02$ & $9.22 \mathrm{E}-01$ & $3.23 \mathrm{E}+09$ & $8.28 \mathrm{E}+08$ & $0.00 \mathrm{E}+00$ \\
\hline 2.000 & 1.750 & $5.72 \mathrm{E}+03$ & $3.47 \mathrm{E}+00$ & $1.18 \mathrm{E}-02$ & $4.68 \mathrm{E}+02$ & 7.99E-01 & $6.62 \mathrm{E}+09$ & $1.58 \mathrm{E}+08$ & $0.00 \mathrm{E}+00$ \\
\hline 2.250 & 2.000 & $3.25 \mathrm{E}+03$ & $1.98 \mathrm{E}+00$ & $6.72 \mathrm{E}-03$ & $2.66 \mathrm{E}+02$ & $4.55 \mathrm{E}-01$ & $2.47 \mathrm{E}+09$ & $1.85 \mathrm{E}+07$ & $0.00 \mathrm{E}+00$ \\
\hline 2.500 & 2.250 & $2.91 \mathrm{E}+03$ & $1.77 \mathrm{E}+00$ & $6.02 \mathrm{E}-03$ & $2.38 \mathrm{E}+02$ & 4.07E-01 & $7.38 \mathrm{E}+08$ & $7.31 \mathrm{E}+04$ & $0.00 \mathrm{E}+00$ \\
\hline 2.750 & 2.500 & $1.61 \mathrm{E}+03$ & $9.90 \mathrm{E}-01$ & $3.34 \mathrm{E}-03$ & $1.32 \mathrm{E}+02$ & $2.26 \mathrm{E}-01$ & $1.57 \mathrm{E}+07$ & $0.00 \mathrm{E}+00$ & $0.00 \mathrm{E}+00$ \\
\hline 3.000 & 2.750 & $1.47 \mathrm{E}+03$ & $9.04 \mathrm{E}-01$ & $3.05 \mathrm{E}-03$ & $1.21 \mathrm{E}+02$ & $2.06 \mathrm{E}-01$ & $3.28 \mathrm{E}+07$ & $0.00 \mathrm{E}+00$ & $0.00 \mathrm{E}+00$ \\
\hline 3.500 & 3.000 & $1.55 \mathrm{E}+03$ & $9.55 \mathrm{E}-01$ & $3.20 \mathrm{E}-03$ & $1.26 \mathrm{E}+02$ & $2.16 \mathrm{E}-01$ & $1.24 \mathrm{E}+07$ & $0.00 \mathrm{E}+00$ & $0.00 \mathrm{E}+00$ \\
\hline 4.000 & 3.500 & $7.75 \mathrm{E}+02$ & $4.83 \mathrm{E}-01$ & $1.60 \mathrm{E}-03$ & $6.34 \mathrm{E}+01$ & $1.09 \mathrm{E}-01$ & $8.99 \mathrm{E}+00$ & $0.00 \mathrm{E}+00$ & $0.00 \mathrm{E}+00$ \\
\hline 4.500 & 4.000 & $3.89 \mathrm{E}+02$ & $2.45 \mathrm{E}-01$ & $8.04 \mathrm{E}-04$ & $3.18 \mathrm{E}+01$ & $5.45 \mathrm{E}-02$ & $4.51 \mathrm{E}+00$ & $0.00 \mathrm{E}+00$ & $0.00 \mathrm{E}+00$ \\
\hline 5.000 & 4.500 & $1.95 \mathrm{E}+02$ & $1.24 \mathrm{E}-01$ & $4.03 \mathrm{E}-04$ & $1.60 \mathrm{E}+01$ & $2.74 \mathrm{E}-02$ & $2.26 \mathrm{E}+00$ & $0.00 \mathrm{E}+00$ & $0.00 \mathrm{E}+00$ \\
\hline 5.500 & 5.000 & $9.78 \mathrm{E}+01$ & $6.30 \mathrm{E}-02$ & $2.02 \mathrm{E}-04$ & $8.01 \mathrm{E}+00$ & $1.38 \mathrm{E}-02$ & $1.13 \mathrm{E}+00$ & $0.00 \mathrm{E}+00$ & $0.00 \mathrm{E}+00$ \\
\hline 6.000 & 5.500 & $4.91 \mathrm{E}+01$ & $3.20 \mathrm{E}-02$ & $1.01 \mathrm{E}-04$ & $4.02 \mathrm{E}+00$ & 6.91E-03 & 5.69E-01 & $0.00 \mathrm{E}+00$ & $0.00 \mathrm{E}+00$ \\
\hline 6.500 & 6.000 & $2.46 \mathrm{E}+01$ & $1.63 \mathrm{E}-02$ & $5.09 \mathrm{E}-05$ & $2.01 \mathrm{E}+00$ & $3.47 \mathrm{E}-03$ & $2.86 \mathrm{E}-01$ & $0.00 \mathrm{E}+00$ & $0.00 \mathrm{E}+00$ \\
\hline 7.000 & 6.500 & $1.23 \mathrm{E}+01$ & $8.34 \mathrm{E}-03$ & $2.55 \mathrm{E}-05$ & $1.01 \mathrm{E}+00$ & $1.74 \mathrm{E}-03$ & $1.43 \mathrm{E}-01$ & $0.00 \mathrm{E}+00$ & $0.00 \mathrm{E}+00$ \\
\hline 7.500 & 7.000 & $6.19 \mathrm{E}+00$ & $4.27 \mathrm{E}-03$ & $1.28 \mathrm{E}-05$ & $5.07 \mathrm{E}-01$ & $8.77 \mathrm{E}-04$ & $7.18 \mathrm{E}-02$ & $0.00 \mathrm{E}+00$ & $0.00 \mathrm{E}+00$ \\
\hline 8.000 & 7.500 & $3.11 \mathrm{E}+00$ & $2.19 \mathrm{E}-03$ & $6.42 \mathrm{E}-06$ & $2.54 \mathrm{E}-01$ & 4.41E-04 & $3.60 \mathrm{E}-02$ & $0.00 \mathrm{E}+00$ & $0.00 \mathrm{E}+00$ \\
\hline 9.000 & 8.000 & $2.32 \mathrm{E}+00$ & $1.70 \mathrm{E}-03$ & 4.79E-06 & $1.90 \mathrm{E}-01$ & $3.30 \mathrm{E}-04$ & 2.69E-02 & $0.00 \mathrm{E}+00$ & $0.00 \mathrm{E}+00$ \\
\hline 10.000 & 9.000 & $5.83 \mathrm{E}-01$ & $4.56 \mathrm{E}-04$ & $1.21 \mathrm{E}-06$ & $4.77 \mathrm{E}-02$ & 8.39E-05 & $6.77 \mathrm{E}-03$ & $0.00 \mathrm{E}+00$ & $0.00 \mathrm{E}+00$ \\
\hline & Total & $8.16 \mathrm{E}+12$ & $2.18 \mathrm{E}+10$ & $6.61 \mathrm{E}+07$ & $1.46 \mathrm{E}+12$ & $1.72 \mathrm{E}+09$ & $1.12 \mathrm{E}+11$ & $4.61 \mathrm{E}+12$ & $6.41 \mathrm{E}+14$ \\
\hline
\end{tabular}


PCP-2011-0001

Revision 0

\begin{tabular}{|c|c|c|c|c|c|c|c|c|c|}
\hline \multicolumn{2}{|c|}{ Energy (MeV) } & \multicolumn{8}{|c|}{ Photon Source (p/s/g) } \\
\hline Upper & Lower & Ac-227 & Am-243 & Np-237 & Pb-210 & Pu-241 & Ra-226 & Sr-90 & Zr-95 \\
\hline 0.020 & 0.010 & $1.75 \mathrm{E}+11$ & $7.36 \mathrm{E}+09$ & $3.52 \mathrm{E}+07$ & $6.47 \mathrm{E}+10$ & $4.03 \mathrm{E}+10$ & $2.09 \mathrm{E}+10$ & $1.70 \mathrm{E}+11$ & $0.00 \mathrm{E}+00$ \\
\hline 0.030 & 0.020 & $2.20 \mathrm{E}+10$ & $8.49 \mathrm{E}+07$ & $4.88 \mathrm{E}+06$ & $9.81 \mathrm{E}+09$ & $7.39 \mathrm{E}+09$ & $4.99 \mathrm{E}+09$ & & \\
\hline 0.040 & 0.030 & $1.33 \mathrm{E}+10$ & $5.18 \mathrm{E}+07$ & $7.73 \mathrm{E}+04$ & $5.83 \mathrm{E}+09$ & $1.46 \mathrm{E}+08$ & $2.98 \mathrm{E}+09$ & $5.64 \mathrm{E}+10$ & $0.00 \mathrm{E}+00$ \\
\hline 0.050 & 0.040 & $2.14 \mathrm{E}+10$ & $4.75 \mathrm{E}+08$ & $9.58 \mathrm{E}+04$ & $1.40 \mathrm{E}+10$ & $8.30 \mathrm{E}+07$ & $3.86 \mathrm{E}+09$ & $4.75 \mathrm{E}+10$ & $0.00 \mathrm{E}+00$ \\
\hline 0.060 & 0.050 & $1.44 \mathrm{E}+10$ & $2.83 \mathrm{E}+07$ & $1.32 \mathrm{E}+05$ & $2.72 \mathrm{E}+09$ & $4.38 \mathrm{E}+10$ & $1.79 \mathrm{E}+09$ & $2.74 \mathrm{E}+10$ & $0.00 \mathrm{E}+00$ \\
\hline 0.070 & 0.060 & $6.77 \mathrm{E}+09$ & $1.13 \mathrm{E}+08$ & $2.77 \mathrm{E}+04$ & $2.55 \mathrm{E}+09$ & $2.43 \mathrm{E}+06$ & $1.33 \mathrm{E}+09$ & $2.60 \mathrm{E}+10$ & $0.00 \mathrm{E}+00$ \\
\hline 0.080 & 0.070 & $1.47 \mathrm{E}+10$ & $4.99 \mathrm{E}+09$ & $3.67 \mathrm{E}+05$ & $1.99 \mathrm{E}+09$ & $2.24 \mathrm{E}+06$ & $7.57 \mathrm{E}+09$ & $2.07 \mathrm{E}+10$ & $0.00 \mathrm{E}+00$ \\
\hline 0.090 & 0.080 & $9.41 \mathrm{E}+10$ & $3.46 \mathrm{E}+07$ & $3.84 \mathrm{E}+06$ & $1.60 \mathrm{E}+09$ & $3.55 \mathrm{E}+05$ & $2.86 \mathrm{E}+09$ & $1.69 \mathrm{E}+10$ & $0.00 \mathrm{E}+00$ \\
\hline 0.100 & 0.090 & $3.05 \mathrm{E}+10$ & $1.19 \mathrm{E}+09$ & $7.68 \mathrm{E}+06$ & $1.62 \mathrm{E}+09$ & $2.66 \mathrm{E}+07$ & $9.38 \mathrm{E}+08$ & $1.75 \mathrm{E}+10$ & $0.00 \mathrm{E}+00$ \\
\hline 0.110 & 0.100 & $3.95 \mathrm{E}+09$ & $3.80 \mathrm{E}+09$ & $5.38 \mathrm{E}+05$ & $8.48 \mathrm{E}+08$ & $2.44 \mathrm{E}+07$ & $4.54 \mathrm{E}+08$ & $9.26 \mathrm{E}+09$ & $0.00 \mathrm{E}+00$ \\
\hline 0.120 & 0.110 & $4.11 \mathrm{E}+09$ & $8.03 \mathrm{E}+08$ & $1.91 \mathrm{E}+06$ & $9.53 \mathrm{E}+08$ & $1.61 \mathrm{E}+06$ & $5.17 \mathrm{E}+08$ & $1.07 \mathrm{E}+10$ & $0.00 \mathrm{E}+00$ \\
\hline 0.130 & 0.120 & $4.40 \mathrm{E}+09$ & $1.11 \mathrm{E}+08$ & $4.03 \mathrm{E}+03$ & $8.77 \mathrm{E}+08$ & $5.74 \mathrm{E}+06$ & $4.75 \mathrm{E}+08$ & $9.86 \mathrm{E}+09$ & $0.00 \mathrm{E}+00$ \\
\hline 0.140 & 0.130 & $1.98 \mathrm{E}+09$ & $2.46 \mathrm{E}+06$ & $4.34 \mathrm{E}+04$ & $6.92 \mathrm{E}+08$ & $1.02 \mathrm{E}+04$ & $4.02 \mathrm{E}+08$ & $8.09 \mathrm{E}+09$ & $0.00 \mathrm{E}+00$ \\
\hline 0.150 & 0.140 & $8.24 \mathrm{E}+09$ & $1.09 \mathrm{E}+07$ & $1.14 \mathrm{E}+05$ & $6.44 \mathrm{E}+08$ & $7.15 \mathrm{E}+05$ & $3.69 \mathrm{E}+08$ & $7.53 \mathrm{E}+09$ & $0.00 \mathrm{E}+00$ \\
\hline 0.160 & 0.150 & $1.36 \mathrm{E}+10$ & $1.52 \mathrm{E}+06$ & $8.65 \mathrm{E}+04$ & $5.18 \mathrm{E}+08$ & $5.30 \mathrm{E}+04$ & $2.91 \mathrm{E}+08$ & $6.32 \mathrm{E}+09$ & $0.00 \mathrm{E}+00$ \\
\hline 0.170 & 0.160 & $1.28 \mathrm{E}+09$ & $2.79 \mathrm{E}+06$ & $2.86 \mathrm{E}+04$ & $4.87 \mathrm{E}+08$ & $3.45 \mathrm{E}+05$ & $2.73 \mathrm{E}+08$ & $5.93 \mathrm{E}+09$ & $0.00 \mathrm{E}+00$ \\
\hline 0.180 & 0.170 & $1.51 \mathrm{E}+09$ & $9.52 \mathrm{E}+05$ & $1.08 \mathrm{E}+04$ & $3.98 \mathrm{E}+08$ & $2.15 \mathrm{E}+04$ & $2.27 \mathrm{E}+08$ & $5.05 \mathrm{E}+09$ & $0.00 \mathrm{E}+00$ \\
\hline 0.190 & 0.180 & $1.00 \mathrm{E}+09$ & $6.73 \mathrm{E}+06$ & $6.81 \mathrm{E}+03$ & $3.76 \mathrm{E}+08$ & $6.36 \mathrm{E}+02$ & \begin{tabular}{|l|}
$1.46 \mathrm{E}+09$ \\
\end{tabular} & $4.78 \mathrm{E}+09$ & $0.00 \mathrm{E}+00$ \\
\hline 0.200 & 0.190 & $1.60 \mathrm{E}+09$ & $1.27 \mathrm{E}+06$ & $7.44 \mathrm{E}+04$ & $6.21 \mathrm{E}+08$ & $3.38 \mathrm{E}+04$ & $3.80 \mathrm{E}+08$ & $8.25 \mathrm{E}+09$ & $0.00 \mathrm{E}+00$ \\
\hline 0.210 & 0.200 & $2.78 \mathrm{E}+09$ & $1.29 \mathrm{E}+08$ & $1.85 \mathrm{E}+04$ & $5.91 \mathrm{E}+08$ & $1.29 \mathrm{E}+06$ & $3.45 \mathrm{E}+08$ & $7.84 \mathrm{E}+09$ & $0.00 \mathrm{E}+00$ \\
\hline 0.220 & 0.210 & $2.51 \mathrm{E}+09$ & $1.22 \mathrm{E}+08$ & $5.06 \mathrm{E}+04$ & $0.00 \mathrm{E}+00$ & $4.67 \mathrm{E}+03$ & $0.00 \mathrm{E}+00$ & $0.00 \mathrm{E}+00$ & $0.00 \mathrm{E}+00$ \\
\hline 0.230 & 0.220 & $1.50 \mathrm{E}+08$ & $8.58 \mathrm{E}+08$ & $2.38 \mathrm{E}+03$ & $0.00 \mathrm{E}+00$ & $4.76 \mathrm{E}+04$ & $0.00 \mathrm{E}+00$ & $0.00 \mathrm{E}+00$ & $0.00 \mathrm{E}+00$ \\
\hline 0.240 & 0.230 & $2.27 \mathrm{E}+10$ & $2.53 \mathrm{E}+00$ & $1.74 \mathrm{E}+04$ & $0.00 \mathrm{E}+00$ & $7.83 \mathrm{E}+03$ & $0.00 \mathrm{E}+00$ & $0.00 \mathrm{E}+00$ & $0.00 \mathrm{E}+00$ \\
\hline 0.250 & 0.240 & $4.94 \mathrm{E}+08$ & $3.72 \mathrm{E}+01$ & $1.69 \mathrm{E}+04$ & $8.64 \mathrm{E}+02$ & $5.35 \mathrm{E}+03$ & $2.60 \mathrm{E}+09$ & $0.00 \mathrm{E}+00$ & $0.00 \mathrm{E}+00$ \\
\hline 0.260 & & $1.73 \mathrm{E}+10$ & $8.77 \mathrm{E}+06$ & $3.61 \mathrm{E}+03$ & $5.90 \mathrm{E}+08$ & $7.59 \mathrm{E}+02$ & $5.58 \mathrm{E}+08$ & $8.59 \mathrm{E}+09$ & $0.00 \mathrm{E}+00$ \\
\hline 0.270 & 0.260 & $2.89 \mathrm{E}+10$ & $6.99 \mathrm{E}+05$ & $2.62 \mathrm{E}+03$ & $5.68 \mathrm{E}+08$ & $5.44 \mathrm{E}+04$ & $3.49 \mathrm{E}+08$ & $8.27 \mathrm{E}+09$ & $0.00 \mathrm{E}+00$ \\
\hline 0.280 & 0.270 & $2.03 \mathrm{E}+10$ & $1.07 \mathrm{E}+09$ & $8.29 \mathrm{E}+04$ & $0.00 \mathrm{E}+00$ & $1.63 \mathrm{E}+04$ & $1.76 \mathrm{E}+08$ & $0.00 \mathrm{E}+00$ & $0.00 \mathrm{E}+00$ \\
\hline 0.290 & & $3.99 \mathrm{E}+09$ & $5.80 \mathrm{E}+07$ & $1.15 \mathrm{E}-01$ & $0.00 \mathrm{E}+00$ & $4.20 \mathrm{E}-03$ & $3.96 \mathrm{E}+07$ & $0.00 \mathrm{E}+00$ & $0.00 \mathrm{E}+00$ \\
\hline 0.300 & 0.290 & $3.24 \mathrm{E}+09$ & $8.71 \mathrm{E}+00$ & $8.89 \mathrm{E}+05$ & $0.00 \mathrm{E}+00$ & $1.01 \mathrm{E}+05$ & $6.77 \mathrm{E}+09$ & $0.00 \mathrm{E}+00$ & $0.00 \mathrm{E}+00$ \\
\hline 0.325 & 0.300 & $1.58 \mathrm{E}+10$ & $1.19 \mathrm{E}+08$ & $1.09 \mathrm{E}+07$ & $6.42 \mathrm{E}+08$ & $1.18 \mathrm{E}+06$ & $4.79 \mathrm{E}+08$ & $1.09 \mathrm{E}+10$ & $0.00 \mathrm{E}+00$ \\
\hline 0.350 & 0.325 & $1.45 \mathrm{E}+10$ & $1.50 \mathrm{E}+08$ & $1.18 \mathrm{E}+06$ & $0.00 \mathrm{E}+00$ & $8.62 \mathrm{E}+05$ & $9.19 \mathrm{E}+07$ & $0.00 \mathrm{E}+00$ & $0.00 \mathrm{E}+00$ \\
\hline 0.375 & 0.350 & $2.55 \mathrm{E}+10$ & $1.73 \mathrm{E}+02$ & $9.19 \mathrm{E}+04$ & $0.00 \mathrm{E}+00$ & $3.25 \mathrm{E}+05$ & $1.27 \mathrm{E}+10$ & $0.00 \mathrm{E}+00$ & $0.00 \mathrm{E}+00$ \\
\hline 0.400 & 0.375 & $1.24 \mathrm{E}+09$ & $2.25 \mathrm{E}+05$ & $4.64 \mathrm{E}+05$ & $3.49 \mathrm{E}+08$ & $2.35 \mathrm{E}+05$ & $5.48 \mathrm{E}+08$ & $7.02 \mathrm{E}+09$ & $0.00 \mathrm{E}+00$ \\
\hline 0.425 & 0.400 & $2.01 \mathrm{E}+10$ & $2.79 \mathrm{E}+02$ & $4.57 \mathrm{E}+05$ & $0.00 \mathrm{E}+00$ & $8.08 \mathrm{E}+04$ & $5.81 \mathrm{E}+07$ & $0.00 \mathrm{E}+00$ & $0.00 \mathrm{E}+00$ \\
\hline 0.450 & 0.425 & $6.73 \mathrm{E}+09$ & $1.23 \mathrm{E}+06$ & $4.59 \mathrm{E}+01$ & $2.08 \mathrm{E}+08$ & $3.28 \mathrm{E}+04$ & $2.22 \mathrm{E}+08$ & $5.06 \mathrm{E}+09$ & $0.00 \mathrm{E}+00$ \\
\hline 0.475 & 0.450 & $5.18 \mathrm{E}+05$ & $1.96 \mathrm{E}+05$ & 7.24E-01 & $0.00 \mathrm{E}+00$ & $2.18 \mathrm{E}+04$ & $2.42 \mathrm{E}+08$ & $0.00 \mathrm{E}+00$ & $0.00 \mathrm{E}+00$ \\
\hline 0.500 & 0.475 & $4.20 \mathrm{E}+08$ & $9.92 \mathrm{E}+05$ & $3.25 \mathrm{E}+00$ & $8.31 \mathrm{E}+07$ & $1.62 \mathrm{E}+03$ & $3.77 \mathrm{E}+08$ & $2.50 \mathrm{E}+09$ & $0.00 \mathrm{E}+00$ \\
\hline 0.525 & 0.500 & $3.92 \mathrm{E}+08$ & $7.63 \mathrm{E}+03$ & $3.09 \mathrm{E}+00$ & $7.90 \mathrm{E}+07$ & $5.26 \mathrm{E}+03$ & $1.25 \mathrm{E}+08$ & $2.38 \mathrm{E}+09$ & $0.00 \mathrm{E}+00$ \\
\hline 0.550 & 0.525 & $1.51 \mathrm{E}+08$ & 9.92E-02 & $4.01 \mathrm{E}-03$ & $0.00 \mathrm{E}+00$ & $1.36 \mathrm{E}+03$ & $1.46 \mathrm{E}+08$ & $0.00 \mathrm{E}+00$ & $0.00 \mathrm{E}+00$ \\
\hline 0.575 & 0.550 & $9.02 \mathrm{E}+06$ & $4.22 \mathrm{E}-02$ & $3.59 \mathrm{E}-04$ & $0.00 \mathrm{E}+00$ & $2.27 \mathrm{E}+03$ & $2.93 \mathrm{E}+07$ & $0.00 \mathrm{E}+00$ & $0.00 \mathrm{E}+00$ \\
\hline 0.600 & 0.575 & $3.78 \mathrm{E}+08$ & $1.56 \mathrm{E}+03$ & $1.62 \mathrm{E}-02$ & $5.05 \mathrm{E}+07$ & $1.35 \mathrm{E}+04$ & $1.95 \mathrm{E}+08$ & $2.10 \mathrm{E}+09$ & $0.00 \mathrm{E}+00$ \\
\hline
\end{tabular}


PCP-2011-0001

Revision 0

\begin{tabular}{|c|c|c|c|c|c|c|c|c|c|}
\hline \multicolumn{2}{|c|}{ Energy (MeV) } & \multicolumn{8}{|c|}{ Photon Source (p/s/g) } \\
\hline Upper & Lower & Ac-227 & Am-243 & Np-237 & Pb-210 & Pu-241 & Ra-226 & Sr-90 & Zr-95 \\
\hline 0.625 & 0.600 & $3.82 \mathrm{E}+08$ & $1.49 \mathrm{E}+03$ & $1.53 \mathrm{E}-02$ & $4.85 \mathrm{E}+07$ & $6.76 \mathrm{E}+04$ & $1.63 \mathrm{E}+10$ & $2.01 \mathrm{E}+09$ & $0.00 \mathrm{E}+00$ \\
\hline 0.650 & 0.625 & $4.45 \mathrm{E}+05$ & $2.18 \mathrm{E}+04$ & $0.00 \mathrm{E}+00$ & $0.00 \mathrm{E}+00$ & $1.01 \mathrm{E}+04$ & $6.09 \mathrm{E}+07$ & $0.00 \mathrm{E}+00$ & $0.00 \mathrm{E}+00$ \\
\hline 0.675 & 0.650 & $6.50 \mathrm{E}+06$ & $8.79 \mathrm{E}+04$ & 2.94E-02 & $0.00 \mathrm{E}+00$ & $4.48 \mathrm{E}+05$ & $5.69 \mathrm{E}+08$ & $0.00 \mathrm{E}+00$ & $0.00 \mathrm{E}+00$ \\
\hline 0.700 & 0.675 & $1.12 \mathrm{E}+08$ & $1.94 \mathrm{E}+01$ & $6.55 \mathrm{E}-03$ & $1.97 \mathrm{E}+07$ & $5.11 \mathrm{E}+04$ & $8.28 \mathrm{E}+07$ & $1.33 \mathrm{E}+09$ & $0.00 \mathrm{E}+00$ \\
\hline 0.725 & 0.700 & $1.04 \mathrm{E}+09$ & $1.97 \mathrm{E}+01$ & $6.58 \mathrm{E}-03$ & $1.90 \mathrm{E}+07$ & $2.31 \mathrm{E}+05$ & $3.87 \mathrm{E}+08$ & $1.28 \mathrm{E}+09$ & $0.00 \mathrm{E}+00$ \\
\hline 0.750 & 0.725 & $2.08 \mathrm{E}+06$ & $1.51 \mathrm{E}+01$ & 5.12E-02 & $4.32 \mathrm{E}+02$ & $1.16 \mathrm{E}+04$ & $3.60 \mathrm{E}+07$ & $0.00 \mathrm{E}+00$ & $0.00 \mathrm{E}+00$ \\
\hline 0.775 & 0.750 & $1.41 \mathrm{E}+09$ & $1.73 \mathrm{E}+01$ & $4.95 \mathrm{E}-02$ & $4.18 \mathrm{E}+02$ & $2.49 \mathrm{E}+04$ & $1.81 \mathrm{E}+09$ & $0.00 \mathrm{E}+00$ & $0.00 \mathrm{E}+00$ \\
\hline 0.800 & 0.775 & $5.49 \mathrm{E}+07$ & 5.53E-02 & $3.08 \mathrm{E}-03$ & $6.88 \mathrm{E}+06$ & $2.72 \mathrm{E}+03$ & $5.28 \mathrm{E}+08$ & $8.59 \mathrm{E}+08$ & $0.00 \mathrm{E}+00$ \\
\hline 0.825 & 0.800 & $5.73 \mathrm{E}+07$ & 5.73E-02 & $1.01 \mathrm{E}-01$ & $8.97 \mathrm{E}+06$ & $3.25 \mathrm{E}+03$ & $5.30 \mathrm{E}+08$ & $8.33 \mathrm{E}+08$ & $0.00 \mathrm{E}+00$ \\
\hline 0.850 & 0.825 & $7.43 \mathrm{E}+09$ & $6.61 \mathrm{E}-02$ & $0.00 \mathrm{E}+00$ & $0.00 \mathrm{E}+00$ & $8.56 \mathrm{E}+02$ & $2.54 \mathrm{E}+08$ & $0.00 \mathrm{E}+00$ & $0.00 \mathrm{E}+00$ \\
\hline 0.875 & 0.850 & $1.36 \mathrm{E}+07$ & 1.19E-08 & $1.97 \mathrm{E}-04$ & $0.00 \mathrm{E}+00$ & $1.86 \mathrm{E}+03$ & $0.00 \mathrm{E}+00$ & $0.00 \mathrm{E}+00$ & $0.00 \mathrm{E}+00$ \\
\hline 0.900 & 0.875 & $5.03 \mathrm{E}+08$ & $2.37 \mathrm{E}-02$ & $1.39 \mathrm{E}-03$ & $1.97 \mathrm{E}+06$ & $3.27 \mathrm{E}+02$ & $1.52 \mathrm{E}+07$ & $5.65 \mathrm{E}+08$ & $0.00 \mathrm{E}+00$ \\
\hline 0.925 & 0.900 & $2.32 \mathrm{E}+07$ & $5.19 \mathrm{E}-03$ & $1.36 \mathrm{E}-03$ & $1.91 \mathrm{E}+06$ & $8.32 \mathrm{E}+02$ & $5.96 \mathrm{E}+07$ & $5.50 \mathrm{E}+08$ & $0.00 \mathrm{E}+00$ \\
\hline 0.950 & 0.925 & $1.73 \mathrm{E}+05$ & $1.09 \mathrm{E}-02$ & $0.00 \mathrm{E}+00$ & $0.00 \mathrm{E}+00$ & $1.25 \mathrm{E}+02$ & $1.12 \mathrm{E}+09$ & $0.00 \mathrm{E}+00$ & $0.00 \mathrm{E}+00$ \\
\hline 0.975 & 0.950 & $4.23 \mathrm{E}+07$ & $1.58 \mathrm{E}-02$ & $0.00 \mathrm{E}+00$ & $0.00 \mathrm{E}+00$ & $6.48 \mathrm{E}+02$ & $1.36 \mathrm{E}+08$ & $0.00 \mathrm{E}+00$ & $0.00 \mathrm{E}+00$ \\
\hline 1.000 & 0.975 & $7.73 \mathrm{E}+06$ & $1.67 \mathrm{E}-02$ & $9.80 \mathrm{E}-04$ & $3.81 \mathrm{E}+05$ & 2.82E-05 & $2.23 \mathrm{E}+07$ & $3.74 \mathrm{E}+08$ & $0.00 \mathrm{E}+00$ \\
\hline 1.250 & 1.000 & $4.03 \mathrm{E}+08$ & $8.21 \mathrm{E}+00$ & $1.23 \mathrm{E}-01$ & $3.90 \mathrm{E}+05$ & $1.87 \mathrm{E}+02$ & $8.90 \mathrm{E}+09$ & $1.15 \mathrm{E}+09$ & $0.00 \mathrm{E}+00$ \\
\hline 1.500 & 1.250 & $1.64 \mathrm{E}+07$ & $6.71 \mathrm{E}+00$ & $2.30 \mathrm{E}-02$ & $1.94 \mathrm{E}+02$ & $9.94 \mathrm{E}+01$ & $3.71 \mathrm{E}+09$ & $3.74 \mathrm{E}+08$ & $0.00 \mathrm{E}+00$ \\
\hline 1.750 & 1.500 & $5.13 \mathrm{E}+02$ & $3.98 \mathrm{E}+00$ & $6.95 \mathrm{E}-01$ & $1.15 \mathrm{E}+02$ & $5.91 \mathrm{E}+01$ & $3.12 \mathrm{E}+09$ & $1.10 \mathrm{E}+08$ & $0.00 \mathrm{E}+00$ \\
\hline 2.000 & 1.750 & $4.45 \mathrm{E}+02$ & $3.45 \mathrm{E}+00$ & $1.18 \mathrm{E}-02$ & $9.97 \mathrm{E}+01$ & $5.12 \mathrm{E}+01$ & $6.39 \mathrm{E}+09$ & $2.11 \mathrm{E}+07$ & $0.00 \mathrm{E}+00$ \\
\hline 2.250 & 2.000 & $2.53 \mathrm{E}+02$ & $1.97 \mathrm{E}+00$ & $6.73 \mathrm{E}-03$ & $5.67 \mathrm{E}+01$ & $2.91 \mathrm{E}+01$ & $2.39 \mathrm{E}+09$ & $2.47 \mathrm{E}+06$ & $0.00 \mathrm{E}+00$ \\
\hline 2.500 & 2.250 & $2.26 \mathrm{E}+02$ & $1.77 \mathrm{E}+00$ & $6.02 \mathrm{E}-03$ & $5.07 \mathrm{E}+01$ & $2.61 \mathrm{E}+01$ & $7.12 \mathrm{E}+08$ & $9.74 \mathrm{E}+03$ & $0.00 \mathrm{E}+00$ \\
\hline 2.750 & 2.500 & $1.26 \mathrm{E}+02$ & 9.85E-01 & $3.34 \mathrm{E}-03$ & $2.81 \mathrm{E}+01$ & $1.45 \mathrm{E}+01$ & $1.51 \mathrm{E}+07$ & $0.00 \mathrm{E}+00$ & $0.00 \mathrm{E}+00$ \\
\hline 3.000 & 2.750 & $1.15 \mathrm{E}+02$ & 8.99E-01 & $3.05 \mathrm{E}-03$ & $2.57 \mathrm{E}+01$ & $1.32 \mathrm{E}+01$ & $3.17 \mathrm{E}+07$ & $0.00 \mathrm{E}+00$ & $0.00 \mathrm{E}+00$ \\
\hline 3.500 & 3.000 & $1.20 \mathrm{E}+02$ & $9.50 \mathrm{E}-01$ & $3.20 \mathrm{E}-03$ & $2.70 \mathrm{E}+01$ & $1.39 \mathrm{E}+01$ & $1.20 \mathrm{E}+07$ & $0.00 \mathrm{E}+00$ & $0.00 \mathrm{E}+00$ \\
\hline 4.000 & 3.500 & $6.03 \mathrm{E}+01$ & $4.80 \mathrm{E}-01$ & $1.60 \mathrm{E}-03$ & $1.35 \mathrm{E}+01$ & $6.96 \mathrm{E}+00$ & $1.07 \mathrm{E}+01$ & $0.00 \mathrm{E}+00$ & $0.00 \mathrm{E}+00$ \\
\hline 4.500 & 4.000 & $3.02 \mathrm{E}+01$ & $2.43 \mathrm{E}-01$ & $8.04 \mathrm{E}-04$ & $6.78 \mathrm{E}+00$ & $3.50 \mathrm{E}+00$ & $5.37 \mathrm{E}+00$ & $0.00 \mathrm{E}+00$ & $0.00 \mathrm{E}+00$ \\
\hline 5.000 & 4.500 & $1.52 \mathrm{E}+01$ & $1.23 \mathrm{E}-01$ & $4.03 \mathrm{E}-04$ & $3.40 \mathrm{E}+00$ & $1.76 \mathrm{E}+00$ & $2.69 \mathrm{E}+00$ & $0.00 \mathrm{E}+00$ & $0.00 \mathrm{E}+00$ \\
\hline 5.500 & 5.000 & $7.61 \mathrm{E}+00$ & $6.26 \mathrm{E}-02$ & $2.02 \mathrm{E}-04$ & $1.71 \mathrm{E}+00$ & $8.82 \mathrm{E}-01$ & $1.35 \mathrm{E}+00$ & $0.00 \mathrm{E}+00$ & $0.00 \mathrm{E}+00$ \\
\hline 6.000 & 5.500 & $3.82 \mathrm{E}+00$ & 3.19E-02 & $1.01 \mathrm{E}-04$ & $8.56 \mathrm{E}-01$ & $4.43 \mathrm{E}-01$ & 6.77E-01 & $0.00 \mathrm{E}+00$ & $0.00 \mathrm{E}+00$ \\
\hline 6.500 & 6.000 & $1.91 \mathrm{E}+00$ & $1.62 \mathrm{E}-02$ & $5.09 \mathrm{E}-05$ & $4.29 \mathrm{E}-01$ & $2.23 \mathrm{E}-01$ & $3.40 \mathrm{E}-01$ & $0.00 \mathrm{E}+00$ & $0.00 \mathrm{E}+00$ \\
\hline 7.000 & 6.500 & $9.60 \mathrm{E}-01$ & $8.29 \mathrm{E}-03$ & $2.55 \mathrm{E}-05$ & $2.15 \mathrm{E}-01$ & $1.12 \mathrm{E}-01$ & \begin{tabular}{|l|}
$1.70 \mathrm{E}-01$ \\
\end{tabular} & $0.00 \mathrm{E}+00$ & $0.00 \mathrm{E}+00$ \\
\hline 7.500 & 7.000 & $4.82 \mathrm{E}-01$ & $4.24 \mathrm{E}-03$ & $1.28 \mathrm{E}-05$ & $1.08 \mathrm{E}-01$ & $5.63 \mathrm{E}-02$ & $8.54 \mathrm{E}-02$ & $0.00 \mathrm{E}+00$ & $0.00 \mathrm{E}+00$ \\
\hline 8.000 & 7.500 & $2.42 \mathrm{E}-01$ & $2.18 \mathrm{E}-03$ & $6.42 \mathrm{E}-06$ & 5.42E-02 & $2.83 \mathrm{E}-02$ & 4.29E-02 & $0.00 \mathrm{E}+00$ & $0.00 \mathrm{E}+00$ \\
\hline 9.000 & 8.000 & \begin{tabular}{|l|l|}
$1.80 \mathrm{E}-01$ \\
\end{tabular} & $1.69 \mathrm{E}-03$ & 4.79E-06 & 4.04E-02 & $2.12 \mathrm{E}-02$ & $3.20 \mathrm{E}-02$ & $0.00 \mathrm{E}+00$ & $0.00 \mathrm{E}+00$ \\
\hline \multirow[t]{2}{*}{10.000} & 9.000 & $4.54 \mathrm{E}-02$ & $4.53 \mathrm{E}-04$ & $1.21 \mathrm{E}-06$ & $1.02 \mathrm{E}-02$ & $5.38 \mathrm{E}-03$ & $8.05 \mathrm{E}-03$ & $0.00 \mathrm{E}+00$ & $0.00 \mathrm{E}+00$ \\
\hline & Total & $6.33 \mathrm{E}+11$ & $2.16 \mathrm{E}+10$ & $6.93 \mathrm{E}+07$ & $1.15 \mathrm{E}+11$ & $9.18 \mathrm{E}+10$ & $1.25 \mathrm{E}+11$ & $6.15 \mathrm{E}+11$ & $0.00 \mathrm{E}+00$ \\
\hline
\end{tabular}


PCP-2011-0001

Revision 0

Table 3-26. Total Photon Source Strength

\begin{tabular}{|c|c|c|c|c|c|}
\hline \multirow{3}{*}{ Isotope } & \multicolumn{5}{|c|}{ Total Photon Source (p/s/g) } \\
\hline & \multirow{2}{*}{ RASTA } & \multicolumn{4}{|c|}{ ORIGEN-S } \\
\hline & & $0.01 \mathrm{~d}$ & $30 \mathrm{~d}$ & $100 \mathrm{~d}$ & $30000 \mathrm{~d}$ \\
\hline Ac-227 & $2.27 \mathrm{E}+10$ & $3.38 \mathrm{E}+10$ & $4.26 \mathrm{E}+12$ & $8.16 \mathrm{E}+12$ & $6.33 \mathrm{E}+11$ \\
\hline Am-241 & $1.36 \mathrm{E}+11$ & $1.03 \mathrm{E}+11$ & $1.03 \mathrm{E}+11$ & $1.03 \mathrm{E}+11$ & $9.07 \mathrm{E}+10$ \\
\hline Am-243 & $6.80 \mathrm{E}+09$ & $7.51 \mathrm{E}+09$ & $2.18 \mathrm{E}+10$ & $2.18 \mathrm{E}+10$ & $2.16 \mathrm{E}+10$ \\
\hline Cd-109 & $9.15 \mathrm{E}+13$ & $8.93 \mathrm{E}+13$ & $8.54 \mathrm{E}+13$ & $7.69 \mathrm{E}+13$ & 2.69E-06 \\
\hline Cf-252 & $1.47 \mathrm{E}+12$ & $1.01 \mathrm{E}+13$ & $9.90 \mathrm{E}+12$ & $9.43 \mathrm{E}+12$ & $1.80 \mathrm{E}+08$ \\
\hline $\mathrm{Cm}-244$ & $3.18 \mathrm{E}+11$ & $3.66 \mathrm{E}+11$ & $3.65 \mathrm{E}+11$ & $3.63 \mathrm{E}+11$ & $1.66 \mathrm{E}+10$ \\
\hline $\mathrm{Cm}-248$ & $2.20 \mathrm{E}+08$ & $1.89 \mathrm{E}+08$ & $1.89 \mathrm{E}+08$ & $1.89 \mathrm{E}+08$ & $1.89 \mathrm{E}+08$ \\
\hline Co-60 & $8.45 \mathrm{E}+13$ & $8.59 \mathrm{E}+13$ & $8.50 \mathrm{E}+13$ & $8.28 \mathrm{E}+13$ & $1.75 \mathrm{E}+09$ \\
\hline Cs-137 & $1.82 \mathrm{E}+11$ & $3.19 \mathrm{E}+12$ & $3.24 \mathrm{E}+12$ & $3.23 \mathrm{E}+12$ & $4.87 \mathrm{E}+11$ \\
\hline Eu-152 & $1.54 \mathrm{E}+13$ & $1.56 \mathrm{E}+13$ & $1.55 \mathrm{E}+13$ & $1.53 \mathrm{E}+13$ & $2.17 \mathrm{E}+11$ \\
\hline $\mathrm{Fe}-59$ & $1.86 \mathrm{E}+15$ & $1.95 \mathrm{E}+15$ & $1.22 \mathrm{E}+15$ & $4.11 \mathrm{E}+14$ & $0.00 \mathrm{E}+00$ \\
\hline Gd-153 & $2.21 \mathrm{E}+14$ & $2.24 \mathrm{E}+14$ & $2.05 \mathrm{E}+14$ & $1.68 \mathrm{E}+14$ & $0.00 \mathrm{E}+00$ \\
\hline Hf-181 & $1.19 \mathrm{E}+15$ & $1.16 \mathrm{E}+15$ & $7.10 \mathrm{E}+14$ & $2.26 \mathrm{E}+14$ & $0.00 \mathrm{E}+00$ \\
\hline Ho- $166 \mathrm{~m}$ & $2.47 \mathrm{E}+11$ & $2.35 \mathrm{E}+11$ & $2.35 \mathrm{E}+11$ & $2.35 \mathrm{E}+11$ & $2.24 \mathrm{E}+11$ \\
\hline Ir-192 & $8.23 \mathrm{E}+14$ & $7.38 \mathrm{E}+13$ & $5.57 \mathrm{E}+13$ & $2.89 \mathrm{E}+13$ & $0.00 \mathrm{E}+00$ \\
\hline Mn-54 & $2.86 \mathrm{E}+14$ & $2.86 \mathrm{E}+14$ & $2.67 \mathrm{E}+14$ & $2.29 E+14$ & $3.35 \mathrm{E}-15$ \\
\hline $\mathrm{Np}-237$ & $2.57 \mathrm{E}+07$ & $2.71 \mathrm{E}+07$ & $4.97 \mathrm{E}+07$ & $6.61 \mathrm{E}+07$ & $6.93 \mathrm{E}+07$ \\
\hline $\mathrm{Pb}-210$ & $6.64 \mathrm{E}+11$ & $7.16 \mathrm{E}+11$ & $1.46 \mathrm{E}+12$ & $1.46 \mathrm{E}+12$ & $1.15 \mathrm{E}+11$ \\
\hline Pm-147 & $3.81 \mathrm{E}+11$ & $7.69 \mathrm{E}+11$ & $7.53 \mathrm{E}+11$ & $7.15 E+11$ & $2.89 \mathrm{E}+02$ \\
\hline Po-210 & $1.76 \mathrm{E}+09$ & $1.76 \mathrm{E}+09$ & $1.52 \mathrm{E}+09$ & $1.07 \mathrm{E}+09$ & $0.00 \mathrm{E}+00$ \\
\hline $\mathrm{Pu}-238$ & $7.15 \mathrm{E}+10$ & $7.69 E+10$ & $7.68 \mathrm{E}+10$ & $7.67 \mathrm{E}+10$ & $4.02 \mathrm{E}+10$ \\
\hline $\mathrm{Pu}-239$ & $9.79 \mathrm{E}+07$ & $1.11 \mathrm{E}+08$ & $1.11 \mathrm{E}+08$ & $1.11 \mathrm{E}+08$ & $1.11 \mathrm{E}+08$ \\
\hline $\mathrm{Pu}-240$ & $9.04 \mathrm{E}+08$ & $8.83 \mathrm{E}+08$ & $8.83 \mathrm{E}+08$ & $8.83 E+08$ & $8.76 \mathrm{E}+08$ \\
\hline $\mathrm{Pu}-241$ & $3.16 \mathrm{E}+08$ & $1.96 \mathrm{E}+08$ & $7.64 \mathrm{E}+08$ & $1.72 \mathrm{E}+09$ & $9.18 \mathrm{E}+10$ \\
\hline $\mathrm{Pu}-242$ & $1.31 \mathrm{E}+07$ & $1.31 \mathrm{E}+07$ & $1.31 \mathrm{E}+07$ & $1.31 \mathrm{E}+07$ & $1.31 \mathrm{E}+07$ \\
\hline Ra-226 & $1.69 \mathrm{E}+09$ & $1.84 \mathrm{E}+09$ & $1.12 \mathrm{E}+11$ & $1.12 \mathrm{E}+11$ & $1.25 \mathrm{E}+11$ \\
\hline Ru-106 & $1.34 \mathrm{E}+10$ & $1.96 \mathrm{E}+14$ & $1.86 \mathrm{E}+14$ & $1.63 \mathrm{E}+14$ & $9.84 \mathrm{E}-11$ \\
\hline Sc-46 & $2.51 \mathrm{E}+15$ & $2.58 \mathrm{E}+15$ & $2.00 \mathrm{E}+15$ & $1.12 \mathrm{E}+15$ & $0.00 \mathrm{E}+00$ \\
\hline $\mathrm{Se}-75$ & $1.19 \mathrm{E}+15$ & $1.18 \mathrm{E}+15$ & $9.96 \mathrm{E}+14$ & $6.64 \mathrm{E}+14$ & $0.00 \mathrm{E}+00$ \\
\hline Sm-145 & $1.61 \mathrm{E}+14$ & $1.61 \mathrm{E}+14$ & $1.51 \mathrm{E}+14$ & $1.32 \mathrm{E}+14$ & $1.81 \mathrm{E}+11$ \\
\hline Sr-90 & $2.04 \mathrm{E}+11$ & $5.93 \mathrm{E}+11$ & $4.64 \mathrm{E}+12$ & $4.61 \mathrm{E}+12$ & $6.15 \mathrm{E}+11$ \\
\hline Tm-170 & $4.75 E+13$ & $4.56 \mathrm{E}+13$ & $3.88 \mathrm{E}+13$ & $2.66 \mathrm{E}+13$ & $0.00 \mathrm{E}+00$ \\
\hline Yb-169 & $2.93 E+15$ & $2.93 E+15$ & $1.53 \mathrm{E}+15$ & $3.36 \mathrm{E}+14$ & $0.00 \mathrm{E}+00$ \\
\hline $\mathrm{Zn}-65$ & $1.62 \mathrm{E}+14$ & $1.63 \mathrm{E}+14$ & $1.51 \mathrm{E}+14$ & $1.23 \mathrm{E}+14$ & $0.00 \mathrm{E}+00$ \\
\hline Zr-95 & $8.10 \mathrm{E}+14$ & $8.31 \mathrm{E}+14$ & $9.10 \mathrm{E}+14$ & $6.41 \mathrm{E}+14$ & $0.00 \mathrm{E}+00$ \\
\hline
\end{tabular}

Shaded values indicate that RASTA source is greater than ORIGEN-S source 
PCP-2011-0001

Revision 0

Table 3-27 Photons from Nuclides with Bremsstrahlung

\begin{tabular}{|c|c|c|c|c|c|c|c|c|c|c|}
\hline \multirow{2}{*}{\multicolumn{2}{|c|}{ Energy (MeV) }} & \multicolumn{9}{|c|}{ Photon Source (photons/sec/g) } \\
\hline & & \multicolumn{3}{|c|}{ Ac-227 } & \multicolumn{3}{|c|}{ Cs-137 } & \multicolumn{3}{|c|}{$\mathrm{Pb}-210$} \\
\hline Upper & Lower & (Decay) & (Brem.) & (Total) & (Decay) & (Brem.) & (Total) & (Decay) & (Brem.) & (Total) \\
\hline $2.00 \mathrm{E}-02$ & $1.00 \mathrm{E}-02$ & $1.11 \mathrm{E}+12$ & $5.10 \mathrm{E}+11$ & $1.62 \mathrm{E}+12$ & $0.00 \mathrm{E}+00$ & $6.73 \mathrm{E}+10$ & $6.73 E+10$ & $2.72 \mathrm{E}+04$ & $1.24 \mathrm{E}+11$ & $1.24 \mathrm{E}+11$ \\
\hline $3.00 \mathrm{E}-02$ & $2.00 \mathrm{E}-02$ & $1.34 \mathrm{E}+10$ & $2.62 \mathrm{E}+11$ & $2.75 \mathrm{E}+11$ & $0.00 \mathrm{E}+00$ & $3.31 \mathrm{E}+10$ & $3.31 \mathrm{E}+10$ & $0.00 \mathrm{E}+00$ & $6.33 \mathrm{E}+10$ & $6.33 \mathrm{E}+10$ \\
\hline $4.00 \mathrm{E}-02$ & $3.00 \mathrm{E}-02$ & $2.18 \mathrm{E}+09$ & $1.66 \mathrm{E}+11$ & $1.68 \mathrm{E}+11$ & $2.14 \mathrm{E}+11$ & $2.02 \mathrm{E}+10$ & $2.34 \mathrm{E}+11$ & $0.00 \mathrm{E}+00$ & $3.98 \mathrm{E}+10$ & $3.98 \mathrm{E}+10$ \\
\hline $5.00 \mathrm{E}-02$ & $4.00 \mathrm{E}-02$ & $1.46 \mathrm{E}+10$ & $1.16 \mathrm{E}+11$ & $1.31 \mathrm{E}+11$ & $0.00 \mathrm{E}+00$ & $1.36 \mathrm{E}+10$ & $1.36 \mathrm{E}+10$ & $0.00 \mathrm{E}+00$ & $2.78 \mathrm{E}+10$ & $2.78 \mathrm{E}+10$ \\
\hline $6.00 \mathrm{E}-02$ & $5.00 \mathrm{E}-02$ & $2.13 \mathrm{E}+11$ & $8.70 \mathrm{E}+10$ & $3.00 \mathrm{E}+11$ & $0.00 \mathrm{E}+00$ & $9.74 \mathrm{E}+09$ & $9.74 \mathrm{E}+09$ & $0.00 \mathrm{E}+00$ & $2.06 \mathrm{E}+10$ & $2.06 \mathrm{E}+10$ \\
\hline $7.00 \mathrm{E}-02$ & $6.00 \mathrm{E}-02$ & $1.17 \mathrm{E}+10$ & $6.77 \mathrm{E}+10$ & $7.95 \mathrm{E}+10$ & $0.00 \mathrm{E}+00$ & $7.27 \mathrm{E}+09$ & $7.27 \mathrm{E}+09$ & $0.00 \mathrm{E}+00$ & $1.59 \mathrm{E}+10$ & $1.59 \mathrm{E}+10$ \\
\hline $8.00 \mathrm{E}-02$ & $.00 \mathrm{E}-02$ & $1.64 \mathrm{E}+11$ & $5.43 \mathrm{E}+10$ & $2.18 \mathrm{E}+11$ & $0.00 \mathrm{E}+00$ & $5.59 \mathrm{E}+09$ & $5.59 \mathrm{E}+09$ & $6.81 \mathrm{E}+04$ & $1.27 \mathrm{E}+10$ & $1.27 \mathrm{E}+10$ \\
\hline $9.00 \mathrm{E}-02$ & 00E-02 & $1.52 \mathrm{E}+11$ & $4.45 \mathrm{E}+10$ & $1.97 \mathrm{E}+11$ & $0.00 \mathrm{E}+00$ & $4.39 \mathrm{E}+09$ & $4.39 \mathrm{E}+09$ & $1.98 \mathrm{E}+04$ & $1.03 \mathrm{E}+10$ & $1.03 \mathrm{E}+10$ \\
\hline $1.00 \mathrm{E}-01$ & & $5.18 \mathrm{E}+10$ & $3.71 \mathrm{E}+10$ & $8.89 \mathrm{E}+10$ & $0.00 \mathrm{E}+00$ & $3.50 \mathrm{E}+09$ & $3.50 \mathrm{E}+09$ & $0.00 \mathrm{E}+00$ & $8.55 \mathrm{E}+09$ & $8.55 \mathrm{E}+09$ \\
\hline $1.10 \mathrm{E}-01$ & $00 \mathrm{E}-01$ & $3.00 \mathrm{E}+10$ & $3.14 \mathrm{E}+10$ & $6.14 \mathrm{E}+10$ & $0.00 \mathrm{E}+00$ & $2.83 \mathrm{E}+09$ & $2.83 \mathrm{E}+09$ & $0.00 \mathrm{E}+00$ & $7.18 \mathrm{E}+09$ & $7.18 \mathrm{E}+09$ \\
\hline $1.20 \mathrm{E}-01$ & & $2.36 \mathrm{E}+10$ & $2.68 \mathrm{E}+10$ & $5.04 \mathrm{E}+10$ & $0.00 \mathrm{E}+00$ & $2.31 \mathrm{E}+09$ & $2.31 \mathrm{E}+09$ & $0.00 \mathrm{E}+00$ & $6.09 \mathrm{E}+09$ & $6.09 \mathrm{E}+09$ \\
\hline $1.30 \mathrm{E}-01$ & & $5.31 \mathrm{E}+08$ & $2.32 \mathrm{E}+10$ & $2.37 \mathrm{E}+10$ & $0.00 \mathrm{E}+00$ & $1.90 \mathrm{E}+09$ & $1.90 \mathrm{E}+09$ & $0.00 \mathrm{E}+00$ & $5.22 \mathrm{E}+09$ & $5.22 \mathrm{E}+09$ \\
\hline $1.40 \mathrm{E}-01$ & & $4.15 \mathrm{E}$ & $2.02 \mathrm{E}+10$ & $2.43 \mathrm{E}+10$ & $0.00 \mathrm{E}+00$ & $1.57 \mathrm{E}+09$ & $1.57 \mathrm{E}+09$ & $0.00 \mathrm{E}+00$ & $4.51 \mathrm{E}+09$ & $4.51 \mathrm{E}+09$ \\
\hline $1.50 \mathrm{E}-01$ & & $4.62 \mathrm{E}+09$ & $1.77 \mathrm{E}+10$ & $2.23 \mathrm{E}+10$ & $0.00 \mathrm{E}+00$ & $1.31 \mathrm{E}+09$ & $1.31 \mathrm{E}+09$ & $0.00 \mathrm{E}+00$ & $3.92 \mathrm{E}+09$ & $3.92 \mathrm{E}+09$ \\
\hline $1.60 \mathrm{E}-01$ & $1.50 \mathrm{E}-01$ & $0.00 \mathrm{E}+00$ & $1.56 \mathrm{E}+10$ & $1.56 \mathrm{E}+10$ & $0.00 \mathrm{E}+00$ & $1.10 \mathrm{E}+09$ & $1.10 \mathrm{E}+09$ & $0.00 \mathrm{E}+00$ & $3.43 \mathrm{E}+09$ & $3.43 \mathrm{E}+09$ \\
\hline $1.70 \mathrm{E}-01$ & $1.60 \mathrm{E}-01$ & $1.04 \mathrm{E}+09$ & $1.38 \mathrm{E}+10$ & $1.48 \mathrm{E}+10$ & $0.00 \mathrm{E}+00$ & $9.24 \mathrm{E}+08$ & $9.24 \mathrm{E}+08$ & $0.00 \mathrm{E}+00$ & $3.02 \mathrm{E}+09$ & $3.02 \mathrm{E}+09$ \\
\hline $1.80 \mathrm{E}-01$ & $1.70 \mathrm{E}-01$ & $1.10 \mathrm{E}+09$ & $1.23 \mathrm{E}+10$ & $1.34 \mathrm{E}+10$ & $0.00 \mathrm{E}+00$ & $7.79 \mathrm{E}+08$ & $7.79 \mathrm{E}+08$ & $0.00 \mathrm{E}+00$ & $2.67 \mathrm{E}+09$ & $2.67 \mathrm{E}+09$ \\
\hline $1.90 \mathrm{E}-01$ & $1.80 \mathrm{E}-01$ & $9.68 \mathrm{E}+08$ & $1.10 \mathrm{E}+10$ & $1.19 \mathrm{E}+10$ & $0.00 \mathrm{E}+00$ & $6.59 \mathrm{E}+08$ & $6.59 \mathrm{E}+08$ & $0.00 \mathrm{E}+00$ & $2.36 \mathrm{E}+09$ & $2.36 \mathrm{E}+09$ \\
\hline $2.00 \mathrm{E}-01$ & $\mathrm{E}-01$ & $3.11 \mathrm{E}+08$ & $9.84 \mathrm{E}+09$ & $1.02 \mathrm{E}+10$ & $0.00 \mathrm{E}+00$ & $5.58 \mathrm{E}+08$ & $5.58 \mathrm{E}+08$ & & $2.10 \mathrm{E}+09$ & \\
\hline $2.10 \mathrm{E}-01$ & & $1.73 \mathrm{E}$ & $8.86 \mathrm{E}+09$ & $2.61 \mathrm{E}+10$ & $0.00 \mathrm{E}+00$ & $4.74 \mathrm{E}+08$ & $4.74 \mathrm{E}+08$ & & $1.88 \mathrm{E}+09$ & \\
\hline $2.20 \mathrm{E}-01$ & & & $7.99 \mathrm{E}+09$ & & $0.00 \mathrm{E}+00$ & $4.03 \mathrm{E}$ & & & $1.68 \mathrm{E}+09$ & \\
\hline $2.30 \mathrm{E}-01$ & & 1.16 & $7.24 \mathrm{E}+09$ & $8.40 \mathrm{E}+09$ & $0.00 \mathrm{E}+00$ & $3.43 \mathrm{E}+08$ & $3.43 \mathrm{E}+08$ & $\overline{0.00}$ & $1.51 \mathrm{E}+09$ & $\mathrm{E}+09$ \\
\hline $2.40 \mathrm{E}-01$ & & 3.031 & $6.56 \mathrm{E}+09$ & $3.10 \mathrm{E}+11$ & $0.00 \mathrm{E}+00$ & $2.93 \mathrm{E}+08$ & $2.93 \mathrm{E}+08$ & 0.00 & $1.36 \mathrm{E}+09$ & $\mathrm{E}+09$ \\
\hline $2.50 \mathrm{E}$ & & -09 & $5.97 \mathrm{E}+09$ & 7.27 & $0.00 \mathrm{E}+00$ & +08 & $2.50 \mathrm{E}+08$ & $\overline{0.0}$ & $1.22 \mathrm{E}+09$ & +09 \\
\hline $2.60 \mathrm{I}$ & & & $5.44 \mathrm{E}+09$ & 2.18 & $0.00 \mathrm{E}+00$ & 2.1 & $2.13 \mathrm{E}+08$ & & $1.11 \mathrm{E}+09$ & +09 \\
\hline $2.70 \mathrm{E}-01$ & & $2.77 \mathrm{E}$ & $4.47 \mathrm{E}+09$ & $7.24 \mathrm{E}+09$ & $0.00 \mathrm{E}+00$ & $1.82 \mathrm{E}+08$ & $1.82 \mathrm{E}+08$ & $8+00$ & $1.00 \mathrm{E}+09$ & $\overline{E+09}$ \\
\hline $2.80 \mathrm{E}-01$ & $2.70 \mathrm{E}-01$ & $2.72 \mathrm{E}+11$ & $4.99 \mathrm{E}+09$ & $2.77 \mathrm{E}+11$ & $0.00 \mathrm{E}+00$ & $1.56 \mathrm{E}+08$ & $1.56 \mathrm{E}+08$ & $0.00 \mathrm{E}+00$ & $9.06 \mathrm{E}+08$ & $9.06 \mathrm{E}+08$ \\
\hline $2.90 \mathrm{E}-01$ & & $4.82 \mathrm{E}+10$ & $3.74 \mathrm{E}+09$ & $5.20 \mathrm{E}+10$ & $0.00 \mathrm{E}+00$ & $1.34 \mathrm{E}+08$ & $1.34 \mathrm{E}+08$ & $0.00 \mathrm{E}+00$ & $8.22 \mathrm{E}+08$ & $8.22 \mathrm{E}+08$ \\
\hline $3.00 \mathrm{E}-01$ & $2.90 \mathrm{E}-01$ & $6.75 \mathrm{E}+10$ & $4.19 \mathrm{E}+09$ & $7.17 \mathrm{E}+10$ & $0.00 \mathrm{E}+00$ & $1.15 \mathrm{E}+08$ & $1.15 \mathrm{E}+08$ & $0.00 \mathrm{E}+00$ & $7.47 \mathrm{E}+08$ & $7.47 \mathrm{E}+08$ \\
\hline $3.25 \mathrm{E}-01$ & & $5.82 \mathrm{E}+10$ & $8.25 \mathrm{E}+09$ & $6.64 \mathrm{E}+10$ & $0.00 \mathrm{E}+00$ & $2.21 \mathrm{E}+08$ & $2.21 \mathrm{E}+08$ & $0.00 \mathrm{E}+00$ & $1.59 \mathrm{E}+09$ & $1.59 \mathrm{E}+09$ \\
\hline $3.50 \mathrm{E}-01$ & $25 \mathrm{E}-01$ & $1.04 \mathrm{E}+11$ & $6.72 \mathrm{E}+09$ & $1.10 \mathrm{E}+11$ & $0.00 \mathrm{E}+00$ & $1.52 \mathrm{E}+08$ & $1.52 \mathrm{E}+08$ & $0.00 \mathrm{E}+00$ & $1.26 \mathrm{E}+09$ & $1.26 \mathrm{E}+09$ \\
\hline $3.75 \mathrm{E}-01$ & & $3.27 \mathrm{E}+11$ & $5.46 \mathrm{E}+09$ & $3.32 \mathrm{E}+11$ & $0.00 \mathrm{E}+00$ & $1.05 \mathrm{E}+08$ & $1.05 \mathrm{E}+08$ & $3.69 \mathrm{E}+01$ & $9.98 \mathrm{E}+08$ & $9.98 \mathrm{E}+08$ \\
\hline $4.00 \mathrm{E}-01$ & & & $4.53 \mathrm{E}+09$ & $6.71 \mathrm{E}+09$ & $0.00 \mathrm{E}+00$ & & $7.58 \mathrm{E}+07$ & $0.00 \mathrm{E}+00$ & $8.06 \mathrm{E}+08$ & $8.06 \mathrm{E}+08$ \\
\hline $4.25 \mathrm{E}-01$ & & & $3.71 \mathrm{E}+09$ & & $0.00 \mathrm{E}+00$ & & $5.51 \mathrm{E}+07$ & & $6.41 \mathrm{E}+08$ & \\
\hline $4.50 \mathrm{E}-01$ & & & $2.99 \mathrm{E}+09$ & 4.03 & $0.00 \mathrm{E}+00$ & 4.04 & $4.04 \mathrm{E}+07$ & +00 & $4.91 \mathrm{E}+08$ & $8+08$ \\
\hline $4.75 \mathrm{E}-01$ & & -06 & $2.34 \mathrm{E}+09$ & 2.34 & $0.00 \mathrm{E}+00$ & 807 & $3.32 \mathrm{E}+07$ & 0.0 & $4.18 \mathrm{E}+08$ & +08 \\
\hline $5.00 \mathrm{E}-01$ & & +07 & $1.93 \mathrm{E}+09$ & 1.961 & $0.00 \mathrm{E}+00$ & $\mathrm{E}+07$ & $2.63 \mathrm{E}+07$ & 0.0 & $3.33 \mathrm{E}+08$ & $3.33 \mathrm{E}+08$ \\
\hline $5.25 \mathrm{E}-01$ & $\overline{E-01}$ & $1.09 \mathrm{E}+09$ & $1.62 \mathrm{E}+09$ & $2.71 \mathrm{E}+09$ & $0.00 \mathrm{E}+00$ & $2.14 \mathrm{E}+07$ & $2.14 \mathrm{E}+07$ & $0.00 \mathrm{E}+00$ & $2.70 \mathrm{E}+08$ & $2.70 \mathrm{E}+08$ \\
\hline $5.50 \mathrm{E}-01$ & & & $1.34 \mathrm{E}+09$ & $1.53 \mathrm{E}+09$ & $0.00 \mathrm{E}+00$ & $1.71 \mathrm{E}+07$ & $1.71 \mathrm{E}+07$ & $\mathrm{E}+00$ & $2.15 \mathrm{E}+08$ & $2.15 \mathrm{E}+08$ \\
\hline $5.75 \mathrm{E}-01$ & & $4.09 \mathrm{E}+08$ & $1.13 \mathrm{E}+09$ & $1.54 \mathrm{E}+09$ & $0.00 \mathrm{E}+00$ & $1.39 \mathrm{E}+07$ & $1.39 \mathrm{E}+07$ & $0.00 \mathrm{E}+00$ & $1.73 \mathrm{E}+08$ & $1.73 \mathrm{E}+08$ \\
\hline $6.00 \mathrm{E}-01$ & & $8.17 \mathrm{E}+06$ & $9.44 \mathrm{E}+08$ & $9.52 \mathrm{E}+08$ & $0.00 \mathrm{E}+00$ & $1.12 \mathrm{E}+07$ & $1.12 \mathrm{E}+07$ & $0.00 \mathrm{E}+00$ & $1.38 \mathrm{E}+08$ & $1.38 \mathrm{E}+08$ \\
\hline $6.25 \mathrm{E}-01$ & $0 \mathrm{E}-01$ & $7.91 \mathrm{E}+08$ & $7.82 \mathrm{E}+08$ & $1.57 \mathrm{E}+09$ & $0.00 \mathrm{E}+00$ & $8.84 \mathrm{E}+06$ & $8.84 \mathrm{E}+06$ & $0.00 \mathrm{E}+00$ & $1.09 \mathrm{E}+08$ & $1.09 \mathrm{E}+08$ \\
\hline $6.50 \mathrm{E}-01$ & $5 \mathrm{E}-01$ & $5.80 \mathrm{E}+06$ & $6.56 \mathrm{E}+08$ & $6.62 \mathrm{E}+08$ & $0.00 \mathrm{E}+00$ & $7.10 \mathrm{E}+06$ & $7.10 \mathrm{E}+06$ & $0.00 \mathrm{E}+00$ & $8.69 \mathrm{E}+07$ & $8.69 \mathrm{E}+07$ \\
\hline $6.75 \mathrm{E}-01$ & $0 \mathrm{E}-01$ & $2.26 \mathrm{E}+08$ & $5.42 \mathrm{E}+08$ & $7.68 \mathrm{E}+08$ & $2.73 \mathrm{E}+12$ & $5.57 \mathrm{E}+06$ & $2.73 \mathrm{E}+12$ & $0.00 \mathrm{E}+00$ & $6.77 \mathrm{E}+07$ & $6.77 \mathrm{E}+07$ \\
\hline $7.00 \mathrm{E}-01$ & & $4.23 \mathrm{E}+08$ & $4.50 \mathrm{E}+08$ & $8.73 \mathrm{E}+08$ & $0.00 \mathrm{E}+00$ & $4.38 \mathrm{E}+06$ & $4.38 \mathrm{E}+06$ & $0.00 \mathrm{E}+00$ & $5.28 \mathrm{E}+07$ & $5.28 \mathrm{E}+07$ \\
\hline $7.25 \mathrm{E}-01$ & & & $3.76 \mathrm{E}+08$ & $9.02 \mathrm{E}+09$ & $0.00 \mathrm{E}+00$ & $3.45 \mathrm{E}+06$ & $3.45 \mathrm{E}+06$ & $0.00 \mathrm{E}+00$ & $4.13 \mathrm{E}+07$ & $4.13 \mathrm{E}+07$ \\
\hline $7.50 \mathrm{E}-01$ & & $2.73 \mathrm{E}+07$ & $3.08 \mathrm{E}+08$ & $3.35 \mathrm{E}+08$ & $0.00 \mathrm{E}+00$ & $2.64 \mathrm{E}+06$ & $2.64 \mathrm{E}+06$ & $0.00 \mathrm{E}+00$ & $3.13 \mathrm{E}+07$ & $3.13 \mathrm{E}+07$ \\
\hline $7.75 \mathrm{E}-01$ & & $1.29 \mathrm{E}+10$ & $2.56 \mathrm{E}+08$ & $1.32 \mathrm{E}+10$ & $0.00 \mathrm{E}+00$ & $2.04 \mathrm{E}+06$ & $2.04 \mathrm{E}+06$ & $0.00 \mathrm{E}+00$ & $2.40 \mathrm{E}+07$ & $2.40 \mathrm{E}+07$ \\
\hline $8.00 \mathrm{E}-01$ & $\mathrm{E}-01$ & $2.41 \mathrm{E}+08$ & $2.08 \mathrm{E}+08$ & $4.49 \mathrm{E}+08$ & $0.00 \mathrm{E}+00$ & $1.53 \mathrm{E}+06$ & $1.53 \mathrm{E}+06$ & $0.00 \mathrm{E}+00$ & $1.78 \mathrm{E}+07$ & $1.78 \mathrm{E}+07$ \\
\hline $8.25 \mathrm{E}-01$ & $8.00 \mathrm{E}-01$ & $1.94 \mathrm{E}+08$ & $1.71 \mathrm{E}+08$ & $3.65 \mathrm{E}+08$ & $0.00 \mathrm{E}+00$ & $1.16 \mathrm{E}+06$ & $1.16 \mathrm{E}+06$ & $1.08 \mathrm{E}+07$ & $1.33 \mathrm{E}+07$ & $2.41 \mathrm{E}+07$ \\
\hline $8.50 \mathrm{E}-01$ & $8.25 \mathrm{E}-01$ & $7.34 \mathrm{E}+10$ & $1.39 \mathrm{E}+08$ & $7.35 \mathrm{E}+10$ & $0.00 \mathrm{E}+00$ & $8.49 \mathrm{E}+05$ & $8.49 \mathrm{E}+05$ & $0.00 \mathrm{E}+00$ & $9.65 \mathrm{E}+06$ & $9.65 \mathrm{E}+06$ \\
\hline $8.75 \mathrm{E}-01$ & $8.50 \mathrm{E}-01$ & $1.23 \mathrm{E}+08$ & $1.11 \mathrm{E}+08$ & $2.35 \mathrm{E}+08$ & $0.00 \mathrm{E}+00$ & $6.04 \mathrm{E}+05$ & $6.04 \mathrm{E}+05$ & $0.00 \mathrm{E}+00$ & $6.76 \mathrm{E}+06$ & $6.76 \mathrm{E}+06$ \\
\hline $9.00 \mathrm{E}-01$ & $8.75 \mathrm{E}-01$ & $6.45 \mathrm{E}+09$ & $9.02 \mathrm{E}+07$ & $6.54 \mathrm{E}+09$ & $0.00 \mathrm{E}+00$ & $4.32 \mathrm{E}+05$ & $4.32 \mathrm{E}+05$ & $0.00 \mathrm{E}+00$ & $4.75 \mathrm{E}+06$ & $4.75 \mathrm{E}+06$ \\
\hline $9.25 \mathrm{E}-01$ & $9.00 \mathrm{E}-01$ & $5.89 \mathrm{E}+07$ & $7.11 \mathrm{E}+07$ & $1.30 \mathrm{E}+08$ & $0.00 \mathrm{E}+00$ & $2.92 \mathrm{E}+05$ & $2.92 \mathrm{E}+05$ & $0.00 \mathrm{E}+00$ & $3.15 \mathrm{E}+06$ & $3.15 \mathrm{E}+06$ \\
\hline $9.50 \mathrm{E}-01$ & $9.25 \mathrm{E}-01$ & $2.28 \mathrm{E}+06$ & $5.62 \mathrm{E}+07$ & $5.85 \mathrm{E}+07$ & $0.00 \mathrm{E}+00$ & $1.94 \mathrm{E}+05$ & $1.94 \mathrm{E}+05$ & $0.00 \mathrm{E}+00$ & $2.04 \mathrm{E}+06$ & $2.04 \mathrm{E}+06$ \\
\hline $9.75 \mathrm{E}-01$ & & $4.60 \mathrm{E}+08$ & $4.44 \mathrm{E}+07$ & $5.04 \mathrm{E}+08$ & $0.00 \mathrm{E}+00$ & $1.26 \mathrm{E}+05$ & $1.26 \mathrm{E}+05$ & $0.00 \mathrm{E}+00$ & $1.29 \mathrm{E}+06$ & $1.29 \mathrm{E}+06$ \\
\hline $1.00 \mathrm{E}+00$ & $9.75 \mathrm{E}-01$ & $1.85 \mathrm{E}+06$ & $3.40 \mathrm{E}+07$ & $3.58 \mathrm{E}+07$ & $0.00 \mathrm{E}+00$ & $7.50 \mathrm{E}+04$ & $7.50 \mathrm{E}+04$ & $0.00 \mathrm{E}+00$ & $7.40 \mathrm{E}+05$ & $7.40 \mathrm{E}+05$ \\
\hline
\end{tabular}


PCP-2011-0001

Revision 0

\begin{tabular}{|c|c|c|c|c|c|c|c|c|c|c|}
\hline \multicolumn{11}{|c|}{ Table 3-27 Photons from Nuclides with Bremsstrahlung } \\
\hline \multirow{2}{*}{\multicolumn{2}{|c|}{ Energy $(\mathrm{MeV})$}} & \multicolumn{9}{|c|}{ Photon Source (photons/sec/g) } \\
\hline & & \multicolumn{3}{|c|}{ Ac-227 } & \multicolumn{3}{|c|}{ Cs-137 } & \multicolumn{3}{|c|}{$\mathrm{Pb}-210$} \\
\hline Upper & Lower & (Decay) & (Brem.) & (Total) & (Decay) & (Brem.) & (Total) & (Decay) & (Brem.) & (Total) \\
\hline $1.25 \mathrm{E}+00$ & $1.00 \mathrm{E}+00$ & $3.14 \mathrm{E}+09$ & $6.33 \mathrm{E}+07$ & $3.21 \mathrm{E}+09$ & $0.00 \mathrm{E}+00$ & $2.77 \mathrm{E}+04$ & $2.77 \mathrm{E}+04$ & $0.00 \mathrm{E}+00$ & $2.48 \mathrm{E}+05$ & $2.48 \mathrm{E}+05$ \\
\hline $1.50 \mathrm{E}+00$ & $1.25 \mathrm{E}+00$ & $1.26 \mathrm{E}+08$ & $2.79 \mathrm{E}+05$ & $1.26 \mathrm{E}+08$ & $0.00 \mathrm{E}+00$ & $0.00 \mathrm{E}+00$ & $0.00 \mathrm{E}+00$ & $0.00 \mathrm{E}+00$ & $2.39 \mathrm{E}+00$ & $2.39 \mathrm{E}+00$ \\
\hline $1.75 \mathrm{E}+00$ & $1.50 \mathrm{E}+00$ & $0.00 \mathrm{E}+00$ & $0.00 \mathrm{E}+00$ & $0.00 \mathrm{E}+00$ & $0.00 \mathrm{E}+00$ & $0.00 \mathrm{E}+00$ & $0.00 \mathrm{E}+00$ & $0.00 \mathrm{E}+00$ & $5.85 \mathrm{E}-05$ & $5.85 \mathrm{E}-05$ \\
\hline $2.00 \mathrm{E}+00$ & $1.75 \mathrm{E}+00$ & $0.00 \mathrm{E}+00$ & $0.00 \mathrm{E}+00$ & $0.00 \mathrm{E}+00$ & $0.00 \mathrm{E}+00$ & $0.00 \mathrm{E}+00$ & $0.00 \mathrm{E}+00$ & $0.00 \mathrm{E}+00$ & $0.00 \mathrm{E}+00$ & $0.00 \mathrm{E}+00$ \\
\hline $2.25 \mathrm{E}+00$ & $2.00 \mathrm{E}+00$ & $0.00 \mathrm{E}+00$ & $0.00 \mathrm{E}+00$ & $0.00 \mathrm{E}+00$ & $0.00 \mathrm{E}+00$ & $0.00 \mathrm{E}+00$ & $0.00 \mathrm{E}+00$ & $0.00 \mathrm{E}+00$ & $0.00 \mathrm{E}+00$ & $0.00 \mathrm{E}+00$ \\
\hline $2.50 \mathrm{E}+00$ & $2.25 \mathrm{E}+00$ & $0.00 \mathrm{E}+00$ & $0.00 \mathrm{E}+00$ & $0.00 \mathrm{E}+00$ & $0.00 \mathrm{E}+00$ & $0.00 \mathrm{E}+00$ & $0.00 \mathrm{E}+00$ & $0.00 \mathrm{E}+00$ & $0.00 \mathrm{E}+00$ & $0.00 \mathrm{E}+00$ \\
\hline $2.75 \mathrm{E}+00$ & $2.50 \mathrm{E}+00$ & $0.00 \mathrm{E}+00$ & $0.00 \mathrm{E}+00$ & $0.00 \mathrm{E}+00$ & $0.00 \mathrm{E}+00$ & $0.00 \mathrm{E}+00$ & $0.00 \mathrm{E}+00$ & $0.00 \mathrm{E}+00$ & $0.00 \mathrm{E}+00$ & $0.00 \mathrm{E}+00$ \\
\hline $3.00 \mathrm{E}+00$ & $2.75 \mathrm{E}+00$ & $0.00 \mathrm{E}+00$ & $0.00 \mathrm{E}+00$ & $0.00 \mathrm{E}+00$ & $0.00 \mathrm{E}+00$ & $0.00 \mathrm{E}+00$ & $0.00 \mathrm{E}+00$ & $0.00 \mathrm{E}+00$ & $0.00 \mathrm{E}+00$ & $0.00 \mathrm{E}+00$ \\
\hline $3.50 \mathrm{E}+00$ & $3.00 \mathrm{E}+00$ & $0.00 \mathrm{E}+00$ & $0.00 \mathrm{E}+00$ & $0.00 \mathrm{E}+00$ & $0.00 \mathrm{E}+00$ & $0.00 \mathrm{E}+00$ & $0.00 \mathrm{E}+00$ & $0.00 \mathrm{E}+00$ & $0.00 \mathrm{E}+00$ & $0.00 \mathrm{E}+00$ \\
\hline $4.00 \mathrm{E}+00$ & $3.50 \mathrm{E}+00$ & $0.00 \mathrm{E}+00$ & $0.00 \mathrm{E}+00$ & $0.00 \mathrm{E}+00$ & $0.00 \mathrm{E}+00$ & $0.00 \mathrm{E}+00$ & $0.00 \mathrm{E}+00$ & $0.00 \mathrm{E}+00$ & $0.00 \mathrm{E}+00$ & $0.00 \mathrm{E}+00$ \\
\hline $4.50 \mathrm{E}+00$ & $4.00 \mathrm{E}+00$ & $0.00 \mathrm{E}+00$ & $0.00 \mathrm{E}+00$ & $0.00 \mathrm{E}+00$ & $0.00 \mathrm{E}+00$ & $0.00 \mathrm{E}+00$ & $0.00 \mathrm{E}+00$ & $0.00 \mathrm{E}+00$ & $0.00 \mathrm{E}+00$ & $0.00 \mathrm{E}+00$ \\
\hline $5.00 \mathrm{E}+00$ & $4.50 \mathrm{E}+00$ & $0.00 \mathrm{E}+00$ & $0.00 \mathrm{E}+00$ & $0.00 \mathrm{E}+00$ & $0.00 \mathrm{E}+00$ & $0.00 \mathrm{E}+00$ & $0.00 \mathrm{E}+00$ & $0.00 \mathrm{E}+00$ & $0.00 \mathrm{E}+00$ & $0.00 \mathrm{E}+00$ \\
\hline $5.50 \mathrm{E}+00$ & $5.00 \mathrm{E}+00$ & $0.00 \mathrm{E}+00$ & $0.00 \mathrm{E}+00$ & $0.00 \mathrm{E}+00$ & $0.00 \mathrm{E}+00$ & $0.00 \mathrm{E}+00$ & $0.00 \mathrm{E}+00$ & $0.00 \mathrm{E}+00$ & $0.00 \mathrm{E}+00$ & $0.00 \mathrm{E}+00$ \\
\hline $6.00 \mathrm{E}+00$ & $5.50 \mathrm{E}+00$ & $0.00 \mathrm{E}+00$ & $0.00 \mathrm{E}+00$ & $0.00 \mathrm{E}+00$ & $0.00 \mathrm{E}+00$ & $0.00 \mathrm{E}+00$ & $0.00 \mathrm{E}+00$ & $0.00 \mathrm{E}+00$ & $0.00 \mathrm{E}+00$ & $0.00 \mathrm{E}+00$ \\
\hline $6.50 \mathrm{E}+00$ & $6.00 \mathrm{E}+00$ & $0.00 \mathrm{E}+00$ & $0.00 \mathrm{E}+00$ & $0.00 \mathrm{E}+00$ & $0.00 \mathrm{E}+00$ & $0.00 \mathrm{E}+00$ & $0.00 \mathrm{E}+00$ & $0.00 \mathrm{E}+00$ & $0.00 \mathrm{E}+00$ & $0.00 \mathrm{E}+00$ \\
\hline $7.00 \mathrm{E}+00$ & $6.50 \mathrm{E}+00$ & $0.00 \mathrm{E}+00$ & $0.00 \mathrm{E}+00$ & $0.00 \mathrm{E}+00$ & $0.00 \mathrm{E}+00$ & $0.00 \mathrm{E}+00$ & $0.00 \mathrm{E}+00$ & $0.00 \mathrm{E}+00$ & $0.00 \mathrm{E}+00$ & $0.00 \mathrm{E}+00$ \\
\hline $7.50 \mathrm{E}+00$ & $7.00 \mathrm{E}+00$ & $0.00 \mathrm{E}+00$ & $0.00 \mathrm{E}+00$ & $0.00 \mathrm{E}+00$ & $0.00 \mathrm{E}+00$ & $0.00 \mathrm{E}+00$ & $0.00 \mathrm{E}+00$ & $0.00 \mathrm{E}+00$ & $0.00 \mathrm{E}+00$ & $0.00 \mathrm{E}+00$ \\
\hline $8.00 \mathrm{E}+00$ & $7.50 \mathrm{E}+00$ & $0.00 \mathrm{E}+00$ & $0.00 \mathrm{E}+00$ & $0.00 \mathrm{E}+00$ & $0.00 \mathrm{E}+00$ & $0.00 \mathrm{E}+00$ & $0.00 \mathrm{E}+00$ & $0.00 \mathrm{E}+00$ & $0.00 \mathrm{E}+00$ & $0.00 \mathrm{E}+00$ \\
\hline $9.00 \mathrm{E}+00$ & $8.00 \mathrm{E}+00$ & $0.00 \mathrm{E}+00$ & $0.00 \mathrm{E}+00$ & $0.00 \mathrm{E}+00$ & $0.00 \mathrm{E}+00$ & $0.00 \mathrm{E}+00$ & $0.00 \mathrm{E}+00$ & $0.00 \mathrm{E}+00$ & $0.00 \mathrm{E}+00$ & $0.00 \mathrm{E}+00$ \\
\hline $1.00 \mathrm{E}+01$ & $9.00 \mathrm{E}+00$ & $0.00 \mathrm{E}+00$ & $0.00 \mathrm{E}+00$ & $0.00 \mathrm{E}+00$ & $0.00 \mathrm{E}+00$ & $0.00 \mathrm{E}+00$ & $0.00 \mathrm{E}+00$ & $0.00 \mathrm{E}+00$ & $0.00 \mathrm{E}+00$ & $0.00 \mathrm{E}+00$ \\
\hline \multicolumn{2}{|c|}{ Total } & $3.64 \mathrm{E}+12$ & $1.63 \mathrm{E}+12$ & $5.27 \mathrm{E}+12$ & $2.94 \mathrm{E}+12$ & $1.82 \mathrm{E}+11$ & $3.12 \mathrm{E}+12$ & $1.09 \mathrm{E}+07$ & $3.83 \mathrm{E}+11$ & $3.83 \mathrm{E}+11$ \\
\hline \multicolumn{2}{|c|}{ Bremsstrahlung Fraction } & & $3.10 \mathrm{E}-01$ & & & $5.83 \mathrm{E}-02$ & & & $1.00 \mathrm{E}+00$ & \\
\hline
\end{tabular}


PCP-2011-0001

Revision 0

\begin{tabular}{|c|c|c|c|c|c|c|c|}
\hline \multicolumn{8}{|c|}{ Table 3-27 Photons from Nuclides with Bremsstrahlung (Continued) } \\
\hline \multirow{2}{*}{\multicolumn{2}{|c|}{ Energy $(\mathrm{MeV})$}} & \multicolumn{6}{|c|}{ Photon Source (photons/sec/g) } \\
\hline & & \multicolumn{3}{|c|}{ Ru-106 } & \multicolumn{3}{|c|}{ Sr-90 } \\
\hline Upper & Lower & (Decay) & (Brem.) & (Total) & (Decay) & (Brem.) & (Total) \\
\hline $2.00 \mathrm{E}-02$ & $1.00 \mathrm{E}-02$ & $0.00 \mathrm{E}+00$ & $1.91 \mathrm{E}+13$ & $1.91 \mathrm{E}+13$ & $5.85 \mathrm{E}+08$ & $5.25 \mathrm{E}+11$ & $5.26 \mathrm{E}+11$ \\
\hline $3.00 \mathrm{E}-02$ & $2.00 \mathrm{E}-02$ & $1.16 \mathrm{E}+11$ & $1.02 \mathrm{E}+13$ & $1.03 \mathrm{E}+13$ & $0.00 \mathrm{E}+00$ & $2.74 \mathrm{E}+11$ & $2.74 \mathrm{E}+11$ \\
\hline $4.00 \mathrm{E}-02$ & $3.00 \mathrm{E}-02$ & $0.00 \mathrm{E}+00$ & $6.67 \mathrm{E}+12$ & $6.67 \mathrm{E}+12$ & $0.00 \mathrm{E}+00$ & $1.76 \mathrm{E}+11$ & $1.76 \mathrm{E}+11$ \\
\hline $5.00 \mathrm{E}-02$ & $4.00 \mathrm{E}-02$ & $0.00 \mathrm{E}+00$ & $4.83 \mathrm{E}+12$ & $4.83 \mathrm{E}+12$ & $0.00 \mathrm{E}+00$ & $1.26 \mathrm{E}+11$ & $1.26 \mathrm{E}+11$ \\
\hline $6.00 \mathrm{E}-02$ & $5.00 \mathrm{E}-02$ & $0.00 \mathrm{E}+00$ & $3.71 \mathrm{E}+12$ & $3.71 \mathrm{E}+12$ & $0.00 \mathrm{E}+00$ & $9.49 \mathrm{E}+10$ & $9.49 \mathrm{E}+10$ \\
\hline $7.00 \mathrm{E}-02$ & $6.00 \mathrm{E}-02$ & $0.00 \mathrm{E}+00$ & $2.97 \mathrm{E}+12$ & $2.97 \mathrm{E}+12$ & $0.00 \mathrm{E}+00$ & $7.48 \mathrm{E}+10$ & $7.48 \mathrm{E}+10$ \\
\hline $8.00 \mathrm{E}-02$ & $7.00 \mathrm{E}-02$ & $0.00 \mathrm{E}+00$ & $2.45 \mathrm{E}+12$ & $2.45 \mathrm{E}+12$ & $0.00 \mathrm{E}+00$ & $6.07 \mathrm{E}+10$ & $6.07 \mathrm{E}+10$ \\
\hline $9.00 \mathrm{E}-02$ & $8.00 \mathrm{E}-02$ & $0.00 \mathrm{E}+00$ & $2.06 \mathrm{E}+12$ & $2.06 \mathrm{E}+12$ & $0.00 \mathrm{E}+00$ & $5.04 \mathrm{E}+10$ & $5.04 \mathrm{E}+10$ \\
\hline $1.00 \mathrm{E}-01$ & $9.00 \mathrm{E}-02$ & $0.00 \mathrm{E}+00$ & $1.76 \mathrm{E}+12$ & $1.76 \mathrm{E}+12$ & $0.00 \mathrm{E}+00$ & $4.25 \mathrm{E}+10$ & $4.25 \mathrm{E}+10$ \\
\hline $1.10 \mathrm{E}-01$ & $1.00 \mathrm{E}-01$ & $0.00 \mathrm{E}+00$ & $1.53 \mathrm{E}+12$ & $1.53 \mathrm{E}+12$ & $0.00 \mathrm{E}+00$ & $3.64 \mathrm{E}+10$ & $3.64 \mathrm{E}+10$ \\
\hline $1.20 \mathrm{E}-01$ & $1.10 \mathrm{E}-01$ & $0.00 \mathrm{E}+00$ & $1.34 \mathrm{E}+12$ & $1.34 \mathrm{E}+12$ & $0.00 \mathrm{E}+00$ & $3.15 \mathrm{E}+10$ & $3.15 \mathrm{E}+10$ \\
\hline $1.30 \mathrm{E}-01$ & $1.20 \mathrm{E}-01$ & $0.00 \mathrm{E}+00$ & $1.19 \mathrm{E}+12$ & $1.19 \mathrm{E}+12$ & $0.00 \mathrm{E}+00$ & $2.76 \mathrm{E}+10$ & \\
\hline $1.40 \mathrm{E}-01$ & $1.30 \mathrm{E}-01$ & $0.00 \mathrm{E}+00$ & $1.06 \mathrm{E}+12$ & & & $2.43 \mathrm{E}+10$ & \\
\hline $1.50 \mathrm{E}-01$ & $1.40 \mathrm{E}-01$ & $0.00 \mathrm{E}+00$ & $9.54 \mathrm{E}+11$ & $9.54 \mathrm{E}+11$ & $0.00 \mathrm{E}+00$ & $2.16 \mathrm{E}+10$ & $2.16 \mathrm{E}+10$ \\
\hline $1.60 \mathrm{E}-01$ & $1.50 \mathrm{E}-01$ & $0.00 \mathrm{E}+00$ & $8.63 \mathrm{E}+11$ & $8.63 \mathrm{E}+11$ & $0.00 \mathrm{E}+00$ & $1.93 \mathrm{E}+10$ & $1.93 \mathrm{E}+10$ \\
\hline $1.70 \mathrm{E}-01$ & $1.60 \mathrm{E}-01$ & $0.00 \mathrm{E}+00$ & $7.84 \mathrm{E}+11$ & $7.84 \mathrm{E}+11$ & $0.00 \mathrm{E}+00$ & $1.73 \mathrm{E}+10$ & $1.73 \mathrm{E}+10$ \\
\hline $1.80 \mathrm{E}-01$ & $1.70 \mathrm{E}-01$ & $0.00 \mathrm{E}+00$ & $7.16 \mathrm{E}+11$ & $7.16 \mathrm{E}+11$ & $0.00 \mathrm{E}+00$ & $1.56 \mathrm{E}+10$ & $1.56 \mathrm{E}+10$ \\
\hline $1.90 \mathrm{E}-01$ & $1.80 \mathrm{E}-01$ & $0.00 \mathrm{E}+00$ & $6.57 \mathrm{E}+11$ & $6.57 \mathrm{E}+11$ & $0.00 \mathrm{E}+00$ & $1.42 \mathrm{E}+10$ & $1.42 \mathrm{E}+10$ \\
\hline $2.00 \mathrm{E}-01$ & $1.90 \mathrm{E}-01$ & $0.00 \mathrm{E}+00$ & $6.04 \mathrm{E}+11$ & $6.04 \mathrm{E}+11$ & $0.00 \mathrm{E}+00$ & $1.29 \mathrm{E}+10$ & $1.29 \mathrm{E}+10$ \\
\hline $2.10 \mathrm{E}-01$ & $2.00 \mathrm{E}-01$ & $0.00 \mathrm{E}+00$ & $5.58 \mathrm{E}+11$ & $5.58 \mathrm{E}+11$ & $0.00 \mathrm{E}+00$ & $1.18 \mathrm{E}+10$ & $1.18 \mathrm{E}+10$ \\
\hline $2.20 \mathrm{E}-01$ & $2.10 \mathrm{E}-01$ & $0.00 \mathrm{E}+00$ & $5.16 \mathrm{E}+11$ & $5.16 \mathrm{E}+11$ & $0.00 \mathrm{E}+00$ & $1.08 \mathrm{E}+10$ & $1.08 \mathrm{E}+10$ \\
\hline $2.30 \mathrm{E}-01$ & $2.20 \mathrm{E}-01$ & $0.00 \mathrm{E}+00$ & $4.80 \mathrm{E}+11$ & $4.80 \mathrm{E}+11$ & $0.00 \mathrm{E}+00$ & $9.92 \mathrm{E}+09$ & $9.92 \mathrm{E}+09$ \\
\hline $2.40 \mathrm{E}-01$ & $2.30 \mathrm{E}-01$ & $0.00 \mathrm{E}+00$ & $4.46 \mathrm{E}+11$ & $4.46 \mathrm{E}+11$ & $0.00 \mathrm{E}+00$ & $9.14 \mathrm{E}+09$ & $9.14 \mathrm{E}+09$ \\
\hline $2.50 \mathrm{E}-01$ & $2.40 \mathrm{E}-01$ & $0.00 \mathrm{E}+00$ & $4.17 \mathrm{E}+11$ & $4.17 \mathrm{E}+11$ & $0.00 \mathrm{E}+00$ & $8.45 \mathrm{E}+09$ & \\
\hline $2.60 \mathrm{E}-01$ & $2.50 \mathrm{E}-01$ & $0.00 \mathrm{E}+00$ & $3.90 \mathrm{E}+11$ & $3.90 \mathrm{E}+11$ & $0.00 \mathrm{E}+00$ & $7.82 \mathrm{E}+09$ & $7.82 \mathrm{E}+09$ \\
\hline $2.70 \mathrm{E}-01$ & $2.60 \mathrm{E}-01$ & $0.00 \mathrm{E}+00$ & $3.65 \mathrm{E}+11$ & & $\mathrm{E}+00$ & $7.26 \mathrm{E}+09$ & $7.26 \mathrm{E}+09$ \\
\hline $2.80 \mathrm{E}-01$ & $2.70 \mathrm{E}-01$ & $1.80 \mathrm{E}+09$ & $3.43 \mathrm{E}+11$ & $3.44 \mathrm{E}+11$ & $\mathrm{E}+00$ & $6.75 \mathrm{E}+09$ & $\overline{\mathrm{E}+09}$ \\
\hline $2.90 \mathrm{E}-01$ & $2.80 \mathrm{E}-01$ & $0.00 \mathrm{E}+00$ & $3.22 \mathrm{E}+11$ & $3.22 \mathrm{E}+11$ & $0.00 \mathrm{E}+00$ & $6.29 \mathrm{E}+09$ & $6.29 \mathrm{E}+09$ \\
\hline $3.00 \mathrm{E}-01$ & $2.90 \mathrm{E}-01$ & $0.00 \mathrm{E}+00$ & $3.03 \mathrm{E}+11$ & $3.03 \mathrm{E}+11$ & $0.00 \mathrm{E}+00$ & $5.87 \mathrm{E}+09$ & $5.87 \mathrm{E}+09$ \\
\hline $3.25 \mathrm{E}-01$ & $3.00 \mathrm{E}-01$ & $5.30 \mathrm{E}+09$ & $6.87 \mathrm{E}+11$ & $6.92 \mathrm{E}+11$ & $\mathrm{E}+00$ & $1.31 \mathrm{E}+10$ & $1.31 \mathrm{E}+10$ \\
\hline $3.50 \mathrm{E}-01$ & $3.25 \mathrm{E}-01$ & $6.20 \mathrm{E}+09$ & $5.98 \mathrm{E}+11$ & $6.05 \mathrm{E}+11$ & $0.00 \mathrm{E}+00$ & $1.12 \mathrm{E}+10$ & $1.12 \mathrm{E}+10$ \\
\hline $3.75 \mathrm{E}-01$ & $3.50 \mathrm{E}-01$ & $0.00 \mathrm{E}+00$ & $5.22 \mathrm{E}+11$ & $5.22 \mathrm{E}+11$ & $0.00 \mathrm{E}+00$ & $9.54 \mathrm{E}+09$ & $9.54 \mathrm{E}+09$ \\
\hline $4.00 \mathrm{E}-01$ & $3.75 \mathrm{E}-01$ & $9.39 \mathrm{E}+09$ & $4.63 \mathrm{E}+11$ & $4.73 \mathrm{E}+11$ & $0.00 \mathrm{E}+00$ & $8.30 \mathrm{E}+09$ & $8.30 \mathrm{E}+09$ \\
\hline $4.25 \mathrm{E}-01$ & $4.00 \mathrm{E}-01$ & $1.07 \mathrm{E}+10$ & $4.09 \mathrm{E}+11$ & $4.20 \mathrm{E}+11$ & $0.00 \mathrm{E}+00$ & $7.19 \mathrm{E}+09$ & $7.19 \mathrm{E}+09$ \\
\hline $4.50 \mathrm{E}-01$ & $4.25 \mathrm{E}-01$ & $1.94 \mathrm{E}+11$ & $4.56 \mathrm{E}+11$ & $6.50 \mathrm{E}+11$ & $0.00 \mathrm{E}+00$ & $9.41 \mathrm{E}+09$ & $9.41 \mathrm{E}+09$ \\
\hline $4.75 \mathrm{E}-01$ & $4.50 \mathrm{E}-01$ & $3.80 \mathrm{E}+09$ & $3.28 \mathrm{E}+11$ & $3.32 \mathrm{E}+11$ & $0.00 \mathrm{E}+00$ & $5.57 \mathrm{E}+09$ & $5.57 \mathrm{E}+09$ \\
\hline $5.00 \mathrm{E}-01$ & $4.75 \mathrm{E}-01$ & $5.17 \mathrm{E}+09$ & $2.94 \mathrm{E}+11$ & $2.99 \mathrm{E}+11$ & $0.00 \mathrm{E}+00$ & $4.89 \mathrm{E}+09$ & $4.89 \mathrm{E}+09$ \\
\hline $5.25 \mathrm{E}-01$ & $5.00 \mathrm{E}-01$ & $2.52 \mathrm{E}+13$ & $2.66 \mathrm{E}+11$ & $2.55 \mathrm{E}+13$ & $\mathrm{E}+00$ & $4.36 \mathrm{E}+09$ & $4.36 \mathrm{E}+09$ \\
\hline $5.50 \mathrm{E}-01$ & $5.25 \mathrm{E}-01$ & $7.61 \mathrm{E}+09$ & $2.40 \mathrm{E}+11$ & $2.48 \mathrm{E}+11$ & $0.00 \mathrm{E}+00$ & $3.85 \mathrm{E}+09$ & $3.85 \mathrm{E}+09$ \\
\hline $5.75 \mathrm{E}-01$ & $5.50 \mathrm{E}-01$ & $9.08 \mathrm{E}+09$ & $2.19 \mathrm{E}+11$ & $2.28 \mathrm{E}+11$ & $0.00 \mathrm{E}+00$ & $3.45 \mathrm{E}+09$ & $3.45 \mathrm{E}+09$ \\
\hline $6.00 \mathrm{E}-01$ & $5.75 \mathrm{E}-01$ & $1.48 \mathrm{E}+10$ & $1.99 \mathrm{E}+11$ & $2.14 \mathrm{E}+11$ & $0.00 \mathrm{E}+00$ & $3.08 \mathrm{E}+09$ & $3.08 \mathrm{E}+09$ \\
\hline $6.25 \mathrm{E}-01$ & $6.00 \mathrm{E}-01$ & $1.31 \mathrm{E}+13$ & $1.81 \mathrm{E}+11$ & $1.33 \mathrm{E}+13$ & $0.00 \mathrm{E}+00$ & $2.74 \mathrm{E}+09$ & $2.74 \mathrm{E}+09$ \\
\hline $6.50 \mathrm{E}-01$ & $6.25 \mathrm{E}-01$ & $2.77 \mathrm{E}+09$ & $1.66 \mathrm{E}+11$ & $1.69 \mathrm{E}+11$ & $0.00 \mathrm{E}+00$ & $2.47 \mathrm{E}+09$ & $2.47 \mathrm{E}+09$ \\
\hline $6.75 \mathrm{E}-01$ & $6.50 \mathrm{E}-01$ & $1.89 \mathrm{E}+10$ & $1.52 \mathrm{E}+11$ & $1.71 \mathrm{E}+11$ & $0.00 \mathrm{E}+00$ & $2.21 \mathrm{E}+09$ & $2.21 \mathrm{E}+09$ \\
\hline $7.00 \mathrm{E}-01$ & $6.75 \mathrm{E}-01$ & $1.20 \mathrm{E}+10$ & $1.39 \mathrm{E}+11$ & $1.51 \mathrm{E}+11$ & $0.00 \mathrm{E}+00$ & $1.99 \mathrm{E}+09$ & $1.99 \mathrm{E}+09$ \\
\hline $7.25 \mathrm{E}-01$ & $7.00 \mathrm{E}-01$ & $2.08 \mathrm{E}+10$ & $1.28 \mathrm{E}+11$ & $1.49 \mathrm{E}+11$ & $0.00 \mathrm{E}+00$ & $1.80 \mathrm{E}+09$ & $1.80 \mathrm{E}+09$ \\
\hline $7.50 \mathrm{E}-01$ & $7.25 \mathrm{E}-01$ & $5.10 \mathrm{E}+09$ & $1.18 \mathrm{E}+11$ & $1.23 \mathrm{E}+11$ & $0.00 \mathrm{E}+00$ & $1.61 \mathrm{E}+09$ & $1.61 \mathrm{E}+09$ \\
\hline $7.75 \mathrm{E}-01$ & $7.50 \mathrm{E}-01$ & $7.96 \mathrm{E}+09$ & $1.09 \mathrm{E}+11$ & $1.17 \mathrm{E}+11$ & $0.00 \mathrm{E}+00$ & $1.46 \mathrm{E}+09$ & $1.46 \mathrm{E}+09$ \\
\hline $8.00 \mathrm{E}-01$ & & $1.79 \mathrm{E}+09$ & $1.00 \mathrm{E}+11$ & & $0.00 \mathrm{E}+00$ & $1.32 \mathrm{E}+09$ & $1.32 \mathrm{E}+09$ \\
\hline $8.25 \mathrm{E}-01$ & $8.00 \mathrm{E}-01$ & $0.00 \mathrm{E}+00$ & $9.28 \mathrm{E}+10$ & $9.28 \mathrm{E}+10$ & $0.00 \mathrm{E}+00$ & $1.19 \mathrm{E}+09$ & $1.19 \mathrm{E}+09$ \\
\hline $8.50 \mathrm{E}-01$ & $8.25 \mathrm{E}-01$ & $0.00 \mathrm{E}+00$ & $8.59 \mathrm{E}+10$ & $8.59 \mathrm{E}+10$ & $0.00 \mathrm{E}+00$ & $1.08 \mathrm{E}+09$ & $1.08 \mathrm{E}+09$ \\
\hline $8.75 \mathrm{E}-01$ & $8.50 \mathrm{E}-01$ & $5.17 \mathrm{E}+11$ & $7.93 \mathrm{E}+10$ & $5.96 \mathrm{E}+11$ & $0.00 \mathrm{E}+00$ & $9.72 \mathrm{E}+08$ & $9.72 \mathrm{E}+08$ \\
\hline $9.00 \mathrm{E}-01$ & $8.75 \mathrm{E}-01$ & $0.00 \mathrm{E}+00$ & $7.37 \mathrm{E}+10$ & $7.37 \mathrm{E}+10$ & $0.00 \mathrm{E}+00$ & $8.83 \mathrm{E}+08$ & $8.83 \mathrm{E}+08$ \\
\hline $9.25 \mathrm{E}-01$ & $9.00 \mathrm{E}-01$ & $0.00 \mathrm{E}+00$ & $6.82 \mathrm{E}+10$ & $6.82 \mathrm{E}+10$ & $0.00 \mathrm{E}+00$ & $7.96 \mathrm{E}+08$ & $7.96 \mathrm{E}+08$ \\
\hline $9.50 \mathrm{E}-01$ & $9.25 \mathrm{E}-01$ & $0.00 \mathrm{E}+00$ & $6.33 \mathrm{E}+10$ & $6.33 \mathrm{E}+10$ & $0.00 \mathrm{E}+00$ & $7.21 \mathrm{E}+08$ & $7.21 \mathrm{E}+08$ \\
\hline $9.75 \mathrm{E}-01$ & $9.50 \mathrm{E}-01$ & $4.83 \mathrm{E}+09$ & $5.90 \mathrm{E}+10$ & $6.38 \mathrm{E}+10$ & $0.00 \mathrm{E}+00$ & $6.55 \mathrm{E}+08$ & $6.55 \mathrm{E}+08$ \\
\hline
\end{tabular}


PCP-2011-0001

Revision 0

\begin{tabular}{|c|c|c|c|c|c|c|c|}
\hline \multicolumn{8}{|c|}{ Table 3-27 Photons from Nuclides with Bremsstrahlung (Continued) } \\
\hline \multirow{2}{*}{\multicolumn{2}{|c|}{ Energy $(\mathrm{MeV})$}} & \multicolumn{6}{|c|}{ Photon Source (photons/sec/g) } \\
\hline & & \multicolumn{3}{|c|}{$\mathrm{Ru}-106$} & \multicolumn{3}{|c|}{ Sr-90 } \\
\hline $1.00 \mathrm{E}+00$ & $9.75 \mathrm{E}-01$ & $0.00 \mathrm{E}+00$ & $5.47 \mathrm{E}+10$ & $5.47 \mathrm{E}+10$ & $0.00 \mathrm{E}+00$ & $5.90 \mathrm{E}+08$ & $5.90 \mathrm{E}+08$ \\
\hline $1.25 \mathrm{E}+00$ & $1.00 \mathrm{E}+00$ & $2.29 \mathrm{E}+12$ & $3.71 \mathrm{E}+11$ & $2.66 \mathrm{E}+12$ & $0.00 \mathrm{E}+00$ & $3.39 \mathrm{E}+09$ & $3.39 \mathrm{E}+09$ \\
\hline $1.50 \mathrm{E}+00$ & $1.25 \mathrm{E}+00$ & $7.26 \mathrm{E}+10$ & $1.86 \mathrm{E}+11$ & $2.59 \mathrm{E}+11$ & $0.00 \mathrm{E}+00$ & $1.15 \mathrm{E}+09$ & $1.15 \mathrm{E}+09$ \\
\hline $1.75 \mathrm{E}+00$ & $1.50 \mathrm{E}+00$ & $2.00 \mathrm{E}+11$ & $9.39 \mathrm{E}+10$ & $2.94 \mathrm{E}+11$ & $0.00 \mathrm{E}+00$ & $3.10 \mathrm{E}+08$ & $3.10 \mathrm{E}+08$ \\
\hline $2.00 \mathrm{E}+00$ & $1.75 \mathrm{E}+00$ & $1.08 \mathrm{E}+11$ & $4.60 \mathrm{E}+10$ & $1.54 \mathrm{E}+11$ & $0.00 \mathrm{E}+00$ & $4.99 \mathrm{E}+07$ & $4.99 \mathrm{E}+07$ \\
\hline $2.25 \mathrm{E}+00$ & $2.00 \mathrm{E}+00$ & $6.16 \mathrm{E}+10$ & $2.13 \mathrm{E}+10$ & $8.29 \mathrm{E}+10$ & $7.51 \mathrm{E}+04$ & $1.50 \mathrm{E}+06$ & $1.57 \mathrm{E}+06$ \\
\hline $2.50 \mathrm{E}+00$ & $2.25 \mathrm{E}+00$ & $7.16 \mathrm{E}+10$ & $8.91 \mathrm{E}+09$ & $8.05 \mathrm{E}+10$ & $0.00 \mathrm{E}+00$ & $4.92 \mathrm{E}+01$ & $4.92 \mathrm{E}+01$ \\
\hline $2.75 \mathrm{E}+00$ & $2.50 \mathrm{E}+00$ & $1.44 \mathrm{E}+10$ & $3.19 \mathrm{E}+09$ & $1.75 \mathrm{E}+10$ & $0.00 \mathrm{E}+00$ & $0.00 \mathrm{E}+00$ & $0.00 \mathrm{E}+00$ \\
\hline $3.00 \mathrm{E}+00$ & $2.75 \mathrm{E}+00$ & $3.43 \mathrm{E}+09$ & $8.71 \mathrm{E}+08$ & $4.31 \mathrm{E}+09$ & $0.00 \mathrm{E}+00$ & $0.00 \mathrm{E}+00$ & $0.00 \mathrm{E}+00$ \\
\hline $3.50 \mathrm{E}+00$ & $3.00 \mathrm{E}+00$ & $2.82 \mathrm{E}+09$ & $7.15 \mathrm{E}+07$ & $2.89 \mathrm{E}+09$ & $0.00 \mathrm{E}+00$ & $0.00 \mathrm{E}+00$ & $0.00 \mathrm{E}+00$ \\
\hline $4.00 \mathrm{E}+00$ & $3.50 \mathrm{E}+00$ & $0.00 \mathrm{E}+00$ & $3.75 \mathrm{E}+02$ & $3.75 \mathrm{E}+02$ & $0.00 \mathrm{E}+00$ & $0.00 \mathrm{E}+00$ & $0.00 \mathrm{E}+00$ \\
\hline $4.50 \mathrm{E}+00$ & $4.00 \mathrm{E}+00$ & $0.00 \mathrm{E}+00$ & $0.00 \mathrm{E}+00$ & $0.00 \mathrm{E}+00$ & $0.00 \mathrm{E}+00$ & $0.00 \mathrm{E}+00$ & $0.00 \mathrm{E}+00$ \\
\hline $5.00 \mathrm{E}+00$ & $4.50 \mathrm{E}+00$ & $0.00 \mathrm{E}+00$ & $0.00 \mathrm{E}+00$ & $0.00 \mathrm{E}+00$ & $0.00 \mathrm{E}+00$ & $0.00 \mathrm{E}+00$ & $0.00 \mathrm{E}+00$ \\
\hline $5.50 \mathrm{E}+00$ & $5.00 \mathrm{E}+00$ & $0.00 \mathrm{E}+00$ & $0.00 \mathrm{E}+00$ & $0.00 \mathrm{E}+00$ & $0.00 \mathrm{E}+00$ & $0.00 \mathrm{E}+00$ & $0.00 \mathrm{E}+00$ \\
\hline $6.00 \mathrm{E}+00$ & $5.50 \mathrm{E}+00$ & $0.00 \mathrm{E}+00$ & $0.00 \mathrm{E}+00$ & $0.00 \mathrm{E}+00$ & $0.00 \mathrm{E}+00$ & $0.00 \mathrm{E}+00$ & $0.00 \mathrm{E}+00$ \\
\hline $6.50 \mathrm{E}+00$ & $6.00 \mathrm{E}+00$ & $0.00 \mathrm{E}+00$ & $0.00 \mathrm{E}+00$ & $0.00 \mathrm{E}+00$ & $0.00 \mathrm{E}+00$ & $0.00 \mathrm{E}+00$ & $0.00 \mathrm{E}+00$ \\
\hline $7.00 \mathrm{E}+00$ & $6.50 \mathrm{E}+00$ & $0.00 \mathrm{E}+00$ & $0.00 \mathrm{E}+00$ & $0.00 \mathrm{E}+00$ & $0.00 \mathrm{E}+00$ & $0.00 \mathrm{E}+00$ & $0.00 \mathrm{E}+00$ \\
\hline $7.50 \mathrm{E}+00$ & $7.00 \mathrm{E}+00$ & $0.00 \mathrm{E}+00$ & $0.00 \mathrm{E}+00$ & $0.00 \mathrm{E}+00$ & $0.00 \mathrm{E}+00$ & $0.00 \mathrm{E}+00$ & $0.00 \mathrm{E}+00$ \\
\hline $8.00 \mathrm{E}+00$ & $7.50 \mathrm{E}+00$ & $0.00 \mathrm{E}+00$ & $0.00 \mathrm{E}+00$ & $0.00 \mathrm{E}+00$ & $0.00 \mathrm{E}+00$ & $0.00 \mathrm{E}+00$ & $0.00 \mathrm{E}+00$ \\
\hline $9.00 \mathrm{E}+00$ & $8.00 \mathrm{E}+00$ & $0.00 \mathrm{E}+00$ & $0.00 \mathrm{E}+00$ & $0.00 \mathrm{E}+00$ & $0.00 \mathrm{E}+00$ & $0.00 \mathrm{E}+00$ & $0.00 \mathrm{E}+00$ \\
\hline $1.00 \mathrm{E}+01$ & $9.00 \mathrm{E}+00$ & $0.00 \mathrm{E}+00$ & $0.00 \mathrm{E}+00$ & $0.00 \mathrm{E}+00$ & $0.00 \mathrm{E}+00$ & $0.00 \mathrm{E}+00$ & $0.00 \mathrm{E}+00$ \\
\hline \multicolumn{2}{|l|}{ Total } & $4.21 \mathrm{E}+13$ & $7.46 \mathrm{E}+13$ & $1.17 \mathrm{E}+14$ & $5.85 \mathrm{E}+08$ & $1.84 \mathrm{E}+12$ & $1.84 \mathrm{E}+12$ \\
\hline \multicolumn{2}{|c|}{ Bremsstrahlung Fraction } & & $6.39 \mathrm{E}-01$ & & & $1.00 \mathrm{E}+00$ & \\
\hline
\end{tabular}


PCP-2011-0001

Revision 0

Table 3-28. Neutron Dose Rate per Unit Neutron Source in Each of the 47 Source Groups No Shielded Container - No Self-shielding

\begin{tabular}{|c|c|c|c|c|c|c|c|c|c|c|}
\hline \multicolumn{2}{|c|}{ Energy (MeV) } & \multicolumn{3}{|c|}{ Bottom Dose Rate (rem/hr) } & \multicolumn{3}{|c|}{ Top Dose Rate (rem/hr) } & \multicolumn{3}{|c|}{ Side Dose Rate (rem $/ \mathrm{hr})$} \\
\hline Upper & Lower & mean & FSD & mean $+3 \pi$ & mean & FSD & mean $+3 \pi$ & mean & FSD & mean $+3 \pi$ \\
\hline $1.00 \mathrm{E}-07$ & $1.00 \mathrm{E}-11$ & $1.12 \mathrm{E}-09$ & $1.20 \mathrm{E}-03$ & $1.13 \mathrm{E}-09$ & $1.78 \mathrm{E}-11$ & $1.04 \mathrm{E}-02$ & $1.83 \mathrm{E}-11$ & $3.76 \mathrm{E}-10$ & $5.00 \mathrm{E}-04$ & $3.77 \mathrm{E}-10$ \\
\hline $4.14 \mathrm{E}-07$ & $1.00 \mathrm{E}-07$ & $1.48 \mathrm{E}-09$ & $1.00 \mathrm{E}-03$ & $1.48 \mathrm{E}-09$ & $3.18 \mathrm{E}-11$ & $7.50 \mathrm{E}-03$ & $3.25 \mathrm{E}-11$ & $5.32 \mathrm{E}-10$ & $4.00 \mathrm{E}-04$ & $5.32 \mathrm{E}-10$ \\
\hline $8.76 \mathrm{E}-07$ & $4.14 \mathrm{E}-07$ & $1.65 \mathrm{E}-09$ & $1.00 \mathrm{E}-03$ & $1.66 \mathrm{E}-09$ & $4.18 \mathrm{E}-11$ & $6.70 \mathrm{E}-03$ & $4.27 \mathrm{E}-11$ & $6.13 \mathrm{E}-10$ & $3.00 \mathrm{E}-04$ & $6.13 \mathrm{E}-10$ \\
\hline $1.86 \mathrm{E}-06$ & $8.76 \mathrm{E}-07$ & $1.78 \mathrm{E}-09$ & $9.00 \mathrm{E}-04$ & $1.79 \mathrm{E}-09$ & $5.02 \mathrm{E}-11$ & $6.20 \mathrm{E}-03$ & $5.12 \mathrm{E}-11$ & $6.73 \mathrm{E}-10$ & $3.00 \mathrm{E}-04$ & $6.73 \mathrm{E}-10$ \\
\hline $5.04 \mathrm{E}-06$ & $1.86 \mathrm{E}-06$ & $1.90 \mathrm{E}-09$ & $9.00 \mathrm{E}-04$ & $1.90 \mathrm{E}-09$ & $5.92 \mathrm{E}-11$ & $5.80 \mathrm{E}-03$ & $6.03 \mathrm{E}-11$ & $7.32 \mathrm{E}-10$ & $3.00 \mathrm{E}-04$ & $7.33 \mathrm{E}-10$ \\
\hline $1.07 \mathrm{E}-05$ & $5.04 \mathrm{E}-06$ & $1.98 \mathrm{E}-09$ & $9.00 \mathrm{E}-04$ & $1.98 \mathrm{E}-09$ & $6.55 \mathrm{E}-11$ & $5.50 \mathrm{E}-03$ & $6.65 \mathrm{E}-11$ & $7.76 \mathrm{E}-10$ & $3.00 \mathrm{E}-04$ & $7.77 \mathrm{E}-10$ \\
\hline $3.73 \mathrm{E}-05$ & $1.07 \mathrm{E}-05$ & $2.03 \mathrm{E}-09$ & $9.00 \mathrm{E}-04$ & $2.03 \mathrm{E}-09$ & $7.29 \mathrm{E}-11$ & $5.20 \mathrm{E}-03$ & $7.40 \mathrm{E}-11$ & $8.15 \mathrm{E}-10$ & $3.00 \mathrm{E}-04$ & $8.16 \mathrm{E}-10$ \\
\hline $1.01 \mathrm{E}-04$ & $3.73 \mathrm{E}-05$ & $2.04 \mathrm{E}-09$ & $8.00 \mathrm{E}-04$ & $2.04 \mathrm{E}-09$ & $7.78 \mathrm{E}-11$ & $5.00 \mathrm{E}-03$ & $7.89 \mathrm{E}-11$ & $8.35 \mathrm{E}-10$ & $3.00 \mathrm{E}-04$ & $8.36 \mathrm{E}-10$ \\
\hline $2.14 \mathrm{E}-04$ & $1.01 \mathrm{E}-04$ & $2.02 \mathrm{E}-09$ & $8.00 \mathrm{E}-04$ & $2.03 \mathrm{E}-09$ & $7.94 \mathrm{E}-11$ & $4.90 \mathrm{E}-03$ & $8.05 \mathrm{E}-11$ & $8.40 \mathrm{E}-10$ & $3.00 \mathrm{E}-04$ & $8.40 \mathrm{E}-10$ \\
\hline $4.54 \mathrm{E}-04$ & $2.14 \mathrm{E}-04$ & $1.98 \mathrm{E}-09$ & $9.00 \mathrm{E}-04$ & $1.99 \mathrm{E}-09$ & $7.89 \mathrm{E}-11$ & $5.10 \mathrm{E}-03$ & $8.02 \mathrm{E}-11$ & $8.22 \mathrm{E}-10$ & $3.00 \mathrm{E}-04$ & $8.23 \mathrm{E}-10$ \\
\hline $1.58 \mathrm{E}-03$ & $4.54 \mathrm{E}-04$ & $1.90 \mathrm{E}-09$ & $8.00 \mathrm{E}-04$ & $1.91 \mathrm{E}-09$ & $7.81 \mathrm{E}-11$ & $4.90 \mathrm{E}-03$ & $7.93 \mathrm{E}-11$ & $8.13 \mathrm{E}-10$ & $3.00 \mathrm{E}-04$ & $8.13 \mathrm{E}-10$ \\
\hline $3.35 \mathrm{E}-03$ & $1.58 \mathrm{E}-03$ & $1.90 \mathrm{E}-09$ & $8.00 \mathrm{E}-04$ & $1.91 \mathrm{E}-09$ & $7.64 \mathrm{E}-11$ & $5.00 \mathrm{E}-03$ & $7.76 \mathrm{E}-11$ & $8.07 \mathrm{E}-10$ & $3.00 \mathrm{E}-04$ & $8.08 \mathrm{E}-10$ \\
\hline $7.10 \mathrm{E}-03$ & $3.35 \mathrm{E}-03$ & $1.88 \mathrm{E}-09$ & $8.00 \mathrm{E}-04$ & $1.88 \mathrm{E}-09$ & $7.92 \mathrm{E}-11$ & $4.90 \mathrm{E}-03$ & $8.03 \mathrm{E}-11$ & $7.99 \mathrm{E}-10$ & $3.00 \mathrm{E}-04$ & $8.00 \mathrm{E}-10$ \\
\hline $1.50 \mathrm{E}-02$ & $7.10 \mathrm{E}-03$ & $1.84 \mathrm{E}-09$ & $8.00 \mathrm{E}-04$ & $1.85 \mathrm{E}-09$ & $8.40 \mathrm{E}-11$ & $4.60 \mathrm{E}-03$ & $8.51 \mathrm{E}-11$ & $7.95 \mathrm{E}-10$ & $3.00 \mathrm{E}-04$ & $7.96 \mathrm{E}-10$ \\
\hline $2.19 \mathrm{E}-02$ & $1.50 \mathrm{E}-02$ & $2.02 \mathrm{E}-09$ & $8.00 \mathrm{E}-04$ & $2.02 \mathrm{E}-09$ & $1.00 \mathrm{E}-10$ & $4.20 \mathrm{E}-03$ & $1.01 \mathrm{E}-10$ & $8.59 \mathrm{E}-10$ & $3.00 \mathrm{E}-04$ & $8.60 \mathrm{E}-10$ \\
\hline $2.42 \mathrm{E}-02$ & $2.19 \mathrm{E}-02$ & $2.11 \mathrm{E}-09$ & $7.00 \mathrm{E}-04$ & $2.12 \mathrm{E}-09$ & $1.30 \mathrm{E}-10$ & $3.60 \mathrm{E}-03$ & $1.31 \mathrm{E}-10$ & $9.17 \mathrm{E}-10$ & $2.00 \mathrm{E}-04$ & $9.17 \mathrm{E}-10$ \\
\hline $2.61 \mathrm{E}-02$ & $2.42 \mathrm{E}-02$ & $2.18 \mathrm{E}-09$ & $7.00 \mathrm{E}-04$ & $2.19 \mathrm{E}-09$ & $1.44 \mathrm{E}-10$ & $3.40 \mathrm{E}-03$ & $1.45 \mathrm{E}-10$ & $9.46 \mathrm{E}-10$ & $2.00 \mathrm{E}-04$ & $9.46 \mathrm{E}-10$ \\
\hline $3.18 \mathrm{E}-02$ & $2.61 \mathrm{E}-02$ & $2.47 \mathrm{E}-09$ & $8.00 \mathrm{E}-04$ & $2.48 \mathrm{E}-09$ & $1.34 \mathrm{E}-10$ & $3.90 \mathrm{E}-03$ & $1.35 \mathrm{E}-10$ & $9.61 \mathrm{E}-10$ & $3.00 \mathrm{E}-04$ & $9.62 \mathrm{E}-10$ \\
\hline $4.09 \mathrm{E}-02$ & $3.18 \mathrm{E}-02$ & $2.96 \mathrm{E}-09$ & $8.00 \mathrm{E}-04$ & $2.97 \mathrm{E}-09$ & $1.41 \mathrm{E}-10$ & $4.40 \mathrm{E}-03$ & $1.43 \mathrm{E}-10$ & $1.02 \mathrm{E}-09$ & $3.00 \mathrm{E}-04$ & $1.03 \mathrm{E}-09$ \\
\hline $6.74 \mathrm{E}-02$ & $4.09 \mathrm{E}-02$ & $3.55 \mathrm{E}-09$ & $8.00 \mathrm{E}-04$ & $3.56 \mathrm{E}-09$ & $1.64 \mathrm{E}-10$ & $4.10 \mathrm{E}-03$ & $1.66 \mathrm{E}-10$ & $1.28 \mathrm{E}-09$ & $3.00 \mathrm{E}-04$ & $1.28 \mathrm{E}-09$ \\
\hline $1.11 \mathrm{E}-01$ & $6.74 \mathrm{E}-02$ & $5.32 \mathrm{E}-09$ & $8.00 \mathrm{E}-04$ & $5.33 \mathrm{E}-09$ & $2.49 \mathrm{E}-10$ & $4.10 \mathrm{E}-03$ & $2.52 \mathrm{E}-10$ & $1.82 \mathrm{E}-09$ & $3.00 \mathrm{E}-04$ & $1.82 \mathrm{E}-09$ \\
\hline $1.83 \mathrm{E}-01$ & $1.11 \mathrm{E}-01$ & $8.71 \mathrm{E}-09$ & $7.00 \mathrm{E}-04$ & $8.72 \mathrm{E}-09$ & $4.26 \mathrm{E}-10$ & $3.80 \mathrm{E}-03$ & $4.31 \mathrm{E}-10$ & $2.93 \mathrm{E}-09$ & $3.00 \mathrm{E}-04$ & $2.93 \mathrm{E}-09$ \\
\hline $2.97 \mathrm{E}-01$ & $1.83 \mathrm{E}-01$ & $1.40 \mathrm{E}-08$ & $7.00 \mathrm{E}-04$ & $1.40 \mathrm{E}-08$ & $6.22 \mathrm{E}-10$ & $4.00 \mathrm{E}-03$ & $6.30 \mathrm{E}-10$ & $4.89 \mathrm{E}-09$ & $3.00 \mathrm{E}-04$ & $4.90 \mathrm{E}-09$ \\
\hline $3.69 \mathrm{E}-01$ & $2.97 \mathrm{E}-01$ & $1.92 \mathrm{E}-08$ & $7.00 \mathrm{E}-04$ & $1.92 \mathrm{E}-08$ & $9.20 \mathrm{E}-10$ & $3.60 \mathrm{E}-03$ & $9.30 \mathrm{E}-10$ & $6.88 \mathrm{E}-09$ & $2.00 \mathrm{E}-04$ & $6.89 \mathrm{E}-09$ \\
\hline $4.98 \mathrm{E}-01$ & $3.69 \mathrm{E}-01$ & $2.51 \mathrm{E}-08$ & $7.00 \mathrm{E}-04$ & $2.51 \mathrm{E}-08$ & $1.09 \mathrm{E}-09$ & $3.90 \mathrm{E}-03$ & $1.10 \mathrm{E}-09$ & $8.97 \mathrm{E}-09$ & $2.00 \mathrm{E}-04$ & $8.98 \mathrm{E}-09$ \\
\hline $6.08 \mathrm{E}-01$ & $4.98 \mathrm{E}-01$ & $3.16 \mathrm{E}-08$ & $6.00 \mathrm{E}-04$ & $3.16 \mathrm{E}-08$ & $1.54 \mathrm{E}-09$ & $3.40 \mathrm{E}-03$ & $1.55 \mathrm{E}-09$ & $1.16 \mathrm{E}-08$ & $2.00 \mathrm{E}-04$ & $1.16 \mathrm{E}-08$ \\
\hline $7.43 \mathrm{E}-01$ & $6.08 \mathrm{E}-01$ & $3.58 \mathrm{E}-08$ & $6.00 \mathrm{E}-04$ & $3.59 \mathrm{E}-08$ & $1.80 \mathrm{E}-09$ & $3.20 \mathrm{E}-03$ & $1.81 \mathrm{E}-09$ & $1.35 \mathrm{E}-08$ & $2.00 \mathrm{E}-04$ & $1.35 \mathrm{E}-08$ \\
\hline $8.21 \mathrm{E}-01$ & $7.43 \mathrm{E}-01$ & $4.04 \mathrm{E}-08$ & $6.00 \mathrm{E}-04$ & $4.05 \mathrm{E}-08$ & $1.78 \mathrm{E}-09$ & $3.30 \mathrm{E}-03$ & $1.80 \mathrm{E}-09$ & $1.50 \mathrm{E}-08$ & $2.00 \mathrm{E}-04$ & $1.50 \mathrm{E}-08$ \\
\hline $1.00 \mathrm{E}+00$ & $8.21 \mathrm{E}-01$ & $4.23 \mathrm{E}-08$ & $6.00 \mathrm{E}-04$ & $4.24 \mathrm{E}-08$ & $2.07 \mathrm{E}-09$ & $3.20 \mathrm{E}-03$ & $2.09 \mathrm{E}-09$ & $1.64 \mathrm{E}-08$ & $2.00 \mathrm{E}-04$ & $1.64 \mathrm{E}-08$ \\
\hline $1.35 \mathrm{E}+00$ & $1.00 \mathrm{E}+00$ & $4.53 \mathrm{E}-08$ & $6.00 \mathrm{E}-04$ & $4.54 \mathrm{E}-08$ & $2.16 \mathrm{E}-09$ & $3.30 \mathrm{E}-03$ & $2.19 \mathrm{E}-09$ & $1.77 \mathrm{E}-08$ & $2.00 \mathrm{E}-04$ & $1.77 \mathrm{E}-08$ \\
\hline $1.65 \mathrm{E}+00$ & $1.35 \mathrm{E}+00$ & $4.62 \mathrm{E}-08$ & $6.00 \mathrm{E}-04$ & $4.63 \mathrm{E}-08$ & $2.14 \mathrm{E}-09$ & $3.30 \mathrm{E}-03$ & $2.16 \mathrm{E}-09$ & $1.82 \mathrm{E}-08$ & $2.00 \mathrm{E}-04$ & $1.82 \mathrm{E}-08$ \\
\hline $1.92 \mathrm{E}+00$ & $1.65 \mathrm{E}+00$ & $4.63 \mathrm{E}-08$ & $6.00 \mathrm{E}-04$ & $4.64 \mathrm{E}-08$ & $2.16 \mathrm{E}-09$ & $3.20 \mathrm{E}-03$ & $2.18 \mathrm{E}-09$ & $1.85 \mathrm{E}-08$ & $2.00 \mathrm{E}-04$ & $1.85 \mathrm{E}-08$ \\
\hline $2.23 \mathrm{E}+00$ & $1.92 \mathrm{E}+00$ & $4.69 \mathrm{E}-08$ & $6.00 \mathrm{E}-04$ & $4.70 \mathrm{E}-08$ & $2.17 \mathrm{E}-09$ & $3.30 \mathrm{E}-03$ & $2.19 \mathrm{E}-09$ & $1.88 \mathrm{E}-08$ & $2.00 \mathrm{E}-04$ & $1.88 \mathrm{E}-08$ \\
\hline $2.35 \mathrm{E}+00$ & $2.23 \mathrm{E}+00$ & $4.67 \mathrm{E}-08$ & $6.00 \mathrm{E}-04$ & $4.68 \mathrm{E}-08$ & $2.14 \mathrm{E}-09$ & $3.20 \mathrm{E}-03$ & $2.16 \mathrm{E}-09$ & $1.87 \mathrm{E}-08$ & $2.00 \mathrm{E}-04$ & $1.88 \mathrm{E}-08$ \\
\hline $2.37 \mathrm{E}+00$ & $2.35 \mathrm{E}+00$ & $4.59 \mathrm{E}-08$ & $6.00 \mathrm{E}-04$ & $4.60 \mathrm{E}-08$ & $2.16 \mathrm{E}-09$ & $3.10 \mathrm{E}-03$ & $2.18 \mathrm{E}-09$ & $1.86 \mathrm{E}-08$ & $2.00 \mathrm{E}-04$ & $1.86 \mathrm{E}-08$ \\
\hline $2.47 \mathrm{E}+00$ & $2.37 \mathrm{E}+00$ & $4.61 \mathrm{E}-08$ & $6.00 \mathrm{E}-04$ & $4.61 \mathrm{E}-08$ & $2.06 \mathrm{E}-09$ & $3.20 \mathrm{E}-03$ & $2.08 \mathrm{E}-09$ & $1.87 \mathrm{E}-08$ & $2.00 \mathrm{E}-04$ & $1.87 \mathrm{E}-08$ \\
\hline $2.73 \mathrm{E}+00$ & $2.47 \mathrm{E}+00$ & $4.69 \mathrm{E}-08$ & $6.00 \mathrm{E}-04$ & $4.69 \mathrm{E}-08$ & $2.10 \mathrm{E}-09$ & $3.40 \mathrm{E}-03$ & $2.12 \mathrm{E}-09$ & $1.88 \mathrm{E}-08$ & $2.00 \mathrm{E}-04$ & $1.88 \mathrm{E}-08$ \\
\hline $3.01 \mathrm{E}+00$ & $2.73 \mathrm{E}+00$ & $4.75 \mathrm{E}-08$ & $6.00 \mathrm{E}-04$ & $4.76 \mathrm{E}-08$ & $2.16 \mathrm{E}-09$ & $3.40 \mathrm{E}-03$ & $2.18 \mathrm{E}-09$ & $1.92 \mathrm{E}-08$ & $2.00 \mathrm{E}-04$ & $1.92 \mathrm{E}-08$ \\
\hline $3.68 \mathrm{E}+00$ & $3.01 \mathrm{E}+00$ & $4.87 \mathrm{E}-08$ & $6.00 \mathrm{E}-04$ & $4.88 \mathrm{E}-08$ & $2.21 \mathrm{E}-09$ & $3.60 \mathrm{E}-03$ & $2.23 \mathrm{E}-09$ & $1.96 \mathrm{E}-08$ & $2.00 \mathrm{E}-04$ & $1.96 \mathrm{E}-08$ \\
\hline $4.97 \mathrm{E}+00$ & $3.68 \mathrm{E}+00$ & $5.20 \mathrm{E}-08$ & $7.00 \mathrm{E}-04$ & $5.21 \mathrm{E}-08$ & $2.31 \mathrm{E}-09$ & $3.60 \mathrm{E}-03$ & $2.34 \mathrm{E}-09$ & $2.09 \mathrm{E}-08$ & $2.00 \mathrm{E}-04$ & $2.09 \mathrm{E}-08$ \\
\hline $6.07 \mathrm{E}+00$ & $4.97 \mathrm{E}+00$ & $5.31 \mathrm{E}-08$ & $7.00 \mathrm{E}-04$ & $5.32 \mathrm{E}-08$ & $2.32 \mathrm{E}-09$ & $3.90 \mathrm{E}-03$ & $2.35 \mathrm{E}-09$ & $2.14 \mathrm{E}-08$ & $2.00 \mathrm{E}-04$ & $2.14 \mathrm{E}-08$ \\
\hline $7.41 \mathrm{E}+00$ & $6.07 \mathrm{E}+00$ & $5.13 \mathrm{E}-08$ & $7.00 \mathrm{E}-04$ & $5.14 \mathrm{E}-08$ & $2.27 \mathrm{E}-09$ & $4.00 \mathrm{E}-03$ & $2.30 \mathrm{E}-09$ & $2.07 \mathrm{E}-08$ & $2.00 \mathrm{E}-04$ & $2.07 \mathrm{E}-08$ \\
\hline $8.61 \mathrm{E}+00$ & $7.41 \mathrm{E}+00$ & $5.03 \mathrm{E}-08$ & $7.00 \mathrm{E}-04$ & $5.04 \mathrm{E}-08$ & $2.25 \mathrm{E}-09$ & $3.80 \mathrm{E}-03$ & $2.27 \mathrm{E}-09$ & $2.04 \mathrm{E}-08$ & $2.00 \mathrm{E}-04$ & $2.04 \mathrm{E}-08$ \\
\hline $1.00 \mathrm{E}+01$ & $8.61 \mathrm{E}+00$ & $4.96 \mathrm{E}-08$ & $7.00 \mathrm{E}-04$ & $4.97 \mathrm{E}-08$ & $2.21 \mathrm{E}-09$ & $3.70 \mathrm{E}-03$ & $2.23 \mathrm{E}-09$ & $2.01 \mathrm{E}-08$ & $2.00 \mathrm{E}-04$ & $2.01 \mathrm{E}-08$ \\
\hline $1.22 \mathrm{E}+01$ & $1.00 \mathrm{E}+01$ & $5.35 \mathrm{E}-08$ & $7.00 \mathrm{E}-04$ & $5.36 \mathrm{E}-08$ & $2.35 \mathrm{E}-09$ & $3.60 \mathrm{E}-03$ & $2.37 \mathrm{E}-09$ & $2.15 \mathrm{E}-08$ & $2.00 \mathrm{E}-04$ & $2.15 \mathrm{E}-08$ \\
\hline $1.42 \mathrm{E}+01$ & $1.22 \mathrm{E}+01$ & $6.24 \mathrm{E}-08$ & $7.00 \mathrm{E}-04$ & $6.25 \mathrm{E}-08$ & $2.70 \mathrm{E}-09$ & $3.50 \mathrm{E}-03$ & $2.73 \mathrm{E}-09$ & $2.48 \mathrm{E}-08$ & $2.00 \mathrm{E}-04$ & $2.49 \mathrm{E}-08$ \\
\hline $1.96 \mathrm{E}+01$ & $1.42 \mathrm{E}+01$ & $7.05 \mathrm{E}-08$ & $6.00 \mathrm{E}-04$ & $7.06 \mathrm{E}-08$ & $3.09 \mathrm{E}-09$ & $3.30 \mathrm{E}-03$ & $3.12 \mathrm{E}-09$ & $2.83 \mathrm{E}-08$ & $2.00 \mathrm{E}-04$ & $2.83 \mathrm{E}-08$ \\
\hline
\end{tabular}


PCP-2011-0001

Revision 0

Table 3-29. Secondary Photon Dose Rate per Unit Neutron Source in Each of the 47 Source Groups - No Shielded Container - No Self-shielding

\begin{tabular}{|c|c|c|c|c|c|c|c|c|c|c|}
\hline \multicolumn{2}{|c|}{ Energy $(\mathrm{MeV})$} & \multicolumn{3}{|c|}{ Bottom Dose Rate (rem/hr) } & \multicolumn{3}{|c|}{ Top Dose Rate $(\mathrm{rem} / \mathrm{hr})$} & \multicolumn{3}{|c|}{ Side Dose Rate $(\mathrm{rem} / \mathrm{hr})$} \\
\hline Upper & Lower & mean & FSD & mean $+3 \pi$ & mean & FSD & mean $+3 \pi$ & mean & FSD & mean $+3 \pi$ \\
\hline $1.00 \mathrm{E}-07$ & $1.00 \mathrm{E}-11$ & $9.00 \mathrm{E}-10$ & 0.0022 & $9.06 \mathrm{E}-10$ & $4.78 \mathrm{E}-11$ & 0.0073 & 4.89E-11 & $4.95 \mathrm{E}-10$ & 0.0006 & $4.95 \mathrm{E}-10$ \\
\hline $4.14 \mathrm{E}-07$ & $1.00 \mathrm{E}-07$ & $5.40 \mathrm{E}-10$ & 0.0029 & $5.45 \mathrm{E}-10$ & $3.79 \mathrm{E}-11$ & 0.0082 & $3.89 \mathrm{E}-11$ & $3.27 \mathrm{E}-10$ & 0.0008 & $3.28 \mathrm{E}-10$ \\
\hline $8.76 \mathrm{E}-07$ & $4.14 \mathrm{E}-07$ & $3.99 \mathrm{E}-10$ & 0.0033 & $4.03 \mathrm{E}-10$ & $3.31 \mathrm{E}-11$ & 0.0082 & $3.39 \mathrm{E}-11$ & $2.54 \mathrm{E}-10$ & 0.0010 & $2.55 \mathrm{E}-10$ \\
\hline $1.86 \mathrm{E}-06$ & $8.76 \mathrm{E}-07$ & $3.13 \mathrm{E}-10$ & 0.0037 & $3.17 \mathrm{E}-10$ & $2.93 \mathrm{E}-11$ & 0.0093 & $3.01 \mathrm{E}-11$ & $2.08 \mathrm{E}-10$ & 0.0010 & $2.09 \mathrm{E}-10$ \\
\hline $5.04 \mathrm{E}-06$ & $1.86 \mathrm{E}-06$ & $2.36 \mathrm{E}-10$ & 0.0042 & $2.39 \mathrm{E}-10$ & $2.49 \mathrm{E}-11$ & 0.0105 & $2.57 \mathrm{E}-11$ & $1.63 \mathrm{E}-10$ & 0.0012 & $1.64 \mathrm{E}-10$ \\
\hline $1.07 \mathrm{E}-05$ & $5.04 \mathrm{E}-06$ & $1.80 \mathrm{E}-10$ & 0.0047 & $1.83 \mathrm{E}-10$ & $2.09 \mathrm{E}-11$ & 0.0106 & $2.16 \mathrm{E}-11$ & $1.30 \mathrm{E}-10$ & 0.0013 & $1.31 \mathrm{E}-10$ \\
\hline $3.73 \mathrm{E}-05$ & $1.07 \mathrm{E}-05$ & $1.27 \mathrm{E}-10$ & 0.0054 & $1.29 \mathrm{E}-10$ & $1.68 \mathrm{E}-11$ & 0.0118 & $1.74 \mathrm{E}-11$ & $9.74 \mathrm{E}-11$ & 0.0015 & $9.78 \mathrm{E}-11$ \\
\hline $1.01 \mathrm{E}-04$ & $3.73 \mathrm{E}-05$ & $9.20 \mathrm{E}-11$ & 0.0063 & $9.38 \mathrm{E}-11$ & $1.37 \mathrm{E}-11$ & 0.0126 & $1.42 \mathrm{E}-11$ & $7.31 \mathrm{E}-11$ & 0.0017 & $7.34 \mathrm{E}-11$ \\
\hline $2.14 \mathrm{E}-04$ & $1.01 \mathrm{E}-04$ & $7.10 \mathrm{E}-11$ & 0.0070 & $7.25 \mathrm{E}-11$ & $1.20 \mathrm{E}-11$ & 0.0143 & $1.26 \mathrm{E}-11$ & $5.87 \mathrm{E}-11$ & 0.0019 & $5.90 \mathrm{E}-11$ \\
\hline $4.54 \mathrm{E}-04$ & $2.14 \mathrm{E}-04$ & $8.49 \mathrm{E}-11$ & 0.0062 & $8.65 \mathrm{E}-11$ & $1.24 \mathrm{E}-11$ & 0.0146 & $1.30 \mathrm{E}-11$ & $6.39 \mathrm{E}-11$ & 0.0018 & $6.42 \mathrm{E}-11$ \\
\hline $1.58 \mathrm{E}-03$ & $4.54 \mathrm{E}-04$ & $7.13 \mathrm{E}-11$ & 0.0063 & $7.26 \mathrm{E}-11$ & $1.03 \mathrm{E}-11$ & 0.0145 & $1.08 \mathrm{E}-11$ & $5.27 \mathrm{E}-11$ & 0.0018 & $5.29 \mathrm{E}-11$ \\
\hline $3.35 \mathrm{E}-03$ & $1.58 \mathrm{E}-03$ & $4.79 \mathrm{E}-11$ & 0.0084 & $4.91 \mathrm{E}-11$ & $8.99 \mathrm{E}-12$ & 0.0155 & $9.41 \mathrm{E}-12$ & $3.83 \mathrm{E}-11$ & 0.0023 & $3.86 \mathrm{E}-11$ \\
\hline $7.10 \mathrm{E}-03$ & $3.35 \mathrm{E}-03$ & $3.87 \mathrm{E}-11$ & 0.0093 & $3.98 \mathrm{E}-11$ & $6.92 \mathrm{E}-12$ & 0.0162 & $7.25 \mathrm{E}-12$ & $3.16 \mathrm{E}-11$ & 0.0025 & $3.18 \mathrm{E}-11$ \\
\hline $1.50 \mathrm{E}-02$ & $7.10 \mathrm{E}-03$ & $3.20 \mathrm{E}-11$ & 0.0100 & $3.29 \mathrm{E}-11$ & $6.09 \mathrm{E}-12$ & 0.0188 & $6.43 \mathrm{E}-12$ & $2.60 \mathrm{E}-11$ & 0.0027 & $2.62 \mathrm{E}-11$ \\
\hline $2.19 \mathrm{E}-02$ & $1.50 \mathrm{E}-02$ & $2.44 \mathrm{E}-11$ & 0.0111 & $2.52 \mathrm{E}-11$ & $5.00 \mathrm{E}-12$ & 0.0190 & $5.29 \mathrm{E}-12$ & $2.07 \mathrm{E}-11$ & 0.0030 & $2.09 \mathrm{E}-11$ \\
\hline $2.42 \mathrm{E}-02$ & $2.19 \mathrm{E}-02$ & $2.19 \mathrm{E}-11$ & 0.0109 & $2.27 \mathrm{E}-11$ & $4.84 \mathrm{E}-12$ & 0.0210 & $5.15 \mathrm{E}-12$ & $1.89 \mathrm{E}-11$ & 0.0030 & $1.91 \mathrm{E}-11$ \\
\hline $2.61 \mathrm{E}-02$ & $2.42 \mathrm{E}-02$ & $2.01 \mathrm{E}-11$ & 0.0116 & $2.08 \mathrm{E}-11$ & $4.66 \mathrm{E}-12$ & 0.0192 & $4.93 \mathrm{E}-12$ & $1.76 \mathrm{E}-11$ & 0.0031 & $1.78 \mathrm{E}-11$ \\
\hline $3.18 \mathrm{E}-02$ & $2.61 \mathrm{E}-02$ & $2.58 \mathrm{E}-11$ & 0.0105 & $2.66 \mathrm{E}-11$ & $5.09 \mathrm{E}-12$ & 0.0193 & $5.39 \mathrm{E}-12$ & $2.07 \mathrm{E}-11$ & 0.0029 & $2.09 \mathrm{E}-11$ \\
\hline $4.09 \mathrm{E}-02$ & $3.18 \mathrm{E}-02$ & $2.87 \mathrm{E}-11$ & 0.0102 & $2.96 \mathrm{E}-11$ & $5.14 \mathrm{E}-12$ & 0.0216 & $5.48 \mathrm{E}-12$ & $2.21 \mathrm{E}-11$ & 0.0029 & $2.23 \mathrm{E}-11$ \\
\hline $6.74 \mathrm{E}-02$ & $4.09 \mathrm{E}-02$ & $1.96 \mathrm{E}-11$ & 0.0122 & $2.03 \mathrm{E}-11$ & $4.26 \mathrm{E}-12$ & 0.0241 & $4.57 \mathrm{E}-12$ & $1.61 \mathrm{E}-11$ & 0.0033 & $1.63 \mathrm{E}-11$ \\
\hline $1.11 \mathrm{E}-01$ & $6.74 \mathrm{E}-02$ & $1.60 \mathrm{E}-11$ & 0.0131 & $1.66 \mathrm{E}-11$ & $3.54 \mathrm{E}-12$ & 0.0243 & $3.80 \mathrm{E}-12$ & $1.33 \mathrm{E}-11$ & 0.0036 & $1.35 \mathrm{E}-11$ \\
\hline $1.83 \mathrm{E}-01$ & $1.11 \mathrm{E}-01$ & $1.23 \mathrm{E}-11$ & 0.0143 & $1.28 \mathrm{E}-11$ & $2.81 \mathrm{E}-12$ & 0.0278 & $3.05 \mathrm{E}-12$ & $1.01 \mathrm{E}-11$ & 0.0040 & $1.03 \mathrm{E}-11$ \\
\hline $2.97 \mathrm{E}-01$ & $1.83 \mathrm{E}-01$ & $8.77 \mathrm{E}-12$ & 0.0159 & $9.19 \mathrm{E}-12$ & $2.27 \mathrm{E}-12$ & 0.0330 & $2.50 \mathrm{E}-12$ & $7.33 \mathrm{E}-12$ & 0.0045 & $7.43 \mathrm{E}-12$ \\
\hline $3.69 \mathrm{E}-01$ & $2.97 \mathrm{E}-01$ & $6.60 \mathrm{E}-12$ & 0.0177 & $6.95 \mathrm{E}-12$ & $1.70 \mathrm{E}-12$ & 0.0297 & $1.85 \mathrm{E}-12$ & $5.55 \mathrm{E}-12$ & 0.0049 & $5.64 \mathrm{E}-12$ \\
\hline $4.98 \mathrm{E}-01$ & $3.69 \mathrm{E}-01$ & $6.19 \mathrm{E}-12$ & 0.0187 & $6.54 \mathrm{E}-12$ & $1.63 \mathrm{E}-12$ & 0.0424 & $1.84 \mathrm{E}-12$ & $5.05 \mathrm{E}-12$ & 0.0053 & $5.13 \mathrm{E}-12$ \\
\hline $6.08 \mathrm{E}-01$ & $4.98 \mathrm{E}-01$ & $5.10 \mathrm{E}-12$ & 0.0204 & $5.42 \mathrm{E}-12$ & $1.14 \mathrm{E}-12$ & 0.0371 & $1.26 \mathrm{E}-12$ & $3.98 \mathrm{E}-12$ & 0.0056 & $4.05 \mathrm{E}-12$ \\
\hline $7.43 \mathrm{E}-01$ & $6.08 \mathrm{E}-01$ & $5.43 \mathrm{E}-12$ & 0.0178 & $5.72 \mathrm{E}-12$ & $1.07 \mathrm{E}-12$ & 0.0388 & $1.20 \mathrm{E}-12$ & $3.88 \mathrm{E}-12$ & 0.0052 & $3.95 \mathrm{E}-12$ \\
\hline $8.21 \mathrm{E}-01$ & $7.43 \mathrm{E}-01$ & $5.88 \mathrm{E}-12$ & 0.0162 & $6.16 \mathrm{E}-12$ & $9.92 \mathrm{E}-13$ & 0.0371 & $1.10 \mathrm{E}-12$ & $4.04 \mathrm{E}-12$ & 0.0048 & $4.10 \mathrm{E}-12$ \\
\hline $1.00 \mathrm{E}+00$ & $8.21 \mathrm{E}-01$ & $9.22 \mathrm{E}-12$ & 0.0100 & $9.50 \mathrm{E}-12$ & $1.13 \mathrm{E}-12$ & 0.0353 & $1.25 \mathrm{E}-12$ & $5.46 \mathrm{E}-12$ & 0.0036 & $5.52 \mathrm{E}-12$ \\
\hline $1.35 \mathrm{E}+00$ & $1.00 \mathrm{E}+00$ & $2.36 \mathrm{E}-11$ & 0.0045 & $2.40 \mathrm{E}-11$ & $1.72 \mathrm{E}-12$ & 0.0225 & $1.84 \mathrm{E}-12$ & $1.23 \mathrm{E}-11$ & 0.0017 & $1.24 \mathrm{E}-11$ \\
\hline $1.65 \mathrm{E}+00$ & $1.35 \mathrm{E}+00$ & $4.18 \mathrm{E}-11$ & 0.0031 & $4.22 \mathrm{E}-11$ & $2.44 \mathrm{E}-12$ & 0.0158 & $2.55 \mathrm{E}-12$ & $2.08 \mathrm{E}-11$ & 0.0011 & $2.08 \mathrm{E}-11$ \\
\hline $1.92 \mathrm{E}+00$ & $1.65 \mathrm{E}+00$ & $5.03 \mathrm{E}-11$ & 0.0029 & $5.07 \mathrm{E}-11$ & $2.90 \mathrm{E}-12$ & 0.0141 & $3.02 \mathrm{E}-12$ & $2.50 \mathrm{E}-11$ & 0.0010 & $2.51 \mathrm{E}-11$ \\
\hline $2.23 \mathrm{E}+00$ & $1.92 \mathrm{E}+00$ & $6.14 \mathrm{E}-11$ & 0.0026 & $6.19 \mathrm{E}-11$ & $3.56 \mathrm{E}-12$ & 0.0137 & $3.71 \mathrm{E}-12$ & $3.04 \mathrm{E}-11$ & 0.0009 & $3.05 \mathrm{E}-11$ \\
\hline $2.35 \mathrm{E}+00$ & $2.23 \mathrm{E}+00$ & $6.45 \mathrm{E}-11$ & 0.0025 & $6.50 \mathrm{E}-11$ & $3.85 \mathrm{E}-12$ & 0.0124 & $3.99 \mathrm{E}-12$ & $3.22 \mathrm{E}-11$ & 0.0009 & $3.23 \mathrm{E}-11$ \\
\hline $2.37 \mathrm{E}+00$ & $2.35 \mathrm{E}+00$ & $6.60 \mathrm{E}-11$ & 0.0025 & $6.65 \mathrm{E}-11$ & $3.84 \mathrm{E}-12$ & 0.0112 & $3.97 \mathrm{E}-12$ & $3.27 \mathrm{E}-11$ & 0.0009 & $3.28 \mathrm{E}-11$ \\
\hline $2.47 \mathrm{E}+00$ & $2.37 \mathrm{E}+00$ & $7.10 \mathrm{E}-11$ & 0.0024 & $7.15 \mathrm{E}-11$ & $4.15 \mathrm{E}-12$ & 0.0117 & $4.30 \mathrm{E}-12$ & $3.52 \mathrm{E}-11$ & 0.0008 & $3.53 \mathrm{E}-11$ \\
\hline $2.73 \mathrm{E}+00$ & $2.47 \mathrm{E}+00$ & $8.38 \mathrm{E}-11$ & 0.0023 & $8.43 \mathrm{E}-11$ & $4.73 \mathrm{E}-12$ & 0.0120 & $4.90 \mathrm{E}-12$ & $4.16 \mathrm{E}-11$ & 0.0008 & $4.17 \mathrm{E}-11$ \\
\hline $3.01 \mathrm{E}+00$ & $2.73 \mathrm{E}+00$ & $9.88 \mathrm{E}-11$ & 0.0021 & $9.94 \mathrm{E}-11$ & $5.41 \mathrm{E}-12$ & 0.0112 & $5.59 \mathrm{E}-12$ & $4.89 \mathrm{E}-11$ & 0.0007 & $4.90 \mathrm{E}-11$ \\
\hline $3.68 \mathrm{E}+00$ & $3.01 \mathrm{E}+00$ & $1.45 \mathrm{E}-10$ & 0.0020 & $1.46 \mathrm{E}-10$ & $7.80 \mathrm{E}-12$ & 0.0098 & $8.03 \mathrm{E}-12$ & $7.13 \mathrm{E}-11$ & 0.0006 & $7.14 \mathrm{E}-11$ \\
\hline $4.97 \mathrm{E}+00$ & $3.68 \mathrm{E}+00$ & $2.10 \mathrm{E}-10$ & 0.0018 & $2.11 \mathrm{E}-10$ & $1.13 \mathrm{E}-11$ & 0.0083 & $1.16 \mathrm{E}-11$ & $1.03 \mathrm{E}-10$ & 0.0006 & $1.03 \mathrm{E}-10$ \\
\hline $6.07 \mathrm{E}+00$ & $4.97 \mathrm{E}+00$ & $2.99 \mathrm{E}-10$ & 0.0018 & $3.00 \mathrm{E}-10$ & $1.58 \mathrm{E}-11$ & 0.0078 & $1.61 \mathrm{E}-11$ & $1.47 \mathrm{E}-10$ & 0.0006 & $1.47 \mathrm{E}-10$ \\
\hline $7.41 \mathrm{E}+00$ & $6.07 \mathrm{E}+00$ & $3.83 \mathrm{E}-10$ & 0.0017 & $3.85 \mathrm{E}-10$ & $2.00 \mathrm{E}-11$ & 0.0076 & $2.05 \mathrm{E}-11$ & $1.89 \mathrm{E}-10$ & 0.0006 & $1.89 \mathrm{E}-10$ \\
\hline $8.61 \mathrm{E}+00$ & $7.41 \mathrm{E}+00$ & $4.68 \mathrm{E}-10$ & 0.0017 & $4.71 \mathrm{E}-10$ & $2.55 \mathrm{E}-11$ & 0.0075 & $2.61 \mathrm{E}-11$ & $2.33 \mathrm{E}-10$ & 0.0005 & $2.33 \mathrm{E}-10$ \\
\hline $1.00 \mathrm{E}+01$ & $8.61 \mathrm{E}+00$ & $5.13 \mathrm{E}-10$ & 0.0017 & $5.16 \mathrm{E}-10$ & $2.85 \mathrm{E}-11$ & 0.0075 & $2.92 \mathrm{E}-11$ & $2.56 \mathrm{E}-10$ & 0.0006 & $2.57 \mathrm{E}-10$ \\
\hline $1.22 \mathrm{E}+01$ & $1.00 \mathrm{E}+01$ & $5.58 \mathrm{E}-10$ & 0.0018 & $5.61 \mathrm{E}-10$ & $3.22 \mathrm{E}-11$ & 0.0076 & $3.30 \mathrm{E}-11$ & $2.80 \mathrm{E}-10$ & 0.0006 & $2.81 \mathrm{E}-10$ \\
\hline $1.42 \mathrm{E}+01$ & $1.22 \mathrm{E}+01$ & $4.87 \mathrm{E}-10$ & 0.0019 & $4.90 \mathrm{E}-10$ & $2.97 \mathrm{E}-11$ & 0.0081 & $3.04 \mathrm{E}-11$ & $2.46 \mathrm{E}-10$ & 0.0006 & $2.47 \mathrm{E}-10$ \\
\hline $1.96 \mathrm{E}+01$ & $1.42 \mathrm{E}+01$ & $3.48 \mathrm{E}-10$ & 0.0019 & $3.50 \mathrm{E}-10$ & $2.16 \mathrm{E}-11$ & 0.0086 & $2.22 \mathrm{E}-11$ & $1.77 \mathrm{E}-10$ & 0.0006 & $1.77 \mathrm{E}-10$ \\
\hline
\end{tabular}


PCP-2011-0001

Revision 0

\section{Table 3-30. Photon Dose Rate per Unit Source in Each of the 77 Source Groups - No} Shielded Container - No Self-shielding

\begin{tabular}{|c|c|c|c|c|c|c|c|c|c|c|}
\hline \multicolumn{2}{|c|}{ Energy $(\mathrm{MeV})$} & \multicolumn{3}{|c|}{ Bottom Dose Rate (rem/hr) } & \multicolumn{3}{|c|}{ Top Dose Rate (rem/hr) } & \multicolumn{3}{|c|}{ Side Dose Rate $(\mathrm{rem} / \mathrm{hr})$} \\
\hline Upper & Lower & mean & FSD & mean $+3 \pi$ & mean & FSD & mean $+3 \pi$ & mean & FSD & mean $+3 \pi$ \\
\hline 0.020 & 0.010 & $0.00 \mathrm{E}+00$ & 0.0000 & $0.00 \mathrm{E}+00$ & $0.00 \mathrm{E}+00$ & 0.0000 & $0.00 \mathrm{E}+00$ & $0.00 \mathrm{E}+00$ & 0.0000 & $0.00 \mathrm{E}+00$ \\
\hline 0.030 & 0.020 & $3.31 \mathrm{E}-29$ & 0.0226 & $3.53 \mathrm{E}-29$ & $0.00 \mathrm{E}+00$ & 0.0000 & $0.00 \mathrm{E}+00$ & $2.47 \mathrm{E}-45$ & 0.0778 & $3.05 \mathrm{E}-45$ \\
\hline 0.040 & 0.030 & $2.41 \mathrm{E}-19$ & 0.0257 & $2.60 \mathrm{E}-19$ & $8.70 \mathrm{E}-44$ & 0.3420 & $1.76 \mathrm{E}-43$ & $2.27 \mathrm{E}-25$ & 0.3778 & $4.85 \mathrm{E}-25$ \\
\hline 0.050 & 0.040 & $1.59 \mathrm{E}-15$ & 0.0328 & $1.75 \mathrm{E}-15$ & $3.35 \mathrm{E}-31$ & 0.2740 & $6.10 \mathrm{E}-31$ & $1.23 \mathrm{E}-18$ & 0.0832 & $1.54 \mathrm{E}-18$ \\
\hline 0.060 & 0.050 & $1.15 \mathrm{E}-13$ & 0.0440 & $1.30 \mathrm{E}-13$ & $2.75 \mathrm{E}-24$ & 0.6894 & $8.43 \mathrm{E}-24$ & $2.58 \mathrm{E}-16$ & 0.0378 & $2.87 \mathrm{E}-16$ \\
\hline 0.070 & 0.060 & $1.06 \mathrm{E}-12$ & 0.0063 & $1.08 \mathrm{E}-12$ & $1.77 \mathrm{E}-21$ & 0.2032 & $2.85 \mathrm{E}-21$ & $6.79 \mathrm{E}-15$ & 0.0111 & $7.01 \mathrm{E}-15$ \\
\hline 0.080 & 0.070 & $4.10 \mathrm{E}-12$ & 0.0051 & $4.17 \mathrm{E}-12$ & $3.92 \mathrm{E}-19$ & 0.2412 & $6.75 \mathrm{E}-19$ & $5.77 \mathrm{E}-14$ & 0.0051 & $5.86 \mathrm{E}-14$ \\
\hline 0.090 & 0.080 & $9.75 \mathrm{E}-12$ & 0.0030 & $9.84 \mathrm{E}-12$ & $7.37 \mathrm{E}-18$ & 0.0885 & $9.33 \mathrm{E}-18$ & $2.45 \mathrm{E}-13$ & 0.0033 & $2.47 \mathrm{E}-13$ \\
\hline 0.100 & 0.090 & $1.73 \mathrm{E}-11$ & 0.0021 & $1.74 \mathrm{E}-11$ & $7.91 \mathrm{E}-17$ & 0.0518 & $9.14 \mathrm{E}-17$ & $6.65 \mathrm{E}-13$ & 0.0020 & $6.69 \mathrm{E}-13$ \\
\hline 0.110 & 0.100 & $2.62 \mathrm{E}-11$ & 0.0022 & $2.63 \mathrm{E}-11$ & $5.50 \mathrm{E}-16$ & 0.1456 & $7.90 \mathrm{E}-16$ & $1.38 \mathrm{E}-12$ & 0.0016 & $1.39 \mathrm{E}-12$ \\
\hline 0.120 & 0.110 & $3.61 \mathrm{E}-11$ & 0.0021 & $3.63 \mathrm{E}-11$ & $2.21 \mathrm{E}-15$ & 0.0967 & $2.85 \mathrm{E}-15$ & $2.39 \mathrm{E}-12$ & 0.0013 & $2.40 \mathrm{E}-12$ \\
\hline 0.130 & 0.120 & $4.67 \mathrm{E}-11$ & 0.0007 & $4.68 \mathrm{E}-11$ & $1.34 \mathrm{E}-14$ & 0.0535 & $1.55 \mathrm{E}-14$ & $4.17 \mathrm{E}-12$ & 0.0005 & $4.18 \mathrm{E}-12$ \\
\hline 0.140 & 0.130 & $5.73 \mathrm{E}-11$ & 0.0007 & $5.74 \mathrm{E}-11$ & $2.48 \mathrm{E}-14$ & 0.0394 & $2.77 \mathrm{E}-14$ & $5.86 \mathrm{E}-12$ & 0.0005 & $5.87 \mathrm{E}-12$ \\
\hline 0.150 & 0.140 & $6.77 \mathrm{E}-11$ & 0.0006 & $6.78 \mathrm{E}-11$ & $4.22 \mathrm{E}-14$ & 0.0297 & $4.59 \mathrm{E}-14$ & $7.69 \mathrm{E}-12$ & 0.0004 & $7.70 \mathrm{E}-12$ \\
\hline 0.160 & 0.150 & $7.80 \mathrm{E}-11$ & 0.0006 & $7.82 \mathrm{E}-11$ & $6.94 \mathrm{E}-14$ & 0.0286 & $7.53 \mathrm{E}-14$ & $9.66 \mathrm{E}-12$ & 0.0004 & $9.67 \mathrm{E}-12$ \\
\hline 0.170 & 0.160 & $8.85 \mathrm{E}-11$ & 0.0006 & $8.87 \mathrm{E}-11$ & $1.00 \mathrm{E}-13$ & 0.0233 & $1.07 \mathrm{E}-13$ & $1.17 \mathrm{E}-11$ & 0.0004 & $1.17 \mathrm{E}-11$ \\
\hline 0.180 & 0.170 & $9.88 \mathrm{E}-11$ & 0.0006 & $9.90 \mathrm{E}-11$ & $1.36 \mathrm{E}-13$ & 0.0192 & $1.43 \mathrm{E}-13$ & $1.39 \mathrm{E}-11$ & 0.0004 & $1.39 \mathrm{E}-11$ \\
\hline 0.190 & 0.180 & $1.09 \mathrm{E}-10$ & 0.0006 & $1.09 \mathrm{E}-10$ & $1.79 \mathrm{E}-13$ & 0.0167 & $1.88 \mathrm{E}-13$ & $1.60 \mathrm{E}-11$ & 0.0003 & $1.60 \mathrm{E}-11$ \\
\hline 0.200 & 0.190 & $1.19 \mathrm{E}-10$ & 0.0006 & $1.19 \mathrm{E}-10$ & $2.33 \mathrm{E}-13$ & 0.0156 & $2.44 \mathrm{E}-13$ & $1.82 \mathrm{E}-11$ & 0.0003 & $1.82 \mathrm{E}-11$ \\
\hline 0.210 & 0.200 & $1.29 \mathrm{E}-10$ & 0.0006 & $1.29 \mathrm{E}-10$ & $2.87 \mathrm{E}-13$ & 0.0142 & $3.00 \mathrm{E}-13$ & $2.04 \mathrm{E}-11$ & 0.0003 & $2.05 \mathrm{E}-11$ \\
\hline 0.220 & 0.210 & $1.38 \mathrm{E}-10$ & 0.0006 & $1.39 \mathrm{E}-10$ & $3.48 \mathrm{E}-13$ & 0.0134 & $3.62 \mathrm{E}-13$ & $2.27 \mathrm{E}-11$ & 0.0003 & $2.27 \mathrm{E}-11$ \\
\hline 0.230 & 0.220 & $1.48 \mathrm{E}-10$ & 0.0006 & $1.48 \mathrm{E}-10$ & $4.10 \mathrm{E}-13$ & 0.0123 & $4.25 \mathrm{E}-13$ & $2.49 \mathrm{E}-11$ & 0.0003 & $2.49 \mathrm{E}-11$ \\
\hline 0.240 & 0.230 & $1.58 \mathrm{E}-10$ & 0.0006 & $1.58 \mathrm{E}-10$ & $4.88 \mathrm{E}-13$ & 0.0116 & $5.05 \mathrm{E}-13$ & $2.71 \mathrm{E}-11$ & 0.0003 & $2.71 \mathrm{E}-11$ \\
\hline 0.250 & 0.240 & $1.67 \mathrm{E}-10$ & 0.0005 & $1.67 \mathrm{E}-10$ & $5.66 \mathrm{E}-13$ & 0.0113 & $5.85 \mathrm{E}-13$ & $2.93 \mathrm{E}-11$ & 0.0003 & $2.94 \mathrm{E}-11$ \\
\hline 0.260 & 0.250 & $1.76 \mathrm{E}-10$ & 0.0005 & $1.76 \mathrm{E}-10$ & $6.35 \mathrm{E}-13$ & 0.0106 & $6.56 \mathrm{E}-13$ & $3.15 \mathrm{E}-11$ & 0.0003 & $3.16 \mathrm{E}-11$ \\
\hline 0.270 & 0.260 & $1.85 \mathrm{E}-10$ & 0.0005 & $1.85 \mathrm{E}-10$ & $7.17 \mathrm{E}-13$ & 0.0101 & $7.38 \mathrm{E}-13$ & $3.37 \mathrm{E}-11$ & 0.0003 & $3.38 \mathrm{E}-11$ \\
\hline 0.280 & 0.270 & $1.94 \mathrm{E}-10$ & 0.0005 & $1.94 \mathrm{E}-10$ & $8.08 \mathrm{E}-13$ & 0.0098 & $8.32 \mathrm{E}-13$ & $3.59 \mathrm{E}-11$ & 0.0003 & $3.60 \mathrm{E}-11$ \\
\hline 0.290 & 0.280 & $2.03 \mathrm{E}-10$ & 0.0005 & $2.03 \mathrm{E}-10$ & $8.94 \mathrm{E}-13$ & 0.0096 & $9.19 \mathrm{E}-13$ & $3.81 \mathrm{E}-11$ & 0.0003 & $3.82 \mathrm{E}-11$ \\
\hline 0.300 & 0.290 & $2.12 \mathrm{E}-10$ & 0.0005 & $2.12 \mathrm{E}-10$ & $9.79 \mathrm{E}-13$ & 0.0092 & $1.01 \mathrm{E}-12$ & $4.03 \mathrm{E}-11$ & 0.0003 & $4.03 \mathrm{E}-11$ \\
\hline 0.325 & 0.300 & $2.26 \mathrm{E}-10$ & 0.0005 & $2.27 \mathrm{E}-10$ & $1.13 \mathrm{E}-12$ & 0.0088 & $1.16 \mathrm{E}-12$ & $4.40 \mathrm{E}-11$ & 0.0003 & $4.41 \mathrm{E}-11$ \\
\hline 0.350 & 0.325 & $2.47 \mathrm{E}-10$ & 0.0005 & $2.47 \mathrm{E}-10$ & $1.38 \mathrm{E}-12$ & 0.0082 & $1.41 \mathrm{E}-12$ & $4.93 \mathrm{E}-11$ & 0.0003 & $4.93 \mathrm{E}-11$ \\
\hline 0.375 & 0.350 & $2.66 \mathrm{E}-10$ & 0.0005 & $2.67 \mathrm{E}-10$ & $1.60 \mathrm{E}-12$ & 0.0074 & $1.63 \mathrm{E}-12$ & $5.44 \mathrm{E}-11$ & 0.0003 & $5.44 \mathrm{E}-11$ \\
\hline 0.400 & 0.375 & $2.85 \mathrm{E}-10$ & 0.0005 & $2.85 \mathrm{E}-10$ & $1.86 \mathrm{E}-12$ & 0.0072 & $1.90 \mathrm{E}-12$ & $5.93 \mathrm{E}-11$ & 0.0002 & $5.94 \mathrm{E}-11$ \\
\hline 0.425 & 0.400 & $3.02 \mathrm{E}-10$ & 0.0005 & $3.03 \mathrm{E}-10$ & $2.10 \mathrm{E}-12$ & 0.0070 & $2.15 \mathrm{E}-12$ & $6.41 \mathrm{E}-11$ & 0.0002 & $6.42 \mathrm{E}-11$ \\
\hline 0.450 & 0.425 & $3.19 \mathrm{E}-10$ & 0.0005 & $3.19 \mathrm{E}-10$ & $2.39 \mathrm{E}-12$ & 0.0067 & $2.43 \mathrm{E}-12$ & $6.88 \mathrm{E}-11$ & 0.0002 & $6.88 \mathrm{E}-11$ \\
\hline 0.475 & 0.450 & $3.35 \mathrm{E}-10$ & 0.0005 & $3.35 \mathrm{E}-10$ & $2.66 \mathrm{E}-12$ & 0.0065 & $2.72 \mathrm{E}-12$ & $7.33 \mathrm{E}-11$ & 0.0002 & $7.34 \mathrm{E}-11$ \\
\hline 0.500 & 0.475 & $3.51 \mathrm{E}-10$ & 0.0005 & $3.51 \mathrm{E}-10$ & $2.92 \mathrm{E}-12$ & 0.0062 & $2.97 \mathrm{E}-12$ & $7.77 \mathrm{E}-11$ & 0.0002 & $7.78 \mathrm{E}-11$ \\
\hline 0.525 & 0.500 & $3.66 \mathrm{E}-10$ & 0.0005 & $3.67 \mathrm{E}-10$ & $3.18 \mathrm{E}-12$ & 0.0060 & $3.24 \mathrm{E}-12$ & $8.22 \mathrm{E}-11$ & 0.0002 & $8.22 \mathrm{E}-11$ \\
\hline
\end{tabular}


PCP-2011-0001

Revision 0

\section{Table 3-30. Photon Dose Rate per Unit Source in Each of the 77 Source Groups - No} Shielded Container - No Self-shielding

\begin{tabular}{|c|c|c|c|c|c|c|c|c|c|c|}
\hline \multicolumn{2}{|c|}{ Energy $(\mathrm{MeV})$} & \multicolumn{3}{|c|}{ Bottom Dose Rate (rem/hr) } & \multicolumn{3}{|c|}{ Top Dose Rate (rem/hr) } & \multicolumn{3}{|c|}{ Side Dose Rate $(\mathrm{rem} / \mathrm{hr})$} \\
\hline Upper & Lower & mean & FSD & mean $+3 \pi$ & mean & FSD & mean $+3 \pi$ & mean & FSD & mean $+3 \pi$ \\
\hline 0.550 & 0.525 & $3.82 \mathrm{E}-10$ & 0.0005 & 3.83E-10 & $3.45 \mathrm{E}-12$ & 0.0057 & $3.51 \mathrm{E}-12$ & $8.66 \mathrm{E}-11$ & 0.0002 & $8.67 \mathrm{E}-11$ \\
\hline 0.575 & 0.550 & $3.97 \mathrm{E}-10$ & 0.0005 & $3.98 \mathrm{E}-10$ & $3.71 \mathrm{E}-12$ & 0.0056 & $3.78 \mathrm{E}-12$ & $9.10 \mathrm{E}-11$ & 0.0002 & $9.11 \mathrm{E}-11$ \\
\hline 0.600 & 0.575 & $4.12 \mathrm{E}-10$ & 0.0005 & $4.13 \mathrm{E}-10$ & $3.97 \mathrm{E}-12$ & 0.0055 & $4.04 \mathrm{E}-12$ & $9.53 \mathrm{E}-11$ & 0.0002 & $9.53 \mathrm{E}-11$ \\
\hline 0.625 & 0.600 & $4.26 \mathrm{E}-10$ & 0.0005 & $4.27 \mathrm{E}-10$ & $4.26 \mathrm{E}-12$ & 0.0053 & $4.32 \mathrm{E}-12$ & $9.94 \mathrm{E}-11$ & 0.0002 & $9.95 \mathrm{E}-11$ \\
\hline 0.650 & 0.625 & $4.39 \mathrm{E}-10$ & 0.0005 & $4.40 \mathrm{E}-10$ & $4.53 \mathrm{E}-12$ & 0.0052 & $4.60 \mathrm{E}-12$ & $1.03 \mathrm{E}-10$ & 0.0002 & $1.04 \mathrm{E}-10$ \\
\hline 0.675 & 0.650 & $4.53 \mathrm{E}-10$ & 0.0005 & $4.53 \mathrm{E}-10$ & $4.81 \mathrm{E}-12$ & 0.0051 & $4.88 \mathrm{E}-12$ & $1.07 \mathrm{E}-10$ & 0.0002 & $1.08 \mathrm{E}-10$ \\
\hline 0.700 & 0.675 & $4.66 \mathrm{E}-10$ & 0.0005 & $4.66 \mathrm{E}-10$ & $5.11 \mathrm{E}-12$ & 0.0050 & $5.19 \mathrm{E}-12$ & $1.11 \mathrm{E}-10$ & 0.0002 & $1.12 \mathrm{E}-10$ \\
\hline 0.725 & 0.700 & $4.79 \mathrm{E}-10$ & 0.0005 & $4.80 \mathrm{E}-10$ & $5.39 \mathrm{E}-12$ & 0.0049 & $5.47 \mathrm{E}-12$ & $1.15 \mathrm{E}-10$ & 0.0002 & $1.15 \mathrm{E}-10$ \\
\hline 0.750 & 0.725 & $4.92 \mathrm{E}-10$ & 0.0005 & $4.93 \mathrm{E}-10$ & $5.68 \mathrm{E}-12$ & 0.0048 & $5.76 \mathrm{E}-12$ & $1.19 \mathrm{E}-10$ & 0.0002 & $1.19 \mathrm{E}-10$ \\
\hline 0.775 & 0.750 & $5.05 \mathrm{E}-10$ & 0.0005 & $5.06 \mathrm{E}-10$ & $5.94 \mathrm{E}-12$ & 0.0048 & $6.03 \mathrm{E}-12$ & $1.23 \mathrm{E}-10$ & 0.0002 & $1.23 \mathrm{E}-10$ \\
\hline 0.800 & 0.775 & $5.18 \mathrm{E}-10$ & 0.0005 & $5.18 \mathrm{E}-10$ & $6.22 \mathrm{E}-12$ & 0.0046 & $6.31 \mathrm{E}-12$ & $1.27 \mathrm{E}-10$ & 0.0002 & $1.27 \mathrm{E}-10$ \\
\hline 0.825 & 0.800 & $5.30 \mathrm{E}-10$ & 0.0005 & $5.31 \mathrm{E}-10$ & $6.52 \mathrm{E}-12$ & 0.0046 & $6.61 \mathrm{E}-12$ & $1.31 \mathrm{E}-10$ & 0.0002 & $1.31 \mathrm{E}-10$ \\
\hline 0.850 & 0.825 & $5.42 \mathrm{E}-10$ & 0.0005 & $5.43 \mathrm{E}-10$ & $6.83 \mathrm{E}-12$ & 0.0046 & $6.93 \mathrm{E}-12$ & $1.35 \mathrm{E}-10$ & 0.0002 & $1.35 \mathrm{E}-10$ \\
\hline 0.875 & 0.850 & $5.54 \mathrm{E}-10$ & 0.0005 & $5.55 \mathrm{E}-10$ & $7.14 \mathrm{E}-12$ & 0.0045 & $7.23 \mathrm{E}-12$ & $1.39 \mathrm{E}-10$ & 0.0002 & $1.39 \mathrm{E}-10$ \\
\hline 0.900 & 0.875 & $5.66 \mathrm{E}-10$ & 0.0005 & $5.67 \mathrm{E}-10$ & $7.41 \mathrm{E}-12$ & 0.0044 & $7.51 \mathrm{E}-12$ & $1.42 \mathrm{E}-10$ & 0.0002 & $1.42 \mathrm{E}-10$ \\
\hline 0.925 & 0.900 & $5.78 \mathrm{E}-10$ & 0.0005 & $5.79 \mathrm{E}-10$ & $7.69 \mathrm{E}-12$ & 0.0043 & $7.79 \mathrm{E}-12$ & $1.46 \mathrm{E}-10$ & 0.0002 & $1.46 \mathrm{E}-10$ \\
\hline 0.950 & 0.925 & $5.90 \mathrm{E}-10$ & 0.0005 & $5.91 \mathrm{E}-10$ & $8.00 \mathrm{E}-12$ & 0.0043 & $8.10 \mathrm{E}-12$ & $1.50 \mathrm{E}-10$ & 0.0002 & $1.50 \mathrm{E}-10$ \\
\hline 0.975 & 0.950 & $6.02 \mathrm{E}-10$ & 0.0005 & $6.03 \mathrm{E}-10$ & $8.31 \mathrm{E}-12$ & 0.0042 & $8.42 \mathrm{E}-12$ & $1.54 \mathrm{E}-10$ & 0.0002 & $1.54 \mathrm{E}-10$ \\
\hline 1.000 & 0.975 & $6.14 \mathrm{E}-10$ & 0.0005 & $6.14 \mathrm{E}-10$ & $8.59 \mathrm{E}-12$ & 0.0042 & $8.70 \mathrm{E}-12$ & $1.57 \mathrm{E}-10$ & 0.0002 & $1.57 \mathrm{E}-10$ \\
\hline 1.250 & 1.000 & $6.74 \mathrm{E}-10$ & 0.0005 & $6.75 \mathrm{E}-10$ & $1.03 \mathrm{E}-11$ & 0.0040 & $1.04 \mathrm{E}-11$ & $1.77 \mathrm{E}-10$ & 0.0002 & $1.77 \mathrm{E}-10$ \\
\hline 1.500 & 1.250 & $7.79 \mathrm{E}-10$ & 0.0005 & $7.80 \mathrm{E}-10$ & $1.32 \mathrm{E}-11$ & 0.0037 & $1.33 \mathrm{E}-11$ & $2.12 \mathrm{E}-10$ & 0.0002 & $2.12 \mathrm{E}-10$ \\
\hline 1.750 & 1.500 & $8.79 \mathrm{E}-10$ & 0.0005 & $8.80 \mathrm{E}-10$ & $1.61 \mathrm{E}-11$ & 0.0034 & $1.62 \mathrm{E}-11$ & $2.45 \mathrm{E}-10$ & 0.0002 & $2.45 \mathrm{E}-10$ \\
\hline 2.000 & 1.750 & $9.72 \mathrm{E}-10$ & 0.0005 & $9.74 \mathrm{E}-10$ & $1.88 \mathrm{E}-11$ & 0.0033 & $1.89 \mathrm{E}-11$ & $2.76 \mathrm{E}-10$ & 0.0002 & $2.77 \mathrm{E}-10$ \\
\hline 2.250 & 2.000 & $1.06 \mathrm{E}-09$ & 0.0005 & $1.06 \mathrm{E}-09$ & $2.14 \mathrm{E}-11$ & 0.0032 & $2.16 \mathrm{E}-11$ & $3.06 \mathrm{E}-10$ & 0.0002 & $3.07 \mathrm{E}-10$ \\
\hline 2.500 & 2.250 & $1.14 \mathrm{E}-09$ & 0.0005 & $1.15 \mathrm{E}-09$ & $2.40 \mathrm{E}-11$ & 0.0032 & $2.42 \mathrm{E}-11$ & $3.35 \mathrm{E}-10$ & 0.0002 & $3.35 \mathrm{E}-10$ \\
\hline 2.750 & 2.500 & $1.23 \mathrm{E}-09$ & 0.0005 & $1.23 \mathrm{E}-09$ & $2.63 \mathrm{E}-11$ & 0.0031 & $2.66 \mathrm{E}-11$ & $3.62 \mathrm{E}-10$ & 0.0002 & $3.63 \mathrm{E}-10$ \\
\hline 3.000 & 2.750 & $1.30 \mathrm{E}-09$ & 0.0005 & $1.31 \mathrm{E}-09$ & $2.86 \mathrm{E}-11$ & 0.0031 & $2.89 \mathrm{E}-11$ & $3.89 \mathrm{E}-10$ & 0.0002 & $3.89 \mathrm{E}-10$ \\
\hline 3.500 & 3.000 & $1.41 \mathrm{E}-09$ & 0.0005 & $1.41 \mathrm{E}-09$ & $3.17 \mathrm{E}-11$ & 0.0030 & $3.20 \mathrm{E}-11$ & $4.26 \mathrm{E}-10$ & 0.0002 & $4.26 \mathrm{E}-10$ \\
\hline 4.000 & 3.500 & $1.55 \mathrm{E}-09$ & 0.0005 & $1.55 \mathrm{E}-09$ & $3.54 \mathrm{E}-11$ & 0.0030 & $3.57 \mathrm{E}-11$ & $4.72 \mathrm{E}-10$ & 0.0002 & $4.73 \mathrm{E}-10$ \\
\hline 4.500 & 4.000 & $1.68 \mathrm{E}-09$ & 0.0005 & $1.68 \mathrm{E}-09$ & $3.90 \mathrm{E}-11$ & 0.0030 & $3.93 \mathrm{E}-11$ & $5.16 \mathrm{E}-10$ & 0.0002 & $5.16 \mathrm{E}-10$ \\
\hline 5.000 & 4.500 & $1.81 \mathrm{E}-09$ & 0.0005 & $1.81 \mathrm{E}-09$ & $4.22 \mathrm{E}-11$ & 0.0030 & $4.25 \mathrm{E}-11$ & $5.57 \mathrm{E}-10$ & 0.0002 & $5.57 \mathrm{E}-10$ \\
\hline 5.500 & 5.000 & 1.93E-09 & 0.0005 & $1.94 \mathrm{E}-09$ & $4.54 \mathrm{E}-11$ & 0.0030 & $4.58 \mathrm{E}-11$ & $5.99 \mathrm{E}-10$ & 0.0002 & $5.99 \mathrm{E}-10$ \\
\hline 6.000 & 5.500 & $2.05 \mathrm{E}-09$ & 0.0005 & $2.06 \mathrm{E}-09$ & $4.82 \mathrm{E}-11$ & 0.0031 & $4.87 \mathrm{E}-11$ & $6.38 \mathrm{E}-10$ & 0.0002 & $6.38 \mathrm{E}-10$ \\
\hline 6.500 & 6.000 & $2.17 \mathrm{E}-09$ & 0.0005 & $2.18 \mathrm{E}-09$ & $5.10 \mathrm{E}-11$ & 0.0031 & $5.14 \mathrm{E}-11$ & $6.76 \mathrm{E}-10$ & 0.0002 & $6.76 \mathrm{E}-10$ \\
\hline 7.000 & 6.500 & $2.29 \mathrm{E}-09$ & 0.0005 & $2.30 \mathrm{E}-09$ & $5.37 \mathrm{E}-11$ & 0.0031 & $5.42 \mathrm{E}-11$ & $7.14 \mathrm{E}-10$ & 0.0002 & $7.14 \mathrm{E}-10$ \\
\hline 7.500 & 7.000 & $2.41 \mathrm{E}-09$ & 0.0005 & $2.41 \mathrm{E}-09$ & $5.63 \mathrm{E}-11$ & 0.0032 & $5.68 \mathrm{E}-11$ & $7.51 \mathrm{E}-10$ & 0.0002 & $7.52 \mathrm{E}-10$ \\
\hline 8.000 & 7.500 & $2.53 \mathrm{E}-09$ & 0.0005 & $2.53 \mathrm{E}-09$ & $5.89 \mathrm{E}-11$ & 0.0032 & $5.95 \mathrm{E}-11$ & $7.89 \mathrm{E}-10$ & 0.0002 & $7.89 \mathrm{E}-10$ \\
\hline 9.000 & 8.000 & $2.70 \mathrm{E}-09$ & 0.0005 & $2.71 \mathrm{E}-09$ & $6.28 \mathrm{E}-11$ & 0.0032 & $6.34 \mathrm{E}-11$ & $8.44 \mathrm{E}-10$ & 0.0002 & $8.44 \mathrm{E}-10$ \\
\hline 10.000 & 9.000 & $2.94 \mathrm{E}-09$ & 0.0005 & $2.95 \mathrm{E}-09$ & $6.81 \mathrm{E}-11$ & 0.0033 & $6.88 \mathrm{E}-11$ & $9.18 \mathrm{E}-10$ & 0.0002 & $9.19 \mathrm{E}-10$ \\
\hline
\end{tabular}


PCP-2011-0001

Revision 0

Table 3-31. Neutron Dose Rate per Unit Neutron Source in Each of the 47 Source Groups No Shielded Container - $\mathrm{PuO}_{2}$ Self-shielding

\begin{tabular}{|c|c|c|c|c|c|c|c|c|c|c|}
\hline \multicolumn{2}{|c|}{ Energy (MeV) } & \multicolumn{3}{|c|}{ Bottom Dose Rate (rem/hr) } & \multicolumn{3}{|c|}{ Top Dose Rate (rem/hr) } & \multicolumn{3}{|c|}{ Side Dose Rate (rem $/ \mathrm{hr})$} \\
\hline Upper & Lower & mean & FSD & mean $+3 \pi$ & mean & FSD & mean $+3 \pi$ & mean & FSD & mean $+3 \pi$ \\
\hline $1.00 \mathrm{E}-07$ & $1.00 \mathrm{E}-11$ & $7.13 \mathrm{E}-08$ & $8.00 \mathrm{E}-04$ & $7.14 \mathrm{E}-08$ & $3.29 \mathrm{E}-09$ & $4.70 \mathrm{E}-03$ & $3.33 \mathrm{E}-09$ & $2.84 \mathrm{E}-08$ & $3.00 \mathrm{E}-04$ & $2.84 \mathrm{E}-08$ \\
\hline $4.14 \mathrm{E}-07$ & $1.00 \mathrm{E}-07$ & $6.64 \mathrm{E}-08$ & $8.00 \mathrm{E}-04$ & $6.66 \mathrm{E}-08$ & $3.06 \mathrm{E}-09$ & $4.40 \mathrm{E}-03$ & $3.10 \mathrm{E}-09$ & $2.65 \mathrm{E}-08$ & $3.00 \mathrm{E}-04$ & $2.65 \mathrm{E}-08$ \\
\hline $8.76 \mathrm{E}-07$ & $4.14 \mathrm{E}-07$ & $3.40 \mathrm{E}-08$ & $1.00 \mathrm{E}-03$ & $3.41 \mathrm{E}-08$ & $1.53 \mathrm{E}-09$ & $5.60 \mathrm{E}-03$ & $1.55 \mathrm{E}-09$ & $1.35 \mathrm{E}-08$ & $4.00 \mathrm{E}-04$ & $1.35 \mathrm{E}-08$ \\
\hline $1.86 \mathrm{E}-06$ & $8.76 \mathrm{E}-07$ & $1.22 \mathrm{E}-08$ & $1.60 \mathrm{E}-03$ & $1.23 \mathrm{E}-08$ & $5.29 \mathrm{E}-10$ & $8.80 \mathrm{E}-03$ & $5.43 \mathrm{E}-10$ & $4.82 \mathrm{E}-09$ & $6.00 \mathrm{E}-04$ & $4.83 \mathrm{E}-09$ \\
\hline $5.04 \mathrm{E}-06$ & $1.86 \mathrm{E}-06$ & $6.13 \mathrm{E}-09$ & $1.90 \mathrm{E}-03$ & $6.17 \mathrm{E}-09$ & $2.55 \mathrm{E}-10$ & $1.11 \mathrm{E}-02$ & $2.64 \mathrm{E}-10$ & $2.43 \mathrm{E}-09$ & $8.00 \mathrm{E}-04$ & $2.44 \mathrm{E}-09$ \\
\hline $1.07 \mathrm{E}-05$ & $5.04 \mathrm{E}-06$ & $1.01 \mathrm{E}-08$ & $1.40 \mathrm{E}-03$ & $1.01 \mathrm{E}-08$ & $4.40 \mathrm{E}-10$ & $8.10 \mathrm{E}-03$ & $4.50 \mathrm{E}-10$ & $4.02 \mathrm{E}-09$ & $6.00 \mathrm{E}-04$ & $4.03 \mathrm{E}-09$ \\
\hline $3.73 \mathrm{E}-05$ & $1.07 \mathrm{E}-05$ & $1.16 \mathrm{E}-08$ & $1.30 \mathrm{E}-03$ & $1.16 \mathrm{E}-08$ & $5.08 \mathrm{E}-10$ & $7.10 \mathrm{E}-03$ & $5.18 \mathrm{E}-10$ & $4.62 \mathrm{E}-09$ & $5.00 \mathrm{E}-04$ & $4.63 \mathrm{E}-09$ \\
\hline $1.01 \mathrm{E}-04$ & $3.73 \mathrm{E}-05$ & $1.53 \mathrm{E}-08$ & $1.30 \mathrm{E}-03$ & $1.54 \mathrm{E}-08$ & $6.86 \mathrm{E}-10$ & $6.90 \mathrm{E}-03$ & $7.01 \mathrm{E}-10$ & $6.12 \mathrm{E}-09$ & $5.00 \mathrm{E}-04$ & $6.13 \mathrm{E}-09$ \\
\hline $2.14 \mathrm{E}-04$ & $1.01 \mathrm{E}-04$ & $8.43 \mathrm{E}-09$ & $1.50 \mathrm{E}-03$ & $8.46 \mathrm{E}-09$ & $3.67 \mathrm{E}-10$ & $8.10 \mathrm{E}-03$ & $3.76 \mathrm{E}-10$ & $3.39 \mathrm{E}-09$ & $6.00 \mathrm{E}-04$ & $3.39 \mathrm{E}-09$ \\
\hline $4.54 \mathrm{E}-04$ & $2.14 \mathrm{E}-04$ & $6.39 \mathrm{E}-09$ & $1.70 \mathrm{E}-03$ & $6.42 \mathrm{E}-09$ & $2.81 \mathrm{E}-10$ & $9.10 \mathrm{E}-03$ & $2.89 \mathrm{E}-10$ & $2.57 \mathrm{E}-09$ & $7.00 \mathrm{E}-04$ & $2.58 \mathrm{E}-09$ \\
\hline $1.58 \mathrm{E}-03$ & $4.54 \mathrm{E}-04$ & $4.57 \mathrm{E}-09$ & $1.80 \mathrm{E}-03$ & $4.60 \mathrm{E}-09$ & $1.99 \mathrm{E}-10$ & $9.60 \mathrm{E}-03$ & $2.04 \mathrm{E}-10$ & $1.88 \mathrm{E}-09$ & $7.00 \mathrm{E}-04$ & $1.89 \mathrm{E}-09$ \\
\hline $3.35 \mathrm{E}-03$ & $1.58 \mathrm{E}-03$ & $3.44 \mathrm{E}-09$ & $1.80 \mathrm{E}-03$ & $3.46 \mathrm{E}-09$ & $1.45 \mathrm{E}-10$ & $1.03 \mathrm{E}-02$ & $1.50 \mathrm{E}-10$ & $1.42 \mathrm{E}-09$ & $7.00 \mathrm{E}-04$ & $1.43 \mathrm{E}-09$ \\
\hline $7.10 \mathrm{E}-03$ & $3.35 \mathrm{E}-03$ & $2.90 \mathrm{E}-09$ & $1.80 \mathrm{E}-03$ & $2.92 \mathrm{E}-09$ & $1.25 \mathrm{E}-10$ & $9.50 \mathrm{E}-03$ & $1.28 \mathrm{E}-10$ & $1.21 \mathrm{E}-09$ & $7.00 \mathrm{E}-04$ & $1.21 \mathrm{E}-09$ \\
\hline $1.50 \mathrm{E}-02$ & $7.10 \mathrm{E}-03$ & $2.69 \mathrm{E}-09$ & $1.80 \mathrm{E}-03$ & $2.70 \mathrm{E}-09$ & $1.23 \mathrm{E}-10$ & $9.90 \mathrm{E}-03$ & $1.27 \mathrm{E}-10$ & $1.14 \mathrm{E}-09$ & $7.00 \mathrm{E}-04$ & $1.14 \mathrm{E}-09$ \\
\hline $2.19 \mathrm{E}-02$ & $1.50 \mathrm{E}-02$ & $2.75 \mathrm{E}-09$ & $1.60 \mathrm{E}-03$ & $2.77 \mathrm{E}-09$ & $1.32 \mathrm{E}-10$ & $8.30 \mathrm{E}-03$ & $1.36 \mathrm{E}-10$ & $1.16 \mathrm{E}-09$ & $6.00 \mathrm{E}-04$ & $1.16 \mathrm{E}-09$ \\
\hline $2.42 \mathrm{E}-02$ & $2.19 \mathrm{E}-02$ & $2.76 \mathrm{E}-09$ & $1.50 \mathrm{E}-03$ & $2.78 \mathrm{E}-09$ & $1.58 \mathrm{E}-10$ & $7.00 \mathrm{E}-03$ & $1.62 \mathrm{E}-10$ & $1.19 \mathrm{E}-09$ & $5.00 \mathrm{E}-04$ & $1.19 \mathrm{E}-09$ \\
\hline $2.61 \mathrm{E}-02$ & $2.42 \mathrm{E}-02$ & $2.84 \mathrm{E}-09$ & $1.50 \mathrm{E}-03$ & $2.85 \mathrm{E}-09$ & $1.73 \mathrm{E}-10$ & $6.50 \mathrm{E}-03$ & $1.77 \mathrm{E}-10$ & $1.22 \mathrm{E}-09$ & $5.00 \mathrm{E}-04$ & $1.22 \mathrm{E}-09$ \\
\hline $3.18 \mathrm{E}-02$ & $2.61 \mathrm{E}-02$ & $3.21 \mathrm{E}-09$ & $1.50 \mathrm{E}-03$ & $3.22 \mathrm{E}-09$ & $1.67 \mathrm{E}-10$ & $6.90 \mathrm{E}-03$ & $1.70 \mathrm{E}-10$ & $1.26 \mathrm{E}-09$ & $6.00 \mathrm{E}-04$ & $1.26 \mathrm{E}-09$ \\
\hline $4.09 \mathrm{E}-02$ & $3.18 \mathrm{E}-02$ & $3.65 \mathrm{E}-09$ & $1.40 \mathrm{E}-03$ & $3.66 \mathrm{E}-09$ & $1.71 \mathrm{E}-10$ & $7.00 \mathrm{E}-03$ & $1.74 \mathrm{E}-10$ & $1.31 \mathrm{E}-09$ & $6.00 \mathrm{E}-04$ & $1.31 \mathrm{E}-09$ \\
\hline $6.74 \mathrm{E}-02$ & $4.09 \mathrm{E}-02$ & $4.17 \mathrm{E}-09$ & $1.10 \mathrm{E}-03$ & $4.18 \mathrm{E}-09$ & $1.92 \mathrm{E}-10$ & $6.40 \mathrm{E}-03$ & $1.96 \mathrm{E}-10$ & $1.55 \mathrm{E}-09$ & $5.00 \mathrm{E}-04$ & $1.55 \mathrm{E}-09$ \\
\hline $1.11 \mathrm{E}-01$ & $6.74 \mathrm{E}-02$ & $5.91 \mathrm{E}-09$ & $1.00 \mathrm{E}-03$ & $5.92 \mathrm{E}-09$ & $2.76 \mathrm{E}-10$ & $5.20 \mathrm{E}-03$ & $2.80 \mathrm{E}-10$ & $2.08 \mathrm{E}-09$ & $4.00 \mathrm{E}-04$ & $2.08 \mathrm{E}-09$ \\
\hline $1.83 \mathrm{E}-01$ & $1.11 \mathrm{E}-01$ & $9.20 \mathrm{E}-09$ & $8.00 \mathrm{E}-04$ & $9.22 \mathrm{E}-09$ & $4.44 \mathrm{E}-10$ & $4.30 \mathrm{E}-03$ & $4.50 \mathrm{E}-10$ & $3.18 \mathrm{E}-09$ & $3.00 \mathrm{E}-04$ & $3.18 \mathrm{E}-09$ \\
\hline $2.97 \mathrm{E}-01$ & $1.83 \mathrm{E}-01$ & $1.44 \mathrm{E}-08$ & $7.00 \mathrm{E}-04$ & $1.44 \mathrm{E}-08$ & $6.42 \mathrm{E}-10$ & $4.20 \mathrm{E}-03$ & $6.51 \mathrm{E}-10$ & $5.13 \mathrm{E}-09$ & $3.00 \mathrm{E}-04$ & $5.13 \mathrm{E}-09$ \\
\hline $3.69 \mathrm{E}-01$ & $2.97 \mathrm{E}-01$ & $1.94 \mathrm{E}-08$ & $7.00 \mathrm{E}-04$ & $1.95 \mathrm{E}-08$ & $9.26 \mathrm{E}-10$ & $3.70 \mathrm{E}-03$ & $9.36 \mathrm{E}-10$ & $7.11 \mathrm{E}-09$ & $3.00 \mathrm{E}-04$ & $7.12 \mathrm{E}-09$ \\
\hline $4.98 \mathrm{E}-01$ & $3.69 \mathrm{E}-01$ & $2.51 \mathrm{E}-08$ & $7.00 \mathrm{E}-04$ & $2.52 \mathrm{E}-08$ & $1.10 \mathrm{E}-09$ & $4.00 \mathrm{E}-03$ & $1.11 \mathrm{E}-09$ & $9.18 \mathrm{E}-09$ & $3.00 \mathrm{E}-04$ & $9.19 \mathrm{E}-09$ \\
\hline $6.08 \mathrm{E}-01$ & $4.98 \mathrm{E}-01$ & $3.17 \mathrm{E}-08$ & $6.00 \mathrm{E}-04$ & $3.17 \mathrm{E}-08$ & $1.53 \mathrm{E}-09$ & $3.50 \mathrm{E}-03$ & $1.54 \mathrm{E}-09$ & $1.18 \mathrm{E}-08$ & $2.00 \mathrm{E}-04$ & $1.18 \mathrm{E}-08$ \\
\hline $7.43 \mathrm{E}-01$ & $6.08 \mathrm{E}-01$ & $3.59 \mathrm{E}-08$ & $6.00 \mathrm{E}-04$ & $3.60 \mathrm{E}-08$ & $1.78 \mathrm{E}-09$ & $3.20 \mathrm{E}-03$ & $1.79 \mathrm{E}-09$ & $1.37 \mathrm{E}-08$ & $2.00 \mathrm{E}-04$ & $1.38 \mathrm{E}-08$ \\
\hline $8.21 \mathrm{E}-01$ & $7.43 \mathrm{E}-01$ & $4.04 \mathrm{E}-08$ & $6.00 \mathrm{E}-04$ & $4.05 \mathrm{E}-08$ & $1.78 \mathrm{E}-09$ & $3.40 \mathrm{E}-03$ & $1.80 \mathrm{E}-09$ & $1.52 \mathrm{E}-08$ & $2.00 \mathrm{E}-04$ & $1.52 \mathrm{E}-08$ \\
\hline $1.00 \mathrm{E}+00$ & $8.21 \mathrm{E}-01$ & $4.23 \mathrm{E}-08$ & $6.00 \mathrm{E}-04$ & $4.24 \mathrm{E}-08$ & $2.04 \mathrm{E}-09$ & $3.30 \mathrm{E}-03$ & $2.06 \mathrm{E}-09$ & $1.66 \mathrm{E}-08$ & $2.00 \mathrm{E}-04$ & $1.66 \mathrm{E}-08$ \\
\hline $1.35 \mathrm{E}+00$ & $1.00 \mathrm{E}+00$ & $4.54 \mathrm{E}-08$ & $6.00 \mathrm{E}-04$ & $4.54 \mathrm{E}-08$ & $2.14 \mathrm{E}-09$ & $3.30 \mathrm{E}-03$ & $2.16 \mathrm{E}-09$ & $1.79 \mathrm{E}-08$ & $2.00 \mathrm{E}-04$ & $1.79 \mathrm{E}-08$ \\
\hline $1.65 \mathrm{E}+00$ & $1.35 \mathrm{E}+00$ & $4.64 \mathrm{E}-08$ & $6.00 \mathrm{E}-04$ & $4.65 \mathrm{E}-08$ & $2.14 \mathrm{E}-09$ & $3.40 \mathrm{E}-03$ & $2.17 \mathrm{E}-09$ & $1.85 \mathrm{E}-08$ & $2.00 \mathrm{E}-04$ & $1.85 \mathrm{E}-08$ \\
\hline $1.92 \mathrm{E}+00$ & $1.65 \mathrm{E}+00$ & $4.65 \mathrm{E}-08$ & $6.00 \mathrm{E}-04$ & $4.66 \mathrm{E}-08$ & $2.15 \mathrm{E}-09$ & $3.30 \mathrm{E}-03$ & $2.17 \mathrm{E}-09$ & $1.88 \mathrm{E}-08$ & $2.00 \mathrm{E}-04$ & $1.88 \mathrm{E}-08$ \\
\hline $2.23 \mathrm{E}+00$ & $1.92 \mathrm{E}+00$ & $4.71 \mathrm{E}-08$ & $6.00 \mathrm{E}-04$ & $4.72 \mathrm{E}-08$ & $2.17 \mathrm{E}-09$ & $3.40 \mathrm{E}-03$ & $2.20 \mathrm{E}-09$ & $1.90 \mathrm{E}-08$ & $2.00 \mathrm{E}-04$ & $1.90 \mathrm{E}-08$ \\
\hline $2.35 \mathrm{E}+00$ & $2.23 \mathrm{E}+00$ & $4.69 \mathrm{E}-08$ & $6.00 \mathrm{E}-04$ & $4.70 \mathrm{E}-08$ & $2.12 \mathrm{E}-09$ & $3.20 \mathrm{E}-03$ & $2.14 \mathrm{E}-09$ & $1.90 \mathrm{E}-08$ & $2.00 \mathrm{E}-04$ & $1.90 \mathrm{E}-08$ \\
\hline $2.37 \mathrm{E}+00$ & $2.35 \mathrm{E}+00$ & $4.62 \mathrm{E}-08$ & $6.00 \mathrm{E}-04$ & $4.62 \mathrm{E}-08$ & $2.17 \mathrm{E}-09$ & $3.20 \mathrm{E}-03$ & $2.19 \mathrm{E}-09$ & $1.88 \mathrm{E}-08$ & $2.00 \mathrm{E}-04$ & $1.88 \mathrm{E}-08$ \\
\hline $2.47 \mathrm{E}+00$ & $2.37 \mathrm{E}+00$ & $4.63 \mathrm{E}-08$ & $6.00 \mathrm{E}-04$ & $4.64 \mathrm{E}-08$ & $2.08 \mathrm{E}-09$ & $3.30 \mathrm{E}-03$ & $2.10 \mathrm{E}-09$ & $1.89 \mathrm{E}-08$ & $2.00 \mathrm{E}-04$ & $1.90 \mathrm{E}-08$ \\
\hline $2.73 \mathrm{E}+00$ & $2.47 \mathrm{E}+00$ & $4.70 \mathrm{E}-08$ & $6.00 \mathrm{E}-04$ & $4.71 \mathrm{E}-08$ & $2.11 \mathrm{E}-09$ & $3.40 \mathrm{E}-03$ & $2.13 \mathrm{E}-09$ & $1.91 \mathrm{E}-08$ & $2.00 \mathrm{E}-04$ & $1.91 \mathrm{E}-08$ \\
\hline $3.01 \mathrm{E}+00$ & $2.73 \mathrm{E}+00$ & $4.78 \mathrm{E}-08$ & $6.00 \mathrm{E}-04$ & $4.78 \mathrm{E}-08$ & $2.15 \mathrm{E}-09$ & $3.40 \mathrm{E}-03$ & $2.17 \mathrm{E}-09$ & $1.94 \mathrm{E}-08$ & $2.00 \mathrm{E}-04$ & $1.94 \mathrm{E}-08$ \\
\hline $3.68 \mathrm{E}+00$ & $3.01 \mathrm{E}+00$ & $4.88 \mathrm{E}-08$ & $7.00 \mathrm{E}-04$ & $4.89 \mathrm{E}-08$ & $2.19 \mathrm{E}-09$ & $3.60 \mathrm{E}-03$ & $2.22 \mathrm{E}-09$ & $1.98 \mathrm{E}-08$ & $2.00 \mathrm{E}-04$ & $1.98 \mathrm{E}-08$ \\
\hline $4.97 \mathrm{E}+00$ & $3.68 \mathrm{E}+00$ & $5.21 \mathrm{E}-08$ & $7.00 \mathrm{E}-04$ & $5.22 \mathrm{E}-08$ & $2.31 \mathrm{E}-09$ & $3.60 \mathrm{E}-03$ & $2.33 \mathrm{E}-09$ & $2.11 \mathrm{E}-08$ & $2.00 \mathrm{E}-04$ & $2.11 \mathrm{E}-08$ \\
\hline $6.07 \mathrm{E}+00$ & $4.97 \mathrm{E}+00$ & $5.33 \mathrm{E}-08$ & $7.00 \mathrm{E}-04$ & $5.34 \mathrm{E}-08$ & $2.34 \mathrm{E}-09$ & $3.90 \mathrm{E}-03$ & $2.37 \mathrm{E}-09$ & $2.16 \mathrm{E}-08$ & $2.00 \mathrm{E}-04$ & $2.16 \mathrm{E}-08$ \\
\hline $7.41 \mathrm{E}+00$ & $6.07 \mathrm{E}+00$ & $5.16 \mathrm{E}-08$ & $7.00 \mathrm{E}-04$ & $5.17 \mathrm{E}-08$ & $2.27 \mathrm{E}-09$ & $3.90 \mathrm{E}-03$ & $2.29 \mathrm{E}-09$ & $2.10 \mathrm{E}-08$ & $2.00 \mathrm{E}-04$ & $2.10 \mathrm{E}-08$ \\
\hline $8.61 \mathrm{E}+00$ & $7.41 \mathrm{E}+00$ & $5.09 \mathrm{E}-08$ & $7.00 \mathrm{E}-04$ & $5.10 \mathrm{E}-08$ & $2.27 \mathrm{E}-09$ & $3.90 \mathrm{E}-03$ & $2.30 \mathrm{E}-09$ & $2.08 \mathrm{E}-08$ & $3.00 \mathrm{E}-04$ & $2.08 \mathrm{E}-08$ \\
\hline $1.00 \mathrm{E}+01$ & $8.61 \mathrm{E}+00$ & $5.02 \mathrm{E}-08$ & $7.00 \mathrm{E}-04$ & $5.03 \mathrm{E}-08$ & $2.24 \mathrm{E}-09$ & $3.80 \mathrm{E}-03$ & $2.27 \mathrm{E}-09$ & $2.05 \mathrm{E}-08$ & $2.00 \mathrm{E}-04$ & $2.05 \mathrm{E}-08$ \\
\hline $1.22 \mathrm{E}+01$ & $1.00 \mathrm{E}+01$ & $5.41 \mathrm{E}-08$ & $7.00 \mathrm{E}-04$ & $5.42 \mathrm{E}-08$ & $2.36 \mathrm{E}-09$ & $3.70 \mathrm{E}-03$ & $2.39 \mathrm{E}-09$ & $2.19 \mathrm{E}-08$ & $2.00 \mathrm{E}-04$ & $2.19 \mathrm{E}-08$ \\
\hline $1.42 \mathrm{E}+01$ & $1.22 \mathrm{E}+01$ & $6.29 \mathrm{E}-08$ & $7.00 \mathrm{E}-04$ & $6.30 \mathrm{E}-08$ & $2.71 \mathrm{E}-09$ & $3.50 \mathrm{E}-03$ & $2.73 \mathrm{E}-09$ & $2.53 \mathrm{E}-08$ & $2.00 \mathrm{E}-04$ & $2.53 \mathrm{E}-08$ \\
\hline $1.96 \mathrm{E}+01$ & $1.42 \mathrm{E}+01$ & $7.11 \mathrm{E}-08$ & $6.00 \mathrm{E}-04$ & $7.12 \mathrm{E}-08$ & $3.08 \mathrm{E}-09$ & $3.30 \mathrm{E}-03$ & $3.11 \mathrm{E}-09$ & $2.88 \mathrm{E}-08$ & $2.00 \mathrm{E}-04$ & $2.88 \mathrm{E}-08$ \\
\hline
\end{tabular}


PCP-2011-0001

Revision 0

Table 3-32. Secondary Photon Dose Rate per Unit Neutron Source in Each of the 47 Source Groups - No Shielded Container - $\mathrm{PuO}_{2}$ Self-shielding

\begin{tabular}{|c|c|c|c|c|c|c|c|c|c|c|}
\hline \multicolumn{2}{|c|}{ Energy $(\mathrm{MeV})$} & \multicolumn{3}{|c|}{ Bottom Dose Rate (rem/hr) } & \multicolumn{3}{|c|}{ Top Dose Rate (rem/hr) } & \multicolumn{3}{|c|}{ Side Dose Rate (rem $/ \mathrm{hr})$} \\
\hline Upper & Lower & mean & FSD & mean $+3 \pi$ & mean & FSD & mean $+3 \pi$ & mean & FSD & mean $+3 \pi$ \\
\hline $1.00 \mathrm{E}-07$ & $1.00 \mathrm{E}-11$ & $2.62 \mathrm{E}-09$ & 0.0012 & $2.63 \mathrm{E}-09$ & $6.01 \mathrm{E}-11$ & 0.0078 & $6.15 \mathrm{E}-11$ & $9.84 \mathrm{E}-10$ & 0.0004 & $9.85 \mathrm{E}-10$ \\
\hline 4.14E-07 & $1.00 \mathrm{E}-07$ & $2.69 \mathrm{E}-09$ & 0.0011 & $2.70 \mathrm{E}-09$ & $6.05 \mathrm{E}-11$ & 0.0076 & $6.19 \mathrm{E}-11$ & $9.95 \mathrm{E}-10$ & 0.0004 & $9.96 \mathrm{E}-10$ \\
\hline $8.76 \mathrm{E}-07$ & $4.14 \mathrm{E}-07$ & $1.41 \mathrm{E}-09$ & 0.0015 & $1.41 \mathrm{E}-09$ & $4.34 \mathrm{E}-11$ & 0.0081 & $4.45 \mathrm{E}-11$ & $5.78 \mathrm{E}-10$ & 0.0006 & $5.79 \mathrm{E}-10$ \\
\hline $1.86 \mathrm{E}-06$ & $8.76 \mathrm{E}-07$ & $6.13 \mathrm{E}-10$ & 0.0023 & $6.17 \mathrm{E}-10$ & $3.25 \mathrm{E}-11$ & 0.0096 & $3.35 \mathrm{E}-11$ & $3.06 \mathrm{E}-10$ & 0.0008 & $3.06 \mathrm{E}-10$ \\
\hline $5.04 \mathrm{E}-06$ & $1.86 \mathrm{E}-06$ & $3.59 \mathrm{E}-10$ & 0.0032 & $3.62 \mathrm{E}-10$ & $2.63 \mathrm{E}-11$ & 0.0098 & $2.70 \mathrm{E}-11$ & $2.03 \mathrm{E}-10$ & 0.0010 & $2.04 \mathrm{E}-10$ \\
\hline $1.07 \mathrm{E}-05$ & $5.04 \mathrm{E}-06$ & $4.88 \mathrm{E}-10$ & 0.0023 & $4.92 \mathrm{E}-10$ & $2.56 \mathrm{E}-11$ & 0.0104 & $2.64 \mathrm{E}-11$ & $2.35 \mathrm{E}-10$ & 0.0009 & $2.35 \mathrm{E}-10$ \\
\hline $3.73 \mathrm{E}-05$ & $1.07 \mathrm{E}-05$ & $5.05 \mathrm{E}-10$ & 0.0021 & $5.09 \mathrm{E}-10$ & $2.34 \mathrm{E}-11$ & 0.0107 & $2.41 \mathrm{E}-11$ & $2.27 \mathrm{E}-10$ & 0.0008 & $2.27 \mathrm{E}-10$ \\
\hline $1.01 \mathrm{E}-04$ & $3.73 \mathrm{E}-05$ & $5.71 \mathrm{E}-10$ & 0.0019 & $5.75 \mathrm{E}-10$ & $2.21 \mathrm{E}-11$ & 0.0107 & $2.28 \mathrm{E}-11$ & $2.41 \mathrm{E}-10$ & 0.0008 & $2.42 \mathrm{E}-10$ \\
\hline $2.14 \mathrm{E}-04$ & $1.01 \mathrm{E}-04$ & $3.29 \mathrm{E}-10$ & 0.0025 & $3.31 \mathrm{E}-10$ & $1.60 \mathrm{E}-11$ & 0.0116 & $1.65 \mathrm{E}-11$ & $1.49 \mathrm{E}-10$ & 0.0010 & $1.50 \mathrm{E}-10$ \\
\hline $4.54 \mathrm{E}-04$ & $2.14 \mathrm{E}-04$ & $2.82 \mathrm{E}-10$ & 0.0027 & $2.84 \mathrm{E}-10$ & $1.56 \mathrm{E}-11$ & 0.0116 & $1.61 \mathrm{E}-11$ & $1.33 \mathrm{E}-10$ & 0.0011 & $1.33 \mathrm{E}-10$ \\
\hline $1.58 \mathrm{E}-03$ & $4.54 \mathrm{E}-04$ & $1.82 \mathrm{E}-10$ & 0.0033 & $1.84 \mathrm{E}-10$ & $1.21 \mathrm{E}-11$ & 0.0127 & $1.26 \mathrm{E}-11$ & $9.17 \mathrm{E}-11$ & 0.0012 & $9.20 \mathrm{E}-11$ \\
\hline $3.35 \mathrm{E}-03$ & $1.58 \mathrm{E}-03$ & $1.16 \mathrm{E}-10$ & 0.0042 & $1.17 \mathrm{E}-10$ & $1.01 \mathrm{E}-11$ & 0.0148 & $1.05 \mathrm{E}-11$ & $6.26 \mathrm{E}-11$ & 0.0016 & $6.29 \mathrm{E}-11$ \\
\hline $7.10 \mathrm{E}-03$ & $3.35 \mathrm{E}-03$ & $8.44 \mathrm{E}-11$ & 0.0048 & $8.56 \mathrm{E}-11$ & $7.95 \mathrm{E}-12$ & 0.0167 & $8.35 \mathrm{E}-12$ & $4.77 \mathrm{E}-11$ & 0.0018 & $4.79 \mathrm{E}-11$ \\
\hline $1.50 \mathrm{E}-02$ & $7.10 \mathrm{E}-03$ & $6.45 \mathrm{E}-11$ & 0.0054 & $6.55 \mathrm{E}-11$ & $6.78 \mathrm{E}-12$ & 0.0168 & $7.12 \mathrm{E}-12$ & $3.79 \mathrm{E}-11$ & 0.0020 & $3.81 \mathrm{E}-11$ \\
\hline $2.19 \mathrm{E}-02$ & $1.50 \mathrm{E}-02$ & $5.13 \mathrm{E}-11$ & 0.0060 & $5.22 \mathrm{E}-11$ & $5.66 \mathrm{E}-12$ & 0.0189 & $5.98 \mathrm{E}-12$ & $3.03 \mathrm{E}-11$ & 0.0022 & $3.05 \mathrm{E}-11$ \\
\hline $2.42 \mathrm{E}-02$ & $2.19 \mathrm{E}-02$ & $4.58 \mathrm{E}-11$ & 0.0059 & $4.66 \mathrm{E}-11$ & $5.42 \mathrm{E}-12$ & 0.0191 & $5.73 \mathrm{E}-12$ & $2.75 \mathrm{E}-11$ & 0.0022 & $2.76 \mathrm{E}-11$ \\
\hline $2.61 \mathrm{E}-02$ & $2.42 \mathrm{E}-02$ & $4.42 \mathrm{E}-11$ & 0.0061 & $4.50 \mathrm{E}-11$ & $5.15 \mathrm{E}-12$ & 0.0196 & $5.46 \mathrm{E}-12$ & $2.62 \mathrm{E}-11$ & 0.0022 & $2.63 \mathrm{E}-11$ \\
\hline $3.18 \mathrm{E}-02$ & $2.61 \mathrm{E}-02$ & $5.10 \mathrm{E}-11$ & 0.0060 & $5.20 \mathrm{E}-11$ & $5.77 \mathrm{E}-12$ & 0.0208 & $6.13 \mathrm{E}-12$ & $2.96 \mathrm{E}-11$ & 0.0022 & $2.98 \mathrm{E}-11$ \\
\hline $4.09 \mathrm{E}-02$ & $3.18 \mathrm{E}-02$ & $5.21 \mathrm{E}-11$ & 0.0062 & $5.30 \mathrm{E}-11$ & $5.37 \mathrm{E}-12$ & 0.0198 & $5.69 \mathrm{E}-12$ & $3.04 \mathrm{E}-11$ & 0.0022 & $3.06 \mathrm{E}-11$ \\
\hline $6.74 \mathrm{E}-02$ & $4.09 \mathrm{E}-02$ & $4.09 \mathrm{E}-11$ & 0.0066 & $4.18 \mathrm{E}-11$ & $4.55 \mathrm{E}-12$ & 0.0206 & $4.83 \mathrm{E}-12$ & $2.38 \mathrm{E}-11$ & 0.0024 & $2.40 \mathrm{E}-11$ \\
\hline $1.11 \mathrm{E}-01$ & $6.74 \mathrm{E}-02$ & $3.64 \mathrm{E}-11$ & 0.0064 & $3.71 \mathrm{E}-11$ & $4.04 \mathrm{E}-12$ & 0.0210 & $4.29 \mathrm{E}-12$ & $2.06 \mathrm{E}-11$ & 0.0024 & $2.08 \mathrm{E}-11$ \\
\hline $1.83 \mathrm{E}-01$ & $1.11 \mathrm{E}-01$ & $3.17 \mathrm{E}-11$ & 0.0067 & $3.23 \mathrm{E}-11$ & $3.27 \mathrm{E}-12$ & 0.0242 & $3.51 \mathrm{E}-12$ & $1.69 \mathrm{E}-11$ & 0.0025 & $1.71 \mathrm{E}-11$ \\
\hline $2.97 \mathrm{E}-01$ & $1.83 \mathrm{E}-01$ & $2.80 \mathrm{E}-11$ & 0.0065 & $2.85 \mathrm{E}-11$ & $2.62 \mathrm{E}-12$ & 0.0275 & $2.84 \mathrm{E}-12$ & $1.42 \mathrm{E}-11$ & 0.0025 & $1.43 \mathrm{E}-11$ \\
\hline $3.69 \mathrm{E}-01$ & $2.97 \mathrm{E}-01$ & $2.55 \mathrm{E}-11$ & 0.0059 & $2.60 \mathrm{E}-11$ & $2.07 \mathrm{E}-12$ & 0.0252 & $2.22 \mathrm{E}-12$ & $1.25 \mathrm{E}-11$ & 0.0025 & $1.26 \mathrm{E}-11$ \\
\hline $4.98 \mathrm{E}-01$ & $3.69 \mathrm{E}-01$ & $2.52 \mathrm{E}-11$ & 0.0059 & $2.56 \mathrm{E}-11$ & $1.96 \mathrm{E}-12$ & 0.0270 & $2.12 \mathrm{E}-12$ & $1.20 \mathrm{E}-11$ & 0.0025 & $1.21 \mathrm{E}-11$ \\
\hline $6.08 \mathrm{E}-01$ & $4.98 \mathrm{E}-01$ & $2.36 \mathrm{E}-11$ & 0.0056 & $2.40 \mathrm{E}-11$ & $1.52 \mathrm{E}-12$ & 0.0268 & $1.64 \mathrm{E}-12$ & $1.08 \mathrm{E}-11$ & 0.0024 & $1.09 \mathrm{E}-11$ \\
\hline $7.43 \mathrm{E}-01$ & $6.08 \mathrm{E}-01$ & $2.43 \mathrm{E}-11$ & 0.0056 & $2.47 \mathrm{E}-11$ & $1.39 \mathrm{E}-12$ & 0.0309 & $1.52 \mathrm{E}-12$ & $1.07 \mathrm{E}-11$ & 0.0023 & $1.08 \mathrm{E}-11$ \\
\hline $8.21 \mathrm{E}-01$ & $7.43 \mathrm{E}-01$ & $2.53 \mathrm{E}-11$ & 0.0055 & $2.57 \mathrm{E}-11$ & $1.49 \mathrm{E}-12$ & 0.0310 & $1.63 \mathrm{E}-12$ & $1.11 \mathrm{E}-11$ & 0.0022 & $1.11 \mathrm{E}-11$ \\
\hline $1.00 \mathrm{E}+00$ & $8.21 \mathrm{E}-01$ & $2.87 \mathrm{E}-11$ & 0.0047 & $2.91 \mathrm{E}-11$ & $1.59 \mathrm{E}-12$ & 0.0271 & $1.72 \mathrm{E}-12$ & $1.26 \mathrm{E}-11$ & 0.0020 & $1.26 \mathrm{E}-11$ \\
\hline $1.35 \mathrm{E}+00$ & $1.00 \mathrm{E}+00$ & $4.53 \mathrm{E}-11$ & 0.0034 & $4.58 \mathrm{E}-11$ & $2.19 \mathrm{E}-12$ & 0.0196 & $2.32 \mathrm{E}-12$ & $2.00 \mathrm{E}-11$ & 0.0013 & $2.01 \mathrm{E}-11$ \\
\hline $1.65 \mathrm{E}+00$ & $1.35 \mathrm{E}+00$ & $6.53 \mathrm{E}-11$ & 0.0028 & $6.58 \mathrm{E}-11$ & $2.81 \mathrm{E}-12$ & 0.0149 & $2.93 \mathrm{E}-12$ & $2.92 \mathrm{E}-11$ & 0.0010 & $2.93 \mathrm{E}-11$ \\
\hline $1.92 \mathrm{E}+00$ & $1.65 \mathrm{E}+00$ & $7.51 \mathrm{E}-11$ & 0.0026 & $7.57 \mathrm{E}-11$ & $3.35 \mathrm{E}-12$ & 0.0134 & $3.48 \mathrm{E}-12$ & $3.40 \mathrm{E}-11$ & 0.0009 & $3.41 \mathrm{E}-11$ \\
\hline $2.23 \mathrm{E}+00$ & $1.92 \mathrm{E}+00$ & $8.77 \mathrm{E}-11$ & 0.0024 & $8.84 \mathrm{E}-11$ & $4.01 \mathrm{E}-12$ & 0.0121 & $4.16 \mathrm{E}-12$ & $3.98 \mathrm{E}-11$ & 0.0008 & $3.99 \mathrm{E}-11$ \\
\hline $2.35 \mathrm{E}+00$ & $2.23 \mathrm{E}+00$ & $9.18 \mathrm{E}-11$ & 0.0023 & $9.25 \mathrm{E}-11$ & $4.31 \mathrm{E}-12$ & 0.0117 & $4.46 \mathrm{E}-12$ & $4.17 \mathrm{E}-11$ & 0.0008 & $4.18 \mathrm{E}-11$ \\
\hline $2.37 \mathrm{E}+00$ & $2.35 \mathrm{E}+00$ & $9.27 \mathrm{E}-11$ & 0.0022 & $9.33 \mathrm{E}-11$ & $4.47 \mathrm{E}-12$ & 0.0121 & $4.63 \mathrm{E}-12$ & $4.22 \mathrm{E}-11$ & 0.0008 & $4.23 \mathrm{E}-11$ \\
\hline $2.47 \mathrm{E}+00$ & $2.37 \mathrm{E}+00$ & $9.81 \mathrm{E}-11$ & 0.0022 & $9.87 \mathrm{E}-11$ & $4.57 \mathrm{E}-12$ & 0.0110 & $4.72 \mathrm{E}-12$ & $4.48 \mathrm{E}-11$ & 0.0008 & $4.49 \mathrm{E}-11$ \\
\hline $2.73 \mathrm{E}+00$ & $2.47 \mathrm{E}+00$ & $1.11 \mathrm{E}-10$ & 0.0021 & $1.12 \mathrm{E}-10$ & $5.12 \mathrm{E}-12$ & 0.0106 & $5.29 \mathrm{E}-12$ & $5.12 \mathrm{E}-11$ & 0.0007 & $5.13 \mathrm{E}-11$ \\
\hline $3.01 \mathrm{E}+00$ & $2.73 \mathrm{E}+00$ & $1.27 \mathrm{E}-10$ & 0.0020 & $1.27 \mathrm{E}-10$ & $5.82 \mathrm{E}-12$ & 0.0101 & $5.99 \mathrm{E}-12$ & $5.86 \mathrm{E}-11$ & 0.0007 & $5.87 \mathrm{E}-11$ \\
\hline $3.68 \mathrm{E}+00$ & $3.01 \mathrm{E}+00$ & $1.73 \mathrm{E}-10$ & 0.0019 & $1.74 \mathrm{E}-10$ & $8.09 \mathrm{E}-12$ & 0.0096 & $8.32 \mathrm{E}-12$ & $8.09 \mathrm{E}-11$ & 0.0006 & $8.11 \mathrm{E}-11$ \\
\hline $4.97 \mathrm{E}+00$ & $3.68 \mathrm{E}+00$ & $2.38 \mathrm{E}-10$ & 0.0018 & $2.39 \mathrm{E}-10$ & $1.17 \mathrm{E}-11$ & 0.0081 & $1.20 \mathrm{E}-11$ & $1.13 \mathrm{E}-10$ & 0.0006 & $1.13 \mathrm{E}-10$ \\
\hline $6.07 \mathrm{E}+00$ & $4.97 \mathrm{E}+00$ & $3.26 \mathrm{E}-10$ & 0.0017 & $3.28 \mathrm{E}-10$ & $1.60 \mathrm{E}-11$ & 0.0078 & $1.64 \mathrm{E}-11$ & $1.56 \mathrm{E}-10$ & 0.0006 & $1.57 \mathrm{E}-10$ \\
\hline $7.41 \mathrm{E}+00$ & $6.07 \mathrm{E}+00$ & $4.11 \mathrm{E}-10$ & 0.0017 & $4.13 \mathrm{E}-10$ & $2.03 \mathrm{E}-11$ & 0.0077 & $2.08 \mathrm{E}-11$ & $1.99 \mathrm{E}-10$ & 0.0006 & $1.99 \mathrm{E}-10$ \\
\hline $8.61 \mathrm{E}+00$ & $7.41 \mathrm{E}+00$ & $4.98 \mathrm{E}-10$ & 0.0017 & $5.01 \mathrm{E}-10$ & $2.56 \mathrm{E}-11$ & 0.0075 & $2.61 \mathrm{E}-11$ & $2.43 \mathrm{E}-10$ & 0.0005 & $2.44 \mathrm{E}-10$ \\
\hline $1.00 \mathrm{E}+01$ & $8.61 \mathrm{E}+00$ & $5.46 \mathrm{E}-10$ & 0.0017 & $5.49 \mathrm{E}-10$ & $2.89 \mathrm{E}-11$ & 0.0076 & $2.95 \mathrm{E}-11$ & $2.67 \mathrm{E}-10$ & 0.0006 & $2.67 \mathrm{E}-10$ \\
\hline $1.22 \mathrm{E}+01$ & $1.00 \mathrm{E}+01$ & $5.93 \mathrm{E}-10$ & 0.0018 & $5.96 \mathrm{E}-10$ & $3.19 \mathrm{E}-11$ & 0.0075 & $3.26 \mathrm{E}-11$ & $2.92 \mathrm{E}-10$ & 0.0006 & $2.92 \mathrm{E}-10$ \\
\hline $1.42 \mathrm{E}+01$ & $1.22 \mathrm{E}+01$ & $5.24 \mathrm{E}-10$ & 0.0018 & $5.27 \mathrm{E}-10$ & $2.92 \mathrm{E}-11$ & 0.0076 & $2.99 \mathrm{E}-11$ & $2.59 \mathrm{E}-10$ & 0.0006 & $2.59 \mathrm{E}-10$ \\
\hline $1.96 \mathrm{E}+01$ & $1.42 \mathrm{E}+01$ & $3.89 \mathrm{E}-10$ & 0.0018 & $3.91 \mathrm{E}-10$ & $2.20 \mathrm{E}-11$ & 0.0082 & $2.25 \mathrm{E}-11$ & $1.91 \mathrm{E}-10$ & 0.0006 & $1.91 \mathrm{E}-10$ \\
\hline
\end{tabular}


PCP-2011-0001

Revision 0

\section{Table 3-33. Photon Dose Rate per Unit Source in Each of the 77 Source Groups - No} Shielded Container $-\mathrm{PuO}_{2}$ Self-shielding

\begin{tabular}{|c|c|c|c|c|c|c|c|c|c|c|}
\hline \multicolumn{2}{|c|}{ Energy $(\mathrm{MeV})$} & \multicolumn{3}{|c|}{ Bottom Dose Rate (rem/hr) } & \multicolumn{3}{|c|}{ Top Dose Rate (rem/hr) } & \multicolumn{3}{|c|}{ Side Dose Rate $(\mathrm{rem} / \mathrm{hr})$} \\
\hline Upper & Lower & mean & FSD & mean $+3 \pi$ & mean & FSD & mean $+3 \pi$ & mean & FSD & mean $+3 \pi$ \\
\hline 0.020 & 0.010 & $0.00 \mathrm{E}+00$ & 0.0000 & $0.00 \mathrm{E}+00$ & $0.00 \mathrm{E}+00$ & 0.0000 & $0.00 \mathrm{E}+00$ & $0.00 \mathrm{E}+00$ & 0.0000 & $0.00 \mathrm{E}+00$ \\
\hline 0.030 & 0.020 & $2.53 \mathrm{E}-30$ & 0.0575 & $2.97 \mathrm{E}-30$ & $0.00 \mathrm{E}+00$ & 0.0000 & $0.00 \mathrm{E}+00$ & $4.60 \mathrm{E}-46$ & 0.2019 & $7.39 \mathrm{E}-46$ \\
\hline 0.040 & 0.030 & $3.94 \mathrm{E}-20$ & 0.0401 & $4.41 \mathrm{E}-20$ & $1.49 \mathrm{E}-44$ & 0.7034 & $4.62 \mathrm{E}-44$ & $3.97 \mathrm{E}-26$ & 0.6744 & $1.20 \mathrm{E}-25$ \\
\hline 0.050 & 0.040 & $4.37 \mathrm{E}-16$ & 0.0224 & $4.67 \mathrm{E}-16$ & $9.25 \mathrm{E}-32$ & 0.3829 & $1.99 \mathrm{E}-31$ & $5.45 \mathrm{E}-19$ & 0.2514 & $9.56 \mathrm{E}-19$ \\
\hline 0.060 & 0.050 & $4.53 \mathrm{E}-14$ & 0.0136 & $4.72 \mathrm{E}-14$ & $1.34 \mathrm{E}-25$ & 0.3352 & $2.69 \mathrm{E}-25$ & $2.00 \mathrm{E}-16$ & 0.2183 & $3.32 \mathrm{E}-16$ \\
\hline 0.070 & 0.060 & $5.81 \mathrm{E}-13$ & 0.0076 & $5.94 \mathrm{E}-13$ & $2.57 \mathrm{E}-21$ & 0.4779 & $6.27 \mathrm{E}-21$ & $4.65 \mathrm{E}-15$ & 0.0176 & $4.89 \mathrm{E}-15$ \\
\hline 0.080 & 0.070 & $2.65 \mathrm{E}-12$ & 0.0060 & $2.70 \mathrm{E}-12$ & $2.17 \mathrm{E}-19$ & 0.1939 & $3.44 \mathrm{E}-19$ & $4.40 \mathrm{E}-14$ & 0.0070 & $4.50 \mathrm{E}-14$ \\
\hline 0.090 & 0.080 & $7.00 \mathrm{E}-12$ & 0.0032 & $7.07 \mathrm{E}-12$ & $2.33 \mathrm{E}-17$ & 0.7250 & $7.39 \mathrm{E}-17$ & $1.99 \mathrm{E}-13$ & 0.0031 & $2.01 \mathrm{E}-13$ \\
\hline 0.100 & 0.090 & $1.34 \mathrm{E}-11$ & 0.0027 & $1.36 \mathrm{E}-11$ & $6.16 \mathrm{E}-17$ & 0.0709 & $7.47 \mathrm{E}-17$ & $5.74 \mathrm{E}-13$ & 0.0022 & $5.77 \mathrm{E}-13$ \\
\hline 0.110 & 0.100 & $2.15 \mathrm{E}-11$ & 0.0028 & $2.16 \mathrm{E}-11$ & $4.33 \mathrm{E}-16$ & 0.0786 & $5.35 \mathrm{E}-16$ & $1.23 \mathrm{E}-12$ & 0.0017 & $1.23 \mathrm{E}-12$ \\
\hline 0.120 & 0.110 & $3.06 \mathrm{E}-11$ & 0.0027 & $3.09 \mathrm{E}-11$ & $1.87 \mathrm{E}-15$ & 0.1071 & $2.47 \mathrm{E}-15$ & $2.18 \mathrm{E}-12$ & 0.0014 & $2.19 \mathrm{E}-12$ \\
\hline 0.130 & 0.120 & 4.12E-11 & 0.0007 & 4.13E-11 & $1.02 \mathrm{E}-14$ & 0.0533 & $1.18 \mathrm{E}-14$ & $3.86 \mathrm{E}-12$ & 0.0006 & $3.87 \mathrm{E}-12$ \\
\hline 0.140 & 0.130 & $5.15 \mathrm{E}-11$ & 0.0007 & $5.16 \mathrm{E}-11$ & $2.02 \mathrm{E}-14$ & 0.0420 & $2.28 \mathrm{E}-14$ & $5.48 \mathrm{E}-12$ & 0.0005 & $5.49 \mathrm{E}-12$ \\
\hline 0.150 & 0.140 & $6.17 \mathrm{E}-11$ & 0.0007 & $6.18 \mathrm{E}-11$ & $3.61 \mathrm{E}-14$ & 0.0402 & $4.05 \mathrm{E}-14$ & $7.27 \mathrm{E}-12$ & 0.0004 & $7.28 \mathrm{E}-12$ \\
\hline 0.160 & 0.150 & $7.19 \mathrm{E}-11$ & 0.0006 & $7.20 \mathrm{E}-11$ & $5.88 \mathrm{E}-14$ & 0.0289 & $6.39 \mathrm{E}-14$ & $9.19 \mathrm{E}-12$ & 0.0004 & $9.20 \mathrm{E}-12$ \\
\hline 0.170 & 0.160 & $8.22 \mathrm{E}-11$ & 0.0006 & $8.23 \mathrm{E}-11$ & $8.98 \mathrm{E}-14$ & 0.0230 & $9.59 \mathrm{E}-14$ & $1.12 \mathrm{E}-11$ & 0.0004 & $1.12 \mathrm{E}-11$ \\
\hline 0.180 & 0.170 & $9.24 \mathrm{E}-11$ & 0.0006 & $9.25 \mathrm{E}-11$ & $1.24 \mathrm{E}-13$ & 0.0195 & $1.32 \mathrm{E}-13$ & $1.33 \mathrm{E}-11$ & 0.0004 & $1.33 \mathrm{E}-11$ \\
\hline 0.190 & 0.180 & $1.02 \mathrm{E}-10$ & 0.0006 & $1.02 \mathrm{E}-10$ & $1.68 \mathrm{E}-13$ & 0.0181 & $1.77 \mathrm{E}-13$ & $1.55 \mathrm{E}-11$ & 0.0003 & $1.55 \mathrm{E}-11$ \\
\hline 0.200 & 0.190 & $1.12 \mathrm{E}-10$ & 0.0006 & $1.12 \mathrm{E}-10$ & $2.19 \mathrm{E}-13$ & 0.0166 & $2.30 \mathrm{E}-13$ & $1.76 \mathrm{E}-11$ & 0.0003 & $1.76 \mathrm{E}-11$ \\
\hline 0.210 & 0.200 & $1.22 \mathrm{E}-10$ & 0.0006 & $1.22 \mathrm{E}-10$ & $2.74 \mathrm{E}-13$ & 0.0148 & $2.86 \mathrm{E}-13$ & $1.98 \mathrm{E}-11$ & 0.0003 & $1.98 \mathrm{E}-11$ \\
\hline 0.220 & 0.210 & $1.31 \mathrm{E}-10$ & 0.0006 & $1.32 \mathrm{E}-10$ & $3.30 \mathrm{E}-13$ & 0.0142 & $3.45 \mathrm{E}-13$ & $2.20 \mathrm{E}-11$ & 0.0003 & $2.20 \mathrm{E}-11$ \\
\hline 0.230 & 0.220 & $1.41 \mathrm{E}-10$ & 0.0006 & $1.41 \mathrm{E}-10$ & $3.99 \mathrm{E}-13$ & 0.0134 & $4.15 \mathrm{E}-13$ & $2.42 \mathrm{E}-11$ & 0.0003 & $2.42 \mathrm{E}-11$ \\
\hline 0.240 & 0.230 & $1.50 \mathrm{E}-10$ & 0.0006 & $1.50 \mathrm{E}-10$ & $4.63 \mathrm{E}-13$ & 0.0123 & $4.80 \mathrm{E}-13$ & $2.64 \mathrm{E}-11$ & 0.0003 & $2.64 \mathrm{E}-11$ \\
\hline 0.250 & 0.240 & $1.59 \mathrm{E}-10$ & 0.0006 & $1.60 \mathrm{E}-10$ & $5.28 \mathrm{E}-13$ & 0.0117 & $5.46 \mathrm{E}-13$ & $2.86 \mathrm{E}-11$ & 0.0003 & $2.86 \mathrm{E}-11$ \\
\hline 0.260 & 0.250 & $1.68 \mathrm{E}-10$ & 0.0006 & $1.69 \mathrm{E}-10$ & $6.01 \mathrm{E}-13$ & 0.0113 & $6.21 \mathrm{E}-13$ & $3.08 \mathrm{E}-11$ & 0.0003 & $3.08 \mathrm{E}-11$ \\
\hline 0.270 & 0.260 & $1.77 \mathrm{E}-10$ & 0.0005 & $1.78 \mathrm{E}-10$ & $6.80 \mathrm{E}-13$ & 0.0107 & $7.02 \mathrm{E}-13$ & $3.30 \mathrm{E}-11$ & 0.0003 & $3.30 \mathrm{E}-11$ \\
\hline 0.280 & 0.270 & $1.86 \mathrm{E}-10$ & 0.0005 & $1.86 \mathrm{E}-10$ & $7.61 \mathrm{E}-13$ & 0.0103 & $7.85 \mathrm{E}-13$ & $3.52 \mathrm{E}-11$ & 0.0003 & $3.52 \mathrm{E}-11$ \\
\hline 0.290 & 0.280 & $1.95 \mathrm{E}-10$ & 0.0005 & $1.95 \mathrm{E}-10$ & $8.35 \mathrm{E}-13$ & 0.0097 & $8.60 \mathrm{E}-13$ & $3.73 \mathrm{E}-11$ & 0.0003 & $3.73 \mathrm{E}-11$ \\
\hline 0.300 & 0.290 & $2.03 \mathrm{E}-10$ & 0.0005 & $2.04 \mathrm{E}-10$ & $9.26 \mathrm{E}-13$ & 0.0094 & $9.52 \mathrm{E}-13$ & $3.95 \mathrm{E}-11$ & 0.0003 & $3.95 \mathrm{E}-11$ \\
\hline 0.325 & 0.300 & $2.18 \mathrm{E}-10$ & 0.0005 & $2.18 \mathrm{E}-10$ & $1.08 \mathrm{E}-12$ & 0.0088 & $1.11 \mathrm{E}-12$ & $4.32 \mathrm{E}-11$ & 0.0003 & $4.32 \mathrm{E}-11$ \\
\hline 0.350 & 0.325 & $2.38 \mathrm{E}-10$ & 0.0005 & $2.38 \mathrm{E}-10$ & $1.30 \mathrm{E}-12$ & 0.0082 & $1.33 \mathrm{E}-12$ & $4.84 \mathrm{E}-11$ & 0.0003 & $4.84 \mathrm{E}-11$ \\
\hline 0.375 & 0.350 & $2.57 \mathrm{E}-10$ & 0.0005 & $2.57 \mathrm{E}-10$ & $1.54 \mathrm{E}-12$ & 0.0078 & $1.58 \mathrm{E}-12$ & $5.34 \mathrm{E}-11$ & 0.0003 & $5.35 \mathrm{E}-11$ \\
\hline 0.400 & 0.375 & $2.75 \mathrm{E}-10$ & 0.0005 & $2.76 \mathrm{E}-10$ & $1.76 \mathrm{E}-12$ & 0.0073 & $1.80 \mathrm{E}-12$ & $5.83 \mathrm{E}-11$ & 0.0003 & $5.84 \mathrm{E}-11$ \\
\hline 0.425 & 0.400 & $2.92 \mathrm{E}-10$ & 0.0005 & $2.93 \mathrm{E}-10$ & $2.02 \mathrm{E}-12$ & 0.0071 & $2.06 \mathrm{E}-12$ & $6.31 \mathrm{E}-11$ & 0.0002 & $6.32 \mathrm{E}-11$ \\
\hline 0.450 & 0.425 & $3.09 \mathrm{E}-10$ & 0.0005 & $3.09 \mathrm{E}-10$ & $2.27 \mathrm{E}-12$ & 0.0068 & $2.32 \mathrm{E}-12$ & $6.77 \mathrm{E}-11$ & 0.0002 & $6.78 \mathrm{E}-11$ \\
\hline 0.475 & 0.450 & $3.25 \mathrm{E}-10$ & 0.0005 & $3.25 \mathrm{E}-10$ & $2.53 \mathrm{E}-12$ & 0.0067 & $2.58 \mathrm{E}-12$ & $7.22 \mathrm{E}-11$ & 0.0002 & $7.23 \mathrm{E}-11$ \\
\hline 0.500 & 0.475 & $3.40 \mathrm{E}-10$ & 0.0005 & $3.40 \mathrm{E}-10$ & $2.79 \mathrm{E}-12$ & 0.0067 & $2.85 \mathrm{E}-12$ & $7.66 \mathrm{E}-11$ & 0.0002 & $7.67 \mathrm{E}-11$ \\
\hline 0.525 & 0.500 & $3.55 \mathrm{E}-10$ & 0.0005 & $3.56 \mathrm{E}-10$ & $3.03 \mathrm{E}-12$ & 0.0061 & $3.09 \mathrm{E}-12$ & $8.10 \mathrm{E}-11$ & 0.0002 & $8.11 \mathrm{E}-11$ \\
\hline
\end{tabular}


PCP-2011-0001

Revision 0

\section{Table 3-33. Photon Dose Rate per Unit Source in Each of the 77 Source Groups - No} Shielded Container $-\mathrm{PuO}_{2}$ Self-shielding

\begin{tabular}{|c|c|c|c|c|c|c|c|c|c|c|}
\hline \multicolumn{2}{|c|}{ Energy $(\mathrm{MeV})$} & \multicolumn{3}{|c|}{ Bottom Dose Rate (rem/hr) } & \multicolumn{3}{|c|}{ Top Dose Rate (rem/hr) } & \multicolumn{3}{|c|}{ Side Dose Rate $(\mathrm{rem} / \mathrm{hr})$} \\
\hline Upper & Lower & mean & FSD & mean $+3 \pi$ & mean & FSD & mean $+3 \pi$ & mean & FSD & mean $+3 \pi$ \\
\hline 0.550 & 0.525 & $3.71 \mathrm{E}-10$ & 0.0005 & $3.71 \mathrm{E}-10$ & $3.29 \mathrm{E}-12$ & 0.0060 & $3.35 \mathrm{E}-12$ & $8.54 \mathrm{E}-11$ & 0.0002 & $8.55 \mathrm{E}-11$ \\
\hline 0.575 & 0.550 & $3.86 \mathrm{E}-10$ & 0.0005 & $3.86 \mathrm{E}-10$ & $3.54 \mathrm{E}-12$ & 0.0057 & $3.60 \mathrm{E}-12$ & $8.98 \mathrm{E}-11$ & 0.0002 & $8.98 \mathrm{E}-11$ \\
\hline 0.600 & 0.575 & $4.00 \mathrm{E}-10$ & 0.0005 & $4.01 \mathrm{E}-10$ & $3.80 \mathrm{E}-12$ & 0.0055 & $3.86 \mathrm{E}-12$ & $9.40 \mathrm{E}-11$ & 0.0002 & $9.41 \mathrm{E}-11$ \\
\hline 0.625 & 0.600 & $4.14 \mathrm{E}-10$ & 0.0005 & $4.15 \mathrm{E}-10$ & $4.09 \mathrm{E}-12$ & 0.0055 & $4.15 \mathrm{E}-12$ & $9.81 \mathrm{E}-11$ & 0.0002 & $9.82 \mathrm{E}-11$ \\
\hline 0.650 & 0.625 & $4.27 \mathrm{E}-10$ & 0.0005 & $4.28 \mathrm{E}-10$ & $4.37 \mathrm{E}-12$ & 0.0054 & $4.44 \mathrm{E}-12$ & $1.02 \mathrm{E}-10$ & 0.0002 & $1.02 \mathrm{E}-10$ \\
\hline 0.675 & 0.650 & $4.40 \mathrm{E}-10$ & 0.0005 & $4.41 \mathrm{E}-10$ & $4.66 \mathrm{E}-12$ & 0.0053 & $4.73 \mathrm{E}-12$ & $1.06 \mathrm{E}-10$ & 0.0002 & $1.06 \mathrm{E}-10$ \\
\hline 0.700 & 0.675 & $4.53 \mathrm{E}-10$ & 0.0005 & $4.54 \mathrm{E}-10$ & $4.92 \mathrm{E}-12$ & 0.0052 & $5.00 \mathrm{E}-12$ & $1.10 \mathrm{E}-10$ & 0.0002 & $1.10 \mathrm{E}-10$ \\
\hline 0.725 & 0.700 & $4.66 \mathrm{E}-10$ & 0.0005 & $4.67 \mathrm{E}-10$ & $5.19 \mathrm{E}-12$ & 0.0051 & $5.27 \mathrm{E}-12$ & $1.14 \mathrm{E}-10$ & 0.0002 & $1.14 \mathrm{E}-10$ \\
\hline 0.750 & 0.725 & $4.79 \mathrm{E}-10$ & 0.0005 & $4.80 \mathrm{E}-10$ & $5.45 \mathrm{E}-12$ & 0.0049 & $5.53 \mathrm{E}-12$ & $1.18 \mathrm{E}-10$ & 0.0002 & $1.18 \mathrm{E}-10$ \\
\hline 0.775 & 0.750 & $4.92 \mathrm{E}-10$ & 0.0005 & $4.92 \mathrm{E}-10$ & $5.71 \mathrm{E}-12$ & 0.0048 & $5.79 \mathrm{E}-12$ & $1.22 \mathrm{E}-10$ & 0.0002 & $1.22 \mathrm{E}-10$ \\
\hline 0.800 & 0.775 & $5.04 \mathrm{E}-10$ & 0.0005 & $5.05 \mathrm{E}-10$ & $5.99 \mathrm{E}-12$ & 0.0048 & $6.07 \mathrm{E}-12$ & $1.26 \mathrm{E}-10$ & 0.0002 & $1.26 \mathrm{E}-10$ \\
\hline 0.825 & 0.800 & $5.16 \mathrm{E}-10$ & 0.0005 & $5.17 \mathrm{E}-10$ & $6.25 \mathrm{E}-12$ & 0.0047 & $6.34 \mathrm{E}-12$ & $1.29 \mathrm{E}-10$ & 0.0002 & $1.30 \mathrm{E}-10$ \\
\hline 0.850 & 0.825 & $5.28 \mathrm{E}-10$ & 0.0005 & $5.29 \mathrm{E}-10$ & $6.53 \mathrm{E}-12$ & 0.0046 & $6.62 \mathrm{E}-12$ & $1.33 \mathrm{E}-10$ & 0.0002 & $1.33 \mathrm{E}-10$ \\
\hline 0.875 & 0.850 & $5.40 \mathrm{E}-10$ & 0.0005 & $5.41 \mathrm{E}-10$ & $6.83 \mathrm{E}-12$ & 0.0045 & $6.93 \mathrm{E}-12$ & $1.37 \mathrm{E}-10$ & 0.0002 & $1.37 \mathrm{E}-10$ \\
\hline 0.900 & 0.875 & $5.52 \mathrm{E}-10$ & 0.0005 & $5.53 \mathrm{E}-10$ & $7.15 \mathrm{E}-12$ & 0.0045 & $7.25 \mathrm{E}-12$ & $1.41 \mathrm{E}-10$ & 0.0002 & $1.41 \mathrm{E}-10$ \\
\hline 0.925 & 0.900 & $5.64 \mathrm{E}-10$ & 0.0005 & $5.65 \mathrm{E}-10$ & $7.41 \mathrm{E}-12$ & 0.0045 & $7.51 \mathrm{E}-12$ & $1.45 \mathrm{E}-10$ & 0.0002 & $1.45 \mathrm{E}-10$ \\
\hline 0.950 & 0.925 & $5.76 \mathrm{E}-10$ & 0.0005 & $5.76 \mathrm{E}-10$ & $7.69 \mathrm{E}-12$ & 0.0043 & $7.79 \mathrm{E}-12$ & $1.48 \mathrm{E}-10$ & 0.0002 & $1.48 \mathrm{E}-10$ \\
\hline 0.975 & 0.950 & $5.87 \mathrm{E}-10$ & 0.0005 & $5.88 \mathrm{E}-10$ & $7.95 \mathrm{E}-12$ & 0.0043 & $8.05 \mathrm{E}-12$ & $1.52 \mathrm{E}-10$ & 0.0002 & $1.52 \mathrm{E}-10$ \\
\hline 1.000 & 0.975 & $5.99 \mathrm{E}-10$ & 0.0005 & $5.99 \mathrm{E}-10$ & $8.21 \mathrm{E}-12$ & 0.0042 & $8.31 \mathrm{E}-12$ & $1.56 \mathrm{E}-10$ & 0.0002 & $1.56 \mathrm{E}-10$ \\
\hline 1.250 & 1.000 & $6.59 \mathrm{E}-10$ & 0.0005 & $6.60 \mathrm{E}-10$ & $9.83 \mathrm{E}-12$ & 0.0040 & $9.94 \mathrm{E}-12$ & $1.75 \mathrm{E}-10$ & 0.0002 & $1.75 \mathrm{E}-10$ \\
\hline 1.500 & 1.250 & $7.63 \mathrm{E}-10$ & 0.0005 & $7.64 \mathrm{E}-10$ & $1.27 \mathrm{E}-11$ & 0.0037 & $1.29 \mathrm{E}-11$ & $2.10 \mathrm{E}-10$ & 0.0002 & $2.10 \mathrm{E}-10$ \\
\hline 1.750 & 1.500 & $8.61 \mathrm{E}-10$ & 0.0005 & $8.62 \mathrm{E}-10$ & $1.55 \mathrm{E}-11$ & 0.0035 & $1.57 \mathrm{E}-11$ & $2.43 \mathrm{E}-10$ & 0.0002 & $2.43 \mathrm{E}-10$ \\
\hline 2.000 & 1.750 & $9.54 \mathrm{E}-10$ & 0.0005 & $9.55 \mathrm{E}-10$ & $1.83 \mathrm{E}-11$ & 0.0034 & $1.85 \mathrm{E}-11$ & $2.74 \mathrm{E}-10$ & 0.0002 & $2.74 \mathrm{E}-10$ \\
\hline 2.250 & 2.000 & 1.04E-09 & 0.0005 & $1.04 \mathrm{E}-09$ & $2.08 \mathrm{E}-11$ & 0.0033 & $2.10 \mathrm{E}-11$ & $3.04 \mathrm{E}-10$ & 0.0002 & $3.04 \mathrm{E}-10$ \\
\hline 2.500 & 2.250 & $1.12 \mathrm{E}-09$ & 0.0005 & $1.13 \mathrm{E}-09$ & $2.34 \mathrm{E}-11$ & 0.0032 & $2.36 \mathrm{E}-11$ & $3.32 \mathrm{E}-10$ & 0.0002 & $3.33 \mathrm{E}-10$ \\
\hline 2.750 & 2.500 & $1.20 \mathrm{E}-09$ & 0.0005 & $1.21 \mathrm{E}-09$ & $2.57 \mathrm{E}-11$ & 0.0032 & $2.60 \mathrm{E}-11$ & $3.60 \mathrm{E}-10$ & 0.0002 & $3.60 \mathrm{E}-10$ \\
\hline 3.000 & 2.750 & $1.28 \mathrm{E}-09$ & 0.0005 & $1.28 \mathrm{E}-09$ & $2.79 \mathrm{E}-11$ & 0.0031 & $2.82 \mathrm{E}-11$ & $3.86 \mathrm{E}-10$ & 0.0002 & $3.86 \mathrm{E}-10$ \\
\hline 3.500 & 3.000 & $1.39 \mathrm{E}-09$ & 0.0005 & $1.39 \mathrm{E}-09$ & $3.09 \mathrm{E}-11$ & 0.0031 & $3.12 \mathrm{E}-11$ & $4.23 \mathrm{E}-10$ & 0.0002 & $4.23 \mathrm{E}-10$ \\
\hline 4.000 & 3.500 & $1.53 \mathrm{E}-09$ & 0.0005 & $1.53 \mathrm{E}-09$ & $3.47 \mathrm{E}-11$ & 0.0031 & $3.50 \mathrm{E}-11$ & $4.69 \mathrm{E}-10$ & 0.0002 & $4.69 \mathrm{E}-10$ \\
\hline 4.500 & 4.000 & $1.66 \mathrm{E}-09$ & 0.0005 & $1.66 \mathrm{E}-09$ & $3.82 \mathrm{E}-11$ & 0.0031 & $3.86 \mathrm{E}-11$ & $5.13 \mathrm{E}-10$ & 0.0002 & $5.13 \mathrm{E}-10$ \\
\hline 5.000 & 4.500 & $1.78 \mathrm{E}-09$ & 0.0005 & $1.78 \mathrm{E}-09$ & $4.14 \mathrm{E}-11$ & 0.0031 & $4.18 \mathrm{E}-11$ & $5.53 \mathrm{E}-10$ & 0.0002 & $5.54 \mathrm{E}-10$ \\
\hline 5.500 & 5.000 & 1.91E-09 & 0.0005 & $1.91 \mathrm{E}-09$ & $4.46 \mathrm{E}-11$ & 0.0031 & $4.50 \mathrm{E}-11$ & $5.95 \mathrm{E}-10$ & 0.0002 & $5.95 \mathrm{E}-10$ \\
\hline 6.000 & 5.500 & $2.02 \mathrm{E}-09$ & 0.0005 & $2.03 \mathrm{E}-09$ & $4.73 \mathrm{E}-11$ & 0.0031 & $4.77 \mathrm{E}-11$ & $6.33 \mathrm{E}-10$ & 0.0002 & $6.34 \mathrm{E}-10$ \\
\hline 6.500 & 6.000 & $2.14 \mathrm{E}-09$ & 0.0005 & $2.14 \mathrm{E}-09$ & $5.01 \mathrm{E}-11$ & 0.0032 & $5.06 \mathrm{E}-11$ & $6.71 \mathrm{E}-10$ & 0.0002 & $6.72 \mathrm{E}-10$ \\
\hline 7.000 & 6.500 & $2.26 \mathrm{E}-09$ & 0.0005 & $2.26 \mathrm{E}-09$ & $5.29 \mathrm{E}-11$ & 0.0032 & $5.34 \mathrm{E}-11$ & $7.09 \mathrm{E}-10$ & 0.0002 & $7.09 \mathrm{E}-10$ \\
\hline 7.500 & 7.000 & $2.37 \mathrm{E}-09$ & 0.0005 & $2.38 \mathrm{E}-09$ & $5.53 \mathrm{E}-11$ & 0.0032 & $5.58 \mathrm{E}-11$ & $7.46 \mathrm{E}-10$ & 0.0002 & $7.46 \mathrm{E}-10$ \\
\hline 8.000 & 7.500 & $2.49 \mathrm{E}-09$ & 0.0005 & $2.49 \mathrm{E}-09$ & $5.80 \mathrm{E}-11$ & 0.0032 & $5.85 \mathrm{E}-11$ & $7.83 \mathrm{E}-10$ & 0.0002 & $7.83 \mathrm{E}-10$ \\
\hline 9.000 & 8.000 & $2.66 \mathrm{E}-09$ & 0.0005 & $2.67 \mathrm{E}-09$ & $6.18 \mathrm{E}-11$ & 0.0033 & $6.24 \mathrm{E}-11$ & $8.38 \mathrm{E}-10$ & 0.0002 & $8.38 \mathrm{E}-10$ \\
\hline 10.000 & 9.000 & $2.90 \mathrm{E}-09$ & 0.0005 & $2.90 \mathrm{E}-09$ & $6.70 \mathrm{E}-11$ & 0.0034 & $6.77 \mathrm{E}-11$ & $9.11 \mathrm{E}-10$ & 0.0002 & $9.12 \mathrm{E}-10$ \\
\hline
\end{tabular}


PCP-2011-0001

Revision 0

\begin{tabular}{|c|c|c|c|c|c|c|c|c|c|c|}
\hline \multicolumn{11}{|c|}{$\begin{array}{c}\text { Table 3-34. Photon Dose Rate per Unit Source in Each of the } 77 \text { Source Groups - SC1 Lead } \\
\text { Shielded Container }\end{array}$} \\
\hline \multicolumn{2}{|c|}{ Energy $(\mathrm{MeV})$} & \multicolumn{3}{|c|}{ Bottom Dose Rate (rem/hr) } & \multicolumn{3}{|c|}{ Top Dose Rate (rem/hr) } & \multicolumn{3}{|c|}{ Side Dose Rate (rem/hr) } \\
\hline Upper & Lower & mean & FSD & mean $+3 \pi$ & mean & FSD & mean $+3 \pi$ & mean & FSD & mean $+3 \pi$ \\
\hline 0.020 & 0.010 & $0.00 \mathrm{E}+00$ & 0.0000 & $0.00 \mathrm{E}+00$ & $0.00 \mathrm{E}+00$ & 0.0000 & $0.00 \mathrm{E}+00$ & $0.00 \mathrm{E}+00$ & 0.0000 & $0.00 \mathrm{E}+00$ \\
\hline 0.030 & 0.020 & $0.00 \mathrm{E}+00$ & 0.0000 & $0.00 \mathrm{E}+00$ & $0.00 \mathrm{E}+00$ & 0.0000 & $0.00 \mathrm{E}+00$ & $0.00 \mathrm{E}+00$ & 0.0000 & $0.00 \mathrm{E}+00$ \\
\hline 0.040 & 0.030 & $0.00 \mathrm{E}+00$ & 0.0000 & $0.00 \mathrm{E}+00$ & $0.00 \mathrm{E}+00$ & 0.0000 & $0.00 \mathrm{E}+00$ & $0.00 \mathrm{E}+00$ & 0.0000 & $0.00 \mathrm{E}+00$ \\
\hline 0.050 & 0.040 & $0.00 \mathrm{E}+00$ & 0.0000 & $0.00 \mathrm{E}+00$ & $0.00 \mathrm{E}+00$ & 0.0000 & $0.00 \mathrm{E}+00$ & $0.00 \mathrm{E}+00$ & 0.0000 & $0.00 \mathrm{E}+00$ \\
\hline 0.060 & 0.050 & $0.00 \mathrm{E}+00$ & 0.0000 & $0.00 \mathrm{E}+00$ & $0.00 \mathrm{E}+00$ & 0.0000 & $0.00 \mathrm{E}+00$ & $0.00 \mathrm{E}+00$ & 0.0000 & $0.00 \mathrm{E}+00$ \\
\hline 0.070 & 0.060 & $0.00 \mathrm{E}+00$ & 0.0000 & $0.00 \mathrm{E}+00$ & $0.00 \mathrm{E}+00$ & 0.0000 & $0.00 \mathrm{E}+00$ & $0.00 \mathrm{E}+00$ & 0.0000 & $0.00 \mathrm{E}+00$ \\
\hline 0.080 & 0.070 & $0.00 \mathrm{E}+00$ & 0.0000 & $0.00 \mathrm{E}+00$ & $0.00 \mathrm{E}+00$ & 0.0000 & $0.00 \mathrm{E}+00$ & $0.00 \mathrm{E}+00$ & 0.0000 & $0.00 \mathrm{E}+00$ \\
\hline 0.090 & 0.080 & $0.00 \mathrm{E}+00$ & 0.0000 & $0.00 \mathrm{E}+00$ & $0.00 \mathrm{E}+00$ & 0.0000 & $0.00 \mathrm{E}+00$ & $0.00 \mathrm{E}+00$ & 0.0000 & $0.00 \mathrm{E}+00$ \\
\hline 0.100 & 0.090 & $0.00 \mathrm{E}+00$ & 0.0000 & $0.00 \mathrm{E}+00$ & $0.00 \mathrm{E}+00$ & 0.0000 & & & & \\
\hline 0.110 & 0.100 & $0.00 \mathrm{E}+1$ & & $00 \mathrm{E}+00$ & $0.00 \mathrm{E}+00$ & 0.0000 & & & & $0.00 \mathrm{E}+00$ \\
\hline 0.120 & 0.110 & $0.00 \mathrm{E}+00$ & 0.0000 & $0.00 \mathrm{E}+00$ & $0.00 \mathrm{E}+00$ & 0.0000 & $0.00 \mathrm{E}+00$ & $0.00 \mathrm{E}+00$ & 0.0000 & $0.00 \mathrm{E}+00$ \\
\hline 0.130 & 0.120 & $0.00 \mathrm{E}+00$ & 0.0000 & $0.00 \mathrm{E}+00$ & $0.00 \mathrm{E}+00$ & 0.0000 & $0.00 \mathrm{E}+00$ & $0.00 \mathrm{E}+00$ & 0.0000 & $0.00 \mathrm{E}+00$ \\
\hline 0.140 & 0.130 & $\mathrm{E}+00$ & 0.0000 & $0.00 \mathrm{E}+00$ & $0.00 \mathrm{E}+00$ & 0.0000 & $\mathrm{E}+00$ & $8+00$ & 0.0000 & $0.00 \mathrm{E}+00$ \\
\hline 0.150 & 0.140 & $\overline{\mathrm{E}+00}$ & 0.0000 & $0.00 \mathrm{E}+00$ & $0.00 \mathrm{E}+00$ & 0.0000 & $0.00 \mathrm{E}+00$ & +00 & 0.0000 & $0.00 \mathrm{E}+00$ \\
\hline 0.160 & 0.150 & $0.00 \mathrm{E}+00$ & 0.0000 & $0.00 \mathrm{E}+00$ & $0.00 \mathrm{E}+00$ & 0.0000 & $0.00 \mathrm{E}+00$ & $\mathrm{E}+00$ & 0.0000 & $0.00 \mathrm{E}+00$ \\
\hline 0.170 & 0.160 & $5.06 \mathrm{E}-50$ & 0.2062 & $8.19 \mathrm{E}-50$ & $3.18 \mathrm{E}-49$ & 0.7881 & $1.07 \mathrm{E}-48$ & $3 \mathrm{E}-50$ & 0.8003 & $9.63 \mathrm{E}-50$ \\
\hline 0.180 & 0.170 & $2.57 \mathrm{E}-44$ & 0.0555 & $3.00 \mathrm{E}-44$ & $2.95 \mathrm{E}-44$ & 0.6366 & $8.57 \mathrm{E}-44$ & $6.00 \mathrm{E}-45$ & 0.0240 & $6.43 \mathrm{E}-45$ \\
\hline 0.190 & 0.180 & $1 \mathrm{E}-40$ & 0.0613 & $3.56 \mathrm{E}-40$ & $2.02 \mathrm{E}-40$ & 0.6295 & $5.82 \mathrm{E}-40$ & $8.28 \mathrm{E}-41$ & 0.0550 & $9.64 \mathrm{E}-41$ \\
\hline 0.200 & 0.190 & $6.70 \mathrm{E}-36$ & 0.8960 & $2.47 \mathrm{E}-35$ & $1.20 \mathrm{E}-37$ & 0.0716 & $1.46 \mathrm{E}-37$ & $2.02 \mathrm{E}-37$ & 0.0328 & $2.22 \mathrm{E}-37$ \\
\hline 0.210 & 0.200 & $4.99 \mathrm{E}-33$ & 0.9071 & $1.86 \mathrm{E}-32$ & $5.97 \mathrm{E}-35$ & 0.0748 & $7.31 \mathrm{E}-35$ & $1.53 \mathrm{E}-34$ & 0.0475 & $1.75 \mathrm{E}-34$ \\
\hline 0.220 & & $1.98 \mathrm{E}-31$ & 0.3913 & $30 \mathrm{E}-31$ & $1.09 \mathrm{E}-32$ & 0.0766 & $1.35 \mathrm{E}-32$ & $3.95 \mathrm{E}-32$ & 0.0350 & $4.36 \mathrm{E}-32$ \\
\hline 0.230 & & $52 \mathrm{E}-29$ & & $61 \mathrm{E}-29$ & $1.32 \mathrm{E}-30$ & 0.1971 & $2.10 \mathrm{E}-30$ & $4.62 \mathrm{E}-30$ & 0.0383 & $5.15 \mathrm{E}-30$ \\
\hline 0.240 & & & & $31 \mathrm{E}-27$ & $6.63 \mathrm{E}-29$ & 0.2470 & $1.15 \mathrm{E}-28$ & & & $3.16 \mathrm{E}-28$ \\
\hline 0.250 & 0.240 & $2 \mathrm{E}-26$ & & $5.46 \mathrm{E}-26$ & $1.96 \mathrm{E}-27$ & 0.2425 & $3.38 \mathrm{E}-27$ & $1.39 \mathrm{E}-26$ & 0.2422 & $2.40 \mathrm{E}-26$ \\
\hline 0.260 & 0.250 & E-25 & & $1.22 \mathrm{E}-24$ & $3.27 \mathrm{E}-26$ & 0.2592 & $5.82 \mathrm{E}-26$ & $\mathrm{E}-25$ & 0.2275 & $5.15 \mathrm{E}-25$ \\
\hline 0.270 & 0.260 & E-23 & 0.1964 & $1.76 \mathrm{E}-23$ & $2.84 \mathrm{E}-25$ & 0.0352 & E-25 & $3 \mathrm{E}-24$ & 0.1723 & $6.87 \mathrm{E}-24$ \\
\hline 0.280 & 0.270 & $3 \mathrm{E}-23$ & 0.0744 & $1.12 \mathrm{E}-22$ & $3.53 \mathrm{E}-24$ & 0.2463 & $6.14 \mathrm{E}-24$ & $4.70 \mathrm{E}-23$ & 0.1831 & $7.28 \mathrm{E}-23$ \\
\hline 0.290 & 0.280 & $8.08 \mathrm{E}-22$ & 0.0962 & $1.04 \mathrm{E}-21$ & $2.40 \mathrm{E}-23$ & 0.1061 & $3.16 \mathrm{E}-23$ & $4.73 \mathrm{E}-22$ & 0.1822 & $7.32 \mathrm{E}-22$ \\
\hline 0.300 & 0.290 & $5.51 \mathrm{E}-21$ & 0.1067 & $7.27 \mathrm{E}-21$ & $1.28 \mathrm{E}-22$ & 0.0916 & $1.64 \mathrm{E}-22$ & $2.98 \mathrm{E}-21$ & 0.2023 & $4.79 \mathrm{E}-21$ \\
\hline 0.325 & 0.300 & $1.20 \mathrm{E}-19$ & 0.1397 & $1.70 \mathrm{E}-19$ & $3.41 \mathrm{E}-21$ & 0.2180 & $5.64 \mathrm{E}-21$ & 5.39E-20 & 0.1426 & $7.70 \mathrm{E}-20$ \\
\hline 0.350 & 0.325 & $2.31 \mathrm{E}-18$ & 0.1039 & $3.03 \mathrm{E}-18$ & $5.38 \mathrm{E}-20$ & 0.1772 & $8.23 \mathrm{E}-20$ & $1.23 \mathrm{E}-18$ & 0.1615 & $1.83 \mathrm{E}-18$ \\
\hline 0.375 & 0.350 & $4.84 \mathrm{E}-17$ & 0.3214 & $9.50 \mathrm{E}-17$ & $4.70 \mathrm{E}-19$ & 0.1507 & $6.82 \mathrm{E}-19$ & $3.09 \mathrm{E}-17$ & 0.3995 & $6.79 \mathrm{E}-17$ \\
\hline 0.400 & 0.375 & $3.91 \mathrm{E}-16$ & 0.2887 & $7.30 \mathrm{E}-16$ & $4.98 \mathrm{E}-18$ & 0.2504 & $8.72 \mathrm{E}-18$ & $1.65 \mathrm{E}-16$ & 0.1177 & $2.24 \mathrm{E}-16$ \\
\hline 0.425 & & & & $2.24 \mathrm{E}-15$ & $2.38 \mathrm{E}-17$ & 0.2609 & $4.24 \mathrm{E}-17$ & $6.13 \mathrm{E}-16$ & 0.0470 & $6.99 \mathrm{E}-16$ \\
\hline 0.450 & & & & & & & $1.96 \mathrm{E}-16$ & & 0.0318 & $2.31 \mathrm{E}-15$ \\
\hline 0.475 & 0.450 & $9.03 \mathrm{E}-15$ & 0.0622 & $1.07 \mathrm{E}-14$ & $2.15 \mathrm{E}-16$ & 0.1594 & $3.17 \mathrm{E}-16$ & $7.06 \mathrm{E}-15$ & 0.1287 & $9.79 \mathrm{E}-15$ \\
\hline 0.500 & 0.475 & $2.07 \mathrm{E}-14$ & 0.0698 & $2.51 \mathrm{E}-14$ & $5.05 \mathrm{E}-16$ & 0.1199 & $6.87 \mathrm{E}-16$ & $1.43 \mathrm{E}-14$ & 0.0194 & $1.52 \mathrm{E}-14$ \\
\hline 0.525 & 0.500 & $4.14 \mathrm{E}-14$ & 0.0224 & $4.42 \mathrm{E}-14$ & $9.44 \mathrm{E}-16$ & 0.0689 & $1.14 \mathrm{E}-15$ & $2.96 \mathrm{E}-14$ & 0.0153 & $3.09 \mathrm{E}-14$ \\
\hline
\end{tabular}


PCP-2011-0001

Revision 0

Table 3-34. Photon Dose Rate per Unit Source in Each of the 77 Source Groups - SC1 Lead Shielded Container

\begin{tabular}{|c|c|c|c|c|c|c|c|c|c|c|}
\hline \multicolumn{2}{|c|}{ Energy $(\mathrm{MeV})$} & \multicolumn{3}{|c|}{ Bottom Dose Rate (rem $/ \mathrm{hr})$} & \multicolumn{3}{|c|}{ Top Dose Rate (rem/hr) } & \multicolumn{3}{|c|}{ Side Dose Rate (rem/hr) } \\
\hline Upper & Lower & mean & FSD & mean $+3 \pi$ & mean & FSD & mean $+3 \pi$ & mean & FSD & mean $+3 \pi$ \\
\hline 0.550 & 0.525 & $7.74 \mathrm{E}-14$ & 0.0211 & $8.23 \mathrm{E}-14$ & $2.90 \mathrm{E}-15$ & 0.2958 & $5.48 \mathrm{E}-15$ & $5.45 \mathrm{E}-14$ & 0.0094 & $\overline{5.61 \mathrm{E}-14}$ \\
\hline 0.575 & 0.550 & $1.37 \mathrm{E}-13$ & 0.0240 & $1.47 \mathrm{E}-13$ & $3.95 \mathrm{E}-15$ & 0.2156 & $6.51 \mathrm{E}-15$ & $9.43 \mathrm{E}-14$ & 0.0093 & $9.69 \mathrm{E}-14$ \\
\hline 0.600 & 0.575 & $2.21 \mathrm{E}-13$ & 0.0202 & $2.34 \mathrm{E}-13$ & $6.32 \mathrm{E}-15$ & 0.1521 & $9.21 \mathrm{E}-15$ & $1.54 \mathrm{E}-13$ & 0.0079 & $1.58 \mathrm{E}-13$ \\
\hline 0.625 & 0.600 & $3.35 \mathrm{E}-13$ & 0.0161 & $3.51 \mathrm{E}-13$ & $9.03 \mathrm{E}-15$ & 0.1125 & $1.21 \mathrm{E}-14$ & $2.36 \mathrm{E}-13$ & 0.0097 & $2.43 \mathrm{E}-13$ \\
\hline 0.650 & 0.625 & $4.72 \mathrm{E}-13$ & 0.0102 & $4.87 \mathrm{E}-13$ & $1.38 \mathrm{E}-14$ & 0.0927 & $1.77 \mathrm{E}-14$ & $3.45 \mathrm{E}-13$ & 0.0103 & $3.56 \mathrm{E}-13$ \\
\hline 0.675 & 0.650 & $6.62 \mathrm{E}-13$ & 0.0098 & $6.81 \mathrm{E}-13$ & $1.90 \mathrm{E}-14$ & 0.0717 & $2.31 \mathrm{E}-14$ & $4.76 \mathrm{E}-13$ & 0.0052 & $4.84 \mathrm{E}-13$ \\
\hline 0.700 & 0.675 & $9.06 \mathrm{E}-13$ & 0.0098 & $9.33 \mathrm{E}-13$ & $2.52 \mathrm{E}-14$ & 0.0578 & $2.95 \mathrm{E}-14$ & $6.48 \mathrm{E}-13$ & 0.0044 & $6.56 \mathrm{E}-13$ \\
\hline 0.725 & 0.700 & $1.18 \mathrm{E}-12$ & 0.0081 & $1.21 \mathrm{E}-12$ & $3.21 \mathrm{E}-14$ & 0.0453 & $3.65 \mathrm{E}-14$ & $8.56 \mathrm{E}-13$ & 0.0045 & $8.67 \mathrm{E}-13$ \\
\hline 0.750 & 0.725 & $1.51 \mathrm{E}-12$ & 0.0068 & $1.54 \mathrm{E}-12$ & $4.09 \mathrm{E}-14$ & 0.0421 & $4.61 \mathrm{E}-14$ & $1.09 \mathrm{E}-12$ & 0.0030 & $1.10 \mathrm{E}-12$ \\
\hline 0.775 & 0.750 & $1.90 \mathrm{E}-12$ & 0.0061 & $1.93 \mathrm{E}-12$ & $4.89 \mathrm{E}-14$ & 0.0312 & $5.34 \mathrm{E}-14$ & $1.37 \mathrm{E}-12$ & 0.0024 & $1.38 \mathrm{E}-12$ \\
\hline 0.800 & 0.775 & $2.33 \mathrm{E}-12$ & 0.0051 & $2.37 \mathrm{E}-12$ & $7.56 \mathrm{E}-14$ & 0.0307 & $8.26 \mathrm{E}-14$ & $1.83 \mathrm{E}-12$ & 0.0013 & $1.83 \mathrm{E}-12$ \\
\hline 0.825 & 0.800 & $2.83 \mathrm{E}-12$ & 0.0047 & $2.87 \mathrm{E}-12$ & $9.10 \mathrm{E}-14$ & 0.0290 & $9.89 \mathrm{E}-14$ & $2.22 \mathrm{E}-12$ & 0.0012 & $2.23 \mathrm{E}-12$ \\
\hline 0.850 & 0.825 & $3.40 \mathrm{E}-12$ & 0.0043 & $3.44 \mathrm{E}-12$ & $1.09 \mathrm{E}-13$ & 0.0258 & $1.18 \mathrm{E}-13$ & $2.66 \mathrm{E}-12$ & 0.0011 & $2.66 \mathrm{E}-12$ \\
\hline 0.875 & 0.850 & $4.00 \mathrm{E}-12$ & 0.0041 & $4.05 \mathrm{E}-12$ & $1.31 \mathrm{E}-13$ & 0.0245 & $1.40 \mathrm{E}-13$ & $3.13 \mathrm{E}-12$ & 0.0011 & $3.14 \mathrm{E}-12$ \\
\hline 0.900 & 0.875 & $4.66 \mathrm{E}-12$ & 0.0039 & $4.71 \mathrm{E}-12$ & $1.56 \mathrm{E}-13$ & 0.0233 & $1.67 \mathrm{E}-13$ & $3.66 \mathrm{E}-12$ & 0.0010 & $3.67 \mathrm{E}-12$ \\
\hline 0.925 & 0.900 & $5.38 \mathrm{E}-12$ & 0.0037 & $5.44 \mathrm{E}-12$ & $1.77 \mathrm{E}-13$ & 0.0216 & $1.89 \mathrm{E}-13$ & $4.23 \mathrm{E}-12$ & 0.0009 & $4.24 \mathrm{E}-12$ \\
\hline 0.950 & 0.925 & $6.15 \mathrm{E}-12$ & 0.0035 & $6.21 \mathrm{E}-12$ & $2.03 \mathrm{E}-13$ & 0.0199 & $2.15 \mathrm{E}-13$ & $4.84 \mathrm{E}-12$ & 0.0009 & $4.86 \mathrm{E}-12$ \\
\hline 0.975 & 0.950 & $6.97 \mathrm{E}-12$ & 0.0033 & $7.04 \mathrm{E}-12$ & $2.32 \mathrm{E}-13$ & 0.0190 & $2.45 \mathrm{E}-13$ & $5.49 \mathrm{E}-12$ & 0.0008 & $5.51 \mathrm{E}-12$ \\
\hline 1.000 & 0.975 & $7.85 \mathrm{E}-12$ & 0.0031 & $7.92 \mathrm{E}-12$ & $2.65 \mathrm{E}-13$ & 0.0182 & $2.79 \mathrm{E}-13$ & $6.18 \mathrm{E}-12$ & 0.0008 & $6.20 \mathrm{E}-12$ \\
\hline 1.250 & 1.000 & $1.36 \mathrm{E}-11$ & 0.0025 & $1.37 \mathrm{E}-11$ & $4.88 \mathrm{E}-13$ & 0.0145 & $5.10 \mathrm{E}-13$ & $1.08 \mathrm{E}-11$ & 0.0007 & $1.08 \mathrm{E}-11$ \\
\hline 1.500 & 1.250 & $2.56 \mathrm{E}-11$ & 0.0021 & $2.57 \mathrm{E}-11$ & $9.91 \mathrm{E}-13$ & 0.0110 & $1.02 \mathrm{E}-12$ & $2.06 \mathrm{E}-11$ & 0.0005 & $2.06 \mathrm{E}-11$ \\
\hline 1.750 & 1.500 & $3.75 \mathrm{E}-11$ & 0.0019 & $3.77 \mathrm{E}-11$ & $1.58 \mathrm{E}-12$ & 0.0094 & $1.63 \mathrm{E}-12$ & $3.06 \mathrm{E}-11$ & 0.0005 & $3.06 \mathrm{E}-11$ \\
\hline 2.000 & 1.750 & $4.85 \mathrm{E}-11$ & 0.0018 & $4.88 \mathrm{E}-11$ & $2.21 \mathrm{E}-12$ & 0.0087 & $2.26 \mathrm{E}-12$ & $3.99 \mathrm{E}-11$ & 0.0004 & $4.00 \mathrm{E}-11$ \\
\hline 2.250 & 2.000 & $5.79 \mathrm{E}-11$ & 0.0017 & $5.82 \mathrm{E}-11$ & $2.78 \mathrm{E}-12$ & 0.0083 & $2.84 \mathrm{E}-12$ & $4.82 \mathrm{E}-11$ & 0.0004 & $4.82 \mathrm{E}-11$ \\
\hline 2.500 & 2.250 & $6.62 \mathrm{E}-11$ & 0.0017 & $6.66 \mathrm{E}-11$ & $3.32 \mathrm{E}-12$ & 0.0081 & $3.40 \mathrm{E}-12$ & $5.55 \mathrm{E}-11$ & 0.0004 & $5.55 \mathrm{E}-11$ \\
\hline 2.750 & 2.500 & $7.33 \mathrm{E}-11$ & 0.0017 & $7.37 \mathrm{E}-11$ & $3.84 \mathrm{E}-12$ & 0.0080 & $3.93 \mathrm{E}-12$ & $6.19 \mathrm{E}-11$ & 0.0004 & $6.19 \mathrm{E}-11$ \\
\hline 3.000 & 2.750 & $7.96 \mathrm{E}-11$ & 0.0018 & $8.00 \mathrm{E}-11$ & $4.27 \mathrm{E}-12$ & 0.0079 & $4.38 \mathrm{E}-12$ & $6.74 \mathrm{E}-11$ & 0.0004 & $6.75 \mathrm{E}-11$ \\
\hline 3.500 & 3.000 & $8.72 \mathrm{E}-11$ & 0.0018 & $8.77 \mathrm{E}-11$ & $4.94 \mathrm{E}-12$ & 0.0079 & $5.06 \mathrm{E}-12$ & $7.43 \mathrm{E}-11$ & 0.0004 & $7.44 \mathrm{E}-11$ \\
\hline 4.000 & 3.500 & $9.53 \mathrm{E}-11$ & 0.0019 & $9.59 \mathrm{E}-11$ & $5.65 \mathrm{E}-12$ & 0.0081 & $5.78 \mathrm{E}-12$ & $8.17 \mathrm{E}-11$ & 0.0005 & $8.18 \mathrm{E}-11$ \\
\hline 4.500 & 4.000 & $1.02 \mathrm{E}-10$ & 0.0019 & $1.02 \mathrm{E}-10$ & $6.24 \mathrm{E}-12$ & 0.0083 & $6.39 \mathrm{E}-12$ & $8.76 \mathrm{E}-11$ & 0.0005 & $8.77 \mathrm{E}-11$ \\
\hline 5.000 & 4.500 & $1.07 \mathrm{E}-10$ & 0.0020 & $1.08 \mathrm{E}-10$ & $6.69 \mathrm{E}-12$ & 0.0085 & $6.86 \mathrm{E}-12$ & $9.23 \mathrm{E}-11$ & 0.0005 & $9.24 \mathrm{E}-11$ \\
\hline 5.500 & 5.000 & $1.12 \mathrm{E}-10$ & 0.0021 & $1.12 \mathrm{E}-10$ & $7.06 \mathrm{E}-12$ & 0.0088 & $7.25 \mathrm{E}-12$ & $9.65 \mathrm{E}-11$ & 0.0005 & $9.66 \mathrm{E}-11$ \\
\hline 6.000 & 5.500 & $1.16 \mathrm{E}-10$ & 0.0022 & $1.17 \mathrm{E}-10$ & $7.39 \mathrm{E}-12$ & 0.0090 & $7.59 \mathrm{E}-12$ & $1.00 \mathrm{E}-10$ & 0.0005 & $1.00 \mathrm{E}-10$ \\
\hline 6.500 & 6.000 & $1.20 \mathrm{E}-10$ & 0.0022 & $1.21 \mathrm{E}-10$ & $7.68 \mathrm{E}-12$ & 0.0093 & $7.89 \mathrm{E}-12$ & $1.04 \mathrm{E}-10$ & 0.0006 & $1.04 \mathrm{E}-10$ \\
\hline 7.000 & 6.500 & $1.24 \mathrm{E}-10$ & 0.0023 & $1.25 \mathrm{E}-10$ & $7.90 \mathrm{E}-12$ & 0.0096 & $8.13 \mathrm{E}-12$ & $1.07 \mathrm{E}-10$ & 0.0006 & $1.08 \mathrm{E}-10$ \\
\hline 7.500 & 7.000 & $1.28 \mathrm{E}-10$ & 0.0024 & $1.29 \mathrm{E}-10$ & $8.15 \mathrm{E}-12$ & 0.0099 & $8.40 \mathrm{E}-12$ & $1.11 \mathrm{E}-10$ & 0.0006 & $1.11 \mathrm{E}-10$ \\
\hline 8.000 & 7.500 & $1.32 \mathrm{E}-10$ & 0.0024 & $1.33 \mathrm{E}-10$ & $8.39 \mathrm{E}-12$ & 0.0102 & $8.65 \mathrm{E}-12$ & $1.15 \mathrm{E}-10$ & 0.0006 & $1.15 \mathrm{E}-10$ \\
\hline 9.000 & 8.000 & $1.39 \mathrm{E}-10$ & 0.0025 & $1.40 \mathrm{E}-10$ & $8.78 \mathrm{E}-12$ & 0.0105 & $9.05 \mathrm{E}-12$ & $1.20 \mathrm{E}-10$ & 0.0006 & $1.21 \mathrm{E}-10$ \\
\hline 10.000 & 9.000 & $1.49 \mathrm{E}-10$ & 0.0026 & $1.50 \mathrm{E}-10$ & $9.47 \mathrm{E}-12$ & 0.0107 & $9.78 \mathrm{E}-12$ & $1.28 \mathrm{E}-10$ & 0.0006 & $1.28 \mathrm{E}-10$ \\
\hline
\end{tabular}


PCP-2011-0001

Revision 0

Table 3-35. Neutron Dose Rate per Unit Neutron Source in Each of the 47 Source Groups SC2 Polyethylene Shielded Container

\begin{tabular}{|c|c|c|c|c|c|c|c|c|c|c|}
\hline \multicolumn{2}{|c|}{ Energy $(\mathrm{MeV})$} & \multicolumn{3}{|c|}{ Bottom Dose Rate (rem/hr) } & \multicolumn{3}{|c|}{ Top Dose Rate (rem/hr) } & \multicolumn{3}{|c|}{ Side Dose Rate (rem $/ \mathrm{hr})$} \\
\hline Upper & Lower & mean & FSD & mean $+3 \pi$ & mean & FSD & mean $+3 \pi$ & mean & FSD & mean $+3 \pi$ \\
\hline $1.00 \mathrm{E}-07$ & $1.00 \mathrm{E}-11$ & $1.90 \mathrm{E}-10$ & 0.0043 & $1.93 \mathrm{E}-10$ & $4.69 \mathrm{E}-12$ & 0.0306 & $5.12 \mathrm{E}-12$ & $1.21 \mathrm{E}-10$ & 0.0012 & $1.22 \mathrm{E}-10$ \\
\hline 4.14E-07 & $1.00 \mathrm{E}-07$ & $2.02 \mathrm{E}-10$ & 0.0042 & $2.05 \mathrm{E}-10$ & $4.79 \mathrm{E}-12$ & 0.0310 & $5.23 \mathrm{E}-12$ & $1.28 \mathrm{E}-10$ & 0.0012 & $1.28 \mathrm{E}-10$ \\
\hline $8.76 \mathrm{E}-07$ & $4.14 \mathrm{E}-07$ & $2.11 \mathrm{E}-10$ & 0.0042 & $2.14 \mathrm{E}-10$ & $4.91 \mathrm{E}-12$ & 0.0321 & $5.39 \mathrm{E}-12$ & $1.32 \mathrm{E}-10$ & 0.0012 & $1.32 \mathrm{E}-10$ \\
\hline $1.86 \mathrm{E}-06$ & $8.76 \mathrm{E}-07$ & $2.19 \mathrm{E}-10$ & 0.0041 & $2.21 \mathrm{E}-10$ & $5.04 \mathrm{E}-12$ & 0.0313 & $5.52 \mathrm{E}-12$ & $1.35 \mathrm{E}-10$ & 0.0012 & $1.36 \mathrm{E}-10$ \\
\hline $5.04 \mathrm{E}-06$ & $1.86 \mathrm{E}-06$ & $2.25 \mathrm{E}-10$ & 0.0040 & $2.28 \mathrm{E}-10$ & $5.19 \mathrm{E}-12$ & 0.0300 & $5.65 \mathrm{E}-12$ & $1.40 \mathrm{E}-10$ & 0.0011 & $1.40 \mathrm{E}-10$ \\
\hline $1.07 \mathrm{E}-05$ & $5.04 \mathrm{E}-06$ & $2.32 \mathrm{E}-10$ & 0.0041 & $2.35 \mathrm{E}-10$ & $5.51 \mathrm{E}-12$ & 0.0325 & $6.05 \mathrm{E}-12$ & $1.44 \mathrm{E}-10$ & 0.0011 & $1.44 \mathrm{E}-10$ \\
\hline $3.73 \mathrm{E}-05$ & $1.07 \mathrm{E}-05$ & $2.42 \mathrm{E}-10$ & 0.0040 & $2.45 \mathrm{E}-10$ & $5.79 \mathrm{E}-12$ & 0.0324 & $6.35 \mathrm{E}-12$ & $1.50 \mathrm{E}-10$ & 0.0011 & $1.50 \mathrm{E}-10$ \\
\hline $1.01 \mathrm{E}-04$ & $3.73 \mathrm{E}-05$ & $2.53 \mathrm{E}-10$ & 0.0040 & $2.56 \mathrm{E}-10$ & $5.73 \mathrm{E}-12$ & 0.0303 & $6.25 \mathrm{E}-12$ & $1.56 \mathrm{E}-10$ & 0.0011 & $1.56 \mathrm{E}-10$ \\
\hline $2.14 \mathrm{E}-04$ & $1.01 \mathrm{E}-04$ & $2.60 \mathrm{E}-10$ & 0.0040 & $2.63 \mathrm{E}-10$ & $5.76 \mathrm{E}-12$ & 0.0301 & $6.28 \mathrm{E}-12$ & $1.61 \mathrm{E}-10$ & 0.0011 & $1.62 \mathrm{E}-10$ \\
\hline $4.54 \mathrm{E}-04$ & $2.14 \mathrm{E}-04$ & $2.66 \mathrm{E}-10$ & 0.0039 & $2.69 \mathrm{E}-10$ & $6.30 \mathrm{E}-12$ & 0.0289 & $6.84 \mathrm{E}-12$ & $1.66 \mathrm{E}-10$ & 0.0011 & $1.66 \mathrm{E}-10$ \\
\hline $1.58 \mathrm{E}-03$ & $4.54 \mathrm{E}-04$ & $2.77 \mathrm{E}-10$ & 0.0040 & $2.80 \mathrm{E}-10$ & $6.42 \mathrm{E}-12$ & 0.0307 & $7.01 \mathrm{E}-12$ & $1.73 \mathrm{E}-10$ & 0.0011 & $1.74 \mathrm{E}-10$ \\
\hline $3.35 \mathrm{E}-03$ & $1.58 \mathrm{E}-03$ & $2.90 \mathrm{E}-10$ & 0.0040 & $2.93 \mathrm{E}-10$ & $6.30 \mathrm{E}-12$ & 0.0283 & $6.83 \mathrm{E}-12$ & $1.80 \mathrm{E}-10$ & 0.0011 & $1.81 \mathrm{E}-10$ \\
\hline $7.10 \mathrm{E}-03$ & $3.35 \mathrm{E}-03$ & $2.97 \mathrm{E}-10$ & 0.0040 & $3.01 \mathrm{E}-10$ & $6.88 \mathrm{E}-12$ & 0.0290 & $7.48 \mathrm{E}-12$ & $1.87 \mathrm{E}-10$ & 0.0011 & $1.88 \mathrm{E}-10$ \\
\hline $1.50 \mathrm{E}-02$ & $7.10 \mathrm{E}-03$ & $3.05 \mathrm{E}-10$ & 0.0039 & $3.09 \mathrm{E}-10$ & $7.15 \mathrm{E}-12$ & 0.0286 & $7.76 \mathrm{E}-12$ & $1.94 \mathrm{E}-10$ & 0.0011 & $1.95 \mathrm{E}-10$ \\
\hline $2.19 \mathrm{E}-02$ & $1.50 \mathrm{E}-02$ & $3.15 \mathrm{E}-10$ & 0.0038 & $3.18 \mathrm{E}-10$ & $7.14 \mathrm{E}-12$ & 0.0260 & $7.70 \mathrm{E}-12$ & $2.00 \mathrm{E}-10$ & 0.0011 & $2.01 \mathrm{E}-10$ \\
\hline $2.42 \mathrm{E}-02$ & $2.19 \mathrm{E}-02$ & $3.18 \mathrm{E}-10$ & 0.0038 & $3.22 \mathrm{E}-10$ & $7.42 \mathrm{E}-12$ & 0.0252 & $7.98 \mathrm{E}-12$ & $2.03 \mathrm{E}-10$ & 0.0011 & $2.04 \mathrm{E}-10$ \\
\hline $2.61 \mathrm{E}-02$ & $2.42 \mathrm{E}-02$ & $3.20 \mathrm{E}-10$ & 0.0038 & $3.24 \mathrm{E}-10$ & $7.49 \mathrm{E}-12$ & 0.0260 & $8.07 \mathrm{E}-12$ & $2.05 \mathrm{E}-10$ & 0.0011 & $2.05 \mathrm{E}-10$ \\
\hline $3.18 \mathrm{E}-02$ & $2.61 \mathrm{E}-02$ & $3.23 \mathrm{E}-10$ & 0.0038 & $3.27 \mathrm{E}-10$ & $7.65 \mathrm{E}-12$ & 0.0277 & $8.29 \mathrm{E}-12$ & $2.06 \mathrm{E}-10$ & 0.0011 & $2.07 \mathrm{E}-10$ \\
\hline $4.09 \mathrm{E}-02$ & $3.18 \mathrm{E}-02$ & $3.29 \mathrm{E}-10$ & 0.0038 & $3.33 \mathrm{E}-10$ & $7.69 \mathrm{E}-12$ & 0.0276 & $8.33 \mathrm{E}-12$ & $2.10 \mathrm{E}-10$ & 0.0011 & $2.11 \mathrm{E}-10$ \\
\hline $6.74 \mathrm{E}-02$ & $4.09 \mathrm{E}-02$ & $3.36 \mathrm{E}-10$ & 0.0038 & $3.40 \mathrm{E}-10$ & $8.10 \mathrm{E}-12$ & 0.0281 & $8.78 \mathrm{E}-12$ & $2.16 \mathrm{E}-10$ & 0.0011 & $2.17 \mathrm{E}-10$ \\
\hline $1.11 \mathrm{E}-01$ & $6.74 \mathrm{E}-02$ & $3.63 \mathrm{E}-10$ & 0.0039 & $3.67 \mathrm{E}-10$ & $8.56 \mathrm{E}-12$ & 0.0288 & $9.30 \mathrm{E}-12$ & $2.31 \mathrm{E}-10$ & 0.0011 & $2.32 \mathrm{E}-10$ \\
\hline $1.83 \mathrm{E}-01$ & $1.11 \mathrm{E}-01$ & $4.18 \mathrm{E}-10$ & 0.0042 & $4.24 \mathrm{E}-10$ & $1.07 \mathrm{E}-11$ & 0.0491 & $1.23 \mathrm{E}-11$ & $2.63 \mathrm{E}-10$ & 0.0011 & $2.64 \mathrm{E}-10$ \\
\hline $2.97 \mathrm{E}-01$ & $1.83 \mathrm{E}-01$ & $5.67 \mathrm{E}-10$ & 0.0050 & $5.76 \mathrm{E}-10$ & $1.49 \mathrm{E}-11$ & 0.0347 & $1.64 \mathrm{E}-11$ & $3.42 \mathrm{E}-10$ & 0.0014 & $3.43 \mathrm{E}-10$ \\
\hline $3.69 \mathrm{E}-01$ & $2.97 \mathrm{E}-01$ & $8.13 \mathrm{E}-10$ & 0.0057 & $8.27 \mathrm{E}-10$ & $2.30 \mathrm{E}-11$ & 0.0457 & $2.61 \mathrm{E}-11$ & $4.71 \mathrm{E}-10$ & 0.0016 & $4.74 \mathrm{E}-10$ \\
\hline $4.98 \mathrm{E}-01$ & $3.69 \mathrm{E}-01$ & $1.21 \mathrm{E}-09$ & 0.0060 & $1.23 \mathrm{E}-09$ & $3.37 \mathrm{E}-11$ & 0.0363 & $3.73 \mathrm{E}-11$ & $6.78 \mathrm{E}-10$ & 0.0017 & $6.81 \mathrm{E}-10$ \\
\hline $6.08 \mathrm{E}-01$ & $4.98 \mathrm{E}-01$ & $1.82 \mathrm{E}-09$ & 0.0055 & $1.85 \mathrm{E}-09$ & $6.06 \mathrm{E}-11$ & 0.0391 & $6.77 \mathrm{E}-11$ & $1.03 \mathrm{E}-09$ & 0.0017 & $1.04 \mathrm{E}-09$ \\
\hline $7.43 \mathrm{E}-01$ & $6.08 \mathrm{E}-01$ & $2.56 \mathrm{E}-09$ & 0.0051 & $2.60 \mathrm{E}-09$ & $8.82 \mathrm{E}-11$ & 0.0268 & $9.53 \mathrm{E}-11$ & $1.47 \mathrm{E}-09$ & 0.0015 & $1.48 \mathrm{E}-09$ \\
\hline $8.21 \mathrm{E}-01$ & $7.43 \mathrm{E}-01$ & $3.24 \mathrm{E}-09$ & 0.0047 & $3.28 \mathrm{E}-09$ & $1.08 \mathrm{E}-10$ & 0.0295 & $1.18 \mathrm{E}-10$ & $1.89 \mathrm{E}-09$ & 0.0015 & $1.90 \mathrm{E}-09$ \\
\hline $1.00 \mathrm{E}+00$ & $8.21 \mathrm{E}-01$ & $4.13 \mathrm{E}-09$ & 0.0044 & 4.19E-09 & $1.56 \mathrm{E}-10$ & 0.0282 & $1.69 \mathrm{E}-10$ & $2.46 \mathrm{E}-09$ & 0.0013 & $2.47 \mathrm{E}-09$ \\
\hline $1.35 \mathrm{E}+00$ & $1.00 \mathrm{E}+00$ & $5.83 \mathrm{E}-09$ & 0.0037 & $5.90 \mathrm{E}-09$ & $2.27 \mathrm{E}-10$ & 0.0242 & $2.43 \mathrm{E}-10$ & $3.59 \mathrm{E}-09$ & 0.0011 & $3.60 \mathrm{E}-09$ \\
\hline $1.65 \mathrm{E}+00$ & $1.35 \mathrm{E}+00$ & 7.67E-09 & 0.0032 & $7.74 \mathrm{E}-09$ & $3.06 \mathrm{E}-10$ & 0.0179 & $3.22 \mathrm{E}-10$ & $4.86 \mathrm{E}-09$ & 0.0009 & $4.87 \mathrm{E}-09$ \\
\hline $1.92 \mathrm{E}+00$ & $1.65 \mathrm{E}+00$ & $8.96 \mathrm{E}-09$ & 0.0029 & $9.03 \mathrm{E}-09$ & $3.92 \mathrm{E}-10$ & 0.0170 & $4.12 \mathrm{E}-10$ & $5.83 \mathrm{E}-09$ & 0.0008 & $5.85 \mathrm{E}-09$ \\
\hline $2.23 \mathrm{E}+00$ & $1.92 \mathrm{E}+00$ & $1.01 \mathrm{E}-08$ & 0.0027 & $1.01 \mathrm{E}-08$ & $4.24 \mathrm{E}-10$ & 0.0138 & $4.42 \mathrm{E}-10$ & $6.62 \mathrm{E}-09$ & 0.0008 & $6.64 \mathrm{E}-09$ \\
\hline $2.35 \mathrm{E}+00$ & $2.23 \mathrm{E}+00$ & $1.08 \mathrm{E}-08$ & 0.0025 & $1.09 \mathrm{E}-08$ & $4.94 \mathrm{E}-10$ & 0.0139 & $5.15 \mathrm{E}-10$ & $7.21 \mathrm{E}-09$ & 0.0007 & $7.23 \mathrm{E}-09$ \\
\hline $2.37 \mathrm{E}+00$ & $2.35 \mathrm{E}+00$ & $1.10 \mathrm{E}-08$ & 0.0025 & $1.11 \mathrm{E}-08$ & $5.23 \mathrm{E}-10$ & 0.0118 & $5.41 \mathrm{E}-10$ & $7.40 \mathrm{E}-09$ & 0.0007 & $7.42 \mathrm{E}-09$ \\
\hline $2.47 \mathrm{E}+00$ & $2.37 \mathrm{E}+00$ & $1.11 \mathrm{E}-08$ & 0.0024 & $1.12 \mathrm{E}-08$ & $5.07 \mathrm{E}-10$ & 0.0127 & $5.26 \mathrm{E}-10$ & $7.54 \mathrm{E}-09$ & 0.0007 & $7.56 \mathrm{E}-09$ \\
\hline $2.73 \mathrm{E}+00$ & $2.47 \mathrm{E}+00$ & $1.17 \mathrm{E}-08$ & 0.0024 & $1.17 \mathrm{E}-08$ & $5.26 \mathrm{E}-10$ & 0.0127 & $5.46 \mathrm{E}-10$ & $7.86 \mathrm{E}-09$ & 0.0007 & 7.87E-09 \\
\hline $3.01 \mathrm{E}+00$ & $2.73 \mathrm{E}+00$ & $1.20 \mathrm{E}-08$ & 0.0023 & $1.21 \mathrm{E}-08$ & $5.53 \mathrm{E}-10$ & 0.0123 & $5.74 \mathrm{E}-10$ & $8.22 \mathrm{E}-09$ & 0.0007 & $8.23 \mathrm{E}-09$ \\
\hline $3.68 \mathrm{E}+00$ & $3.01 \mathrm{E}+00$ & $1.31 \mathrm{E}-08$ & 0.0022 & $1.32 \mathrm{E}-08$ & $6.02 \mathrm{E}-10$ & 0.0111 & $6.22 \mathrm{E}-10$ & $9.05 \mathrm{E}-09$ & 0.0006 & $9.06 \mathrm{E}-09$ \\
\hline $4.97 \mathrm{E}+00$ & $3.68 \mathrm{E}+00$ & $1.59 \mathrm{E}-08$ & 0.0019 & $1.60 \mathrm{E}-08$ & $8.20 \mathrm{E}-10$ & 0.0095 & $8.44 \mathrm{E}-10$ & $1.12 \mathrm{E}-08$ & 0.0005 & $1.12 \mathrm{E}-08$ \\
\hline $6.07 \mathrm{E}+00$ & $4.97 \mathrm{E}+00$ & $1.79 \mathrm{E}-08$ & 0.0017 & $1.80 \mathrm{E}-08$ & $1.06 \mathrm{E}-09$ & 0.0077 & $1.09 \mathrm{E}-09$ & $1.29 \mathrm{E}-08$ & 0.0005 & $1.29 \mathrm{E}-08$ \\
\hline $7.41 \mathrm{E}+00$ & $6.07 \mathrm{E}+00$ & $1.86 \mathrm{E}-08$ & 0.0016 & $1.87 \mathrm{E}-08$ & $1.22 \mathrm{E}-09$ & 0.0070 & $1.25 \mathrm{E}-09$ & $1.36 \mathrm{E}-08$ & 0.0004 & $1.36 \mathrm{E}-08$ \\
\hline $8.61 \mathrm{E}+00$ & $7.41 \mathrm{E}+00$ & $1.86 \mathrm{E}-08$ & 0.0016 & $1.86 \mathrm{E}-08$ & $1.27 \mathrm{E}-09$ & 0.0067 & $1.30 \mathrm{E}-09$ & $1.38 \mathrm{E}-08$ & 0.0004 & $1.38 \mathrm{E}-08$ \\
\hline $1.00 \mathrm{E}+01$ & $8.61 \mathrm{E}+00$ & $1.87 \mathrm{E}-08$ & 0.0015 & $1.88 \mathrm{E}-08$ & $1.40 \mathrm{E}-09$ & 0.0059 & $1.42 \mathrm{E}-09$ & $1.40 \mathrm{E}-08$ & 0.0004 & $1.41 \mathrm{E}-08$ \\
\hline $1.22 \mathrm{E}+01$ & $1.00 \mathrm{E}+01$ & $2.03 \mathrm{E}-08$ & 0.0014 & $2.04 \mathrm{E}-08$ & $1.64 \mathrm{E}-09$ & 0.0054 & $1.67 \mathrm{E}-09$ & $1.54 \mathrm{E}-08$ & 0.0004 & $1.54 \mathrm{E}-08$ \\
\hline $1.42 \mathrm{E}+01$ & $1.22 \mathrm{E}+01$ & $2.32 \mathrm{E}-08$ & 0.0013 & $2.32 \mathrm{E}-08$ & $1.99 \mathrm{E}-09$ & 0.0048 & $2.02 \mathrm{E}-09$ & $1.76 \mathrm{E}-08$ & 0.0003 & $1.76 \mathrm{E}-08$ \\
\hline $1.96 \mathrm{E}+01$ & $1.42 \mathrm{E}+01$ & $2.65 \mathrm{E}-08$ & 0.0013 & $2.66 \mathrm{E}-08$ & $2.48 \mathrm{E}-09$ & 0.0044 & $2.52 \mathrm{E}-09$ & $2.04 \mathrm{E}-08$ & 0.0003 & $2.04 \mathrm{E}-08$ \\
\hline
\end{tabular}


PCP-2011-0001

Revision 0

Table 3-36. Secondary Photon Dose Rate per Unit Neutron Source in Each of the 47 Source Groups - SC2 Polyethylene Shielded Container

\begin{tabular}{|c|c|c|c|c|c|c|c|c|c|c|}
\hline \multicolumn{2}{|c|}{ Energy $(\mathrm{MeV})$} & \multicolumn{3}{|c|}{ Bottom Dose Rate (rem/hr) } & \multicolumn{3}{|c|}{ Top Dose Rate (rem/hr) } & \multicolumn{3}{|c|}{ Side Dose Rate (rem $/ \mathrm{hr})$} \\
\hline Upper & Lower & mean & FSD & mean $+3 \pi$ & mean & FSD & mean $+3 \pi$ & mean & FSD & mean $+3 \pi$ \\
\hline $1.00 \mathrm{E}-07$ & $1.00 \mathrm{E}-11$ & $3.76 \mathrm{E}-10$ & 0.0042 & $3.81 \mathrm{E}-10$ & $2.30 \mathrm{E}-11$ & 0.0151 & $2.41 \mathrm{E}-11$ & $3.17 \mathrm{E}-10$ & 0.0011 & $3.18 \mathrm{E}-10$ \\
\hline 4.14E-07 & $1.00 \mathrm{E}-07$ & $3.88 \mathrm{E}-10$ & 0.0045 & $3.93 \mathrm{E}-10$ & $2.30 \mathrm{E}-11$ & 0.0145 & $2.40 \mathrm{E}-11$ & $3.22 \mathrm{E}-10$ & 0.0011 & $3.23 \mathrm{E}-10$ \\
\hline $8.76 \mathrm{E}-07$ & $4.14 \mathrm{E}-07$ & $3.87 \mathrm{E}-10$ & 0.0043 & $3.92 \mathrm{E}-10$ & $2.35 \mathrm{E}-11$ & 0.0159 & $2.46 \mathrm{E}-11$ & $3.25 \mathrm{E}-10$ & 0.0011 & $3.26 \mathrm{E}-10$ \\
\hline $1.86 \mathrm{E}-06$ & $8.76 \mathrm{E}-07$ & $3.91 \mathrm{E}-10$ & 0.0042 & $3.96 \mathrm{E}-10$ & $2.34 \mathrm{E}-11$ & 0.0152 & $2.45 \mathrm{E}-11$ & $3.27 \mathrm{E}-10$ & 0.0011 & $3.28 \mathrm{E}-10$ \\
\hline $5.04 \mathrm{E}-06$ & $1.86 \mathrm{E}-06$ & 3.94E-10 & 0.0043 & $4.00 \mathrm{E}-10$ & $2.39 \mathrm{E}-11$ & 0.0147 & $2.49 \mathrm{E}-11$ & $3.28 \mathrm{E}-10$ & 0.0011 & $3.29 \mathrm{E}-10$ \\
\hline $1.07 \mathrm{E}-05$ & $5.04 \mathrm{E}-06$ & $3.97 \mathrm{E}-10$ & 0.0044 & $4.03 \mathrm{E}-10$ & $2.35 \mathrm{E}-11$ & 0.0140 & $2.45 \mathrm{E}-11$ & $3.30 \mathrm{E}-10$ & 0.0011 & $3.31 \mathrm{E}-10$ \\
\hline $3.73 \mathrm{E}-05$ & $1.07 \mathrm{E}-05$ & $3.94 \mathrm{E}-10$ & 0.0043 & $3.99 \mathrm{E}-10$ & $2.42 \mathrm{E}-11$ & 0.0148 & $2.53 \mathrm{E}-11$ & $3.31 \mathrm{E}-10$ & 0.0011 & $3.32 \mathrm{E}-10$ \\
\hline $1.01 \mathrm{E}-04$ & $3.73 \mathrm{E}-05$ & $3.99 \mathrm{E}-10$ & 0.0044 & $4.04 \mathrm{E}-10$ & $2.35 \mathrm{E}-11$ & 0.0139 & $2.44 \mathrm{E}-11$ & $3.33 \mathrm{E}-10$ & 0.0011 & $3.34 \mathrm{E}-10$ \\
\hline $2.14 \mathrm{E}-04$ & $1.01 \mathrm{E}-04$ & $3.99 \mathrm{E}-10$ & 0.0044 & $4.04 \mathrm{E}-10$ & $2.43 \mathrm{E}-11$ & 0.0158 & $2.55 \mathrm{E}-11$ & $3.33 \mathrm{E}-10$ & 0.0011 & $3.34 \mathrm{E}-10$ \\
\hline $4.54 \mathrm{E}-04$ & $2.14 \mathrm{E}-04$ & $3.95 \mathrm{E}-10$ & 0.0043 & $4.00 \mathrm{E}-10$ & $2.45 \mathrm{E}-11$ & 0.0152 & $2.56 \mathrm{E}-11$ & $3.33 \mathrm{E}-10$ & 0.0011 & $3.34 \mathrm{E}-10$ \\
\hline $1.58 \mathrm{E}-03$ & $4.54 \mathrm{E}-04$ & $3.95 \mathrm{E}-10$ & 0.0044 & $4.00 \mathrm{E}-10$ & $2.43 \mathrm{E}-11$ & 0.0157 & $2.55 \mathrm{E}-11$ & $3.34 \mathrm{E}-10$ & 0.0012 & $3.35 \mathrm{E}-10$ \\
\hline $3.35 \mathrm{E}-03$ & $1.58 \mathrm{E}-03$ & $3.94 \mathrm{E}-10$ & 0.0044 & $3.99 \mathrm{E}-10$ & $2.37 \mathrm{E}-11$ & 0.0146 & $2.47 \mathrm{E}-11$ & $3.32 \mathrm{E}-10$ & 0.0012 & $3.34 \mathrm{E}-10$ \\
\hline $7.10 \mathrm{E}-03$ & $3.35 \mathrm{E}-03$ & $3.93 \mathrm{E}-10$ & 0.0045 & $3.99 \mathrm{E}-10$ & $2.37 \mathrm{E}-11$ & 0.0161 & $2.49 \mathrm{E}-11$ & $3.32 \mathrm{E}-10$ & 0.0012 & $3.33 \mathrm{E}-10$ \\
\hline $1.50 \mathrm{E}-02$ & $7.10 \mathrm{E}-03$ & $3.88 \mathrm{E}-10$ & 0.0046 & $3.93 \mathrm{E}-10$ & $2.39 \mathrm{E}-11$ & 0.0159 & $2.50 \mathrm{E}-11$ & $3.30 \mathrm{E}-10$ & 0.0012 & $3.31 \mathrm{E}-10$ \\
\hline $2.19 \mathrm{E}-02$ & $1.50 \mathrm{E}-02$ & $3.90 \mathrm{E}-10$ & 0.0044 & $3.95 \mathrm{E}-10$ & $2.39 \mathrm{E}-11$ & 0.0138 & $2.49 \mathrm{E}-11$ & $3.29 \mathrm{E}-10$ & 0.0012 & $3.30 \mathrm{E}-10$ \\
\hline $2.42 \mathrm{E}-02$ & $2.19 \mathrm{E}-02$ & $3.88 \mathrm{E}-10$ & 0.0045 & $3.94 \mathrm{E}-10$ & $2.38 \mathrm{E}-11$ & 0.0140 & $2.48 \mathrm{E}-11$ & $3.29 \mathrm{E}-10$ & 0.0012 & $3.30 \mathrm{E}-10$ \\
\hline $2.61 \mathrm{E}-02$ & $2.42 \mathrm{E}-02$ & $3.88 \mathrm{E}-10$ & 0.0046 & $3.93 \mathrm{E}-10$ & $2.41 \mathrm{E}-11$ & 0.0142 & $2.51 \mathrm{E}-11$ & $3.28 \mathrm{E}-10$ & 0.0012 & $3.30 \mathrm{E}-10$ \\
\hline $3.18 \mathrm{E}-02$ & $2.61 \mathrm{E}-02$ & $3.86 \mathrm{E}-10$ & 0.0045 & $3.91 \mathrm{E}-10$ & $2.39 \mathrm{E}-11$ & 0.0139 & $2.49 \mathrm{E}-11$ & $3.27 \mathrm{E}-10$ & 0.0012 & $3.28 \mathrm{E}-10$ \\
\hline $4.09 \mathrm{E}-02$ & $3.18 \mathrm{E}-02$ & $3.86 \mathrm{E}-10$ & 0.0046 & $3.91 \mathrm{E}-10$ & $2.38 \mathrm{E}-11$ & 0.0153 & $2.49 \mathrm{E}-11$ & $3.27 \mathrm{E}-10$ & 0.0012 & $3.28 \mathrm{E}-10$ \\
\hline $6.74 \mathrm{E}-02$ & $4.09 \mathrm{E}-02$ & $3.81 \mathrm{E}-10$ & 0.0046 & $3.86 \mathrm{E}-10$ & $2.39 \mathrm{E}-11$ & 0.0167 & $2.51 \mathrm{E}-11$ & $3.25 \mathrm{E}-10$ & 0.0012 & $3.26 \mathrm{E}-10$ \\
\hline $1.11 \mathrm{E}-01$ & $6.74 \mathrm{E}-02$ & $3.74 \mathrm{E}-10$ & 0.0045 & $3.79 \mathrm{E}-10$ & $2.38 \mathrm{E}-11$ & 0.0150 & $2.49 \mathrm{E}-11$ & $3.21 \mathrm{E}-10$ & 0.0012 & $3.22 \mathrm{E}-10$ \\
\hline $1.83 \mathrm{E}-01$ & $1.11 \mathrm{E}-01$ & $3.66 \mathrm{E}-10$ & 0.0045 & $3.71 \mathrm{E}-10$ & $2.30 \mathrm{E}-11$ & 0.0149 & $2.40 \mathrm{E}-11$ & $3.12 \mathrm{E}-10$ & 0.0012 & $3.13 \mathrm{E}-10$ \\
\hline $2.97 \mathrm{E}-01$ & $1.83 \mathrm{E}-01$ & $3.49 \mathrm{E}-10$ & 0.0046 & $3.54 \mathrm{E}-10$ & $2.29 \mathrm{E}-11$ & 0.0145 & $2.39 \mathrm{E}-11$ & $3.00 \mathrm{E}-10$ & 0.0012 & $3.01 \mathrm{E}-10$ \\
\hline $3.69 \mathrm{E}-01$ & $2.97 \mathrm{E}-01$ & $3.36 \mathrm{E}-10$ & 0.0046 & $3.40 \mathrm{E}-10$ & $2.20 \mathrm{E}-11$ & 0.0165 & $2.31 \mathrm{E}-11$ & $2.89 \mathrm{E}-10$ & 0.0012 & $2.90 \mathrm{E}-10$ \\
\hline $4.98 \mathrm{E}-01$ & $3.69 \mathrm{E}-01$ & $3.20 \mathrm{E}-10$ & 0.0045 & $3.24 \mathrm{E}-10$ & $2.17 \mathrm{E}-11$ & 0.0149 & $2.27 \mathrm{E}-11$ & $2.76 \mathrm{E}-10$ & 0.0012 & $2.77 \mathrm{E}-10$ \\
\hline $6.08 \mathrm{E}-01$ & $4.98 \mathrm{E}-01$ & $3.05 \mathrm{E}-10$ & 0.0048 & $3.09 \mathrm{E}-10$ & $2.07 \mathrm{E}-11$ & 0.0156 & $2.16 \mathrm{E}-11$ & $2.61 \mathrm{E}-10$ & 0.0012 & $2.62 \mathrm{E}-10$ \\
\hline $7.43 \mathrm{E}-01$ & $6.08 \mathrm{E}-01$ & $2.87 \mathrm{E}-10$ & 0.0047 & $2.91 \mathrm{E}-10$ & $2.01 \mathrm{E}-11$ & 0.0169 & $2.11 \mathrm{E}-11$ & $2.48 \mathrm{E}-10$ & 0.0012 & $2.49 \mathrm{E}-10$ \\
\hline $8.21 \mathrm{E}-01$ & $7.43 \mathrm{E}-01$ & $2.74 \mathrm{E}-10$ & 0.0048 & $2.78 \mathrm{E}-10$ & $1.96 \mathrm{E}-11$ & 0.0162 & $2.05 \mathrm{E}-11$ & $2.37 \mathrm{E}-10$ & 0.0012 & $2.38 \mathrm{E}-10$ \\
\hline $1.00 \mathrm{E}+00$ & $8.21 \mathrm{E}-01$ & $2.61 \mathrm{E}-10$ & 0.0049 & $2.65 \mathrm{E}-10$ & $1.89 \mathrm{E}-11$ & 0.0155 & $1.97 \mathrm{E}-11$ & $2.25 \mathrm{E}-10$ & 0.0013 & $2.26 \mathrm{E}-10$ \\
\hline $1.35 \mathrm{E}+00$ & $1.00 \mathrm{E}+00$ & $2.36 \mathrm{E}-10$ & 0.0049 & $2.40 \mathrm{E}-10$ & $1.73 \mathrm{E}-11$ & 0.0142 & $1.80 \mathrm{E}-11$ & $2.05 \mathrm{E}-10$ & 0.0013 & $2.06 \mathrm{E}-10$ \\
\hline $1.65 \mathrm{E}+00$ & $1.35 \mathrm{E}+00$ & $2.12 \mathrm{E}-10$ & 0.0051 & $2.15 \mathrm{E}-10$ & $1.60 \mathrm{E}-11$ & 0.0161 & $1.68 \mathrm{E}-11$ & $1.84 \mathrm{E}-10$ & 0.0013 & $1.85 \mathrm{E}-10$ \\
\hline $1.92 \mathrm{E}+00$ & $1.65 \mathrm{E}+00$ & $1.94 \mathrm{E}-10$ & 0.0050 & $1.96 \mathrm{E}-10$ & $1.51 \mathrm{E}-11$ & 0.0164 & $1.58 \mathrm{E}-11$ & $1.70 \mathrm{E}-10$ & 0.0013 & $1.70 \mathrm{E}-10$ \\
\hline $2.23 \mathrm{E}+00$ & $1.92 \mathrm{E}+00$ & $1.81 \mathrm{E}-10$ & 0.0049 & $1.84 \mathrm{E}-10$ & $1.43 \mathrm{E}-11$ & 0.0166 & $1.50 \mathrm{E}-11$ & $1.59 \mathrm{E}-10$ & 0.0013 & $1.60 \mathrm{E}-10$ \\
\hline $2.35 \mathrm{E}+00$ & $2.23 \mathrm{E}+00$ & $1.74 \mathrm{E}-10$ & 0.0052 & $1.77 \mathrm{E}-10$ & $1.37 \mathrm{E}-11$ & 0.0172 & $1.44 \mathrm{E}-11$ & $1.51 \mathrm{E}-10$ & 0.0013 & $1.52 \mathrm{E}-10$ \\
\hline $2.37 \mathrm{E}+00$ & $2.35 \mathrm{E}+00$ & $1.71 \mathrm{E}-10$ & 0.0053 & $1.73 \mathrm{E}-10$ & $1.37 \mathrm{E}-11$ & 0.0179 & $1.44 \mathrm{E}-11$ & $1.49 \mathrm{E}-10$ & 0.0013 & $1.49 \mathrm{E}-10$ \\
\hline $2.47 \mathrm{E}+00$ & $2.37 \mathrm{E}+00$ & $1.67 \mathrm{E}-10$ & 0.0050 & $1.69 \mathrm{E}-10$ & $1.34 \mathrm{E}-11$ & 0.0145 & $1.40 \mathrm{E}-11$ & $1.47 \mathrm{E}-10$ & 0.0013 & $1.48 \mathrm{E}-10$ \\
\hline $2.73 \mathrm{E}+00$ & $2.47 \mathrm{E}+00$ & $1.64 \mathrm{E}-10$ & 0.0051 & $1.66 \mathrm{E}-10$ & $1.30 \mathrm{E}-11$ & 0.0152 & $1.36 \mathrm{E}-11$ & $1.44 \mathrm{E}-10$ & 0.0013 & $1.45 \mathrm{E}-10$ \\
\hline $3.01 \mathrm{E}+00$ & $2.73 \mathrm{E}+00$ & $1.62 \mathrm{E}-10$ & 0.0050 & $1.64 \mathrm{E}-10$ & $1.31 \mathrm{E}-11$ & 0.0163 & $1.38 \mathrm{E}-11$ & $1.42 \mathrm{E}-10$ & 0.0013 & $1.43 \mathrm{E}-10$ \\
\hline $3.68 \mathrm{E}+00$ & $3.01 \mathrm{E}+00$ & $1.56 \mathrm{E}-10$ & 0.0048 & $1.59 \mathrm{E}-10$ & $1.31 \mathrm{E}-11$ & 0.0163 & $1.38 \mathrm{E}-11$ & $1.39 \mathrm{E}-10$ & 0.0013 & $1.39 \mathrm{E}-10$ \\
\hline $4.97 \mathrm{E}+00$ & $3.68 \mathrm{E}+00$ & $1.49 \mathrm{E}-10$ & 0.0046 & $1.51 \mathrm{E}-10$ & $1.23 \mathrm{E}-11$ & 0.0138 & $1.28 \mathrm{E}-11$ & $1.31 \mathrm{E}-10$ & 0.0012 & $1.32 \mathrm{E}-10$ \\
\hline $6.07 \mathrm{E}+00$ & $4.97 \mathrm{E}+00$ & $1.68 \mathrm{E}-10$ & 0.0040 & $1.70 \mathrm{E}-10$ & $1.45 \mathrm{E}-11$ & 0.0127 & $1.50 \mathrm{E}-11$ & $1.47 \mathrm{E}-10$ & 0.0010 & $1.47 \mathrm{E}-10$ \\
\hline $7.41 \mathrm{E}+00$ & $6.07 \mathrm{E}+00$ & $1.94 \mathrm{E}-10$ & 0.0035 & $1.96 \mathrm{E}-10$ & $1.67 \mathrm{E}-11$ & 0.0111 & $1.73 \mathrm{E}-11$ & $1.70 \mathrm{E}-10$ & 0.0009 & $1.70 \mathrm{E}-10$ \\
\hline $8.61 \mathrm{E}+00$ & $7.41 \mathrm{E}+00$ & $2.26 \mathrm{E}-10$ & 0.0032 & $2.29 \mathrm{E}-10$ & $1.99 \mathrm{E}-11$ & 0.0105 & $2.05 \mathrm{E}-11$ & $1.97 \mathrm{E}-10$ & 0.0008 & $1.98 \mathrm{E}-10$ \\
\hline $1.00 \mathrm{E}+01$ & $8.61 \mathrm{E}+00$ & $2.36 \mathrm{E}-10$ & 0.0031 & $2.38 \mathrm{E}-10$ & $2.20 \mathrm{E}-11$ & 0.0101 & $2.26 \mathrm{E}-11$ & $2.07 \mathrm{E}-10$ & 0.0008 & $2.07 \mathrm{E}-10$ \\
\hline $1.22 \mathrm{E}+01$ & $1.00 \mathrm{E}+01$ & $2.62 \mathrm{E}-10$ & 0.0031 & $2.65 \mathrm{E}-10$ & $2.57 \mathrm{E}-11$ & 0.0096 & $2.64 \mathrm{E}-11$ & $2.31 \mathrm{E}-10$ & 0.0008 & $2.31 \mathrm{E}-10$ \\
\hline $1.42 \mathrm{E}+01$ & $1.22 \mathrm{E}+01$ & $2.46 \mathrm{E}-10$ & 0.0030 & $2.49 \mathrm{E}-10$ & $2.54 \mathrm{E}-11$ & 0.0103 & $2.62 \mathrm{E}-11$ & $2.19 \mathrm{E}-10$ & 0.0008 & $2.19 \mathrm{E}-10$ \\
\hline $1.96 \mathrm{E}+01$ & $1.42 \mathrm{E}+01$ & $1.95 \mathrm{E}-10$ & 0.0031 & $1.97 \mathrm{E}-10$ & $2.13 \mathrm{E}-11$ & 0.0097 & $2.20 \mathrm{E}-11$ & $1.73 \mathrm{E}-10$ & 0.0008 & $1.74 \mathrm{E}-10$ \\
\hline
\end{tabular}


PCP-2011-0001

Revision 0

Table 3-37. Photon Dose Rate per Unit Source in Each of the 77 Source Groups - SC2 Polyethylene Shielded Container

\begin{tabular}{|c|c|c|c|c|c|c|c|c|c|c|}
\hline \multicolumn{2}{|c|}{ Energy $(\mathrm{MeV})$} & \multicolumn{3}{|c|}{ Bottom Dose Rate (rem/hr) } & \multicolumn{3}{|c|}{ Top Dose Rate (rem/hr) } & \multicolumn{3}{|c|}{ Side Dose Rate $(\mathrm{rem} / \mathrm{hr})$} \\
\hline Upper & Lower & mean & FSD & mean $+3 \pi$ & mean & FSD & mean $+3 \pi$ & mean & FSD & mean $+3 \pi$ \\
\hline 0.020 & 0.010 & $0.00 \mathrm{E}+00$ & 0.0000 & $0.00 \mathrm{E}+00$ & $0.00 \mathrm{E}+00$ & 0.0000 & $0.00 \mathrm{E}+00$ & $0.00 \mathrm{E}+00$ & 0.0000 & $0.00 \mathrm{E}+00$ \\
\hline 0.030 & 0.020 & $3.52 \mathrm{E}-30$ & 0.0060 & $3.58 \mathrm{E}-30$ & $0.00 \mathrm{E}+00$ & 0.0000 & $0.00 \mathrm{E}+00$ & $4.28 \mathrm{E}-43$ & 0.0552 & $4.99 \mathrm{E}-43$ \\
\hline 0.040 & 0.030 & $3.81 \mathrm{E}-20$ & 0.2247 & $6.38 \mathrm{E}-20$ & $2.34 \mathrm{E}-47$ & 0.9962 & $9.33 \mathrm{E}-47$ & $2.59 \mathrm{E}-26$ & 0.3118 & $5.02 \mathrm{E}-26$ \\
\hline 0.050 & 0.040 & $2.37 \mathrm{E}-16$ & 0.0712 & $2.88 \mathrm{E}-16$ & $4.69 \mathrm{E}-34$ & 0.0952 & $6.03 \mathrm{E}-34$ & $1.85 \mathrm{E}-19$ & 0.6205 & $5.30 \mathrm{E}-19$ \\
\hline 0.060 & 0.050 & $1.88 \mathrm{E}-14$ & 0.0419 & $2.12 \mathrm{E}-14$ & $6.67 \mathrm{E}-26$ & 0.6540 & $1.98 \mathrm{E}-25$ & $1.47 \mathrm{E}-16$ & 0.2077 & $2.39 \mathrm{E}-16$ \\
\hline 0.070 & 0.060 & $1.85 \mathrm{E}-13$ & 0.0127 & $1.92 \mathrm{E}-13$ & $1.47 \mathrm{E}-20$ & 0.6896 & $4.50 \mathrm{E}-20$ & $5.85 \mathrm{E}-15$ & 0.0157 & $6.12 \mathrm{E}-15$ \\
\hline 0.080 & 0.070 & $7.95 \mathrm{E}-13$ & 0.0122 & $8.24 \mathrm{E}-13$ & $2.11 \mathrm{E}-19$ & 0.1175 & $2.85 \mathrm{E}-19$ & $6.10 \mathrm{E}-14$ & 0.0096 & $6.27 \mathrm{E}-14$ \\
\hline 0.090 & 0.080 & $1.96 \mathrm{E}-12$ & 0.0073 & $2.01 \mathrm{E}-12$ & $9.44 \mathrm{E}-18$ & 0.2279 & $1.59 \mathrm{E}-17$ & $2.74 \mathrm{E}-13$ & 0.0043 & $2.77 \mathrm{E}-13$ \\
\hline 0.100 & 0.090 & $3.67 \mathrm{E}-12$ & 0.0077 & $3.76 \mathrm{E}-12$ & $6.88 \mathrm{E}-17$ & 0.1360 & $9.69 \mathrm{E}-17$ & $7.37 \mathrm{E}-13$ & 0.0024 & $7.42 \mathrm{E}-13$ \\
\hline 0.110 & 0.100 & $5.71 \mathrm{E}-12$ & 0.0056 & $5.81 \mathrm{E}-12$ & $3.65 \mathrm{E}-16$ & 0.1064 & $4.81 \mathrm{E}-16$ & $1.51 \mathrm{E}-12$ & 0.0020 & $1.51 \mathrm{E}-12$ \\
\hline 0.120 & 0.110 & $8.20 \mathrm{E}-12$ & 0.0061 & $8.35 \mathrm{E}-12$ & $1.39 \mathrm{E}-15$ & 0.1684 & $2.09 \mathrm{E}-15$ & $2.57 \mathrm{E}-12$ & 0.0017 & $2.59 \mathrm{E}-12$ \\
\hline 0.130 & 0.120 & $1.07 \mathrm{E}-11$ & 0.0036 & $1.09 \mathrm{E}-11$ & $5.72 \mathrm{E}-15$ & 0.4214 & $1.30 \mathrm{E}-14$ & $3.88 \mathrm{E}-12$ & 0.0015 & $3.90 \mathrm{E}-12$ \\
\hline 0.140 & 0.130 & $1.33 \mathrm{E}-11$ & 0.0015 & $1.34 \mathrm{E}-11$ & $1.50 \mathrm{E}-14$ & 0.0466 & $1.70 \mathrm{E}-14$ & $5.85 \mathrm{E}-12$ & 0.0005 & $5.85 \mathrm{E}-12$ \\
\hline 0.150 & 0.140 & $1.61 \mathrm{E}-11$ & 0.0014 & $1.62 \mathrm{E}-11$ & $2.41 \mathrm{E}-14$ & 0.0385 & $2.69 \mathrm{E}-14$ & $7.62 \mathrm{E}-12$ & 0.0005 & $7.64 \mathrm{E}-12$ \\
\hline 0.160 & 0.150 & $1.90 \mathrm{E}-11$ & 0.0013 & $1.90 \mathrm{E}-11$ & $4.21 \mathrm{E}-14$ & 0.0339 & $4.64 \mathrm{E}-14$ & $9.52 \mathrm{E}-12$ & 0.0004 & $9.53 \mathrm{E}-12$ \\
\hline 0.170 & 0.160 & $2.19 \mathrm{E}-11$ & 0.0013 & $2.20 \mathrm{E}-11$ & $6.22 \mathrm{E}-14$ & 0.0292 & $6.77 \mathrm{E}-14$ & $1.15 \mathrm{E}-11$ & 0.0004 & $1.15 \mathrm{E}-11$ \\
\hline 0.180 & 0.170 & $2.49 \mathrm{E}-11$ & 0.0012 & $2.50 \mathrm{E}-11$ & $8.56 \mathrm{E}-14$ & 0.0254 & $9.22 \mathrm{E}-14$ & $1.36 \mathrm{E}-11$ & 0.0004 & $1.36 \mathrm{E}-11$ \\
\hline 0.190 & 0.180 & $2.78 \mathrm{E}-11$ & 0.0012 & $2.79 \mathrm{E}-11$ & $1.14 \mathrm{E}-13$ & 0.0240 & $1.23 \mathrm{E}-13$ & $1.57 \mathrm{E}-11$ & 0.0004 & $1.57 \mathrm{E}-11$ \\
\hline 0.200 & 0.190 & $3.08 \mathrm{E}-11$ & 0.0012 & $3.09 \mathrm{E}-11$ & $1.48 \mathrm{E}-13$ & 0.0210 & $1.57 \mathrm{E}-13$ & $1.79 \mathrm{E}-11$ & 0.0003 & $1.79 \mathrm{E}-11$ \\
\hline 0.210 & 0.200 & $3.38 \mathrm{E}-11$ & 0.0011 & $3.39 \mathrm{E}-11$ & $1.81 \mathrm{E}-13$ & 0.0181 & $1.90 \mathrm{E}-13$ & $2.00 \mathrm{E}-11$ & 0.0003 & $2.01 \mathrm{E}-11$ \\
\hline 0.220 & 0.210 & $3.68 \mathrm{E}-11$ & 0.0011 & $3.69 \mathrm{E}-11$ & $2.24 \mathrm{E}-13$ & 0.0169 & $2.35 \mathrm{E}-13$ & $2.23 \mathrm{E}-11$ & 0.0003 & $2.23 \mathrm{E}-11$ \\
\hline 0.230 & 0.220 & $3.98 \mathrm{E}-11$ & 0.0011 & $3.99 \mathrm{E}-11$ & $2.70 \mathrm{E}-13$ & 0.0159 & $2.82 \mathrm{E}-13$ & $2.45 \mathrm{E}-11$ & 0.0003 & $2.45 \mathrm{E}-11$ \\
\hline 0.240 & 0.230 & $4.28 \mathrm{E}-11$ & 0.0011 & $4.29 \mathrm{E}-11$ & $3.19 \mathrm{E}-13$ & 0.0148 & $3.33 \mathrm{E}-13$ & $2.67 \mathrm{E}-11$ & 0.0003 & $2.67 \mathrm{E}-11$ \\
\hline 0.250 & 0.240 & $4.57 \mathrm{E}-11$ & 0.0011 & $4.59 \mathrm{E}-11$ & $3.69 \mathrm{E}-13$ & 0.0137 & $3.84 \mathrm{E}-13$ & $2.89 \mathrm{E}-11$ & 0.0003 & $2.90 \mathrm{E}-11$ \\
\hline 0.260 & 0.250 & $4.87 \mathrm{E}-11$ & 0.0011 & $4.89 \mathrm{E}-11$ & $4.27 \mathrm{E}-13$ & 0.0135 & $4.45 \mathrm{E}-13$ & $3.12 \mathrm{E}-11$ & 0.0003 & $3.12 \mathrm{E}-11$ \\
\hline 0.270 & 0.260 & $5.16 \mathrm{E}-11$ & 0.0010 & $5.18 \mathrm{E}-11$ & $4.81 \mathrm{E}-13$ & 0.0128 & $4.99 \mathrm{E}-13$ & $3.34 \mathrm{E}-11$ & 0.0003 & $3.34 \mathrm{E}-11$ \\
\hline 0.280 & 0.270 & $5.45 \mathrm{E}-11$ & 0.0010 & $5.47 \mathrm{E}-11$ & $5.35 \mathrm{E}-13$ & 0.0121 & $5.55 \mathrm{E}-13$ & $3.56 \mathrm{E}-11$ & 0.0003 & $3.57 \mathrm{E}-11$ \\
\hline 0.290 & 0.280 & $5.75 \mathrm{E}-11$ & 0.0010 & $5.76 \mathrm{E}-11$ & $5.93 \mathrm{E}-13$ & 0.0117 & $6.14 \mathrm{E}-13$ & $3.79 \mathrm{E}-11$ & 0.0003 & $3.79 \mathrm{E}-11$ \\
\hline 0.300 & 0.290 & $6.04 \mathrm{E}-11$ & 0.0010 & $6.06 \mathrm{E}-11$ & $6.57 \mathrm{E}-13$ & 0.0110 & $6.79 \mathrm{E}-13$ & $4.01 \mathrm{E}-11$ & 0.0003 & $4.01 \mathrm{E}-11$ \\
\hline 0.325 & 0.300 & $6.53 \mathrm{E}-11$ & 0.0010 & $6.55 \mathrm{E}-11$ & $7.73 \mathrm{E}-13$ & 0.0105 & $7.98 \mathrm{E}-13$ & $4.39 \mathrm{E}-11$ & 0.0003 & $4.39 \mathrm{E}-11$ \\
\hline 0.350 & 0.325 & $7.22 \mathrm{E}-11$ & 0.0010 & $7.25 \mathrm{E}-11$ & $9.48 \mathrm{E}-13$ & 0.0096 & $9.76 \mathrm{E}-13$ & $4.93 \mathrm{E}-11$ & 0.0003 & $4.94 \mathrm{E}-11$ \\
\hline 0.375 & 0.350 & $7.90 \mathrm{E}-11$ & 0.0010 & $7.92 \mathrm{E}-11$ & $1.14 \mathrm{E}-12$ & 0.0091 & $1.17 \mathrm{E}-12$ & $5.47 \mathrm{E}-11$ & 0.0003 & $5.47 \mathrm{E}-11$ \\
\hline 0.400 & 0.375 & $8.54 \mathrm{E}-11$ & 0.0010 & $8.57 \mathrm{E}-11$ & $1.34 \mathrm{E}-12$ & 0.0086 & $1.37 \mathrm{E}-12$ & $5.98 \mathrm{E}-11$ & 0.0003 & $5.99 \mathrm{E}-11$ \\
\hline 0.425 & 0.400 & $9.17 \mathrm{E}-11$ & 0.0009 & $9.19 \mathrm{E}-11$ & $1.54 \mathrm{E}-12$ & 0.0082 & $1.58 \mathrm{E}-12$ & $6.49 \mathrm{E}-11$ & 0.0002 & $6.49 \mathrm{E}-11$ \\
\hline 0.450 & 0.425 & $9.77 \mathrm{E}-11$ & 0.0009 & $9.79 \mathrm{E}-11$ & $1.77 \mathrm{E}-12$ & 0.0079 & $1.81 \mathrm{E}-12$ & $6.98 \mathrm{E}-11$ & 0.0002 & $6.98 \mathrm{E}-11$ \\
\hline 0.475 & 0.450 & $1.03 \mathrm{E}-10$ & 0.0009 & $1.04 \mathrm{E}-10$ & $1.98 \mathrm{E}-12$ & 0.0076 & $2.03 \mathrm{E}-12$ & $7.46 \mathrm{E}-11$ & 0.0002 & $7.46 \mathrm{E}-11$ \\
\hline 0.500 & 0.475 & $1.09 \mathrm{E}-10$ & 0.0009 & $1.09 \mathrm{E}-10$ & $2.20 \mathrm{E}-12$ & 0.0076 & $2.25 \mathrm{E}-12$ & $7.92 \mathrm{E}-11$ & 0.0002 & $7.93 \mathrm{E}-11$ \\
\hline 0.525 & 0.500 & $1.15 \mathrm{E}-10$ & 0.0009 & $1.15 \mathrm{E}-10$ & $2.45 \mathrm{E}-12$ & 0.0070 & $2.50 \mathrm{E}-12$ & $8.39 \mathrm{E}-11$ & 0.0002 & $8.39 \mathrm{E}-11$ \\
\hline
\end{tabular}


PCP-2011-0001

Revision 0

Table 3-37. Photon Dose Rate per Unit Source in Each of the 77 Source Groups - SC2 Polyethylene Shielded Container

\begin{tabular}{|c|c|c|c|c|c|c|c|c|c|c|}
\hline \multicolumn{2}{|c|}{ Energy $(\mathrm{MeV})$} & \multicolumn{3}{|c|}{ Bottom Dose Rate (rem/hr) } & \multicolumn{3}{|c|}{ Top Dose Rate (rem/hr) } & \multicolumn{3}{|c|}{ Side Dose Rate $(\mathrm{rem} / \mathrm{hr})$} \\
\hline Upper & Lower & mean & FSD & mean $+3 \pi$ & mean & FSD & mean $+3 \pi$ & mean & FSD & mean $+3 \pi$ \\
\hline 0.550 & 0.525 & $1.21 \mathrm{E}-10$ & 0.0009 & $1.21 \mathrm{E}-10$ & $2.69 \mathrm{E}-12$ & 0.0068 & $2.74 \mathrm{E}-12$ & $8.86 \mathrm{E}-11$ & 0.0002 & $8.86 \mathrm{E}-11$ \\
\hline 0.575 & 0.550 & $1.26 \mathrm{E}-10$ & 0.0009 & $1.26 \mathrm{E}-10$ & $2.95 \mathrm{E}-12$ & 0.0066 & $3.00 \mathrm{E}-12$ & $9.32 \mathrm{E}-11$ & 0.0002 & $9.33 \mathrm{E}-11$ \\
\hline 0.600 & 0.575 & $1.31 \mathrm{E}-10$ & 0.0009 & $1.32 \mathrm{E}-10$ & $3.16 \mathrm{E}-12$ & 0.0063 & $3.22 \mathrm{E}-12$ & $9.77 \mathrm{E}-11$ & 0.0002 & $9.78 \mathrm{E}-11$ \\
\hline 0.625 & 0.600 & $1.37 \mathrm{E}-10$ & 0.0009 & $1.37 \mathrm{E}-10$ & $3.41 \mathrm{E}-12$ & 0.0061 & $3.47 \mathrm{E}-12$ & $1.02 \mathrm{E}-10$ & 0.0002 & $1.02 \mathrm{E}-10$ \\
\hline 0.650 & 0.625 & $1.41 \mathrm{E}-10$ & 0.0009 & $1.42 \mathrm{E}-10$ & $3.68 \mathrm{E}-12$ & 0.0059 & $3.74 \mathrm{E}-12$ & $1.06 \mathrm{E}-10$ & 0.0002 & $1.06 \mathrm{E}-10$ \\
\hline 0.675 & 0.650 & $1.46 \mathrm{E}-10$ & 0.0009 & $1.47 \mathrm{E}-10$ & $3.96 \mathrm{E}-12$ & 0.0058 & $4.02 \mathrm{E}-12$ & $1.11 \mathrm{E}-10$ & 0.0002 & $1.11 \mathrm{E}-10$ \\
\hline 0.700 & 0.675 & $1.51 \mathrm{E}-10$ & 0.0009 & $1.52 \mathrm{E}-10$ & $4.23 \mathrm{E}-12$ & 0.0056 & $4.30 \mathrm{E}-12$ & $1.15 \mathrm{E}-10$ & 0.0002 & $1.15 \mathrm{E}-10$ \\
\hline 0.725 & 0.700 & $1.56 \mathrm{E}-10$ & 0.0009 & $1.57 \mathrm{E}-10$ & $4.50 \mathrm{E}-12$ & 0.0055 & $4.57 \mathrm{E}-12$ & $1.19 \mathrm{E}-10$ & 0.0002 & $1.19 \mathrm{E}-10$ \\
\hline 0.750 & 0.725 & $1.61 \mathrm{E}-10$ & 0.0009 & $1.61 \mathrm{E}-10$ & $4.76 \mathrm{E}-12$ & 0.0054 & $4.84 \mathrm{E}-12$ & $1.23 \mathrm{E}-10$ & 0.0002 & $1.23 \mathrm{E}-10$ \\
\hline 0.775 & 0.750 & $1.66 \mathrm{E}-10$ & 0.0009 & $1.66 \mathrm{E}-10$ & $5.05 \mathrm{E}-12$ & 0.0053 & $5.13 \mathrm{E}-12$ & $1.27 \mathrm{E}-10$ & 0.0002 & $1.27 \mathrm{E}-10$ \\
\hline 0.800 & 0.775 & $1.70 \mathrm{E}-10$ & 0.0008 & $1.71 \mathrm{E}-10$ & $5.34 \mathrm{E}-12$ & 0.0051 & $5.42 \mathrm{E}-12$ & $1.31 \mathrm{E}-10$ & 0.0002 & $1.31 \mathrm{E}-10$ \\
\hline 0.825 & 0.800 & $1.75 \mathrm{E}-10$ & 0.0008 & $1.75 \mathrm{E}-10$ & $5.61 \mathrm{E}-12$ & 0.0051 & $5.70 \mathrm{E}-12$ & $1.35 \mathrm{E}-10$ & 0.0002 & $1.35 \mathrm{E}-10$ \\
\hline 0.850 & 0.825 & $1.80 \mathrm{E}-10$ & 0.0008 & $1.80 \mathrm{E}-10$ & $5.89 \mathrm{E}-12$ & 0.0049 & $5.98 \mathrm{E}-12$ & $1.39 \mathrm{E}-10$ & 0.0002 & $1.39 \mathrm{E}-10$ \\
\hline 0.875 & 0.850 & $1.84 \mathrm{E}-10$ & 0.0008 & $1.85 \mathrm{E}-10$ & $6.20 \mathrm{E}-12$ & 0.0049 & $6.29 \mathrm{E}-12$ & $1.43 \mathrm{E}-10$ & 0.0002 & $1.43 \mathrm{E}-10$ \\
\hline 0.900 & 0.875 & $1.89 \mathrm{E}-10$ & 0.0008 & $1.89 \mathrm{E}-10$ & $6.47 \mathrm{E}-12$ & 0.0048 & $6.56 \mathrm{E}-12$ & $1.47 \mathrm{E}-10$ & 0.0002 & $1.47 \mathrm{E}-10$ \\
\hline 0.925 & 0.900 & $1.93 \mathrm{E}-10$ & 0.0008 & $1.93 \mathrm{E}-10$ & $6.78 \mathrm{E}-12$ & 0.0047 & $6.88 \mathrm{E}-12$ & $1.51 \mathrm{E}-10$ & 0.0002 & $1.51 \mathrm{E}-10$ \\
\hline 0.950 & 0.925 & $1.97 \mathrm{E}-10$ & 0.0008 & $1.98 \mathrm{E}-10$ & $7.08 \mathrm{E}-12$ & 0.0046 & $7.18 \mathrm{E}-12$ & $1.55 \mathrm{E}-10$ & 0.0002 & $1.55 \mathrm{E}-10$ \\
\hline 0.975 & 0.950 & $2.02 \mathrm{E}-10$ & 0.0008 & $2.02 \mathrm{E}-10$ & $7.35 \mathrm{E}-12$ & 0.0045 & $7.45 \mathrm{E}-12$ & $1.59 \mathrm{E}-10$ & 0.0002 & $1.59 \mathrm{E}-10$ \\
\hline 1.000 & 0.975 & $2.06 \mathrm{E}-10$ & 0.0008 & $2.06 \mathrm{E}-10$ & $7.65 \mathrm{E}-12$ & 0.0045 & $7.76 \mathrm{E}-12$ & $1.63 \mathrm{E}-10$ & 0.0002 & $1.63 \mathrm{E}-10$ \\
\hline 1.250 & 1.000 & $2.28 \mathrm{E}-10$ & 0.0008 & $2.29 \mathrm{E}-10$ & $9.45 \mathrm{E}-12$ & 0.0042 & $9.57 \mathrm{E}-12$ & $1.83 \mathrm{E}-10$ & 0.0002 & $1.83 \mathrm{E}-10$ \\
\hline 1.500 & 1.250 & $2.68 \mathrm{E}-10$ & 0.0008 & $2.68 \mathrm{E}-10$ & $1.27 \mathrm{E}-11$ & 0.0038 & $1.28 \mathrm{E}-11$ & $2.19 \mathrm{E}-10$ & 0.0002 & $2.19 \mathrm{E}-10$ \\
\hline 1.750 & 1.500 & $3.05 \mathrm{E}-10$ & 0.0008 & $3.05 \mathrm{E}-10$ & $1.60 \mathrm{E}-11$ & 0.0035 & $1.62 \mathrm{E}-11$ & $2.53 \mathrm{E}-10$ & 0.0002 & $2.53 \mathrm{E}-10$ \\
\hline 2.000 & 1.750 & $3.40 \mathrm{E}-10$ & 0.0008 & $3.41 \mathrm{E}-10$ & $1.92 \mathrm{E}-11$ & 0.0034 & $1.94 \mathrm{E}-11$ & $2.85 \mathrm{E}-10$ & 0.0002 & $2.86 \mathrm{E}-10$ \\
\hline 2.250 & 2.000 & $3.73 \mathrm{E}-10$ & 0.0008 & $3.74 \mathrm{E}-10$ & $2.24 \mathrm{E}-11$ & 0.0032 & $2.26 \mathrm{E}-11$ & $3.17 \mathrm{E}-10$ & 0.0002 & $3.17 \mathrm{E}-10$ \\
\hline 2.500 & 2.250 & $4.04 \mathrm{E}-10$ & 0.0008 & $4.05 \mathrm{E}-10$ & $2.54 \mathrm{E}-11$ & 0.0031 & $2.56 \mathrm{E}-11$ & $3.46 \mathrm{E}-10$ & 0.0002 & $3.46 \mathrm{E}-10$ \\
\hline 2.750 & 2.500 & $4.35 \mathrm{E}-10$ & 0.0008 & $4.36 \mathrm{E}-10$ & $2.83 \mathrm{E}-11$ & 0.0030 & $2.86 \mathrm{E}-11$ & $3.75 \mathrm{E}-10$ & 0.0002 & $3.75 \mathrm{E}-10$ \\
\hline 3.000 & 2.750 & $4.64 \mathrm{E}-10$ & 0.0008 & $4.65 \mathrm{E}-10$ & $3.12 \mathrm{E}-11$ & 0.0030 & $3.15 \mathrm{E}-11$ & $4.02 \mathrm{E}-10$ & 0.0002 & $4.03 \mathrm{E}-10$ \\
\hline 3.500 & 3.000 & $5.05 \mathrm{E}-10$ & 0.0008 & $5.07 \mathrm{E}-10$ & $3.51 \mathrm{E}-11$ & 0.0029 & $3.54 \mathrm{E}-11$ & $4.41 \mathrm{E}-10$ & 0.0002 & $4.42 \mathrm{E}-10$ \\
\hline 4.000 & 3.500 & $5.58 \mathrm{E}-10$ & 0.0008 & $5.59 \mathrm{E}-10$ & $3.99 \mathrm{E}-11$ & 0.0029 & $4.03 \mathrm{E}-11$ & $4.91 \mathrm{E}-10$ & 0.0002 & $4.91 \mathrm{E}-10$ \\
\hline 4.500 & 4.000 & $6.07 \mathrm{E}-10$ & 0.0008 & $6.09 \mathrm{E}-10$ & $4.45 \mathrm{E}-11$ & 0.0029 & $4.49 \mathrm{E}-11$ & $5.37 \mathrm{E}-10$ & 0.0002 & $5.38 \mathrm{E}-10$ \\
\hline 5.000 & 4.500 & $6.54 \mathrm{E}-10$ & 0.0008 & $6.56 \mathrm{E}-10$ & $4.86 \mathrm{E}-11$ & 0.0028 & $4.90 \mathrm{E}-11$ & $5.82 \mathrm{E}-10$ & 0.0002 & $5.82 \mathrm{E}-10$ \\
\hline 5.500 & 5.000 & $7.03 \mathrm{E}-10$ & 0.0008 & $7.05 \mathrm{E}-10$ & $5.27 \mathrm{E}-11$ & 0.0028 & $5.31 \mathrm{E}-11$ & $6.27 \mathrm{E}-10$ & 0.0002 & $6.27 \mathrm{E}-10$ \\
\hline 6.000 & 5.500 & $7.48 \mathrm{E}-10$ & 0.0008 & $7.50 \mathrm{E}-10$ & $5.63 \mathrm{E}-11$ & 0.0029 & $5.68 \mathrm{E}-11$ & $6.69 \mathrm{E}-10$ & 0.0002 & $6.69 \mathrm{E}-10$ \\
\hline 6.500 & 6.000 & $7.93 \mathrm{E}-10$ & 0.0008 & $7.95 \mathrm{E}-10$ & $5.99 \mathrm{E}-11$ & 0.0029 & $6.04 \mathrm{E}-11$ & $7.10 \mathrm{E}-10$ & 0.0002 & $7.10 \mathrm{E}-10$ \\
\hline 7.000 & 6.500 & $8.38 \mathrm{E}-10$ & 0.0008 & $8.40 \mathrm{E}-10$ & $6.34 \mathrm{E}-11$ & 0.0029 & $6.39 \mathrm{E}-11$ & $7.51 \mathrm{E}-10$ & 0.0002 & $7.52 \mathrm{E}-10$ \\
\hline 7.500 & 7.000 & $8.83 \mathrm{E}-10$ & 0.0008 & $8.85 \mathrm{E}-10$ & $6.66 \mathrm{E}-11$ & 0.0029 & $6.72 \mathrm{E}-11$ & $7.92 \mathrm{E}-10$ & 0.0002 & $7.92 \mathrm{E}-10$ \\
\hline 8.000 & 7.500 & $9.27 \mathrm{E}-10$ & 0.0008 & $9.29 \mathrm{E}-10$ & $7.00 \mathrm{E}-11$ & 0.0030 & $7.06 \mathrm{E}-11$ & $8.33 \mathrm{E}-10$ & 0.0002 & $8.33 \mathrm{E}-10$ \\
\hline 9.000 & 8.000 & $9.93 \mathrm{E}-10$ & 0.0009 & $9.96 \mathrm{E}-10$ & $7.48 \mathrm{E}-11$ & 0.0030 & $7.54 \mathrm{E}-11$ & $8.93 \mathrm{E}-10$ & 0.0002 & $8.94 \mathrm{E}-10$ \\
\hline 10.000 & 9.000 & $1.08 \mathrm{E}-09$ & 0.0009 & 1.09E-09 & $8.14 \mathrm{E}-11$ & 0.0030 & $8.22 \mathrm{E}-11$ & $9.75 \mathrm{E}-10$ & 0.0002 & $9.75 \mathrm{E}-10$ \\
\hline
\end{tabular}


PCP-2011-0001

Revision 0

Table 3-38. Photon Dose Rate per Unit Source in Each of the 77 Source Groups - SC3 Tungsten Shielded Container

\begin{tabular}{|c|c|c|c|c|c|c|c|c|c|c|}
\hline \multicolumn{2}{|c|}{ Energy $(\mathrm{MeV})$} & \multicolumn{3}{|c|}{ Bottom Dose Rate $(\mathrm{rem} / \mathrm{hr})$} & \multicolumn{3}{|c|}{ Top Dose Rate (rem/hr) } & \multicolumn{3}{|c|}{ Side Dose Rate (rem/hr) } \\
\hline Upper & Lower & mean & FSD & mean $+3 \pi$ & mean & FSD & mean $+3 \pi$ & mean & FSD & mean $+3 \pi$ \\
\hline 0.020 & 0.010 & $0.00 \mathrm{E}+00$ & 0.0000 & $0.00 \mathrm{E}+00$ & $0.00 \mathrm{E}+00$ & 0.0000 & $0.00 \mathrm{E}+00$ & $0.00 \mathrm{E}+00$ & 0.0000 & $0.00 \mathrm{E}+00$ \\
\hline 0.030 & 0.020 & $0.00 \mathrm{E}+00$ & 0.0000 & $0.00 \mathrm{E}+00$ & $0.00 \mathrm{E}+00$ & 0.0000 & $0.00 \mathrm{E}+00$ & $0.00 \mathrm{E}+00$ & 0.0000 & $0.00 \mathrm{E}+00$ \\
\hline 0.040 & 0.030 & $0.00 \mathrm{E}+00$ & 0.0000 & $0.00 \mathrm{E}+00$ & $0.00 \mathrm{E}+00$ & 0.0000 & $0.00 \mathrm{E}+00$ & $0.00 \mathrm{E}+00$ & 0.0000 & $0.00 \mathrm{E}+00$ \\
\hline 0.050 & 0.040 & $0.00 \mathrm{E}+00$ & 0.0000 & $0.00 \mathrm{E}+00$ & $0.00 \mathrm{E}+00$ & 0.0000 & $0.00 \mathrm{E}+00$ & $0.00 \mathrm{E}+00$ & 0.0000 & $0.00 \mathrm{E}+00$ \\
\hline 0.060 & 0.050 & $0.00 \mathrm{E}+00$ & 0.0000 & $0.00 \mathrm{E}+00$ & $0.00 \mathrm{E}+00$ & 0.0000 & $0.00 \mathrm{E}+00$ & $0.00 \mathrm{E}+00$ & 0.0000 & $0.00 \mathrm{E}+00$ \\
\hline 0.070 & 0.060 & $0.00 \mathrm{E}+00$ & 0.0000 & $0.00 \mathrm{E}+00$ & $0.00 \mathrm{E}+00$ & 0.0000 & $0.00 \mathrm{E}+00$ & $0.00 \mathrm{E}+00$ & 0.0000 & $0.00 \mathrm{E}+00$ \\
\hline 0.080 & 0.070 & $0.00 \mathrm{E}+00$ & 0.0000 & $0.00 \mathrm{E}+00$ & $0.00 \mathrm{E}+00$ & 0.0000 & $0.00 \mathrm{E}+00$ & $0.00 \mathrm{E}+00$ & 0.0000 & $0.00 \mathrm{E}+00$ \\
\hline 0.090 & 0.080 & $0.00 \mathrm{E}+00$ & 0.0000 & $0.00 \mathrm{E}+00$ & $0.00 \mathrm{E}+00$ & 0.0000 & $0.00 \mathrm{E}+00$ & $0.00 \mathrm{E}+00$ & 0.0000 & $0.00 \mathrm{E}+00$ \\
\hline 0.100 & 0.090 & $0.00 \mathrm{E}+00$ & 0.0000 & $0.00 \mathrm{E}+00$ & $0.00 \mathrm{E}+00$ & 0.0000 & $0.00 \mathrm{E}+00$ & $0.00 \mathrm{E}+00$ & 0.0000 & $0.00 \mathrm{E}+00$ \\
\hline 0.110 & 0.100 & $0.00 \mathrm{E}+00$ & 0.0000 & $0.00 \mathrm{E}+00$ & $0.00 \mathrm{E}+00$ & 0.0000 & $0.00 \mathrm{E}+00$ & $0.00 \mathrm{E}+00$ & 0.0000 & $0.00 \mathrm{E}+00$ \\
\hline 0.120 & 0.110 & $0.00 \mathrm{E}+00$ & 0.0000 & $0.00 \mathrm{E}+00$ & $0.00 \mathrm{E}+00$ & 0.0000 & $0.00 \mathrm{E}+00$ & $0.00 \mathrm{E}+00$ & 0.0000 & $0.00 \mathrm{E}+00$ \\
\hline 0.130 & 0.120 & $0.00 \mathrm{E}+00$ & 0.0000 & $0.00 \mathrm{E}+00$ & $0.00 \mathrm{E}+00$ & 0.0000 & $0.00 \mathrm{E}+00$ & $0.00 \mathrm{E}+00$ & 0.0000 & $0.00 \mathrm{E}+00$ \\
\hline 0.140 & 0.130 & $0.00 \mathrm{E}+00$ & 0.0000 & $0.00 \mathrm{E}+00$ & $0.00 \mathrm{E}+00$ & 0.0000 & $0.00 \mathrm{E}+00$ & $0.00 \mathrm{E}+00$ & 0.0000 & $0.00 \mathrm{E}+00$ \\
\hline 0.150 & 0.140 & $0.00 \mathrm{E}+00$ & 0.0000 & $0.00 \mathrm{E}+00$ & $0.00 \mathrm{E}+00$ & 0.0000 & $0.00 \mathrm{E}+00$ & $0.00 \mathrm{E}+00$ & 0.0000 & $0.00 \mathrm{E}+00$ \\
\hline 0.160 & 0.150 & $0.00 \mathrm{E}+00$ & 0.0000 & $0.00 \mathrm{E}+00$ & $0.00 \mathrm{E}+00$ & 0.0000 & $0.00 \mathrm{E}+00$ & $0.00 \mathrm{E}+00$ & 0.0000 & $0.00 \mathrm{E}+00$ \\
\hline 0.170 & 0.160 & $0.00 \mathrm{E}+00$ & 0.0000 & $0.00 \mathrm{E}+00$ & $0.00 \mathrm{E}+00$ & 0.0000 & $0.00 \mathrm{E}+00$ & $0.00 \mathrm{E}+00$ & 0.0000 & $0.00 \mathrm{E}+00$ \\
\hline 0.180 & 0.170 & $1.04 \mathrm{E}-48$ & 0.1447 & $1.49 \mathrm{E}-48$ & $8.94 \mathrm{E}-52$ & 0.7976 & $3.03 \mathrm{E}-51$ & $1.89 \mathrm{E}-49$ & 0.2113 & $3.09 \mathrm{E}-49$ \\
\hline 0.190 & 0.180 & $9.73 \mathrm{E}-44$ & 0.0643 & $1.16 \mathrm{E}-43$ & $1.10 \mathrm{E}-46$ & 0.0773 & $1.36 \mathrm{E}-46$ & $2.85 \mathrm{E}-44$ & 0.0722 & $3.47 \mathrm{E}-44$ \\
\hline 0.200 & 0.190 & $5.38 \mathrm{E}-40$ & 0.1536 & $7.86 \mathrm{E}-40$ & $7.68 \mathrm{E}-43$ & 0.1125 & $1.03 \mathrm{E}-42$ & $2.23 \mathrm{E}-40$ & 0.2699 & 4.04E-40 \\
\hline 0.210 & 0.200 & $7.56 \mathrm{E}-37$ & 0.1521 & $1.10 \mathrm{E}-36$ & $1.21 \mathrm{E}-39$ & 0.1501 & $1.75 \mathrm{E}-39$ & $3.32 \mathrm{E}-37$ & 0.2488 & $5.80 \mathrm{E}-37$ \\
\hline 0.220 & 0.210 & $2.84 \mathrm{E}-34$ & 0.0834 & $3.54 \mathrm{E}-34$ & $5.11 \mathrm{E}-37$ & 0.0207 & $5.42 \mathrm{E}-37$ & $1.53 \mathrm{E}-34$ & 0.2292 & $2.59 \mathrm{E}-34$ \\
\hline 0.230 & 0.220 & $4.71 \mathrm{E}-32$ & 0.0410 & $5.29 \mathrm{E}-32$ & $1.03 \mathrm{E}-34$ & 0.0321 & $1.13 \mathrm{E}-34$ & $2.70 \mathrm{E}-32$ & 0.2140 & $4.43 \mathrm{E}-32$ \\
\hline 0.240 & 0.230 & $3.81 \mathrm{E}-30$ & 0.0373 & $4.23 \mathrm{E}-30$ & $1.07 \mathrm{E}-32$ & 0.1187 & $1.45 \mathrm{E}-32$ & $4.03 \mathrm{E}-30$ & 0.6073 & $1.14 \mathrm{E}-29$ \\
\hline 0.250 & 0.240 & $1.76 \mathrm{E}-28$ & 0.0723 & $2.14 \mathrm{E}-28$ & $4.92 \mathrm{E}-31$ & 0.0606 & $5.81 \mathrm{E}-31$ & $1.84 \mathrm{E}-28$ & 0.5969 & $5.13 \mathrm{E}-28$ \\
\hline 0.260 & 0.250 & $4.96 \mathrm{E}-27$ & 0.0665 & $5.95 \mathrm{E}-27$ & $1.42 \mathrm{E}-29$ & 0.0431 & $1.60 \mathrm{E}-29$ & $2.39 \mathrm{E}-27$ & 0.1109 & $3.18 \mathrm{E}-27$ \\
\hline 0.270 & 0.260 & $9.05 \mathrm{E}-26$ & 0.0511 & $1.04 \mathrm{E}-25$ & $3.23 \mathrm{E}-28$ & 0.1042 & $4.24 \mathrm{E}-28$ & $5.09 \mathrm{E}-26$ & 0.1150 & $6.85 \mathrm{E}-26$ \\
\hline 0.280 & 0.270 & $1.40 \mathrm{E}-24$ & 0.0874 & $1.77 \mathrm{E}-24$ & $1.95 \mathrm{E}-26$ & 0.7501 & $6.35 \mathrm{E}-26$ & $5.74 \mathrm{E}-25$ & 0.0965 & $7.40 \mathrm{E}-25$ \\
\hline 0.290 & 0.280 & $1.19 \mathrm{E}-23$ & 0.0618 & $1.41 \mathrm{E}-23$ & $2.12 \mathrm{E}-25$ & 0.7354 & $6.79 \mathrm{E}-25$ & $5.30 \mathrm{E}-24$ & 0.0789 & $6.56 \mathrm{E}-24$ \\
\hline 0.300 & 0.290 & $9.83 \mathrm{E}-23$ & 0.1050 & $1.29 \mathrm{E}-22$ & $5.79 \mathrm{E}-24$ & 0.9148 & $2.17 \mathrm{E}-23$ & $4.10 \mathrm{E}-23$ & 0.0775 & $5.05 \mathrm{E}-23$ \\
\hline 0.325 & 0.300 & $1.85 \mathrm{E}-20$ & 0.8130 & $6.37 \mathrm{E}-20$ & $1.01 \mathrm{E}-22$ & 0.7844 & $3.39 \mathrm{E}-22$ & $1.62 \mathrm{E}-21$ & 0.1849 & $2.52 \mathrm{E}-21$ \\
\hline 0.350 & 0.325 & $1.77 \mathrm{E}-19$ & 0.3870 & $3.83 \mathrm{E}-19$ & $4.29 \mathrm{E}-22$ & 0.2049 & $6.93 \mathrm{E}-22$ & $4.87 \mathrm{E}-20$ & 0.1893 & $7.63 \mathrm{E}-20$ \\
\hline 0.375 & 0.350 & $3.34 \mathrm{E}-17$ & 0.9626 & $1.30 \mathrm{E}-16$ & $6.69 \mathrm{E}-21$ & 0.2626 & $1.20 \mathrm{E}-20$ & $9.10 \mathrm{E}-19$ & 0.3402 & $1.84 \mathrm{E}-18$ \\
\hline 0.400 & 0.375 & $7.17 \mathrm{E}-18$ & 0.0608 & $8.47 \mathrm{E}-18$ & $5.93 \mathrm{E}-20$ & 0.1877 & $9.27 \mathrm{E}-20$ & $1.15 \mathrm{E}-17$ & 0.2242 & $1.93 \mathrm{E}-17$ \\
\hline 0.425 & 0.400 & $5.68 \mathrm{E}-17$ & 0.1548 & $8.32 \mathrm{E}-17$ & $4.50 \mathrm{E}-19$ & 0.2960 & $8.50 \mathrm{E}-19$ & $4.22 \mathrm{E}-17$ & 0.1040 & $5.54 \mathrm{E}-17$ \\
\hline 0.450 & 0.425 & $2.15 \mathrm{E}-16$ & 0.0814 & $2.67 \mathrm{E}-16$ & $3.04 \mathrm{E}-18$ & 0.4133 & $6.80 \mathrm{E}-18$ & $2.13 \mathrm{E}-16$ & 0.1303 & $2.96 \mathrm{E}-16$ \\
\hline 0.475 & 0.450 & $7.58 \mathrm{E}-16$ & 0.0821 & $9.44 \mathrm{E}-16$ & $1.29 \mathrm{E}-17$ & 0.3041 & $2.47 \mathrm{E}-17$ & $5.86 \mathrm{E}-16$ & 0.0938 & $7.52 \mathrm{E}-16$ \\
\hline 0.500 & 0.475 & $4.39 \mathrm{E}-15$ & 0.4714 & $1.06 \mathrm{E}-14$ & $3.74 \mathrm{E}-17$ & 0.3228 & $7.37 \mathrm{E}-17$ & $1.48 \mathrm{E}-15$ & 0.0523 & $1.71 \mathrm{E}-15$ \\
\hline 0.525 & 0.500 & $4.56 \mathrm{E}-15$ & 0.0713 & $5.54 \mathrm{E}-15$ & $6.34 \mathrm{E}-17$ & 0.1714 & $9.59 \mathrm{E}-17$ & $3.22 \mathrm{E}-15$ & 0.0306 & $3.51 \mathrm{E}-15$ \\
\hline
\end{tabular}


PCP-2011-0001

Revision 0

Table 3-38. Photon Dose Rate per Unit Source in Each of the 77 Source Groups - SC3 Tungsten Shielded Container

\begin{tabular}{|c|c|c|c|c|c|c|c|c|c|c|}
\hline \multicolumn{2}{|c|}{ Energy $(\mathrm{MeV})$} & \multicolumn{3}{|c|}{ Bottom Dose Rate (rem/hr) } & \multicolumn{3}{|c|}{ Top Dose Rate (rem/hr) } & \multicolumn{3}{|c|}{ Side Dose Rate $(\mathrm{rem} / \mathrm{hr})$} \\
\hline Upper & Lower & mean & FSD & mean $+3 \pi$ & mean & FSD & mean $+3 \pi$ & mean & FSD & mean $+3 \pi$ \\
\hline 0.550 & 0.525 & $8.67 \mathrm{E}-15$ & 0.0482 & $9.93 \mathrm{E}-15$ & $1.25 \mathrm{E}-16$ & 0.1497 & $1.81 \mathrm{E}-16$ & $6.78 \mathrm{E}-15$ & 0.0295 & $7.38 \mathrm{E}-15$ \\
\hline 0.575 & 0.550 & $1.84 \mathrm{E}-14$ & 0.0842 & $2.30 \mathrm{E}-14$ & $3.27 \mathrm{E}-16$ & 0.2457 & $5.69 \mathrm{E}-16$ & $1.21 \mathrm{E}-14$ & 0.0167 & $1.27 \mathrm{E}-14$ \\
\hline 0.600 & 0.575 & $2.83 \mathrm{E}-14$ & 0.0423 & $3.19 \mathrm{E}-14$ & $4.73 \mathrm{E}-16$ & 0.1674 & $7.10 \mathrm{E}-16$ & $2.05 \mathrm{E}-14$ & 0.0137 & $2.14 \mathrm{E}-14$ \\
\hline 0.625 & 0.600 & $4.67 \mathrm{E}-14$ & 0.0448 & $5.30 \mathrm{E}-14$ & $8.07 \mathrm{E}-16$ & 0.1555 & $1.18 \mathrm{E}-15$ & $3.46 \mathrm{E}-14$ & 0.0158 & $3.63 \mathrm{E}-14$ \\
\hline 0.650 & 0.625 & $6.77 \mathrm{E}-14$ & 0.0276 & $7.33 \mathrm{E}-14$ & $1.22 \mathrm{E}-15$ & 0.1116 & $1.63 \mathrm{E}-15$ & $5.72 \mathrm{E}-14$ & 0.1071 & $7.56 \mathrm{E}-14$ \\
\hline 0.675 & 0.650 & $1.02 \mathrm{E}-13$ & 0.0278 & $1.11 \mathrm{E}-13$ & $1.60 \mathrm{E}-15$ & 0.0566 & $1.87 \mathrm{E}-15$ & $7.40 \mathrm{E}-14$ & 0.0086 & $7.59 \mathrm{E}-14$ \\
\hline 0.700 & 0.675 & $1.39 \mathrm{E}-13$ & 0.0159 & $1.45 \mathrm{E}-13$ & $2.25 \mathrm{E}-15$ & 0.0367 & $2.49 \mathrm{E}-15$ & $1.08 \mathrm{E}-13$ & 0.0237 & $1.15 \mathrm{E}-13$ \\
\hline 0.725 & 0.700 & $1.91 \mathrm{E}-13$ & 0.0232 & $2.05 \mathrm{E}-13$ & $4.33 \mathrm{E}-15$ & 0.2626 & $7.75 \mathrm{E}-15$ & $1.45 \mathrm{E}-13$ & 0.0093 & $1.49 \mathrm{E}-13$ \\
\hline 0.750 & 0.725 & $2.44 \mathrm{E}-13$ & 0.0166 & $2.57 \mathrm{E}-13$ & $5.82 \mathrm{E}-15$ & 0.1515 & $8.47 \mathrm{E}-15$ & $2.06 \mathrm{E}-13$ & 0.0042 & $2.09 \mathrm{E}-13$ \\
\hline 0.775 & 0.750 & $3.23 \mathrm{E}-13$ & 0.0147 & $3.37 \mathrm{E}-13$ & $8.03 \mathrm{E}-15$ & 0.1258 & $1.11 \mathrm{E}-14$ & $2.68 \mathrm{E}-13$ & 0.0037 & $2.71 \mathrm{E}-13$ \\
\hline 0.800 & 0.775 & $4.18 \mathrm{E}-13$ & 0.0131 & $4.34 \mathrm{E}-13$ & $1.11 \mathrm{E}-14$ & 0.1002 & $1.45 \mathrm{E}-14$ & $3.41 \mathrm{E}-13$ & 0.0033 & $3.44 \mathrm{E}-13$ \\
\hline 0.825 & 0.800 & $5.23 \mathrm{E}-13$ & 0.0119 & $5.42 \mathrm{E}-13$ & $1.50 \mathrm{E}-14$ & 0.0876 & $1.90 \mathrm{E}-14$ & $4.25 \mathrm{E}-13$ & 0.0030 & $4.29 \mathrm{E}-13$ \\
\hline 0.850 & 0.825 & $6.36 \mathrm{E}-13$ & 0.0108 & $6.56 \mathrm{E}-13$ & $1.74 \mathrm{E}-14$ & 0.0778 & $2.14 \mathrm{E}-14$ & $5.23 \mathrm{E}-13$ & 0.0027 & $5.28 \mathrm{E}-13$ \\
\hline 0.875 & 0.850 & $7.76 \mathrm{E}-13$ & 0.0099 & $7.99 \mathrm{E}-13$ & $2.05 \mathrm{E}-14$ & 0.0696 & $2.47 \mathrm{E}-14$ & $6.35 \mathrm{E}-13$ & 0.0025 & $6.40 \mathrm{E}-13$ \\
\hline 0.900 & 0.875 & $9.23 \mathrm{E}-13$ & 0.0092 & $9.48 \mathrm{E}-13$ & $2.66 \mathrm{E}-14$ & 0.0603 & $3.14 \mathrm{E}-14$ & $7.61 \mathrm{E}-13$ & 0.0023 & $7.67 \mathrm{E}-13$ \\
\hline 0.925 & 0.900 & $1.11 \mathrm{E}-12$ & 0.0084 & $1.14 \mathrm{E}-12$ & $3.07 \mathrm{E}-14$ & 0.0577 & $3.60 \mathrm{E}-14$ & $9.03 \mathrm{E}-13$ & 0.0022 & $9.09 \mathrm{E}-13$ \\
\hline 0.950 & 0.925 & $1.29 \mathrm{E}-12$ & 0.0080 & $1.32 \mathrm{E}-12$ & $3.72 \mathrm{E}-14$ & 0.0560 & $4.35 \mathrm{E}-14$ & $1.06 \mathrm{E}-12$ & 0.0020 & $1.07 \mathrm{E}-12$ \\
\hline 0.975 & 0.950 & $1.50 \mathrm{E}-12$ & 0.0075 & $1.53 \mathrm{E}-12$ & $4.16 \mathrm{E}-14$ & 0.0530 & $4.82 \mathrm{E}-14$ & $1.23 \mathrm{E}-12$ & 0.0019 & $1.24 \mathrm{E}-12$ \\
\hline 1.000 & 0.975 & $1.72 \mathrm{E}-12$ & 0.0071 & $1.76 \mathrm{E}-12$ & $4.97 \mathrm{E}-14$ & 0.0517 & $5.74 \mathrm{E}-14$ & $1.42 \mathrm{E}-12$ & 0.0018 & $1.43 \mathrm{E}-12$ \\
\hline 1.250 & 1.000 & $3.34 \mathrm{E}-12$ & 0.0055 & $3.39 \mathrm{E}-12$ & $1.07 \mathrm{E}-13$ & 0.0334 & $1.18 \mathrm{E}-13$ & $2.78 \mathrm{E}-12$ & 0.0014 & $2.79 \mathrm{E}-12$ \\
\hline 1.500 & 1.250 & $7.19 \mathrm{E}-12$ & 0.0041 & $7.28 \mathrm{E}-12$ & $2.55 \mathrm{E}-13$ & 0.0235 & $2.73 \mathrm{E}-13$ & $6.06 \mathrm{E}-12$ & 0.0010 & $6.08 \mathrm{E}-12$ \\
\hline 1.750 & 1.500 & $1.18 \mathrm{E}-11$ & 0.0035 & $1.19 \mathrm{E}-11$ & $4.63 \mathrm{E}-13$ & 0.0182 & $4.88 \mathrm{E}-13$ & $9.93 \mathrm{E}-12$ & 0.0009 & $9.96 \mathrm{E}-12$ \\
\hline 2.000 & 1.750 & $1.64 \mathrm{E}-11$ & 0.0032 & $1.65 \mathrm{E}-11$ & $6.88 \mathrm{E}-13$ & 0.0160 & $7.21 \mathrm{E}-13$ & $1.39 \mathrm{E}-11$ & 0.0008 & $1.39 \mathrm{E}-11$ \\
\hline 2.250 & 2.000 & $2.05 \mathrm{E}-11$ & 0.0030 & $2.07 \mathrm{E}-11$ & $9.32 \mathrm{E}-13$ & 0.0152 & $9.74 \mathrm{E}-13$ & $1.76 \mathrm{E}-11$ & 0.0008 & $1.76 \mathrm{E}-11$ \\
\hline 2.500 & 2.250 & $2.44 \mathrm{E}-11$ & 0.0030 & $2.46 \mathrm{E}-11$ & $1.09 \mathrm{E}-12$ & 0.0147 & $1.13 \mathrm{E}-12$ & $2.10 \mathrm{E}-11$ & 0.0007 & $2.10 \mathrm{E}-11$ \\
\hline 2.750 & 2.500 & $2.78 \mathrm{E}-11$ & 0.0029 & $2.81 \mathrm{E}-11$ & $1.30 \mathrm{E}-12$ & 0.0140 & $1.36 \mathrm{E}-12$ & $2.40 \mathrm{E}-11$ & 0.0007 & $2.41 \mathrm{E}-11$ \\
\hline 3.000 & 2.750 & $3.07 \mathrm{E}-11$ & 0.0030 & $3.09 \mathrm{E}-11$ & $1.52 \mathrm{E}-12$ & 0.0139 & $1.58 \mathrm{E}-12$ & $2.66 \mathrm{E}-11$ & 0.0007 & $2.67 \mathrm{E}-11$ \\
\hline 3.500 & 3.000 & $3.42 \mathrm{E}-11$ & 0.0030 & $3.46 \mathrm{E}-11$ & $1.75 \mathrm{E}-12$ & 0.0138 & $1.82 \mathrm{E}-12$ & $2.98 \mathrm{E}-11$ & 0.0007 & $2.99 \mathrm{E}-11$ \\
\hline 4.000 & 3.500 & $3.80 \mathrm{E}-11$ & 0.0031 & $3.83 \mathrm{E}-11$ & $2.02 \mathrm{E}-12$ & 0.0139 & $2.11 \mathrm{E}-12$ & $3.31 \mathrm{E}-11$ & 0.0008 & $3.32 \mathrm{E}-11$ \\
\hline 4.500 & 4.000 & $4.05 \mathrm{E}-11$ & 0.0032 & $4.09 \mathrm{E}-11$ & $2.21 \mathrm{E}-12$ & 0.0143 & $2.31 \mathrm{E}-12$ & $3.56 \mathrm{E}-11$ & 0.0008 & $3.57 \mathrm{E}-11$ \\
\hline 5.000 & 4.500 & $4.23 \mathrm{E}-11$ & 0.0033 & $4.27 \mathrm{E}-11$ & $2.37 \mathrm{E}-12$ & 0.0148 & $2.47 \mathrm{E}-12$ & $3.74 \mathrm{E}-11$ & 0.0008 & $3.75 \mathrm{E}-11$ \\
\hline 5.500 & 5.000 & $4.40 \mathrm{E}-11$ & 0.0035 & $4.44 \mathrm{E}-11$ & $2.52 \mathrm{E}-12$ & 0.0152 & $2.63 \mathrm{E}-12$ & $3.89 \mathrm{E}-11$ & 0.0009 & $3.90 \mathrm{E}-11$ \\
\hline 6.000 & 5.500 & $4.52 \mathrm{E}-11$ & 0.0036 & $4.57 \mathrm{E}-11$ & $2.58 \mathrm{E}-12$ & 0.0157 & $2.71 \mathrm{E}-12$ & $4.02 \mathrm{E}-11$ & 0.0009 & $4.03 \mathrm{E}-11$ \\
\hline 6.500 & 6.000 & $4.65 \mathrm{E}-11$ & 0.0037 & $4.70 \mathrm{E}-11$ & $2.68 \mathrm{E}-12$ & 0.0162 & $2.81 \mathrm{E}-12$ & $4.12 \mathrm{E}-11$ & 0.0009 & $4.13 \mathrm{E}-11$ \\
\hline 7.000 & 6.500 & $4.79 \mathrm{E}-11$ & 0.0038 & $4.84 \mathrm{E}-11$ & $2.77 \mathrm{E}-12$ & 0.0168 & $2.91 \mathrm{E}-12$ & $4.23 \mathrm{E}-11$ & 0.0009 & $4.24 \mathrm{E}-11$ \\
\hline 7.500 & 7.000 & $4.91 \mathrm{E}-11$ & 0.0040 & $4.97 \mathrm{E}-11$ & $2.82 \mathrm{E}-12$ & 0.0173 & $2.96 \mathrm{E}-12$ & $4.33 \mathrm{E}-11$ & 0.0010 & $4.35 \mathrm{E}-11$ \\
\hline 8.000 & 7.500 & $5.03 \mathrm{E}-11$ & 0.0041 & $5.09 \mathrm{E}-11$ & $2.87 \mathrm{E}-12$ & 0.0177 & $3.02 \mathrm{E}-12$ & $4.44 \mathrm{E}-11$ & 0.0010 & $4.45 \mathrm{E}-11$ \\
\hline 9.000 & 8.000 & $5.20 \mathrm{E}-11$ & 0.0042 & $5.27 \mathrm{E}-11$ & $3.02 \mathrm{E}-12$ & 0.0182 & $3.19 \mathrm{E}-12$ & $4.61 \mathrm{E}-11$ & 0.0010 & $4.62 \mathrm{E}-11$ \\
\hline 10.000 & 9.000 & $5.52 \mathrm{E}-11$ & 0.0043 & $5.59 \mathrm{E}-11$ & $3.12 \mathrm{E}-12$ & 0.0189 & $3.30 \mathrm{E}-12$ & $4.85 \mathrm{E}-11$ & 0.0011 & $4.87 \mathrm{E}-11$ \\
\hline
\end{tabular}


PCP-2011-0001

Revision 0

Table 3-39. Actinide Total Dose Rate - No Shielded Container

\begin{tabular}{|c|c|c|c|c|c|}
\hline \multirow{2}{*}{$\% \mathrm{Be}$} & \multicolumn{5}{|c|}{ Dose Rate from 1 gram of Isotope (rem $/ \mathrm{hr})$} \\
\hline & Am-241 & $\mathrm{Am}-243$ & Cf-252 & $\mathrm{Cm}-244$ & $\mathrm{Cm}-248$ \\
\hline \multicolumn{6}{|c|}{ Neutron } \\
\hline 0 & $5.31 \mathrm{E}-08$ & $1.67 \mathrm{E}-07$ & $1.00 \mathrm{E}+05$ & $4.72 \mathrm{E}-01$ & $1.72 \mathrm{E}+00$ \\
\hline 10 & $1.27 \mathrm{E}-01$ & $6.47 \mathrm{E}-03$ & & $4.17 \mathrm{E}+00$ & \\
\hline 20 & $2.03 \mathrm{E}-01$ & $1.03 \mathrm{E}-02$ & & $6.36 \mathrm{E}+00$ & \\
\hline 30 & $2.53 \mathrm{E}-01$ & $1.28 \mathrm{E}-02$ & & $7.82 \mathrm{E}+00$ & \\
\hline 40 & $2.89 \mathrm{E}-01$ & $1.46 \mathrm{E}-02$ & & $8.85 \mathrm{E}+00$ & \\
\hline 50 & $3.16 \mathrm{E}-01$ & $1.60 \mathrm{E}-02$ & & $9.63 \mathrm{E}+00$ & \\
\hline 60 & $3.36 \mathrm{E}-01$ & $1.70 \mathrm{E}-02$ & & $1.02 \mathrm{E}+01$ & \\
\hline 70 & $3.53 \mathrm{E}-01$ & $1.78 \mathrm{E}-02$ & & $1.07 \mathrm{E}+01$ & \\
\hline 80 & $3.66 \mathrm{E}-01$ & $1.85 \mathrm{E}-02$ & & $1.11 \mathrm{E}+01$ & \\
\hline 90 & $3.78 \mathrm{E}-01$ & $1.91 \mathrm{E}-02$ & & $1.14 \mathrm{E}+01$ & \\
\hline \multicolumn{6}{|c|}{ Secondary Photon } \\
\hline 0 & $1.04 \mathrm{E}-10$ & $3.04 \mathrm{E}-10$ & $2.15 \mathrm{E}+02$ & $9.00 \mathrm{E}-04$ & $2.96 \mathrm{E}-03$ \\
\hline 10 & $6.60 \mathrm{E}-04$ & $3.28 \mathrm{E}-05$ & & $2.10 \mathrm{E}-02$ & \\
\hline 20 & $1.05 \mathrm{E}-03$ & $5.22 \mathrm{E}-05$ & & $3.28 \mathrm{E}-02$ & \\
\hline 30 & $1.31 \mathrm{E}-03$ & $6.50 \mathrm{E}-05$ & & $4.07 \mathrm{E}-02$ & \\
\hline 40 & $1.50 \mathrm{E}-03$ & $7.41 \mathrm{E}-05$ & & $4.63 \mathrm{E}-02$ & \\
\hline 50 & $1.64 \mathrm{E}-03$ & $8.09 \mathrm{E}-05$ & & $5.05 \mathrm{E}-02$ & \\
\hline 60 & $1.75 \mathrm{E}-03$ & $8.62 \mathrm{E}-05$ & & $5.38 \mathrm{E}-02$ & \\
\hline 70 & $1.83 \mathrm{E}-03$ & $9.04 \mathrm{E}-05$ & & $5.64 \mathrm{E}-02$ & \\
\hline 80 & $1.90 \mathrm{E}-03$ & $9.39 \mathrm{E}-05$ & & $5.86 \mathrm{E}-02$ & \\
\hline 90 & $1.96 \mathrm{E}-03$ & $9.68 \mathrm{E}-05$ & & $6.03 \mathrm{E}-02$ & \\
\hline \multicolumn{6}{|c|}{ Primary Photon } \\
\hline 0 & $9.11 \mathrm{E}-03$ & $2.52 \mathrm{E}-02$ & $4.38 \mathrm{E}+03$ & $3.54 \mathrm{E}-02$ & $9.24 \mathrm{E}-02$ \\
\hline 10 & $9.11 \mathrm{E}-03$ & $2.52 \mathrm{E}-02$ & & $3.54 \mathrm{E}-02$ & \\
\hline 20 & $9.11 \mathrm{E}-03$ & $2.52 \mathrm{E}-02$ & & $3.54 \mathrm{E}-02$ & \\
\hline 30 & $9.11 \mathrm{E}-03$ & $2.52 \mathrm{E}-02$ & & $3.54 \mathrm{E}-02$ & \\
\hline 40 & $9.11 \mathrm{E}-03$ & $2.52 \mathrm{E}-02$ & & $3.54 \mathrm{E}-02$ & \\
\hline 50 & $9.11 \mathrm{E}-03$ & $2.52 \mathrm{E}-02$ & & $3.54 \mathrm{E}-02$ & \\
\hline 60 & $9.11 \mathrm{E}-03$ & $2.52 \mathrm{E}-02$ & & $3.54 \mathrm{E}-02$ & \\
\hline 70 & $9.11 \mathrm{E}-03$ & $2.52 \mathrm{E}-02$ & & $3.54 \mathrm{E}-02$ & \\
\hline 80 & $9.11 \mathrm{E}-03$ & $2.52 \mathrm{E}-02$ & & $3.54 \mathrm{E}-02$ & \\
\hline 90 & $9.11 \mathrm{E}-03$ & $2.52 \mathrm{E}-02$ & & $3.54 \mathrm{E}-02$ & \\
\hline \multicolumn{6}{|c|}{ Total } \\
\hline 0 & $9.11 \mathrm{E}-03$ & $2.52 \mathrm{E}-02$ & $1.05 \mathrm{E}+05$ & $5.08 \mathrm{E}-01$ & $1.82 \mathrm{E}+00$ \\
\hline 10 & $1.37 \mathrm{E}-01$ & $3.17 \mathrm{E}-02$ & & $4.23 \mathrm{E}+00$ & \\
\hline 20 & $2.13 \mathrm{E}-01$ & $3.56 \mathrm{E}-02$ & & $6.43 \mathrm{E}+00$ & \\
\hline 30 & $2.64 \mathrm{E}-01$ & $3.81 \mathrm{E}-02$ & & $7.90 \mathrm{E}+00$ & \\
\hline 40 & $2.99 \mathrm{E}-01$ & $3.99 \mathrm{E}-02$ & & $8.94 \mathrm{E}+00$ & \\
\hline 50 & $3.26 \mathrm{E}-01$ & $4.13 \mathrm{E}-02$ & & $9.71 \mathrm{E}+00$ & \\
\hline 60 & $3.47 \mathrm{E}-01$ & $4.23 \mathrm{E}-02$ & & $1.03 \mathrm{E}+01$ & \\
\hline 70 & $3.64 \mathrm{E}-01$ & $4.31 \mathrm{E}-02$ & & $1.08 \mathrm{E}+01$ & \\
\hline 80 & $3.77 \mathrm{E}-01$ & $4.38 \mathrm{E}-02$ & & $1.12 \mathrm{E}+01$ & \\
\hline 90 & $3.89 \mathrm{E}-01$ & $4.44 \mathrm{E}-02$ & & $1.15 \mathrm{E}+01$ & \\
\hline
\end{tabular}

Note: $90 \%$ Beryllium is modeled as 1 gram of actinide and 0.9 grams of beryllium. 
PCP-2011-0001

Revision 0

Table 3-39. Actinide Total Dose Rate - No Shielded Container (Continued)

\begin{tabular}{|c|c|c|c|c|c|c|}
\hline \multirow{2}{*}{$\% \mathrm{Be}$} & \multicolumn{6}{|c|}{ Dose Rate from 1 gram of Isotope (rem $/ \mathrm{hr}$ ) } \\
\hline & Np-237 & $\mathrm{Pu}-238$ & Pu-239 & $\mathrm{Pu}-240$ & $\mathrm{Pu}-241$ & $\mathrm{Pu}-242$ \\
\hline \multicolumn{7}{|c|}{ Neutron } \\
\hline 0 & $4.74 \mathrm{E}-12$ & $1.10 \mathrm{E}-04$ & $9.28 \mathrm{E}-10$ & $4.38 \mathrm{E}-05$ & $2.09 \mathrm{E}-09$ & 7.34E-05 \\
\hline 10 & $1.51 \mathrm{E}-05$ & $6.37 \mathrm{E}-01$ & $1.84 \mathrm{E}-03$ & $6.84 \mathrm{E}-03$ & $6.12 \mathrm{E}-05$ & $1.71 \mathrm{E}-04$ \\
\hline 20 & $2.40 \mathrm{E}-05$ & $1.02 \mathrm{E}+00$ & $2.93 \mathrm{E}-03$ & $1.09 \mathrm{E}-02$ & $9.72 \mathrm{E}-05$ & $2.29 \mathrm{E}-04$ \\
\hline 30 & $2.99 \mathrm{E}-05$ & $1.27 \mathrm{E}+00$ & $3.65 \mathrm{E}-03$ & $1.35 \mathrm{E}-02$ & $1.21 \mathrm{E}-04$ & $2.67 \mathrm{E}-04$ \\
\hline 40 & $3.41 \mathrm{E}-05$ & $1.45 \mathrm{E}+00$ & $4.16 \mathrm{E}-03$ & $1.54 \mathrm{E}-02$ & $1.38 \mathrm{E}-04$ & $2.94 \mathrm{E}-04$ \\
\hline 50 & $3.73 \mathrm{E}-05$ & $1.58 \mathrm{E}+00$ & $4.54 \mathrm{E}-03$ & $1.68 \mathrm{E}-02$ & $1.50 \mathrm{E}-04$ & $3.14 \mathrm{E}-04$ \\
\hline 60 & $3.97 \mathrm{E}-05$ & $1.69 \mathrm{E}+00$ & $4.84 \mathrm{E}-03$ & $1.79 \mathrm{E}-02$ & $1.60 \mathrm{E}-04$ & $3.30 \mathrm{E}-04$ \\
\hline 70 & $4.16 \mathrm{E}-05$ & $1.77 \mathrm{E}+00$ & $5.08 \mathrm{E}-03$ & $1.88 \mathrm{E}-02$ & $1.68 \mathrm{E}-04$ & $3.42 \mathrm{E}-04$ \\
\hline 80 & $4.32 \mathrm{E}-05$ & $1.84 \mathrm{E}+00$ & $5.27 \mathrm{E}-03$ & $1.95 \mathrm{E}-02$ & $1.74 \mathrm{E}-04$ & $3.52 \mathrm{E}-04$ \\
\hline 90 & $4.45 \mathrm{E}-05$ & $1.89 \mathrm{E}+00$ & $5.43 \mathrm{E}-03$ & $2.01 \mathrm{E}-02$ & $1.80 \mathrm{E}-04$ & $3.60 \mathrm{E}-04$ \\
\hline \multicolumn{7}{|c|}{ Secondary Photon } \\
\hline 0 & $8.34 \mathrm{E}-15$ & $1.98 \mathrm{E}-07$ & $1.73 \mathrm{E}-12$ & $7.42 \mathrm{E}-08$ & $3.71 \mathrm{E}-12$ & $1.27 \mathrm{E}-07$ \\
\hline 10 & $7.44 \mathrm{E}-08$ & $3.31 \mathrm{E}-03$ & $9.21 \mathrm{E}-06$ & $3.42 \mathrm{E}-05$ & $3.03 \mathrm{E}-07$ & $6.12 \mathrm{E}-07$ \\
\hline 20 & $1.18 \mathrm{E}-07$ & $5.28 \mathrm{E}-03$ & $1.47 \mathrm{E}-05$ & $5.44 \mathrm{E}-05$ & $4.81 \mathrm{E}-07$ & $8.98 \mathrm{E}-07$ \\
\hline 30 & $1.48 \mathrm{E}-07$ & $6.59 \mathrm{E}-03$ & $1.83 \mathrm{E}-05$ & $6.77 \mathrm{E}-05$ & $5.99 \mathrm{E}-07$ & $1.09 \mathrm{E}-06$ \\
\hline 40 & $1.68 \mathrm{E}-07$ & 7.52E-03 & $2.09 \mathrm{E}-05$ & 7.72E-05 & $6.82 \mathrm{E}-07$ & $1.22 \mathrm{E}-06$ \\
\hline 50 & $1.84 \mathrm{E}-07$ & $8.22 \mathrm{E}-03$ & $2.28 \mathrm{E}-05$ & $8.43 \mathrm{E}-05$ & $7.44 \mathrm{E}-07$ & $1.32 \mathrm{E}-06$ \\
\hline 60 & $1.96 \mathrm{E}-07$ & $8.76 \mathrm{E}-03$ & $2.43 \mathrm{E}-05$ & $8.97 \mathrm{E}-05$ & $7.93 \mathrm{E}-07$ & $1.39 \mathrm{E}-06$ \\
\hline 70 & $2.05 \mathrm{E}-07$ & $9.20 \mathrm{E}-03$ & $2.55 \mathrm{E}-05$ & $9.41 \mathrm{E}-05$ & $8.31 \mathrm{E}-07$ & $1.46 \mathrm{E}-06$ \\
\hline 80 & $2.13 \mathrm{E}-07$ & $9.55 \mathrm{E}-03$ & $2.64 \mathrm{E}-05$ & $9.77 \mathrm{E}-05$ & $8.62 \mathrm{E}-07$ & $1.51 \mathrm{E}-06$ \\
\hline 90 & $2.20 \mathrm{E}-07$ & $9.85 \mathrm{E}-03$ & $2.73 \mathrm{E}-05$ & $1.01 \mathrm{E}-04$ & $8.88 \mathrm{E}-07$ & $1.55 \mathrm{E}-06$ \\
\hline \multicolumn{7}{|c|}{ Primary Photon } \\
\hline 0 & $1.14 \mathrm{E}-04$ & $1.69 \mathrm{E}-03$ & $5.71 \mathrm{E}-05$ & $2.34 \mathrm{E}-05$ & $1.53 \mathrm{E}-03$ & $5.87 \mathrm{E}-06$ \\
\hline 10 & $1.14 \mathrm{E}-04$ & $1.69 \mathrm{E}-03$ & $5.71 \mathrm{E}-05$ & $2.34 \mathrm{E}-05$ & $1.53 \mathrm{E}-03$ & $5.87 \mathrm{E}-06$ \\
\hline 20 & $1.14 \mathrm{E}-04$ & $1.69 \mathrm{E}-03$ & $5.71 \mathrm{E}-05$ & $2.34 \mathrm{E}-05$ & $1.53 \mathrm{E}-03$ & $5.87 \mathrm{E}-06$ \\
\hline 30 & $1.14 \mathrm{E}-04$ & $1.69 \mathrm{E}-03$ & $5.71 \mathrm{E}-05$ & $2.34 \mathrm{E}-05$ & $1.53 \mathrm{E}-03$ & $5.87 \mathrm{E}-06$ \\
\hline 40 & $1.14 \mathrm{E}-04$ & $1.69 \mathrm{E}-03$ & $5.71 \mathrm{E}-05$ & $2.34 \mathrm{E}-05$ & $1.53 \mathrm{E}-03$ & $5.87 \mathrm{E}-06$ \\
\hline 50 & $1.14 \mathrm{E}-04$ & $1.69 \mathrm{E}-03$ & $5.71 \mathrm{E}-05$ & $2.34 \mathrm{E}-05$ & $1.53 \mathrm{E}-03$ & $5.87 \mathrm{E}-06$ \\
\hline 60 & $1.14 \mathrm{E}-04$ & $1.69 \mathrm{E}-03$ & $5.71 \mathrm{E}-05$ & $2.34 \mathrm{E}-05$ & $1.53 \mathrm{E}-03$ & $5.87 \mathrm{E}-06$ \\
\hline 70 & $1.14 \mathrm{E}-04$ & $1.69 \mathrm{E}-03$ & $5.71 \mathrm{E}-05$ & $2.34 \mathrm{E}-05$ & $1.53 \mathrm{E}-03$ & $5.87 \mathrm{E}-06$ \\
\hline 80 & $1.14 \mathrm{E}-04$ & $1.69 \mathrm{E}-03$ & $5.71 \mathrm{E}-05$ & $2.34 \mathrm{E}-05$ & $1.53 \mathrm{E}-03$ & $5.87 \mathrm{E}-06$ \\
\hline 90 & $1.14 \mathrm{E}-04$ & $1.69 \mathrm{E}-03$ & $5.71 \mathrm{E}-05$ & $2.34 \mathrm{E}-05$ & $1.53 \mathrm{E}-03$ & $5.87 \mathrm{E}-06$ \\
\hline \multicolumn{7}{|c|}{ Total } \\
\hline 0 & $1.14 \mathrm{E}-04$ & $1.80 \mathrm{E}-03$ & $5.71 \mathrm{E}-05$ & $6.74 \mathrm{E}-05$ & $1.53 \mathrm{E}-03$ & $7.94 \mathrm{E}-05$ \\
\hline 10 & $1.29 \mathrm{E}-04$ & $6.42 \mathrm{E}-01$ & $1.90 \mathrm{E}-03$ & $6.90 \mathrm{E}-03$ & $1.59 \mathrm{E}-03$ & $1.78 \mathrm{E}-04$ \\
\hline 20 & $1.38 \mathrm{E}-04$ & $1.02 \mathrm{E}+00$ & $3.00 \mathrm{E}-03$ & $1.09 \mathrm{E}-02$ & $1.63 \mathrm{E}-03$ & $2.36 \mathrm{E}-04$ \\
\hline 30 & $1.44 \mathrm{E}-04$ & $1.28 \mathrm{E}+00$ & $3.72 \mathrm{E}-03$ & $1.36 \mathrm{E}-02$ & $1.65 \mathrm{E}-03$ & $2.74 \mathrm{E}-04$ \\
\hline 40 & $1.48 \mathrm{E}-04$ & $1.46 \mathrm{E}+00$ & $4.24 \mathrm{E}-03$ & $1.55 \mathrm{E}-02$ & $1.67 \mathrm{E}-03$ & $3.01 \mathrm{E}-04$ \\
\hline 50 & $1.51 \mathrm{E}-04$ & $1.59 \mathrm{E}+00$ & $4.62 \mathrm{E}-03$ & $1.69 \mathrm{E}-02$ & $1.68 \mathrm{E}-03$ & $3.21 \mathrm{E}-04$ \\
\hline 60 & $1.54 \mathrm{E}-04$ & $1.70 \mathrm{E}+00$ & $4.92 \mathrm{E}-03$ & $1.80 \mathrm{E}-02$ & $1.69 \mathrm{E}-03$ & $3.37 \mathrm{E}-04$ \\
\hline 70 & $1.56 \mathrm{E}-04$ & $1.78 \mathrm{E}+00$ & $5.16 \mathrm{E}-03$ & $1.89 \mathrm{E}-02$ & $1.70 \mathrm{E}-03$ & $3.49 \mathrm{E}-04$ \\
\hline 80 & $1.57 \mathrm{E}-04$ & $1.85 \mathrm{E}+00$ & $5.36 \mathrm{E}-03$ & $1.96 \mathrm{E}-02$ & $1.71 \mathrm{E}-03$ & $3.59 \mathrm{E}-04$ \\
\hline 90 & $1.59 \mathrm{E}-04$ & $1.90 \mathrm{E}+00$ & $5.52 \mathrm{E}-03$ & $2.02 \mathrm{E}-02$ & $1.71 \mathrm{E}-03$ & $3.68 \mathrm{E}-04$ \\
\hline
\end{tabular}

Note: $90 \%$ Beryllium is modeled as 1 gram of actinide and 0.9 grams of beryllium. 
PCP-2011-0001

Revision 0

Table 3-40. Effect of $\mathrm{PuO}_{2}$ Self-shielding

\begin{tabular}{|c|c|c|c|c|c|c|c|c|c|}
\hline \multirow{2}{*}{ Isotope } & \multicolumn{9}{|c|}{ Dose Rate from 1 gram of Isotope (rem/hr) } \\
\hline & $10 \% \mathrm{Be}$ & $20 \% \mathrm{Be}$ & $30 \% \mathrm{Be}$ & $40 \% \mathrm{Be}$ & $50 \% \mathrm{Be}$ & $60 \% \mathrm{Be}$ & $70 \% \mathrm{Be}$ & $80 \% \mathrm{Be}$ & $90 \% \mathrm{Be}$ \\
\hline \multicolumn{10}{|c|}{ No Self-shielding } \\
\hline$\overline{\mathrm{Am}-241}$ & $1.37 \mathrm{E}-01$ & $2.13 \mathrm{E}-01$ & $2.64 \mathrm{E}-01$ & $2.99 \mathrm{E}-01$ & $3.26 \mathrm{E}-01$ & $3.47 \mathrm{E}-01$ & $3.64 \mathrm{E}-01$ & $3.77 \mathrm{E}-01$ & $3.89 \mathrm{E}-01$ \\
\hline Am-243 & $7.59 \mathrm{E}-01$ & $8.35 \mathrm{E}-01$ & $8.86 \mathrm{E}-01$ & $9.21 \mathrm{E}-01$ & $9.48 \mathrm{E}-01$ & $9.69 \mathrm{E}-01$ & $9.86 \mathrm{E}-01$ & $9.99 \mathrm{E}-01$ & $1.01 \mathrm{E}+00$ \\
\hline $\mathrm{Cm}-244$ & $4.23 \mathrm{E}+00$ & $6.43 \mathrm{E}+00$ & $7.90 \mathrm{E}+00$ & $8.94 \mathrm{E}+00$ & $9.71 \mathrm{E}+00$ & $1.03 \mathrm{E}+01$ & $1.08 \mathrm{E}+01$ & $1.12 \mathrm{E}+01$ & $1.15 \mathrm{E}+01$ \\
\hline $\mathrm{Np}-237$ & $3.58 \mathrm{E}-03$ & $3.59 \mathrm{E}-03$ & $3.60 \mathrm{E}-03$ & $3.60 \mathrm{E}-03$ & $3.61 \mathrm{E}-03$ & $3.61 \mathrm{E}-03$ & $3.61 \mathrm{E}-03$ & $3.61 \mathrm{E}-03$ & $3.61 \mathrm{E}-03$ \\
\hline $\mathrm{Pu}-238$ & $6.42 \mathrm{E}-01$ & $1.02 \mathrm{E}+00$ & $1.28 \mathrm{E}+00$ & $1.46 \mathrm{E}+00$ & $1.59 \mathrm{E}+00$ & $1.70 \mathrm{E}+00$ & $1.78 \mathrm{E}+00$ & $1.85 \mathrm{E}+00$ & $1.90 \mathrm{E}+00$ \\
\hline $\mathrm{Pu}-239$ & $1.91 \mathrm{E}-03$ & $3.00 \mathrm{E}-03$ & $3.73 \mathrm{E}-03$ & $4.24 \mathrm{E}-03$ & $4.63 \mathrm{E}-03$ & $4.93 \mathrm{E}-03$ & $5.16 \mathrm{E}-03$ & $5.36 \mathrm{E}-03$ & $5.52 \mathrm{E}-03$ \\
\hline $\mathrm{Pu}-240$ & $6.90 \mathrm{E}-03$ & $1.09 \mathrm{E}-02$ & $1.36 \mathrm{E}-02$ & $1.55 \mathrm{E}-02$ & $1.69 \mathrm{E}-02$ & $1.80 \mathrm{E}-02$ & $1.89 \mathrm{E}-02$ & $1.96 \mathrm{E}-02$ & $2.02 \mathrm{E}-02$ \\
\hline $\mathrm{Pu}-241$ & $1.23 \mathrm{E}-01$ & $1.90 \mathrm{E}-01$ & $2.35 \mathrm{E}-01$ & $2.67 \mathrm{E}-01$ & $2.91 \mathrm{E}-01$ & $3.09 \mathrm{E}-01$ & $3.24 \mathrm{E}-01$ & $3.36 \mathrm{E}-01$ & $3.46 \mathrm{E}-01$ \\
\hline $\mathrm{Pu}-242$ & $1.78 \mathrm{E}-04$ & $2.36 \mathrm{E}-04$ & $2.74 \mathrm{E}-04$ & $3.01 \mathrm{E}-04$ & $3.21 \mathrm{E}-04$ & $3.37 \mathrm{E}-04$ & $3.49 \mathrm{E}-04$ & $3.59 \mathrm{E}-04$ & $3.68 \mathrm{E}-04$ \\
\hline \multicolumn{10}{|c|}{ Self-shielding by $\mathrm{PuO} 2$} \\
\hline Am-241 & $1.38 \mathrm{E}-01$ & $2.14 \mathrm{E}-01$ & $2.65 \mathrm{E}-01$ & $3.01 \mathrm{E}-01$ & $3.28 \mathrm{E}-01$ & $3.49 \mathrm{E}-01$ & $3.66 \mathrm{E}-01$ & $3.79 \mathrm{E}-01$ & $3.91 \mathrm{E}-01$ \\
\hline $\mathrm{Am}-243$ & $7.60 \mathrm{E}-01$ & $8.36 \mathrm{E}-01$ & $8.87 \mathrm{E}-01$ & $9.23 \mathrm{E}-01$ & $9.50 \mathrm{E}-01$ & $9.71 \mathrm{E}-01$ & $9.88 \mathrm{E}-01$ & $1.00 \mathrm{E}+00$ & $1.01 \mathrm{E}+00$ \\
\hline $\mathrm{Cm}-244$ & $4.25 \mathrm{E}+00$ & $6.47 \mathrm{E}+00$ & $7.94 \mathrm{E}+00$ & $8.99 \mathrm{E}+00$ & $9.77 \mathrm{E}+00$ & $1.04 \mathrm{E}+01$ & $1.09 \mathrm{E}+01$ & $1.13 \mathrm{E}+01$ & $1.16 \mathrm{E}+01$ \\
\hline $\mathrm{Np}-237$ & $3.58 \mathrm{E}-03$ & $3.59 \mathrm{E}-03$ & $3.60 \mathrm{E}-03$ & $3.60 \mathrm{E}-03$ & $3.61 \mathrm{E}-03$ & $3.61 \mathrm{E}-03$ & $3.61 \mathrm{E}-03$ & $3.61 \mathrm{E}-03$ & $3.61 \mathrm{E}-03$ \\
\hline $\mathrm{Pu}-238$ & $6.45 \mathrm{E}-01$ & $1.03 \mathrm{E}+00$ & $1.28 \mathrm{E}+00$ & $1.46 \mathrm{E}+00$ & $1.60 \mathrm{E}+00$ & $1.71 \mathrm{E}+00$ & $1.79 \mathrm{E}+00$ & $1.86 \mathrm{E}+00$ & $1.92 \mathrm{E}+00$ \\
\hline $\mathrm{Pu}-239$ & $1.92 \mathrm{E}-03$ & $3.02 \mathrm{E}-03$ & $3.75 \mathrm{E}-03$ & $4.27 \mathrm{E}-03$ & $4.65 \mathrm{E}-03$ & $4.95 \mathrm{E}-03$ & $5.19 \mathrm{E}-03$ & $5.39 \mathrm{E}-03$ & $5.55 \mathrm{E}-03$ \\
\hline $\mathrm{Pu}-240$ & $6.93 \mathrm{E}-03$ & $1.10 \mathrm{E}-02$ & $1.37 \mathrm{E}-02$ & $1.56 \mathrm{E}-02$ & $1.70 \mathrm{E}-02$ & $1.81 \mathrm{E}-02$ & $1.90 \mathrm{E}-02$ & $1.97 \mathrm{E}-02$ & $2.03 \mathrm{E}-02$ \\
\hline $\mathrm{Pu}-241$ & $1.23 \mathrm{E}-01$ & $1.91 \mathrm{E}-01$ & $2.37 \mathrm{E}-01$ & $2.68 \mathrm{E}-01$ & $2.92 \mathrm{E}-01$ & $3.11 \mathrm{E}-01$ & $3.26 \mathrm{E}-01$ & $3.38 \mathrm{E}-01$ & $3.48 \mathrm{E}-01$ \\
\hline $\mathrm{Pu}-242$ & $1.79 \mathrm{E}-04$ & $2.37 \mathrm{E}-04$ & $2.75 \mathrm{E}-04$ & $3.03 \mathrm{E}-04$ & $3.23 \mathrm{E}-04$ & $3.39 \mathrm{E}-04$ & $3.51 \mathrm{E}-04$ & $3.61 \mathrm{E}-04$ & $3.70 \mathrm{E}-04$ \\
\hline
\end{tabular}

Note: $90 \%$ Beryllium is modeled as 1 gram of actinide and 0.9 grams of beryllium. 
PCP-2011-0001

Revision 0

Table 3-41. Actinide Total Dose Rate - SC2 Polyethylene Shielded Container

\begin{tabular}{|c|c|c|c|c|c|}
\hline \multirow{2}{*}{$\% \mathrm{Be}$} & \multicolumn{5}{|c|}{ Dose Rate from 1 gram of Isotope (rem $/ \mathrm{hr})$} \\
\hline & Am-241 & Am-243 & Cf-252 & $\mathrm{Cm}-244$ & $\mathrm{Cm}-248$ \\
\hline \multicolumn{6}{|c|}{ Neutron } \\
\hline 0 & $1.085 \mathrm{E}-08$ & $5.581 \mathrm{E}-09$ & $2.119 \mathrm{E}+04$ & $9.547 \mathrm{E}-02$ & $3.349 \mathrm{E}-01$ \\
\hline 10 & $3.905 \mathrm{E}-02$ & $1.968 \mathrm{E}-03$ & & $1.250 \mathrm{E}+00$ & \\
\hline 20 & $6.229 \mathrm{E}-02$ & $3.132 \mathrm{E}-03$ & & $1.933 \mathrm{E}+00$ & \\
\hline 30 & $7.770 \mathrm{E}-02$ & $3.901 \mathrm{E}-03$ & & $2.388 \mathrm{E}+00$ & \\
\hline 40 & $8.866 \mathrm{E}-02$ & $4.447 \mathrm{E}-03$ & & $2.710 \mathrm{E}+00$ & \\
\hline 50 & $9.688 \mathrm{E}-02$ & $4.855 \mathrm{E}-03$ & & $2.952 \mathrm{E}+00$ & \\
\hline 60 & $1.032 \mathrm{E}-01$ & $5.171 \mathrm{E}-03$ & & $3.142 \mathrm{E}+00$ & \\
\hline 70 & $1.083 \mathrm{E}-01$ & $5.424 \mathrm{E}-03$ & & $3.290 \mathrm{E}+00$ & \\
\hline 80 & $1.125 \mathrm{E}-01$ & $5.630 \mathrm{E}-03$ & & $3.413 \mathrm{E}+00$ & \\
\hline 90 & $1.159 \mathrm{E}-01$ & $5.802 \mathrm{E}-03$ & & $3.514 \mathrm{E}+00$ & \\
\hline \multicolumn{6}{|c|}{ Secondary Photon } \\
\hline 0 & $2.735 \mathrm{E}-10$ & $1.477 \mathrm{E}-10$ & $5.029 \mathrm{E}+02$ & $2.448 \mathrm{E}-03$ & $9.236 \mathrm{E}-03$ \\
\hline 10 & $4.750 \mathrm{E}-04$ & $2.423 \mathrm{E}-05$ & & $1.627 \mathrm{E}-02$ & \\
\hline 20 & $7.575 \mathrm{E}-04$ & $3.856 \mathrm{E}-05$ & & $2.444 \mathrm{E}-02$ & \\
\hline 30 & $9.448 \mathrm{E}-04$ & $4.803 \mathrm{E}-05$ & & $2.989 \mathrm{E}-02$ & \\
\hline 40 & $1.078 \mathrm{E}-03$ & $5.475 \mathrm{E}-05$ & & $3.375 \mathrm{E}-02$ & \\
\hline 50 & $1.178 \mathrm{E}-03$ & $5.977 \mathrm{E}-05$ & & $3.663 \mathrm{E}-02$ & \\
\hline 60 & $1.255 \mathrm{E}-03$ & $6.366 \mathrm{E}-05$ & & $3.890 \mathrm{E}-02$ & \\
\hline 70 & $1.317 \mathrm{E}-03$ & $6.677 \mathrm{E}-05$ & & $4.067 \mathrm{E}-02$ & \\
\hline 80 & $1.367 \mathrm{E}-03$ & $6.931 \mathrm{E}-05$ & & $4.214 \mathrm{E}-02$ & \\
\hline 90 & $1.409 \mathrm{E}-03$ & $7.141 \mathrm{E}-05$ & & $4.335 \mathrm{E}-02$ & \\
\hline \multicolumn{6}{|c|}{ Primary Photon } \\
\hline 0 & $1.727 \mathrm{E}-03$ & $5.191 \mathrm{E}-03$ & $1.464 \mathrm{E}+03$ & $1.148 \mathrm{E}-02$ & $3.088 \mathrm{E}-02$ \\
\hline 10 & $1.727 \mathrm{E}-03$ & $5.191 \mathrm{E}-03$ & & $1.148 \mathrm{E}-02$ & \\
\hline 20 & $1.727 \mathrm{E}-03$ & $5.191 \mathrm{E}-03$ & & $1.148 \mathrm{E}-02$ & \\
\hline 30 & $1.727 \mathrm{E}-03$ & $5.191 \mathrm{E}-03$ & & $1.148 \mathrm{E}-02$ & \\
\hline 40 & $1.727 \mathrm{E}-03$ & 5.191E-03 & & $1.148 \mathrm{E}-02$ & \\
\hline 50 & $1.727 \mathrm{E}-03$ & $5.191 \mathrm{E}-03$ & & $1.148 \mathrm{E}-02$ & \\
\hline 60 & $1.727 \mathrm{E}-03$ & $5.191 \mathrm{E}-03$ & & $1.148 \mathrm{E}-02$ & \\
\hline 70 & $1.727 \mathrm{E}-03$ & $5.191 \mathrm{E}-03$ & & $1.148 \mathrm{E}-02$ & \\
\hline 80 & $1.727 \mathrm{E}-03$ & $5.191 \mathrm{E}-03$ & & $1.148 \mathrm{E}-02$ & \\
\hline 90 & $1.727 \mathrm{E}-03$ & $5.191 \mathrm{E}-03$ & & $1.148 \mathrm{E}-02$ & \\
\hline \multicolumn{6}{|c|}{ Total } \\
\hline 0 & $1.727 \mathrm{E}-03$ & $5.191 \mathrm{E}-03$ & $2.316 \mathrm{E}+04$ & $1.094 \mathrm{E}-01$ & $3.750 \mathrm{E}-01$ \\
\hline 10 & $4.126 \mathrm{E}-02$ & $7.183 \mathrm{E}-03$ & & $1.278 \mathrm{E}+00$ & \\
\hline 20 & $6.478 \mathrm{E}-02$ & $8.362 \mathrm{E}-03$ & & $1.969 \mathrm{E}+00$ & \\
\hline 30 & $8.037 \mathrm{E}-02$ & $9.140 \mathrm{E}-03$ & & $2.429 \mathrm{E}+00$ & \\
\hline 40 & $9.147 \mathrm{E}-02$ & $9.693 \mathrm{E}-03$ & & $2.756 \mathrm{E}+00$ & \\
\hline 50 & $9.978 \mathrm{E}-02$ & $1.011 \mathrm{E}-02$ & & $3.000 \mathrm{E}+00$ & \\
\hline 60 & $1.062 \mathrm{E}-01$ & $1.043 \mathrm{E}-02$ & & $3.192 \mathrm{E}+00$ & \\
\hline 70 & $1.114 \mathrm{E}-01$ & $1.068 \mathrm{E}-02$ & & $3.342 \mathrm{E}+00$ & \\
\hline 80 & $1.156 \mathrm{E}-01$ & $1.089 \mathrm{E}-02$ & & $3.466 \mathrm{E}+00$ & \\
\hline 90 & $1.191 \mathrm{E}-01$ & $1.106 \mathrm{E}-02$ & & $3.569 \mathrm{E}+00$ & \\
\hline
\end{tabular}

Note: $90 \%$ Beryllium is modeled as 1 gram of actinide and 0.9 grams of beryllium. 
PCP-2011-0001

Revision 0

Table 3-41. Actinide Total Dose Rate - SC2 Polyethylene Shielded Container (Continued)

\begin{tabular}{|c|c|c|c|c|c|c|}
\hline \multirow{2}{*}{$\% \mathrm{Be}$} & \multicolumn{6}{|c|}{ Dose Rate from 1 gram of Isotope (rem/hr) } \\
\hline & Np-237 & $\mathrm{Pu}-238$ & $\mathrm{Pu}-239$ & $\mathrm{Pu}-240$ & $\mathrm{Pu}-241$ & $\mathrm{Pu}-242$ \\
\hline \multicolumn{7}{|c|}{ Neutron } \\
\hline 0 & $9.294 \mathrm{E}-13$ & $2.182 \mathrm{E}-05$ & $1.269 \mathrm{E}-10$ & $8.470 \mathrm{E}-06$ & $1.718 \mathrm{E}-11$ & $1.429 \mathrm{E}-05$ \\
\hline 10 & $4.519 \mathrm{E}-06$ & $1.956 \mathrm{E}-01$ & $5.567 \mathrm{E}-04$ & $2.065 \mathrm{E}-03$ & $1.884 \mathrm{E}-05$ & $4.372 \mathrm{E}-05$ \\
\hline 20 & $7.196 \mathrm{E}-06$ & $3.121 \mathrm{E}-01$ & $8.868 \mathrm{E}-04$ & $3.281 \mathrm{E}-03$ & $2.994 \mathrm{E}-05$ & $6.103 \mathrm{E}-05$ \\
\hline 30 & $8.967 \mathrm{E}-06$ & $3.894 \mathrm{E}-01$ & $1.105 \mathrm{E}-03$ & $4.086 \mathrm{E}-03$ & $3.726 \mathrm{E}-05$ & $7.243 \mathrm{E}-05$ \\
\hline 40 & $1.022 \mathrm{E}-05$ & $4.445 \mathrm{E}-01$ & $1.260 \mathrm{E}-03$ & $4.657 \mathrm{E}-03$ & $4.244 \mathrm{E}-05$ & $8.050 \mathrm{E}-05$ \\
\hline 50 & $1.116 \mathrm{E}-05$ & $4.857 \mathrm{E}-01$ & $1.376 \mathrm{E}-03$ & $5.083 \mathrm{E}-03$ & $4.631 \mathrm{E}-05$ & $8.652 \mathrm{E}-05$ \\
\hline 60 & $1.189 \mathrm{E}-05$ & $5.177 \mathrm{E}-01$ & $1.466 \mathrm{E}-03$ & $5.414 \mathrm{E}-03$ & $4.931 \mathrm{E}-05$ & $9.118 \mathrm{E}-05$ \\
\hline 70 & $1.247 \mathrm{E}-05$ & $5.433 \mathrm{E}-01$ & $1.538 \mathrm{E}-03$ & $5.678 \mathrm{E}-03$ & $5.170 \mathrm{E}-05$ & $9.489 \mathrm{E}-05$ \\
\hline 80 & $1.295 \mathrm{E}-05$ & $5.642 \mathrm{E}-01$ & $1.596 \mathrm{E}-03$ & $5.894 \mathrm{E}-03$ & $5.365 \mathrm{E}-05$ & $9.792 \mathrm{E}-05$ \\
\hline 90 & $1.334 \mathrm{E}-05$ & $5.816 \mathrm{E}-01$ & $1.645 \mathrm{E}-03$ & $6.073 \mathrm{E}-03$ & $5.527 \mathrm{E}-05$ & $1.004 \mathrm{E}-04$ \\
\hline \multicolumn{7}{|c|}{ Secondary Photon } \\
\hline 0 & $2.525 \mathrm{E}-14$ & $5.826 \mathrm{E}-07$ & $3.313 \mathrm{E}-12$ & $2.359 \mathrm{E}-07$ & $4.638 \mathrm{E}-13$ & $3.931 \mathrm{E}-07$ \\
\hline 10 & $5.745 \mathrm{E}-08$ & $2.378 \mathrm{E}-03$ & $6.912 \mathrm{E}-06$ & $2.575 \mathrm{E}-05$ & $2.382 \mathrm{E}-07$ & $7.652 \mathrm{E}-07$ \\
\hline 20 & $9.148 \mathrm{E}-08$ & $3.794 \mathrm{E}-03$ & $1.101 \mathrm{E}-05$ & $4.085 \mathrm{E}-05$ & $3.785 \mathrm{E}-07$ & $9.841 \mathrm{E}-07$ \\
\hline 30 & $1.140 \mathrm{E}-07$ & $4.733 \mathrm{E}-03$ & $1.372 \mathrm{E}-05$ & $5.083 \mathrm{E}-05$ & $4.710 \mathrm{E}-07$ & $1.128 \mathrm{E}-06$ \\
\hline 40 & $1.300 \mathrm{E}-07$ & $5.402 \mathrm{E}-03$ & $1.564 \mathrm{E}-05$ & $5.791 \mathrm{E}-05$ & $5.366 \mathrm{E}-07$ & $1.230 \mathrm{E}-06$ \\
\hline 50 & $1.419 \mathrm{E}-07$ & $5.903 \mathrm{E}-03$ & $1.708 \mathrm{E}-05$ & $6.320 \mathrm{E}-05$ & $5.855 \mathrm{E}-07$ & $1.306 \mathrm{E}-06$ \\
\hline 60 & $1.512 \mathrm{E}-07$ & $6.292 \mathrm{E}-03$ & $1.820 \mathrm{E}-05$ & $6.730 \mathrm{E}-05$ & $6.234 \mathrm{E}-07$ & $1.365 \mathrm{E}-06$ \\
\hline 70 & $1.586 \mathrm{E}-07$ & $6.602 \mathrm{E}-03$ & $1.909 \mathrm{E}-05$ & $7.058 \mathrm{E}-05$ & $6.536 \mathrm{E}-07$ & $1.412 \mathrm{E}-06$ \\
\hline 80 & $1.646 \mathrm{E}-07$ & $6.856 \mathrm{E}-03$ & $1.981 \mathrm{E}-05$ & $7.325 \mathrm{E}-05$ & $6.782 \mathrm{E}-07$ & $1.450 \mathrm{E}-06$ \\
\hline 90 & $1.697 \mathrm{E}-07$ & $7.067 \mathrm{E}-03$ & $2.042 \mathrm{E}-05$ & $7.547 \mathrm{E}-05$ & $6.987 \mathrm{E}-07$ & $1.482 \mathrm{E}-06$ \\
\hline \multicolumn{7}{|c|}{ Primary Photon } \\
\hline 0 & $2.597 \mathrm{E}-05$ & $4.015 \mathrm{E}-04$ & $1.606 \mathrm{E}-05$ & $5.726 \mathrm{E}-06$ & $3.478 \mathrm{E}-04$ & $1.947 \mathrm{E}-06$ \\
\hline 10 & $2.597 \mathrm{E}-05$ & $4.015 \mathrm{E}-04$ & $1.606 \mathrm{E}-05$ & $5.726 \mathrm{E}-06$ & $3.478 \mathrm{E}-04$ & $1.947 \mathrm{E}-06$ \\
\hline 20 & $2.597 \mathrm{E}-05$ & $4.015 \mathrm{E}-04$ & $1.606 \mathrm{E}-05$ & $5.726 \mathrm{E}-06$ & $3.478 \mathrm{E}-04$ & $1.947 \mathrm{E}-06$ \\
\hline 30 & $2.597 \mathrm{E}-05$ & $4.015 \mathrm{E}-04$ & $1.606 \mathrm{E}-05$ & $5.726 \mathrm{E}-06$ & $3.478 \mathrm{E}-04$ & $1.947 \mathrm{E}-06$ \\
\hline 40 & $2.597 \mathrm{E}-05$ & $4.015 \mathrm{E}-04$ & $1.606 \mathrm{E}-05$ & $5.726 \mathrm{E}-06$ & $3.478 \mathrm{E}-04$ & $1.947 \mathrm{E}-06$ \\
\hline 50 & $2.597 \mathrm{E}-05$ & $4.015 \mathrm{E}-04$ & $1.606 \mathrm{E}-05$ & $5.726 \mathrm{E}-06$ & $3.478 \mathrm{E}-04$ & $1.947 \mathrm{E}-06$ \\
\hline 60 & $2.597 \mathrm{E}-05$ & $4.015 \mathrm{E}-04$ & $1.606 \mathrm{E}-05$ & $5.726 \mathrm{E}-06$ & $3.478 \mathrm{E}-04$ & $1.947 \mathrm{E}-06$ \\
\hline 70 & $2.597 \mathrm{E}-05$ & $4.015 \mathrm{E}-04$ & $1.606 \mathrm{E}-05$ & $5.726 \mathrm{E}-06$ & $3.478 \mathrm{E}-04$ & $1.947 \mathrm{E}-06$ \\
\hline 80 & $2.597 \mathrm{E}-05$ & $4.015 \mathrm{E}-04$ & $1.606 \mathrm{E}-05$ & $5.726 \mathrm{E}-06$ & $3.478 \mathrm{E}-04$ & $1.947 \mathrm{E}-06$ \\
\hline 90 & $2.597 \mathrm{E}-05$ & $4.015 \mathrm{E}-04$ & $1.606 \mathrm{E}-05$ & $5.726 \mathrm{E}-06$ & $3.478 \mathrm{E}-04$ & $1.947 \mathrm{E}-06$ \\
\hline \multicolumn{7}{|c|}{ Total } \\
\hline 0 & $2.597 \mathrm{E}-05$ & $4.239 \mathrm{E}-04$ & $1.606 \mathrm{E}-05$ & $1.443 \mathrm{E}-05$ & $3.478 \mathrm{E}-04$ & $1.663 \mathrm{E}-05$ \\
\hline 10 & $3.055 \mathrm{E}-05$ & $1.983 \mathrm{E}-01$ & $5.797 \mathrm{E}-04$ & $2.096 \mathrm{E}-03$ & $3.669 \mathrm{E}-04$ & 4.644E-05 \\
\hline 20 & $3.326 \mathrm{E}-05$ & $3.163 \mathrm{E}-01$ & $9.138 \mathrm{E}-04$ & $3.328 \mathrm{E}-03$ & $3.782 \mathrm{E}-04$ & $6.396 \mathrm{E}-05$ \\
\hline 30 & $3.505 \mathrm{E}-05$ & $3.945 \mathrm{E}-01$ & $1.135 \mathrm{E}-03$ & $4.142 \mathrm{E}-03$ & $3.856 \mathrm{E}-04$ & $7.551 \mathrm{E}-05$ \\
\hline 40 & $3.632 \mathrm{E}-05$ & $4.503 \mathrm{E}-01$ & $1.292 \mathrm{E}-03$ & $4.720 \mathrm{E}-03$ & $3.908 \mathrm{E}-04$ & $8.367 \mathrm{E}-05$ \\
\hline 50 & $3.727 \mathrm{E}-05$ & $4.920 \mathrm{E}-01$ & $1.409 \mathrm{E}-03$ & $5.152 \mathrm{E}-03$ & $3.947 \mathrm{E}-04$ & $8.977 \mathrm{E}-05$ \\
\hline 60 & $3.801 \mathrm{E}-05$ & $5.244 \mathrm{E}-01$ & $1.500 \mathrm{E}-03$ & $5.487 \mathrm{E}-03$ & $3.978 \mathrm{E}-04$ & $9.449 \mathrm{E}-05$ \\
\hline 70 & $3.860 \mathrm{E}-05$ & $5.503 \mathrm{E}-01$ & $1.573 \mathrm{E}-03$ & $5.754 \mathrm{E}-03$ & $4.002 \mathrm{E}-04$ & $9.824 \mathrm{E}-05$ \\
\hline 80 & $3.908 \mathrm{E}-05$ & $5.715 \mathrm{E}-01$ & $1.632 \mathrm{E}-03$ & $5.972 \mathrm{E}-03$ & $4.022 \mathrm{E}-04$ & $1.013 \mathrm{E}-04$ \\
\hline 90 & $3.948 \mathrm{E}-05$ & $5.891 \mathrm{E}-01$ & $1.682 \mathrm{E}-03$ & $6.154 \mathrm{E}-03$ & $4.038 \mathrm{E}-04$ & $1.039 \mathrm{E}-04$ \\
\hline
\end{tabular}

Note: $90 \%$ Beryllium is modeled as 1 gram of actinide and 0.9 grams of beryllium. 
PCP-2011-0001

Revision 0

Table 3-42. Dose Rate from Actinides as a Function of Time

\begin{tabular}{|c|c|c|c|c|c|c|c|c|}
\hline \multirow{3}{*}{ Isotope } & \multicolumn{8}{|c|}{ Dose Rate from 1 gram of Isotope $(\mathrm{rem} / \mathrm{hr})$} \\
\hline & \multicolumn{4}{|c|}{ No Beryllium } & \multicolumn{4}{|c|}{$90 \%$ Beryllium Added } \\
\hline & 0 days & 30 days & 100 days & 30000 days & 0 days & 30 days & 100 days & 30000 days \\
\hline \multicolumn{9}{|c|}{ No Shielded Container } \\
\hline Am-241 & $9.11 \mathrm{E}-03$ & $9.11 \mathrm{E}-03$ & $9.11 \mathrm{E}-03$ & $8.43 \mathrm{E}-03$ & $3.89 \mathrm{E}-01$ & $3.89 \mathrm{E}-01$ & $3.88 \mathrm{E}-01$ & $3.41 \mathrm{E}-01$ \\
\hline Am-243 & $2.52 \mathrm{E}-02$ & $6.31 \mathrm{E}-01$ & $6.31 \mathrm{E}-01$ & $6.26 \mathrm{E}-01$ & $4.44 \mathrm{E}-02$ & $1.01 \mathrm{E}+00$ & $6.50 \mathrm{E}-01$ & $6.45 \mathrm{E}-01$ \\
\hline Cf-252 & $1.05 \mathrm{E}+05$ & $1.03 \mathrm{E}+05$ & $9.79 \mathrm{E}+04$ & $4.38 \mathrm{E}+03$ & & & & \\
\hline $\mathrm{Cm}-244$ & $5.08 \mathrm{E}-01$ & $5.07 \mathrm{E}-01$ & $5.03 \mathrm{E}-01$ & $2.19 \mathrm{E}-02$ & $1.15 \mathrm{E}+01$ & $1.15 \mathrm{E}+01$ & $1.14 \mathrm{E}+01$ & $5.15 \mathrm{E}-01$ \\
\hline $\mathrm{Cm}-248$ & $1.82 \mathrm{E}+00$ & $1.82 \mathrm{E}+00$ & $1.82 \mathrm{E}+00$ & $1.82 \mathrm{E}+00$ & & & & \\
\hline $\mathrm{Np}-237$ & $1.14 \mathrm{E}-04$ & $4.93 \mathrm{E}-03$ & $3.31 \mathrm{E}-03$ & $3.57 \mathrm{E}-03$ & $1.59 \mathrm{E}-04$ & $2.01 \mathrm{E}-03$ & $3.36 \mathrm{E}-03$ & $3.61 \mathrm{E}-03$ \\
\hline $\mathrm{Pu}-238$ & $1.80 \mathrm{E}-03$ & $1.80 \mathrm{E}-03$ & $1.79 \mathrm{E}-03$ & $9.42 \mathrm{E}-04$ & $1.90 \mathrm{E}+00$ & $1.90 \mathrm{E}+00$ & $1.90 \mathrm{E}+00$ & $9.95 \mathrm{E}-01$ \\
\hline $\mathrm{Pu}-239$ & $5.71 \mathrm{E}-05$ & $5.73 \mathrm{E}-05$ & $5.73 \mathrm{E}-05$ & $5.70 \mathrm{E}-05$ & $5.52 \mathrm{E}-03$ & $5.52 \mathrm{E}-03$ & $5.52 \mathrm{E}-03$ & $5.51 \mathrm{E}-03$ \\
\hline $\mathrm{Pu}-240$ & $6.74 \mathrm{E}-05$ & $6.73 \mathrm{E}-05$ & $6.73 \mathrm{E}-05$ & $6.68 \mathrm{E}-05$ & $2.02 \mathrm{E}-02$ & $2.02 \mathrm{E}-02$ & $2.02 \mathrm{E}-02$ & $2.00 \mathrm{E}-02$ \\
\hline $\mathrm{Pu}-241$ & $1.53 \mathrm{E}-03$ & $5.81 \mathrm{E}-03$ & $6.04 \mathrm{E}-03$ & $8.54 \mathrm{E}-03$ & $1.71 \mathrm{E}-03$ & $7.50 \mathrm{E}-03$ & $1.12 \mathrm{E}-02$ & $3.46 \mathrm{E}-01$ \\
\hline $\mathrm{Pu}-242$ & 7.94E-05 & $7.93 \mathrm{E}-05$ & 7.93E-05 & 7.94E-05 & $3.68 \mathrm{E}-04$ & $3.68 \mathrm{E}-04$ & $3.68 \mathrm{E}-04$ & $3.68 \mathrm{E}-04$ \\
\hline \multicolumn{9}{|c|}{ SC2 Polyethylene Shielded Container } \\
\hline Am-241 & $1.73 \mathrm{E}-03$ & $1.73 \mathrm{E}-03$ & $1.73 \mathrm{E}-03$ & $1.64 \mathrm{E}-03$ & $1.19 \mathrm{E}-01$ & $1.19 \mathrm{E}-01$ & $1.19 \mathrm{E}-01$ & $1.05 \mathrm{E}-01$ \\
\hline Am-243 & 5.19E-03 & $1.65 \mathrm{E}-01$ & $1.65 \mathrm{E}-01$ & $1.64 \mathrm{E}-01$ & $1.11 \mathrm{E}-02$ & $1.71 \mathrm{E}-01$ & $1.71 \mathrm{E}-01$ & $1.69 \mathrm{E}-01$ \\
\hline Cf-252 & $2.32 \mathrm{E}+04$ & $2.27 \mathrm{E}+04$ & $2.17 \mathrm{E}+04$ & $1.46 \mathrm{E}+03$ & & & & \\
\hline $\mathrm{Cm}-244$ & $1.09 \mathrm{E}-01$ & $1.09 \mathrm{E}-01$ & $1.08 \mathrm{E}-01$ & $4.72 \mathrm{E}-03$ & $3.57 \mathrm{E}+00$ & $3.56 \mathrm{E}+00$ & $3.53 \mathrm{E}+00$ & $1.59 \mathrm{E}-01$ \\
\hline $\mathrm{Cm}-248$ & $3.75 \mathrm{E}-01$ & $3.75 \mathrm{E}-01$ & $3.75 \mathrm{E}-01$ & $3.75 \mathrm{E}-01$ & & & & \\
\hline $\mathrm{Np}-237$ & $2.60 \mathrm{E}-05$ & $5.57 \mathrm{E}-04$ & $9.41 \mathrm{E}-04$ & $1.01 \mathrm{E}-03$ & $3.95 \mathrm{E}-05$ & $5.70 \mathrm{E}-04$ & $9.55 \mathrm{E}-04$ & $1.03 \mathrm{E}-03$ \\
\hline $\mathrm{Pu}-238$ & $4.24 \mathrm{E}-04$ & $4.24 \mathrm{E}-04$ & $4.23 \mathrm{E}-04$ & $2.22 \mathrm{E}-04$ & $5.89 \mathrm{E}-01$ & $5.89 \mathrm{E}-01$ & $5.88 \mathrm{E}-01$ & $3.08 \mathrm{E}-01$ \\
\hline Pu-239 & $1.61 \mathrm{E}-05$ & $1.61 \mathrm{E}-05$ & $1.61 \mathrm{E}-05$ & $1.60 \mathrm{E}-05$ & $1.68 \mathrm{E}-03$ & $1.68 \mathrm{E}-03$ & $1.68 \mathrm{E}-03$ & $1.68 \mathrm{E}-03$ \\
\hline $\mathrm{Pu}-240$ & $1.44 \mathrm{E}-05$ & $1.44 \mathrm{E}-05$ & $1.44 \mathrm{E}-05$ & $1.43 \mathrm{E}-05$ & $6.15 \mathrm{E}-03$ & $6.15 \mathrm{E}-03$ & $6.15 \mathrm{E}-03$ & $6.10 \mathrm{E}-03$ \\
\hline $\mathrm{Pu}-241$ & $3.48 \mathrm{E}-04$ & $1.43 \mathrm{E}-03$ & $1.49 \mathrm{E}-03$ & $1.66 \mathrm{E}-03$ & $4.04 \mathrm{E}-04$ & $1.95 \mathrm{E}-03$ & $3.09 \mathrm{E}-03$ & $1.06 \mathrm{E}-01$ \\
\hline $\mathrm{Pu}-242$ & $1.66 \mathrm{E}-05$ & $1.66 \mathrm{E}-05$ & $1.66 \mathrm{E}-05$ & $1.66 \mathrm{E}-05$ & $1.04 \mathrm{E}-04$ & $1.04 \mathrm{E}-04$ & $1.04 \mathrm{E}-04$ & $1.04 \mathrm{E}-04$ \\
\hline
\end{tabular}

Note: $90 \%$ Beryllium is modeled as 1 gram of actinide and 0.9 grams of beryllium. 
PCP-2011-0001

Revision 0

Table 3-43. Primary Photon Dose Rate

\begin{tabular}{|c|c|c|c|c|}
\hline \multirow[b]{2}{*}{ Isotope } & \multicolumn{4}{|c|}{ Dose Rate from 1 gram of Isotope (rem/hr) } \\
\hline & $\begin{array}{c}\text { No } \\
\text { Shielded } \\
\text { Container } \\
\end{array}$ & $\begin{array}{l}\text { SC1 Lead } \\
\text { Shielded } \\
\text { Container }\end{array}$ & $\begin{array}{c}\text { SC2 Polyethylene } \\
\text { Shielded } \\
\text { Container }\end{array}$ & $\begin{array}{c}\text { SC3 Tungsten } \\
\text { Shielded } \\
\text { Container }\end{array}$ \\
\hline Ac- 227 & $4.84 \mathrm{E}+02$ & $3.80 \mathrm{E}-01$ & $1.41 \mathrm{E}+02$ & 7.48E-02 \\
\hline $\mathrm{Am}-241$ & $9.11 \mathrm{E}-03$ & $8.87 \mathrm{E}-07$ & $1.73 \mathrm{E}-03$ & $1.51 \mathrm{E}-07$ \\
\hline Am-243 & $6.31 \mathrm{E}-01$ & $1.06 \mathrm{E}-07$ & $1.65 \mathrm{E}-01$ & $2.32 \mathrm{E}-08$ \\
\hline Cd-109 & $3.52 \mathrm{E}+01$ & $0.00 \mathrm{E}+00$ & $7.18 \mathrm{E}+00$ & $0.00 \mathrm{E}+00$ \\
\hline Cf-252 & $4.38 \mathrm{E}+03$ & $1.15 \mathrm{E}+02$ & $1.46 \mathrm{E}+03$ & $3.90 \mathrm{E}+01$ \\
\hline $\mathrm{Cm}-244$ & $3.54 \mathrm{E}-02$ & 7.68E-04 & $1.15 \mathrm{E}-02$ & $2.58 \mathrm{E}-04$ \\
\hline $\mathrm{Cm}-248$ & $1.02 \mathrm{E}-01$ & $2.55 \mathrm{E}-03$ & $3.40 \mathrm{E}-02$ & $8.56 \mathrm{E}-04$ \\
\hline Co-60 & $6.10 \mathrm{E}+04$ & $1.64 \mathrm{E}+03$ & $2.09 \mathrm{E}+04$ & $4.43 \mathrm{E}+02$ \\
\hline Cs-137 & $1.24 \mathrm{E}+03$ & $1.86 \mathrm{E}+00$ & $4.01 \mathrm{E}+02$ & $3.03 \mathrm{E}-01$ \\
\hline Eu-152 & $4.52 \mathrm{E}+03$ & $7.54 \mathrm{E}+01$ & $1.49 \mathrm{E}+03$ & $1.97 \mathrm{E}+01$ \\
\hline Fe-59 & $1.28 \mathrm{E}+06$ & $3.32 \mathrm{E}+04$ & $4.37 \mathrm{E}+05$ & $8.90 \mathrm{E}+03$ \\
\hline Gd-153 & $1.44 \mathrm{E}+03$ & $3.34 \mathrm{E}-33$ & $3.14 \mathrm{E}+02$ & $5.80 \mathrm{E}-38$ \\
\hline Hf-181 & $2.21 \mathrm{E}+05$ & $1.33 \mathrm{E}+01$ & $6.69 \mathrm{E}+04$ & $5.45 \mathrm{E}+00$ \\
\hline Ho-166m & $7.56 \mathrm{E}+01$ & $2.78 \mathrm{E}-01$ & $2.41 \mathrm{E}+01$ & $5.47 \mathrm{E}-02$ \\
\hline Ir-192 & $1.98 \mathrm{E}+05$ & $2.92 \mathrm{E}+01$ & $5.93 \mathrm{E}+04$ & $4.83 \mathrm{E}+00$ \\
\hline Mn-54 & $1.55 \mathrm{E}+05$ & $9.84 \mathrm{E}+02$ & $5.15 \mathrm{E}+04$ & $1.88 \mathrm{E}+02$ \\
\hline $\mathrm{Np}-237$ & $3.57 \mathrm{E}-03$ & $1.38 \mathrm{E}-09$ & $1.01 \mathrm{E}-03$ & $5.66 \mathrm{E}-11$ \\
\hline $\mathrm{Pb}-210$ & $7.12 \mathrm{E}+00$ & $6.67 \mathrm{E}-04$ & $1.92 \mathrm{E}+00$ & $1.16 \mathrm{E}-04$ \\
\hline Pm-147 & $7.50 \mathrm{E}-01$ & $9.72 \mathrm{E}-22$ & $1.66 \mathrm{E}-01$ & $3.21 \mathrm{E}-22$ \\
\hline Po-210 & $9.25 \mathrm{E}-01$ & $5.02 \mathrm{E}-03$ & $3.06 \mathrm{E}-01$ & $9.51 \mathrm{E}-04$ \\
\hline $\mathrm{Pu}-238$ & $1.69 \mathrm{E}-03$ & $1.19 \mathrm{E}-06$ & $4.02 \mathrm{E}-04$ & $2.73 \mathrm{E}-07$ \\
\hline Pu-239 & $5.71 \mathrm{E}-05$ & $2.37 \mathrm{E}-09$ & $1.61 \mathrm{E}-05$ & $4.43 \mathrm{E}-10$ \\
\hline $\mathrm{Pu}-240$ & $2.34 \mathrm{E}-05$ & $9.24 \mathrm{E}-08$ & $5.73 \mathrm{E}-06$ & $3.11 \mathrm{E}-08$ \\
\hline $\mathrm{Pu}-241$ & $8.54 \mathrm{E}-03$ & 7.87E-07 & $1.66 \mathrm{E}-03$ & $1.34 \mathrm{E}-07$ \\
\hline $\mathrm{Pu}-242$ & $5.87 \mathrm{E}-06$ & $1.51 \mathrm{E}-07$ & $1.95 \mathrm{E}-06$ & $5.11 \mathrm{E}-08$ \\
\hline Ra-226 & $3.79 \mathrm{E}+01$ & $8.61 \mathrm{E}-01$ & $1.25 \mathrm{E}+01$ & $2.73 \mathrm{E}-01$ \\
\hline Ru-106 & $2.15 \mathrm{E}+04$ & $8.59 \mathrm{E}+01$ & $6.79 \mathrm{E}+03$ & $2.35 \mathrm{E}+01$ \\
\hline Sc-46 & $1.56 \mathrm{E}+06$ & $2.31 \mathrm{E}+04$ & $5.24 \mathrm{E}+05$ & $5.43 \mathrm{E}+03$ \\
\hline Se-75 & $1.30 \mathrm{E}+05$ & $1.25 \mathrm{E}-01$ & $3.56 \mathrm{E}+04$ & 8.07E-03 \\
\hline Sm-145 & $1.39 \mathrm{E}+01$ & $8.54 \mathrm{E}-05$ & $2.63 \mathrm{E}+00$ & $3.61 \mathrm{E}-05$ \\
\hline Sr-90 & $6.92 \mathrm{E}+01$ & $1.42 \mathrm{E}-01$ & $2.00 \mathrm{E}+01$ & $3.43 \mathrm{E}-02$ \\
\hline Tm-170 & $7.43 \mathrm{E}+02$ & $2.73 \mathrm{E}-02$ & $1.97 \mathrm{E}+02$ & $4.52 \mathrm{E}-03$ \\
\hline Yb-169 & $9.43 \mathrm{E}+04$ & $2.05 \mathrm{E}-02$ & $2.46 \mathrm{E}+04$ & $3.15 \mathrm{E}-03$ \\
\hline $\mathrm{Zn}-65$ & $1.06 \mathrm{E}+05$ & $2.10 \mathrm{E}+03$ & $3.60 \mathrm{E}+04$ & $5.19 \mathrm{E}+02$ \\
\hline Zr-95 & $4.33 \mathrm{E}+05$ & $1.49 \mathrm{E}+03$ & $1.42 \mathrm{E}+05$ & $2.59 \mathrm{E}+02$ \\
\hline
\end{tabular}


PCP-2011-0001

Revision 0

Table 3-44. Effect of Self-shielding by Iron on Primary Photon Dose Rate

\begin{tabular}{|c|c|c|}
\hline \multirow{2}{*}{ Isotope } & \multicolumn{2}{|c|}{ Dose Rate from 1 gram of Isotope (rem/hr) } \\
\hline & No Self-shielding & Self-shielding by Iron \\
\hline Ac-227 & $4.84 \mathrm{E}+02$ & $4.64 \mathrm{E}+02$ \\
\hline $\mathrm{Am}-241$ & $9.11 \mathrm{E}-03$ & $4.69 \mathrm{E}-03$ \\
\hline Am-243 & $6.31 \mathrm{E}-01$ & $5.76 \mathrm{E}-01$ \\
\hline Cd-109 & $3.52 \mathrm{E}+01$ & $2.53 \mathrm{E}+01$ \\
\hline Cf-252 & $4.38 \mathrm{E}+03$ & $4.27 \mathrm{E}+03$ \\
\hline $\mathrm{Cm}-244$ & $3.17 \mathrm{E}-02$ & $3.09 \mathrm{E}-02$ \\
\hline $\mathrm{Cm}-248$ & $1.02 \mathrm{E}-01$ & $9.927 \mathrm{E}-02$ \\
\hline Co-60 & $6.10 \mathrm{E}+04$ & $5.97 \mathrm{E}+04$ \\
\hline Cs-137 & $1.24 \mathrm{E}+03$ & $1.20 \mathrm{E}+03$ \\
\hline Eu-152 & $4.52 \mathrm{E}+03$ & $4.39 \mathrm{E}+03$ \\
\hline Fe-59 & $1.28 \mathrm{E}+06$ & $1.25 \mathrm{E}+06$ \\
\hline Gd-153 & $1.44 \mathrm{E}+03$ & $1.15 \mathrm{E}+03$ \\
\hline Hf-181 & $2.21 \mathrm{E}+05$ & $2.12 \mathrm{E}+05$ \\
\hline Ho-166m & $7.56 \mathrm{E}+01$ & $7.326 \mathrm{E}+011$ \\
\hline Ir-192 & $1.98 \mathrm{E}+05$ & $1.91 \mathrm{E}+05$ \\
\hline Mn-54 & $1.55 \mathrm{E}+05$ & $1.51 \mathrm{E}+05$ \\
\hline $\mathrm{Np}-237$ & $3.57 \mathrm{E}-03$ & $3.39 \mathrm{E}-03$ \\
\hline $\mathrm{Pb}-210$ & $7.12 \mathrm{E}+00$ & $6.63 \mathrm{E}+00$ \\
\hline Pm-147 & $7.50 \mathrm{E}-01$ & $6.10 \mathrm{E}-01$ \\
\hline Po-210 & $9.25 \mathrm{E}-01$ & $9.01 \mathrm{E}-01$ \\
\hline $\mathrm{Pu}-238$ & $1.69 \mathrm{E}-03$ & $1.44 \mathrm{E}-03$ \\
\hline $\mathrm{Pu}-239$ & $5.71 \mathrm{E}-05$ & $5.36 \mathrm{E}-05$ \\
\hline $\mathrm{Pu}-240$ & $2.34 \mathrm{E}-05$ & $2.02 \mathrm{E}-05$ \\
\hline $\mathrm{Pu}-241$ & $8.54 \mathrm{E}-03$ & $4.58 \mathrm{E}-03$ \\
\hline $\mathrm{Pu}-242$ & $5.87 \mathrm{E}-06$ & $5.71 \mathrm{E}-06$ \\
\hline Ra-226 & $3.79 \mathrm{E}+01$ & $3.69 \mathrm{E}+01$ \\
\hline Ru-106 & $2.15 \mathrm{E}+04$ & $2.08 \mathrm{E}+04$ \\
\hline Sc-46 & $1.56 \mathrm{E}+06$ & $1.52 \mathrm{E}+06$ \\
\hline Se-75 & $1.30 \mathrm{E}+05$ & $1.23 \mathrm{E}+05$ \\
\hline Sm-145 & $1.39 \mathrm{E}+01$ & $8.13 \mathrm{E}+00$ \\
\hline Sr-90 & $6.92 \mathrm{E}+01$ & $6.57 \mathrm{E}+01$ \\
\hline Tm-170 & $7.43 \mathrm{E}+02$ & $6.89 \mathrm{E}+02$ \\
\hline Yb-169 & $9.43 \mathrm{E}+04$ & $8.80 \mathrm{E}+04$ \\
\hline Zn-65 & $1.06 \mathrm{E}+05$ & $1.04 \mathrm{E}+05$ \\
\hline Zr-95 & $4.33 \mathrm{E}+05$ & $4.21 \mathrm{E}+05$ \\
\hline
\end{tabular}


PCP-2011-0001

Revision 0

Table 3-45. Maximum Dose Rate without Impurities

\begin{tabular}{|c|c|c|c|c|}
\hline \multirow[b]{2}{*}{ Isotope } & \multicolumn{4}{|c|}{ Dose Rate from 1 gram of Isotope (rem/hr) } \\
\hline & $\begin{array}{c}\text { No } \\
\text { Shielded } \\
\text { Container }\end{array}$ & $\begin{array}{c}\text { SC1 Lead } \\
\text { Shielded } \\
\text { Container }\end{array}$ & $\begin{array}{c}\text { SC2 Polyethylene } \\
\text { Shielded } \\
\text { Container }\end{array}$ & $\begin{array}{c}\text { SC3 Tungsten } \\
\text { Shielded } \\
\text { Container }\end{array}$ \\
\hline Ac-227 & $4.84 \mathrm{E}+02$ & $3.80 \mathrm{E}-01$ & $1.41 \mathrm{E}+02$ & $7.48 \mathrm{E}-02$ \\
\hline Am-241 & $9.11 \mathrm{E}-03$ & & $1.73 \mathrm{E}-03$ & \\
\hline Am-243 & $6.31 \mathrm{E}-01$ & & $1.65 \mathrm{E}-01$ & \\
\hline Cd-109 & $3.52 \mathrm{E}+01$ & $0.00 \mathrm{E}+00$ & $7.18 \mathrm{E}+00$ & $0.00 \mathrm{E}+00$ \\
\hline Cf-252 & $1.05 \mathrm{E}+05$ & & $2.32 \mathrm{E}+04$ & \\
\hline $\mathrm{Cm}-244$ & $5.08 \mathrm{E}-01$ & & 1.09E-01 & \\
\hline $\mathrm{Cm}-248$ & $1.83 \mathrm{E}+00$ & & $3.78 \mathrm{E}-01$ & \\
\hline Co-60 & $6.10 \mathrm{E}+04$ & $1.64 \mathrm{E}+03$ & $2.09 \mathrm{E}+04$ & $4.43 \mathrm{E}+02$ \\
\hline Cs-137 & $1.24 \mathrm{E}+03$ & $1.86 \mathrm{E}+00$ & $4.01 \mathrm{E}+02$ & $3.03 \mathrm{E}-01$ \\
\hline Eu-152 & $4.52 \mathrm{E}+03$ & $7.54 \mathrm{E}+01$ & $1.49 \mathrm{E}+03$ & $1.97 \mathrm{E}+01$ \\
\hline Fe-59 & $1.28 \mathrm{E}+06$ & $3.32 \mathrm{E}+04$ & $4.37 \mathrm{E}+05$ & $8.90 \mathrm{E}+03$ \\
\hline Gd-153 & $1.44 \mathrm{E}+03$ & $3.34 \mathrm{E}-33$ & $3.14 \mathrm{E}+02$ & $5.80 \mathrm{E}-38$ \\
\hline Hf-181 & $2.21 \mathrm{E}+05$ & $1.33 \mathrm{E}+01$ & $6.69 \mathrm{E}+04$ & $5.45 \mathrm{E}+00$ \\
\hline Ho-166m & $7.56 \mathrm{E}+01$ & $2.78 \mathrm{E}-01$ & $2.41 \mathrm{E}+01$ & $5.47 \mathrm{E}-02$ \\
\hline Ir-192 & $1.98 \mathrm{E}+05$ & $2.92 \mathrm{E}+01$ & $5.93 \mathrm{E}+04$ & $4.83 \mathrm{E}+00$ \\
\hline Mn-54 & $1.55 \mathrm{E}+05$ & $9.84 \mathrm{E}+02$ & $5.15 \mathrm{E}+04$ & $1.88 \mathrm{E}+02$ \\
\hline $\mathrm{Np}-237$ & $6.27 \mathrm{E}-03$ & & $1.01 \mathrm{E}-03$ & \\
\hline $\mathrm{Pb}-210$ & $7.12 \mathrm{E}+00$ & $6.67 \mathrm{E}-04$ & $1.92 \mathrm{E}+00$ & $1.16 \mathrm{E}-04$ \\
\hline Pm-147 & $7.50 \mathrm{E}-01$ & $9.72 \mathrm{E}-22$ & $1.66 \mathrm{E}-01$ & $3.21 \mathrm{E}-22$ \\
\hline Po-210 & $9.25 \mathrm{E}-01$ & $5.02 \mathrm{E}-03$ & $3.06 \mathrm{E}-01$ & $9.51 \mathrm{E}-04$ \\
\hline $\mathrm{Pu}-238$ & $1.80 \mathrm{E}-03$ & & 4.24E-04 & \\
\hline Pu-239 & $5.73 \mathrm{E}-05$ & & $1.61 \mathrm{E}-05$ & \\
\hline $\mathrm{Pu}-240$ & $6.74 \mathrm{E}-05$ & & $1.44 \mathrm{E}-05$ & \\
\hline $\mathrm{Pu}-241$ & $8.54 \mathrm{E}-03$ & & $1.66 \mathrm{E}-03$ & \\
\hline $\mathrm{Pu}-242$ & 7.94E-05 & & $1.66 \mathrm{E}-05$ & \\
\hline Ra-226 & $3.79 \mathrm{E}+01$ & $8.61 \mathrm{E}-01$ & $1.25 \mathrm{E}+01$ & $2.73 \mathrm{E}-01$ \\
\hline Ru-106 & $2.15 \mathrm{E}+04$ & $8.59 \mathrm{E}+01$ & $6.79 \mathrm{E}+03$ & $2.35 \mathrm{E}+01$ \\
\hline $\mathrm{Sc}-46$ & $1.56 \mathrm{E}+06$ & $2.31 \mathrm{E}+04$ & $5.24 \mathrm{E}+05$ & $5.43 \mathrm{E}+03$ \\
\hline Se-75 & $1.30 \mathrm{E}+05$ & $1.25 \mathrm{E}-01$ & $3.56 \mathrm{E}+04$ & 8.07E-03 \\
\hline Sm-145 & $1.39 \mathrm{E}+01$ & $8.54 \mathrm{E}-05$ & $2.63 \mathrm{E}+00$ & $3.61 \mathrm{E}-05$ \\
\hline Sr-90 & $6.92 \mathrm{E}+01$ & $1.42 \mathrm{E}-01$ & $2.00 \mathrm{E}+01$ & $3.43 \mathrm{E}-02$ \\
\hline Tm-170 & $7.43 \mathrm{E}+02$ & $2.73 \mathrm{E}-02$ & $1.97 \mathrm{E}+02$ & $4.52 \mathrm{E}-03$ \\
\hline Yb-169 & $9.43 \mathrm{E}+04$ & $2.05 \mathrm{E}-02$ & $2.46 \mathrm{E}+04$ & $3.15 \mathrm{E}-03$ \\
\hline $\mathrm{Zn}-65$ & $1.06 \mathrm{E}+05$ & $2.10 \mathrm{E}+03$ & $3.60 \mathrm{E}+04$ & $5.19 \mathrm{E}+02$ \\
\hline Zr-95 & $4.33 \mathrm{E}+05$ & $1.49 \mathrm{E}+03$ & $1.42 \mathrm{E}+05$ & $2.59 \mathrm{E}+02$ \\
\hline
\end{tabular}


PCP-2011-0001

Revision 0

Table 3-46. Maximum Dose Rate with Impurities

\begin{tabular}{|c|c|c|c|c|c|c|c|c|c|}
\hline \multirow{2}{*}{ Isotope } & \multicolumn{9}{|c|}{ Dose Rate from 1 gram of Isotope (rem/hr) } \\
\hline & $10 \% \mathrm{Be}$ & $20 \% \mathrm{Be}$ & $30 \% \mathrm{Be}$ & $40 \% \mathrm{Be}$ & $50 \% \mathrm{Be}$ & $60 \% \mathrm{Be}$ & $70 \% \mathrm{Be}$ & $80 \% \mathrm{Be}$ & $90 \% \mathrm{Be}$ \\
\hline \multicolumn{10}{|c|}{ No Shielded Container } \\
\hline Am-241 & $1.37 \mathrm{E}-01$ & $2.13 \mathrm{E}-01$ & $2.64 \mathrm{E}-01$ & $2.99 \mathrm{E}-01$ & $3.26 \mathrm{E}-01$ & $3.47 \mathrm{E}-01$ & $3.64 \mathrm{E}-01$ & $3.77 \mathrm{E}-01$ & $3.89 \mathrm{E}-01$ \\
\hline Am-243 & $7.59 \mathrm{E}-01$ & $8.35 \mathrm{E}-01$ & $8.86 \mathrm{E}-01$ & $9.21 \mathrm{E}-01$ & $9.48 \mathrm{E}-01$ & $9.69 \mathrm{E}-01$ & $9.86 \mathrm{E}-01$ & $9.99 \mathrm{E}-01$ & $1.01 \mathrm{E}+00$ \\
\hline $\mathrm{Cm}-244$ & $4.23 \mathrm{E}+00$ & $6.43 \mathrm{E}+00$ & $7.90 \mathrm{E}+00$ & $8.94 \mathrm{E}+00$ & $9.71 \mathrm{E}+00$ & $1.03 \mathrm{E}+01$ & $1.08 \mathrm{E}+01$ & $1.12 \mathrm{E}+01$ & $1.15 \mathrm{E}+01$ \\
\hline Np-237 & $3.58 \mathrm{E}-03$ & $3.59 \mathrm{E}-03$ & $3.60 \mathrm{E}-03$ & $3.60 \mathrm{E}-03$ & $3.61 \mathrm{E}-03$ & $3.61 \mathrm{E}-03$ & $3.61 \mathrm{E}-03$ & $3.61 \mathrm{E}-03$ & $3.61 \mathrm{E}-03$ \\
\hline $\mathrm{Pu}-238$ & $6.42 \mathrm{E}-01$ & $1.02 \mathrm{E}+00$ & $1.28 \mathrm{E}+00$ & $1.46 \mathrm{E}+00$ & $1.59 \mathrm{E}+00$ & $1.70 \mathrm{E}+00$ & $1.78 \mathrm{E}+00$ & $1.85 \mathrm{E}+00$ & $1.90 \mathrm{E}+00$ \\
\hline $\mathrm{Pu}-239$ & $1.91 \mathrm{E}-03$ & $3.00 \mathrm{E}-03$ & $3.73 \mathrm{E}-03$ & $4.24 \mathrm{E}-03$ & $4.63 \mathrm{E}-03$ & $4.93 \mathrm{E}-03$ & $5.16 \mathrm{E}-03$ & $5.36 \mathrm{E}-03$ & $5.52 \mathrm{E}-03$ \\
\hline $\mathrm{Pu}-240$ & $6.90 \mathrm{E}-03$ & $1.09 \mathrm{E}-02$ & $1.36 \mathrm{E}-02$ & $1.55 \mathrm{E}-02$ & $1.69 \mathrm{E}-02$ & $1.80 \mathrm{E}-02$ & $1.89 \mathrm{E}-02$ & $1.96 \mathrm{E}-02$ & $2.02 \mathrm{E}-02$ \\
\hline $\mathrm{Pu}-241$ & $1.23 \mathrm{E}-01$ & $1.90 \mathrm{E}-01$ & $2.35 \mathrm{E}-01$ & $2.67 \mathrm{E}-01$ & $2.91 \mathrm{E}-01$ & 3.09E-01 & $3.24 \mathrm{E}-01$ & $3.36 \mathrm{E}-01$ & $3.46 \mathrm{E}-01$ \\
\hline $\mathrm{Pu}-242$ & $1.78 \mathrm{E}-04$ & $2.36 \mathrm{E}-04$ & $2.74 \mathrm{E}-04$ & $3.01 \mathrm{E}-04$ & $3.21 \mathrm{E}-04$ & $3.37 \mathrm{E}-04$ & $3.49 \mathrm{E}-04$ & $3.59 \mathrm{E}-04$ & $3.68 \mathrm{E}-04$ \\
\hline \multicolumn{10}{|c|}{ SC2 Polyethylene Shielded Container } \\
\hline $\mathrm{Am}-241$ & $4.13 \mathrm{E}-02$ & $6.48 \mathrm{E}-02$ & $8.04 \mathrm{E}-02$ & $9.15 \mathrm{E}-02$ & $9.98 \mathrm{E}-02$ & $1.06 \mathrm{E}-01$ & $1.11 \mathrm{E}-01$ & $1.16 \mathrm{E}-01$ & $1.19 \mathrm{E}-01$ \\
\hline Am-243 & $1.67 \mathrm{E}-01$ & $1.68 \mathrm{E}-01$ & $1.69 \mathrm{E}-01$ & $1.70 \mathrm{E}-01$ & $1.70 \mathrm{E}-01$ & $1.70 \mathrm{E}-01$ & $1.70 \mathrm{E}-01$ & $1.71 \mathrm{E}-01$ & $1.71 \mathrm{E}-01$ \\
\hline $\mathrm{Cm}-244$ & $1.28 \mathrm{E}+00$ & $1.97 \mathrm{E}+00$ & $2.43 \mathrm{E}+00$ & $2.76 \mathrm{E}+00$ & $3.00 \mathrm{E}+00$ & $3.19 \mathrm{E}+00$ & $3.34 \mathrm{E}+00$ & $3.47 \mathrm{E}+00$ & $3.57 \mathrm{E}+00$ \\
\hline Np-237 & $1.02 \mathrm{E}-03$ & $1.02 \mathrm{E}-03$ & $1.02 \mathrm{E}-03$ & $1.03 \mathrm{E}-03$ & $1.03 \mathrm{E}-03$ & $1.03 \mathrm{E}-03$ & $1.03 \mathrm{E}-03$ & $1.03 \mathrm{E}-03$ & $1.03 \mathrm{E}-03$ \\
\hline Pu-238 & $1.98 \mathrm{E}-01$ & $3.16 \mathrm{E}-01$ & $3.95 \mathrm{E}-01$ & $4.50 \mathrm{E}-01$ & $4.92 \mathrm{E}-01$ & $5.24 \mathrm{E}-01$ & $5.50 \mathrm{E}-01$ & $5.71 \mathrm{E}-01$ & $5.89 \mathrm{E}-01$ \\
\hline Pu-239 & $5.80 \mathrm{E}-04$ & $9.14 \mathrm{E}-04$ & $1.13 \mathrm{E}-03$ & $1.29 \mathrm{E}-03$ & $1.41 \mathrm{E}-03$ & $1.50 \mathrm{E}-03$ & $1.57 \mathrm{E}-03$ & $1.63 \mathrm{E}-03$ & $1.68 \mathrm{E}-03$ \\
\hline $\mathrm{Pu}-240$ & $2.10 \mathrm{E}-03$ & $3.33 \mathrm{E}-03$ & $4.14 \mathrm{E}-03$ & $4.72 \mathrm{E}-03$ & $5.15 \mathrm{E}-03$ & 5.49E-03 & $5.75 \mathrm{E}-03$ & $5.97 \mathrm{E}-03$ & $6.15 \mathrm{E}-03$ \\
\hline $\mathrm{Pu}-241$ & $3.70 \mathrm{E}-02$ & $5.79 \mathrm{E}-02$ & $7.17 \mathrm{E}-02$ & $8.16 \mathrm{E}-02$ & $8.89 \mathrm{E}-02$ & $9.46 \mathrm{E}-02$ & $9.92 \mathrm{E}-02$ & $1.03 \mathrm{E}-01$ & $1.06 \mathrm{E}-01$ \\
\hline $\mathrm{Pu}-242$ & $4.64 \mathrm{E}-05$ & $6.40 \mathrm{E}-05$ & $7.55 \mathrm{E}-05$ & $8.37 \mathrm{E}-05$ & $8.98 \mathrm{E}-05$ & $9.45 \mathrm{E}-05$ & $9.82 \mathrm{E}-05$ & $1.01 \mathrm{E}-04$ & $1.04 \mathrm{E}-04$ \\
\hline
\end{tabular}

Note: $90 \%$ Beryllium is modeled as 1 gram of actinide and 0.9 grams of beryllium. 
PCP-2011-0001

Revision 0

Table 3-47. Allowed Mass for Shipment - NCT without Impurities

\begin{tabular}{|c|c|c|c|c|}
\hline \multirow[b]{2}{*}{ Isotope } & \multicolumn{4}{|c|}{ Allowed Mass of Isotope $(\mathrm{g})$} \\
\hline & $\begin{array}{c}\text { No } \\
\text { Shielded } \\
\text { Container }\end{array}$ & $\begin{array}{c}\text { SC1 Lead } \\
\text { Shielded } \\
\text { Container }\end{array}$ & $\begin{array}{c}\text { SC2 Polyethylene } \\
\text { Shielded } \\
\text { Container }\end{array}$ & $\begin{array}{c}\text { SC3 Tungsten } \\
\text { Shielded } \\
\text { Container }\end{array}$ \\
\hline Ac-227 & $4.1 \mathrm{E}-04$ & $5.3 \mathrm{E}-01$ & $1.4 \mathrm{E}-03$ & $2.7 \mathrm{E}+00$ \\
\hline Am-241 & $2.2 \mathrm{E}+01$ & & $1.2 \mathrm{E}+02$ & \\
\hline Am-243 & $3.2 \mathrm{E}-01$ & & $1.2 \mathrm{E}+00$ & \\
\hline Cd-109 & $5.7 \mathrm{E}-03$ & Unlimited $^{*}$ & $2.8 \mathrm{E}-02$ & Unlimited $^{*}$ \\
\hline $\mathrm{Cf}-252$ & $1.9 \mathrm{E}-06$ & & $8.6 \mathrm{E}-06$ & \\
\hline $\mathrm{Cm}-244$ & $3.9 \mathrm{E}-01$ & & $1.8 \mathrm{E}+00$ & \\
\hline $\mathrm{Cm}-248$ & $1.1 \mathrm{E}-01$ & & $5.3 \mathrm{E}-01$ & \\
\hline Co-60 & $3.3 \mathrm{E}-06$ & $1.2 \mathrm{E}-04$ & $9.6 \mathrm{E}-06$ & $4.5 \mathrm{E}-04$ \\
\hline Cs-137 & $1.6 \mathrm{E}-04$ & $1.1 \mathrm{E}-01$ & $5.0 \mathrm{E}-04$ & $6.6 \mathrm{E}-01$ \\
\hline Eu-152 & $4.4 \mathrm{E}-05$ & $2.7 \mathrm{E}-03$ & $1.3 \mathrm{E}-04$ & $1.0 \mathrm{E}-02$ \\
\hline $\mathrm{Fe}-59$ & $1.6 \mathrm{E}-07$ & $6.0 \mathrm{E}-06$ & $4.6 \mathrm{E}-07$ & $2.2 \mathrm{E}-05$ \\
\hline Gd-153 & $1.4 \mathrm{E}-04$ & Unlimited $^{*}$ & $6.4 \mathrm{E}-04$ & Unlimited $^{*}$ \\
\hline Hf-181 & $9.1 \mathrm{E}-07$ & $1.5 \mathrm{E}-02$ & $3.0 \mathrm{E}-06$ & $3.7 \mathrm{E}-02$ \\
\hline Ho-166m & $2.6 \mathrm{E}-03$ & $7.2 \mathrm{E}-01$ & $8.3 \mathrm{E}-03$ & $3.7 \mathrm{E}+00$ \\
\hline Ir-192 & $1.0 \mathrm{E}-06$ & $6.8 \mathrm{E}-03$ & $3.4 \mathrm{E}-06$ & $4.1 \mathrm{E}-02$ \\
\hline Mn-54 & $1.3 \mathrm{E}-06$ & $2.0 \mathrm{E}-04$ & $3.9 \mathrm{E}-06$ & $1.1 \mathrm{E}-03$ \\
\hline $\mathrm{Np}-237$ & $3.2 \mathrm{E}+01$ & & $2.0 \mathrm{E}+02$ & \\
\hline $\mathrm{Pb}-210$ & $2.8 \mathrm{E}-02$ & $3.0 \mathrm{E}+02$ & $1.0 \mathrm{E}-01$ & $1.7 \mathrm{E}+03$ \\
\hline Pm-147 & $2.7 \mathrm{E}-01$ & Unlimited $^{*}$ & $1.2 \mathrm{E}+00$ & Unlimited $^{*}$ \\
\hline Po-210 & $2.2 \mathrm{E}-01$ & $4.0 \mathrm{E}+01$ & $6.5 \mathrm{E}-01$ & $2.1 \mathrm{E}+02$ \\
\hline $\mathrm{Pu}-238$ & $1.1 \mathrm{E}+02$ & & $4.7 \mathrm{E}+02$ & \\
\hline $\mathrm{Pu}-239$ & $3.5 \mathrm{E}+03$ & & $1.2 \mathrm{E}+04$ & \\
\hline $\mathrm{Pu}-240$ & $3.0 \mathrm{E}+03$ & & $1.4 \mathrm{E}+04$ & \\
\hline $\mathrm{Pu}-241$ & $2.3 \mathrm{E}+01$ & & $1.2 \mathrm{E}+02$ & \\
\hline $\mathrm{Pu}-242$ & $2.5 \mathrm{E}+03$ & & $1.2 \mathrm{E}+04$ & \\
\hline Ra-226 & $5.3 \mathrm{E}-03$ & $2.3 \mathrm{E}-01$ & $1.6 \mathrm{E}-02$ & $7.3 \mathrm{E}-01$ \\
\hline Ru-106 & $9.3 \mathrm{E}-06$ & $2.3 \mathrm{E}-03$ & $2.9 \mathrm{E}-05$ & $8.5 \mathrm{E}-03$ \\
\hline $\mathrm{Sc}-46$ & $1.3 \mathrm{E}-07$ & $8.7 \mathrm{E}-06$ & $3.8 \mathrm{E}-07$ & $3.7 \mathrm{E}-05$ \\
\hline Se-75 & $1.5 \mathrm{E}-06$ & $1.6 \mathrm{E}+00$ & $5.6 \mathrm{E}-06$ & $2.5 \mathrm{E}+01$ \\
\hline Sm-145 & $1.4 \mathrm{E}-02$ & $2.3 \mathrm{E}+03$ & $7.6 \mathrm{E}-02$ & $5.5 \mathrm{E}+03$ \\
\hline Sr-90 & $2.9 \mathrm{E}-03$ & $1.4 \mathrm{E}+00$ & $1.0 \mathrm{E}-02$ & $5.8 \mathrm{E}+00$ \\
\hline Tm-170 & $2.7 \mathrm{E}-04$ & $7.3 \mathrm{E}+00$ & $1.0 \mathrm{E}-03$ & $4.4 \mathrm{E}+01$ \\
\hline $\mathrm{Yb}-169$ & $2.1 \mathrm{E}-06$ & $9.8 \mathrm{E}+00$ & $8.1 \mathrm{E}-06$ & $6.3 \mathrm{E}+01$ \\
\hline $\mathrm{Zn}-65$ & $1.9 \mathrm{E}-06$ & $9.5 \mathrm{E}-05$ & $5.5 \mathrm{E}-06$ & $3.9 \mathrm{E}-04$ \\
\hline Zr-95 & $4.6 \mathrm{E}-07$ & $1.3 \mathrm{E}-04$ & $1.4 \mathrm{E}-06$ & $7.7 \mathrm{E}-04$ \\
\hline
\end{tabular}

* Unlimited denotes that the allowed mass of the isotope is restricted only by the capacity of the container. 
PCP-2011-0001

Revision 0

Table 3-48. Allowed Mass for Shipment - Exclusive Use without Impurities

\begin{tabular}{|c|c|c|c|c|}
\hline \multirow[b]{2}{*}{ Isotope } & \multicolumn{4}{|c|}{ Allowed Mass of Isotope $(\mathrm{g})$} \\
\hline & $\begin{array}{c}\text { No } \\
\text { Shielded } \\
\text { Container }\end{array}$ & $\begin{array}{c}\text { SC1 Lead } \\
\text { Shielded } \\
\text { Container }\end{array}$ & $\begin{array}{c}\text { SC2 Polyethylene } \\
\text { Shielded } \\
\text { Container }\end{array}$ & $\begin{array}{c}\text { SC3 Tungsten } \\
\text { Shielded } \\
\text { Container }\end{array}$ \\
\hline Ac- 227 & $2.1 \mathrm{E}-03$ & $2.6 \mathrm{E}+00$ & $7.1 \mathrm{E}-03$ & $1.3 \mathrm{E}+01$ \\
\hline Am-241 & $1.1 \mathrm{E}+02$ & & $5.8 \mathrm{E}+02$ & \\
\hline Am-243 & $1.6 \mathrm{E}+00$ & & $6.1 \mathrm{E}+00$ & \\
\hline Cd-109 & $2.8 \mathrm{E}-02$ & Unlimited $^{*}$ & $1.4 \mathrm{E}-01$ & Unlimited $^{*}$ \\
\hline Cf-252 & $9.5 \mathrm{E}-06$ & & $4.3 \mathrm{E}-05$ & \\
\hline $\mathrm{Cm}-244$ & $2.0 \mathrm{E}+00$ & & $9.1 \mathrm{E}+00$ & \\
\hline $\mathrm{Cm}-248$ & $5.5 \mathrm{E}-01$ & & $2.6 \mathrm{E}+00$ & \\
\hline Co-60 & $1.6 \mathrm{E}-05$ & $6.1 \mathrm{E}-04$ & $4.8 \mathrm{E}-05$ & $2.3 \mathrm{E}-03$ \\
\hline Cs-137 & $8.1 \mathrm{E}-04$ & $5.4 \mathrm{E}-01$ & $2.5 \mathrm{E}-03$ & $3.3 \mathrm{E}+00$ \\
\hline Eu-152 & $2.2 \mathrm{E}-04$ & $1.3 \mathrm{E}-02$ & $6.7 \mathrm{E}-04$ & $5.1 \mathrm{E}-02$ \\
\hline Fe-59 & $7.8 \mathrm{E}-07$ & $3.0 \mathrm{E}-05$ & $2.3 \mathrm{E}-06$ & $1.1 \mathrm{E}-04$ \\
\hline Gd-153 & $6.9 \mathrm{E}-04$ & Unlimited $^{*}$ & $3.2 \mathrm{E}-03$ & Unlimited $^{*}$ \\
\hline Hf-181 & $4.5 \mathrm{E}-06$ & $7.5 \mathrm{E}-02$ & $1.5 \mathrm{E}-05$ & $1.8 \mathrm{E}-01$ \\
\hline Ho-166m & $1.3 \mathrm{E}-02$ & $3.6 \mathrm{E}+00$ & $4.2 \mathrm{E}-02$ & $1.8 \mathrm{E}+01$ \\
\hline Ir-192 & $5.0 \mathrm{E}-06$ & $3.4 \mathrm{E}-02$ & $1.7 \mathrm{E}-05$ & $2.1 \mathrm{E}-01$ \\
\hline $\mathrm{Mn}-54$ & $6.4 \mathrm{E}-06$ & $1.0 \mathrm{E}-03$ & $1.9 \mathrm{E}-05$ & $5.3 \mathrm{E}-03$ \\
\hline $\mathrm{Np}-237$ & $1.6 \mathrm{E}+02$ & & $9.9 \mathrm{E}+02$ & \\
\hline $\mathrm{Pb}-210$ & $1.4 \mathrm{E}-01$ & $1.5 \mathrm{E}+03$ & $5.2 \mathrm{E}-01$ & $8.6 \mathrm{E}+03$ \\
\hline Pm-147 & $1.3 \mathrm{E}+00$ & Unlimited $^{*}$ & $6.0 \mathrm{E}+00$ & Unlimited $^{*}$ \\
\hline Po-210 & $1.1 \mathrm{E}+00$ & $2.0 \mathrm{E}+02$ & $3.3 \mathrm{E}+00$ & $1.1 \mathrm{E}+03$ \\
\hline $\mathrm{Pu}-238$ & $5.6 \mathrm{E}+02$ & & $2.4 \mathrm{E}+03$ & \\
\hline $\mathrm{Pu}-239$ & $1.7 \mathrm{E}+04$ & & $6.2 \mathrm{E}+04$ & \\
\hline $\mathrm{Pu}-240$ & $1.5 \mathrm{E}+04$ & & $6.9 \mathrm{E}+04$ & \\
\hline $\mathrm{Pu}-241$ & $1.2 \mathrm{E}+02$ & & $6.0 \mathrm{E}+02$ & \\
\hline $\mathrm{Pu}-242$ & $1.3 \mathrm{E}+04$ & & $6.0 \mathrm{E}+04$ & \\
\hline Ra-226 & $2.6 \mathrm{E}-02$ & $1.2 \mathrm{E}+00$ & $8.0 \mathrm{E}-02$ & $3.7 \mathrm{E}+00$ \\
\hline Ru-106 & $4.7 \mathrm{E}-05$ & $1.2 \mathrm{E}-02$ & $1.5 \mathrm{E}-04$ & $4.3 \mathrm{E}-02$ \\
\hline Sc-46 & $6.4 \mathrm{E}-07$ & $4.3 \mathrm{E}-05$ & $1.9 \mathrm{E}-06$ & $1.8 \mathrm{E}-04$ \\
\hline $\mathrm{Se}-75$ & $7.7 \mathrm{E}-06$ & $8.0 \mathrm{E}+00$ & $2.8 \mathrm{E}-05$ & $1.2 \mathrm{E}+02$ \\
\hline Sm-145 & $7.2 \mathrm{E}-02$ & $1.2 \mathrm{E}+04$ & $3.8 \mathrm{E}-01$ & $2.8 \mathrm{E}+04$ \\
\hline Sr-90 & $1.4 \mathrm{E}-02$ & $7.1 \mathrm{E}+00$ & $5.0 \mathrm{E}-02$ & $2.9 \mathrm{E}+01$ \\
\hline Tm-170 & $1.3 \mathrm{E}-03$ & $3.7 \mathrm{E}+01$ & $5.1 \mathrm{E}-03$ & $2.2 \mathrm{E}+02$ \\
\hline Yb-169 & $1.1 \mathrm{E}-05$ & $4.9 \mathrm{E}+01$ & $4.1 \mathrm{E}-05$ & $3.2 \mathrm{E}+02$ \\
\hline $\mathrm{Zn}-65$ & $9.4 \mathrm{E}-06$ & $4.8 \mathrm{E}-04$ & $2.8 \mathrm{E}-05$ & $1.9 \mathrm{E}-03$ \\
\hline $\mathrm{Zr}-95$ & $2.3 \mathrm{E}-06$ & $6.7 \mathrm{E}-04$ & $7.0 \mathrm{E}-06$ & $3.9 \mathrm{E}-03$ \\
\hline
\end{tabular}

* Unlimited denotes that the allowed mass of the isotope is restricted only by the capacity of the container. 
PCP-2011-0001

Revision 0

Table 3-49. Allowed Actinide Mass for Shipment - NCT with Impurities

\begin{tabular}{|c|c|c|c|c|c|c|c|c|c|}
\hline \multirow{2}{*}{ Isotope } & \multicolumn{9}{|c|}{ Allowed Mass of Isotope $(\mathrm{g})$} \\
\hline & $10 \% \mathrm{Be}$ & $20 \% \mathrm{Be}$ & $30 \% \mathrm{Be}$ & $40 \% \mathrm{Be}$ & $50 \% \mathrm{Be}$ & $60 \% \mathrm{Be}$ & $70 \% \mathrm{Be}$ & $80 \% \mathrm{Be}$ & $90 \% \mathrm{Be}$ \\
\hline & \multicolumn{9}{|c|}{ No Shielded Container } \\
\hline Am-241 & $1.5 \mathrm{E}+00$ & $9.4 \mathrm{E}-01$ & $7.6 \mathrm{E}-01$ & $6.7 \mathrm{E}-01$ & $6.1 \mathrm{E}-01$ & $5.8 \mathrm{E}-01$ & $5.5 \mathrm{E}-01$ & $5.3 \mathrm{E}-01$ & $5.1 \mathrm{E}-01$ \\
\hline Am-243 & $2.6 \mathrm{E}-01$ & $2.4 \mathrm{E}-01$ & $2.3 \mathrm{E}-01$ & $2.2 \mathrm{E}-01$ & $2.1 \mathrm{E}-01$ & $2.1 \mathrm{E}-01$ & $2.0 \mathrm{E}-01$ & $2.0 \mathrm{E}-01$ & $2.0 \mathrm{E}-01$ \\
\hline Cm-244 & $4.7 \mathrm{E}-02$ & $3.1 \mathrm{E}-02$ & $2.5 \mathrm{E}-02$ & $2.2 \mathrm{E}-02$ & $2.1 \mathrm{E}-02$ & $1.9 \mathrm{E}-02$ & $1.9 \mathrm{E}-02$ & $1.8 \mathrm{E}-02$ & $1.7 \mathrm{E}-02$ \\
\hline $\mathrm{Np}-237$ & $5.6 \mathrm{E}+01$ & $5.6 \mathrm{E}+01$ & $5.6 \mathrm{E}+01$ & $5.6 \mathrm{E}+01$ & $5.5 \mathrm{E}+01$ & $5.5 \mathrm{E}+01$ & $5.5 \mathrm{E}+01$ & $5.5 \mathrm{E}+01$ & $5.5 \mathrm{E}+01$ \\
\hline $\mathrm{Pu}-238$ & $3.1 \mathrm{E}-01$ & $2.0 \mathrm{E}-01$ & $1.6 \mathrm{E}-01$ & $1.4 \mathrm{E}-01$ & $1.3 \mathrm{E}-01$ & $1.2 \mathrm{E}-01$ & $1.1 \mathrm{E}-01$ & $1.1 \mathrm{E}-01$ & $1.0 \mathrm{E}-01$ \\
\hline $\mathrm{Pu}-239$ & $1.0 \mathrm{E}+02$ & $6.7 \mathrm{E}+01$ & $5.4 \mathrm{E}+01$ & $4.7 \mathrm{E}+01$ & $4.3 \mathrm{E}+01$ & $4.1 \mathrm{E}+01$ & $3.9 \mathrm{E}+01$ & $3.7 \mathrm{E}+01$ & $3.6 \mathrm{E}+01$ \\
\hline $\mathrm{Pu}-240$ & $2.9 \mathrm{E}+01$ & $1.8 \mathrm{E}+01$ & $1.5 \mathrm{E}+01$ & $1.3 \mathrm{E}+01$ & $1.2 \mathrm{E}+01$ & $1.1 \mathrm{E}+01$ & $1.1 \mathrm{E}+01$ & $1.0 \mathrm{E}+01$ & $9.9 \mathrm{E}+00$ \\
\hline Pu-241 & $1.6 \mathrm{E}+00$ & $1.1 \mathrm{E}+00$ & $8.5 \mathrm{E}-01$ & $7.5 \mathrm{E}-01$ & $6.9 \mathrm{E}-01$ & $6.5 \mathrm{E}-01$ & $6.2 \mathrm{E}-01$ & $6.0 \mathrm{E}-01$ & $5.8 \mathrm{E}-01$ \\
\hline \multirow[t]{2}{*}{$\mathrm{Pu}-242$} & $1.1 \mathrm{E}+03$ & $8.5 \mathrm{E}+02$ & $7.3 \mathrm{E}+02$ & $6.6 \mathrm{E}+02$ & $6.2 \mathrm{E}+02$ & $5.9 \mathrm{E}+02$ & $5.7 \mathrm{E}+02$ & $5.6 \mathrm{E}+02$ & $5.4 \mathrm{E}+02$ \\
\hline & \multicolumn{9}{|c|}{ SC2 Polyethylene Shielded Container } \\
\hline Am-241 & $4.8 \mathrm{E}+00$ & $3.1 \mathrm{E}+00$ & $2.5 \mathrm{E}+00$ & $2.2 \mathrm{E}+00$ & $2.0 \mathrm{E}+00$ & $1.9 \mathrm{E}+00$ & $1.8 \mathrm{E}+00$ & $1.7 \mathrm{E}+00$ & $1.7 \mathrm{E}+00$ \\
\hline Am-243 & $1.2 \mathrm{E}+00$ & $1.2 \mathrm{E}+00$ & $1.2 \mathrm{E}+00$ & $1.2 \mathrm{E}+00$ & $1.2 \mathrm{E}+00$ & $1.2 \mathrm{E}+00$ & $1.2 \mathrm{E}+00$ & $1.2 \mathrm{E}+00$ & $1.2 \mathrm{E}+00$ \\
\hline $\mathrm{Cm}-244$ & $1.6 \mathrm{E}-01$ & $1.0 \mathrm{E}-01$ & $8.2 \mathrm{E}-02$ & $7.3 \mathrm{E}-02$ & $6.7 \mathrm{E}-02$ & $6.3 \mathrm{E}-02$ & $6.0 \mathrm{E}-02$ & $5.8 \mathrm{E}-02$ & $5.6 \mathrm{E}-02$ \\
\hline Np-237 & $2.0 \mathrm{E}+02$ & $2.0 \mathrm{E}+02$ & $2.0 \mathrm{E}+02$ & $2.0 \mathrm{E}+02$ & $1.9 \mathrm{E}+02$ & $1.9 \mathrm{E}+02$ & $1.9 \mathrm{E}+02$ & $1.9 \mathrm{E}+02$ & $1.9 \mathrm{E}+02$ \\
\hline $\mathrm{Pu}-238$ & $1.0 \mathrm{E}+00$ & $6.3 \mathrm{E}-01$ & $5.1 \mathrm{E}-01$ & $4.4 \mathrm{E}-01$ & $4.1 \mathrm{E}-01$ & $3.8 \mathrm{E}-01$ & $3.6 \mathrm{E}-01$ & $3.5 \mathrm{E}-01$ & $3.4 \mathrm{E}-01$ \\
\hline Pu-239 & $3.4 \mathrm{E}+02$ & $2.2 \mathrm{E}+02$ & $1.8 \mathrm{E}+02$ & $1.5 \mathrm{E}+02$ & $1.4 \mathrm{E}+02$ & $1.3 \mathrm{E}+02$ & $1.3 \mathrm{E}+02$ & $1.2 \mathrm{E}+02$ & $1.2 \mathrm{E}+02$ \\
\hline $\mathrm{Pu}-240$ & $9.5 \mathrm{E}+01$ & $6.0 \mathrm{E}+01$ & $4.8 \mathrm{E}+01$ & $4.2 \mathrm{E}+01$ & $3.9 \mathrm{E}+01$ & $3.6 \mathrm{E}+01$ & $3.5 \mathrm{E}+01$ & $3.3 \mathrm{E}+01$ & $3.2 \mathrm{E}+01$ \\
\hline $\mathrm{Pu}-241$ & $5.4 \mathrm{E}+00$ & $3.5 \mathrm{E}+00$ & $2.8 \mathrm{E}+00$ & $2.5 \mathrm{E}+00$ & $2.2 \mathrm{E}+00$ & $2.1 \mathrm{E}+00$ & $2.0 \mathrm{E}+00$ & $1.9 \mathrm{E}+00$ & $1.9 \mathrm{E}+00$ \\
\hline $\mathrm{Pu}-242$ & $4.3 \mathrm{E}+03$ & $3.1 \mathrm{E}+03$ & $2.6 \mathrm{E}+03$ & $2.4 \mathrm{E}+03$ & $2.2 \mathrm{E}+03$ & $2.1 \mathrm{E}+03$ & $2.0 \mathrm{E}+03$ & $2.0 \mathrm{E}+03$ & $1.9 \mathrm{E}+03$ \\
\hline
\end{tabular}

Note: $90 \%$ Beryllium is modeled as 1 gram of actinide and 0.9 grams of beryllium.

Table 3-50. Allowed Actinide Mass for Shipment - Exclusive Use with Impurities

\begin{tabular}{|c|c|c|c|c|c|c|c|c|c|}
\hline \multirow{2}{*}{ Isotope } & \multicolumn{9}{|c|}{ Allowed Mass of Isotope $(\mathrm{g})$} \\
\hline & $10 \% \mathrm{Be}$ & $20 \% \mathrm{Be}$ & $30 \% \mathrm{Be}$ & $40 \% \mathrm{Be}$ & $50 \% \mathrm{Be}$ & $60 \% \mathrm{Be}$ & $70 \% \mathrm{Be}$ & $80 \% \mathrm{Be}$ & $90 \% \mathrm{Be}$ \\
\hline & \multicolumn{9}{|c|}{ No Shielded Container } \\
\hline Am-241 & $7.3 \mathrm{E}+00$ & $4.7 \mathrm{E}+00$ & $3.8 \mathrm{E}+00$ & $3.3 \mathrm{E}+00$ & $3.1 \mathrm{E}+00$ & $2.9 \mathrm{E}+00$ & $2.7 \mathrm{E}+00$ & $2.7 \mathrm{E}+00$ & $2.6 \mathrm{E}+00$ \\
\hline Am-243 & $1.3 \mathrm{E}+00$ & $1.2 \mathrm{E}+00$ & $1.1 \mathrm{E}+00$ & $1.1 \mathrm{E}+00$ & $1.1 \mathrm{E}+00$ & $1.0 \mathrm{E}+00$ & $1.0 \mathrm{E}+00$ & $1.0 \mathrm{E}+00$ & $9.9 \mathrm{E}-01$ \\
\hline $\mathrm{Cm}-244$ & $2.4 \mathrm{E}-01$ & $1.6 \mathrm{E}-01$ & $1.3 \mathrm{E}-01$ & $1.1 \mathrm{E}-01$ & $1.0 \mathrm{E}-01$ & $9.7 \mathrm{E}-02$ & $9.3 \mathrm{E}-02$ & $8.9 \mathrm{E}-02$ & $8.7 \mathrm{E}-02$ \\
\hline $\mathrm{Np}-237$ & $2.8 \mathrm{E}+02$ & $2.8 \mathrm{E}+02$ & $2.8 \mathrm{E}+02$ & $2.8 \mathrm{E}+02$ & $2.8 \mathrm{E}+02$ & $2.8 \mathrm{E}+02$ & $2.8 \mathrm{E}+02$ & $2.8 \mathrm{E}+02$ & $2.8 \mathrm{E}+02$ \\
\hline $\mathrm{Pu}-238$ & $1.6 \mathrm{E}+00$ & $9.8 \mathrm{E}-01$ & $7.8 \mathrm{E}-01$ & $6.9 \mathrm{E}-01$ & $6.3 \mathrm{E}-01$ & $5.9 \mathrm{E}-01$ & $5.6 \mathrm{E}-01$ & $5.4 \mathrm{E}-01$ & $5.2 \mathrm{E}-01$ \\
\hline Pu-239 & $5.2 \mathrm{E}+02$ & $3.3 \mathrm{E}+02$ & $2.7 \mathrm{E}+02$ & $2.4 \mathrm{E}+02$ & $2.2 \mathrm{E}+02$ & $2.0 \mathrm{E}+02$ & $1.9 \mathrm{E}+02$ & $1.9 \mathrm{E}+02$ & $1.8 \mathrm{E}+02$ \\
\hline $\mathrm{Pu}-240$ & $1.5 \mathrm{E}+02$ & $9.1 \mathrm{E}+01$ & $7.4 \mathrm{E}+01$ & $6.5 \mathrm{E}+01$ & $5.9 \mathrm{E}+01$ & $5.6 \mathrm{E}+01$ & $5.3 \mathrm{E}+01$ & $5.1 \mathrm{E}+01$ & $5.0 \mathrm{E}+01$ \\
\hline $\mathrm{Pu}-241$ & $8.1 \mathrm{E}+00$ & $5.3 \mathrm{E}+00$ & $4.3 \mathrm{E}+00$ & $3.7 \mathrm{E}+00$ & $3.4 \mathrm{E}+00$ & $3.2 \mathrm{E}+00$ & $3.1 \mathrm{E}+00$ & $3.0 \mathrm{E}+00$ & $2.9 \mathrm{E}+00$ \\
\hline \multirow[t]{2}{*}{$\mathrm{Pu}-242$} & $5.6 \mathrm{E}+03$ & $4.2 \mathrm{E}+03$ & $3.6 \mathrm{E}+03$ & $3.3 \mathrm{E}+03$ & $3.1 \mathrm{E}+03$ & $3.0 \mathrm{E}+03$ & $2.9 \mathrm{E}+03$ & $2.8 \mathrm{E}+03$ & $2.7 \mathrm{E}+03$ \\
\hline & \multicolumn{9}{|c|}{ SC2 Polyethylene Shielded Container } \\
\hline$\overline{\mathrm{Am}-241}$ & $2.4 \mathrm{E}+01$ & $1.5 \mathrm{E}+01$ & $1.2 \mathrm{E}+01$ & $1.1 \mathrm{E}+01$ & $1.0 \mathrm{E}+01$ & $9.4 \mathrm{E}+00$ & $9.0 \mathrm{E}+00$ & $8.7 \mathrm{E}+00$ & $8.4 \mathrm{E}+00$ \\
\hline Am-243 & $6.0 \mathrm{E}+00$ & $5.9 \mathrm{E}+00$ & $5.9 \mathrm{E}+00$ & $5.9 \mathrm{E}+00$ & $5.9 \mathrm{E}+00$ & $5.9 \mathrm{E}+00$ & $5.9 \mathrm{E}+00$ & $5.9 \mathrm{E}+00$ & $5.9 \mathrm{E}+00$ \\
\hline $\mathrm{Cm}-244$ & $7.8 \mathrm{E}-01$ & $5.1 \mathrm{E}-01$ & $4.1 \mathrm{E}-01$ & $3.6 \mathrm{E}-01$ & $3.3 \mathrm{E}-01$ & $3.1 \mathrm{E}-01$ & $3.0 \mathrm{E}-01$ & $2.9 \mathrm{E}-01$ & $2.8 \mathrm{E}-01$ \\
\hline $\mathrm{Np}-237$ & $9.8 \mathrm{E}+02$ & $9.8 \mathrm{E}+02$ & $9.8 \mathrm{E}+02$ & $9.8 \mathrm{E}+02$ & $9.7 \mathrm{E}+02$ & $9.7 \mathrm{E}+02$ & $9.7 \mathrm{E}+02$ & $9.7 \mathrm{E}+02$ & $9.7 \mathrm{E}+02$ \\
\hline $\mathrm{Pu}-238$ & $5.0 \mathrm{E}+00$ & $3.2 \mathrm{E}+00$ & $2.5 \mathrm{E}+00$ & $2.2 \mathrm{E}+00$ & $2.0 \mathrm{E}+00$ & $1.9 \mathrm{E}+00$ & $1.8 \mathrm{E}+00$ & $1.7 \mathrm{E}+00$ & $1.7 \mathrm{E}+00$ \\
\hline Pu-239 & $1.7 \mathrm{E}+03$ & $1.1 \mathrm{E}+03$ & $8.8 \mathrm{E}+02$ & $7.7 \mathrm{E}+02$ & $7.1 \mathrm{E}+02$ & $6.7 \mathrm{E}+02$ & $6.4 \mathrm{E}+02$ & $6.1 \mathrm{E}+02$ & $5.9 \mathrm{E}+02$ \\
\hline $\mathrm{Pu}-240$ & $4.8 \mathrm{E}+02$ & $3.0 \mathrm{E}+02$ & $2.4 \mathrm{E}+02$ & $2.1 \mathrm{E}+02$ & $1.9 \mathrm{E}+02$ & $1.8 \mathrm{E}+02$ & $1.7 \mathrm{E}+02$ & $1.7 \mathrm{E}+02$ & $1.6 \mathrm{E}+02$ \\
\hline $\mathrm{Pu}-241$ & $2.7 \mathrm{E}+01$ & $1.7 \mathrm{E}+01$ & $1.4 \mathrm{E}+01$ & $1.2 \mathrm{E}+01$ & $1.1 \mathrm{E}+01$ & $1.1 \mathrm{E}+01$ & $1.0 \mathrm{E}+01$ & $9.7 \mathrm{E}+00$ & $9.4 \mathrm{E}+00$ \\
\hline $\mathrm{Pu}-242$ & $2.2 \mathrm{E}+04$ & $1.6 \mathrm{E}+04$ & $1.3 \mathrm{E}+04$ & $1.2 \mathrm{E}+04$ & $1.1 \mathrm{E}+04$ & $1.1 \mathrm{E}+04$ & $1.0 \mathrm{E}+04$ & $9.9 \mathrm{E}+03$ & $9.6 \mathrm{E}+03$ \\
\hline
\end{tabular}

Note: $90 \%$ Beryllium is modeled as 1 gram of actinide and 0.9 grams of beryllium. 
PCP-2011-0001

Revision 0

\subsection{Conclusions}

Bounding shielding calculations for a set of proposed SGQ isotopes were performed using the MCNP transport code to develop a set of response multipliers for "dose per particle" for each neutron and photon spectral group. The source spectrum for each isotope generated using the ORIGEN-S and RASTA computer codes was folded with the response multipliers to generate the dose rate per gram of each isotope in the 9977 shipping package and its associated shielded containers.

Table 3-47 through Table 3-50 present the maximum amount of a single isotope that could be shipped in the Model 9977 Package (or packagings having the same or larger external dimensions as well as similar structural materials) and have the external radiation level within the regulatory dose limits at the surface of the package. The estimates of the mass limits presented would also serve as conservative limits for both the Models 9975 and 9978 packages. If a package contains a mixture of isotopes, the acceptability for shipment can be determined by a sum of fractions approach.

For the cases of actinides mixed with light elements, beryllium represents the bounding light element. The added beryllium (10 to 90 percent of the actinide mass) in the cases studied represents between 9 and 47 percent concentration of the total mixture mass. For beryllium concentrations larger than 50 percent, the increase in the neutron source term and dose rate tend to increase at a much lower rate than at concentrations lower than 50\%. Previous studies [LLNLTR-461255] have indicated that the limiting mass drops by approximately $55 \%$ for an increase in concentration from 10 to 30 percent, while the decrease is only about 11 percent when the concentration changes from 70 to 90 percent. The intimately mixed actinide-beryllium form used in these models is very conservative and thus the limits presented in this report are practical bounds on the mass that can be safely shipped.

Some of the quantities that were derived may not really qualify to be termed as "small gram." However, several of these instances are when there is a shielded container employed within the Model 9977 packaging and consequently only the amount that would fit into the cavity of the shielded container would be shippable. Consequently, it may be fair to term these smaller amounts as the real small gram quantity limit for the Model 9977 Package (or packagings having the same or larger external dimensions) from a standpoint of the external radiation levels allowed under 10CFR Part 71 regulations.

It should be noted that the SGQ masses presented in this report represent limits that would comply with the external radiation limits under 10CFR Part 71. They do not necessarily bound lower limits that may be required to comply with other factors such as heat load of the package.

\subsection{References}

49 CFR 173.403, Title 49 - Transportation. Part 173 - Shippers General Requirements For Shipments And Packagings. Section 403 Definitions, US GPO, Washington, DC, October 2009.

AEA Technology QSA, Sources, Catalog available on the internet at http://www.public.asu.edu/ gbadams/spr06/334/XraySources.pdf and http://www.hightechsource.co.uk/generalapplications.html.

ANS-1981, Radioactive Sources, Their Manufacture and Characteristics, Janzow, E. F., Presented at the ANS Nuclear Technology Exhibit, Beijing, China, October 1981.

ANSI/ANS-6.1.1-1977, Neutron and Gamma-Ray Flux-to-Dose Rate Factors, American Nuclear Society, Le Grange Park, IL, March 1977. 
PCP-2011-0001

Revision 0

DLC-75, BUGLE-80, Coupled 47 Neutron, 20 Gamma, P3 Cross-Section Library for LWR Shielding Calculations, ORNL Radiation Shielding Information Center, 1980.

HW-82784, BREMRAD, A Computer Code for External and Internal Bremsstrahlung Calculations, Van Tuyl, H. H., Battelle-Northwest Laboratories, Richland, WA, September 1964.

LA 12827 M, Criticality Calculations with MCNP: A Primer, Harmon II, C. D., Busch, R. D., Briesmeister, J. F., and Forster, R.A., Los Alamos National Laboratory, Los Alamos, NM, August 1994.

LA-13639, SOURCES AA: A Code for Calculating! .n) Spontaneous Fission, and Delayed Neutron Sources and Spectra, Wilson, W. B., et al, Los Alamos National Laboratory, Los Alamos, NM, September 1999.

LA-UR-03-1987, MCNP - A General Monte Carlo N-Particle Transport Code, Version 5, X-5 Monte Carlo Team, Los Alamos National Laboratory, Los Alamos, NM, April 24, 2003.

LLNL-TR-461255, Definition of "Small Gram Quantity Contents" for Type B Radioactive Material Transportation Packages: Activity-Based Content Limits, Sitaraman, S., et al., Lawrence Livermore National Laboratory, Livermore, CA, October 2010.

Midwest Tungsten Service, Tungsten Alloy.

ORNL/TM-2005/39, Version 5, Volume II, Section F7, ORIGEN-S: Scale System Module to Calculate Fuel Depletion, Actinide Transmutation, Fission Product Buildup and Decay, and Associated Radiation Source Terms, Hermann, O. W. and Westfall, R. M., Oak Ridge National Laboratory, Oak Ridge, TN, April 2005.

ORNL/TM-2005/39, Version 5, Volumes I-III SCALE: A Modular Code System for Performing Standardized Computer Analyses for Licensing Evaluation, Hermann, O. W. and Westfall, R. M., Oak Ridge National Laboratory, Oak Ridge, TN, April 2005.

R-R1-G-00037, Revision 0, 9977 Small Gram Quantity Shielded Container, Type 1, Savannah River National Laboratory, Aiken, SC, November 2009.

R-R1-G-00038, Revision 0, Small Gram Quantity Shielded Container, Type 2, Savannah River National Laboratory, Aiken, SC, November 2009.

R-R1-G-00039, Revision 0, Small Gram Quantity Shielded Container, Type 3, Savannah River National Laboratory, Aiken, SC, November 2009.

SRNS-RP-2008-00117, Revision 0, Software Configuration And Control Guidance For MCNP On SRS Personal Computers (U), Bridges, A. H. and Joshi, J. N., Savannah River Nuclear Solutions, Aiken, SC, November 2008.

SRNS-RP-2008-00150, Revision 0, Software Configuration And Control Guidance For SCALE On SRS Personal Computers (U), Bridges, A. H. and Joshi, J. N., Savannah River Nuclear Solutions, Aiken, SC, November 2008.

SRNS-RP-2008-00151, Revision 0, SCALE Test Report for SRS Personal Computers (U), Bridges, A. H. and Joshi, J. N., Savannah River Nuclear Solutions, Aiken, SC, November 2008.

SRNS-RP-2009-00275, Revision 0, Radiation Source Term Analysis Code RASTA User Guide (U), Nathan, S. J., Savannah River Nuclear Solutions, Aiken, SC, March 2009. 
PCP-2011-0001

Revision 0

SRNS-RP-2009-00276, Revision 0, Software Configuration And Control Guidance For RASTA On SRS Personal Computers (U), Nathan, S. J., Savannah River Nuclear Solutions, Aiken, SC, March 2009.

SRNS-RP-2009-00277, Revision 0, RASTA Validation Report for SRNS Personal Computers (U), Nathan, S. J., Savannah River Nuclear Solutions, Aiken, SC, March 2009.

SRNS-RP-2009-00285, Revision 0, Monte Carlo N-Particle Transport Code System MCNP5 Shielding Validation Report (U), Nathan, S. J., Savannah River Nuclear Solutions, Aiken, SC, April 2009.

WSMS CRT 02 0060, Revision 1, Radiological Engineering Shielding Material Composition, Barnett, M. H., Westinghouse Safety Management Solutions, Aiken, SC, September 2003. 
PCP-2011-0001

Revision 0

\section{Appendix A Radiation Source Input Files}

ORIGEN-S input files were divided into three categories: light element (origenle.nnn.in), actinides without beryllium (origenac.nnn.in), and actinides with beryllium (origen_be.nnn.yy.in). These files are described in Table A-1.

RASTA input files were divided into two sets to avoid problems with the maximum number of groups allowed in RASTA. Cases were run without beryllium (rastax.nnn.in) and with beryllium (rasta_bex.nnn.yy.in) where $x$ is either $1(10 \mathrm{MeV} \geq \mathrm{E} \geq 0.45 \mathrm{MeV})$ or $2(0.45 \mathrm{MeV} \geq \mathrm{E} \geq$ $0.01 \mathrm{MeV})$. These files are described in Table A-2.

Sample listings are provided below for files marked with an asterisk $(*)$. 
PCP-2011-0001

Revision 0

Table A-1 ORIGEN-S Input Files

\begin{tabular}{|c|c|}
\hline File Name & Description \\
\hline origenle.101.in & Cd-109 \\
\hline origenle.102.in* & Co-60 \\
\hline origenle.103.in & Cs-137 \\
\hline origenle.104.in & Eu-152 \\
\hline origenle.105.in & Fe-59 \\
\hline origenle.106.in & Gd-153 \\
\hline origenle.107.in & Hf-181 \\
\hline origenle.108.in & Ho- $166 \mathrm{~m}$ \\
\hline origenle.109.in & Ir-192 \\
\hline origenle.110.in & $\mathrm{Mn}-54$ \\
\hline origenle.112.in & Pm-147 \\
\hline origenle.113.in & $\mathrm{Ru}-106$ \\
\hline origenle.114.in & Sc-46 \\
\hline origenle.115.in & Se-75 \\
\hline origenle.116.in & Sm-145 \\
\hline origenle.117.in & Sr-90 \\
\hline origenle.118.in & Tm-170 \\
\hline origenle.119.in & Yb-169 \\
\hline origenle.120.in & $\mathrm{Zn}-65$ \\
\hline origenle.121.in & Zr-95 \\
\hline origenle.122.in & $\mathrm{Pd}-103$ \\
\hline origenac.101.in & Ac- 227 \\
\hline origenac.102.in* & Am-241 \\
\hline origenac.103.in & Am-243 \\
\hline origenac.104.in & Cf-252 \\
\hline origenac.105.in & $\mathrm{Cm}-244$ \\
\hline origenac.106.in & $\mathrm{Cm}-248$ \\
\hline origenac.107.in & $\mathrm{Np}-237$ \\
\hline origenac.108.in & $\mathrm{Pb}-210$ \\
\hline origenac.109.in & Po-210 \\
\hline origenac.110.in & $\mathrm{Pu}-238$ \\
\hline origenac.111.in & $\mathrm{Pu}-239$ \\
\hline origenac.112.in & $\mathrm{Pu}-240$ \\
\hline origenac.113.in & $\mathrm{Pu}-241$ \\
\hline origenac.114.in & $\mathrm{Pu}-242$ \\
\hline origenac.115.in & Ra-226 \\
\hline origen_be.137.yy.in & Am-241 with yy percent added beryllium \\
\hline origen_be.138.yy.in & Am-243 with yy percent added beryllium \\
\hline origen_be.139.yy.in & Cm-244 with yy percent added beryllium \\
\hline origen_be.140.yy.in & Np-237 with yy percent added beryllium \\
\hline origen be.141.yy.in* & Pu-238 with yy percent added beryllium \\
\hline origen_be.142.yy.in & Pu-239 with yy percent added beryllium \\
\hline origen_be.143.yy.in & Pu-240 with yy percent added beryllium \\
\hline origen_be.144.yy.in & Pu-241 with yy percent added beryllium \\
\hline origen_be.145.yy.in & Pu-242 with yy percent added beryllium \\
\hline
\end{tabular}

Note: yy $\%$ Beryllium is modeled as 1 gram of actinide and 0.yy grams of beryllium. 
PCP-2011-0001

Revision 0

Table A-2. RASTA Input Files

\begin{tabular}{|c|c|}
\hline File Name & Description \\
\hline rastax.101.in & Ac-227 \\
\hline rastax.102.in* & Am-241 \\
\hline rastax.103.in & Am-243 \\
\hline rastax.104.in & Cd-109 \\
\hline rastax.105.in & Cf-252 \\
\hline rastax.106.in & $\mathrm{Cm}-244$ \\
\hline rastax.107.in & $\mathrm{Cm}-248$ \\
\hline rastax.108.in* & Co-60 \\
\hline rastax.109.in & Cs-137 \\
\hline rastax.110.in & Eu-152 \\
\hline rastax.111.in & Fe-59 \\
\hline rastax.112.in & Gd-153 \\
\hline rastax.113.in & Hf-181 \\
\hline rastax.114.in & Ho-166m \\
\hline rastax.115.in & Ir-192 \\
\hline rastax.116.in & Mn-54 \\
\hline rastax.117.in & Np-237 \\
\hline rastax.118.in & $\mathrm{Pb}-210$ \\
\hline rastax.119.in & $\mathrm{Pm}-147$ \\
\hline rastax.120.in & Po-210 \\
\hline rastax.121.in & $\mathrm{Pu}-238$ \\
\hline rastax.122.in & $\mathrm{Pu}-239$ \\
\hline rastax.123.in & $\mathrm{Pu}-240$ \\
\hline rastax.124.in & $\mathrm{Pu}-241$ \\
\hline rastax.125.in & $\mathrm{Pu}-242$ \\
\hline rastax.126.in & Ra-226 \\
\hline rastax.127.in & Ru-106 \\
\hline rastax.128.in & Sc-46 \\
\hline rastax.129.in & Se-75 \\
\hline rastax.130.in & Sm-145 \\
\hline rastax.131.in & Sr-90 \\
\hline rastax.132.in & Tm-170 \\
\hline rastax.133.in & Yb-169 \\
\hline rastax.134.in & $\mathrm{Zn}-65$ \\
\hline rastax.135.in & Zr-95 \\
\hline rastax.136.in & Pd-103 \\
\hline rasta_bex.137.yy.in & Am-241 with yy percent added beryllium \\
\hline rasta_bex.138.yy.in & Am-243 with yy percent added beryllium \\
\hline rasta bex.139.yy.in & $\mathrm{Cm}-244$ with yy percent added beryllium \\
\hline rasta_bex.140.yy.in & Np-237 with yy percent added beryllium \\
\hline rasta_bex.141.yy.in* & Pu-238 with yy percent added beryllium \\
\hline rasta_bex.142.yy.in & $\mathrm{Pu}-239$ with yy percent added beryllium \\
\hline rasta_bex.143.yy.in & Pu-240 with yy percent added beryllium \\
\hline rasta bex.144.yy.in & Pu-241 with yy percent added beryllium \\
\hline rasta_bex.145.yy.in & Pu-242 with yy percent added beryllium \\
\hline
\end{tabular}

Note: yy $\%$ Beryllium is modeled as 1 gram of actinide and 0.yy grams of beryllium. 
PCP-2011-0001

Revision 0

Table A-3. RASTA Input Files for Bremsstrahlung Cases

\begin{tabular}{||l|l|}
\hline File Name & Description \\
\hline ac227_x & Ac-227 \\
\hline cs137_0dx & Cs-137 \\
\hline pb210_x & Pb-210 \\
\hline ru106_0dx* & Ru-106 \\
\hline sr90_30dx & Sr-90 \\
\hline
\end{tabular}


PCP-2011-0001

Revision 0

File origenle.102.in

'This SCALE input file was generated by

'OrigenArp Version 6.0.13.12 January 12, 2010

=origens

$0 \$ \$$ a11 71 e $t$

Decay Case Co-60

3\$\$ 21110 a16 2 a33 77 e $t$

$35 \$ \$ 0 t$

$54 \$ \$$ a 8 1 a 110

$56 \$ \$$ a2 14 a 6 a 10 a 0 13 1 a15 3 a17 2 e

$57 * * 0$ a3 $1 e-05 e$

$95 \$ 1 t$

Case $1 \quad$ Co-60

0 MTU

$60 * * 0.010 .03 \quad 0.1 \quad 0.3 \quad 1 \quad 3 \quad 1030 \quad 100 \quad 300 \quad 10003000 \quad 10000 \quad 30000$

$61 * * \mathrm{f} 1 \mathrm{e}-20$

$65 \$ \$$

'Gram-Atoms Grams Curies Watts-All Watts-Gamma

$\begin{array}{llllllllll}3 z & 1 & 0 & 0 & 1 & 0 & 0 & 3 z & 3 z & 6 z \\ 3 z & 0 & 0 & 0 & 0 & 0 & 0 & 3 z & 3 z & 6 z \\ 3 z & 0 & 0 & 0 & 0 & 0 & 0 & 3 z & 3 z & 6 z\end{array}$

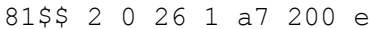

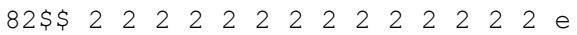

$83 * \star$

$1.0000000 \mathrm{E}+079.0000000 \mathrm{E}+06 \quad 8.0000000 \mathrm{E}+067.5000000 \mathrm{E}+067.0000000 \mathrm{E}+06$

$6.5000000 \mathrm{E}+06 \quad 6.0000000 \mathrm{E}+06 \quad 5.5000000 \mathrm{E}+06 \quad 5.0000000 \mathrm{E}+06 \quad 4.5000000 \mathrm{E}+06$

$4.0000000 \mathrm{E}+06 \quad 3.5000000 \mathrm{E}+06 \quad 3.0000000 \mathrm{E}+06 \quad 2.7500000 \mathrm{E}+06 \quad 2.5000000 \mathrm{E}+06$

$2.2500000 \mathrm{E}+06 \quad 2.0000000 \mathrm{E}+06 \quad 1.7500000 \mathrm{E}+06 \quad 1.5000000 \mathrm{E}+06 \quad 1.2500000 \mathrm{E}+06$

$1.0000000 \mathrm{E}+06 \quad 9.7500000 \mathrm{E}+05 \quad 9.5000000 \mathrm{E}+05 \quad 9.2500000 \mathrm{E}+05 \quad 9.0000000 \mathrm{E}+05$

$8.7500000 \mathrm{E}+058.5000000 \mathrm{E}+05 \quad 8.2500000 \mathrm{E}+05 \quad 8.0000000 \mathrm{E}+05 \quad 7.7500000 \mathrm{E}+05$

$7.5000000 \mathrm{E}+05 \quad 7.2500000 \mathrm{E}+05 \quad 7.0000000 \mathrm{E}+05 \quad 6.7500000 \mathrm{E}+05 \quad 6.5000000 \mathrm{E}+05$

6.2500000E+05 6.0000000E+05 5.7500000E+05 5.5000000E+05 5.2500000E+05

$5.0000000 \mathrm{E}+05 \quad 4.7500000 \mathrm{E}+05 \quad 4.5000000 \mathrm{E}+05 \quad 4.2500000 \mathrm{E}+05 \quad 4.0000000 \mathrm{E}+05$

$3.7500000 \mathrm{E}+053.5000000 \mathrm{E}+05 \quad 3.2500000 \mathrm{E}+05 \quad 3.0000000 \mathrm{E}+052.9000000 \mathrm{E}+05$

$2.8000000 \mathrm{E}+052.7000000 \mathrm{E}+05 \quad 2.6000000 \mathrm{E}+05 \quad 2.5000000 \mathrm{E}+052.4000000 \mathrm{E}+05$

$2.3000000 \mathrm{E}+05 \quad 2.2000000 \mathrm{E}+05 \quad 2.1000000 \mathrm{E}+05 \quad 2.0000000 \mathrm{E}+05 \quad 1.9000000 \mathrm{E}+05$

$1.8000000 \mathrm{E}+051.7000000 \mathrm{E}+051.6000000 \mathrm{E}+05 \quad 1.5000000 \mathrm{E}+051.4000000 \mathrm{E}+05$

$1.3000000 \mathrm{E}+051.2000000 \mathrm{E}+05 \quad 1.1000000 \mathrm{E}+05 \quad 1.0000000 \mathrm{E}+059.0000000 \mathrm{E}+04$

8.0000000E+04 7.0000000E+04 6.0000000E+04 5.0000000E+04 4.0000000E+04

3.0000000E+04 2.0000000E+04 1.0000000E+04 e

$73 \$ \$ 270600$

$74 * * 1$

$75 \$ \$ 1$

56\$\$ 0 o a10 1 e t

56\$\$ 0 a a10 2 e $t$

$56 \$ \$ 0$ a 0103 e $t$

$56 \$ \$ 0$ a 0 a 4 e t

$56 \$ \$ 0$ a $010 \quad 5$ e $t$

$56 \$ \$ 0$ a 0106 e $t$

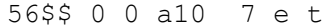

$56 \$ \$ 0$ a 0 (0 8 e

$56 \$ \$ 0$ a 0 10 9 e $t$

$56 \$ \$ 0$ a 0 10 10 e t

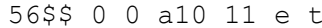

56\$\$ 0 a a 1012 e t

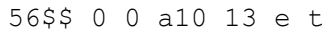

$56 \$ \$ 0$ a 0 10 14 e $t$

$56 \$$ fo $t$

end

=shell

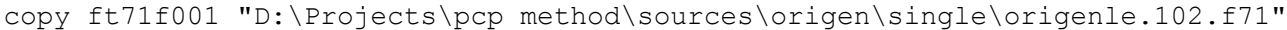
del ft71f001

end 
PCP-2011-0001

Revision 0

File origenac.102.in

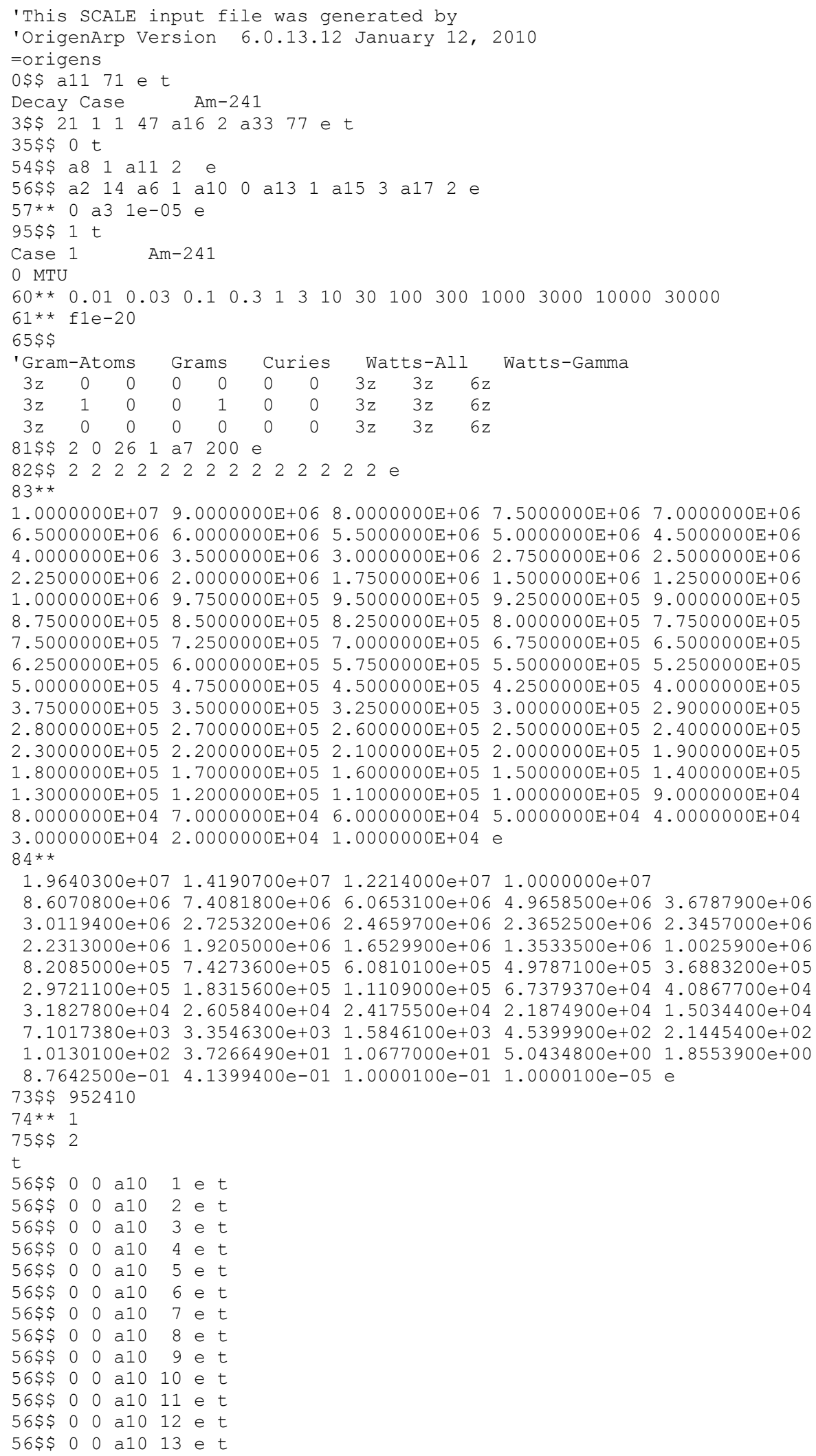


PCP-2011-0001

Revision 0

56\$\$ 0 a a 014 e t

$56 \$ \$ 0 t$

end

=shell

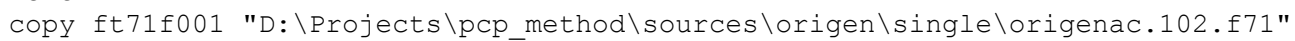
del ft71f001

end

\section{File origen_be.141.90.in}

'This SCALE input file was generated by

'OrigenArp Version 6.0.13.12 January 12, 2010

=origens

$0 \$ \$$ a11 71 e $t$

Decay Case $\mathrm{Pu}-238 \mathrm{Be}$

$3 \$ \$ 211147$ a16 2 a33 77 e $t$

$35 \$ \$ 0 t$

$54 \$ \$$ a 8 1 a11 2 e

56\$\$ a2 14 a6 1 a10 0 a13 2 a15 3 a17 2 e

$57 * \star 0$ a3 $1 e-05$ e

$95 \$ \$ 1$

case 1 Pu-238Be

0 MTU

$60 * * 0.010 .03 \quad 0.1 \quad 0.3 \quad 1 \quad 3 \quad 10 \quad 30100300 \quad 1000 \quad 3000 \quad 10000 \quad 30000$

$61 * \star \mathrm{f} 1 \mathrm{e}-20$

$65 \$ \$$

'Gram-Atoms Grams Curies Watts-All Watts-Gamma

$\begin{array}{llllllllll}3 z & 0 & 0 & 0 & 0 & 0 & 0 & 3 z & 3 z & 6 z\end{array}$

$\begin{array}{llllllllll}3 z & 1 & 0 & 0 & 1 & 0 & 0 & 3 z & 3 z & 6 z\end{array}$

$\begin{array}{llllllllll}3 z & 0 & 0 & 0 & 0 & 0 & 0 & 3 z & 3 z & 6 z\end{array}$

$81 \$ \$ 20026$ a7 200 e

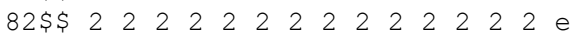

$83 * \star$

$1.0000000 \mathrm{E}+079.0000000 \mathrm{E}+06 \quad 8.0000000 \mathrm{E}+06 \quad 7.5000000 \mathrm{E}+067.0000000 \mathrm{E}+06$

$6.5000000 \mathrm{E}+06 \quad 6.0000000 \mathrm{E}+06 \quad 5.5000000 \mathrm{E}+06 \quad 5.0000000 \mathrm{E}+06 \quad 4.5000000 \mathrm{E}+06$

$4.0000000 \mathrm{E}+06 \quad 3.5000000 \mathrm{E}+06 \quad 3.0000000 \mathrm{E}+06 \quad 2.7500000 \mathrm{E}+062.5000000 \mathrm{E}+06$

$2.2500000 \mathrm{E}+06 \quad 2.0000000 \mathrm{E}+06 \quad 1.7500000 \mathrm{E}+06 \quad 1.5000000 \mathrm{E}+06 \quad 1.2500000 \mathrm{E}+06$

$1.0000000 \mathrm{E}+06 \quad 9.7500000 \mathrm{E}+05 \quad 9.5000000 \mathrm{E}+05 \quad 9.2500000 \mathrm{E}+05 \quad 9.0000000 \mathrm{E}+05$

$8.7500000 \mathrm{E}+058.5000000 \mathrm{E}+058.2500000 \mathrm{E}+05 \quad 8.0000000 \mathrm{E}+05 \quad 7.7500000 \mathrm{E}+05$

$7.5000000 \mathrm{E}+05 \quad 7.2500000 \mathrm{E}+05 \quad 7.0000000 \mathrm{E}+05 \quad 6.7500000 \mathrm{E}+05 \quad 6.5000000 \mathrm{E}+05$

6.2500000E+05 6.0000000E+05 5.7500000E+05 5.5000000E+05 5.2500000E+05

$5.0000000 \mathrm{E}+05 \quad 4.7500000 \mathrm{E}+05 \quad 4.5000000 \mathrm{E}+05 \quad 4.2500000 \mathrm{E}+05 \quad 4.0000000 \mathrm{E}+05$

$3.7500000 \mathrm{E}+053.5000000 \mathrm{E}+05 \quad 3.2500000 \mathrm{E}+05 \quad 3.0000000 \mathrm{E}+052.9000000 \mathrm{E}+05$

$2.8000000 \mathrm{E}+05 \quad 2.7000000 \mathrm{E}+05 \quad 2.6000000 \mathrm{E}+05 \quad 2.5000000 \mathrm{E}+05 \quad 2.4000000 \mathrm{E}+05$

$2.3000000 \mathrm{E}+052.2000000 \mathrm{E}+05 \quad 2.1000000 \mathrm{E}+05 \quad 2.0000000 \mathrm{E}+051.9000000 \mathrm{E}+05$

$1.8000000 \mathrm{E}+051.7000000 \mathrm{E}+05 \quad 1.6000000 \mathrm{E}+05 \quad 1.5000000 \mathrm{E}+05 \quad 1.4000000 \mathrm{E}+05$

1.3000000E+05 1.2000000E+05 1.1000000E+05 1.0000000E+05 9.0000000E+04

8.0000000E+04 7.0000000E+04 6.0000000E+04 5.0000000E+04 4.0000000E+04

$3.0000000 E+042.0000000 E+04 \quad 1.0000000 E+04 \mathrm{e}$

$84 \star \star$

$1.9640300 e+07 \quad 1.4190700 e+07 \quad 1.2214000 e+07 \quad 1.0000000 e+07$

$8.6070800 e+067.4081800 e+06 \quad 6.0653100 e+06 \quad 4.9658500 e+06 \quad 3.6787900 e+06$

$3.0119400 e+062.7253200 e+06 \quad 2.4659700 e+06 \quad 2.3652500 e+062.3457000 e+06$

$2.2313000 e+061.9205000 e+06 \quad 1.6529900 e+06 \quad 1.3533500 e+06 \quad 1.0025900 e+06$

$8.2085000 e+05 \quad 7.4273600 e+05 \quad 6.0810100 e+05 \quad 4.9787100 e+05 \quad 3.6883200 e+05$

$2.9721100 e+051.8315600 e+05 \quad 1.1109000 e+05 \quad 6.7379370 e+04 \quad 4.0867700 e+04$

$3.1827800 e+04 \quad 2.6058400 e+04 \quad 2.4175500 e+04 \quad 2.1874900 e+04 \quad 1.5034400 e+04$

$7.1017380 e+03 \quad 3.3546300 e+031.5846100 e+03 \quad 4.5399900 e+02 \quad 2.1445400 e+02$

$1.0130100 e+023.7266490 e+011.0677000 e+015.0434800 e+001.8553900 e+00$

$8.7642500 \mathrm{e}-014.1399400 \mathrm{e}-011.0000100 \mathrm{e}-011.0000100 \mathrm{e}-05 \mathrm{e}$

$73 \$ \$ 94238040000$

$74 * * 10.9$

$75 \$ 24$

$t$

$56 \$ \$ 0 \quad 0$ a10 1 e $t$

$56 \$ \$ 0$ a 0102 e $t$

$56 \$ \$ 0$ a 0 10 3 e $t$

56\$\$ 0 a a 0 e t

56\$\$ 0 a a 0 e t

$56 \$ 00$ a 106 e $t$ 
PCP-2011-0001

Revision 0

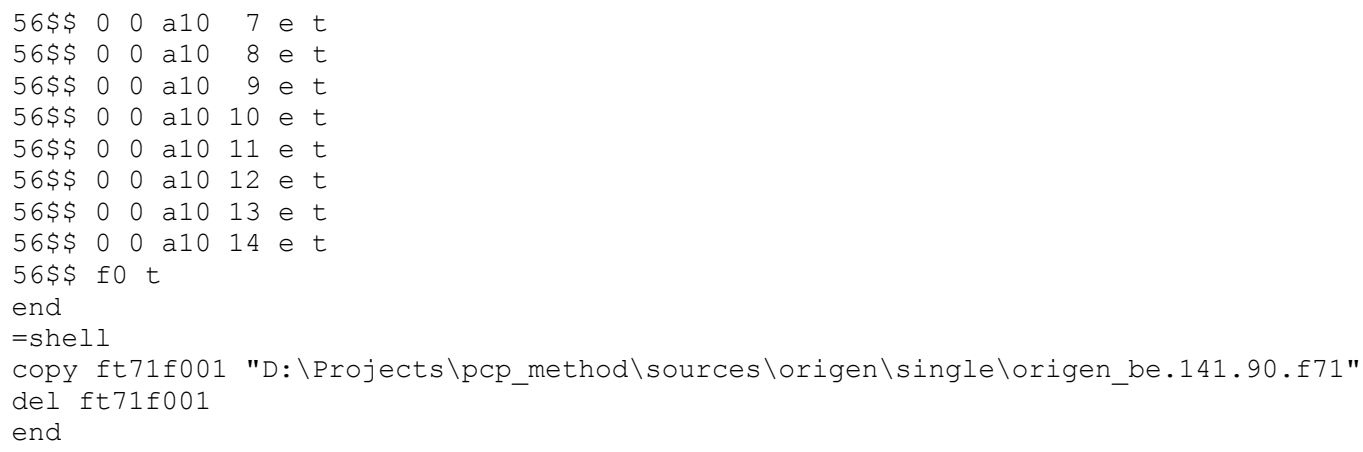

\section{File rasta1.102.in}

SGQ PCP Methodology

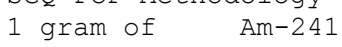

1.500

2.750

2.500

0.825

0.800

0.650

0.500

0.600

0.725

0.700

0.525

0.475

\section{File rasta2.108.in}

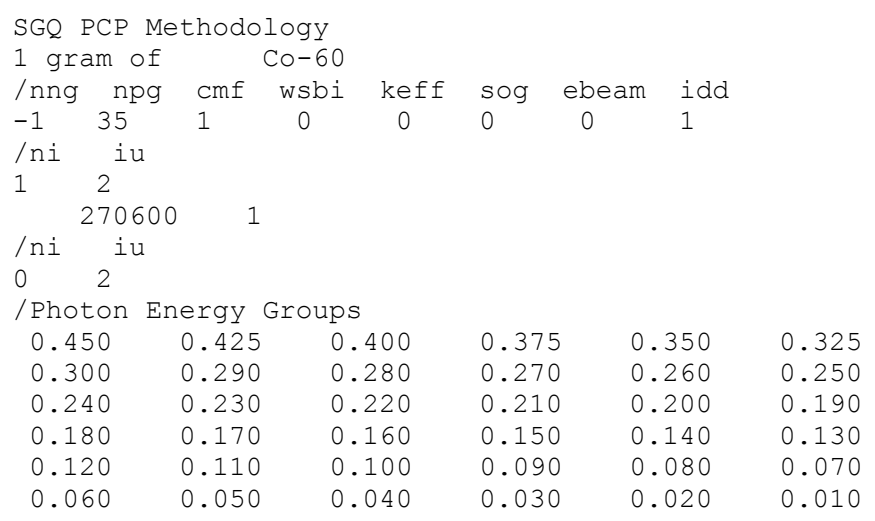


PCP-2011-0001

Revision 0

File rasta_be1.141.90.in

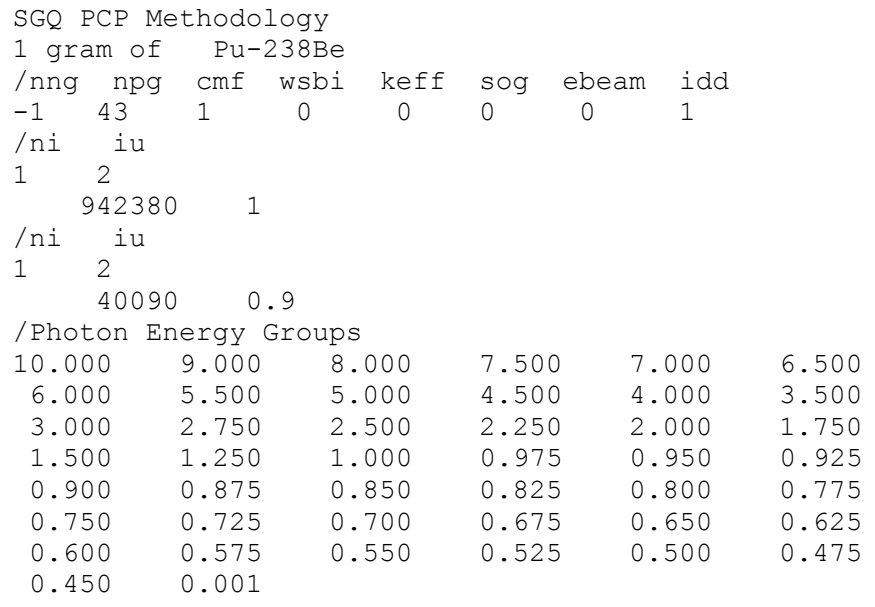

\section{File ru106_0d2}

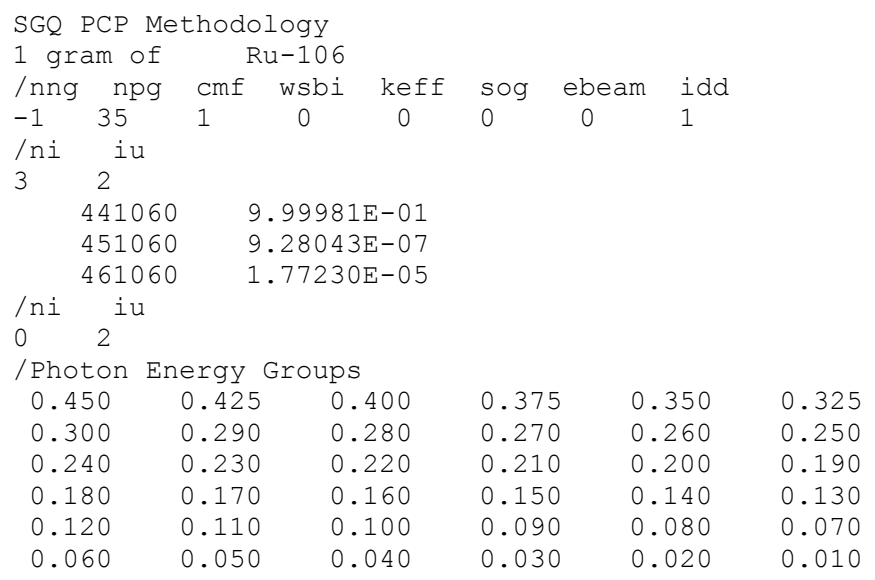


PCP-2011-0001

Revision 0

Appendix B Radiation Transport Input Files 
PCP-2011-0001

Revision 0

The MCNP input files are described in Table B-1. The file naming convention used is case.grp.in where "case" is the case name and "grp" is one hundred plus the group number.

Sample listings are provided below for files marked with an asterisk (*).

\section{Table B-1. MCNP Input Files}

\begin{tabular}{|c|c|}
\hline File Name & Description \\
\hline \multicolumn{2}{|r|}{ No Shielded Container } \\
\hline neut.1nn.in & Neutron sources - surface detectors no self-shielding, $\mathrm{nn}=1-47$ \\
\hline neutpuo.1nn.in* & Neutron sources - surface detectors $\mathrm{PuO}_{2}$ self-shielding, $\mathrm{nn}=1-47$ \\
\hline phot.1pp.in & Photon sources - surface detectors no self-shielding, $\mathrm{pp}=1-77$ \\
\hline photpt.1pp.in & Photon sources - point detectors no self-shielding, $p p=1-11$ \\
\hline photfe.1pp.in & Photon sources - surface detectors iron self-shielding, $\mathrm{pp}=1-77$ \\
\hline photfept.1pp.in & Photon sources - point detectors iron self-shielding, $p p=1-11$ \\
\hline \multicolumn{2}{|r|}{ SC1 Lead Shielded Container } \\
\hline sc1.1pp.in* & Photon sources - surface detectors no self-shielding, $\mathrm{pp}=1-77$ \\
\hline sc1pt.1pp.in & Photon sources - point detectors no self-shielding, $\mathrm{pp}=1-48$ \\
\hline \multicolumn{2}{|r|}{ SC2 Polyethylene Shielded Container } \\
\hline sc2n.1nn.in* & Neutron sources - surface detectors no self-shielding, $\mathrm{nn}=1-47$ \\
\hline sc2.1pp.in & Photon sources - surface detectors no self-shielding, $\mathrm{pp}=1-77$ \\
\hline sc2pt.1pp.in & Photon sources - point detectors no self-shielding, $\mathrm{pp}=1-12$ \\
\hline \multicolumn{2}{|r|}{ SC3 Tungsten Shielded Container } \\
\hline sc3.1pp.in & Photon sources - surface detectors no self-shielding, $\mathrm{pp}=1-77$ \\
\hline sc3pt.1pp.in* & Photon sources - point detectors no self-shielding, $p p=1-46$ \\
\hline
\end{tabular}


File neutpuo.147.in

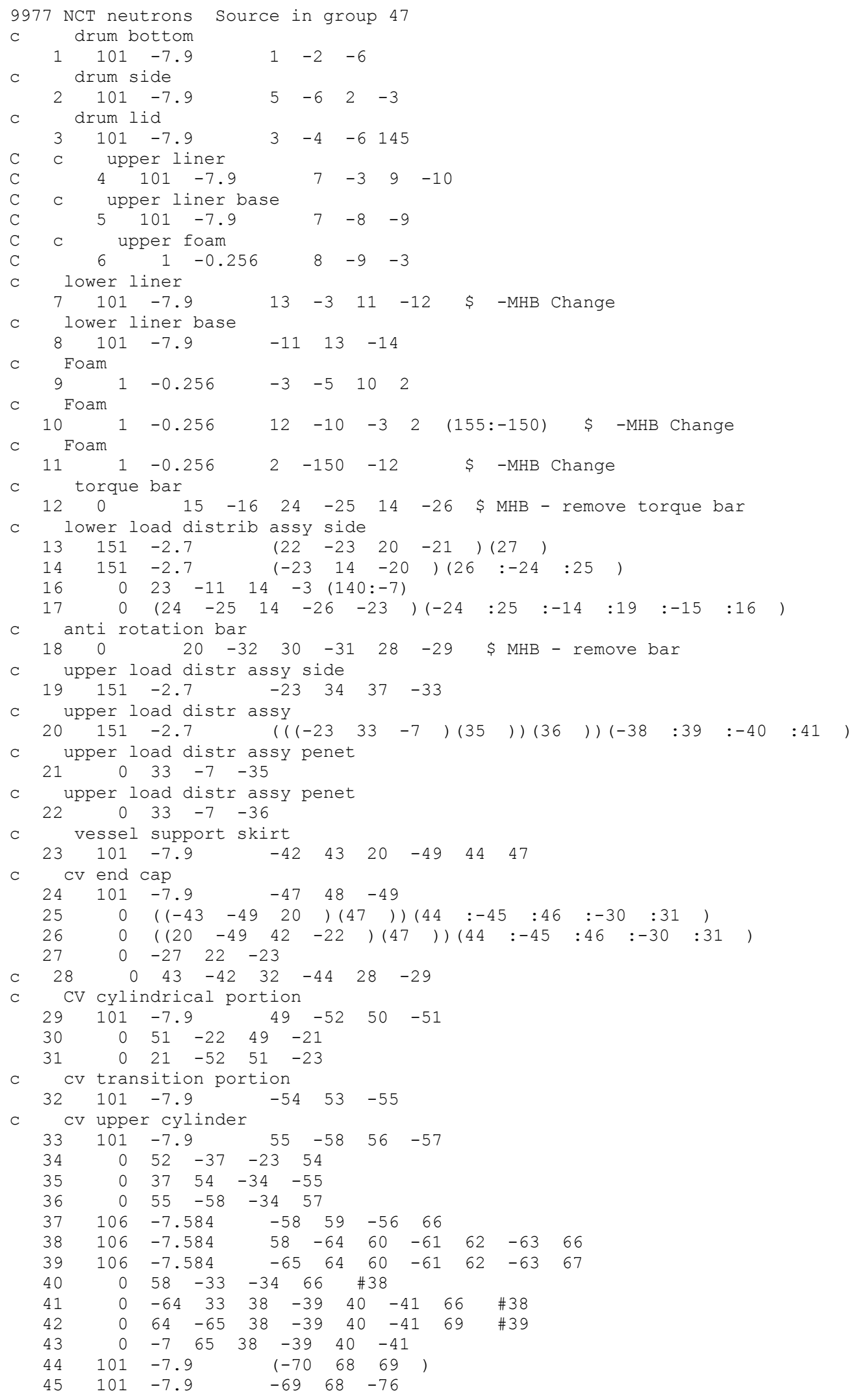


PCP-2011-0001

Revision 0

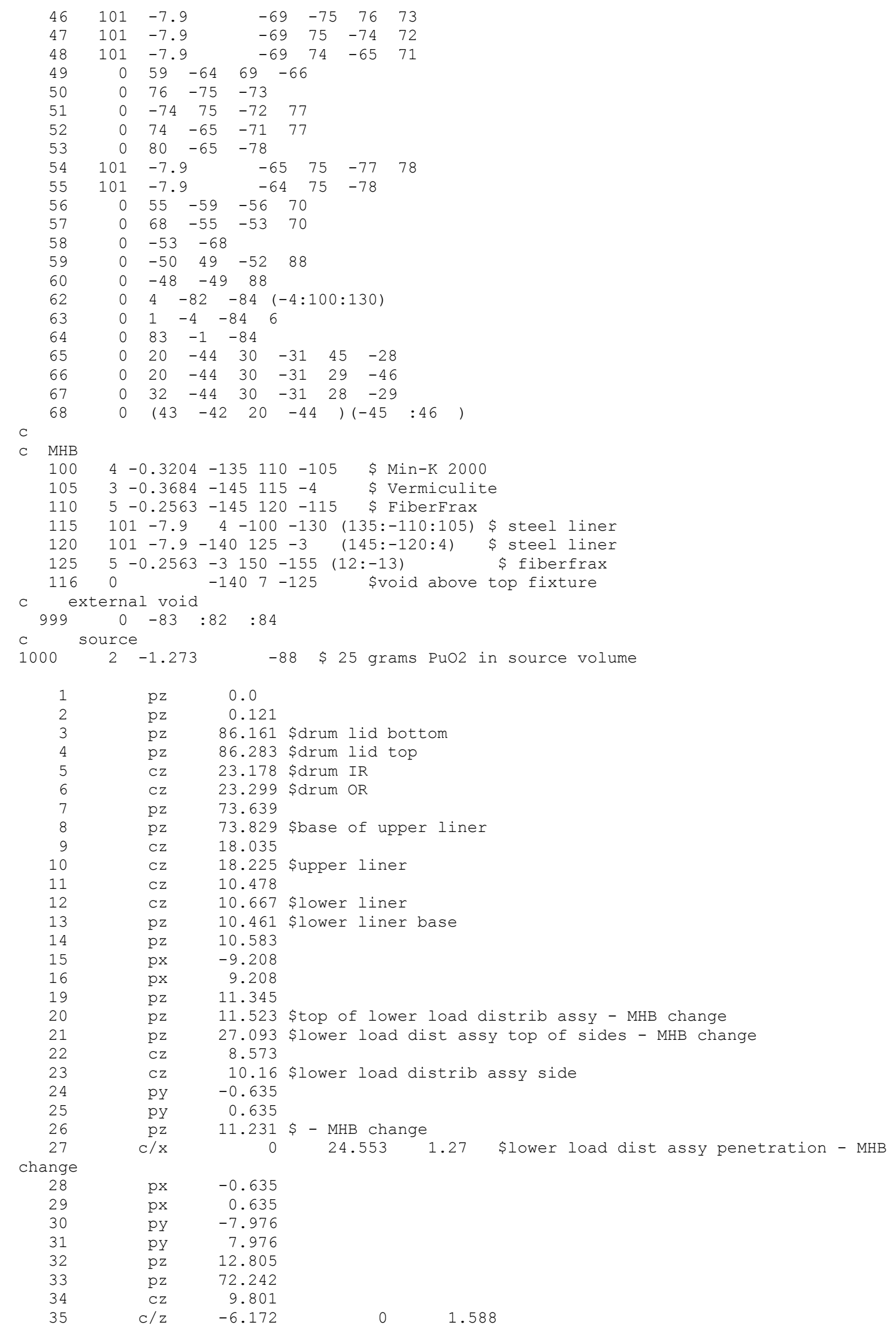


PCP-2011-0001

Revision 0

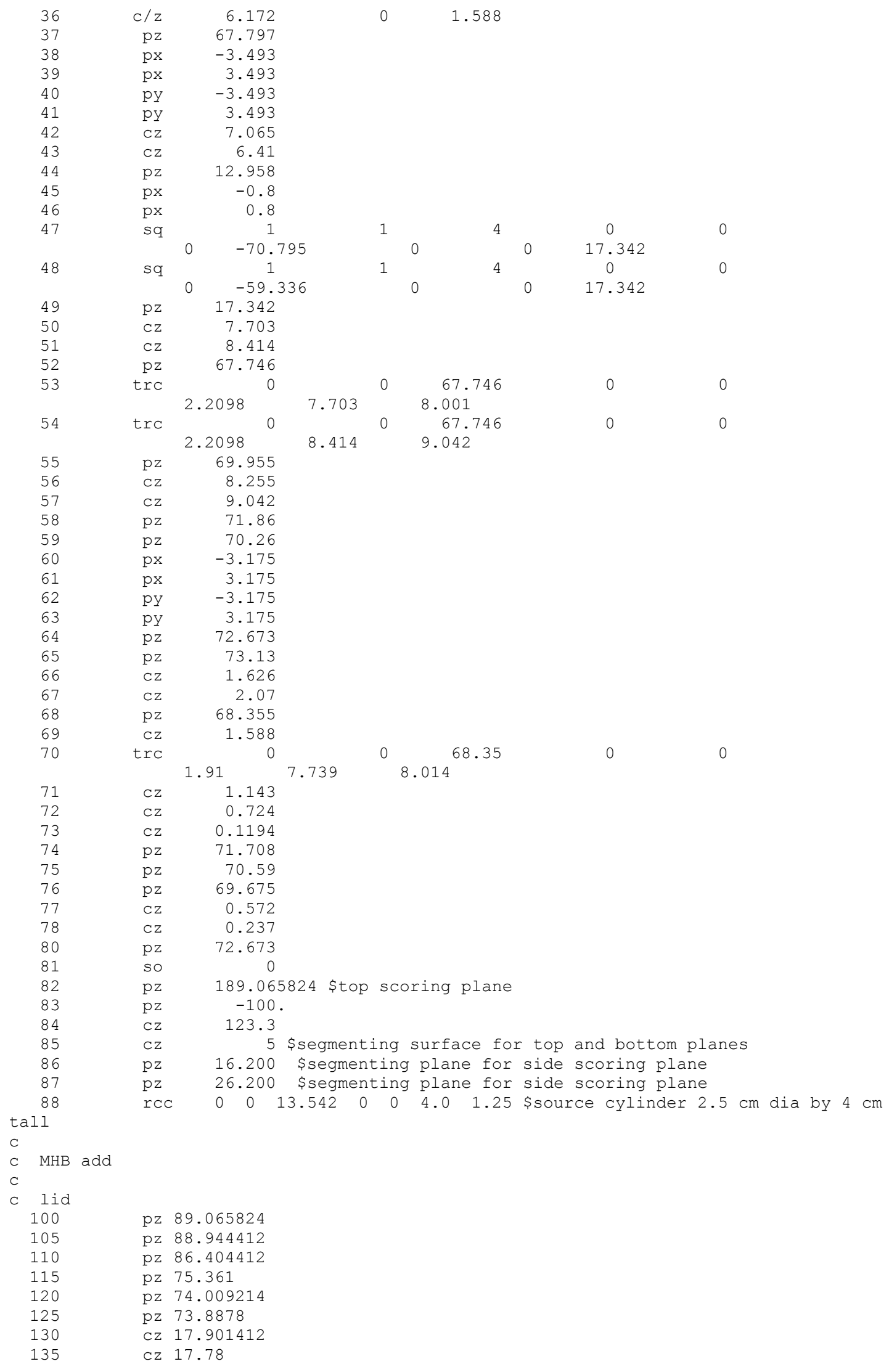


PCP-2011-0001

Revision 0

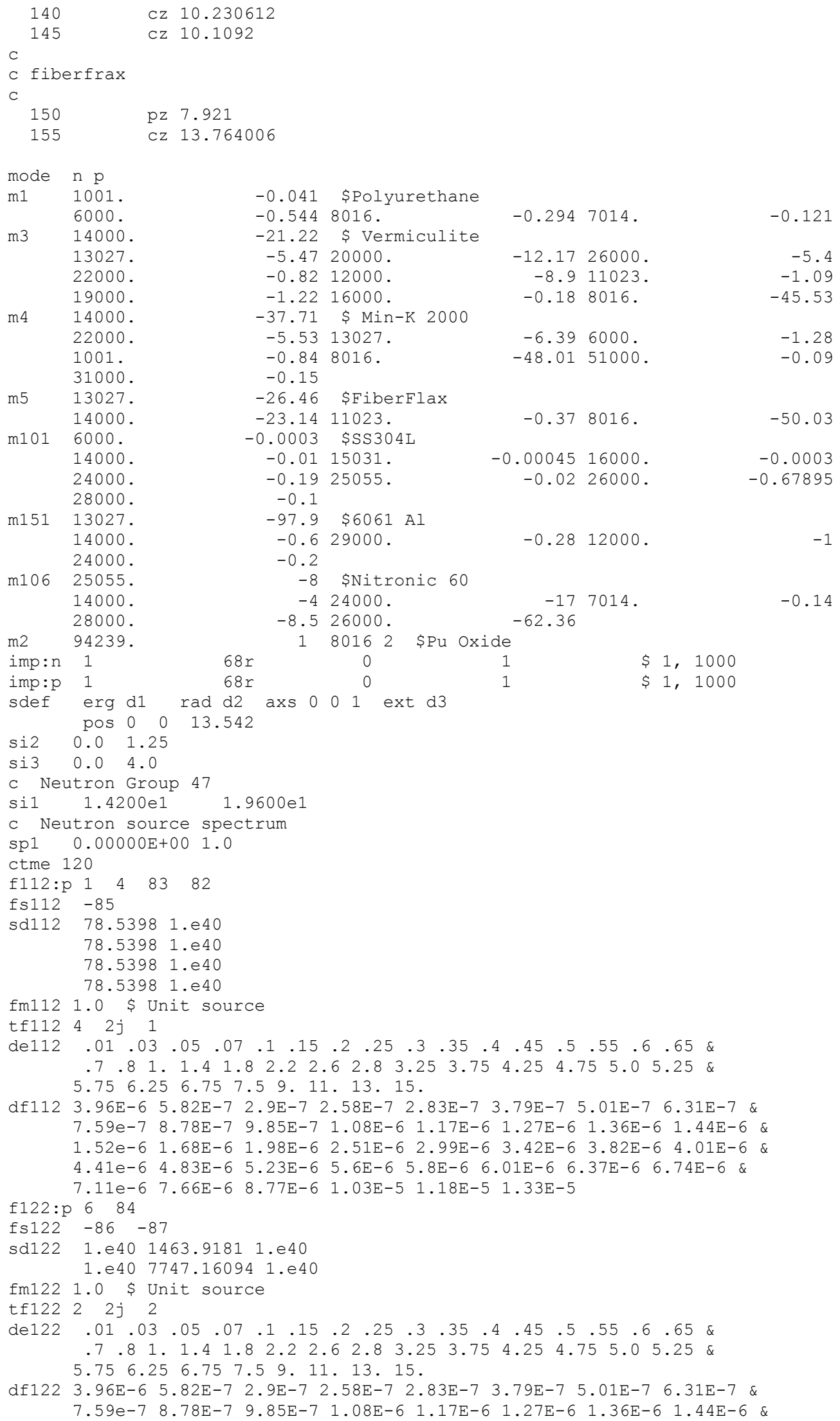


PCP-2011-0001

Revision 0

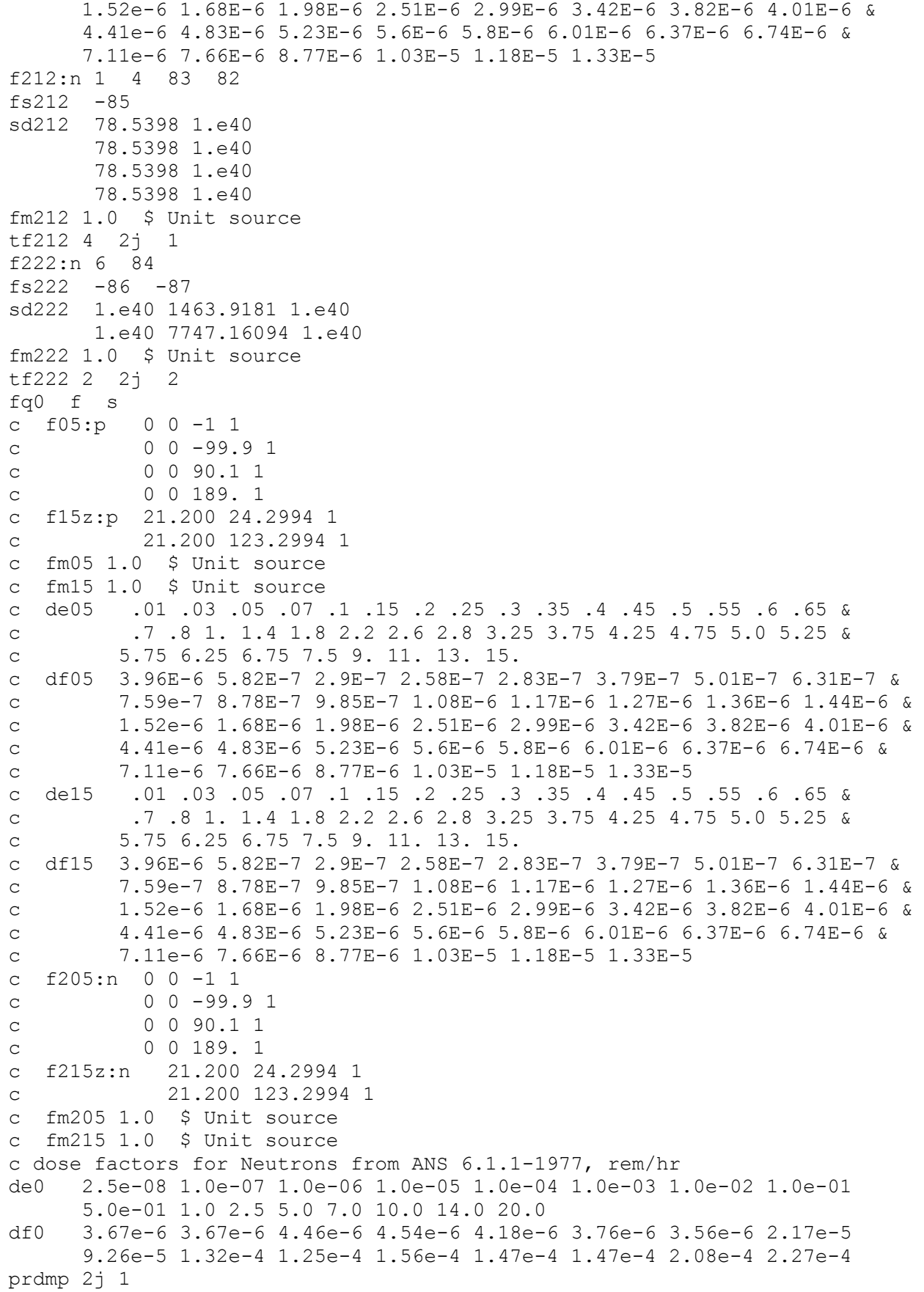

\section{File sc1.101.in}

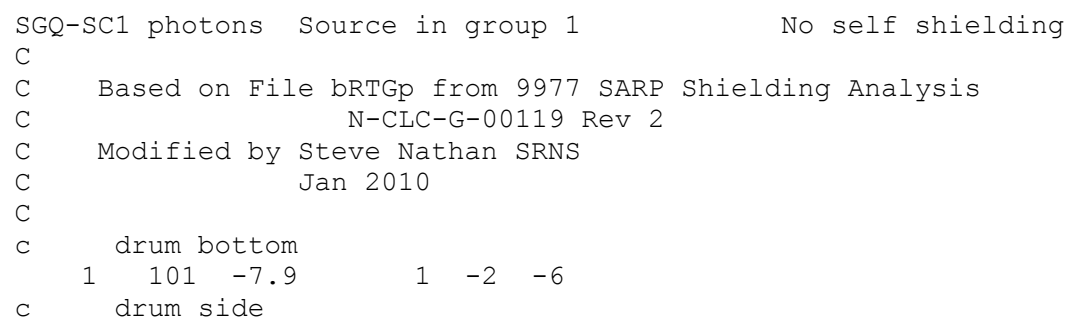




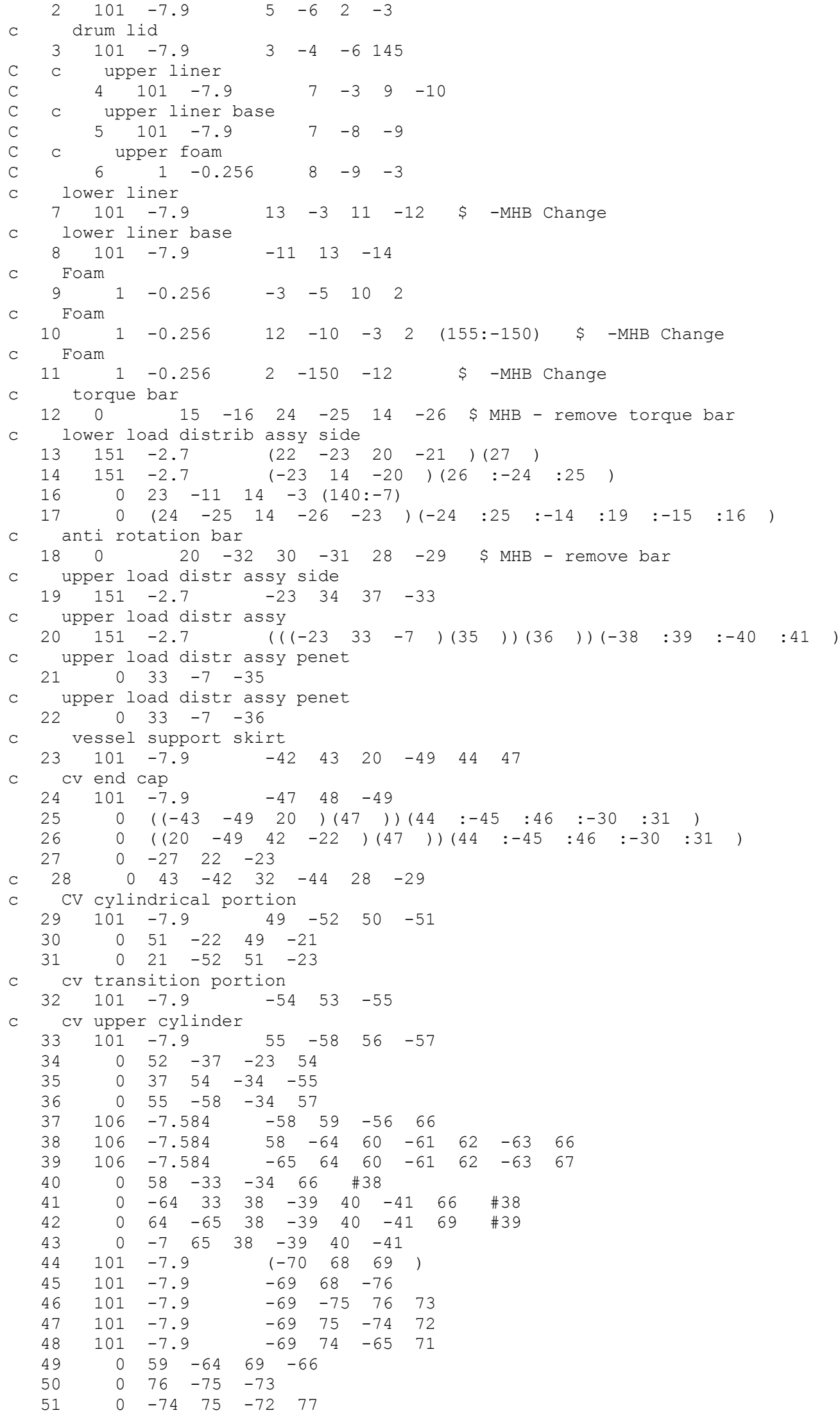


PCP-2011-0001

Revision 0

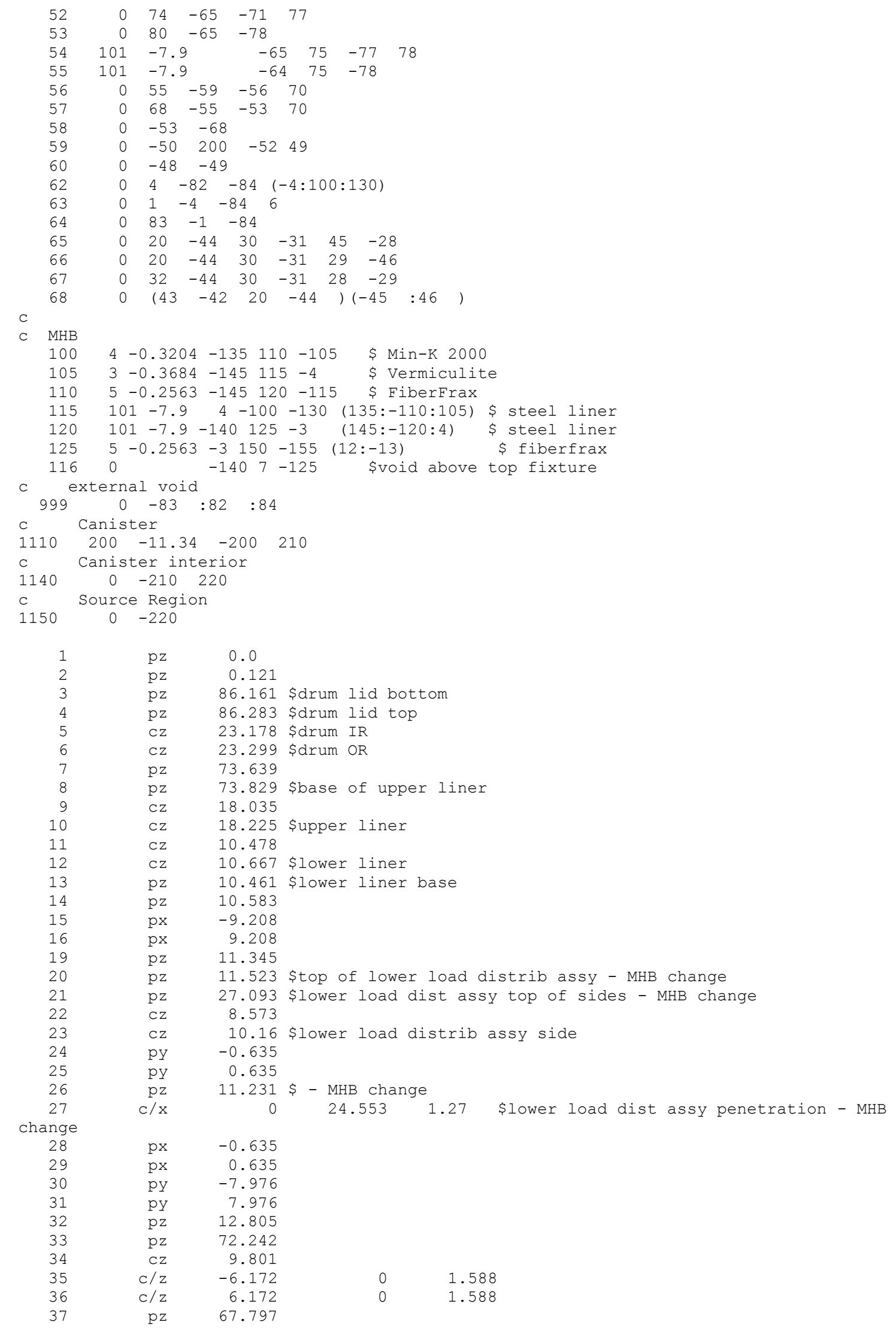


PCP-2011-0001

Revision 0

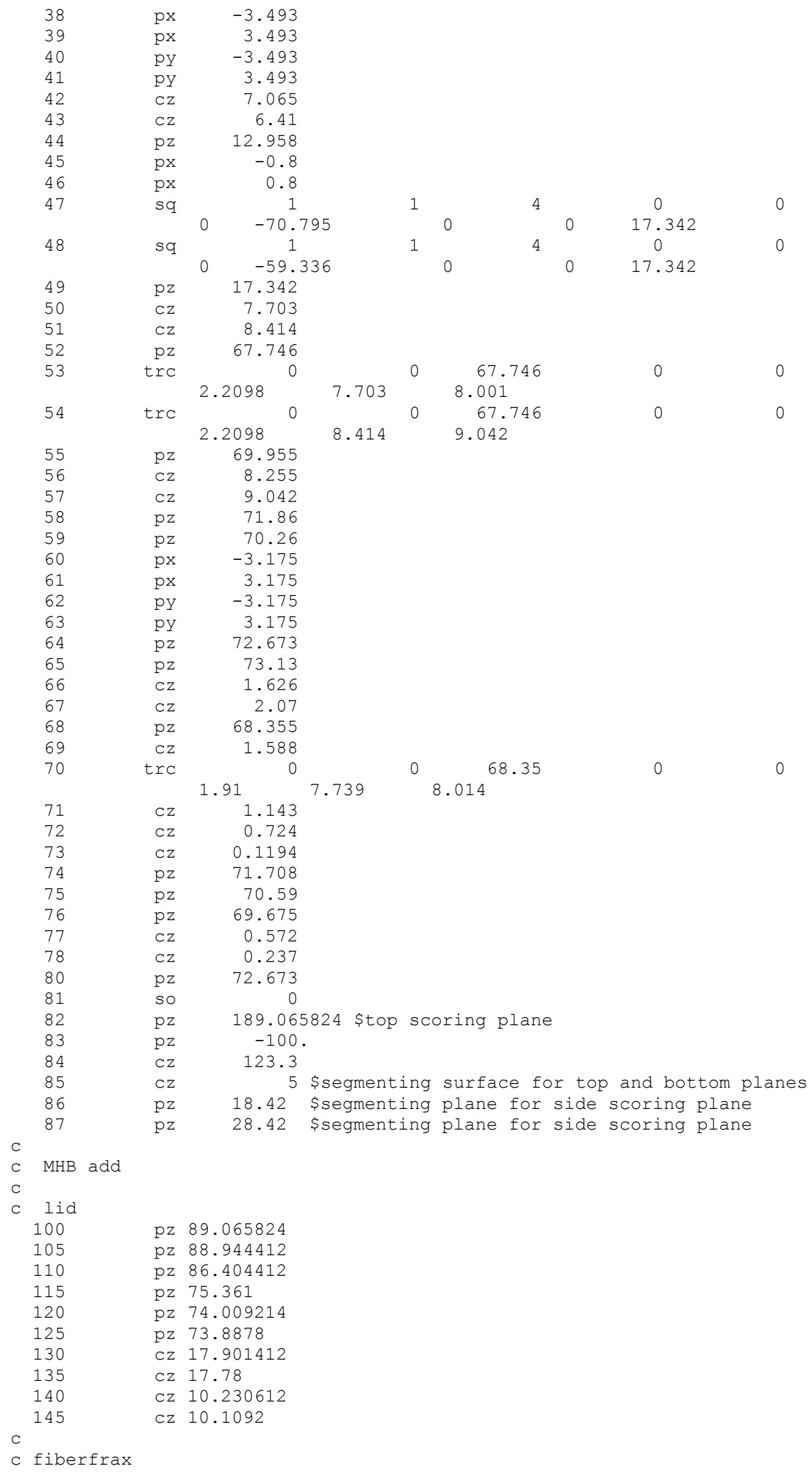


PCP-2011-0001

Revision 0

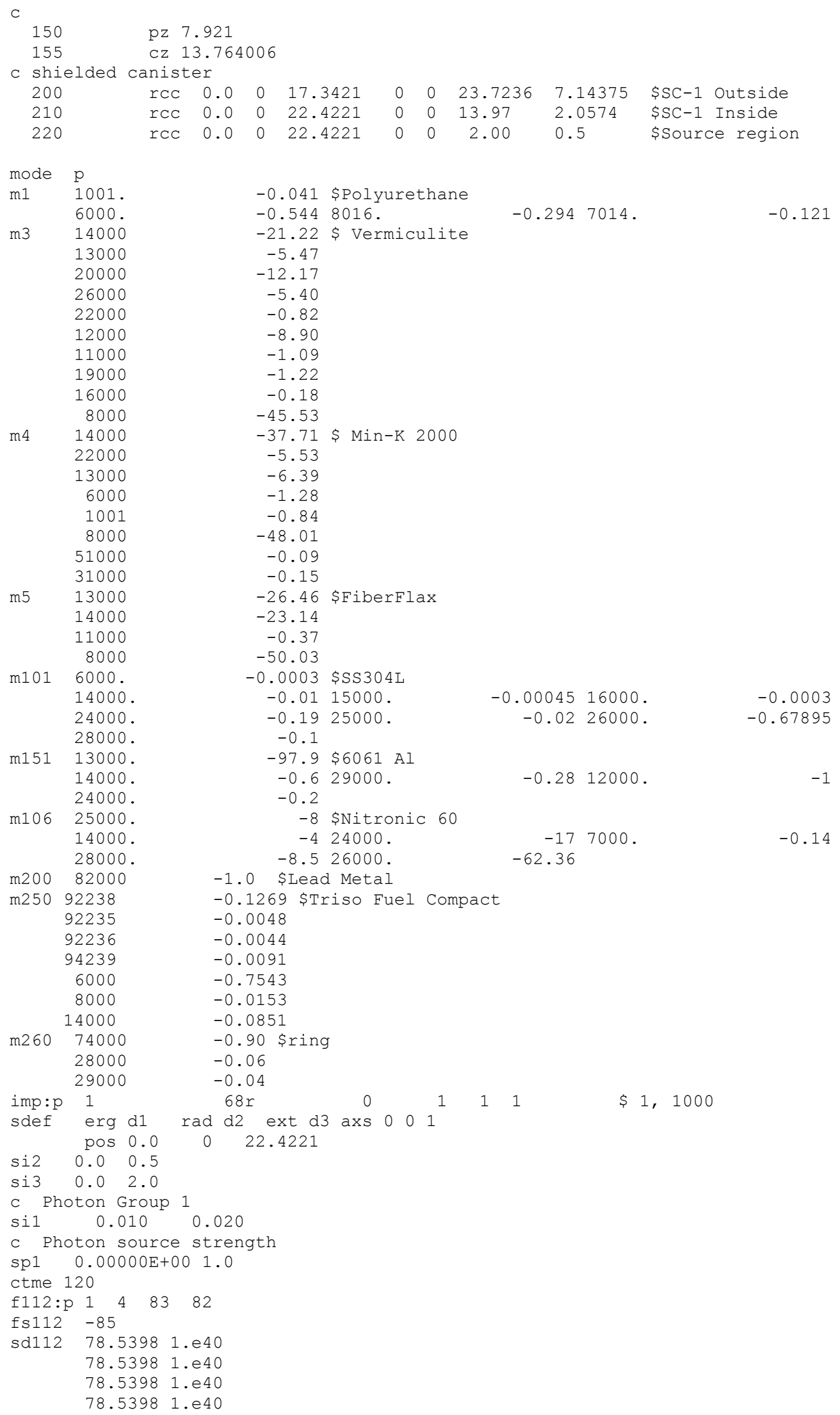




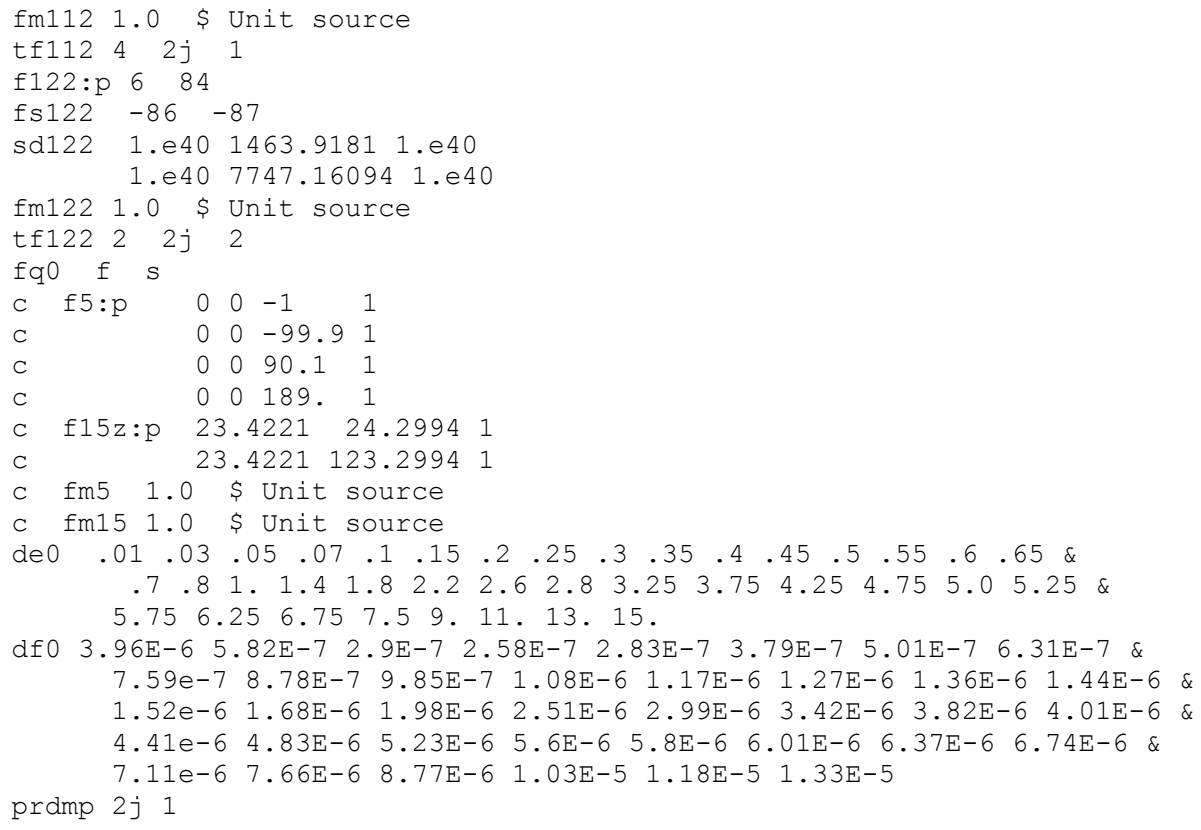

\section{File sc2n.107.in}

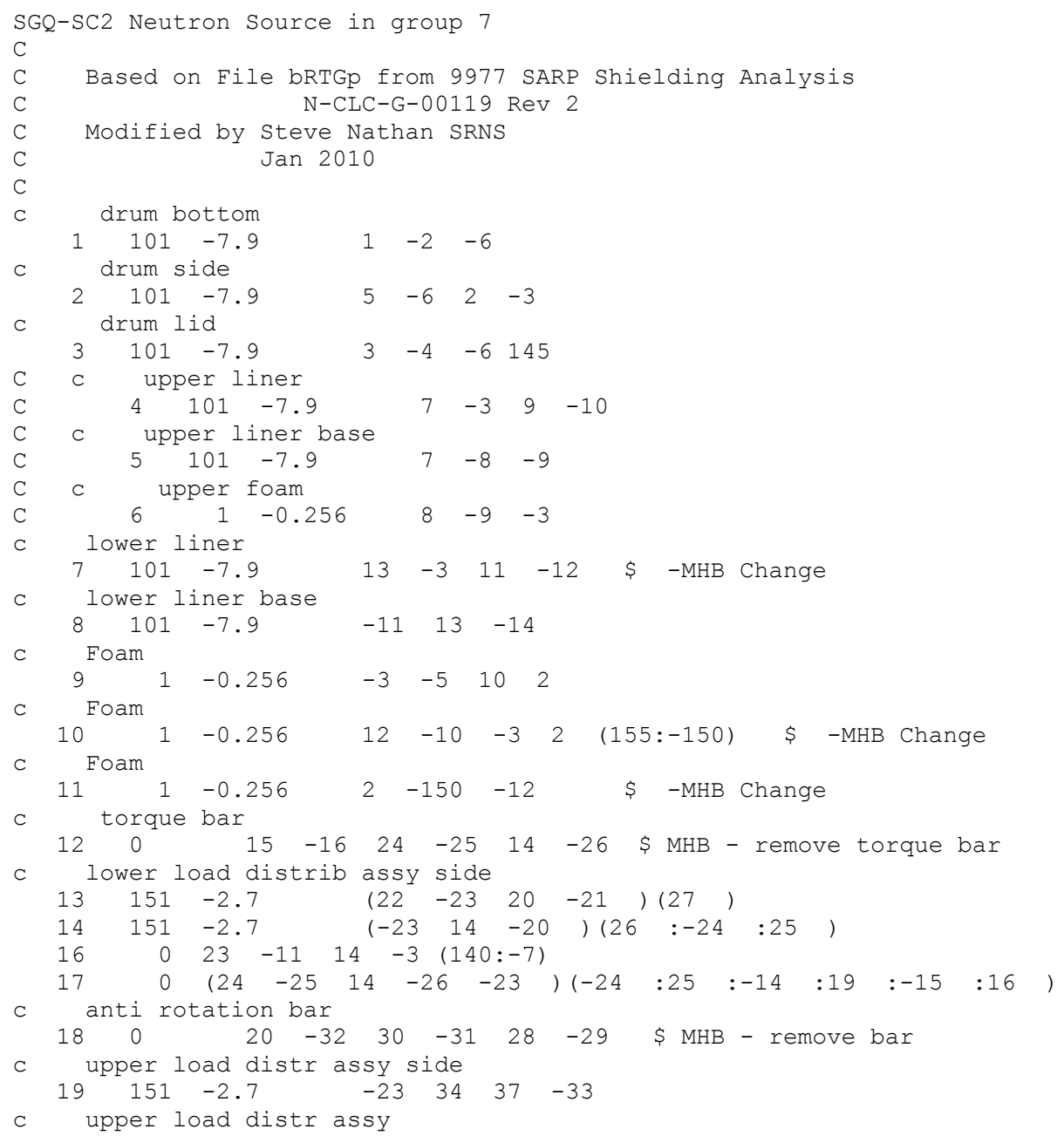


PCP-2011-0001

Revision 0

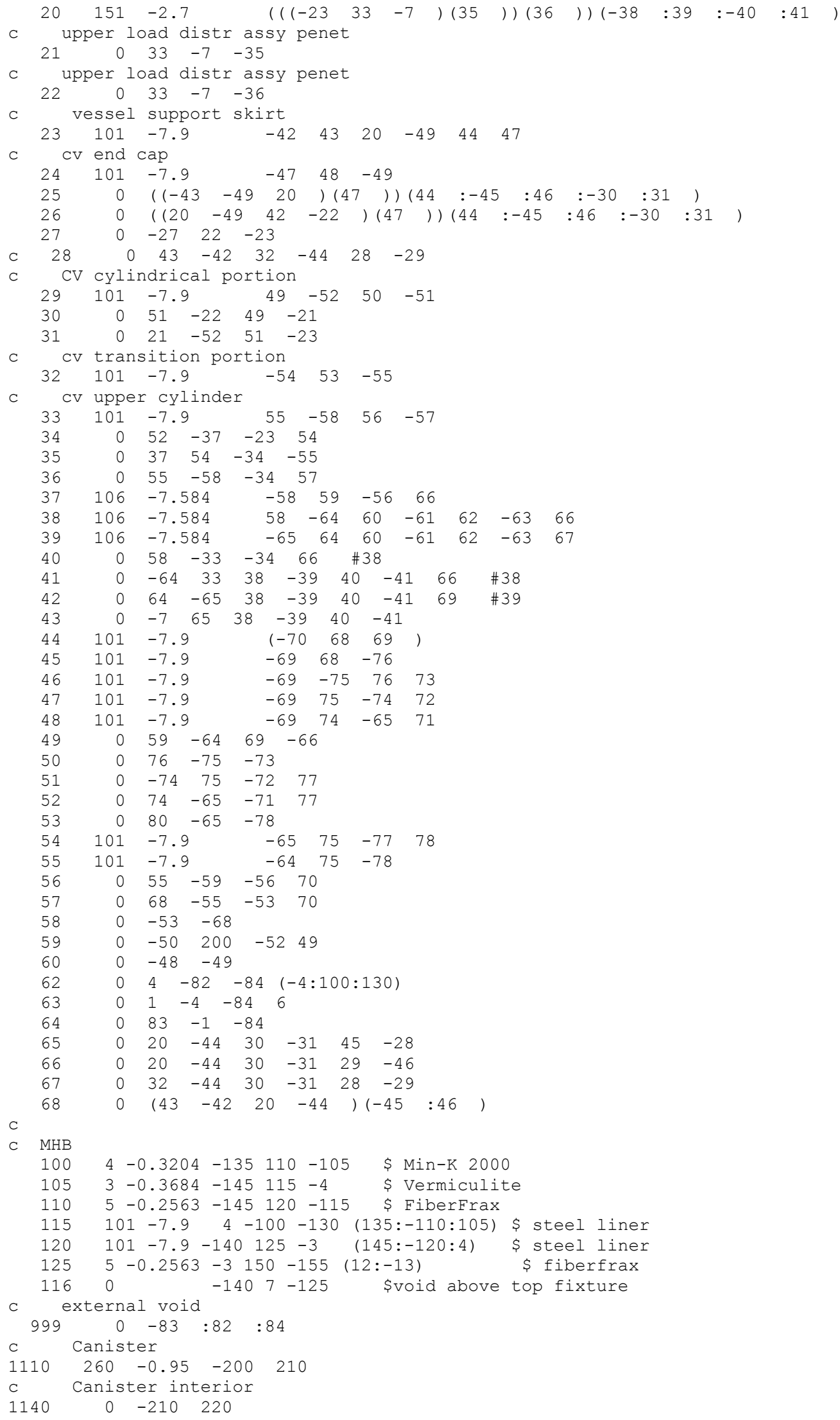


PCP-2011-0001

Revision 0

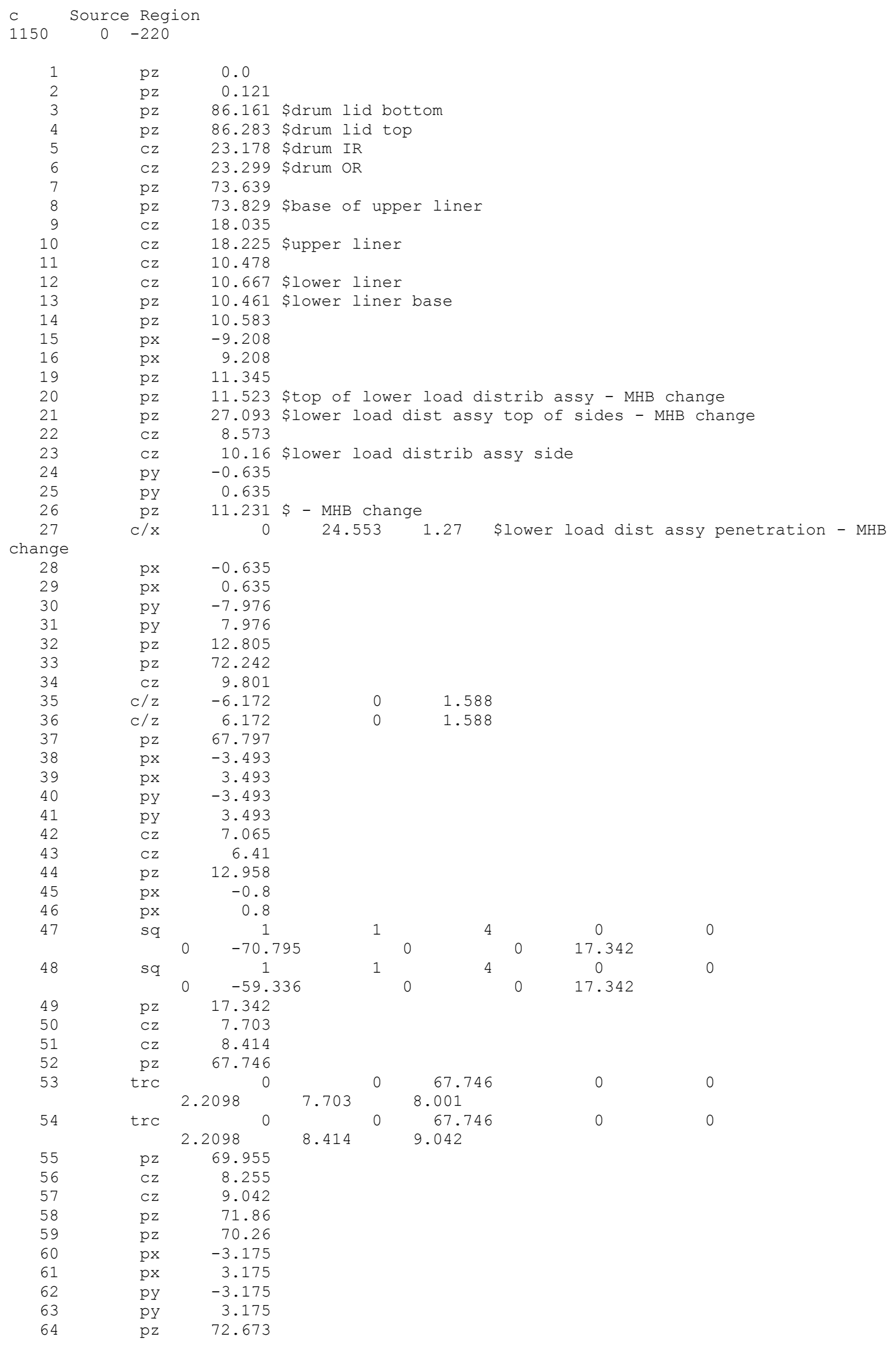


PCP-2011-0001

Revision 0

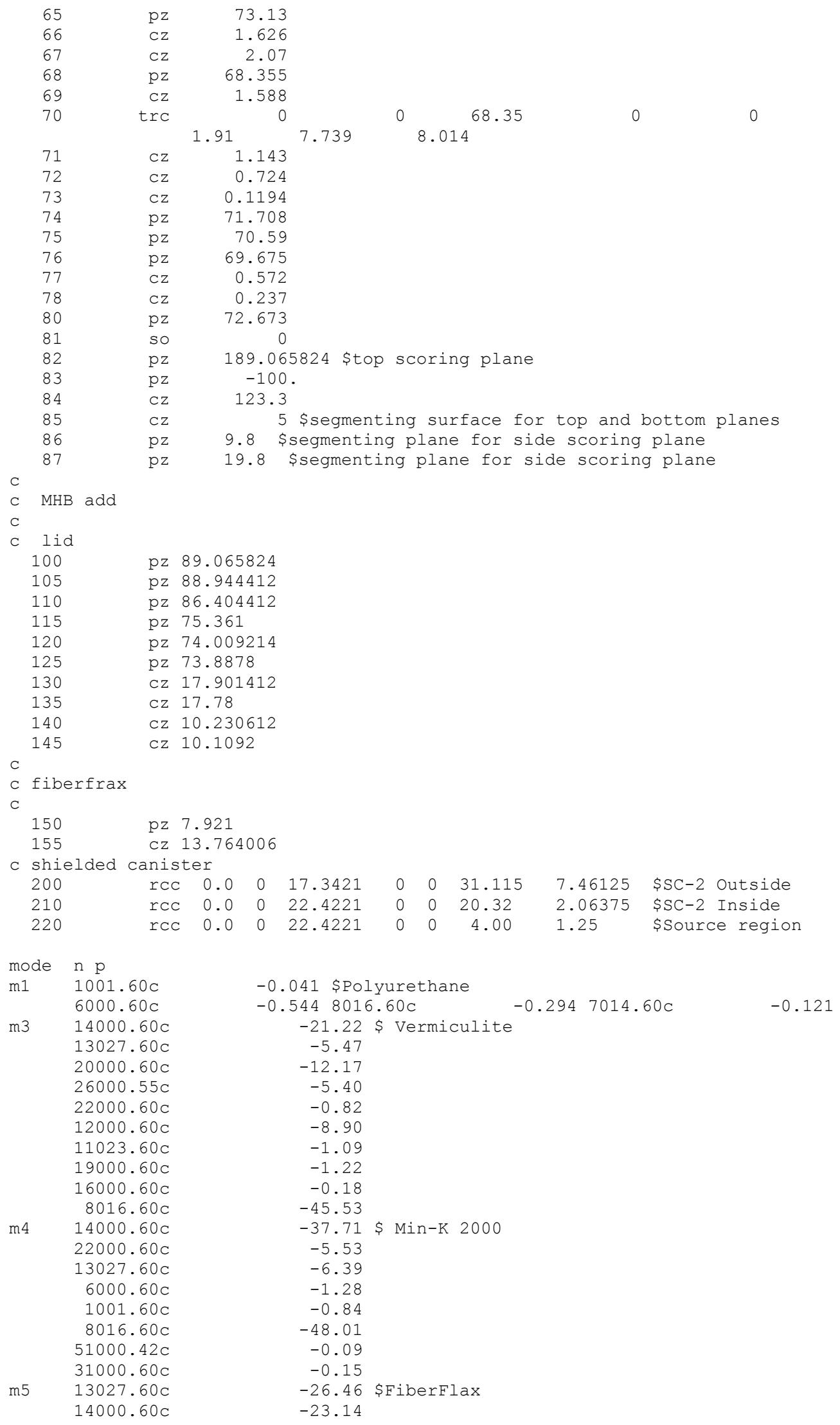


PCP-2011-0001

Revision 0

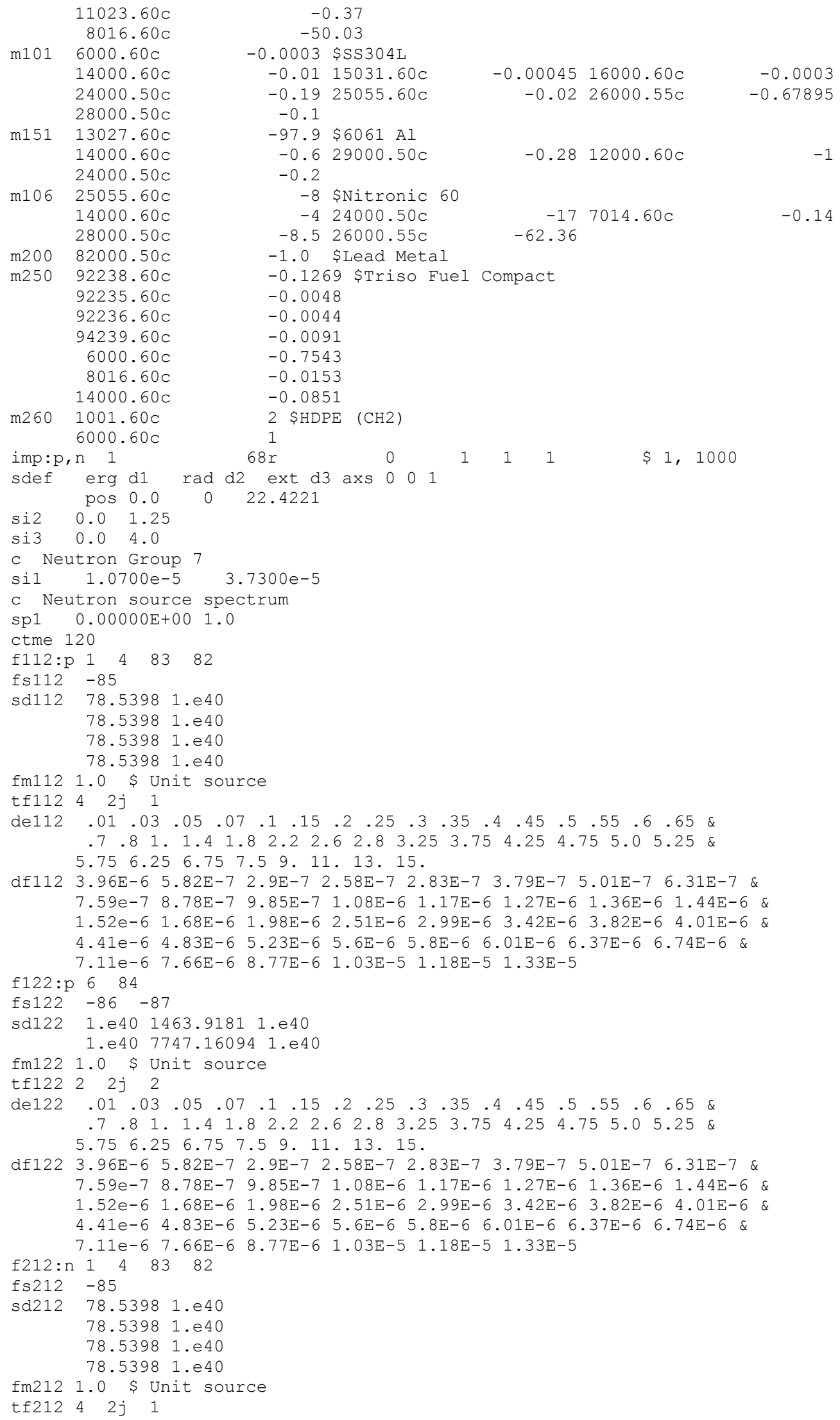


PCP-2011-0001

Revision 0

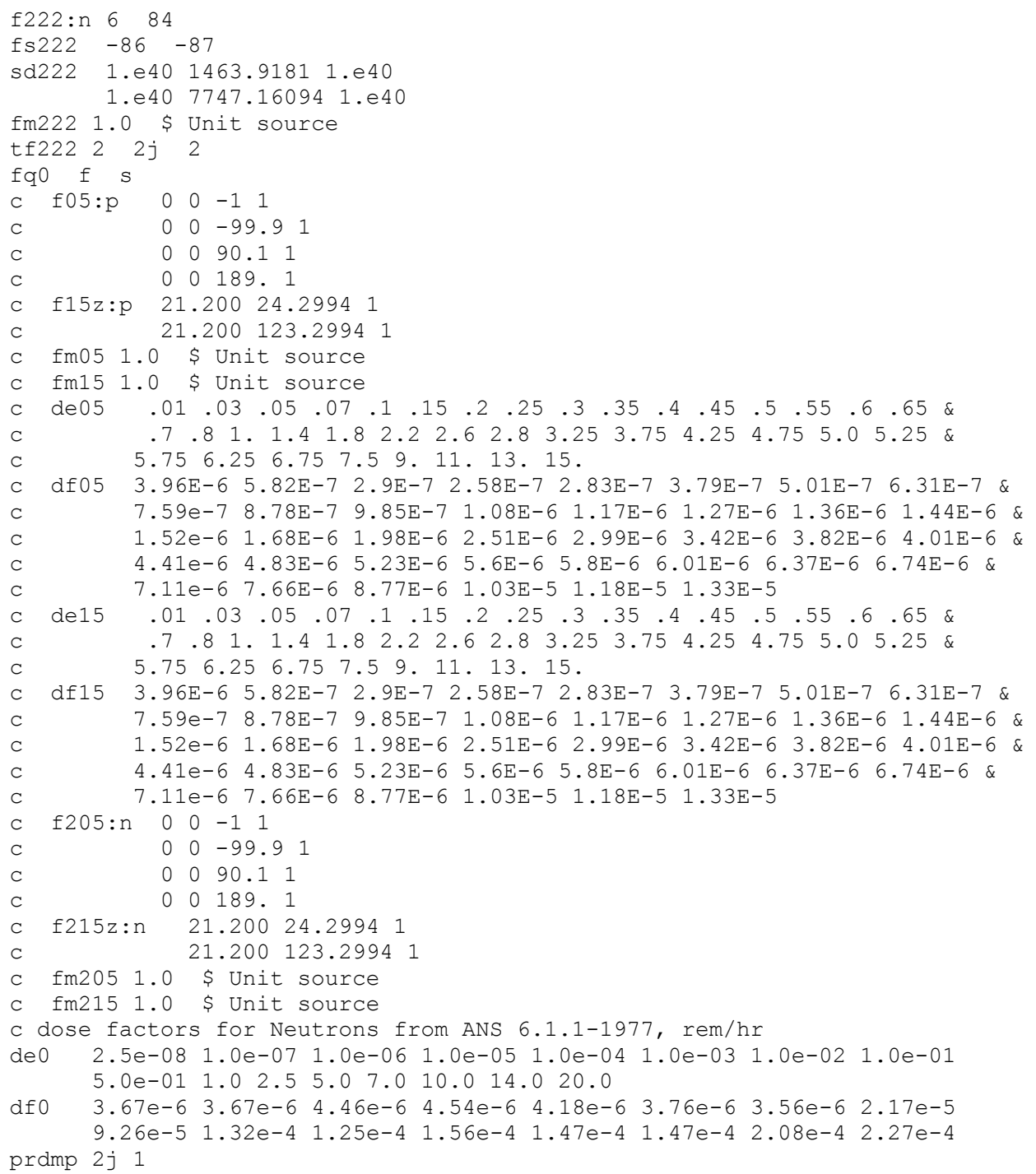

\section{File sc3pt.120.in}

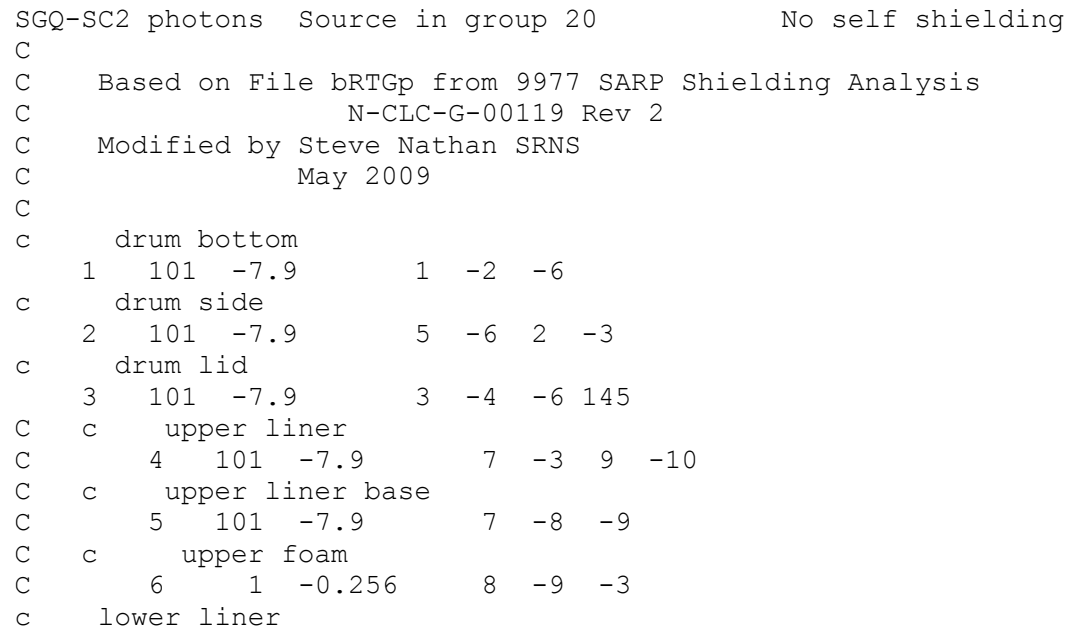




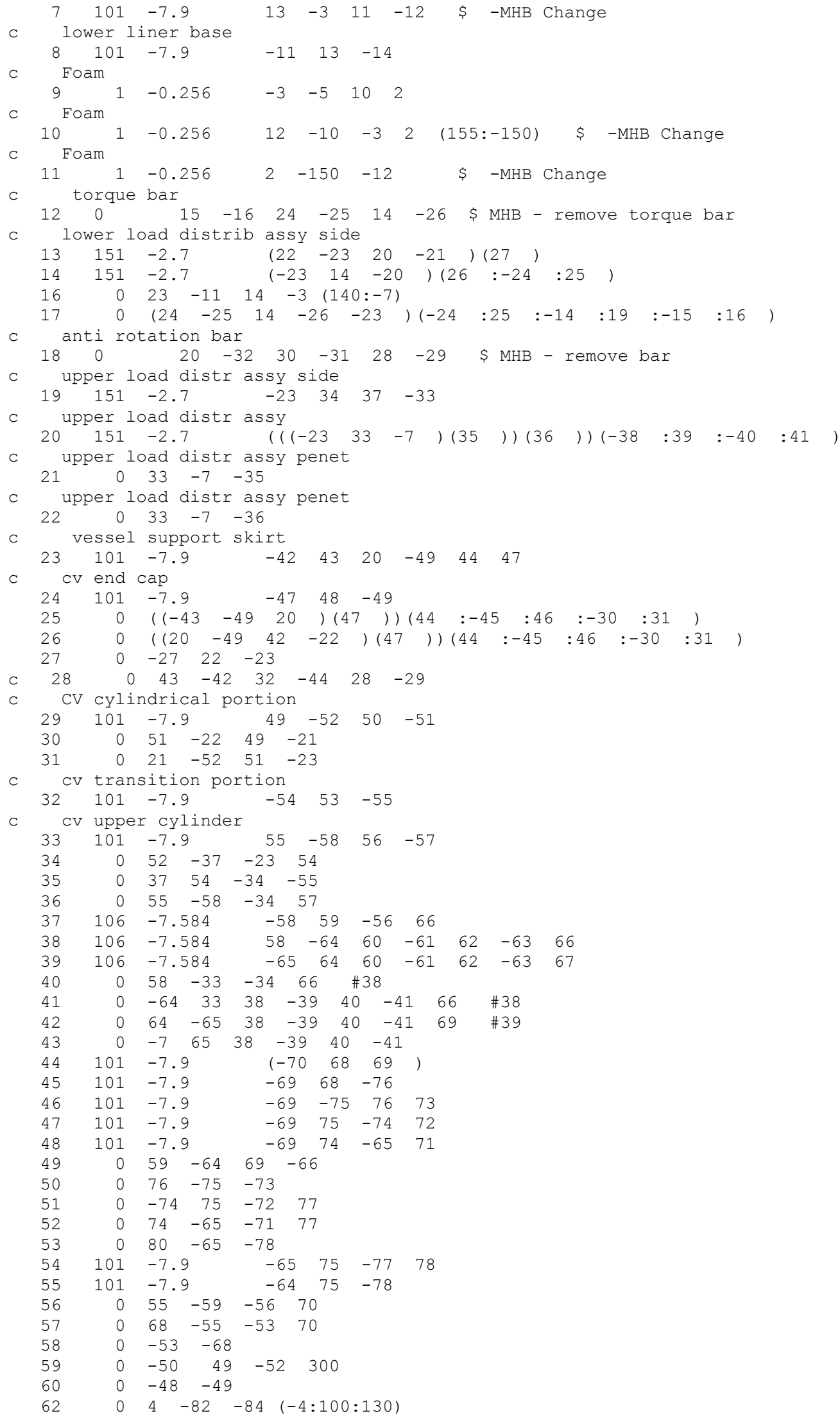


PCP-2011-0001

Revision 0

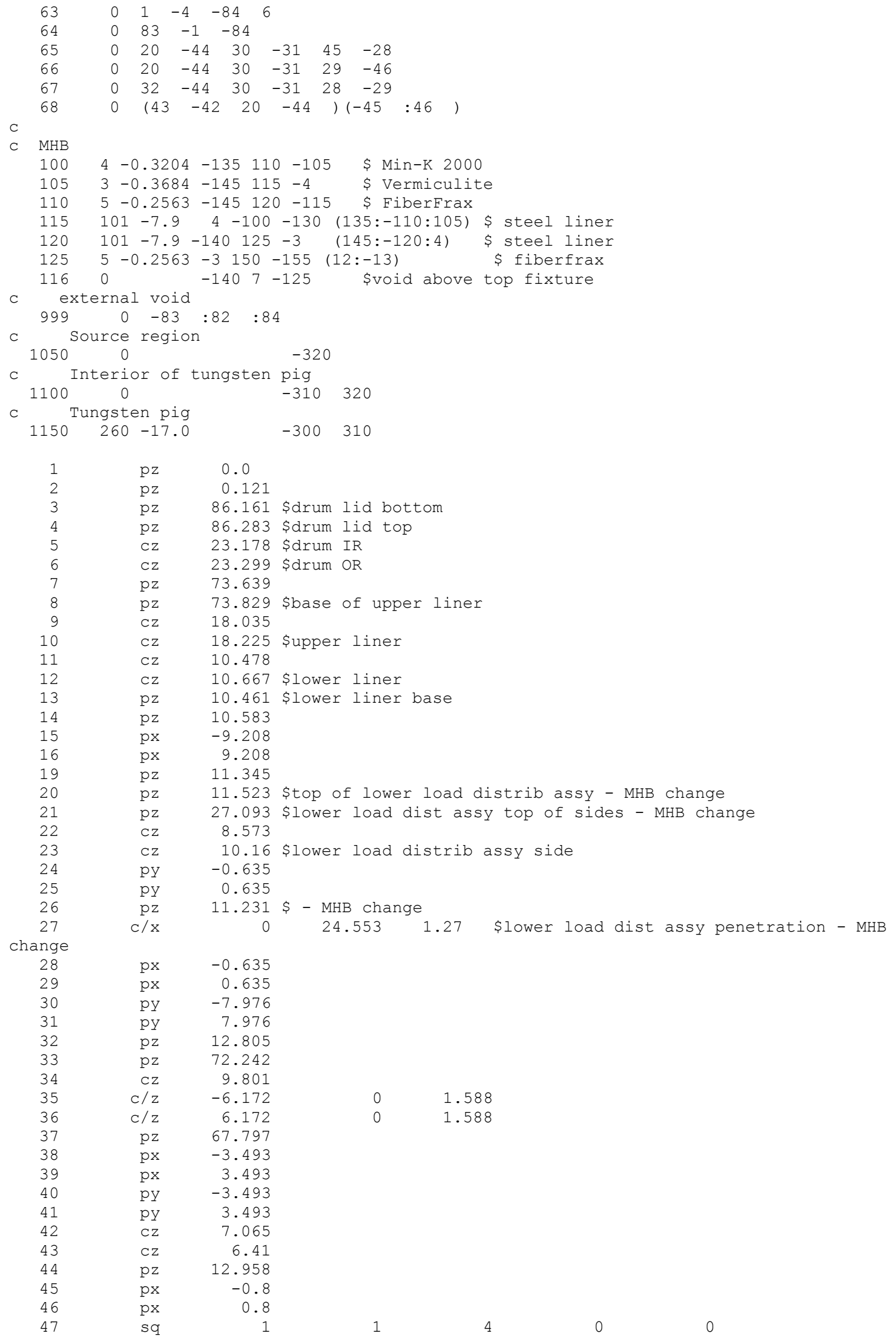


PCP-2011-0001

Revision 0

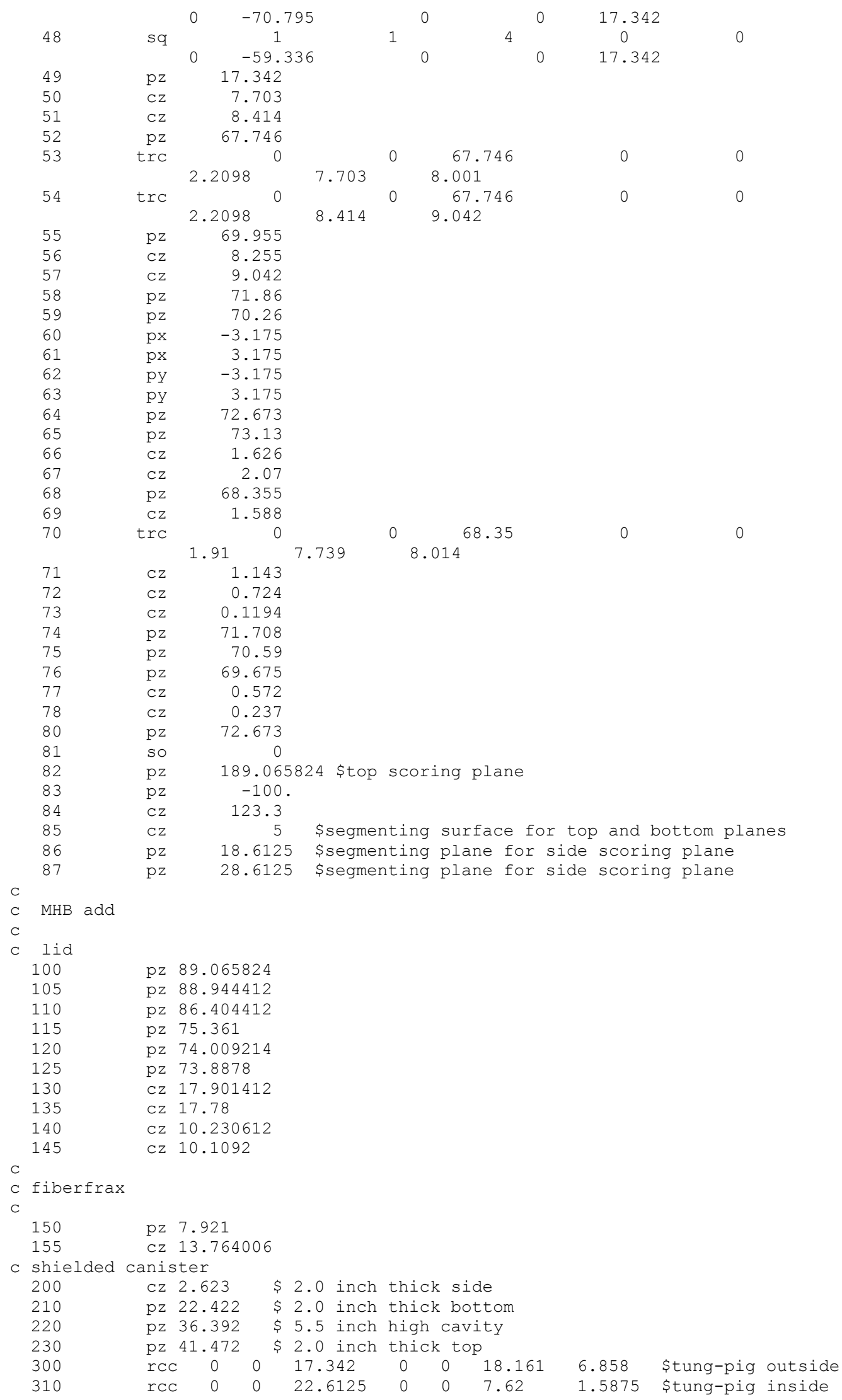


PCP-2011-0001

Revision 0

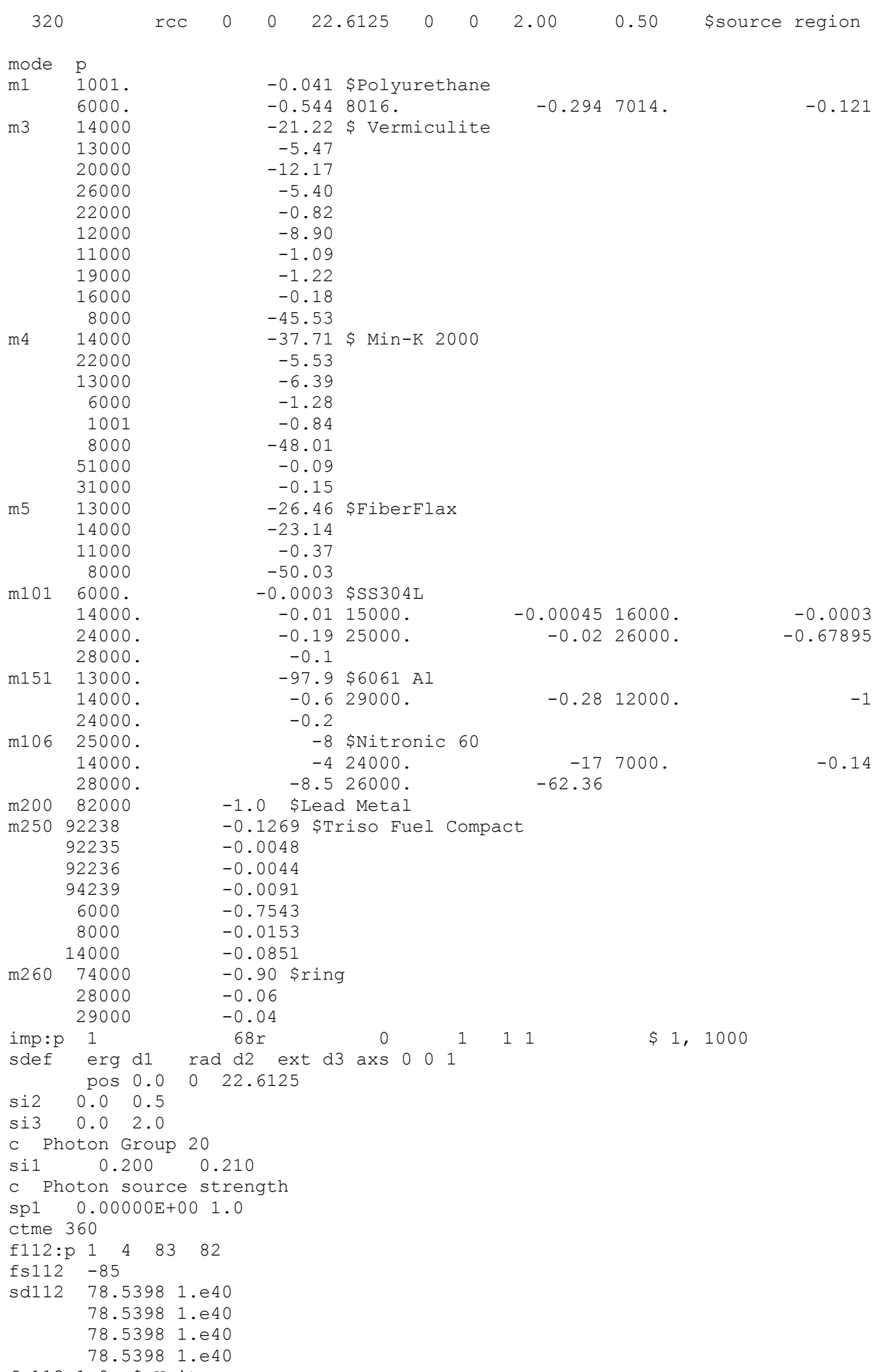


PCP-2011-0001

Revision 0

fm122 1.0 \$ Unit source

$\operatorname{tf} 122 \quad 1 \quad 2 j \quad 2$

fq0 f $s$

$\mathrm{f5}: \mathrm{p} \quad 0 \quad 0 \quad-1 \quad 1$

$\begin{array}{lllll}0 & 0 & -99.9 & 1\end{array}$

$\begin{array}{llll}0 & 0 & 90.1 & 1\end{array}$

$\begin{array}{lllll}0 & 0 & 189 . & 1\end{array}$

f15z:p $\quad 23.6125 \quad 24.2994 \quad 1$

23.6125123 .29941

fm5 1.0 \$ Unit source

fm15 1.0 \$ Unit source

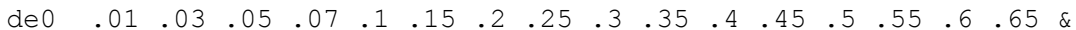

$\begin{array}{llllllllllllllllllll}.7 & .8 & 1 . & 1.4 & 1.8 & 2.2 & 2.6 & 2.8 & 3.25 & 3.75 & 4.25 & 4.75 & 5.0 & 5.25 & \&\end{array}$

$5.756 .256 .75 \quad 7.5 \quad 9.11 .13 .15$.

df0 $3.96 \mathrm{E}-6 \quad 5.82 \mathrm{E}-7 \quad 2.9 \mathrm{E}-7 \quad 2.58 \mathrm{E}-7 \quad 2.83 \mathrm{E}-7 \quad 3.79 \mathrm{E}-7 \quad 5.01 \mathrm{E}-7 \quad 6.31 \mathrm{E}-7 \quad$ \& $7.59 \mathrm{e}-7 \quad 8.78 \mathrm{E}-7 \quad 9.85 \mathrm{E}-7 \quad 1.08 \mathrm{E}-6 \quad 1.17 \mathrm{E}-6 \quad 1.27 \mathrm{E}-6 \quad 1.36 \mathrm{E}-6 \quad 1.44 \mathrm{E}-6 \quad \&$ $1.52 \mathrm{e}-6 \quad 1.68 \mathrm{E}-6 \quad 1.98 \mathrm{E}-6 \quad 2.51 \mathrm{E}-6 \quad 2.99 \mathrm{E}-6 \quad 3.42 \mathrm{E}-6 \quad 3.82 \mathrm{E}-6 \quad 4.01 \mathrm{E}-6 \quad$ \& $4.41 \mathrm{e}-6 \quad 4.83 \mathrm{E}-6 \quad 5.23 \mathrm{E}-6 \quad 5.6 \mathrm{E}-6 \quad 5.8 \mathrm{E}-6 \quad 6.01 \mathrm{E}-6 \quad 6.37 \mathrm{E}-6 \quad 6.74 \mathrm{E}-6 \quad \alpha$ $7.11 \mathrm{e}-6 \quad 7.66 \mathrm{E}-6 \quad 8.77 \mathrm{E}-6 \quad 1.03 \mathrm{E}-5 \quad 1.18 \mathrm{E}-5 \quad 1.33 \mathrm{E}-5$

prdmp $2 j 1$ 
PCP-2011-0001

Revision 0

Appendix C Python Scripts 
PCP-2011-0001

Revision 0

The Python scripts used to extract data from the MCNP output files are listed in Table C-1.

Sample listings are provided below for files marked with an asterisk (*).

Table C-1. Python Scripts

\begin{tabular}{|c|c|}
\hline File Name & Description \\
\hline mctal_reader_ver01.py* & $\begin{array}{l}\text { Python script to read an MCNP MCTAL file and create a dictionary containing } \\
\text { the tally data for surface tallies. }\end{array}$ \\
\hline mctal_reader_ver02.py* & $\begin{array}{l}\text { Python script to read an MCNP MCTAL file and create a dictionary containing } \\
\text { the tally data for point detector tallies. }\end{array}$ \\
\hline neutpuo_tally_212.py & $\begin{array}{l}\text { Python script to read an MCNP MCTAL file and extract data for surface tally } \\
212 \text {, neutron top and bottom surfaces from MCNP case neutpuo. }\end{array}$ \\
\hline neutpuo_tally_222.py* & $\begin{array}{l}\text { Python script to read an MCNP MCTAL file and extract data for surface tally } \\
222 \text {, neutron side surface from MCNP case neutpuo. }\end{array}$ \\
\hline neutpuo_tally_112.py & $\begin{array}{l}\text { Python script to read an MCNP MCTAL file and extract data for surface tally } \\
112 \text {, secondary photon top and bottom surfaces from MCNP case neutpuo. }\end{array}$ \\
\hline neutpuo_tally_122.py & $\begin{array}{l}\text { Python script to read an MCNP MCTAL file and extract data for surface tally } \\
122 \text {, secondary photon side surface from MCNP case neutpuo. }\end{array}$ \\
\hline neut_tally_212.py & $\begin{array}{l}\text { Python script to read an MCNP MCTAL file and extract data for surface tally } \\
212 \text {, neutron top and bottom surfaces from MCNP case neut. }\end{array}$ \\
\hline neut_tally_222.py & $\begin{array}{l}\text { Python script to read an MCNP MCTAL file and extract data for surface tally } \\
222 \text {, neutron side surface from MCNP case neut. }\end{array}$ \\
\hline neut_tally_112.py & $\begin{array}{l}\text { Python script to read an MCNP MCTAL file and extract data for surface tally } \\
112 \text {, secondary photon top and bottom surfaces from MCNP case neut. }\end{array}$ \\
\hline neut_tally_122.py & $\begin{array}{l}\text { Python script to read an MCNP MCTAL file and extract data for surface tally } \\
112 \text {, secondary photon side surface from MCNP case neut. }\end{array}$ \\
\hline photfe_tally_112.py & $\begin{array}{l}\text { Python script to read an MCNP MCTAL file and extract data for surface tally } \\
112 \text {, photon top and bottom surfaces from MCNP case photfe. }\end{array}$ \\
\hline photfe_tally_122.py & $\begin{array}{l}\text { Python script to read an MCNP MCTAL file and extract data for surface tally } \\
122 \text {, photon side surface from MCNP case photfe. }\end{array}$ \\
\hline phot_tally_112.py & $\begin{array}{l}\text { Python script to read an MCNP MCTAL file and extract data for surface tally } \\
112 \text {, photon top and bottom surfaces from MCNP case phot. }\end{array}$ \\
\hline phot_tally_122.py & $\begin{array}{l}\text { Python script to read an MCNP MCTAL file and extract data for surface tally } \\
122 \text {, photon side surface from MCNP case phot. }\end{array}$ \\
\hline photfept_15.py & $\begin{array}{l}\text { Python script to read an MCNP MCTAL file and extract data for point detector } \\
\text { tally } 15 \text {, photon side surface from MCNP case photfept. }\end{array}$ \\
\hline photfept_5.py & $\begin{array}{l}\text { Python script to read an MCNP MCTAL file and extract data for point detector } \\
\text { tally 5, photon top and bottom surfaces from MCNP case photfept. }\end{array}$ \\
\hline photpt_15.py & $\begin{array}{l}\text { Python script to read an MCNP MCTAL file and extract data for point detector } \\
\text { tally } 15 \text {, photon side surface from MCNP case photpt. }\end{array}$ \\
\hline photpt_5.py & $\begin{array}{l}\text { Python script to read an MCNP MCTAL file and extract data for point detector } \\
\text { tally 5, photon top and bottom surfaces from MCNP case photpt. }\end{array}$ \\
\hline sc1_tally_112.py & $\begin{array}{l}\text { Python script to read an MCNP MCTAL file and extract data for surface tally } \\
112 \text {, photon top and bottom surfaces from MCNP case } \mathrm{sc} 1 \text {. }\end{array}$ \\
\hline sc1_tally_122.py & $\begin{array}{l}\text { Python script to read an MCNP MCTAL file and extract data for surface tally } \\
122 \text {, photon side surface from MCNP case sc1. }\end{array}$ \\
\hline sc1pt_15.py & $\begin{array}{l}\text { Python script to read an MCNP MCTAL file and extract data for point detector } \\
\text { tally } 15 \text {, photon side surface from MCNP case sc1pt. }\end{array}$ \\
\hline sc1pt_5.py* & $\begin{array}{l}\text { Python script to read an MCNP MCTAL file and extract data for point detector } \\
\text { tally 5, photon top and bottom surfaces from MCNP case sc } 1 \mathrm{pt} \text {. }\end{array}$ \\
\hline
\end{tabular}


PCP-2011-0001

Revision 0

\begin{tabular}{||l|l||}
\hline File Name & Description \\
\hline sc2n_tally_112.py & $\begin{array}{l}\text { Python script to read an MCNP MCTAL file and extract data for surface tally } \\
112, \text { secondary photon top and bottom surfaces from MCNP case sc2n. }\end{array}$ \\
\hline \hline sc2n_tally_122.py & $\begin{array}{l}\text { Python script to read an MCNP MCTAL file and extract data for surface tally } \\
\text { 122, secondary photon side surface from MCNP case sc2n. }\end{array}$ \\
\hline sc2n_tally_212.py & $\begin{array}{l}\text { Python script to read an MCNP MCTAL file and extract data for surface tally } \\
\text { 212, neutron top and bottom surfaces from MCNP case sc2n. }\end{array}$ \\
\hline sc2n_tally_222.py & $\begin{array}{l}\text { Python script to read an MCNP MCTAL file and extract data for surface tally } \\
\text { 222, neutron side surface from MCNP case sc2n. }\end{array}$ \\
\hline sc2_tally_112.py & $\begin{array}{l}\text { Python script to read an MCNP MCTAL file and extract data for surface tally } \\
112, \text { photon top and bottom surfaces from MCNP case sc2p. }\end{array}$ \\
\hline sc2_tally_122.py & $\begin{array}{l}\text { Python script to read an MCNP MCTAL file and extract data for surface tally } \\
\text { 122,photon side surface from MCNP case sc2p. }\end{array}$ \\
\hline sc2ppt_15.py & $\begin{array}{l}\text { Python script to read an MCNP MCTAL file and extract data for point detector } \\
\text { tally 15, photon side surface from MCNP case sc2ppt. }\end{array}$ \\
\hline sc2ppt_5.py & $\begin{array}{l}\text { Python script to read an MCNP MCTAL file and extract data for point detector } \\
\text { tally 5, photon top and bottom surfaces from MCNP case sc2ppt. }\end{array}$ \\
\hline sc3_tally_112.py & $\begin{array}{l}\text { Python script to read an MCNP MCTAL file and extract data for surface tally } \\
112, \text { photon top and bottom surfaces from MCNP case sc3. }\end{array}$ \\
\hline sc3_tally_122.py & $\begin{array}{l}\text { Python script to read an MCNP MCTAL file and extract data for surface tally } \\
\text { 122, photon side surface from MCNP case sc3. }\end{array}$ \\
\hline sc3pt_15.py & $\begin{array}{l}\text { Python script to read an MCNP MCTAL file and extract data for point detector } \\
\text { tally 15, photon side surface from MCNP case sc3pt. }\end{array}$ \\
\hline sc3pt_5.py & $\begin{array}{l}\text { Python script to read an MCNP MCTAL file and extract data for point detector } \\
\text { tally 5, photon top and bottom surfaces from MCNP case sc3pt. }\end{array}$ \\
\hline
\end{tabular}


PCP-2011-0001

Revision 0

\section{Python Script mctal_reader_ver01.py}

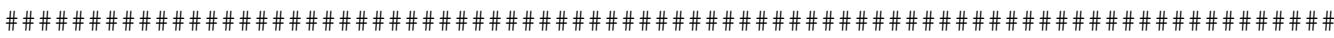

Python script to read an MCNP mctal file and create a dictionary containing \# the tally data. This is not a 'fully-functional' mctal parser. It is only \# set up to handle tallies with cell/surface bins and segmenting surfaces. \# There is some coding included that recognizes the presence of other types \# of bins (e.g., energy), but the dictionary that is returned has only been \# tested and used with tallies that have surface bins and segmenting surfaces. \# The structure of the MCNP mctal file is described on pages B-25 through B-28 \# of the MCNP manual.

Version 0.1 (Developmental)

$2010 / 12 / 22$

J. M. Risner

Oak Ridge National Laboratory

Reactor and Nuclear Systems Division / Radiation Transport

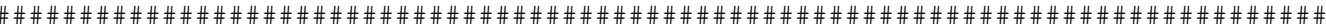

\# The arguments passed to this procedure are the name of the MCNP mctal file \# and the number of the tally for which the data will be extracted.

def get_tally( mctal_file, requested_tally ) :

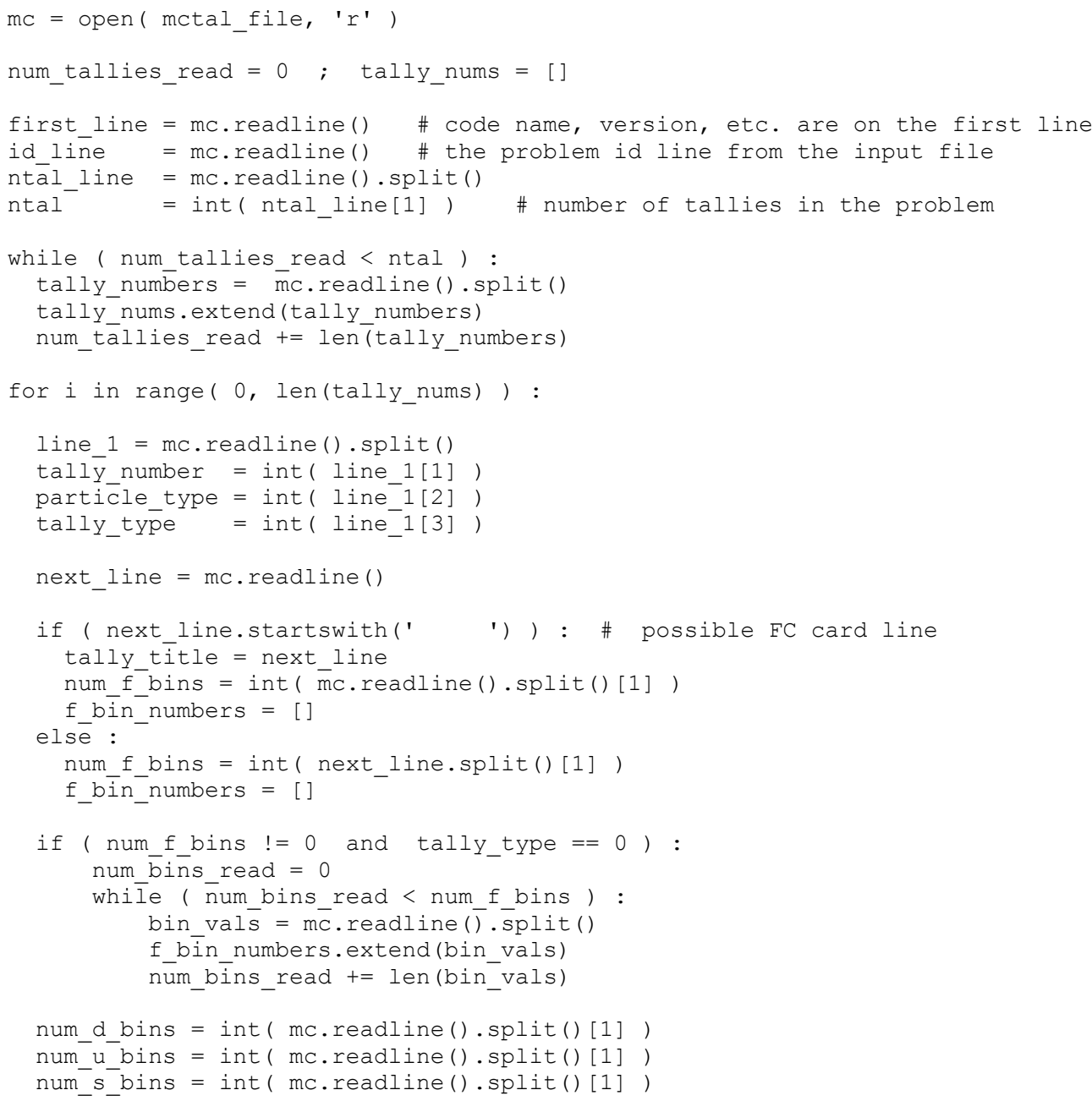


PCP-2011-0001

Revision 0

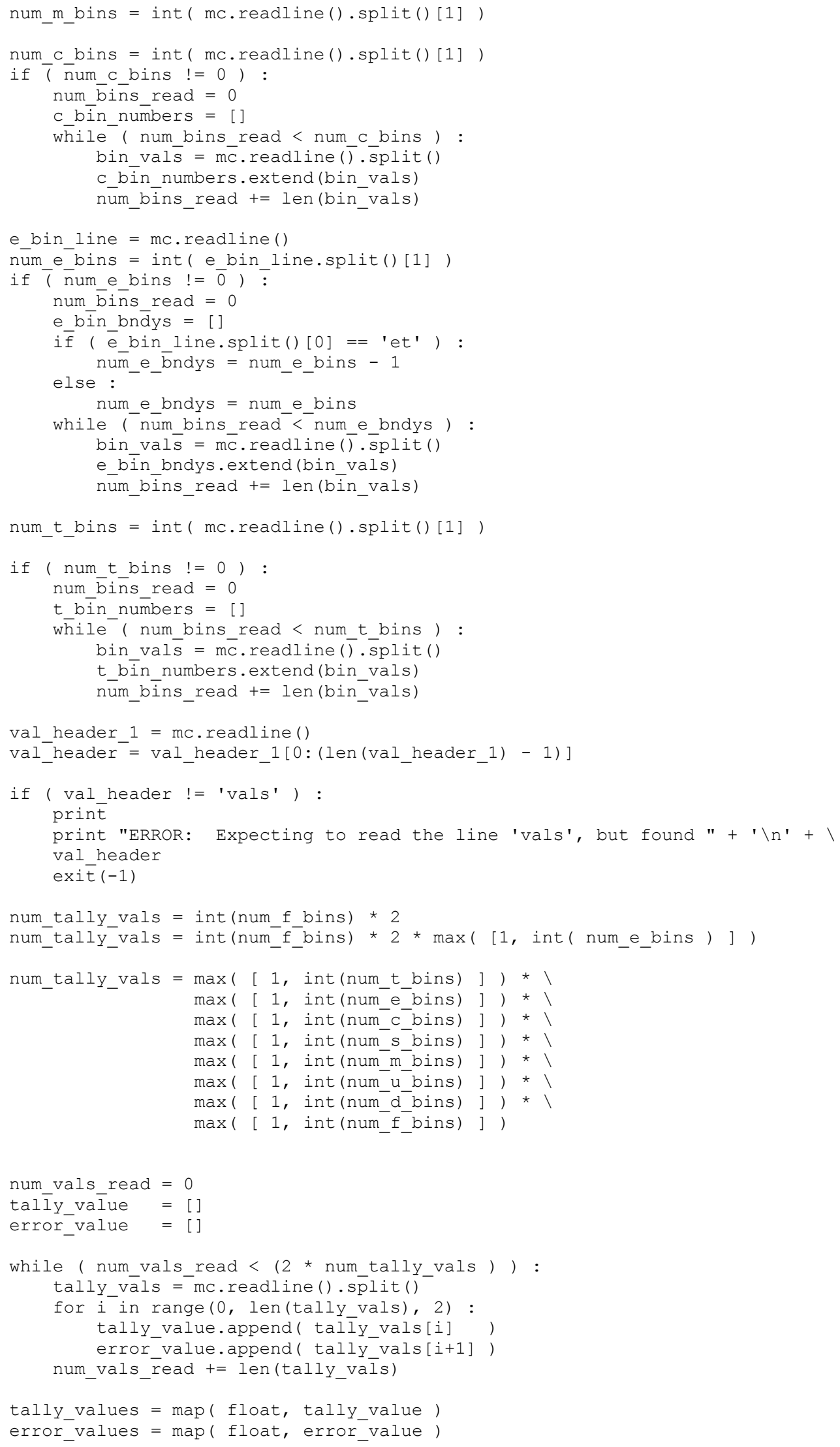


PCP-2011-0001

Revision 0

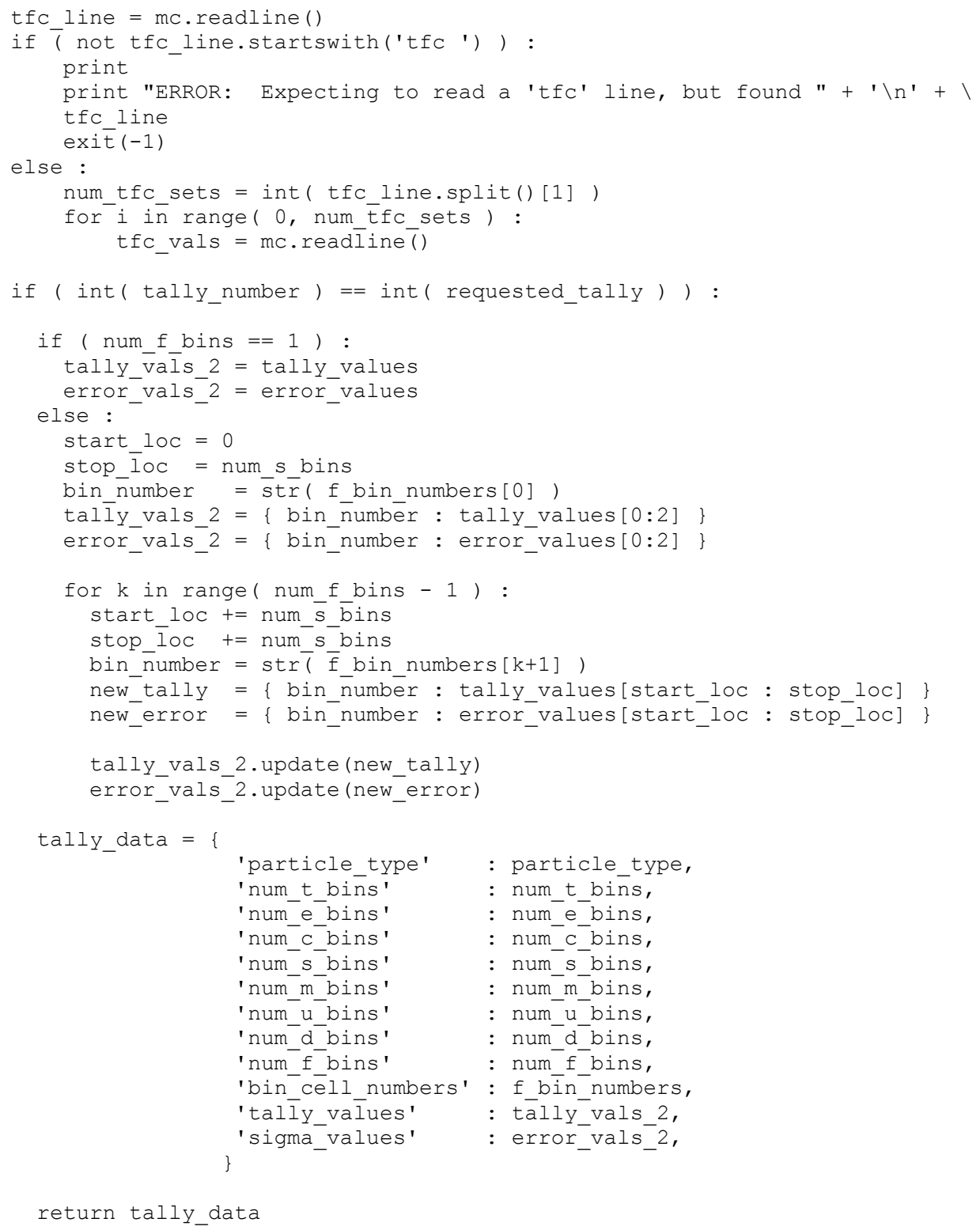

\section{Python Script mctal_reader_ver02.py}

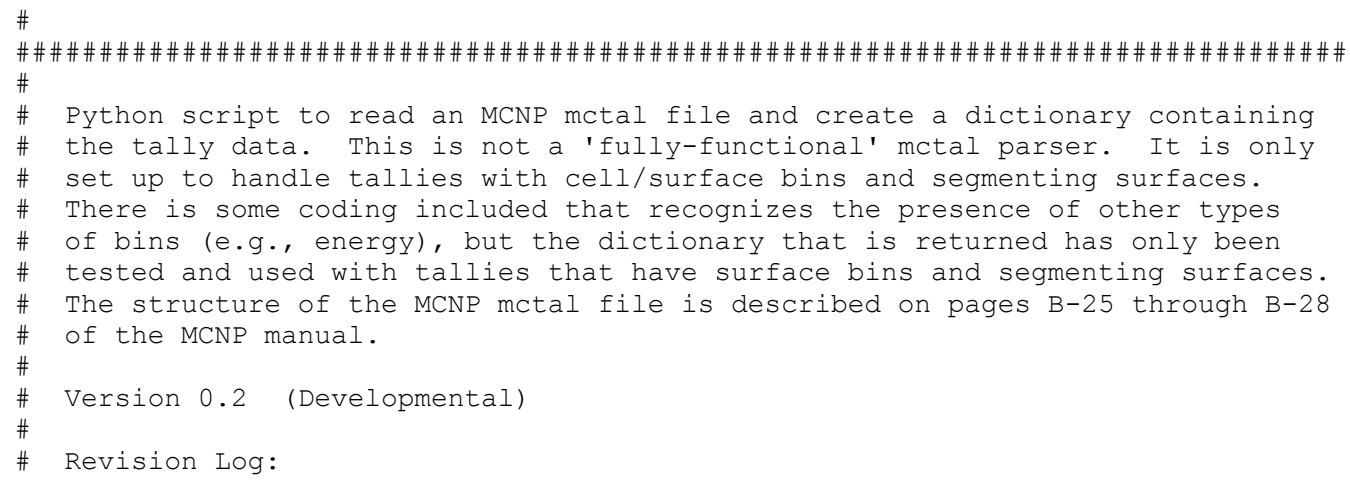


PCP-2011-0001

Revision 0

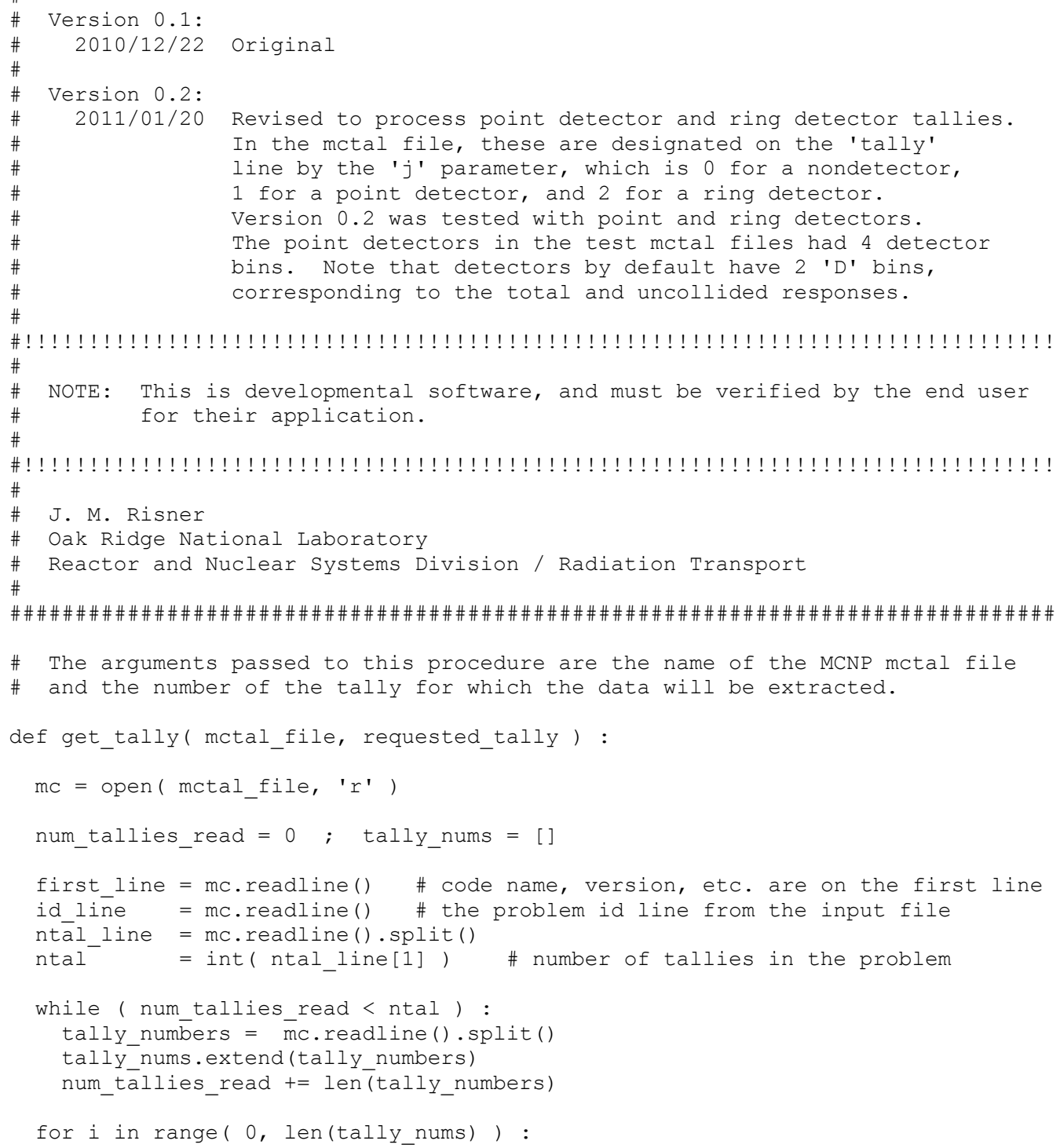


PCP-2011-0001

Revision 0

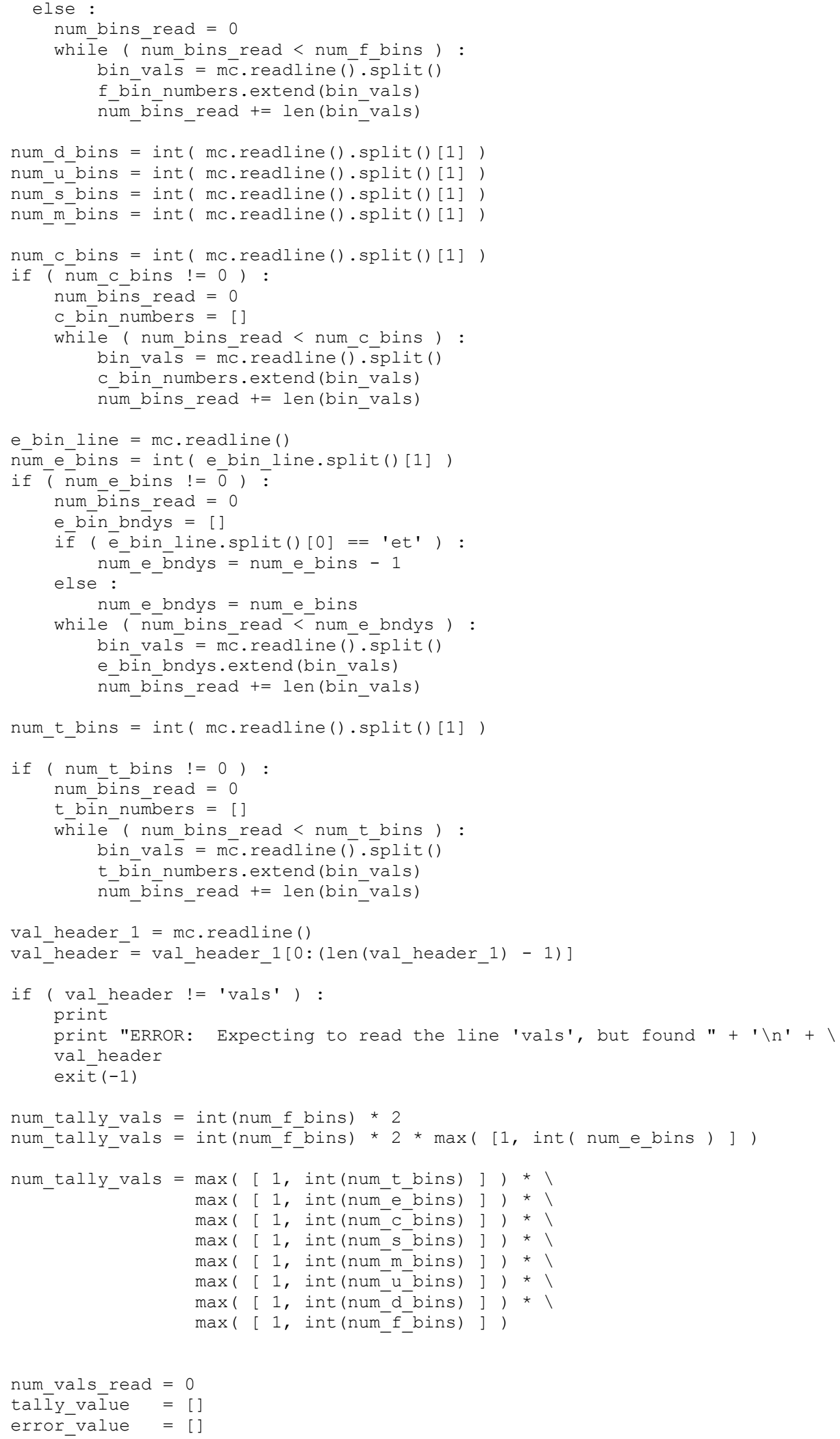


PCP-2011-0001

Revision 0

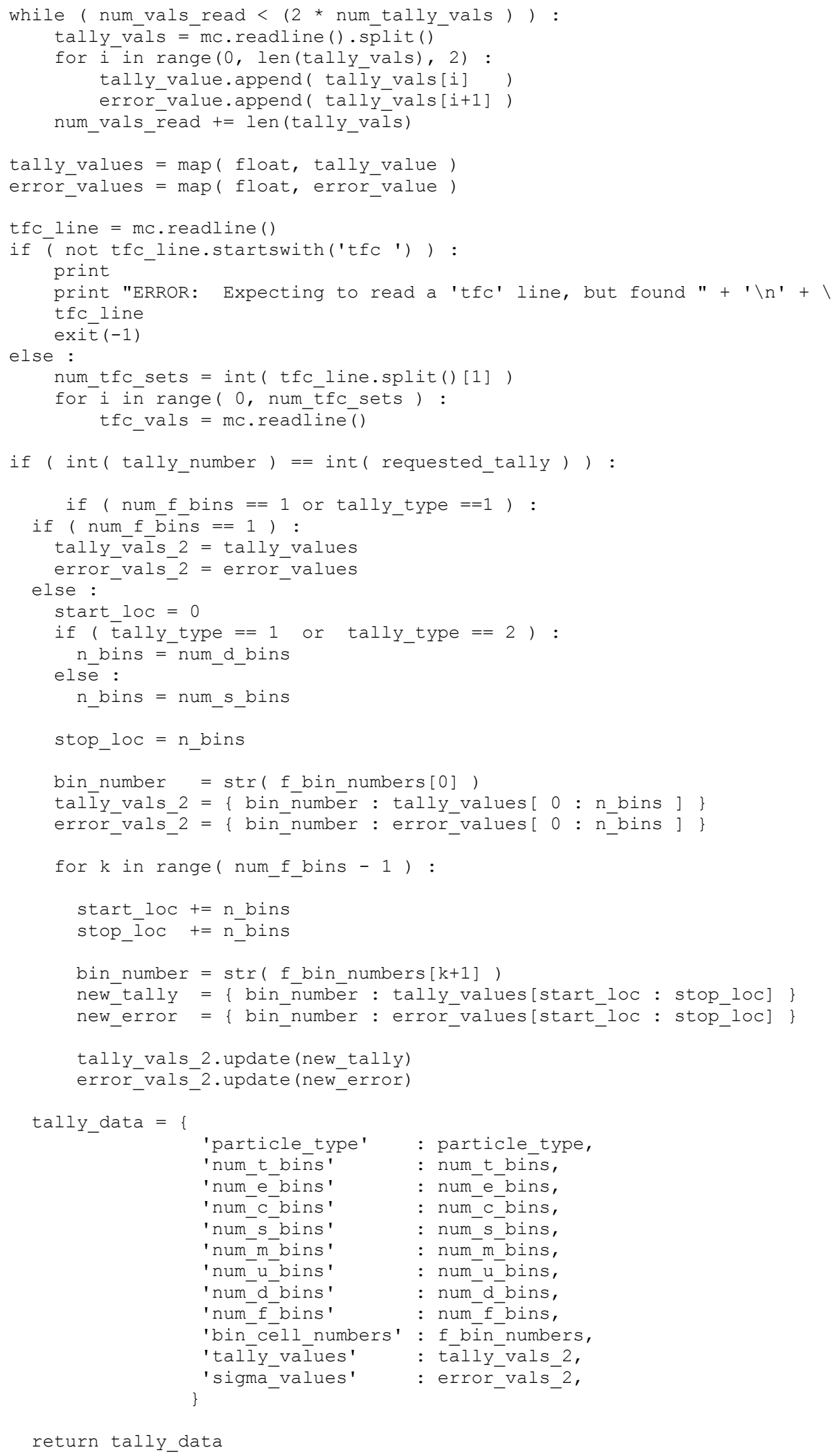


PCP-2011-0001

Revision 0

\section{Python Script neutpuo_tally_222.py}

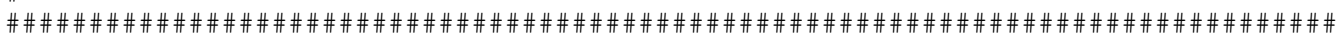

\# Python script to read an MCNP mctal file and extract data for \# a specified tally.

\# This script was developed for use in analyzing small gram quantity (SGQ) shipments in the 9977 shipping container.

Version 0.1 (Developmental)

$2010 / 12 / 22$

J. M. Risner

Oak Ridge National Laboratory

Reactor and Nuclear Systems Division / Radiation Transport

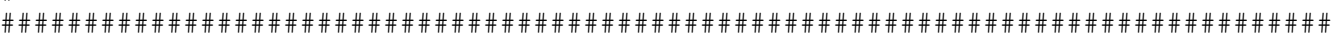
import mctal_reader_ver01 as mctal_reader

\# Set the 'base name' format for the tally files. The group information \# is appended to the end of the 'base name'.

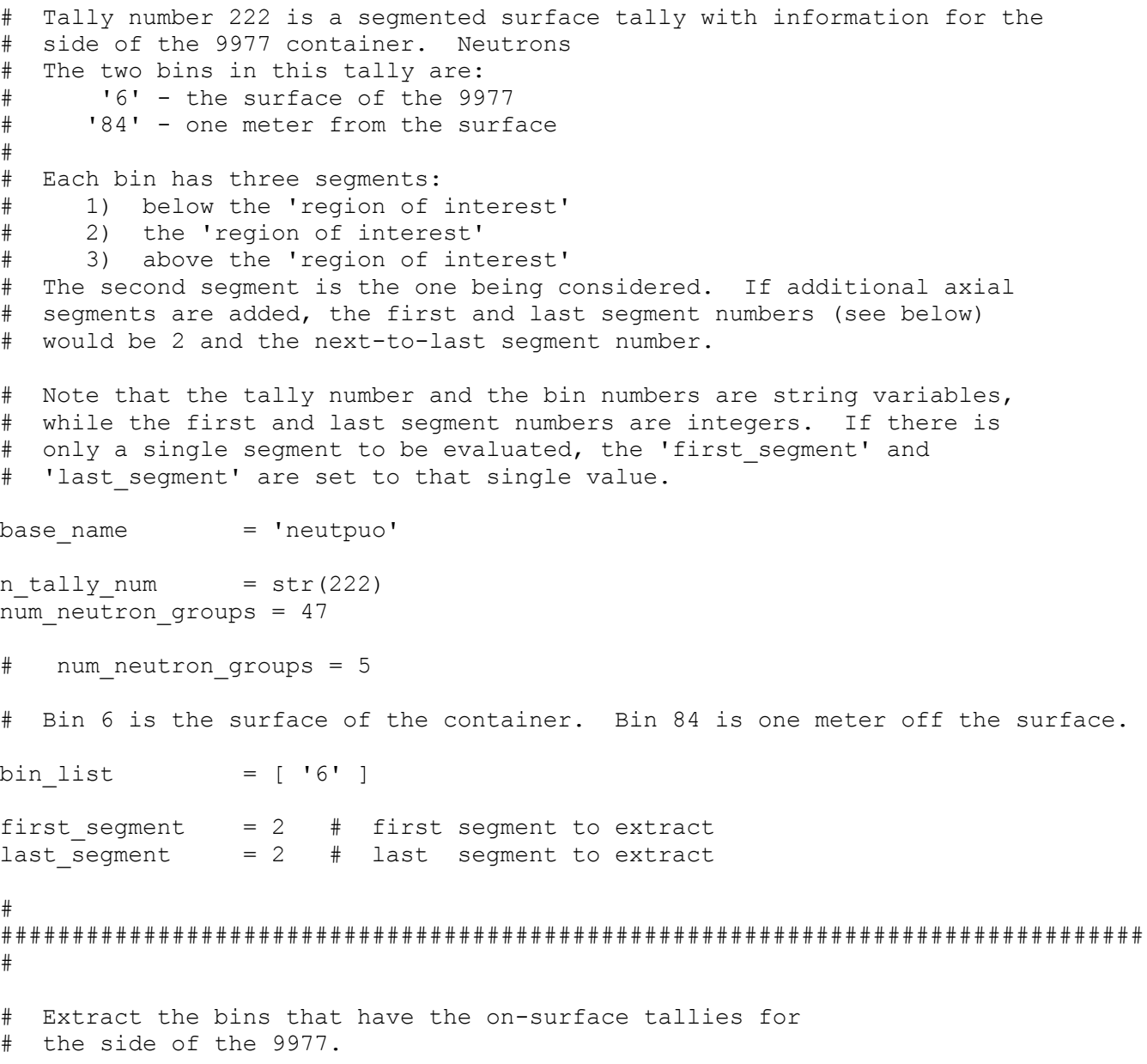


PCP-2011-0001

Revision 0

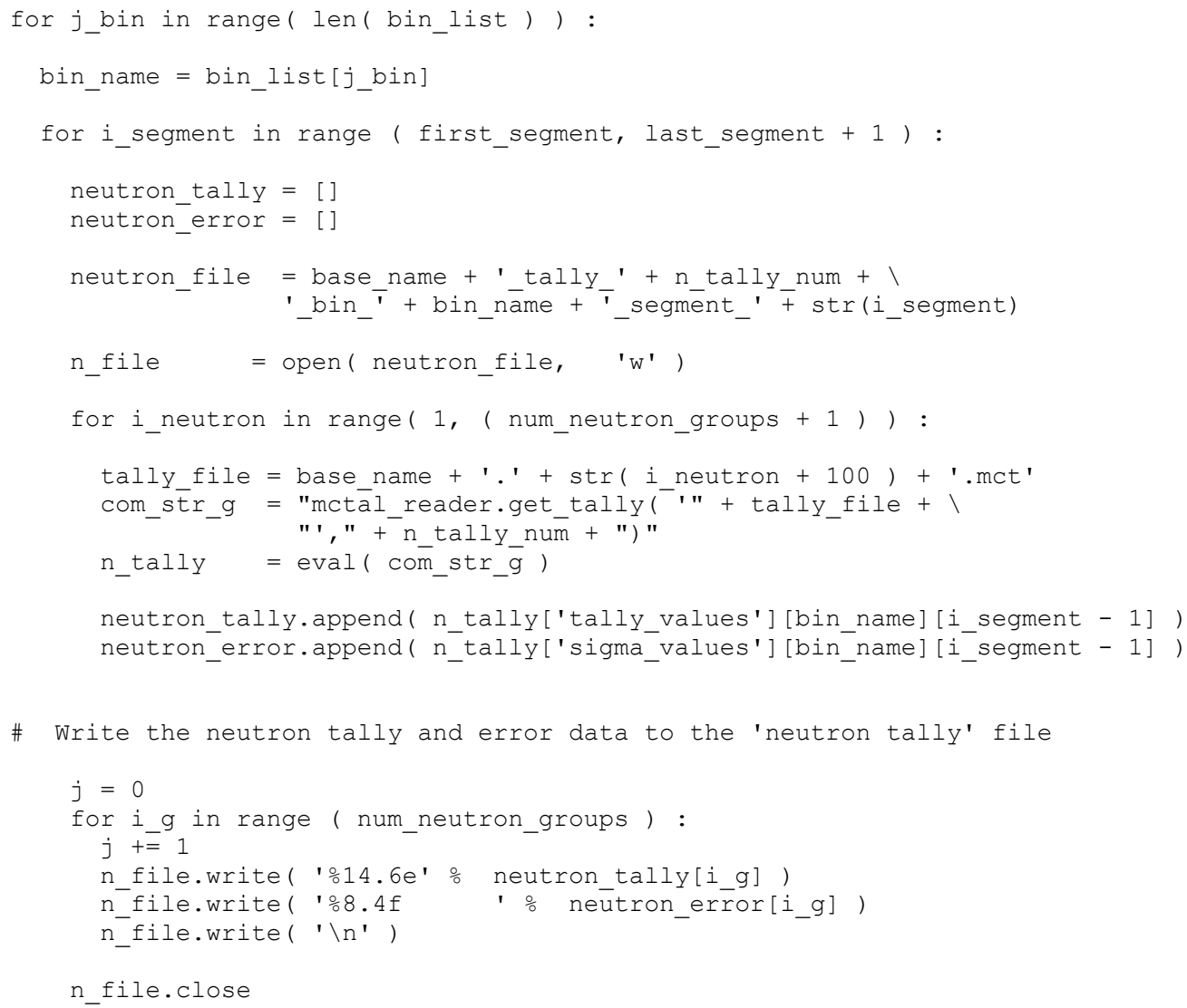

\section{Python Script sc1pt_5.py}

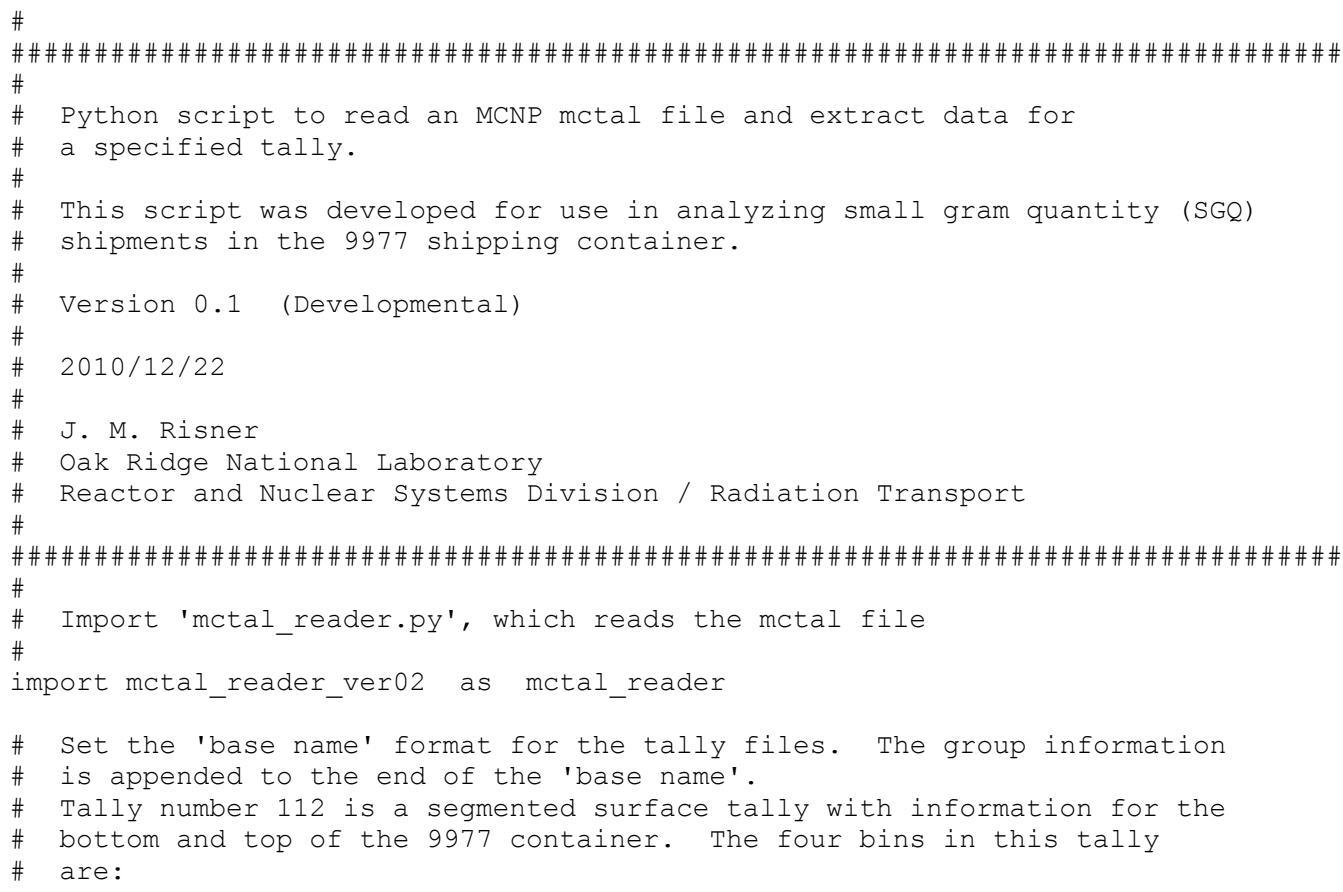


PCP-2011-0001

Revision 0

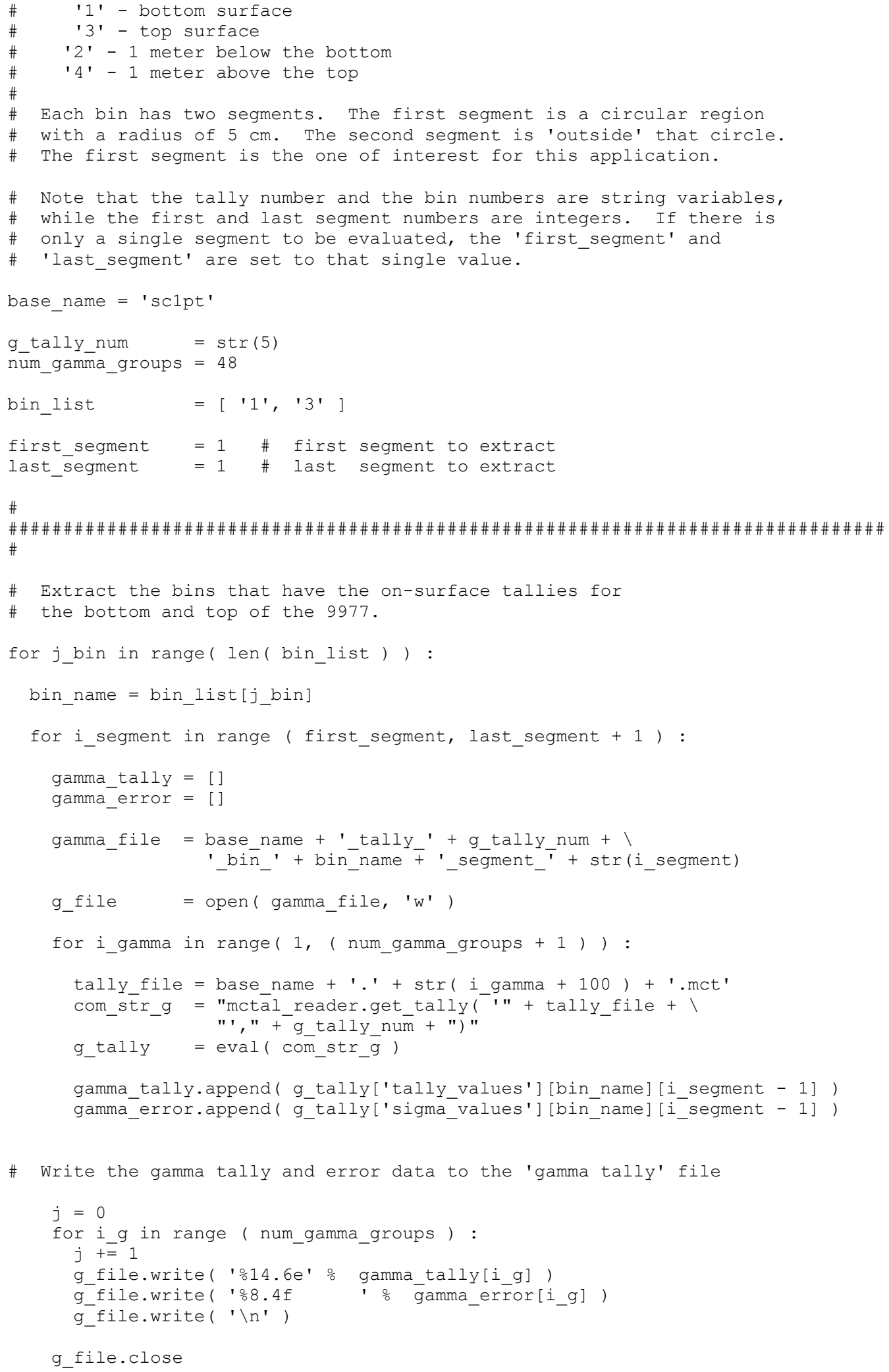




\section{Distribution:}

S. L. Tibrea, SRNL

J. S. Bellamy, SRNL

J. L. England, SRNL

J. Brotherton, SRNS

D. G. Erickson, SRNS

G. Abramczyk, SRNL

P. S. Blanton, SRNL

D. R. Leduc, SRNL

B. M. Loftin, SRNL

J. L. Murphy, SRNL

A. H. Bridges, SRNS

B. Anderson, LLNL

D. Biswas, LLNL

S. Kim, LLNL 